\title{
Auf der Suche nach der Hawaiischen Nation
}

\section{Autonomiepolitik und kulturelle Revitalisierung im} US-Bundesstaat Hawai`i

\author{
Dissertation \\ zur Erlangung des sozialwissenschaftlichen Doktorgrades der \\ Sozialwissenschaftlichen Fakultät der Georg-August-Universität \\ Göttingen \\ vorgelegt von \\ Ulrich Menter \\ aus Osnabrück \\ Göttingen 2009
}


1. Gutachterin:

Frau Prof. Dr. Brigitta Hauser-Schäublin

2. Gutachterin:

Frau Prof. Dr. Elfriede Hermann

Tag der mündlichen Prüfung: 09.11.2009 
Für Hermann Krogmann 



\section{Inhaltsverzeichnis}

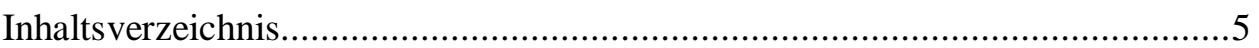

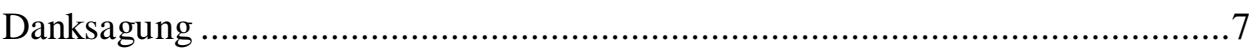

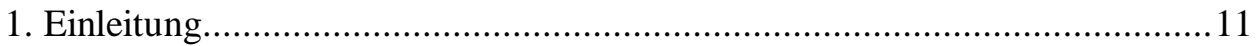

1.1 Hawai'i: Paradies in der Südsee? ...........................................................11

1.2 Themenstellung und Aufbau der Arbeit ..................................................15

2. Historischer Überblick: Geschichten des Verlustes, Geschichten des

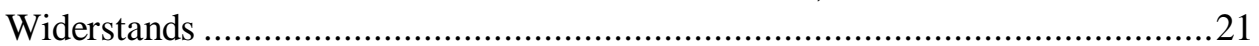

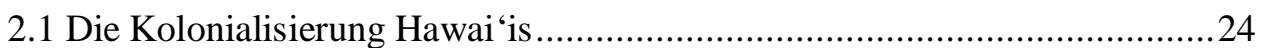

2.1.1 Das frühe Königreich Hawai‘i $(1791$ - 1849)..........................................25

2.1.2 Das Ende des Königreichs Hawai‘i $(1850$ - 1893) ....................................36

2.1.3 Republik, Territorium, Bundesstaat.......................................................43

2.2 Hawaiischer Widerstand im 19. und 20. Jahrhundert...................................55

3. Demographie, Ethnizität und „Race“: Wer sind die Hawaiier? .......................73

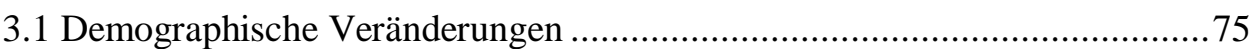

3.2 „Hawai ‘i“ und „Hawaiians“: Aus- und Abgrenzungen ...................................85

3.3 „Local“ - eine übergreifende ethnische/kulturelle Kategorie ...........................97

4. Politische Gruppierungen und staatliche Institutionen ..................................105

4.1 Die Hawaiische Autonomiebewegung …...............................................106

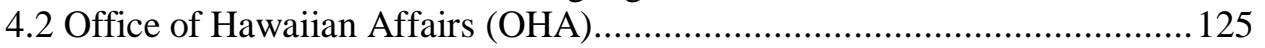

4.3 Hawaiian Sovereignty Elections Council (HSEC) .....................................131

4.4 Der Bundesstaat Hawai`i und die „Hawaiian Sovereignty“ ........................136

5. Politisierung des Kulturellen - Kulturalisierung des Politischen..................143

5.1 'Ōlelo Hawai'i, die hawaiische Sprache......................................................146

5.2 Hula: Kommerz, Spiritualität und Widerstand .........................................153 
5.3 Hawaiische Hochseefahrt: Hōkūle‘a und Hawai‘ iloa .................................165

6. Das Gedenkjahr 1993: Brennpunkte symbolischer Politik..........................177

6.1 Die Gedenkfeiern an den Umsturz von 1893: ‘Onipa'a ............................. 180

6.2 The People’s International Tribunal Hawai‘i.............................................. 198

6.3 Autonomiebewegung, Bundesstaat und USA im Gedenkjahr 1993............ 207

7. Konflikte um Landrechte und Institutionen.............................................219

7.1 Hawaiisches Land damals, hawaiisches Land heute ...................................220

7.2 Die staatlichen Treuhandgebiete im Bundesstaat Hawai‘ $i$..........................224

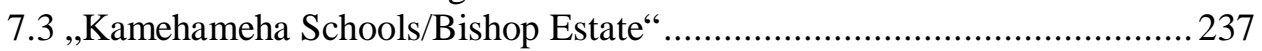

8. Ethnie oder Nation: hawaiische Rechts- und Identitätsdiskurse ................... 251

8.1 Autonomie oder Souveränität, Nationalität oder Ethnizität?..................... 253

8.2 Hawaiische Gruppenrechte auf dem Prüfstand ......................................... 263

8.3 „Federal Recognition“: Ende des politischen Widerstandes?......................274

9. Widerstand und autonome Praxis: Zeitgenössische hawaiische Kunst im Spannungsfeld von Politik und „Contemporary Art“ ..................................... 281

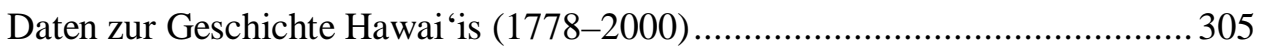

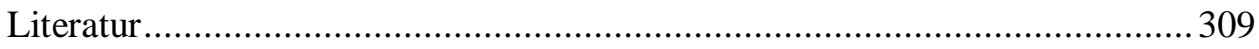




\section{Danksagung}

In der Zeit von der ersten Idee bis zur Ausführung und schließlich der Publikation dieser Arbeit haben viele Menschen mit Informationen und Ratschlägen, mit motivierendem Zuspruch, aber auch mit praktischer Unterstützung zu ihrem Gelingen beigetragen. Ihnen allen sei hier von Herzen gedankt.

Mein besonderer Dank gilt all jenen Hawaiierinnen und Hawaiiern, die mir als Vertreter ihrer jeweiligen Institutionen bzw. Organisationen oder als Privatpersonen die Situation der hawaiischen Bevölkerung sowie verschiedene Wege zu einer Verbesserung ihrer Lage eindrücklich vor Augen führten. Stellvertretend nennen möchte ich Hāli'imaile Andrade, Kawai Aona-Ueoka und 'Imaikalani Kalāhele, die als beteiligte Künstlerinnen und Künstler die Ausstellung „Ho'okū' $\bar{e}^{\text {“ }} 1998$ nach Stuttgart begleiteten. Von ihnen habe ich sowohl in Stuttgart wie auch später in Honolulu vieles über Geschichte und Politik Hawai'is gelernt, vor allem aber die Bedeutung und den Sinn des einfachen Wortes Aloha erfahren. Mahalo me ke aloha pumehana!

An der University of Hawai' $i$ in Mānoa und Hilo hatte ich vielfältige Gelegenheit, mich mit der akademischen Seite der Hawaiian Sovereignty auseinanderzusetzen und meine Gesprächspartner begegneten mir immer wieder mit großer Offenheit. Meine Arbeit basiert auf zahlreichen schriftlichen Quellen und wäre sicher nicht möglich gewesen ohne die große Hilfsbereitschaft aller Mitarbeiterinnen und Mitarbeiter der Hawai‘i-Pacific Collection der Hamilton Library (UH Mānoa) wie auch der Legal Reference Library (Hawai'‘i State Capitol). Ihnen allen gilt mein aufrichtiger Dank. Und schließlich danke ich Dr. Rainer Buschmann, der sich bei meinen beiden ersten Reisen 1995 und 1997 immer wieder Zeit für Diskussionen zum Thema nahm, mir aber auch mit Rat und Tat die schnelle Eingewöhnung in das Leben in Hawai'i erleichterte.

Es gab und gibt viele unterschiedliche Sichtweisen auf die politischen und sozialen Entwicklungen in Hawai'i. Alle Gesprächspartner in Hawai'i ließen mich mit großer Selbstverständlichkeit an ihrer jeweiligen Sicht der 
Dinge teilhaben. Die hier vorliegende Arbeit verdankt diesen Gesprächen sehr viel, ihre Ergebnisse sind aber allein von mir zu verantworten.

Ein ganz besonderer Dank gilt Dr. Ingrid Heermann, die nicht nur 1998 die Ausstellung „Ho'okū' ‘ $e^{‘}$ ins Linden-Museum Stuttgart holte, sondern auch an ersten Vorüberlegungen zu dieser Arbeit beteiligt war. Die Idee einer Forschung zur politischen Bewegung der indigenen Bevölkerung Hawai'is entstand schon 1994 im Zuge einer Ausstellungsplanung, und das LindenMuseum, mit Unterstützung der Robert Bosch Stiftung und Anthony Meyers, ermöglichte mir 1995 den ersten Forschungsaufenthalt in Hawai‘i. Ich hatte in den Folgejahren wiederholt das große Vergnügen, mit Dr. Heermann zusammenarbeiten zu können. Auch wenn es dabei um ganz andere Themen ging und die Arbeit an der Dissertation hinter der Museumsarbeit zurückstehen musste, rief sie mir immer wieder meine unvollendete Hawai'i-Forschung in Erinnerung. Nicht nur hierfür schulde ich ihr großen Dank.

Der größte Dank gebührt meiner Betreuerin, Prof. Dr. Brigitta HauserSchäublin. Schon bei unserem ersten Gespräch über die mögliche Betreuung meiner Dissertation zeigte sie sich aufgeschlossen für die von mir vorgebrachte Themenstellung, und 2004 konnte ich die Arbeit als Doktorand an der Universität Göttingen aufnehmen. In der Folgezeit gab sie mir zahlreiche Hinweise, die mir bei der Eingrenzung der Untersuchung und Schärfung der Themenstellung eine sehr große Hilfe waren. Über die gesamte, nicht eben kurze Zeit der Fertigstellung meiner Dissertation erlahmte ihr Interesse nicht, und mit großem pädagogischen Geschick half sie mir mehr als einmal über Klippen und Durststrecken einer Doktorarbeit hinweg. Als nach Abgabe der Arbeit und erfolgter Doktorprüfung im Jahr 2009 die neuen Aufgaben meiner beruflichen Tätigkeit die Publikation der Dissertation in den Hintergrund zu drängen drohten, fand sie auch hier den richtigen Ton und Zeitpunkt, um freundlich-streng den Abschluss anzumahnen. Für ihr wissenschaftliches Interesse und ihre immer konstruktive Kritik, für Ermutigung und manches mahnende Wort danke ich Frau Prof. Dr. Hauser-Schäublin von ganzem Herzen.

Ebenfalls großer Dank gilt Prof. Dr. Elfriede Hermann, die ohne Zögern die Aufgabe der Zweitgutachterin übernahm und stets großes Interesse am Thema meiner Arbeit zeigte. Ich danke ihr für viele anregende und konstruktive Diskussionen über und in Hawai' $i$, wo ich sie 2006 bei der Organisation des von ihr geleiteten Symposiums „Changing Contexts, Shifting Meanings“ an der Honolulu Academy of Arts unterstützen durfte.

Eine große Hilfe war die Zusammenarbeit mit Dr. Karin Klenke und Dr. Stefanie Steinebach in unserer kleinen „Taskforce Diss“, in der wissenschaftliche Diskussion, gegenseitige Motivation und gemeinsamer Kaffeegenuss eine kongeniale Verbindung eingingen. Danke!

Es war nicht immer einfach, die Arbeit an der Dissertation neben meiner Tätigkeit als Ethnologe im Museum mit dem nötigen Nachdruck weiter- 
und fortzuführen. Freunde und Freundinnen, Kolleginnen und Kollegen erlebten geduldig die Entstehung dieser Arbeit und haben mir mit Zuversicht und ermahnenden Hinweisen, mit persönlicher Zuwendung, aber auch mit so manchem von mir vielleicht nicht gern gehörten, aber wirksamen Scherz über „Schaffenskrisen“ hinweggeholfen. Ihnen allen möchte ich hier, wenn noch nicht anders geschehen, ganz besonders herzlich danken!

Meine Familie hat mich in dem Vorhaben der vorliegenden Arbeit immer ohne jede Einschränkung unterstützt. Der Dank insbesondere an meine Mutter Friede Anne Menter und meine Großmutter Frieda Flügelmann kann deshalb gar nicht groß genug bemessen sein.

Von Anfang an konnte ich auf die fast bedingungslose Unterstützung Hermann Krogmanns zählen, die über den Kontext dieser Arbeit weit hinausreicht. Seine zumeist ruhige und gleichmütige Begleitung meiner Arbeit, aber auch der sehr kritische Austausch über so manche Themenstellung war mir eine sehr große Hilfe. Ihm möchte ich dieses Buch widmen. 



\section{Einleitung}

\subsection{Hawai`i: Paradies in der Südsee?}

„Fünf Stunden Flug entfernt von der nordamerikanischen Westküste liegen in der Mitte des Pazifischen Ozeans die idyllischen Sonneninseln von Hawaii, die eigentlich die Gipfel einer über 3000 km langen Gebirgskette unter der Oberfläche des Pazifiks bilden. Die Inselgruppe besteht aus mehr als 20 Inseln. Acht davon bilden den Staat Hawaii: Oahu mit der Hauptstadt Honolulu, Kauai, Maui, Hawai'i und die kleineren Inseln Molokai, Lanai, Niihau und Kahoolawe. In ganz Hawaii sagt man ,Aloha', denn dieses Wort hat mehrere Bedeutungen. Es sagt nicht nur schlicht ,Hallo', sondern auch ,Auf Wiedersehen' und ,Ich liebe Dich'. Hawaiianer lassen Blumen sprechen und als Ausdruck besonderer Herzlichkeit werden dem Besucher beim Empfang ,Leis’, so heißen die bunten Blumenkränze, umgehängt“. ${ }^{1}$

Als einer der mehr als 6 Millionen Besucher, die während der 1990er jedes Jahr auf die hawaiischen Inseln kamen, traf ich 1995 an einem späten Abend in Honolulu ein. ${ }^{2}$ Es sollte mein erster Aufenthalt auf diesen Inseln sein, die in der Vorstellung vieler - und in einem gewissen Maße auch in meiner eigenen - für paradiesische Landschaften und unbeschwertes Leben standen bzw. stehen. Ich erreichte diese fernab der Kontinente liegenden Inseln nicht unvorbereitet: schließlich sollte die Reise der Vorbereitung meiner Forschungsarbeit über das „Hawaiian Sovereignty Movement“ und dessen Forderungen nach politischer und kultureller Unabhängigkeit dienen. Ich hatte Literatur über die traditionelle

\footnotetext{
${ }^{1}$ Informationsbroschüre des Hawaii Visitors Bureau in Deutschland, ca. 1994.

${ }^{2}$ Diesem ersten sechswöchigen Forschungsaufenthalt 1995 folgten 1997 und 2006 zwei weitere sechs bzw. acht Wochen dauernde Reisen nach Hawai'i.
} 
hawaiische Gesellschaft und ihre Transformation im Laufe des 19. Jahrhunderts ebenso gelesen wie die politische Geschichte des Archipels nach dem Untergang des hawaiischen Königreichs und der Machtübernahme durch USamerikanische Geschäftsleute. Soweit dies von Deutschland aus möglich war, hatte ich mich zudem mit der Struktur und den politischen Ansprüchen der hawaiischen Bewegung befasst und erste Gesprächstermine mit Wissenschaftlern und Vertretern hawaiischer Institutionen vereinbart. Mir war natürlich bekannt, dass der „American Way of Life“ das Leben im US-Bundesstaat Hawai'i prägte und dass die Suche nach der Südseeidylle, wie sie sich trotzdem in meiner Vorstellung fand, wohl vergeblich sein würde.

Der amerikanische Schriftsteller und Reisende Mark Twain beschrieb Hawai' $i$ als „the loveliest fleet of islands that lies anchored in any ocean“. ${ }^{3}$ Bis heute gehört dieser Satz zum Kernbestand der Hawai'i-Werbung - steht er doch, gewissermaßen in kondensierter Form, für eine paradiesische Einzigartigkeit dieser nördlich des Äquators gelegenen Inseln. Im Werk Mark Twains, der 1866 als Korrespondent vier Monate auf den Inseln verbrachte und in späteren Jahren noch einmal dorthin zurückkehrte, findet sich mehr als einmal dieser verklärte und verklärende Blick auf die hawaiischen Inseln. Doch Twain verweist in seinen Texten auch auf die Veränderungen, die Hawai'i im 19. Jahrhundert erfuhr (Twain 1990) - und benutzt ja selbst in dem so häufig wiedergegebenen Bild von den paradiesischen Inseln die Metapher einer vor Anker liegenden Flotte: „... oceanic symbols of progress, commerce, the American Navy in the Pacific, and civilization in general“ (Sumida 1991:55).

Hawai' $i$ ist bis heute kein eindeutiger Ort: Schon meine erste Taxifahrt vom Flughafen $\mathrm{zu}$ dem angemieteten Apartment in den Bergen oberhalb Honolulus, in einem Tal mit dem exotisch klingenden Namen Nu'uanu (hier führte und gewann Kamehameha I. mit seinen Kriegern im Jahre 1795 die entscheidende Schlacht gegen die Verteidiger der Insel O‘ahu und legte damit die Grundlage für das vereinigte hawaiische Königreich), lieferte widerstreitende Eindrücke. Das selbstverständlich amerikanisch anmutende Taxi fuhr über einen mehrspurigen Highway aus Betonplatten, und Verkehrsschilder und Leitplanken gestalteten eine Szenerie, die ebenso gut nach Illinois oder New York gepasst hätte. Zugleich strömte aber die laue Luft einer pazifischen Nacht durch die heruntergelassenen Fenster und merkwürdig unpassende Kokospalmen reckten zwischen flachen Industriebauten ihre Fiederblätter in den dunklen Himmel.

Am Morgen nach meiner Ankunft wanderte ich entlang des Pali Highways von den Bergen an die Küste - der Weg führte aus einer amerikanischen Vorstadtsiedlung, vorbei an architektonischen Zeugnissen der hawaiischen Geschichte, in die von Hochhausarchitektur geprägte Innenstadt von Honolulu,

${ }^{3}$ Zitiert nach Sumida 1991:55. 
wo der in einem nachempfundenen Rokoko-Stil erbaute, 1882 fertig gestellte 'Iolani Palast an das letzte Jahrzehnt des Königreichs Hawai'i erinnert. Im historischen Zentrum um den Palast drängten sich Touristen aus Japan und den USA, die ansonsten aber kaum im Stadtbild auffielen - verbrachten doch die meisten von ihnen ihren Aufenthalt außerhalb organisierter Besichtigungsfahrten in Waikīkī, dem heutigen Touristenviertel der Millionenstadt Honolulu. Allerdings blieb auf meiner Wanderung auch das „Hawaiische“ zunächst seltsam fern: überall wurde amerikanisches Englisch gesprochen, die Menschen schienen überwiegend asiatischer oder euro-amerikanischer Herkunft zu sein und abgesehen von den allgegenwärtigen „Aloha Shirts“ (oder „Hawai'i Hemden“, wie sie in Deutschland bekannt sind) sowie vereinzelten Muumuus, den bis zum Knöchel reichenden, bunt gemusterten und an die von den protestantischen Missionaren propagierte Frauenkleidung erinnernden Baumwollkleidern, entsprach die Kleidung der vorbeieilenden Passanten dem, was in jeder beliebigen amerikanischen Großstadt auch nicht anders zu erwarten gewesen wäre.

Die von mir empfundene Ambivalenz des Ortes, die Diskrepanz zwischen einer hawaiischen Vergangenheit, von der Literatur und architektonische Zeugnisse berichten, und der Gegenwart des Geschäftszentrums von Honolulu hat ihre Ursache in den tief greifenden Veränderungen der Gesellschaft Hawai'is seit der europäischen Entdeckung der Inseln durch James Cook im Jahre 1778. Schon mit den ersten europäischen Seefahrern kamen bis dahin unbekannte, sich verheerend auswirkende Krankheiten auf die Inseln; christliche Missionare brachten eine neue Religion, die - gefördert durch den hawaiischen Adel - bald die ideologische Grundlage des Gemeinwesens bildete; neue Rechtsvorstellungen ersetzten die traditionelle Rechtsordnung, euroamerikanische Pflanzer und Geschäftsleute machten Zucker zum Hauptwirtschaftsgut der Inseln und führten Hawai' $i$ in die ökonomische und politische Abhängigkeit von den USA. Die neu entstehenden Plantagen benötigten Arbeitskräfte, die man aus Asien holte und die auf den Inseln eine neue Heimat fanden, was die Bevölkerungsstruktur Hawai'is grundlegend verändern sollte. Unter Mitwirkung von US-Streitkräften stürzten im Jahre 1893 amerikanische Geschäftsleute die letzte hawaiische Monarchin: Der entscheidende Schritt zur Verdrängung der Hawaiier von den Schaltstellen der Macht und zur Integration Hawai' is in die USA war damit getan.

Im heutigen US-Bundesstaat Hawai'i sind die indigenen Hawaiier eine wachsende, hinsichtlich ihrer Abstammung und sozialen Zugehörigkeit sehr heterogene Minderheit, deren sozio-ökonomische Kennzahlen sie aber noch immer als eine sozial marginalisierte Gruppe innerhalb der Gesamtbevölkerung Hawai'is ausweisen. Auch wenn hawaiische Musik die Käufer in jedem Supermarkt berieselte, hawaiische Ortsbezeichnungen und Straßennamen ein spezifisches Lokalgefühl suggerierten, Versatzstücke traditioneller hawaiischer 
Kultur in Souvenirläden, Restaurants und Hotelanlagen omnipräsent waren und die beiden großen Tageszeitungen fast täglich die politischen Forderungen hawaiischer Gruppierungen meldeten und kommentierten, blieben die indigenen Hawaiier für mich doch zunächst auf seltsame Weise verborgen.

Bald sollte sich zeigen, dass die Ambivalenz des Ortes „Hawai'i“, wie er bei meiner ersten Begegnung wahrgenommen wurde und wie er in freilich anderer Weise im Werk Mark Twains begegnet, sich gewissermaßen in der zunehmend in mein Blickfeld tretenden hawaiischen Bevölkerung selbst spiegelte - auch wenn die „Hawaiians“ im Sprachgebrauch des Inselstaates terminologisch klar von der übrigen Bevölkerung abgegrenzt sind bzw. sich selbst von ihr abgrenzen. ${ }^{4}$ Jenseits der sprachlichen Bestimmung fehlt jedoch jegliche Eindeutigkeit: der allergrößte Teil der Hawaiier kann auf multiethnische Zugehörigkeiten verweisen, Hawaiier gehören zu allen Bevölkerungsschichten und sind bei politischen und sozialen Auseinandersetzungen häufig in allen beteiligten Konfliktparteien vertreten.

Bisweilen kann Musik die Annäherung an ein Thema erleichtern, zumal in den Texten der hawaiischen Popmusik der 1990er Jahre häufig Themen der politischen Gegenwart im Vordergrund standen. Der 1997 verstorbene Israel Kamakawiwo'ole, einer der bekanntesten hawaiischen Sänger, sprach in seinem 1993 veröffentlichten Lied „Hawai‘i ’78“ zahlreiche Themen an, die im Diskurs über die hawaiischen Forderungen nach kultureller und politischer Eigenständigkeit einen hohen Stellenwert haben.

Er lässt die hawaiischen Monarchen der Vergangenheit - gemeint sind wohl Kamehameha I. und Lili'uokalani, die letzte Vertreterin der hawaiischen Monarchie und der erste Herrscher über den gesamten Archipel - für einen Tag in die Gegenwart Hawai'is kommen und konfrontiert sie mit dem modernen Inselstaat: „Could you just imagine if they were around / and saw highways on their sacred grounds / how would they feel about this modern city life. / Tears would come from each others eyes / as they would stop to realize / that our people are in great, great danger now. “ ${ }^{5}$ Sie sähen Autobahnen, die über die Überreste alter Tempelanlagen führen; Land, das sich nicht mehr im Besitz der hawaiischen Bevölkerung befindet und den wirtschaftlichen Zielen von Spekulanten geopfert wird sowie den Verlust von traditionellem Lebensstil und alter Religion.

Liest man den Text jedoch auf eine andere Weise, so zeigen sich ebenso einige der zahlreichen Brüche, die die jüngere Geschichte der Hawaiier kennzeichnen: Als Kamehameha den Archipel eroberte und das Königreich

\footnotetext{
${ }^{4}$ Die Begriffe „Hawaiier“ und „hawaiisch“ verwende ich in dieser Arbeit - so wie auch in Hawai‘i üblich - ausschließlich in Bezug auf jenen Teil der Inselbevölkerung, der seine Abstammung auf die polynesischen Bewohner des Archipels vor 1778, dem Jahr der europäischen Entdeckung, zurückführt.

${ }^{5}$ Israel Kamakawiwo‘ole, Facing Future (The Mountain Apple Company 1993).
} 
begründete, tat er dies gegen den erbitterten Widerstand der (hawaiischen) Bewohner auf den übrigen Inseln; die offizielle Religion mit ihren Göttern und organisierten Priesterschaften löste der hawaiische Hochadel noch vor der Ankunft der ersten amerikanischen Missionare auf; der hawaiische Adel war in entscheidendem Maße an der Privatisierung des Landes beteiligt; und schließlich sind es auch die Hawaiier selbst, die den modernen Lebensstil, zu dem auch die Autobahnen in Honolulu gehören, pflegen und nicht mehr missen mögen. Menschen hawaiischer Abstammung finden sich nicht nur auf Seiten der Bewegung für politische und kulturelle Autonomie, die Bewegung agiert vielmehr in einem Umfeld, in dem auch auf der Gegenseite Hawaiier stehen oder, in der Vergangenheit, für die Entstehung der beklagten Missstände zumindest mitverantwortlich zeichnen müssen.

Der Text verweist vor allem aber auch auf die großen Konfliktfelder und Politikbereiche, die für das „Hawaiian Sovereignty Movement“ bis heute von zentraler Bedeutung sind: so steht das Bild von der gefährdeten hawaiischen Bevölkerung für die Benachteiligungen, die von den Hawaiiern als Minderheit im Staat Hawai'i bis heute erfahren werden - und damit letztlich für die Frage nach der Verantwortlichkeit für diese Entwicklung. Ebenso liefern die politischen, rechtlichen und sozialen Aspekte der nach wie vor ungelösten Landrechtsfragen immer wieder neuen Konfliktstoff. Schließlich stellt der Versuch der Wiedererlangung politischer Macht und kultureller Deutungshoheit eine Triebfeder der politischen Bewegung dar. Im Folgenden werde ich meine Untersuchung der hawaiischen Bewegung und ihres historischen, politischen und kulturellen Umfeldes näher vorstellen.

\subsection{Themenstellung und Aufbau der Arbeit}

Das in dieser Arbeit betrachtete „Hawaiian Sovereignty Movement“ umfasst eine Vielzahl von Gruppierungen und Organisationen, die wiederum ein weites Spektrum politischer Ziele repräsentieren. Der Begriff „Sovereignty“ (Souveränität) hat in diesem Zusammenhang im öffentlichen Diskurs Hawai'is eine spezifische Bedeutung gewonnen, die im weitesten Sinne alle hawaiischen Bemühungen um politische Abgrenzung von Bundesstaat und USA umfasst. Da diese Bemühungen nur in wenigen Fällen die tatsächliche Eigenstaatlichkeit Hawai' is (Souveränität) zum Ziel haben, sondern in aller Regel die weitgehende kulturelle, politische und ökonomische Eigenständigkeit der Hawaiier innerhalb der USA (Autonomie) anstreben, verwende ich in dieser Untersuchung zwar auch den in Hawai`i gebräuchlichen Namen „Hawaiian Sovereignty Movement“, bevorzuge aber anstelle der wörtlichen Übersetzung die Bezeichnung „Hawaiische Autonomiebewegung“. Wie sich zeigen wird, bestimmen hier differenzierte Vorstellungen politischer und kultureller Autonomie, d.h. einer 
weitgehenden Selbständigkeit ohne vollständige Herauslösung aus den bestehenden staatlichen Strukturen, das Gesamtbild.

Die Entwicklung der politischen Bewegung verlief annähernd parallel zu einer seit den 1970er Jahren andauernden Revitalisierung wesentlicher Elemente hawaiischer Kultur. Beide Prozesse fanden vor dem Hintergrund einer maßgeblich von US-amerikanischen Normen geprägten Gesellschaft statt, die Hawai‘i durch ihre multiethnische Zusammensetzung zu einem Sonderfall unter den US-Bundesstaaten macht. Aufgrund der historischen und demographischen Entwicklungen in Hawai'i verfügt der allergrößte Teil der Hawaiier heute über multiple ethnische wie kulturelle Identifikationsoptionen.

Im Mittelpunkt der folgenden Analyse und Betrachtung der hawaiischen Bewegung steht daher die Frage nach der Ethnisierung und ihren speziellen Faktoren sowie die Untersuchung der Revitalisierung von Kultur und deren politische Nutzung und Instrumentalisierung durch die Bewegung. Ein weiterer Akzent dieser Untersuchung liegt auf den Strategien, die von den verschiedenen Akteuren, den Vertretern der Autonomiebewegung ebenso wie den Vertretern staatlicher und halbstaatlicher Institutionen, im Kontext unterschiedlicher gesellschaftlicher Konfliktfelder angewendet werden. Das Ziel der Arbeit ist somit eine möglichst umfassende Darstellung der spezifisch hawaiischen Aspekte dieser ethnisch-politischen Bewegung im US-Bundesstaat Hawai'i. Die Forderungen und Ziele des „Hawaiian Sovereignty Movements“ prägten in vielen Bereichen die politische Diskussion im Hawai`i der 1990er Jahre. Die vorliegende Arbeit versteht sich daher nicht zuletzt auch als ein Beitrag zur neueren Geschichtsschreibung Hawai‘ is.

Schon erste Gespräche an der University of Hawai'i in Honolulu und Hilo machten deutlich, wie groß die Distanz zwischen offiziellen Vertretern des „Hawaiian Sovereignty Movements“ und nicht-hawaiischen Forschern und Untersuchern waren. Vonseiten der Bewegungsprotagonisten wurde die Beteiligung „,amerikanischer“ (nicht-hawaiischer) Forscher als unberechtigte Einmischung angesehen - „Western Science“ war offenbar Teil eines in den späten 1980er und frühen 1990er Jahren aufgebauten und aufrechterhaltenen Feindbildes der Bewegung. Es nimmt deshalb nicht wunder, dass sich bis zum Ende der 1990er Jahre nur wenige Arbeiten kritisch mit dem „Hawaiian Sovereignty Movement“ und der eng mit ihm verflochtenen „Hawaiian Renaissance“, der seit den 1960er Jahren andauernden kulturellen Revitalisierung, befassten. ${ }^{6}$ Seither setzen sich auch immer mehr Autoren hawaiischer Abstammung mit „hawaiischen“ Themen auseinander, doch liegen die Schwerpunkte der neueren Literatur vor allem auf Fragen der Historiographie, der kulturellen Revitalisierung und Identitätsbildung, der (historischen) Rechtsdiskussion sowie der Dar-

${ }^{6}$ Zu nennen sind hier z.B. Jocelyn Linnekin (1983, 1990b, 1992) oder Jonathan Friedman (1992, 1993). 
stellung konkreter Ziele der hawaiischen Bewegung (z.B. McGregor 2007; Silva 2004; Coffman 2003, o.J.; Halualani 2002; Osorio 2002, 2001; Merry 2000, 1997; Wilson 2000; Trask, H.-K. 1999; Wood 1999; Hartwell 1996; Hasager u. Friedman [Hg.] 1994; Dudley u. Agard 1993; Kame‘eleihiwa 1992; Parker 1989).

Den theoretischen Rahmen der Untersuchung stecken zwei Themen der kultur- und sozialwissenschaftlichen Diskussion ab: Zum einen ist dies die Untersuchung von Ethnizität im Sinne einer politisch nutzbaren ethnisch begründeten Identität sowie der mit diesem Konzept verbundenen Konfliktpotenziale, zum anderen ist es die Politisierung von Kultur und eine Politik der Kulturen bzw. Identitäten (hierzu u.a. Groenemeyer 2003; Hormel u. Scherr 2003; Kaschuba 2001; Niethammer 2000; Smith 1994; Orywal u. Hackstein 1993; Nagel 1993; Heckmann 1992; Olzak 1983). Im Vordergrund stehen dabei Politisierungs- bzw. Deutungsprozesse kultureller Symbole, ein Aspekt, dem in der spezifischen Situation Hawai'is besonderes Gewicht zukommt - werden doch z.B. durch die Tourismusindustrie Einzelaspekte „traditioneller“ hawaiischer bzw. einer hybriden „polynesischen“ Kultur propagiert und so wichtige Eckpunkte für die Außenwahrnehmung des „Hawaiischen“ gesetzt. Die Untersuchung richtet den Blick immer wieder und in unterschiedlichen Kontexten auf die von der Bewegung eingesetzten Strategien zur Sicherung und Bekräftigung des eigenen „Kulturellen Kapitals“ (Esser 1996) - ist doch der Erfolg im Streit um die Definitionsmacht über hawaiische Identität, Kultur und Geschichte eines der vorrangigen Ziele der politischen Bewegung und ihrer Vertreter. ${ }^{7}$

Die Dissertation gliedert sich in vier Hauptteile, die jeweils mehrere Kapitel umfassen und unterschiedliche Aspekte der hawaiischen Bewegung in den Vordergrund stellen: einen historischen Überblick, die Darstellung und Analyse der am Autonomiediskurs beteiligten Gruppierungen und Institutionen, die Untersuchung der Bewegungspraxis (wobei der zeitliche Schwerpunkt auf den 1990er Jahren liegt) und schließlich eine Fortführung der Untersuchung in die Jahre nach der Jahrtausendwende.

Die Kapitel im Einzelnen: Eine historische Darstellung in Hinblick auf den „Gegenwartsstatus“ der Hawaiier legt in Kapitel 2 die Grundlagen für das

\footnotetext{
${ }^{7}$ In anderen Siedlergesellschaften, in diesem Kontext sind vor allem Nordamerika und Neuseeland zu nennen, haben sich ebenfalls ethnische politische Bewegungen herausgebildet, deren Ziele und Strategien Ähnlichkeiten mit dem „Hawaiian Sovereignty Movement“ zeigen. Insbesondere die Politik der Māori in Aotearoa/Neuseeland weist, trotz der sehr unterschiedlichen rechtlichen und historischen Ausgangsposition, Parallelen auf (siehe hierzu u.a.: Fleras u. Spoonley 1999; Durie 1998; Sharp 1997; Melbourne 1995; Cox 1993; Hazlehurst 1993; für Nordamerika siehe z.B. Sturm 2002; Washburn 1996; Nagel 1995; Cornell 1988). Anknüpfungspunkte finden sich auch in Europa, wo ethnische Bewegungen in verschiedenen Staaten versuchen, politische und kulturelle Eigenständigkeit zu erreichen bzw. zu erweitern (siehe z.B. Böhnke 2002; Cudic 2001; Toivanen 2001; Alsheimer, Moosmüller u. Roth [Hg.] 2000; Kokot u. Dracklé [Hg.] 1996).
} 
Verständnis heutiger hawaiischer Autonomie- bzw. Kulturpolitik und stellt Verbindungen zum hawaiischen Widerstand im 19. Jahrhundert her, in dessen Nachfolge sich Vertreter des „Hawaiian Sovereignty Movements“ sehen. Aufbauend auf diesem Blick zurück auf die politische Entwicklung stehen in Kapitel 3 und 4 zunächst die Sozialgeschichte und die mit ihr verbundenen demographischen Prozesse im Vordergrund. Letztere sind im Kontext dieser Arbeit insbesondere für die Eigen- bzw. Fremdwahrnehmung der Hawaiier und die mit dem Attribut „hawaiisch“ verbundenen Aus- und Abgrenzungen von großer Bedeutung. Bei der in Kapitel 4 folgenden Darstellung und Analyse der politischen Repräsentanten der hawaiischen Bevölkerung, sei es durch die wesentlichen Gruppierungen der Autonomiebewegung oder die staatlichen bzw. quasi-staatlichen Institutionen, liegt der Schwerpunkt auf den diversen Deutungen des „Hawaiischen“: ebendiese „Hawaiianness“ und ihr jeweiliges Verständnis bietet ein anschauliches Beispiel dafür, wie unscharf die Abgrenzungen zwischen politischen Gegnern im gesellschaftlichen und kulturellen Kontext Hawai‘is sein können.

Kapitel 5 richtet den Fokus auf die eng mit der politischen Bewegung verknüpfte kulturelle Revitalisierung. Mit den Themen Sprache, Tanz und Seefahrt stellt das Kapitel drei Kernsymbole des hawaiischen Selbstverständnisses heraus und zeichnet ihre von zahlreichen Brüchen und Zäsuren gekennzeichnete Entwicklung seit dem 19. Jahrhundert nach. Dabei liegt der Schwerpunkt auf dem Zusammenspiel (erneuerter) kultureller Phänomene mit politischen Forderungen bzw. ihrem Einsatz zur Durchsetzung dieser Forderungen. Hierbei werden auch die Schnittstellen zwischen Politik, Kultur und Ökonomie hinsichtlich der Auseinandersetzungen um die Deutungshoheit über hawaiische Traditionen sichtbar. Im Anschluss bietet Kapitel 6, das sich vor allem mit den Ereignissen des Gedenkjahres 1993 befasst, eine synchrone Betrachtung von Strategien und Aktionen der am Autonomiediskurs beteiligten Akteure. In einem solchen synchronen Querschnitt erscheint die eingangs vorgestellte Chronologie der hawaiischen Geschichte erneut als ein gewordenes Ganzes, gewissermaßen als zusammengefasster Bestand des Gegenwartswissens und bildet den Hintergrund für die Entfaltung und das Verständnis der jeweils aktuellen Ereignisse. Stand im vorangegangenen Kapitel die Symbolpolitik des Staates wie auch der Bewegung im Vordergrund, so nimmt Kapitel 7 mit den großen Landkonflikten der 1990er Jahre ein Thema auf, das die politische Diskussion dieses Jahrzehnts weithin bestimmte. Das Land nimmt in der politischen, ökonomischen und kulturellen Argumentation der Bewegung einen herausragenden Stellenwert ein - anhand der (auch innerhawaiischen) Auseinandersetzungen zeigt sich die starke Ideologisierung des Kernsymbols „Land“ sowie dessen Potential für die politische Nutzung über die unmittelbare Landrechtsfrage hinaus.

In den beiden Schlusskapiteln werden die komplementären Stränge der Autonomiebewegung (Politik und Kultur) noch einmal aufgegriffen und in die 
Gegenwart weitergeführt. Kapitel 8 befasst sich mit den Debatten um die Verfasstheit eines zukünftigen hawaiischen Gemeinwesens bzw. des Verhältnisses, insbesondere des Rechtsverhältnisses zu den USA, wobei im Hintergrund dieser vor allem innerhalb der hawaiischen Gemeinschaft geführten Kontroversen immer auch die Frage nach den Grundlagen einer gemeinsamen hawaiischen Identität steht. Dabei treten divergierende Deutungen der hawaiischen Geschichte und grundsätzliche Unterschiede in der Definition des „HawaiischSeins“ zutage. Kapitel 9 widmet sich schließlich einer zunehmend selbstverständlichen kollektiven Praxis kultureller Autonomie. Insbesondere Künstler und Autoren haben sich in den vergangenen Jahren sichtbar von den Bemühungen der kulturellen Revitalisierung und den legitimierenden Rückgriffen auf das Überkommene emanzipiert. Zugleich werden politische Inhalte selbstverständlich transportiert und vermittelt - die Ideen der Autonomie haben in Wissenschaft und Kulturszenen Fuß gefasst. Die Diskussion einer Transformation des politischen Widerstands hin zu einer autonomen Praxis bzw. der Übergänge zwischen politischen und kulturellen Autonomiebestrebungen schließt diese Untersuchung des „Hawaiian Sovereignty Movements“ ab.

Bei den von mir im Rahmen dieser Arbeit verwendeten Quellen handelt es sich vor allem um gedruckte Texte. Zwar wurden von mir während meiner Aufenthalte in Hawai'i Interviews und Gespräche mit Vertreterinnen und Vertretern der Autonomiebewegung sowie mit Repräsentanten der wichtigsten hawaiischen oder staatlichen Institutionen geführt. Darüber hinaus konnte ich an verschiedenen Veranstaltungen hawaiischer Organisationen teilnehmen, doch treten diese Quellen in aller Regel hinter den schriftlich vorliegenden Daten zurück. Aufgrund der hohen Professionalisierung und starken Politisierung der gesamten Bewegung wie auch der ihr nahe stehenden Personen und Gruppierungen differieren die in Interviews gemachten Aussagen nicht oder nur in sehr geringem Maße von den schriftlichen Quellen, die häufig vom gleichen Personenkreis verfasst wurden. Hinzu kommt, dass - abgesehen von den Vertretern der staatlichen Institutionen - insgesamt eine eher geringe Bereitschaft vorhanden war, mit mir in meiner Rolle als Wissenschaftler zu sprechen, d.h. mit jemandem, der seine Aufgabe darin sah, die Bewegung kritisch zu untersuchen und auch zu hinterfragen. Diese Ablehnung gab es bei anderen, eher privaten Gesprächsgelegenheiten nicht - eben weil diese nicht den Weg in meine Aufzeichnungen finden sollten. Nicht zuletzt wegen meiner relativ kurzen Aufenthalte in Hawai'i konnte zudem nicht der gesamte relevante Personenkreis interviewt werden, so dass ich im Allgemeinen auf Gesprächsinformationen verzichte und sie nur in wenigen Fällen zur Ergänzung der übrigen Quellen verwende. Da es sich bei allen im Rahmen dieser Untersuchung präsentierten Themen um öffentlich geführte Auseinandersetzungen über „offizielle“ Versionen von Geschichte und Kultur handelt (gewissermaßen eine öffentliche Verhandlung von Identität), die in verschiedenen Medien präsentiert und 
als Texte verbreitet wurden, können sie auch anhand dieser schriftlicher Quellen beobachtet, gegenübergestellt und analysiert werden (Dracklé u. Kokot 1996:8-9; Schmidtke u. Ruzza 1993).

Die Hawai'i-Pacific Collection der Hamilton Library an der University of Hawai'i at Mānoa, die William S. Richardson School of Law Library, Mānoa, sowie die Bibliothek des Legal Reference Bureau (Hawai'i State Capitol) haben umfassendes Quellenmaterial zu diversen Aspekten des „Hawaiian Sovereignty Movements“ in ihren Beständen. Als Primärquellen gesichtet habe ich darüber hinaus die beiden großen, in Honolulu erscheinenden Tageszeitungen des Staates (Honolulu Star-Bulletin, Honolulu Advertiser) sowie kleinere Zeitschriften und Zeitungen. Veröffentlichungen des Office of Hawaiian Affairs (die Monatszeitung Ka Wai Ola o OHA und weitere Publikationen) und anderer Institutionen (Native Hawaiian Advisory Council, Department of Hawaiian Home Lands u.a.) reflektieren die Positionen dieser staatlichhawaiischen Institutionen. Veröffentlichungen verschiedener Gruppierungen der hawaiischen Bewegung liegen in gedruckter Form vor (Flugblätter, Programme, Zeitungen); eine wichtige Quelle ist darüber hinaus das Internet: verschiedene Gruppierungen unterhielten zumindest zeitweise und zum Teil bis heute sehr umfangreiche Websites, auf denen sie ihre jeweiligen Positionen vertraten und vertreten. Die benutzten Quellen sind in der Literaturliste verzeichnet. Artikel und Kommentare aus Tageszeitungen werden unter Angabe des Titels, der jeweiligen Zeitung, des Erscheinungsdatums sowie der Seitenzahl ausschließlich in den entsprechenden Fußnoten bibliographiert (soweit bekannt, wird auch der jeweilige Autor genannt; allerdings erscheinen insbesondere Leitartikel und Kommentare ohne Namensnennung).

Die Schreibung hawaiischer Wörter und Ausdrücke in dieser Arbeit richtet sich nach der von Pukui und Elbert in ihrem Hawaiian Dictionary (1986) festgelegten Orthographie, die heute in Hawai' $i$ allgemein Verwendung findet. In der älteren Literatur und bei zahlreichen Eigennamen wurden insbesondere die diakritischen Zeichen, der Längenstrich kahakō und das Zeichen für den Verschlusslaut 'okina, nicht verwendet, so dass sich in Zitaten oder Bezeichnungen auch im vorliegenden Text zum Beispiel Aina statt 'Äina (Land) oder Hawaii statt Hawai'i findet. Kursivschreibungen bleiben im Wesentlichen den im Text verwendeten hawaiischen Wörtern vorbehalten, wobei Eigennamen, Namen von Institutionen etc. oder geographische Bezeichnungen nicht hervorgehoben werden. Abschließend sei angemerkt, dass die von mir verwendeten männlichen Bezeichnungen immer auch ihren weiblichen Gegenpart gleichberechtigt einschließen. 


\section{Historischer Überblick: Geschichten des Verlus- tes, Geschichten des Widerstands}

Am 19. Januar des Jahres 1778 gingen die Schiffe des britischen Kapitäns James Cook vor Waimea an der Südwestküste Kaua'is vor Anker - dieser erste Kontakt der Inselbewohner mit Europäern beendete zugleich viele Jahrhunderte eines abgeschlossenen insularen Daseins, nachdem vor wohl fast 2000 Jahren polynesische Seefahrer von den Marquesas gen Norden aufbrachen und den entlegenen Archipel im Nordpazifik entdeckten. Noch im gleichen Jahr 1778 kehrte Cook nach Hawai'i zurück, ${ }^{8}$ erkundete die Inselgruppe von See aus und landete schließlich am 17. Januar 1779 in der Kealakekua Bucht an der Westküste der Insel Hawai'i, wo er und seine Mannschaft über mehrere Wochen intensiven Kontakt mit der lokalen Bevölkerung hatten und wo James Cook am 14. Februar 1779 bei einer Auseinandersetzung mit Hawaiiern den Tod fand. ${ }^{9}$

Die Entdeckungen Cooks katapultierten die Gesellschaft Hawai‘is, die am Übergang zum 19. Jahrhundert vom Machtkampf hawaiischer Adliger gezeichnet war, in wenigen Jahrzehnten in eine bereits global agierende Weltwirtschaft sowie in die Interessensphären der europäischen und amerikanischen Großmächte. So war die Wende zum 19. Jahrhundert nicht nur der Ausgangspunkt des geeinten und souveränen Königreichs Hawai'i, dessen Ende 1893 einen der zentralen Bezugspunkte der Autonomiebewegung darstellt, sondern

\footnotetext{
${ }^{8}$ Nach der Landung an der Küste Kaua'is brachen die Seefahrer zur Nordwestküste Nordamerikas auf, wo Cook nach der Nordwestpassage suchen sollte und im Laufe des Jahres 1778 die Küste Alaskas erforschte.

${ }^{9}$ Eine Zusammenschau der Fahrten Cooks im hawaiischen Archipel findet sich z.B. bei Salmond (2004:378ff.) und Thomas (2003:355ff.). Die Ursachen des Todes von James Cook (wobei das Zusammentreffen seiner Ankunft mit dem Beginn des Makahiki-Festes und seine mögliche Identifikation mit dem hawaiischen Gott Lono an zentraler Stelle stehen) waren Gegenstand einer kontrovers geführten Debatte (siehe hierzu im Überblick Thomas [2003:443] und als Hauptwerke Sahlins 1985, 1995 sowie Obeyesekere 1992).
} 
zugleich der Beginn einer massiven Umstrukturierung der Gesellschaft, an deren Ende der Verlust politischer wie kultureller Eigenständigkeit des hawaiischen Bevölkerungsteils auf den Inseln stand. Anders als in den von europäischen Mutterländern und/oder zugewanderten Siedler-Eliten regierten Kolonien gingen die maßgebliche Veränderungen in Hawai'i nicht auf das überwiegend einseitige Handeln einer Kolonialmacht bzw. deren Vertreter zurück (die großen Mächte des 18. und 19. Jahrhunderts schienen sich erst recht spät für die einsamen Inseln zu interessieren), sie entwickelten sich vielmehr aus einem von unterschiedlichen Interessen und Motiven getragenen Zusammenspiel des hawaiischen Adels, der immerhin bis zur Mitte des 19. Jahrhunderts de facto die Politik des Inselreiches dominierte, und der zunehmend zahlreicher auf dem Archipel vertretenen Ausländer.

Die neuere Geschichte Hawai'is seit 1779, in deren Verlauf sich jene Prozesse vollziehen, die zu gravierenden ökonomischen sowie politischen Verschiebungen und schließlich zu einem auch formalen Ende der hawaiischen Herrschaft über die Inseln führten, ist bis heute von unterschiedlichen Darstellungen und Deutungen geprägt. Wichtige Quellen zur Geschichte des hawaiischen Königreichs sowie des graduellen Übergangs zu einer politischen wie ökonomischen Dominanz der euro-amerikanischen Zuwanderer sind die Werke hawaiischer Autoren des 19. Jahrhunderts, deren bekannteste David Malo (ca. 1793-1853), John Papa I'‘'̄ (1800-1870) und Samuel Mānaiakalani Kamakau (1815-1876) sind. Sie blickten zurück auf die Zeit vor den Europäern und beschrieben die von ihnen erlebten Veränderungen in der hawaiischen Gesellschaft; doch sahen sie auf ihre eigene Gesellschaft bereits durch den Filter des Christentums und der von missionarischer Unterweisung geprägten Moralvorstellungen. ${ }^{10}$ Für den Großteil der heute vorliegenden Standardwerke zur Geschichte Hawai'is im 19. Jahrhunderts ist die euro-amerikanische Perspektive der Ausgangspunkt historischer Beschreibung und Analyse, zeitgenössische hawaiische Standpunkte bleiben eher unterrepräsentiert. ${ }^{11}$ Es nimmt daher nicht wunder, dass in den letzten Jahren Historiker hawaiischer Herkunft dieses kritische Jahrhundert mit dem Blick auf vielfach unberücksichtigte hawaiischsprachige Quellen und hawaiische Deutungen politischer Ereignisse

\footnotetext{
${ }^{10}$ Es liegen heute in Übersetzungen vor: Kamakau (1991a, b; 1992a, b), Malo (1992) und $\bar{I}^{‘} \overline{1}$ (1991).

${ }^{11} \mathrm{Zu}$ nennen sind hier v.a. Ralph Kuykendall, dessen dreibändige History of the Hawaiian Kingdom (1947-1967) noch immer von großer Bedeutung ist, sowie Gavan Daws, der mit Shoal of time: a history of the Hawaiian Islands (1968) eine Betrachtung der historischen Entwicklung bis zur Staatswerdung 1959 vorlegte. Eine kritischere Geschichtsschreibung findet sich u.a. bei Tom Coffman, der mit Nation within (o.J.) und The island edge of America (2003) eine politische Geschichte Hawai‘ is seit dem Umsturz vorlegte.
} 
erneut untersuchen und dabei auch deutliche Aussagen zur gegenwärtigen Situation der Hawaiier nicht scheuen. ${ }^{12}$

Für das (Selbst-) Verständnis der Autonomiebewegung von den 1990er Jahren bis zur Gegenwart, ihres Strebens nach politischer Souveränität und kultureller Selbstbestimmung, sind die politischen, kulturellen und wirtschaftlichen Entwicklungen des 19. Jahrhunderts von entscheidender Bedeutung. ${ }^{13}$ Unterschiedliche Lesarten der hawaiischen Geschichte spielten und spielen in der Auseinandersetzung um die hawaiische Autonomie oder Souveränität eine große Rolle. Ich werde daher im folgenden historischen Überblick zunächst versuchen, zentrale Entwicklungsstränge der politischen und der davon nicht zu trennenden ökonomischen Geschichte Hawai' is seit dem Ende des 18. Jahrhunderts aufzuzeigen. Ebenso möchte ich darlegen, dass diese Prozesse nicht ohne den zum Teil offenen Widerstand einer sich im Laufe des Jahrhunderts immer weiter von jeglicher Möglichkeit der Einflussnahme auf Monarchen und Regierung entfernenden Bevölkerung vonstatten gingen. Dieser durchaus kontinuierliche hawaiische Widerstand wird durch die gegenwärtige Autonomiebewegung, deren Entwicklung seit den 1970er Jahren ich zum Gegenstand des zweiten Teils dieses Kapitels machen werde, bis heute als Bezugspunkt in Anspruch genommen.

\footnotetext{
${ }^{12}$ Mit der Untersuchung Native land and foreign desires (1992) legte Lilikalā Kame'eleihiwa eine Neudeutung der Landprivatisierung von 1848 vor; Noenoe K. Silvas Aloha betrayed (2004) untersucht den indigenen Widerstand gegen die Machtübernahme der Haole und in seinem Buch Dismembering Lāhui (2002) analysiert Jonathan Kamakawiwo‘ole Osorio den Einfluss des euroamerikanischen Rechtes auf die Auflösung des hawaiischen Königreiches.

${ }^{13}$ Ebenfalls von nicht zu unterschätzender Bedeutung für die sozialen und politischen Entwicklungen in Hawaii waren die tief greifenden demographischen Verschiebungen, auf die ich in Kapitel 3.1 eingehen werde.
} 


\subsection{Die Kolonialisierung Hawai' is}

„They said they had heard that in several countries where foreigners had intermingled with the aboriginal natives, the latter had soon disappeared; and should missionaries come to live at Waiakea, perhaps the land would ultimately become theirs, and the kanaka maore (aborigines) cease to be its occupiers“ (Ellis 1827:312).

Die Befürchtungen der Hawaiier, von denen der britische Missionar William Ellis schon im Jahre 1827 berichtete, sollten sich im Laufe des 19. Jahrhunderts als nur allzu wahr herausstellen. ${ }^{14}$ Die Zahl der Hawaiier nahm nicht nur extrem ab, die Bevölkerung wurde auch von dem Land, das sie von alters her nutzte, vertrieben. Auch wenn Hawai'i bis zu seiner Eingliederung in die USA am Ende des Jahrhunderts ein formal souveränes Mitglied der internationalen Staatengemeinschaft blieb, entwickelte es sich in der Zeit der Monarchie zunehmend zu einer de-facto-Kolonie der Vereinigten Staaten, bis es dann als US-Territorium und schließlich als Bundesstaat völlig von den USA vereinnahmt wurde. ${ }^{15}$

Die politische Geschichte Hawai'is seit dem ersten Kontakt mit Europäern kann der Übersichtlichkeit halber in drei größere Zeiträume unterteilt werden:

1. Die Schaffung und Konsolidierung des Königreiches (Ende des 18. bis zur Mitte des 19. Jahrhunderts),

2. eine Phase zunehmenden Einflusses der Zuwanderer und der USA, die mit dem Sturz der Monarchie und der Gründung der Republik endet (ca. 1850 bis zum Ende des 19. Jahrhunderts) und schließlich

3. die bis zur Gegenwart andauernde Integration des Archipels in die politischen, militärischen und wirtschaftlichen Strukturen der Vereinigten Staaten.

\footnotetext{
${ }^{14}$ Ellis selbst hielt diese Befürchtungen für unbegründet und führte sie auf die Kenntnis der Hawaiier von Entwicklungen in Südamerika und in der Karibik zurück.

${ }^{15}$ Die Geschichte Hawai' is als faktische US-Kolonie ist vielen Amerikanern, die sich grundsätzlich scheuen, die eigene, aus einer Revolution gegen das „Mutterland“ Großbritannien hervorgegangene Nation als potenzielle und tatsächliche Kolonialmacht zu sehen, praktisch unbekannt. Hierfür ist auch eine Episode charakteristisch, wie ich sie bei einem Besuch in der Dauerausstellung des Bishop Museums 1995 in Honolulu erlebte: Vier ältere amerikanische Touristinnen betrachteten zugleich mit mir die Vitrine, in der die Entmachtung Lili‘uokalanis im Jahre 1893, ihr anschließender Arrest im 'Iolani Palast und der Übergang von der Monarchie zur Republik Hawai'i anhand von Fotografien und Objekten dargestellt war, wobei der begleitende Text keinen dezidierten Bezug auf die Beteiligung US-amerikanischer Kräfte an dieser „Revolution“ nahm. Eine der Damen kommentierte die Präsentation dann unter dem mitfühlenden Gemurmel der anderen mit den Worten: „Oh those British...“ - als ich sie auf ihren Irrtum hinwies, reagierten sie verwundert bis ungläubig.
} 
Die nachstehende Darstellung dieser historischen Abschnitte soll zum einen die Autonomiebewegung und ihre Zielsetzungen historisch kontextualisieren, zum anderen aber auch Grundlagen für das nähere Verständnis der Besonderheiten dieses Inselstaates legen.

\subsubsection{Das frühe Königreich Hawai‘i (1791 - 1849)}

\section{Indigener Expansionismus}

Als James Cook und seine Mannschaft 1778 die Insel Kaua'i erreichten, war der hawaiische Archipel noch in die mehr oder weniger den größeren Inseln entsprechenden Machtbereiche von vier ranghohen Adeligen, Ali ‘ ${ }^{\prime}$ nui, geteilt. Die Europäer trafen auf eine streng hierarchisch gegliederte und in zwei Klassen geteilte Gesellschaft, in der die Mitglieder eines durch Genealogien gestuften Geburtsadels (Ali 'i) der übrigen Bevölkerung (Maka 'áinana), in der Mehrheit Bauern und Fischer, gegenüberstanden. ${ }^{16}$

Die Rivalität zwischen den hohen Adligen war eine der Triebfedern des politischen Wandels auf den Inseln, und als der Ali ‘ $i$ nui der Insel Hawai' ${ }^{\prime}$ i im Jahre 1782 starb, nutzte ein bis dahin politisch eher unbedeutender Ali ' $i$ mit dem Namen Kamehameha die Gelegenheit, sich gegen den Nachfolger zu wenden und selbst zum Ali ‘ $i$ nui aufzusteigen. Wohl eher zufällig lagen die bevorzugten Ankerplätze der europäischen Seefahrer (wie z.B. die von Cook besuchte Kealakekua-Bucht) in seinem Machtbereich, womit Kamehameha eine privilegierte Position im einige Jahre später einsetzenden Handel mit europäischen Schiffsbesatzungen gesichert war.

Schon 1785, nur drei Jahre nach dem Sieg Kamehamehas, ankerte das erste Schiff in Hawai'i, das sich - mit Fellen beladen - auf der Fahrt von der Nordwestküste Amerikas zum Handelsplatz Guangzhou (Kanton) in China befand. In den beiden folgenden Jahrzehnten entwickelte sich dann ein schwungvoller Fellhandel zwischen der amerikanischen Nordwestküste und China (mit Hawai' $i$ als günstig gelegenem Proviantierungsort), der zunächst von den Briten, spätestens seit der Jahrhundertwende aber von den Amerikanern dominiert wurde (Kuykendall 1947:84-5; Daws 1968:32-3).

Auf den Inseln selbst monopolisierten die hawaiischen Adligen das Geschäft mit den Fellhändlern und verschafften sich so europäische Güter und Waffen. Für Kamehameha brachte der privilegierte Zugang zu den Technologien der europäischen und amerikanischen Seefahrer große Vorteile bei der Verfolgung seiner hegemonialen Ziele. Sahlins (1992:3) zufolge waren die neu eingeführten Kanonen und Gewehre sogar entscheidend für seine kriegerischen

\footnotetext{
${ }^{16}$ Neben dem Adel und der gemeinen Bevölkerung existierte noch eine „unterste“ Bevölkerungsschicht, die Kauā, was Pukui und Elbert (1986) als „Ausgestoßene“, „Unberührbare“ oder „Sklaven“ übersetzen. Eine weitere gesellschaftliche Klasse bildeten möglicherweise die religiösen Funktionsträger, die die Schranke zwischen Ali' $i$ und Maka'äinana überwanden (siehe hierzu Davenport 1969:4); zum hawaiischen Rangsystem siehe v.a. Sahlins (1958) und Valeri (1972).
} 
Erfolge der kommenden Jahre. ${ }^{17}$ Nachdem er 1791 die Insel Hawai ${ }^{`} i$ erobert hatte, nahm er 1795 Maui und Moloka'i ein, bevor seine Krieger noch im gleichen Jahr die Verteidiger der Insel O`ahu über das Kliff des Nu'uanu Pali in den sicheren Tod trieben. Mit der Eroberung O`ahus stieg Kamehameha zum mächtigsten Ali ‘i nui der gesamten Inselkette auf ${ }^{18}$ und wurde zum Begründer einer hawaiischen Monarchie, die bis 1893 Bestand haben sollte.

In den ersten Jahrzehnten nach dem Zusammentreffen von Hawaiiern und Europäern kamen immer mehr fremde Seefahrer auf die Inseln, die Kontakte mit den Besuchern intensivierten sich und zahlreiche Hawaiier, die auf den Handelsschiffen anheuerten, berichteten bei ihrer Rückkehr über die Welt jenseits des Meeres. Grundlagen und Strukturen der politischen wie auch der wirtschaftlichen Macht auf den Inseln blieben in diesen Jahrzehnten aber weiterhin hawaiischen Traditionen verpflichtet und die wachsenden Ansprüche und immer unverhohlener eingeforderten Normen der weißen Einwanderer, der Haole $^{19}$, wurden im Rahmen überkommener Deutungsmuster interpretiert und in die hawaiische Weltordnung integriert.

Als erster Ali ${ }^{i} i$ in historischer Zeit brachte Kamehameha alle Inseln unter eine zentrale Kontrolle und führte als $M \bar{c}{ }^{\top} \bar{\imath}$ (König) ${ }^{20}$ tief greifende Neuerungen ein - so übertrug er die Verwaltung ganzer Inseln dem neu geschaffenen Amt des Kia ‘äina (,Gouverneur“) und durchbrach mit der Vergabe von Land als vererbbarem Eigentum (an seine Verbündeten) das traditionelle Land-

\footnotetext{
${ }^{17}$ Kamehameha nutzte nicht nur westliche Technologie, er umgab sich auch sehr früh mit europäischen Beratern, deren bekannteste wohl John Young (ca. 1749-1835), dessen hawaiischer Name 'Olohana lautete, und Isaac Davis (ca. 1758-1810) waren, zwei britische Strandläufer, die seit 1790 auf Hawai`i lebten und zu den wichtigsten politischen Ratgebern Kamehamehas avancierten. Beide hatten im frühen Königreich Hawai‘i u.a. als Gouverneure der Inseln O‘ahu und Hawai' $i$ bedeutende Stellungen inne und waren in die adelige hawaiische Gesellschaft aufgenommen worden.

Ein weiteres Beispiel für Kamehamehas strategische Nutzung der Kontakte zu den Haole, den Fremden, ist die so genannte „Zession“ der Insel Hawai'i an Großbritannien während des Aufenthaltes von Kapitän George Vancouver (1792-1794). Kamehameha bezweckte ein Bündnis mit den mächtigen Briten, das er im Rahmen traditioneller hawaiischer Usancen anstrebte und das ihm in den bevorstehenden Eroberungszügen nutzen sollte, während Vancouver, gemäß seines westlichen Verständnisses von Politik und Völkerrecht, von einer tatsächlichen Abtretungserklärung sprach (Kuykendall 1947:41-2; cf. Mykkänen 2003:38). Die Britische Krone hat diese „Abtretungserklärung“ von 1794 allerdings offiziell nie zur Kenntnis genommen.

${ }^{18}$ Erst 15 Jahre später (1810) ordneten sich die nördlichen Inseln Kaua‘i und Ni‘ihau kampflos der Oberherrschaft Kamehamehas unter.

${ }^{19}$ Das hawaiische Wort Haole bedeutet wörtlich „ohne Atem“ und wird bis heute von Hawaiiern zur Bezeichnung von Euroamerikanern benutzt - eine genaue Etymologie des Wortes ist nicht bekannt (vgl. Pukui u. Elbert 1986:58).

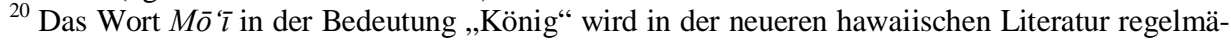
ßig zur Bezeichnung der regierenden Ali $i$ verwendet, ist aber wohl relativ neuen Ursprungs: im Wörterbuch von Pukui und Elbert wird auf das Fehlen früher Quellen vor 1832 hingewiesen (1986:251).
} 
recht (Kame'eleihiwa 1992:51-2). Er war einerseits traditionelles hawaiisches Oberhaupt und zugleich auch Wegbereiter von Umwälzungen, wie z.B. der weitgehenden Übernahme westlicher Konzepte und der Transformation des politischen Lebens im Sinne eines europäischen Politik- und Rechtsverständnisses, die er voraussehen konnte. $^{21}$

\section{Die Abschaffung der alten Ordnung}

Nach dem Tod Kamehamehas am 8. Mai 1819 stieg seine Witwe Ka'ahumanu zur zentralen Protagonistin gesellschaftlicher Umbrüche auf, die das soziale, religiöse und politische Leben in Hawai'i grundsätzlich verändern sollten. ${ }^{22}$ Unterstützt von hochrangigen Adligen überzeugte sie den jungen Kamehameha II., das 'Aikapu zu brechen - das Verbot gemeinsamen Speisens von Männern und Frauen, in dem sich die religiös begründeten Regeln des sozialen und politischen Miteinanders bündelten. Schon bald nach dieser öffentlichen und folgenlosen Demonstration des Ungehorsams gegen die Götter ergingen Anordnungen, überall auf den Inseln die Tempelstätten (Heiau) sowie die Standbilder der Gottheiten zu zerstören.

Die Folgen dieser Loslösung eines Großteils des Adels von der Religion waren einschneidend, auch wenn damit nicht die hawaiische Religion in toto abgeschafft war, wie Kame‘eleihiwa (1992:68) konstatierte:

„This event did not mean that the maka áinana relinquished their own form of religion, for 'Aumakua (family guardian) worship has persisted until today. However, the state religion which ordered the Hawaiian universe was abandoned, removing the foundation upon which Hawaiian society had been established.“

Während dem Gemeinwesen also die religiöse Basis entzogen wurde, hatten die Statusverhältnisse zwischen Ali ‘i und Maka 'äinana weiter Bestand - der Adel blieb so in einer Machtposition gegenüber der gemeinen Bevölkerung, ohne dass die traditionellen, in der Religion gründenden Regeln des Ausgleichs weiter Geltung hatten.

Die der Aufhebung des Kapu durch Ka'ahumanu zugrunde liegenden Motive wurden in der von euro-amerikanischen Autoren verfassten historischen Literatur vor allem eigennützigen Zielen der Regentin zugeschrieben: so sah Daws (1968:55f.) ihr Machtstreben als Ursache, hätten die Regeln des Kapu doch die volle Teilhabe von Frauen an der Macht verhindert. 1992 stellte

\footnotetext{
${ }^{21}$ Siehe zum Verhältnis von Kultur und Politik im Hawai‘i der ersten Hälfte des 19. Jahrhunderts die Studie von Mykkänen (2003).

${ }^{22}$ Nachfolger Kamehamehas war sein Sohn Liholiho, der unter dem Namen Kamehameha II. bis 1824 regierte. Ka'ahumanu rief sich selbst zur Regentin aus und hatte das von ihr neu geschaffene Amt der Kuhina nui bis zu ihrem Tod im Jahre 1832 inne (cf. Sahlins 1992:60-1).
} 
die hawaiische Historikerin Lilikalā Kame'eleihiwa (1992:79-82) dann den Bruch des 'Aikapu in einen ganz anderen Zusammenhang: ${ }^{23}$ vor dem Hintergrund des enormen Bevölkerungsverlustes (bis 1823 hatte sich die Bevölkerungszahl der Inseln mehr als halbiert) ${ }^{24}$ und der zunehmenden Auflösung überkommener Regeln stellte sich die Frage nach den Grundlagen des Gemeinwesens neu. Und vielleicht sahen Ka'ahumanu und ihre Gefolgsleute, nachdem die hawaiischen Götter und ihre Regeln das Sterben der hawaiischen Bevölkerung nicht aufhalten konnten, in der Lebensweise der Haole die wahrhaftigere Alternative:

„White people had broken every kapu of the old Akua and yet they did not die. Hawaiians adhered to the 'Aikapu and died by the thousands. White people in Hawai'i got drunk, endulged in sensual pleasures, respected only those Hawaiians who acquired Western goods, and did not seem to possess any Akua. Yet white people seemed to hold the secret of life“" (Kame‘ eleihiwa 1992:82).

Doch es waren nicht nur die alten Götter, die durch den Kapu-Bruch schon bald der Vergangenheit angehören sollten, wie Jonathan Kamakawiwo'ole Osorio, Historiker und Direktor des Center for Hawaiian Studies an der University of Hawai'i, Mānoa, herausstellt (2002:11): „... by this same act, she [Ka'ahumanu; U.M.] reduced herself and her chiefly relatives to the status of human beings, no longer a divinty“ - der Adel selbst begab sich so eines der wesentlichen Faktoren für seine gegenüber der übrigen Bevölkerung privilegierte Stellung.

Hinzu kam, dass Kamehameha II. bei seinem Amtsantritt auf eine Neuverteilung des Landes verzichtete, die bestehenden Landgebiete der Ali ${ }^{i} i$ intakt ließ und auf diese Weise auch seine Position als $M \bar{c}{ }^{\prime} \bar{l}$ schwächte:

„The king remained in principle the supreme lord of all lands (haku'äina) with rights, therefore, to benefit from them or from the people's labor. These rights were not canceled de jure until the Great Māhele of 1848. Yet as the chiefs' tenures were already secure, the royal dues became unenforceable“ (Sahlins 1992:62).

Diese gesellschaftlichen Umbrüche fallen zeitlich zusammen mit einem wirtschaftlichen Wandel, der einen zusätzlichen Einfluss auf die Neuorientierung

\footnotetext{
${ }^{23}$ Kame 'eleihiwa steht mit ihrem Buch „Native lands and foreign desires“ (1992) am Beginn der seit den 1990er Jahren beginnenden hawaiischen Neubetrachtung der politischen Entwicklungen des 19. Jahrhunderts.

${ }^{24}$ Eine ausführliche Betrachtung der demographischen Veränderungen und Verschiebungen in Hawaii erfolgt in Kapitel 3.1.
} 
der hawaiischen Adligen in der veränderten Gesellschaft hatten. In den ersten beiden Jahrzehnten des 19. Jahrhunderts verlor der Fellhandel zwischen Alaska und China, der bis 1810 die wirtschaftlichen Beziehungen mit den Haole bestimmte, an Bedeutung und machte dem Handel mit Sandelholz, einem hawaiischen Produkt, Platz. Den Ali ‘ $i$ boten sich hierdurch neue Möglichkeiten des Statusgewinns außerhalb des traditionellen Rangsystems. Galt zu Lebzeiten Kamehamehas noch das Кapu, das den Handel mit Sandelholz gewissermaßen einem königlichen Monopol unterwarf, so expandierte der Handel nach 1819 und der Aufhebung der religiösen Restriktionen ungebremst.

Wie sehr die hawaiischen Ali' $i$, unter deren Aufsicht die Ausbeutung von menschlicher Arbeitskraft und Holzbeständen voranschritt, bemüht waren, ihren Platz in der neuen Wirtschaftsstruktur zu suchen, zeigt auch die ungewöhnlich hohe Zahl von mehr als 80 Schiffen europäischer Bauart, die von ihnen noch vor 1830 erworben und u.a. für Handelsfahrten eingesetzt wurden. Zwar verschafften sich auch andernorts im Pazifik indigene Inselbewohner Zugang zu europäischen Transportmitteln, doch geschah dies nirgends in auch nur annähernder Größenordnung (Mills 2003:66 ff.). Ein Teil des hawaiischen Adels sicherte sich so einen Zugang zu europäischen Luxusgütern, für deren Erwerb sie sich aber auch zunehmend bei ihren europäischen Handelpartnern verschuldeten und so ihre Stellung im wirtschaftlichen Gefüge des beginnenden 19. Jahrhunderts gefährdeten. ${ }^{25}$ Nachdem durch den Raubbau und die vollständige Zerstörung der Ressourcen der Handel mit Sandelholz im Jahre 1829 zusammenbrach, blieben viele hawaiische Adlige völlig überschuldet zurück.

\section{Religion und die Festschreibung weltlichen Rechts}

Für den hawaiischen Adel, der sich ja von den Göttern herleitete, war die Religion mit ihren Prinzipien und Ritualen ein wesentliches Mittel zur Manifestation und Ausübung seiner Macht. Für die nur ein Jahr nach dem Ende der offiziellen Religion auf den Inseln eintreffenden Missionare des American Board of Commissions for Foreign Missions (ABCFM) aus Neuengland stellte die Annäherung an den Adel und dessen weltliche Macht hingegen ein probates Mittel zur Verbreitung ihres eigenen Glaubens dar. ${ }^{26}$ Sehr bald schon nach ihrer An-

\footnotetext{
${ }^{25}$ Zur Aufblähung des Sandelholzhandels und des einhergehenden Erwerbs von Luxusgütern durch die Ali ${ }^{\prime} i$ siehe ausführlich Sahlins (1990; 1992:57-67).

${ }^{26}$ Es ist hinsichtlich des zeitlichen Zusammenfalls der Vorgänge in Hawai`i und dem Kommen der Missionare des ABCFM von Interesse, dass diese Missionare (unter den insgesamt 16 Mitgliedern der Gruppe befanden sich allerdings nur zwei Pastoren, Hiram Bingham und Asa Thurston) nicht aufgrund eigener Überlegungen nach Hawai'i aufgebrochen waren. Diese gehen vielmehr auf die Bekehrung eines jungen Hawaiiers, 'Ōpūkaha'ia, zurück, dessen Taufe bereits 1815 in Torringford, Massachusetts, stattfand. Der wahrscheinlich 1792 geborene 'Ōpūkaha'ia, durch seine Arbeit auf einem Handelsschiff nach Neuengland gekommen, besuchte dort die Missionsschule und starb hier noch im Jahre 1818. Statt seiner begleiteten vier andere junge Hawaiier die Missionarsgruppe auf ihrer 1819 beginnenden Reise zu den hawaiischen Inseln. Die
} 
kunft suchten die höchstrangigen Mitglieder der hawaiischen Gesellschaft um Ka'ahumanu und Keōpūolani, die Mutter des amtierenden $M \bar{o}{ }^{'} \bar{\imath}$, den Kontakt zu den Missionaren, und es bildete sich eine Allianz, die wesentlich zum durchgreifenden Erfolg der christlichen Missionierung auf den hawaiischen Inseln beitrug. ${ }^{27}$ Die hawaiischen Adligen nutzten die Einführung der neuen Religion durchaus im eigenen Interesse, wie Kame‘eleihiwa am Beispiel Ka'ahumanus zeigt:

„She [Ka'ahumanu; U.M.] rewrote the equation that formerly had rested upon the 'Aikapu, replacing the old Akua with Jehovah, and making Christian laws the new kapu, complete with the sanctity of the Sabbath and the ritual prayers of New England Calvinism. The new religion may not have been compatible with Hawaiian culture, but its symbols filled the void left by the loss of 'Aikapu and the rituals of state religion“ (Kame'eleihiwa 1992:154)

Mit der Mission kamen aber nicht nur eine neue Religion und ihre kirchlichreligiösen Regeln, die sich ja nicht ausschließlich auf die Feiertagsheiligung beschränkten: mit der Regulierung der Kleidung, des sexuellen Umgangs und kultureller Traditionen griffen die Missionare vielmehr tief in das hawaiische Leben ein. Darüber hinaus propagierten sie ein euro-amerikanisches Rechtsund Politikverständnis, dessen Rezeption auf hawaiischer Seite durch die enge Verbindung mit dem herrschenden Adel weit reichende Folgen haben sollte. Durch die Entwicklung eines hawaiischen Alphabets, die Produktion zunächst vor allem religiöser Schriften, die Gründung von Schulen und die weitgehende Alphabetisierung der hawaiischen Bevölkerung schon in den ersten Jahrzehnten des 19. Jahrhunderts schufen sie überdies ein Medium, mit dem die Verbreitung des von ihnen favorisierten Gedankenguts intensiviert werden konnte. 28

Geschichte um 'Ōpūkaha'ia findet sich ausführlich bei Schweizer (2005:83-88) und auch bei Silva (2004:30-1).

${ }^{27}$ Zur frühen Mission in Hawai'i siehe Kuykendall 1947:100-16; Daws 1968:61 ff.; zum Verhältnis Ka'ahumanus zur Mission siehe auch Sahlins 1992:67-73. Zur engen Verbindung zwischen Ali ' $i$ und Mission sowie den daraus resultierenden Abgrenzungsproblemen der Missionare siehe Mykkänen (2003:80-3).

${ }^{28}$ Die Übersetzungen der Missionare sorgten auch für Bedeutungsverschiebungen in der hawaiischen Sprache, wie Noenoe Silva am Beispiel des Wortes „pono“ (2004:32-3) aufzeigt. Im religiösen und später auch im weltlichen Schrifttum nahm pono die Bedeutung von „Rechtschaffenheit“ („righteousness“) an, ein Sinngehalt, der dem hawaiischen Wort zuvor fremd war. Silva schreibt hierzu: „Whereas pono had been used previously to describe the ideal behavior of ali'i and concepts such as balance, completeness, and material well-being, it now took on the foreign connotation of conforming to Christian morality“ (2004:33). 
Die Festschreibung religiöser Regeln als weltliches Gesetz erfolgte bereits ab 1825 zunächst gegen den Widerstand ausländischer Inselbewohner und Seeleute. Denn die von der Mission angeregten und vom hawaiischen Adel festgeschriebenen, auf dem Dekalog beruhenden moralischen Regeln bedeuteten eine massive Einschränkung ihrer bis dahin recht frei verfügbaren Vergnügungen. Für die hawaiischen Gesetzesmacher bedeutete die Einführung und Durchsetzung der neuen Regeln aber auch den Beginn einer Politik im europäischen Sinne, wie Mykkänen (2003:94-8) am Beispiel des Prostitutionsverbots aufzeigt: die erzwungene Konfrontation und Auseinandersetzung mit einer öffentlichen Diskussion widerstreitender Standpunkte stellte einen entscheidenden Wendepunkt in der politischen Geschichte des Königreichs dar. ${ }^{29}$

In dieser Zeit begann die Geschichte des Königreichs als moderne, an westlichen Vorbildern orientierte Nation, die sich als souveränes Mitglied im Kreise der Nationalstaaten begriff. ${ }^{30}$ Bis zur Mitte des 19. Jahrhunderts gewann die Bedeutung des geschriebenen Rechts zunehmend an Bedeutung und ersetzte nach und nach die traditionellen Regeln gesellschaftlichen Zusammenlebens in Hawai ' ${ }^{31}{ }^{31}$ Bereits 1839 unterzeichnete Kamehameha III. das erste umfassende Gesetzeswerk des Königreichs, dessen Präambel die Vorherrschaft des Rechts verankerte: „God had bestowed certain rights alike on all men and all chiefs, and all people of all lands“ (zit. nach Osorio 2002:25). Der Text, verfasst von William Richards, Missionar und wichtigster Berater des Königs, war ein Vorläufer der Verfassung von 1840, die das Königreich in eine konstitutionelle Monarchie nach europäischem Vorbild umwandelte. Erstmalig konnten Maka 'äinana als gewählte Vertreter Einfluss auf Regierung und Gesetzgebung nehmen - allerdings galt dies auch für naturalisierte Haole, deren Anliegen sich deutlich von den Interessen der frühen Missionare abhoben:

„Within a decade [gemeint ist hier das Jahrzehnt von 1840 bis 1850; U.M.] the government had indeed changed its class as well as its ethnic character. The missionary statesmen co-opted by the alii to control the

\footnotetext{
29 „Strange as it may sound, politics in a genuinely comparative sense was first a matter of putting a stop to an established practice of carnal love being a commodity. ... The presence of conflicting foreign parties - missionaries and sailors - produced a conflicting exchange of ideas, which were synthesized under the concept of politics. The events in the narrative illustrate how this unique conflict ... gave birth to conceptual distinctions which resulted in the creation of politics as an object for talk in and about Hawaii““ (Mykkänen 2003:97-8).

${ }^{30}$ Bereits 1826 schlossen das Königreich Hawai`i und die USA einen Freundschafts- und Handelsvertrag („Articles of arrangement“), der US-Bürgern weitgehende Rechte in dem Inselstaat einräumte, allerdings von den USA nicht ratifiziert wurde. Entsprechende Verträge mit Großbritannien und Frankreich folgten 1836 bzw. 1839 (Kuykendall 1947:98-9, 148, 166-7).

${ }^{31}$ Osorio (2002) stellt diesen durch das schriftlich niedergelegte Recht beförderten gesellschaftlichen Riss in den Vordergrund seiner historischen Analyse des Königreichs Hawaii (vergl. auch Osorio 2003).
} 
foreign sector had been superseded by Haole of a different ilk ...These men, when they did not directly represent the ,monied estates' of the foreign bourgeoisie, were at least prepared to compromise with them in the name of ,progress"“ (Sahlins 1992:131).

Der Geltungsanspruch der Neubürger wuchs mit der Zunahme ihrer ökonomischen Aktivitäten in Hawai ' ${ }^{3} .{ }^{32}$ Ihr Verlangen nach sicherem Landbesitz fand seine Ergänzung in dem Streben zahlreicher Ali ${ }^{i} i$ nach stärkerer Partizipation im kapitalistischen Wirtschaftssystem, was ihnen die Ausschaltung niederer Instanzen des traditionellen Landrechts wünschenswert erschienen ließ. ${ }^{33} \mathrm{Im}$ Jahre 1845 setzte die Legislatur dann einen Prozess in Gang, an dessen Ende die Umwandlung allen Landbesitzes in frei handelbares privates Eigentum stand und damit der vollständige Bruch mit dem überlieferten Landrecht.

\section{Die Privatisierung des Landbesitzes}

Neben Kai, dem Ozean, stellte 'Áina, das Land, zum einen die unveräußerliche und aufgrund der insularen Gegebenheiten begrenzte Grundlage fast aller wirtschaftlichen Tätigkeit dar, es war zum anderen aber auch die Basis politischer Macht. Die gesellschaftlichen Auswirkungen des vor-europäischen Landrechtssystems werden von der hawaiischen Historikerin Lilikalā Kame'eleihiwa mit einem Bild der Einheit von Land, Ali ‘ $i$ und gemeiner Bevölkerung beschrieben:

„In practical terms, the maka 'áinana fed and clothed the Ali ' $i$ Nui, who provided the organization required to produce enough food to sustain an ever-increasing population. Should a makaāinana fail to cultivate or mālama [pflegen, erhalten; U.M.] his portion of 'Āina, that was grounds for dismissal. By the same token, should a konohiki fail in proper direction of the maka'äinana, he too would be dismissed - for his own failure to mālama. The Ali ‘i Nui were no better off in this respect, for if any famine affected the 'Aina, they could be ousted for failing to mālama their religious duties. Hence, to Mālama 'Aina was by extension to care for the maka 'äinana and the Ali ${ }^{\circ} i$, for in the Hawaiian metaphor, these three components are mystically one and the same” (Kame‘eleihiwa 1992:30-1).

\footnotetext{
${ }^{32}$ Bereits 1835 hatte die erste kommerzielle Zuckerrohrpflanzung in Kōloa, Kaua‘i, den Betrieb aufgenommen.

${ }^{33}$ Das traditionelle hawaiische Landrecht sah eine „Verwaltungspyramide“ über dem jeweiligen Landabschnitt vor, an deren Spitze letztlich der $M \bar{o} ‘ \grave{\imath}$ stand. Durch diese Strukturen blieben in dem neuen Wirtschaftssystem immer größere Teile der Gewinns aus der Landnutzung auf den unteren Ebenen „hängen“, was das Interesse an einer Umwandlung des überkommenen Landrechts insbesondere bei den hohen Ali ${ }^{`} i$ wachsen ließ.
} 
Ein solches Idealbild eng verwobener Beziehungen zwischen den Menschen und dem von ihnen bewohnten und bearbeitetem Land blendet Konflikte der um Status konkurrierenden Ali ‘i oder Differenzen zwischen Maka 'äinana und den ihnen übergeordneten Konohiki aus: Im Vordergrund steht vielmehr die Vorstellung eines gesellschaftlichen Zusammenlebens, in dem 'Āina, das Land, der Dreh- und Angelpunkt ist, über den alle Mitglieder der Gesellschaft und alle Lebensbereiche miteinander verbunden sind. Dieses Gesellschaftsmodell, wenn es denn in dieser Ausprägung je so existiert hat, ${ }^{34}$ fand spätestens in der Mitte des 19. Jahrhunderts sein Ende: In den Jahren von 1848 bis 1855 legte die große Landteilung Māhele die Grundlage für privates Landeigentum. Die Privatisierung des Landes war ein zentrales Element der massiven Umgestaltung Hawai'is durch eine immer umfassendere Implementierung euroamerikanischer Rechtsnormen. In der Folge sollten zudem weit gestreute Landverkäufe zu einer deutlichen Verkleinerung der sich in hawaiischer Hand befindlichen Landflächen führen. Doch nicht nur die Besitzverhältnisse, auch das traditionelle Verhältnis zwischen den Bevölkerungsgruppen wandelte sich durch die Privatisierung des Landes:

„There can be no question that the Mahele, which allowed private ownership of land, also established the indigenous occupants, both Ali' $i$ and Maka'ainana, as competitors rather than as caretakers of the 'aina. After 1850, Native people were forced to appeal to the courts to allow them to fish, to gain access to irrigation water, and even to farm and to graze lands that were unoccupied. For the most part, these decisions, even when they resulted in favor of the hoa'äina, ${ }^{35}$ only conferred rights that they had once universally enjoyed. Thus, law became the arbiter between a family that the law itself estranged" (Osorio 2002:55).

Nicht nur, dass die Māhele das traditionell enge Beziehungsgeflecht zwischen Adel und der Allgemeinbevölkerung endgültig auflöste, sie hinterließ auch eine sehr ungleiche Verteilung des Landbesitzes: ${ }^{36}$ Während der König ca. 405.000 ha der gesamten Landfläche des Archipels zu seinen Kronlanden zählen konnte (und zusätzliche 608.000 ha als Regierungsland deklarierte), wurden weitere 608.000 ha insgesamt 251 Ali $` i$ zuerkannt. 80.000 Maka áinana erhielten im

\footnotetext{
${ }^{34}$ Siehe z.B. Parker (1989:10-15) für eine neutralere Darstellung der Beziehungen zwischen den gesellschaftlichen Gruppen und des hawaiischen Landrechts.

35 „Freunde des Landes“: Bezeichnung für jene Maka'āinana, denen gewisse Landrechte im Rahmen der Kuleana Gesetzgebung von 1850, die Landansprüche der nicht-adligen Bevölkerung regeln sollte, zugesprochen wurden (Osorio 2002:53; Lucas 1995:27).

${ }^{36}$ Zur Transformation des hawaiischen Königreichs durch die eingeführten Rechtsnormen der Missionare bzw. euro-amerikanischer Siedler siehe z.B. Merry (2000:35 ff.).
} 
Rahmen der staatlichen Landteilung insgesamt weniger als 11.500 ha (Osorio 2002:44). ${ }^{37}$ In den Jahrzehnten nach der Māhele gelangte darüber hinaus wertvolles Land in die Hände der wirtschaftlich immer erfolgreicheren euroamerikanischen Einwanderer, die 1896 bei einem Bevölkerungsanteil von nur 7\% bereits einen Anteil von 58,4\% am Gesamtwert aller Immobilien im Königreich hielten (Kreisel 1984:139). ${ }^{38}$ In den folgenden Jahrzehnten verschoben sich die Besitzverhältnisse weiter zum Nachteil der hawaiischen Bevölkerung und heute befindet sich der geringste Teil des Landes im Eigentum einzelner privater hawaiischer Landeigner.

Doch Land diente im alten Hawai'i nicht nur dem Nahrungserwerb oder als Pfand für die Durchsetzung politischer Interessen, es war nicht nur durch die im Lauf der Zeit sich wandelnden Grenzen adliger Einflussbereiche, durch die Demarkierungen der traditionellen Landnutzungsflächen ahupua'a und 'ili, ${ }^{39}$ nach mauka oder makai (berg- bzw. seewärts) organisiert und gegliedert. Es war auch eine Landschaft der Götter, legendärer Helden und ihrer Taten, bedeutender historischer Ereignisse und herausragender Naturdenkmäler, die in den Orts- und Flurbezeichnungen auf den hawaiischen Inseln bis auf den heutigen Tag wiederkehren und von einer besonderen Einbindung des Landes in das Leben der Menschen und der Existenz einer ausdifferenzierten sozialen und auch ideologischen Landschaft auf allen Inseln des Archipels zeugen. ${ }^{40}$

Das Zusammenwirken des Königs, der Ali ${ }^{i} i$ und der an sicherem Eigentum interessierten Haole in der Frage der Landrechte stellt einen Schwerpunkt der Untersuchungen gegenwärtiger hawaiischer Historiker dar. ${ }^{41} \mathrm{Ihr}$ Interesse gilt dabei möglichen rationalen Gründen, die über das von den Histo-

\footnotetext{
${ }^{37}$ Die geringe Fläche dieses so genannten Kuleana-Landes wurde wenigstens z.T. durch dessen hohe wirtschaftliche Nutzbarkeit ausgeglichen (Chinen 1958:31).

${ }^{38}$ Ausgenommen sind hier die portugiesischen Einwanderer, die fast ausschließlich im Rahmen der staatlich gesteuerten Einwanderung als Plantagenarbeiter auf die Inseln kamen. Sie hatten in aller Regel keinen Landbesitz und stellten 1896 fast 14\% der Gesamtbevölkerung (Lind 1967:28).

${ }^{39}$ Zur Terminologie des traditionellen hawaiischen Landrechts siehe Lucas 1995; hier die Seiten $4,40$.

${ }^{40} \mathrm{Zu}$ mythologischen und legendären Bezügen siehe Pukui, Elbert und Mookini 1974; Kanahele (1986:184) weist auf die große Bedeutung der Ortsnamen in den von Pukui (1997) gesammelten hawaiischen Sinnsprüchen hin. In der zweiten Hälfte des 20. Jahrhunderts erfuhr das hawaiische Land in seiner traditionellen Bedeutung, freilich nurmehr als abstrakte Referenz und ohne seine realen Bezüge und alten rechtlichen Grundlagen, eine umfassende Ideologisierung insofern, als der hawaiische Begriff für Land, das Wort 'Äina, im Zuge der Politisierung hawaiischer Kultur immer stärker mit emotionalen, auf eine hawaiische Identität bezogenen Inhalten befrachtet wurde.

${ }^{41}$ Bereits im Vorfeld der Gesetzesänderungen kam es zu erheblichen Protesten in der hawaiischen Bevölkerung, die, wie ich im Abschnitt 2.2 zeigen werde, den Prozess sehr kritisch begleitete.
} 
rikern der „alten Schule“ ${ }^{\text {42 }}$ angeführte Eigeninteresse der Adligen hinaus deren Mitwirkung an der Landgesetzgebung legitimiert. ${ }^{43}$ So interpretiert Osorio die Landgesetzgebungen von 1848-1855 als eine hawaiische Reaktion auf eine zuvor nie da gewesene Situation, eine „,...foreign solution to the problem of managing lands increasingly emptied of people“ (2002:49).

Die erste Hälfte des 19. Jh. sah also die Begründung und Konsolidierung der hawaiischen Monarchie unter Kamehameha I., die Abschaffung der traditionellen religiösen Grundlagen durch seine direkten Nachfolger, den Übergang von der absoluten Herrschaft des $M \bar{o}{ }^{`} \bar{l}$ zur konstitutionellen Monarchie und schließlich das Ende des traditionellen Landrechtsystems. Missionare und ihre Haole-Gegenspieler konnten zwar ihren Einfluss auf die Entscheidungen des $M \bar{o} ' \bar{l}$ und der hochrangigen Ali ‘ $i$ steigern, doch blieben diese einstweilen der bestimmende politische Machtfaktor.

\footnotetext{
${ }^{42}$ Siehe z.B. Kuykendall 1947; Chinen 1958; Daws 1968.

${ }^{43}$ Siehe z.B. Silva 2004; Osorio 2002; Kame‘eleihiwa 1992.
} 


\subsubsection{Das Ende des Königreichs Hawai`i $(1850$ - 1893)}

\section{Die zunehmende Macht der Ökonomie}

Mit der Aufgabe des traditionellen Landrechts und damit der Privatisierung des Landbesitzes hatten die Gesetzgeber einer durchgreifenden Ökonomisierung der hawaiischen Gesellschaft den Weg geebnet. Aus den USA zugewanderte Zuckerpflanzer versuchten immer stärkeren Einfluss auf die Geschicke des Inselstaates zu nehmen und schreckten dabei auch vor immer deutlicheren Machtproben nicht zurück. 1853 versuchten Haole erstmals die Regierungsgewalt an sich zu reißen, was zu einer politisch so unübersichtlichen Situation führte, dass Kamehameha III. die Annexion Hawai' is durch die Vereinigten Staaten zur Diskussion stellte - eine Vorstellung, die bei den hawaiischen Mitgliedern der Legislatur jedoch keine Zustimmung fand. ${ }^{44}$

In der politischen Diskussion des Königreichs gewann die Frage eines so genannten Reziprozitätsvertrages mit den USA an Bedeutung. Dieser Vertrag sollte die wirtschaftliche Position des Königreichs in der neuen, inzwischen maßgeblich von den Interessen der Pflanzer bestimmten ökonomischen Ordnung sichern. ${ }^{45}$ Unter König William Lunalilo, der 1873 als erster von der Legislatur gewählter Monarch den hawaiischen Thron bestieg, gewann die Frage eines Gegenseitigkeitsvertrages mit den Vereinigten Staaten neue Dynamik. Allerdings bestanden die USA auf einer Gegenleistung für ihren Verzicht auf Einfuhrzölle und Lunalilo formulierte die Möglichkeit einer Abtretung von Land im Gebiet von Pu'uloa, dem heutigen Pearl Harbor. Der Protest gegen den Vorschlag, hawaiisches Land unter die Verwaltung einer anderen Nation zu stellen, war aufseiten der hawaiischen Bevölkerung so deutlich und so stark, dass König und Kabinett sich von diesem Vorschlag distanzieren mussten (Kuykendall 1953:249-57; Daws 1968:191-2).

Im Wahlkampf um die Nachfolge Lunalilos, der zwischen Emma Na'ea Kaleleonālani Rooke (der Witwe Kamehamehas IV., genannt Queen

${ }^{44}$ Einer der bekanntesten Gegner dieser Politik war John Papa İ‘ ${ }^{‘}$ (1800-1870), Vertreter des Oberhauses und Richter am obersten Gerichtshof ( $\left.\overline{\mathrm{I}}^{\prime} \overline{\mathrm{i}} 1993\right)$. Auch die beiden Prinzen Alexander Liholiho und Lot Kamehameha waren ausgesprochene Gegner einer Annexion Hawai'is durch die USA. Wie verworren die damalige Situation war, zeigt auch die Drohung des US-Gesandten mit der möglichen Gewaltanwendung durch die ansässigen Amerikaner, auf die der Außenminister Hawai' 'is mit einem Hilfeersuchen an die „Drei Mächte“ (Großbritannien, Frankreich und eben die USA) und deren im Hafen von Honolulu liegenden Kriegsschiffe reagierte. Dieser Schutz wurde gewährt und die Frage einer Annexion trat für die folgenden Jahrzehnte in den Hintergrund (Kuykendall 1947:416-27; Daws 1968:148-53).

${ }^{45}$ Die wachsende Bedeutung des Zuckers für die sich zunehmend an euro-amerikanischen Maßstäben der Ökonomie orientierende hawaiische Wirtschaft belegen die folgenden Zahlen: von 1854 bis 1863 (dem Jahr des Regierungsantritts Kamehamehas V.) steigerte sich der Zuckerexport von ca. 264 t (581.777 lbs) auf ca. 2.404 t (5.295.121 lbs). Am Ende der neunjährigen Regierungszeit Kamehamehas V., er starb 1872, exportierte das Königreich ca. 7.716 t (16.995.402 lbs) Zucker vor allem in die USA (Kuykendall 1953:141). 
Emma) und David Kalākaua ausgefochten wurde, standen sich 1874 gegensätzliche Standpunkte gegenüber: ${ }^{46}$ Während Queen Emma mit ihrem Wahlspruch „Hawai'i for Hawaiians“ schon früh einen deutlich anti-amerikanischen Standpunkt bezogen hatte, sich gegen jegliche weitere politische Zugeständnisse an die Haole-Bevölkerung aussprach und zudem von ranghöherer Abstammung als ihr Gegner war, bemühte sich Kalākaua um die Unterstützung der Pflanzer und anderer Haole, was ihm schließlich den Wahlsieg eintrug. Nach dessen Bekanntgabe kam es zu Tumulten und Angriffen von Hawaiiern auf hawaiische Abgeordnete, und es waren amerikanische und britische Soldaten, die diesen Aufstand der Anhänger Queen Emmas niederschlugen. Osorio sieht diesen Aufruhr als Ausdruck dafür, dass weite Teile der hawaiischen Bevölkerung, vielfach noch den traditionellen Gesellschaftsvorstellungen verhaftet, sich in der zweiten Hälfte des 19. Jahrhunderts der eigenen Machtlosigkeit und der zunehmenden Entfremdung von den Regierenden bewusst wurden und schreibt hierzu:

„This was not some senseless violence. It was too partisan, too intentional for that. Even the police officers sent to establish control were reported to have ,removed their badges' and joined in the fray themselves. The attack on the legislators was not quite a revolution, but it certainly was an extraordinary statement from a peope who had suffered a government increasingly distant from them and had responded with patience and petition for thirty-two years. The idea that their own countrymen would support an inferior $\mathrm{Mo}^{\top} \overline{1}$ was more than they could bear“ (Osorio 2002:157).

Im Jahre 1876 unterzeichnete Kalākaua schließlich den Vertrag auf Gegenseitigkeit, der in einem vom US-Senat eingefügten Zusatz dem Königreich Hawai' $i$ untersagte, irgendwelche staatlichen Landflächen anderen Nationen als den USA zu überlassen. Während dieser Vertrag für die Zuckerindustrie im Königreich Hawai'i einen enormen wirtschaftlichen Aufschwung bedeutete, stellte er zugleich den bis dahin nachhaltigsten Eingriff in die eigenstaatliche Souveränität des Königreichs dar. Nicht nur, dass die USA sich die militärische Dominanz über die Inseln sicherten, mit dem Vertrag und den durch ihn gegebenen wirtschaftlichen Vorteilen war auch der Weg in die ökonomische Abhängigkeit von den Zuckerabnehmern auf dem amerikanischen Festland vorgezeichnet. $^{47}$

\footnotetext{
${ }^{46}$ Siehe hierzu ausführlich Osorio (2002:151 ff.), auf den ich mich im Folgenden beziehe.

${ }^{47}$ Für die USA stand die Sicherung des eigenen militärisch-ökonomischen Einflusses über den Archipel im Vordergrund des Vertrages. Insbesondere die Klausel zur Abwehr etwaiger exterritorialer Basen oder Niederlassungen anderer Staaten hat nicht unwesentlich zur schließlich erfolgten Ratifizierung durch die USA beigetragen (La Croix u. Grandy 1997:168).
} 
Doch es war nicht nur der Gegenseitigkeitsvertrag mit den USA, der aus heutiger Sicht die Selbständigkeit des hawaiischen Königreichs erstmals maßgeblich infrage stellte: mit der Wahl David Kalākauas zum König, so Osorio (2002:157), wurde auch die Institution des $M \bar{o}^{\prime} \bar{l}$ selbst untergraben: „His [Kalākauas; U.M.] weakness, as far as some of the Natives were concerned, was not his character or his leadership, but his genealogy. Even in 1874, the kānaka still insisted that their Mō'î be more than the government's chief executive.“

\section{Die Regierungszeit Kalākauas}

Kalākaua begann seine Regierungszeit in den Augen vieler Hawaiier mit dem Makel der Abhängigkeit von Haole-Pflanzern. Nicht zuletzt als Reaktion hierauf stellte er schon bald die Förderung hawaiischer Kultur, vernachlässigter oder bisher gar untersagter Künste in den Vordergrund seines Wirkens. Er setzte sich für den lange aus dem öffentlichen Leben verbannten Hula ein, förderte die traditionellen hawaiischen Heilkünste und betrieb die Errichtung eines „Native Hawaiian Board of Health“. 1886 begründete er die Hale Nauā, eine Gesellschaft zur Erforschung hawaiischer Religion und Genealogien, und unter seiner Ägide wurde das „Kumulipo“, der hawaiische Schöpfungsgesang, erstmalig in schriftlicher Form festgehalten.

Diese kulturpolitischen Akzente trugen zu einer wachsenden Distanz zwischen dem Monarchen und seinen Haole-Unterstützern bei. ${ }^{48}$ Insbesondere der Bau des 'Iolani Palastes in Honolulu, für viele Hawaiier bis heute ein zentrales Symbol für die Eigenständigkeit und Souveränität des Königreichs, und die zweiwöchigen Krönungszeremonien nach seiner Fertigstellung im Jahre 1882 ließen Vorwürfe der Verschwendung öffentlicher Mittel und der Korruption laut werden, die nicht nur Haole verstimmten, sondern auch die Entstehung einer hawaiischen Opposition gegenüber dem König, dem $M \bar{o} \mathfrak{‘}$ '̆ beförderten - etwas zuvor noch nie da Gewesenes. Viele der hawaiischen Oppositionellen rekrutierten sich aus den Reihen der Anhänger Queen Emmas und die gemeinsame Ablehnung Kalākauas und seiner Politik führten sie und die Gegner auf Seiten der nicht-hawaiischen Bevölkerung näher zusammen. ${ }^{49}$

1887 gründeten einflussreiche Haole die „Hawaiian League“, die schon bald 400 Mitglieder zählte und mit den bereits 1884 als Schützenverein gegründeten „Honolulu Rifles“ auch über einen paramilitärischen Arm verfügte. Noch im Gründungsjahr zwang die „Hawaiian League“ den König eine neue Verfassung zu unterzeichnen, die zum einen seine Rechte einschränkte, zum

\footnotetext{
${ }^{48}$ Ein fast skuriler Zwischenfall waren die Planungen Kalākauas für eine polynesische Allianz unter der Führung Hawai'is, wobei sein Versuch, auf die politischen Entwicklungen im Sāmoa des Jahres 1887 Einfluss zu nehmen, fast einen Konflikt mit dem Deutschen Reich heraufbeschworen hätte (siehe hierzu ausführlich Kuykendall 1967:322-39).

${ }^{49}$ Siehe hierzu Osorio (2002:159-66).
} 
anderen einen Großteil der Hawaiier durch hohe Eigentumsqualifikationen von den Wahlen ausschloss. ${ }^{50}$ Diese Verfassung von 1887, aufgrund ihrer Erzwingung durch die bewaffneten „Honolulu Rifles“ schon bald als „BajonettVerfassung“ tituliert, stieß auf großen Widerstand in der hawaiischen Bevölkerung, der sich in Massenversammlungen und Petitionen an den König äußerte, jedoch letztlich folgenlos blieb. ${ }^{51}$

Schon bei der Wahl von 1887 zeigte sich die volle Wirkung der neuen Regeln: Zwar trat eine hawaiische Opposition an, die ihre Stimmenzahl gegenüber der letzten Wahl sogar noch steigern konnte - jedoch reichten diese Stimmen nicht aus, die durch die Verfassung verbreiterte Wahlbasis der „Reformer" zu überstimmen, zumal sich in deren Lager auch namhafte hawaiische Gegner der Politik Kalākauas fanden. Jonathan Osorio fasst das Ergebnis dieser Wahl zusammen:

„The turnabout between the dominance of the king's party and the dominance of the haole party was abrupt and demonstrated the swift transformation of electoral power generated by the new constitution. [...] Altogether, Natives made up three of the twenty-four nobles in the 1887 session and eleven of twenty-four representatives. [...] ,Good government' (that is, government by a firm white hand) had finally been achieved. The instruments that had made it possible were the drastic changes to the power of the king, the transformation of the legislative assembly into a body that represented the business community, and equally fra-reaching change in the definition of the electorate. However, it also came as the result of tremendous loss of Native confidence in themselves and a very real confusion of what it meant to be Native“ (Osorio 2002:247-8).

\footnotetext{
${ }^{50}$ Die neue Verfassung übertrug zum einen die Rechte der Exekutive vom König auf das Kabinett, zum anderen machte sie alle Mitglieder des Kabinetts, das durch einfache Mehrheit der Legislatur aufgelöst werden konnte, zu stimmberechtigten Mitgliedern des Oberhauses. Hinsichtlich des Wahlrechtes sind die folgenden bemerkenswerten Änderungen anzuführen: euroamerikanische Ausländer mussten keine Bürger des Königreichs werden, um das Wahlrecht auszuüben. Die Wählerschaft wurde auf Männer hawaiischer oder europäischer Abstammung begrenzt, die zudem über ein nicht unwesentliches Vermögen oder Einkommen verfügen mussten (cf. Silva 2004:126). Es sei hier auf den geringen Anteil der Haole an der Bevölkerung Hawai' is hingewiesen: Zwischen 1884 und 1890 lag der Anteil der Weißen an der Gesamtbevölkerung bei ca. 20\%, wobei allerdings der Großteil der Weißen Portugiesen waren (ca. 13\% der Gesamtbevölkerung), die in diesem Zeitraum als Plantagenarbeiter nach Hawai'i gebracht wurden (Lind 1967:28). Durch den Ausschluss aller Asiaten vom Wahlrecht blieben auch die inzwischen in großer Zahl auf den Inseln lebenden Kontraktarbeiter von der politischen Einflussnahme ausgeschlossen (siehe Kapitel 3.1).

${ }^{51}$ Siehe ausführlich bei McGregor-Alegado (1979).
} 
Ebenfalls 1887 wurde der Gegenseitigkeitsvertrag mit den USA verlängert, die schließlich auch die Verfügungsgewalt über das Gebiet des heutigen Pearl Harbor erhielten - was die politische Situation in Hawai‘i zusätzlich polarisierte:

„The effect of the treaty was to lay bare the division of political interest in which the native Hawaiian population became opposed to more concessions while planters and merchants were willing to take political action to trade a portion of Hawaii's sovereignty in exchange for greater security of wealth“ (La Croix u. Grandy 1997:181).

Die Unausgewogenheit dieses Tausches (Souveränität gegen Gewinnsicherung) erwies sich schon wenige Jahre später, als 1890 in den USA das so genannte McKinley-Gesetz in Kraft trat. Mit diesem Gesetz wurden zwar alle US-Zölle auf Zuckerimporte aufgehoben, zugleich aber auch die Subventionierung der heimischen Zuckerproduktion eingeführt - eine wirtschaftliche Katastrophe für die Zuckerpflanzer in Hawai ‘ $i{ }^{52}$

\section{Lili ‘uokalani und das Ende der hawaiischen Monarchie}

Als verfassungsmäßige Nachfolgerin Kalākauas bestieg dessen Schwester Lili‘uokalani am 29. Januar 1891 den Thron. Sie machte keinen Hehl aus ihrer Ablehnung der so genannten Bajonett-Verfassung und hatte die Absicht, die Monarchie wieder zu stärken und das Wahlrecht, das mit der BajonettVerfassung ja auf alle steuerzahlenden männlichen Einwohner gleich welcher Staatsangehörigkeit ausgedehnt worden war, wieder auf Bürger des Königreichs zu beschränken. Doch schlossen sich auch die Gegner einer solchen Politik zusammen: der Gedanke einer Annexion des Archipels durch die Vereinigten Staaten fiel bei der Haole-Bevölkerung nicht zuletzt durch den wirtschaftlichen Einbruch des vorangegangenen Jahres auf immer fruchtbareren Boden und schon zu Beginn des Jahres 1892 gründeten Haole einen geheimen „Annexation Club“ (Kuykendall 1967:532-41).

Nach den ebenfalls 1892 abgehaltenen Wahlen zeigte sich die hawaiische Seite in mehrere Fraktionen zersplittert und im Parlament ergaben sich keine eindeutigen Stimmverhältnisse. Als die Königin die Sitzungsperiode am 14. Januar 1893 beendete, blieb die Verfassungsfrage offen. Lili‘uokalani plante nun den Erlass einer neuen Verfassung, so wie auch die Verfassungen von 1864 und 1887 formal vom Monarchen erlassen worden waren. ${ }^{53}$

\footnotetext{
${ }^{52}$ Am ersten Geltungstag des McKinley-Gesetzes fielen die Preise für Zucker um 38\%; der Wert der Exporte aus Hawai'i nahm von US\$ 13 Mio. im Jahre 1890 auf US\$ 10 Mio. 1891 ab und erreichte 1892 einen Tiefstand von US\$ 8 Mio. (La Croix u. Grandy 1997:183).

${ }^{53}$ Die wichtigsten von der neuen Verfassung vorgesehenen Änderungen betrafen das Wahlrecht (nur noch männliche Staatsbürger sollten wahlberechtigt ein und die Mitglieder des Oberhauses
} 
Die beabsichtigte Proklamation der Verfassung bot Vertretern der HaoleMinderheit einen Anlass, die faktisch bereits vorhandene politische Dominanz der weißen Bevölkerung auch de jure durch die eigene Machtübernahme und Abschaffung der Monarchie zu manifestieren. Trotz einer Verschiebung der ursprünglich noch für den Nachmittag des 14. Januars vorgesehenen Verfassungsproklamation begannen sich die Ereignisse in Honolulu zu überstürzen. Ein „Committee of Safety“, rekrutiert aus Mitgliedern des Annexation Club, sollte auf den „revolutionären Akt“ der Königin mit der Gründung und Einsetzung einer „provisorischen Regierung“ reagieren - dies war bereits im Vorfeld mit dem US-Gesandten in Hawai'i, John L. Stevens, der seine volle Unterstützung zusicherte, abgeklärt worden. Auf Veranlassung Stevens’ landeten am 16. Januar bewaffnete Truppen der im Hafen liegenden U.S.S. Boston in Honolulu und am Abend des folgenden Tages, dem 17. Januar 1893, trat die Königin unter dem Druck der Truppen und unter Protest zurück. In ihrer dem „Committee of Safety“ übermittelten Erklärung, bis heute eines der zentralen Dokumente des hawaiischen Widerstandes, heißt es:

„I, Liliuokalani, by the Grace of God and under the Constitution of the Kingdom, Queen, do hereby solemnly protest against any and all acts done against myself and the constitutional Government of the Hawaiian Kingdom by certain persons claiming to have established a provisional government of and for this kingdom.

That I yield to the superior force of the United States of America, whose minister plenipotentiary, His Excellency John L. Stevens, has caused United States troops to be landed at Honolulu and declared that he would support the said provisional government.

Now, to avoid any collision of armed forces and perhaps the loss of life, I do under this protest, and impelled by said force, yield my authority until such time as the Government of the United States shall, upon the facts being presented to it, undo the action of its representatives and reinstate me in the authority which I claim as the constitutional sovereign of the Hawaiian Islands“" (zit. nach Kuykendall 1967:603).

Lili'uokalani machte in dieser Erklärung deutlich, dass sie sich weiterhin als Souverän des Königreichs sah und ihr erzwungenes Nachgeben als nur vorübergehend betrachtete; zugleich appellierte sie an die Vereinigten Staaten, den Rechtszustand auf den Inseln wiederherzustellen. Dennoch wurde am 1. Februar die Flagge der Vereinigten Staaten über dem Regierungsgebäude aufgezo-

wieder von der Königin auf Lebenszeit ernannt werden) sowie die Restauration der Unabhängigkeit des Monarchen vom Kabinett (Kuykendall 1967:586). 
gen: auf „dringende Bitten“ der provisorischen Regierung und mit der Begründung, dass sie sich nicht in der Lage sähe, die öffentliche Ordnung in der Stadt aufrechtzuerhalten (wobei es zu keinen offenen Protesten und Drohungen gekommen war), hatte der US-Gesandte Stevens Hawai'i unter den vorübergehenden „Schutz“ der Vereinigten Staaten gestellt (Kuykendall 1967:607-9).

Offener Widerstand von hawaiischer Seite blieb weitgehend aus, sieht man vom Protest gegen die Ableistung eines von der Provisorischen Regierung verlangten Treueeides ab. Auch ließen sich nur noch wenige Hawaiier als Wähler registrieren, da auch hierfür ein Eid auf die neue Regierung verlangt wurde, und schließlich fanden sich nur noch wenige Hawaiier bereit, den Eid als Geschworene zu leisten (Russ 1992:21-3, 43-4, 213). Eines der bis heute bekanntesten Beispiele für den eher stillen Protest der Hawaiier gegen den Sturz der Regierung ist das Lied „Kaulana nā pua“, das Ellen Kekoahiwaikalani Wright Prendergast im Januar 1893 aus Anlass der Treueeid-Verweigerung fast der gesamten „Royal Hawaiian Band“ schrieb. ${ }^{54}$ Im Text des Liedes heißt es u.a.:

„Kaulana nā pua a'o Hawai'i / Kūpa'a mahope o ka 'āina / Hiki mai ka 'elele o ka loko 'ino / Palapala 'ānunu me ka pākaha. // Famous are the children of Hawai'i / Ever loyal to the land / When the evil-hearted messenger comes / With his greedy document of extortion“ (Übers. N. Mahoe u. S. Elbert, zit. nach Nordyke u. Nayes 1993:28-9).

Bis heute wird dieses Lied, nach einer Textzeile auch als „Mele 'ai pōhaku“ (,Stone-eating song“) bezeichnet, als Ausdruck des Protestes gesungen (Nordyke u. Nayes 1993:32-3; Silva 2004:134-5).

Während man in Washington bereits über eine mögliche Annexion Hawai'is durch die USA verhandelte, sandte Lili'uokalani eine Abordnung in die amerikanische Hauptstadt, um gegen die aktuellen Entwicklungen zu protestieren (Kuykendall 1967:609-18). US-Präsident S. Grover Cleveland schickte hierauf einen Gesandten nach Honolulu, dessen Bericht die Grundlage für Präsident Clevelands „Message to Congress Relating to the Hawaiian Islands“ vom 18. Dezember 1893 darstellte. Eine ihrer meistzitierten Passagen lautet:

„[...] By an act of war committed with the participation of a diplomatic representative of the United States and without authority of Congress, the Government of a feeble but friendly and confiding people has been overthrown. A substantial wrong has thus been done which a due re-

\footnotetext{
${ }^{54}$ Hier sollte darauf hingewiesen werden, dass Musik, Tanz und Lied hawaiische Medien des Protestes waren, die der Bevölkerung ermöglichten, ihrer Ablehnung und ihrem Widerstand Ausdruck zu verleihen, ohne Repressalien befürchten zu müssen - die Texte der Lieder waren für den allergrößten Teil der zugewanderten Bevölkerung, die des Hawaiischen unkundig blieb, unverständlich.
} 
gard for our national character as well as the rights of the injured people requires we should endeavour to repair [...]“ (zit. nach OHA o.J.:12).

Die Reaktion der Provisorischen Regierung fiel eindeutig aus: sie teilte dem Präsidenten mit, dass sie ,... respectfully and unhesitatingly declines to entertain the proposition of the President of the United States that it should surrender its authority to the ex-Queen“ (zit. nach Kuykendall 1967:646). Am 4. Juli 1894, dem amerikanischen Unabhängigkeitstag, proklamierte man die „Republic of Hawaii“, die von den USA (entgegen den Aussagen des Präsidenten) und Großbritannien umgehend anerkannt wurde (Daws 1968:281).

Politisches Ziel der nun in Hawai'i herrschenden Oligarchie aus Kaufleuten und Pflanzern blieb die Annexion der Inseln durch die USA: Zwar hatte der amerikanische Kongress bereits im Frühjahr des Jahres 1894 Resolutionen verabschiedet, die andere Nationen vor der Einmischung in die inneren Angelegenheiten Hawai' 'is warnten, da dies als ,unfreundlicher Akt“ gegen die USA gelten würde (Kuykendall 1967:649-50) - es sollte aber noch weitere vier Jahre dauern, bis sich die USA bereit fanden, dem konkreten Gedanken einer Annexion Hawai' is näher zu treten.

\subsubsection{Republik, Territorium, Bundesstaat}

Die Republik auf dem Weg nach Amerika

Die hawaiische Bevölkerung brachte ihre Ablehnung der neuen Verhältnisse immer wieder zum Ausdruck und die hawaiischen Zeitungen Honolulus nahmen trotz eingeschränkter Pressefreiheit eindeutig Stellung gegen die neue Regierung. Wie Noenoe Silva (2004:123 ff.) zeigen kann, blieben die kontinuierlichen Proteste großer hawaiischer politischer Organisationen wie Hui Kālai'āina oder Hui Aloha 'Āina von der etablierten Geschichtsschreibung bisher weitgehend unberücksichtigt, da Nachrichten über ihre Aktivitäten vor allem von hawaiischsprachigen Medien aufgegriffen und verbreitet wurden. Zentrale Quellen lagen daher bis zur Jahrhundertwende fast ausschließlich in hawaiischer Sprache vor und wurden weitgehend ignoriert:

„The lack of historical reference to such a large and organized resistance is typical of colonial situations, in which the archive in the language of the colonizer is privileged to a high degree over that of the vernacular - that is the language of the native people“ (Silva 2004:125).

Hinreichend Resonanz in den nicht-hawaiischen Medien fand hingegen ein für den hawaiischen Widerstand sehr ungewöhnliches Unternehmen, das zugleich einen Hinweis auf die sichere Position der provisorischen Regierung und des 
von ihr ausgerufenen Übergangsstaates gibt: 1895 versuchten hawaiische Monarchisten, die Königin mit Waffengewalt erneut an die Macht zu bringen - ein Versuch, der angesichts der überlegenen Regierungstruppen chancenlos war und einmalig blieb. Sowohl den Aufständischen wie der ehemaligen Königin wurde der Prozess gemacht, doch trotz der verhängten langjährigen Freiheitsstrafen (und sogar einiger Todesurteile) kamen alle bereits im folgenden Jahr wieder auf freien Fuß und auch Lili'uokalani, die im 'Iolani Palast unter Arrest gestellt wurde, erhielt ihre vollen Bürgerrechte zurück. ${ }^{55}$

Trotz allen Bemühungen von Seiten der „Republic of Hawaii“ schienen die USA zunächst wenig gewillt, sich die Inseln einzuverleiben; und als die Annexion 1898 - mehr als fünf Jahre nach dem Sturz der Monarchie - schließlich erfolgte, wurde einmal mehr deutlich, wie abhängig die Inseln von sie nur mittelbar berührenden außenpolitischen Interessen Amerikas waren. Als der amtierende US-Präsident William McKinley am 7. Juli 1898 die Annexionserklärung des Kongresses unterzeichnete, geschah dies nicht zuletzt wegen der durch die amerikanische Besetzung der Philippinen immens gewachsenen strategischen Bedeutung der Inseln.

Die deutliche Ablehnung der Annexion durch die hawaiische Bevölkerung ${ }^{56}$ zeigte sich auch anlässlich der entsprechenden Feierlichkeiten in Honolulu: wie die Presse berichtete, blieben die eingeladenen Mitglieder des Königshauses der Feier allesamt fern und auch die hawaiische Bevölkerung fehlte der Presse zufolge in den Besucherrängen (Russ 1992:365-9). Die Schilderung eines amerikanischen Besuchers der Feierlichkeiten verdeutlicht den von Hawaiiern geleisteten passiven Widerstand gegen die politischen Entwicklungen:

„The band of Hawaiian damsels ... who were to have lowered for the last time the Hawaiian flag, as the government band played for the last time officially the ponoi, ${ }^{57}$ would not lower it. The band refused to play

\footnotetext{
${ }^{55}$ Zur so genannten Konterrevolution von 1895, die von dem in Italien ausgebildeten Hawaiier Charles Wilcox angeführt wurde, siehe Daws 1968:282-4; ausführlich bei Andrade 1996:149-66; Russ 1992:51-87; zur Sichtweise der Königin siehe Lili‘uokalani 1990:262-77.

${ }^{56}$ Im Vorjahr der Annexion fanden große Protestversammlungen statt, die auch von der regierungsfreundlichen Presse nicht ignoriert werden konnten. Die bedeutendsten hawaiischen politischen Vereinigungen, Hui Kālai'āina und die Hawaiian Patriotic League (Hui Aloha 'Āina), sandten eine gemeinsame Petition an den US-Präsidenten, in der ein Referendum der hawaiischen Bevölkerung über die bevorstehende Besitzergreifung gefordert wurde. Wie die Reise einer hawaiischen Delegation nach Washington und ein an den US-Gesandten in Hawai 'i gerichtetes Memorandum blieb auch diese Petition unbeachtet und folgenlos (vgl. Russ 1992:206-13).

${ }^{57}$ Gemeint ist die Hymne des Königreichs „Hawai`i pono“i“, deren Text König Kalākaua verfasste und deren Melodie der Hofmusikmeister und Leiter der „Royal Hawaiian Band“ Heinrich Berger (1844 geboren in Berlin, gestorben 1829 in Honolulu) komponierte. „Hawai‘i pono‘i“ dient heute als Hymne des Bundesstaates Hawaii.
} 
the ponoi, and loud weeping was the only music contributed by the natives“ (L. A. Beardslee, zit. nach Russ 1992:368-9).

\section{Politik und Wirtschaft im US-Territorium Hawai ' $i$}

Nach der formalen Übergabe der Souveränität und Gründung des USTerritoriums ergab sich eine für die hawaiische Bevölkerung durchaus positive Situation: Mit der Verabschiedung des „Organic Act“, des Grundgesetzes für das Territorium Hawai‘i, erhielten alle ehemaligen Bürger der „Republic of Hawaii“ die eingeschränkte US-Staatsbürgerschaft und somit galt für alle volljährigen Männer das uneingeschränkte lokale Wahlrecht. Schon in den Wahlen von $1900^{58}$ machte sich diese Änderung des Wahlrechts deutlich bemerkbar, als die von Liliu'okalani unterstützte „Home Rule Party“ mit dem Slogan „Nānā $i \mathrm{ka}$ 'ili“" (,Schau auf die Haut“, also: Wähle hawaiisch!) überlegen die Wahl gewann. ${ }^{59}$ Die Stimmen der hawaiischen Bevölkerung blieben noch lange Zeit wahlentscheidend:

„To a large extent, Island politics was native politics. The Hawaiians and part-Hawaiians had a clear majority of voters through the 1922 election, and more than any other group until 1938. In every election, Hawaiians and part-Hawaiians comprised more than half of the candidates for office. The Hawaiian voter turnout was substantially higher than that for other groups, including the haoles“" (Fuchs 1961:161).

Allerdings gelang es der hawaiischen Wählermehrheit nicht, diese Stimmenmehrheit in konkrete Politik umzusetzen - wichtige Gesetzesvorhaben blieben unerledigt: Zum einen soll die Uneinigkeit der hawaiischen Parlamentarier (ein bis heute immer wieder aufgegriffener Vorwurf) hierzu beigetragen haben, sicher hat aber das Vetorecht des Gouverneurs Sanford B. Dole viele Vorhaben verhindert. Auch im US-Kongress wurden Vorschläge des Delegierten aus Hawai‘'i, Robert W. Wilcox (der Anführer der Rebellion von 1893) nicht weiterverfolgt (Fuchs 1961:157-8; Andrade 1996:199-209, 223).

Einen starken und bestimmenden Einfluss auf die Politik im USTerritorium übten hingegen die Repräsentanten der großen Konzerne aus, die sich im Zuge des Wachstums der Zuckerindustrie bis 1910 in Hawai'i herausgebildet hatten. Hervorzuheben ist hierbei der überproportional große Einfluss, den die Nachfahren der nicht einmal ein Jahrhundert zuvor nach Hawai'i gekommenen protestantischen Missionare in den Aufsichtsräten der so genannten

\footnotetext{
${ }^{58} \mathrm{Zu}$ den Entwicklungen zwischen 1898 und 1900 und den politischen Aktivitäten hawaiischer Vertreter siehe Andrade 1996:181-90.

${ }^{59}$ Siehe hierzu Andrade 1996:193-8, Fuchs 1961:157 und Daws 1968:294.
} 
„Big Five“, den fünf maßgeblichen Firmen, ausübten. ${ }^{60}$ Diese ökonomisch und personell eng miteinander verwobenen Unternehmen sollten über lange Zeit das wirtschaftliche und letztlich auch das politische Leben auf den Inseln bestimmen.

„By a kind of inevitable extension they came to control ... every business associated with sugar: banking; insurance; utilities; wholesale and retail merchandising; railroad transportation in the islands; shipping between islands and between the islands and California. The agencies, established in the nineteenth century to serve the plantations, had become the tail that wagged the dog" (Daws 1968:312).

Der politische Arm dieser Oligarchie war die Republikanische Partei in Hawai'i, deren Geschichte im frühen 20. Jahrhundert mit dem Namen des hawaiischen Adligen Jonah Kūhiō Kalaniana'ole (1871-1922) verbunden ist auch dies ein Beispiel für die seit dem 19. Jahrhundert bestehenden Verflechtungen zwischen hawaiischem Adel und amerikanischen (Wirtschafts-) Interessen. Für die Partei stellte die Kooption des Prinzen, der für seine Teilnahme am Aufstand von 1895 noch inhaftiert worden war, den letztlich erfolgreichen Versuch dar, hawaiische Wählerstimmen zurückzugewinnen; Prinz Kūhiō hingegen hoffte, als Delegierter des Territoriums im US-Kongress, eine Funktion, die er von 1902 bis zu seinem Tode innehatte, eigene Ziele durchsetzen zu können (Fuchs 1961:159-60, 162; Andrade 1996:241-7).

Ein politischer Sieg des Prinzen war sicherlich die „County Home Rule“, d.h. die Schaffung von Bezirksverwaltungen, was zu einer Dezentralisierung der Verwaltung und somit zu neuen politischen Posten führte, die überwiegend von Hawaiiern eingenommen wurden. Am engsten jedoch bleibt der Name Kūhiōs bis heute mit dem Hawaiian Homes Commission Act von 1920 verbunden, der zwar Land für die Ansiedlung von Hawaiiern zur Verfügung stellte, zugleich aber den Haole die weitaus bedeutenderen wirtschaftlichen Vorteile verschaffte.

\section{Land der Hawaiier - Land für Hawaiier}

Die Entstehungsgeschichte des Hawaiian Homes Commission Act liefert ein bezeichnendes Beispiel für das zweifelhafte Bemühen hawaiischer Politiker der 1920er und 1930er Jahre, in einem von ethnischen Wahlblöcken bestimmten

\footnotetext{
${ }^{60}$ Zu den „Big Five“ zählen American Factors (Amfac, ehem. Hackfeld \& Co., die als deutsche Firma nach dem Ersten Weltkrieg enteignet wurde), Castle \& Cooke, Alexander \& Baldwin, Theo. Davies \& Co. sowie C. Brewer \& Co. Zu Entwicklung und Bedeutung siehe z.B. Kent 1983:69-91; für das Beispiel Amfac siehe Cooper u. Daws 1990:208-35.
} 
politischen Klima zwischen den Ansprüchen ihrer hawaiischen Klientel und den Zielen der mächtigen Haole-Oligarchie zu vermitteln.

Ausgangspunkt der Gesetzgebung war das ehemalige Kron- und Regierungsland, dessen Verwaltung durch die Annexion neu geregelt wurde. So heißt es hierzu in der am 7. Juni 1898 von Präsident William McKinley unterzeichneten so genannten „Newlands Resolution“61, die den Übergang der Regierungsgewalt von der Provisorischen Regierung der Republik Hawaii an die USA regelte:

„Whereas the Government of the Republic of Hawaii having, in due form, signified its consent [...] to cede and transfer to the United States the absolute fee and ownership of all public, Government, or Crown lands, public buildings or edifices, ports, harbors, military equipment, and all other public property of every kind and description belonging to the Government of the Hawaiian Islands, together with every right and appurtenance thereunto appertaining ...“ (Newlands 1898).

Gleichzeitig schränken die weiteren Bestimmungen die Verfügungsgewalt der Vereinigten Staaten über das öffentliche Land aber erheblich ein:

„The existing laws of the United States relative to public lands shall not apply to such lands in the Hawaiian Islands; but the Congress of the United States shall enact special laws for their management and disposition: Provided, that all revenue from or proceeds of the same, except as regards such part thereof as may be used or occupied for the civil, military, or naval purposes of the United States, or may be assigned for the use of the local government, shall be used solely for the benefit of the inhabitants of the Hawaiian Islands for educational and other public purposes“ (Newlands 1898).

Mit dem „Organic Act“ vom 30. April 1900, dem Grundlagenvertrag des neuen US-Territoriums, wurde der Besitztitel für das im öffentlichen Besitz befindliche Land den USA zugesprochen, während die Territorialregierung für jegliche Landgesetzgebung und Verwaltung zuständig war. Dies bedeutete zugleich, dass die Gesetze der Republik Hawaii im Wesentlichen Bestand hatten (cf. MacKenzie 1991c:15f.; Parker 1989:15; House Majority Staff Office 1993:25f.). ${ }^{62}$

\footnotetext{
${ }^{61}$ Diese Annexionsresolution, benannt nach ihrem maßgeblichen Urheber, dem Kongressmitglied Francis G. Newlands, wird in der Literatur vielfach auch als „Joint Resolution“ bezeichnet (siehe z.B. MacKenzie 1991c:15f.).

${ }^{62}$ Der besondere Charakter dieser Landflächen - bis heute als „Ceded Lands Trust“ bzw. „Public Lands Trust“ bezeichnet - blieb auch nach der vollständigen Aufnahme Hawai 'is in die USA
} 
Kūhiō und seine Anhänger, die in der „Ahahui Pu'uhonua O Nā Hawai'i“', der „Hawaiian Protective Association“ organisiert waren, legten 1920 den ersten Entwurf eines Gesetzes vor, mit dem die Rücksiedlung von Hawaiiern auf landwirtschaftlich nutzbare Parzellen des ehemaligen Kronlandes ermöglicht werden sollte. ${ }^{63}$ In dieser ersten Fassung hieß es:

„WHEREAS, there is now available or soon to become available large tracts of public lands under the control of the United States of America from which suitable areas could readily be set aside permanently as government lands subject to long term leases and renewals of leases for the encouragement of associations or colonies of individuals of Hawaiian blood for mutual growth and help to bring a rehabilitation of their race and to furnish an incentive for the preservation of the best characteristics of an independent citizenship of Hawaiian blood [...] (zitiert nach McGregor 1990:15).

Es ist hier nicht der Ort, die komplexen Interessenverflechtungen und Begleitumstände, die mit der Home Lands-Gesetzgebung verbunden waren, ausführlich darzustellen (siehe hierzu zusammenfassend Murakami 1991:43-8); und so soll es genügen festzustellen, dass der 1921 schließlich verabschiedete „Hawaiian Homes Commission Act“, der auf eine weitere Vorlage Kūhiōs zurückging, außer der tatsächlichen Bereitstellung von Land für die Ansiedlung von Hawaiiern nur noch wenig mit dem ersten Entwurf zu tun hatte (U.S. Department of the Interior 2000:32). Das Gesetz beschränkte das vergabefähige Land auf 200.000 acres (81.000 ha) minderer oder gar schlechter Güte ${ }^{64}$ und grenzte den Kreis der Berechtigten durch die Schaffung einer Kategorie „Native Hawaiians" von zumindest 50\% hawaiischer Abstammung ein (siehe hierzu Kapitel 3.2). Zugleich wurde für die Zuckerproduktion nutzbares Land von der

1959 erhalten. Der Admissions Act vom 18. März 1959 sieht die Rückübertragung dieses öffentlichen Landes an den Bundesstaat Hawai`i vor, betont zugleich dessen treuhänderische Funktion und bestimmt in Paragraph 5(f) des Vertrages: „The lands [...] shall be held ... as a public trust for the support of the public schools and other public educational institutions, fort he betterment of the conditions of native Hawaiians, as defined in the Hawaiian Homes Commission Act, 1920 [...] Such lands, proceeds, and income shall be managed and disposed of for one or more of the foregoing purposes in such manner as the constitution and laws of said State [Hawai'i; U.M.] may provide, and their use for any other object shall constitute a breach of trust for which suit may be brought by the United States“ (U.S. Government 1959). Allerdings dauerte es bis 1978, um die Treuhandschaft des Bundesstaates Hawai'i auch in der Staatsverfassung zu präzisieren.

${ }^{63}$ Ich beziehe mich im Folgenden auf die Darstellung von McGregor (1990), die auch ausführliche Informationen über den Hintergrund der Ahahui Pu'uhonua O Nā Hawai'i gibt (1990:1-5).

${ }^{64}$ So bestanden ca. 22.000 ha aus baren Lavafeldern, ca. 3.000 ha lagen auf steilen Abhängen und auf einem Großteil der Flächen fehlte der Zugang zu ausreichend Wasser (U.S. Department of the Interior 2000:32-3). 
Vergabe ausgenommen und die geltenden Restriktionen für den Erwerb der Nutzungsrechte an großen Landflächen für die Plantagen aufgehoben, so dass auch die herrschende Zuckeroligarchie in Hawai‘i das „Gesetz zur Rehabilitation der hawaiischen Bevölkerung“ als Sieg für sich verbuchen konnte.

Prinz Kūhiō, der hawaiische Initiator des ursprünglichen „Hawaiian Rehabilitation Act“ und maßgeblich beteiligt an der endgültigen Auswahl der in den Geltungsbereich der Endfassung des „Hawaiian Homes Commission Act“ einbezogenen Landflächen, trat weiterhin für das Gesetz ein - gegen skeptische Stimmen aus der hawaiischen Bevölkerung, aber auch gegen Einwände aus der Politik. So griff er im Wahlkampf auf kulturelle Vorstellungen der USamerikanischen Gesellschaft zurück, indem er eine schon von den Missionaren verbreitete Kritik an einer angeblich zu passiven hawaiischen Lebenshaltung anklingen ließ, um das Gesetz gegenüber seiner Wählerschaft zu begründen:

„Much has been said that the Hawaiians are not getting the best lands. [...] I want to tell you that Congress does not believe and never will believe as a policy in homesteading land worth from $\$ 500$ to $\$ 1,000$ an acre. That is not the American way. What made the American people great was the work of its pioneers in developing that which was worth nothing.

Too many Hawaiians have said in effect: ,Give us the best land you've got, give us all the money you can, feed us poi and fish, and we'll be happy.' I want to tell you that you never will succeed unless you get out and hustle“ (zitiert nach McGregor 1990:25).

In einer anderen Rede weist Kūhiō Kalaniana'ole auf den von ihm geschlossenen Kompromiss mit den Interessen der Zuckerindustrie und die damit einhergehende Verwässerung des Gesetzes hin: „If I were to attempt in Congress to take away cane lands for the Hawaiian people there would be a terrible row; one would never hear the last about“ (ebd.).

Trotz seiner zweifelhaften Rolle beim Zustandekommen der Gesetzgebung für die Hawaiian Home Lands wird Kūhīo Kalanianiana'ole heute von Hawaiiern gefeiert - zumal von Nutznießern des Hawaiian Homes Commission Act und den von ihm begründeten Hawaiian Civic Clubs; letztere erinnern gemeinsam mit den verschiedenen Homestead Organizations am Prince Kūhiō Day, einem staatlichen Feiertag am 26. März, mit Paraden an seine Verdienste. Für diese positive Einstellung gegenüber Kalaniana'ole und seinen Leistungen soll stellvertretend auch das folgende Zitat von Katherine Maunakea, 1907 geborene Lehrerin und Poetin und im Jahre 1993 Bewohnerin von Hawaiian Home Land in Nānākuli, stehen: 
„... it occured to me that there was no song written for Prince Kūhiō the man who was God's gift to Hawai' $i$ as our delegate to Congress for 20 years and who fought for issues favoring all of Hawai' $i$ as well as his own people. Here we were, living on this precious land he worked so hard to get for us, and I felt I just had to learn more about him. I joined the Hawaiian Civic Clubs; he was the one who started the Civic Club movement“ (Maunakea 1993:28-9).

Auch nach dem Tod Kalaniana'oles bestimmten die großen Wirtschaftsunternehmen und die Republikanische Partei die politischen Entwicklungen in Hawai'i. Wie effektiv dies geschah, zeigt z.B. die politische Bevormundung der immer größeren Zahl wahlberechtigter Plantagenarbeiter japanischer Herkunft, die aufgrund ihrer sozialen Situation der Demokratischen Partei zuneigten: ${ }^{65}$ Verwalter der Plantagen verhinderten Auftritte von Kandidaten der Demokratischen Partei in den Pflanzungen, ihre Parteigänger mussten mit dem Verlust des Arbeitsplatzes rechnen und selbst über die Stimmabgabe wachte die Oligarchie (Fuchs 1961:179-88). Die lückenlose politische Kontrolle der Plantagenarbeiter sicherte der Republikanischen Partei, die durch Haole und große Teile der hawaiischen Wahlberechtigten unterstützt wurde, auch in den Wahlbezirken mit überwiegend japanischer Bevölkerung Stimmanteile von nicht weniger als 70\%. Der Sozialhistoriker Lawrence Fuchs bringt dieses Phänomen auf den Punkt:

„Throughout the late 1920's and during the '30‘s, most of the heavily Japanese districts on the outer islands were nearly as solidly Republican as the silk-stocking haole precincts in Honolulu“ (Fuchs 1961:180).

Während der Jahrzehnte vor dem Zweiten Weltkrieg war das Territorium ökonomisch vollkommen von den USA abhängig: gingen 1914 noch 95,5\% der Exporte in die USA, waren es zwanzig Jahre später bereits 98,6\%. Bei den Importen zeigte sich ein ähnliches Bild: von 1914 bis 1934 wuchs der Anteil der aus den USA stammenden Waren von 80,4\% auf 92\% (Kent 1983:89). Und wieder war es ein US-Gesetz zum Schutz der heimischen Zuckerindustrie, das den Zuckerbaronen wie schon 1890 vor Augen führte, wie abhängig sie von Entscheidungen waren, die sie nicht beeinflussen konnten. Das „JonesCostigan“-Gesetz von 1934 stellte Hawai'i, wie auch die besetzten Philippinen und Puerto Rico, ausländischen Zuckerlieferanten gleich und senkte damit die Hawai'i zustehende Exportquote um 10\%. Es waren wirtschaftliche Interessen,

${ }^{65}$ In den zwei Jahrzehnten von 1920 bis 1940 wuchs die Zahl der Wahlberechtigten japanischer Abstammung von 658 Personen auf 27.107, d.h. 31\% aller Wahlberechtigten in Hawai‘i, an (Lind 1967:97). 
die einflussreiche Kreise nach der Annexion durch die USA nun die völlige Integration als US-Bundesstaat ins Auge fassen ließen (Daws 1968:332-3; Fuchs 1961:408-9).

\section{Hawai 'i als Bundesstaat der USA}

Bereits unmittelbar nach dem Zweiten Weltkrieg (und wohl auch unter dem Eindruck der Bedeutung, die Hawai'i im Zweiten Weltkrieg für die Amerikaner gewonnen hatte) wurde die Aufnahme Hawai'is als Bundesstaat in den Vereinigten Staaten wie in Hawai'i selbst als realistische Zukunftsperspektive des Archipels diskutiert. Allerdings gab es bis zu dem entscheidenden Referendum auch eine starke Opposition in der Bevölkerung Hawai'is, wie eine Umfrage aus dem Jahre 1959 zeigt: Ihr zufolge sprachen sich immerhin 32\% aller Hawaiier, 34\% der Haole und 37\% der Bürger portugiesischer Abstammung ${ }^{66}$ gegen den Status eines Bundesstaates aus (Fuchs 1961:413). Alice Kamokilaikawai Campbell, zu jener Zeit Senatorin im Territorialsenat, gab bei einer Anhörung des US-Kongresses 1946 eine Einstellung zu Protokoll, die vielleicht von vielen anderen Hawaiiern geteilt wurde:

„I do not feel ... [that] we should forfeit the traditional rights and privileges of the natives of our islands for a mere thimbleful of votes in Congress, that we, the lovers of Hawaii from long association with it should sacrifice our birthright for the greed of alien desires to remain on our shores, that we should satisfy the thirst for power and control of some inflated industrialists and politicians who hide under the guise of friends of Hawaii, yet still keeping an eagle eye on the financial and political pressure button of subjugation over the people in general of these islands“" (Alice K. Campbell, zitiert nach Whitehead 1993:50).

Hingegen findet sich eine deutliche Opposition - auch eine hawaiische Opposition - in den Ergebnissen des offiziellen Referendums über den Beitritt Hawai‘ is zu den Vereinigten Staaten im Jahre 1959 nur begrenzt wieder. Für die heutige Autonomiebewegung und ihre Vertreter zeigt sich hier ein nicht zu unterschätzendes Dilemma, wenn sie damit argumentiert, dass die Integration in die USA gegen den Willen der hawaiischen Bevölkerung zustande kam. Bei einer Wahlbeteiligung von $90 \%$ der registrierten Wählerschaft stimmten 132.773 für und nur 7.971 gegen die Bundesstaatlichkeit Hawai' is (Whitehead 1993:43):

\footnotetext{
${ }^{66}$ Portugiesen stellten als einzige Europäer ein signifikantes Kontingent von Kontraktarbeitern und werden aufgrund dieses sozialen/kulturellen Unterschiedes z.T. bis heute als eigenständige Kategorie in den Statistiken Hawaiis geführt.
} 
„The votes on every major island in the Territory, despite the complex ethnic tensions intertwined with the statehood issue, overwhelmingly voted yes. The final count was seventeen to one, with prostatehood victories in every representative district, and significant antistatehood sentiment expressed only in small Portuguese and Hawaiian precincts. The only one of the Islands ' 240 precincts to reject statehood was tiny Niihau, all of whose 107 registered voters were Hawaiian or partHawaiian“ (Fuchs 1961:414).

Auch die Annahme, hawaiische Gegner des Beitritts seien der Abstimmung einfach ferngeblieben, ist nicht unumstritten: zwar hatten insgesamt ca. 17\% der Wähler mit „Nein“ oder gar nicht abgestimmt, aber von diesen Wählern gehörte ein großer Teil anderen ethnischen Gruppen an. Wie Bell (1984:277) darüber hinaus feststellte, gab es die geringsten Abweichungen zwischen pro und contra (und damit auch eine große Zahl von Gegenstimmen) in den besonders wohlhabenden Bezirken O‘ahus: „Those who had profited most under territorial rule were the most reluctant to encourage its dissolution" ${ }^{67}$ Eine andere Möglichkeit, die fehlende Ablehnung der nationalen Integration durch die Hawaiier zu erklären, ziehen die Autoren Michael Kioni Dudley und Keoni Agard in Betracht- schließlich eröffneten die Fragen des Referendums der hawaiischen Wählerschaft nur begrenzte Wahlmöglichkeiten:

„One could only choose between having Statehood or continuing the American Territorial Government. It was an American question asked of Americans: ,What kind of Americanism do you chose?' There was no box for checking ,None of the above““(Dudley u. Agard 1993:74).

Mit der Aufnahme Hawai'is in die USA und dem neu gewonnenen Status eines politisch gleichberechtigten Bundesstaates entfiel auch die Beobachtung durch die Vereinten Nationen, auf deren Liste der „Non-self-governing Territories“ (abhängige Gebiete, deren Entkolonialisierung anzustreben sei) der Archipel bis 1959 geführt wurde (United Nations 2002). Die Hawaiier, vor 66 Jahren noch die Träger eines souveränen Staates, schienen auf den Status einer der Minderheiten, die das Staatsvolk des neuen Bundesstaates ausmachten, reduziert und endgültig als eigenständige politische Kraft entmachtet zu sein.

Tief greifende Veränderungen in der Politik Hawaiis zeichneten sich bereits $1954 \mathrm{ab}$, als die Stimmen der so genannten „Minderheiten“ (damit waren die Wahlberechtigten japanischer, philippinischer, chinesischer und auch hawaiischer Abstammung gemeint) der Demokratischen Partei zum Sieg in der

${ }^{67} \mathrm{Zu}$ einer historischen Betrachtung des politischen Prozesses der hawaiischen Bundesstaatlichkeit siehe Bell 1984. 
territorialen Legislatur verhalfen. Die Partei trat mit dem Vorhaben einer Bodenreform an, d.h. sie wollte Grundbesitz auch für kleine Farmer und Hausbauer ermöglichen, was eine Zerschlagung des großen Landbesitzes auf den Inseln bedeutet hätte. Eine Umsetzung der Pläne erfolgte nicht; vielmehr entwickelte sich in den Jahren nach dem Sieg der Demokraten (sie bestimmten immerhin bis zum Jahr 2002 die Politik in Hawai‘i) eine intensive „Zusammenarbeit“ zwischen demokratischen Politikern und finanzstarken Investoren (Kent 1983:160-1; Cooper u. Daws 1990:39-41, 42-85). Die bereits zu Zeiten der Republik bestehende Konzentration des Landbesitzes blieb im Wesentlichen auch nach dem Beitritt zu den Vereinigten Staaten erhalten: 1964/65 waren 487.412 ha (1.203.487 acres) oder 29,3\% der Gesamtfläche des Staates Eigentum der sieben größten Landbesitzer, 1995 waren es noch 403.207 ha (995.574 acres) bzw. 24,2\%. (State of Hawaii 1996:192). ${ }^{68}$ Zahlen aus dem Jahr 2003 weisen den acht großen privaten Landbesitzern noch $20 \%$ der gesamten Landfläche des Staates zu (State of Hawaii 2006:sec. 6, 6.07).

In den 1970er Jahren heizten die verstärkte Zuwanderung aus den kontinentalen USA, der Kapitalzufluss aus den USA und dem Ausland sowie die enorm wachsende Bedeutung der Tourismusindustrie (die zur neuen wirtschaftlichen Basis des Bundesstaates wurde) die Bodenspekulation auf den Inseln an. ${ }^{69}$ Besuchten 1965 noch weniger als 700.000 Menschen die Inseln, so waren es 1970 bereits fast 1,75 Millionen, mehr als 3,9 Millionen im Jahr 1980 und fast 7 Millionen Touristen im Jahr 1990 (State of Hawaii 1996:199). Die Ausgaben der Touristen in Hawai'i wuchsen im gleichen Zeitraum von US\$ 595 Mio. (1970) auf US\$ 9,738 Milliarden im Jahr 1990 (State of Hawaii 1996:215).$^{70}$ Der hierdurch ausgelöste Investitionsboom konzentrierte sich zunächst auf Waikīkî, das seit langem erschlossene touristische Zentrum der Insel O`ahu - doch schon bald gerieten immer neue Gebiete in das Visier der Investoren. Ein herausragendes Beispiel ist die Entwicklung der Tourismusregion von Kā'anapali auf der Insel Maui, wo schon 1969 ein erstes Hotel errichtet wurde. Zu Beginn der 1970er Jahre war bereits eine Fläche von ca. 132 ha für den Tourismus erschlossen und bis zur Mitte der 1980er Jahre hatte sich die für den Fremdenverkehr genutzte Fläche fast verdoppelt (Cooper u. Daws 1990:278-9). ${ }^{71}$

\footnotetext{
${ }^{68}$ Die Zahlen für das Jahr 1988 zeigen, dass insgesamt 1.541 .175 acres (624.176 ha) bzw. 38,2\% des Landes im Besitz des Bundes, des Staates oder der Counties waren. An Private verpachtetes Land ist hiervon bereits ausgenommen (State of Hawaii 1996:190).

${ }^{69}$ Zur Bodenspekulation in Hawai'i siehe Cooper u. Daws 1990, bes. S. 69 ff., 259 ff., 278 ff.

${ }^{70}$ Nachdem die Zahl der Touristen seit 1990 zunächst zurückgegangen war, zählte Hawaii im Jahre 2006 fast 7,5 Mio. Besucher, die mehr als 12 Milliarden US\$ ausgaben (State of Hawaii 2006:sec. 7, 7.03, 7.28).

${ }^{71}$ Die Tourismusindustrie hat die alten Hauptwirtschaftszweige der Zuckerproduktion und Ananaspflanzungen endgültig verdrängt: 2005 stand den Ausgaben der Touristen in Höhe von
} 
Durch (Zwangs-) Räumungen ganzer Siedlungen, durch Zersiedelung des Landes und durch den Bau neuer Straßen zur Erschließung immer neuer Baugebiete prägte dieser Immobilienboom das soziale und politische Klima im Hawai` $\mathrm{i}$ der 1970er Jahre. Unüberhörbar wandte sich der Protest gegen politische Repräsentanten, Investoren und Konzerne. In dieser Atmosphäre des Widerstands gegen Landnahme und Verdrängung der lokalen Bevölkerung entwickelten sich die Vorläufer des „Hawaiian Movement“, der hawaiischen Autonomiebewegung. Als eine politische Kraft, die ihre Ziele außerhalb des etablierten politischen Systems durchzusetzen versuchte, betraten 70 Jahre nach dem Sieg der Home Rule Party neue hawaiische Organisationen die politische Bühne Hawai'is. ${ }^{72}$

ca. 12 Milliarden US\$ eine Zuckerproduktion im Wert von nur noch 92,4 Millionen US\$ (in der Mitte der 1970er Jahre waren es noch fast 700 Millionen US\$) und eine Ananasernte im Wert von ca. 113 Millionen US\$ gegenüber. Ein sehr wichtiger Wirtschaftsfaktor sind auch die Ausgaben des US-Militärs, die 2004 eine Höhe von ca. 4,75 Milliarden US\$ erreichten (State of Hawaii 2006:sec. 13, 13.01).

${ }^{72} \mathrm{Zu}$ den Protesten der 70er Jahre und den Anfängen der hawaiischen Bewegung siehe Trask 1987; Dudley u. Agard 1993:107-9; Kent 1983:158-9; Ethnic Studies 1974:17-8. 


\subsection{Hawaiischer Widerstand im 19. und 20. Jahrhundert}

Petitionen und Versammlungen - hawaiischer Protest im 19. Jahrhundert Wenn der vorangegangene Abschnitt die Stationen und markanten Wendepunkte der politischen Geschichte Hawai' is im 19. Jahrhundert nachzeichnete und dabei die Reaktionen der hawaiischen Bevölkerung, wo nötig, mit einbezog, so wird der vorliegende Abschnitt sich in der Hauptsache mit diesen Reaktionen beschäftigen und die jeweiligen Formen und Abstufungen eines hawaiischen Widerstandes zum Gegenstand seiner Darstellung machen.

So ist schon früh ein passiver Widerstand der einfachen Bevölkerung der maka 'äinana gegen die ihnen von den ali' $i$ abverlangten, dem neuen kapitalistischen Wirtschaftssystem geschuldeten Arbeitsanforderungen zu verzeichnen. Aktiven Widerstand leisteten sie z.B. gegen das von ali $` i$ und Missionaren gemeinsam durchgesetzte Verbote des Hula.

Unter dem Eindruck der Verfassung von 1840 und des stets wachsenden Fremdeinflusses nahm der Protest im Jahre 1845 eine andere Form und Qualität an. An König Kamehameha III. gerichtete Petitionen, einige von mehr als 1000 Hawaiiern unterzeichnet, gaben dem Wunsch der maka 'äinana Ausdruck, den Ausländern keine weiteren Zugeständnisse zu machen. Sahlins fasst den Tenor dieser zahlreichen Petitionen zusammen: ${ }^{74}$

„From all quarters of the Islands, over mass collections of signatures, the people made pleas to the king and governing ali ${ }^{\prime} i$, sometimes with great eloquence, rehearsing especially a triad of objections: to the presence of foreigners in government, to the sale of land to foreigners, and to the naturalization of foreigners as Hawaiian citizens" (Sahlins 1992:130).

Wie Osorio (2002:31) feststellt, sprach aus all diesen Gesuchen vor allem die Sorge der maka 'äinana, durch die von König und Adel unter Anleitung der Missionare betriebene Politik ihre traditionellen Landrechte zu verlieren.

Fast ein halbes Jahrhundert später führte der breite Protest gegen die unter Androhung von Waffengewalt erzwungene Verfassung zur Gründung der Hui Kālai'āina, an deren erster öffentlicher Zusammenkunft bis zu 1.500 Menschen teilgenommen haben sollen. Als politische Partei der Hawaiier gewann

\footnotetext{
${ }^{73} \mathrm{Zu}$ diesen frühen Widerstandsphänomenen in Hawai‘i siehe Ralston (1984:33-6) und Sahlins (1992:108, 113).

${ }^{74}$ Für Beispiele dieser Petitionen von 1845 siehe McGregor (2007:197-8), Silva (2004:38-9), Osorio (2002:30-1) und Sahlins (1992:130-1); auch Kuykendall (1947:257-60) und Ralston (1984:35-6).
} 
Hui Kālai ‘aina auch die Legislatur-Wahlen von 1890, bei denen es ihr in der Haupsache um den Erhalt der Monarchie und die Stärkung der hawaiischen Wählerschaft ging (Silva 2004:127). Hui Kālai‘āina blieb über den Sturz der Monarchie hinaus ein Sprachrohr hawaiischer Interessen - auch wenn sie durch die jeweils geltenden parlamentarischen Regeln und interne Differenzen nur selten in der Lage war, diese durchzusetzen.

Im Streit gegen die unpopuläre Verfassung kam es auch zu einem ersten Versuch hawaiischer Nationalisten, ihre Ziele mit Waffengewalt zu erzwingen. ${ }^{75}$ Am 30. Juli 1889 besetzten bewaffnete Widerständler unter Führung von Robert Kalanihiapo Wilcox den 'Iolani Palast in Honolulu, bevor angelandete US-Truppen sie überwältigten. Dabei fanden acht der Aufständischen den Tod, 12 wurden verwundet und insgesamt 70 wegen Verrat, Verschwörung oder Rebellion angeklagt. Nur drei der Angeklagten wurde tatsächlich der Prozess gemacht: unter ihnen auch Wilcox, der jedoch von einem hawaiischen Geschworenengericht freigesprochen wurde. ${ }^{76}$ Auffällig ist die fehlende breite Unterstützung des Aufstandes durch die hawaiische Bevölkerung, was McGregor-Alegado in ihrer Analyse des Widerstandes gegen die Verfassung von 1887 zu erklären sucht:

„The ,Rebellion“ of 1889 must be viewed as one event in a continued process of Hawaiian resistance to the erosion of both their culture and their political power. Seen in this light, it illustrates some of the main obstacles in that movement. Grass roots political activism was not a part of the Hawaiian tradition, and its lack left the ,rebels' of 1889 helpless in the face of what was, after all, a comparatively small, but determined force opposing them. Mistrust of self-promoting leaders may also have played a part in keeping potential supporters of the uprising from uniting around Wilcox and his companions. Kalākaua like his predecessors on the throne, had made his terms with the foreigners and had a stake in the continuation of this arrangement ... Finally, the monarchy failed to gain support for its defense except a small sector of middle and upper middle class Hawaiians. This may have stemmed from the failure of past monarchs to protect the interests of the Hawaiian people“ (McGregor-Alegado 1979:127).

\footnotetext{
${ }^{75}$ Ein zweiter, ebenfalls erfolgloser Versuch fand 1895 mit dem Ziel statt, die abgesetzte Königin wieder an die Macht zu bringen.

${ }^{76}$ Es fällt auf, dass sowohl hier wie auch bei den Prozessen nach dem missglückten Versuch Wilcox' von 1895 die Königin wieder an die Macht zu bringen, hawaiische Gerichte noch sehr wohl in der Lage sind, auf die Bestrafung dieser gegen den herrschenden Status Quo gerichteten Taten zu verzichten. Inwieweit dies ein Spezifikum der damaligen staatlichen Situation in Hawai'i oder ein Beispiel für die Konfliktbewältigung in kleinen Gesellschaften ist, muss hier offen bleiben.
} 
Der hawaiische Widerstand blieb im Wesentlichen gewaltfrei und gewann in der kurzen Regierungszeit Lili‘uokalanis beständig an Kraft. Nur kurze Zeit nach ihrem Sturz gründeten sich zwei weitere hawaiische politische Organisationen: die Hui Hawai'i Aloha 'Āina und die Hui Hawai'i Aloha 'Āina o Nā Wāhine, in der sich hawaiische Frauen zusammengeschlossen hatten. Die im hawaiischen Namen der Organisationen enthaltene Wendung „Aloha 'Äina“, „Liebe zum Land“, drückt eine Vorstellung aus, die sich in vielen Sprichwörtern ('Ōlelo No 'eau) wieder findet und die erst zum Ende des 19. Jahrhunderts im Englischen mit „,patriotic“ übersetzt wird. ${ }^{77}$ Die beiden genannten Organisationen werden denn auch sowohl in der zeitgenössischen als auch in der aktuellen Literatur mit dem Namen „Hawaiian Patriotic League“ bezeichnet. Noenoe Silva (2004:131-2) geht in ihrer Studie des hawaiischen Widerstands im 19. Jahrhundert auf diese Namensgebung ein und weist auf die sehr unterschiedlichen Konnotationen der beiden Begriffe „Aloha 'Äina“ und „patriotic“ ${ }^{78}$ sowie die enge Beziehung des hawaiischen Terminus zu traditionellen kosmologischen Vorstellungen hin. In der Wendung „ka poe aloha 'äina“ (,the people who love the land") wurde der Begriff „Aloha 'Āina“ auch für die Verteidiger und Verfechter der Souveränität des hawaiischen Königreichs verwendet und ist heute in seinen unterschiedlichen, bisweilen unscharfen Bedeutungen im Kontext der Autonomiebewegung weiterhin in Gebrauch.

Nach Gründung der Republik verlagerten sich die Äußerungen des Widerstandes zu einem großen Teil in die hawaiischsprachige Presse und in der Folge wurden Herausgeber mit Strafverfahren überzogen und einige Zeitungen mussten gar ihr Erscheinen einstellen. Allein schon die hawaiische Sprache war inzwischen zu einem Medium des Widerstandes geworden: wie Chapin (1996:99) feststellt, verzichteten die Zeitungen der Opposition zunehmend auf englische Texte und erschienen schließlich fast ausschließlich in hawaiischer Sprache, die ihre Bedeutung als offizielle Sprache gegen Ende des 19. Jahrhunderts bereits verloren hatte. Die Zeitungen veröffentlichten auch die Lieder (mele), die den missglückten Aufstand von 1895 feierten und die zum Ende des Jahres 1895 im „Buke Mele Lāhui““79 gesammelt erschienen. In den mele äußerte sich ein wachsender Nationalismus, der vor dem Hintergrund einer Konfrontation von Royalisten und Befürwortern der Annexion in der politischen Diskussion immer deutlicher an Boden gewann (Stillmann 1989:19).

Im zeitlichen Vorfeld der Annexion protestierten die politischen Vertreter der hawaiischen Bevölkerung auf das schärfste gegen die Pläne der „Republik Hawai'i“‘. So riefen die großen Organisationen zu Versammlungen auf,

\footnotetext{
${ }^{77}$ Cf. Pukui u. Elbert (1986:21); Pukui, Elbert u. Mookini (1974:268-9).

${ }^{78}$ So ist der hawaiische Begriff im Gegensatz zu seinen europäischen Analogien nicht geschlechtsbezogen und hat darüber hinaus eine völlig andere Herleitung, die sich nicht auf eine staatliche Organisationsform bezieht.

79 „Buch der Lieder der Nation“ [U.M.]
} 
an denen tausende teilnahmen. Und wieder unterzeichneten viele tausend Hawaiier eine Petition der Hui Aloha 'Āina, die dem US-Kongress von einer Delegation überbracht wurde. Sie beinhaltete die Forderung nach einem Referendum unter der hawaiischen Bevölkerung - die Regierung einer kleinen Minderheit habe nicht das Recht, über die Souveränität Hawai‘is zu entscheiden, so die Verfasser. Die Vorsitzenden der Hui Aloha 'Āina sandten auch ein Memorandum an den US-Gesandten in Hawai' $i$, in dem es u.a. heißt:

„The Declaration of American Independence expresses that Governments derive their just powers from the consent of the governed; Therefore, Be it RESOLVED: That as the representatives of a large and influential body of native Hawaiians, we solemnly protest against annexation in the manner proposed and without reference to the consent of the people of the Hawaiian Islands“ (zitiert nach Russ 1992:364-5).

Wie der Wortlaut dieses Zitates, die Geschichte der Petitionen und Versammlungen sowie auch die interne Struktur von Organisationen wie Hui Kalai‘āina und Hui Aloha 'Āina zeigen, waren Forderungen und Formen des Widerstandes - sieht man einmal von den an die hawaiische Bevölkerung gerichteten mele $\mathrm{ab}$ - an westlichen Rechts- und Politikvorstellungen orientiert. ${ }^{80}$ Dies kann angesichts einer mehr als 70-jährigen Über- und Umformung zumindest der in den Städten lebenden hawaiischen Gesellschaft durch euroamerikanische Normen nicht wirklich verwundern. Auch die ehemalige Königin Lili‘uokalani, die mit einer Delegation nach Washington und Boston gereist war, um Unterstützung gegen die Annexion Hawai' is zu gewinnen, präsentiert sich im Rahmen der amerikanischer Konventionen als gebildete und wohlhabende Frau - nicht zuletzt um den von der US-Presse geschürten Vorurteilen über die hawaiische Bevölkerung und ihre Königin entgegenzuwirken. ${ }^{81}$

Für die heutige Autonomiebewegung ist die von den Politikern und Politikerinnen des 19. Jahrhunderts geleistete Arbeit insoweit von bleibender Bedeutung, als völkerrechtliche wie auch im Rahmen nationaler Gesetze einklagbare Ansprüche auf Eigenständigkeit und Souveränität weiterhin Bestand haben. Auch wenn die ablehnende Haltung der hawaiischen Bevölkerung nicht in Statistiken erfasst wurde, so zeigen doch die Reaktionen ihrer maßgeblichen Vertreter eine durchgängige und deutliche Ablehnung aller von Haole und den

\footnotetext{
${ }^{80}$ Silva (2004:162) weist darauf hin, dass sich fast alle Quellen auf das Geschehen in Honolulu sowie einigen anderen größeren Orten in Hawai‘i beziehen; wie (und ob) sich der hawaiische Widerstand in den eher traditionell geprägten ruralen Gebieten des Archipels äußerte ist bisher noch nicht erschlossen.

${ }^{81}$ Zum Aufenthalt Lili‘uokalanis in den USA und den von der Presse vermittelten Bildern siehe ausführlich Silva (2004:164 ff.).
} 
USA eingeleiteten Schritte zur Einschränkung und letztlich Aufhebung der Souveränität der Monarchie und des hawaiischen Staates.

1998 jährte sich das Datum der Annexion zum 100. Male. Aus diesem Anlass befasste sich „Self-Determination“, der Newsletter des Kanaka Maoli Tribunal Kōmike (eine Gruppierung der heutigen Autonomiebewegung), mit dem Widerstand der Jahre 1897 bis 1898 und schrieb in seinem Aufmacher:

„After 100 years, our people are still fighting U.S. colonialism ... This issue of Self-Determination is a tribute to our Kanaka Maoli people's resistance and our continuing struggle against those who deny our rights to land, self-determination and sovereignty“ (Self-Determination, March 1998).

Nach der Annexion verlagerten sich Protest und hawaiische Politik in die Strukturen der Legislative und der Verwaltung des Territoriums, ein politischer Widerstand wie in den Jahren zuvor ist nicht mehr dokumentiert. ${ }^{82}$ Erst zum Ende der 1960er Jahre regte sich wieder öffentlicher Protest und eine hawaiische Bewegung formulierte erneut Forderungen nach kultureller Eigenständigkeit und politischer Autonomie. Im Folgenden werde ich die Entwicklung dieser Bewegung, die sich selbst durchaus in der Tradition des Widerstandes im 19. Jahrhundert sieht, nachzeichnen.

\section{(Neu-) Anfänge in den 1970er Jahren}

Schon seit den 1960er Jahren lenkte die Revitalisierung hawaiischen Handwerks und hawaiischer Künste den Blick auf die Hawaiier und ihre vielfach vergessen geglaubten Traditionen. Diese „Hawaiian Renaissance“ war zugleich Resultat und Inspiration eines erneuerten Selbstbewusstseins der hawaiischen Minderheit, das sich in den von sozialen Umbrüchen gekennzeichneten 1970er Jahren zunehmend in politischen Forderungen äußerte.

Große Teile der Bevölkerung des Bundesstaates profitierten zu Beginn dieses Jahrzehnts noch einmal besonders von einem „Wirtschaftswunder“, das sich auf die ökonomischen Umstrukturierungen im Zuge der Staatswerdung 1959 zurückführen ließ. Andere, unter ihnen der größte Teil der Hawaiier, blieben in der sozialen und ökonomischen Entwicklung der Inselgesellschaft immer weiter zurück - auch das „Neue Hawai' i“ (Kent 1983:3) hatte sich nicht von einer ethnischen Stratifizierung befreit, die von den aufeinander folgenden Einwanderungsphasen des 19. Jahrhunderts geprägt war und die das soziale Leben seit den 1950er Jahren weiterhin bestimmte (cf. McGregor-Alegado

\footnotetext{
${ }^{82}$ Inwieweit hier eventuelle neuere historische Forschungen weitere Erkenntnisse hinsichtlich einer Fortdauer des außerparlamentarischen hawaiischen Widerstandes in der Zeit zwischen Annexion und Aufnahme in die USA liefern können, bleibt abzuwarten.
} 
1980:34). Nutznießer der gewandelten Verhältnisse waren wiederum die Haole und die seit den Tagen des Territoriums in der sozialen Schichtung der Gesellschaft Hawai'is aufgestiegenen Bürger chinesischer, japanischer und koreanischer Abstammung, während ein großer Teil der Hawaiier, aber auch der Filipinos und Samoaner von den ökonomischen Entwicklungen kaum profitierten.

Insbesondere der immense Landbedarf der Tourismus- sowie der Immobilienindustrie sollte zu Zusammenstößen zwischen Industrievertretern und staatlichen Ordnungshütern auf der einen und betroffenen Landnutzern auf der anderen Seite führen. Letztere fanden Unterstützung bei einem großen Protestpotential, das weit über den Kreis der unmittelbar von Kündigungen und Landnahme Betroffenen hinausreichte - die bedeutenden sozialen Bewegungen in den USA der 1950er und 1960er Jahre, die Bürgerrechtsbewegung, die AntiVietnamkriegsbewegung sowie die Bewegungen der Native Americans und der Afroamerikaner, waren auch in Hawai'i nicht ohne Resonanz geblieben.

Eine der frühesten und zugleich bedeutendsten Auseinandersetzungen im Kontext der Umnutzung von Land war der 1970 beginnende Konflikt um die Räumung und anschließende Neubebauung von Siedlungsland im Kalama Tal an der Südostspitze der Insel O`ahu - ein Konflikt, dem verschiedene Autoren eine Katalysatorfunktion für die Entwicklung einer politischen Bewegung der Hawaiier zusprechen. ${ }^{83}$ Das gesamte Kalama Valley gehörte zum Grundbesitz des Bishop Estate. Die Tatsache, dass der Bishop Estate, durch die testamentarischen Verfügungen einer Urenkelin Kamehamehas I. errichtet und mit dem alleinigen Stiftungsziel der Ausbildung hawaiischer Kinder und Jugendlicher, als grundsätzlich hawaiische Institution galt (s. Kau 1991:281-4), trug zu dem besonderen Charakter dieses Landkonfliktes bei.

Noch vor der ersten Räumungsaktion im Kalama Valley gründete sich im Umfeld der Universität von Hawai'i in Mānoa das Kōkua Kalama Committee ${ }^{84}$, dessen Mitglieder überwiegend hawaiischer Abstammung waren. Wie Haunani-Kay Trask schreibt, war die Ausweitung des Konfliktes im politischen Umfeld der beginnenden 1970er Jahre durchaus vorhersehbar:

„The historic link between campus activists and a grass-roots community could have been predicted by any keen observer of the time. On the verge of victory in their anti-war offensive, students had already made the connection between American cultural, political, and economic exploitation of Third World People abroad and the same kind of exploitation of local people of color at home. All that was missing was a community“ (H.-K. Trask 1987:133).

\footnotetext{
${ }^{83}$ Zu nennen sind hier vor allem H.-K. Trask (1987), die Autoren des Textes Ethnic Studies 221 (1974) sowie Milner (2006), auf die ich mich im Folgenden beziehe.

${ }^{84}$ Kōkua: Hilfe, Unterstützung [U.M.].
} 
Wie Trask hier feststellt, spielten zu diesem frühen Zeitpunkt Aspekte der Ethnizität keine oder nur eine untergeordnete Rolle. Vielmehr forderte die Konstellation dieses Konfliktes - hier der mächtige Bishop Estate im Bunde mit finanzkräftigen US-Investoren, dort die machtlosen Vertreter eines immer mehr der Vergangenheit zugehörigen ländlichen Lebensstils - die Assoziation mit dem Kampf gegen die US-amerikanischen Hegemonialbestrebungen geradezu heraus.

„With Kalama Valley, you really had a landmark struggle. You had the antiwar movement, SDS [Students for a Democratic Society; U.M.], The Resistance, Youth Action, all now looking at this small valley across from Sandy Beach, on 'Ehukai Road. Bishop Estate - a huge landowner, allegedly acting for Native Hawaiians, but actually an instrument of colonialization - was letting Kaiser, a major company, have that land to develop into affluent housing. Because Kaiser got major defense department contracts, the antiwar movement saw that taking Kaiser on in Kalama Valley, where they were destroying the lifestyle of yet another people, was a very important way of connecting issues“ (John Witeck, in: Mast u. Mast 1996:345). ${ }^{85}$

Nach verschiedenen aufeinander folgenden Räumungsaktionen verschärfte sich der Konflikt im Frühjahr 1971 mit der Besetzung des Tales, in dem nur noch sechs der ursprünglichen Bewohner ausharrten, durch verschiedene Unterstützergruppen. Tonangebend war hierbei das frühere Kōkua Kalama Committee, das sich inzwischen in Kōkua Hawai' $i$ umbenannt hatte: ein bezeichnender Hinweis auf die weiter gefasste Bedeutung, die der Auseinandersetzung im Kalama Valley inzwischen beigemessen wurde. Auch an der Besetzung beteiligt waren „The Hawaiians“, eine gleichfalls 1970 gegründete Gruppierung mit einer ausschließlich hawaiischen Mitgliedschaft. ${ }^{86}$ Als Kōkua Hawai' i schließlich alle an den Protesten beteiligten Haole aufforderte, das Tal zu verlassen, bedeutete dies jedoch noch keine Hawaiisierung des Konfliktes: vielmehr war dies Ausdruck einer kulturellen Differenzierung zwischen Haole auf der einen und den „Locals“, den Angehörigen der unterprivilegierten ethnischen Gruppen, auf der anderen Seite. ${ }^{87}$ Diese Abweisung der euro-amerikanischen Mitstreiter sollte die Ablehnung des mit ihnen assoziierten „weißen“ US-

\footnotetext{
${ }^{85}$ John Witeck, Assistant Director der Gewerkschaft United Public Worker Hawai ‘i, war zur Zeit der Kalama Valley-Proteste Leiter der Youth Action, einer Organisation in kirchlicher Trägerschaft, die zur „Antiwar“-Bewegung gehörte und soziale Projekte unterstützte.

${ }^{86}$ „The Hawaiians“ verfolgten hauptsächlich das Ziel, hawaiische Belange in der Politik des Staates deutlicher hervorzuheben sowie die staatliche Vergabe von Siedlungsland an Hawaiier zu beschleunigen (Ethnic Studies 221 1974:19-25).

${ }^{87}$ Zum Konzept der sozialen und kulturellen Kategorie „Local“ in Hawai‘i siehe Kapitel 3.3.
} 
amerikanischen Lebensstils, zu dem eben auch die geplante Eigenheimsiedlung gehörte, verdeutlichen und zugleich die mediale Aufmerksamkeit auf die „traditionelle“, mit der Natur und Einfachheit assoziierte Lebensweise der sozial benachteiligten lokalen Bevölkerung lenken (cf. H.-K. Trask 1987:146).

In den Auseinandersetzungen um die Landnutzung im Kalama Valley, die letztlich im Sinne der Landeigner und Investoren entschieden wurden, klingen bereits die wesentlichen Themen einer hawaiischen Bewegung an, die sich im Laufe der 1970er Jahre immer deutlicher aus dem Spektrum der gegen das politische und soziale Establishment gerichteten Gruppierungen herauskristallisierte:

- Eine starke Konzentration auf das Land und seine Nutzung, aus der sich in der Folgezeit eine immer deutlichere Forderung nach hawaiischer Autonomie und Souveränität entwickeln sollte;

- eine betonte Abgrenzung von den Haole und zunehmend auch von der nicht-hawaiischen lokalen Bevölkerung, d.h. die Entwicklung einer ethnisch geprägten Bewegung und die Ethnisierung eines als grundsätzlich empfundenen Konfliktes zwischen den wirtschaftlichen Interessen einer kleinen Minderheit und der Mehrheit der Bevölkerung in Hawai'i;

- das Beharren auf den überkommenen Vorrechten der Hawaiier als ersten Bewohnern des Archipels und Nachfahren der Bürger des souveränen Königreichs Hawai‘i;

- und schließlich der am Beispiel Kalama Valley bereits deutlich zutage tretende Konflikt zwischen Hawaiiern unterschiedlicher Lebenssphären, der mit der wachsenden Ausdifferenzierung der Bewegung noch zunehmen wird.

Diesen letzten Punkt fasst Haunani-Kay Trask noch einmal prägnant am Beispiel des Kalama Valley-Konfliktes zusammen:

„The painful experience of class distinctions became the agonizing reality of class confrontation within the Hawaiian community itself. Not only was the Bishop Estate perceived as a Hawaiian trust evicting Hawaiian people, but the individuals who drove the bulldozers and carried away the ,trespassers“ were Hawaiian“ (H.-K. Trask 1987:143).

Vergleicht man die beiden hier genannten Gruppierungen Kōkua Hawai'i (bzw. Kōkua Kalama) und „The Hawaiians“, so fallen wesentliche Unterschiede auf: bei der erstgenannten handelt es sich um eine zwar von Hawaiiern geprägte, aber hinsichtlich ihrer Anhängerschaft um keine spezifisch hawaiische Organisation, während letztere eine ausschließlich hawaiische Mitgliedschaft hatte und sich mit „hawaiischen“ Themen wie der Wohnungsnot der Hawaiier und 
hawaiischer Selbstbestimmung befasste. „The Hawaiians“, in Waimānalo, einer überwiegend von Hawaiiern bewohnten Siedlung auf der Nordseite O`ahus gegründet, zählte darüber hinaus zu ihren Mitgliedern überwiegend „lowincome, disadvantaged and powerless Hawaiians" (Ethnic Studies 221 1974:21), hingegen rekrutierten sich die Anhänger von Kōkua Hawai'i überwiegend aus dem akademischen Umfeld der University of Hawai' $i$ in Honolulu. McGregor-Alegado (1980:44) bezeichnet denn auch „The Hawaiians“ als „the first Hawaiian political organization of the seventies". ${ }^{88}$

Die Protestbewegung entwickelte sich in der ersten Hälfte der 1970er Jahre analog zu den bereits genannten Gruppierungen weiter: Neben Protestgruppen, die an konkrete Ereignisse und Gemeinden gebunden waren, in der Regel relativ eng umrissene Ziele verfolgten und häufig über eine multiethnische Migliedschaft verfügten, entstanden weitere Gruppierungen mit hawaiischer Mitgliedschaft, die umfassendere politische Ziele auf der Basis ethnisch und historisch begründeter Rechte der Hawaiier auf Land verfolgten. ${ }^{89}$ Die bereits mehrfach zitierte Vordenkerin der Bewegung, Haunani-Kay Trask, schreibt hierzu: „This call for land arises out of an understanding of the native claims of Hawaiians as the indigenous people of Hawaii ... The rights of ,locals" were not thereby opposed. But Hawaiians ' historic and cultural claims to the land as the first and original claimants were increasingly seen, at least by Hawaiians, as primary“ (H.-K. Trask 1984:121-2 [Hervorhebungen im Original]).

Ein Jahr nach der Gründung von Kōkua Kalama und „The Hawaiians“ wurde 1971 der Congress of Hawaiian People ins Leben gerufen. ${ }^{90}$ Seine Mitglieder gehörten überwiegend dem hawaiischen Mittelstand an und sahen ihre Aufgabe zunächst vor allem in der Lobbyarbeit für die Ernennung eines hawaiischstämmigen Treuhänders des Bishop Estate (Kamehameha Schools), dessen bisherige Treuhänder sich nicht unbedingt durch Engagement für die hawaiische Minderheit ausgezeichnet hatten, sowie in der Ausweitung hawaiischen Einflusses auf die Innenpolitik des Bundesstaates. Weitere Gründungen politischer Aktionsgruppen mit ähnlichen Zielen erfolgten 1973: zu nennen sind das Homerule Movement, dessen Name sich auf die gleichnamige hawaiische Partei des 19. Jahrhunderts bezieht, und die Hawaiian Coalition, ein in-

\footnotetext{
${ }^{88}$ Ausführlich zu „The Hawaiians“ siehe Ethnic Studies 221 (1974:19-25).

${ }^{89}$ Im Folgenden verzichte ich weitestgehend auf eine Darstellung lokaler Protest- und Widerstandsgruppen; es sei hier aber darauf hingewiesen, daß die Bedeutung dieser Proteste für die Entwicklung der politischen Bewegung gerade in den 70er Jahren nicht unterbewertet werden darf. In einer Aufstellung von „Native Hawaiian Struggles“ listet Minerbi (1994) für die Zeit von 1970 bis 1980 allein auf O`ahu acht bedeutende Protestereignisse auf.

${ }^{90} \mathrm{Zu}$ dieser und den im Folgenden genannten Organisationen siehe Ethnic Studies 221 (1974); McGregor-Alegado (1980:44 ff.); H.-K. Trask (1984:122-3).
} 
formeller Zusammenschluß verschiedener kleinerer Gruppen, deren Bestreben die Förderung des politischen Bewusstseins der hawaiischen Bevölkerung war. 1971 verabschiedete der US-Kongress den „Alaska Native Claims Settlement Act (ANCSA)“, der die Ansprüche der indigenen Bevölkerung Alaskas auf das Land des Bundesstaates abschließend regelte. ${ }^{91}$ In Hawai`i war die Gründung einer Gruppierung mit dem eingängigen und bezugreichen Akronym A.L.O.H.A. (Aboriginal Lands of Hawaiian Ancestry) ein Echo dieser Gesetzgebung, verfolgte A.L.O.H.A. doch als erste hawaiische Organisation das konkrete Ziel einer Reparationsleistung der Vereinigten Staaten an die Hawaiier. Diese Zahlung sollte den Landverlust durch die von der Republik Hawaii veranlassten Abtretungen an die US-Regierung (Ceded Lands von 1898) entschädigen. Auf Betreiben von A.L.O.H.A. brachten die Abgeordneten des Bundesstaates Hawai'i im US-Repräsentantenhaus 1974 eine so genannte „Native Hawaiian Claims Bill“ in den amerikanischen Kongress ein, der allerdings kein Erfolg beschieden war. ${ }^{92}$

Nach mehreren erfolglosen Vorlagen des Gesetzentwurfs gaben der Abgeordnete Spark Matsunaga und der US-Senator für Hawai‘ $i$, Daniel Inouye, den Anstoß für die Einrichtung der „Hawaiian Native Claims Settlement Study Commission“ zur Klärung der hawaiischen Entschädigungsansprüche durch den US-Kongress - diesen Wechsel der Initiative in die allgemeine, nicht explizit hawaiische Politik unterstützte A.L.O.H.A. zunächst nur zögerlich. 1980 verabschiedete der Kongress dann unter Präsident James Carter die notwendigen Gesetze zur Errichtung der „Native Hawaiians Study Commission“, deren Personal von der Nachfolgeregierung unter Ronald Reagan allerdings vollständig umbesetzt wurde. Der 1983 veröffentlichte Abschlussbericht der Kommission kam zu keinem gemeinsamen Ergebnis - die drei aus Hawai‘i stammenden Kommissionsmitglieder (Kina'u Boyd Kamali‘i, Winona K. Beamer und $\mathrm{H}$. Rodger Betts) verfassten einen Minderheitsbericht (insgesamt verfügte die Kommission über neun Mitglieder), der in grundsätzlichen Fragen vom Mehrheitsvotum $^{93}$ abweicht (Parker 1989:136-7; House Majority Staff Office 1993:15-7). In ihrer Einleitung zu dieser „Dissenting Study“ schreiben sie:

\footnotetext{
${ }^{91}$ Insgesamt wurde der indigenen Bevölkerung Alaskas durch das Gesetz ein Neuntel der Gesamtfläche des Staates (16,2 Mio. ha), eine einmalige Zahlung von US\$ 962.500.000 sowie die Zahlung einer Abgabe in Höhe von 2\% auf die Gewinne aus der Ausbeutung von Bodenschätzen und der Nutzung von Land des Bundes zugesprochen. Die Besitztitel an dem Land werden von insgesamt 12 regionalen und 200 kommunalen Körperschaften gehalten; zur Geschichte des ANCSA, der eine gewisse Vorbildfunktion für die Meinungsbildung in Hawai'i hatte, siehe Parker (1989:142-6) sowie University of Alaska Anchorage Justice Center (2001), Alaska Native Knowledge Network (2006) und Landye Bennett Blumstein (2007).

${ }^{92}$ Der Entwurf verlangte jährliche Zahlungen von US\$ 100 Mio. über einen Zeitraum von 10 Jahren an eine neu zu schaffende „Hawaiian Native Corporation“, die das Geld für die Hawaiier verwalten sollte (Parker 1989:135-6).

${ }^{93}$ Native Hawaiians Study Commission (1983a)
} 
„Lacking the needed critical and probing analysis, the findings and conclusions contained in Volume I argue that the United States bears no legal or moral responsibility or culpability for the actions of American officials at that time [1893; U.M.].

WE DISAGREE.

Further, Volume I asserts that, regardless of this rejection of legal or ethical accountability by the United States, that [sic!] Native Hawaiians were not deprived of any compensable interest represented by the Kingdom of Hawai' $i$. Thus, even if the United States were to admit any responsibility for the overthrow of the Kingdom of Hawai' $i$ - a position which they deny - then there would still be no basis for claims by Native Hawaiians.

AGAIN, WE DISAGREE.

Moreover, the majority of the Commission's membership is of the opinion that departures from standards of proper international behavior or of traditional assent to transfers of land and sovereignty - as understood and practiced in American precedents - also do not bear any violation or abridgement of Native Hawaiian interests or rights.

WE DISAGREE“ (Native Hawaiians Study Commission 1983b).

Sie benennen hier einen der problematischsten Punkte im Verhältnis zwischen hawaiischer Bevölkerung und der Regierung der Vereinigten Staaten, die anders als z.B. im Falle der Native Americans in den kontinentalen USA oder der indigenen Bevölkerung Alaskas - nach wie vor keine Sonderbeziehung zu den Hawaiiern und daraus resultierende Verpflichtungen und Rechtsansprüche anerkennt. Hierbei steht insbesondere die Frage nach der Anerkennung einer offiziellen Beteiligung der USA am Umsturz von 1893 zur Diskussion, die auch durch die von Präsident Clinton 1993 unterzeichnete so genannte „Apology Resolution“ (s.u.) letztlich nicht beantwortet wird. Die Berichte bilden einen vorläufigen Abschluss der Bemühungen um Anerkennung und Entschädigung durch die Bundesregierung. Erst im Jahr 2000 bemühte sich der US-Senator Daniel Akaka erneut, eine unter hawaiischen Vertretern umstrittene „Federal Recognition“ zu erreichen. ${ }^{94}$

Als erste hawaiische Gruppierung setzte sich 'Ohana O Hawai'i, 1974 von Peggy Ha'o Ross gegründet, das Ziel der Wiedererrichtung des Hawaiischen Königreichs und nahm zugleich für sich in Anspruch, dessen einzige

\footnotetext{
${ }^{94}$ Die Organisation A.L.O.H.A., die zwar viele Mitglieder, aber wenig Aktive zählte, löste sich, als die Initiatorin Louisa K. Rice Hawai`i verließ, faktisch auf. 1994 rief Louisa Rice DeMello A.L.O.H.A. wieder ins Leben, wobei die Gruppierung allerdings keine Entschädigungsforderungen mehr erhob, sondern die Restitution von Land verlangte („Land-rights group ALOHA revived by its founder“; Mark Matsunada [HA 21.11.1994:A3]).
} 
rechtmäßige Vertretung zu sein. 1980 erklärte Ross schließlich einseitig die Unabhängigkeit Hawai'is von den USA, zur Untermauerung dieses Anspruchs hatte die Organisation ihre Forderungen sowohl bei den Vereinten Nationen als auch beim Internationalen Gerichtshof in Den Haag eingebracht - die allerdings, genauso wie die USA selbst, nicht darauf reagierten (Heckathorn 1988:58).

Schon früh wurde die unbewohnte Insel Kaho'olawe, die kleinste der acht Hauptinseln des Archipels, von den neu entstehenden hawaiischen Gruppierungen als Symbol des Widerstands genutzt: seit 1941 von der US Armee (später der US Navy) verwaltet und zunächst als Truppenübungsplatz, dann als Zielort für Bombenabwürfe genutzt, bot Kaho'olawe bereits für A.L.O.H.A. eine Möglichkeit, Aufmerksamkeit für ihre Reparationsforderungen zu erreichen, während die Landrechtsorganisation Hui Ala Loa von der Nachbarinsel Moloka'i die Zerstörung der Insel beendet sehen wollte. Mit der Protect Kaho'olawe 'Ohana (PKO) entstand 1976 dann ein neuer Typ hawaiischer Gruppierungen: Als erste in der Reihe der Neugründungen verband die PKO in ihrem Programm klare politische Forderungen - die Rückgabe der Insel Kaho'olawe an die Hawaiier - mit konkreten Vorstellungen „hawaiischer Werte“ - so z.B. „Aloha 'Āina“ - und kontrastierte letztere mit „westlichen“, destruktiven Landnutzungsvorstellungen wie sie Kaho'olawe drastisch vor Augen führte (H.-K. Trask 1984:123). Die intensiven Bemühungen der PKO führten schließlich 1980 zur Beendigung der Bombenabwürfe und ein Jahrzehnt darauf zur Einrichtung der Kaho'olawe Island Conveyance Commission und der Rückübertragung der Insel an den Staat Hawai'i (Protect Kaho'olawe 'Ohana 1998).

Diese kurze Vorstellung der wichtigsten politischen Gruppierungen der 1970er Jahre sowie die große Zahl der hier nicht berücksichtigten lokal orientierten Landrechtsgruppen (siehe hierzu Minerbi 1994) zeigt die wachsende Wahrnehmung eigener Rechte durch die hawaiische Minderheit. Dennoch kommt es, trotz der frühen Zusammenarbeit verschiedener Protest- und Lobbygruppen in Bündnissen wie dem Congress of Hawaiian People, zu keiner Konsolidierung einer „hawaiischen Bewegung“: es bleibt zunächst bei einer relativ großen Zahl politisch aktiver Gruppen, die ein breites Spektrum von Zielen auf verschiedene Weise (juristisch, wie z.B. bei der Forderung nach Reparationen, oder kulturell begründet, so durch die Annahme eines Wertes wie Aloha 'Äina) zu erreichen suchen. Eine sich bereits früh abzeichnende Tendenz ist die immer stärkere Ethnisierung der Konflikte. Noch 1974 schrieben die in ihrer Mehrzahl hawaiischen Autoren einer kurzen Darstellung der damals aktiven hawaiischen Organisationen und der von ihnen angegangenen Probleme:

„The issues facing Hawaiians are essentially the same as those facing people of many other races. In addition, not all Hawaiians are con- 
fronted with problems, only a certain class of Hawaiians are. The work of the Hawaiian organizations has been aimed at raising the conditions of the Hawaiians. In working towards that end, it becomes evident that 1. these problems do not only affect Hawaiians...

2. there are concerns that are common to all people of Hawaii who are being oppressed...

3. the more people there are working together, the more can be accomplished. In unity, there is strength.

...Only together can we win. Imua! ${ }^{90 ، ~(E t h n i c ~ S t u d i e s ~} 221$ 1974:71)

Bis zum Ende des Jahrzehnts hatte sich eine inzwischen breit gefächerte hawaiische Bewegung von ihren „linken“ Wurzeln (wie sie z.B. bei Kōkua Kalama zu erkennen sind) entfernt: An die Stelle einer gesellschaftlichen Analyse, in der die Probleme eines sehr großen Teiles der Hawaiier als Aspekt der Unterdrückung aller besitzlosen Bevölkerungsteile durch die herrschende Klasse erklärt wurden, trat eine Deutung der hawaiischen Geschichte und der hawaiischen Gesellschaft, die allen Hawaiiern als indigener Bevölkerung eine gemeinsame, besondere Kultur und eine daraus resultierende Bindung an das Land zuschrieb. Hieraus folgten Exklusivrechte der Hawaiier und Lösungsstrategien für die sozialen Probleme der hawaiischen Minderheit, bei denen Landrechte und eine stärkere Achtung hawaiischer Werte eine wesentliche Rolle spielen sollten (cf. H.-K. Trask 1984:126-7).

Die Aktivitäten der unterschiedlichen hawaiischen Gruppierungen hatten bis zum Ende der 1970er Jahre bereits soviel öffentliche Aufmerksamkeit erregt, dass die mit der Aktualisierung der Staatsverfassung betraute Versammlung von 1978 an den Problemen der hawaiischen Bevölkerungsminderheit und den Forderungen diverser Organisationen nicht vorbeisehen konnte, zumal Hawaiier in diesem Gremium 19\% aller Delegierten und somit die drittstärkste ethnische Gruppe unter ihnen stellten (Lee 1993:16). ${ }^{96}$ Die von hawaiischen Delegierten eingebrachten Vorschläge wurden zum Teil sehr kontrovers diskutiert - in ihrem Kompendium zur Verfassung des Staates Hawai‘i schreibt Lee zusammenfassend:

„Among the most controversial changes were those in the Hawaiian affairs package which received a substantial number of ,no' votes. These amendments created an Office of Hawaiian Affairs, enhanced the Hawaiian Lands rehabilitation program, required the teaching of Hawaiian culture in the public schools, established Hawaiian as an official lan-

\footnotetext{
${ }^{95}$ I mua!: Vorwärts!

96 Die größte Gruppe stellten die Delegierten japanischer Abstammung mit 39\%, die „Caucasians“ (Weiße) waren mit 27\% die zweitgrößte Delegiertengruppe.
} 
guage, protected traditional and customary rights, and limited the use of adverse possession for acquiring title to land. In addition, constitutional status was granted to the state motto, written in Hawaiian, as well as to the edict of King Kamehameha called the ,Law of the Splintered Paddle““ (Lee 1993:19). ${ }^{97}$

Von den hier genannten Verfassungsänderungen ist im Kontext dieser Arbeit die Schaffung des Office of Hawaiian Affairs (OHA) von größter Bedeutung: die unabhängige Behörde, der mit 20\% der staatlichen jährlichen Einkünfte aus dem „Public Land Trust“ erhebliche Geldmittel zugeteilt wurden, sollte unter hawaiischer Leitung selbständig Förderprogramme für Bürger hawaiischer Abstammung entwickeln und durchführen (Hawaii Revised Statutes o.J.:§ 1013.5). Darüber hinaus war das Office of Hawaiian Affairs auch als Träger staatlicher wie nichtstaatlicher Programme und Förderungsfonds vorgesehen und sollte als Empfangsberechtigter über eventuelle Reparationszahlungen an die Hawaiier verfügen können (Hawaii Revised Statutes o.J.:\$10-3).

Das Office of Hawaiian Affairs nahm seine Arbeit im Jahre 1980 auf damit steht am Ende der 1970er Jahre, an deren Beginn kleine, oft lokal organisierte hawaiische Gruppen Widerstand gegen Landrechtsverletzungen und soziale Benachteiligung leisteten, die Gründung einer quasi-staatlichen Institution, die über substantielle finanzielle Mittel verfügen konnte und für sich in Anspruch nahm, für fast alle Belange der hawaiischen Bevölkerung zuständig zu sein. Das Office of Hawaiian Affairs gewann schnell an Einfluss und bald schon betrachtete es sich als einen der wichtigsten Akteure in der sich immer deutlicher abzeichnenden Auseinandersetzung um die „Hawaiian Sovereignty“. Diesem Anspruch mochte ein großer Teil der in den 1980er Jahren entstehenden Gruppierungen (nunmehr immer häufiger als „Hawaiian Sovereignty Movement“ bezeichnet) nicht folgen und ging auf Distanz zu diesem, wie sie es nannten, Vertreter des Staates.

Die Forderung nach „Sovereignty“ - die hawaiische Bewegung seit 1980

Die Bedeutung „hawaiischer“ Themen in der öffentlichen Wahrnehmung der 1980er Jahre lässt sich an einer wachsenden Zahl entsprechender Veröffentlichungen in den Medien des Bundesstaates ablesen. Eine Auswertung des Index für die beiden großen Tageszeitungen Hawai' is (Hawaii State Library 19291994) zeigt, dass die Berichterstattung in der zweiten Hälfte dieses Jahrzehntes

97 Die Aufnahme des Māmalahoe Kānāwai (Law of the Splintered Paddle) ist eine eher symbolische Reverenz an die hawaiische Geschichte: Artikel IX, Absatz 10 der hawaiischen Verfassung besagt: „The law of the splintered paddle, mamala-hoe kanawai, decreed by Kamehameha I - Let every elderly person, woman and child lie by the roadside in safety - shall be a unique and living symbol of the State‘s concern for public safety. [...]“ (Hawai'i Constitution 1997). 
zum einen deutlich umfangreicher und zum anderen auch kontinuierlicher war, d.h. weniger abhängig von spezifischen Ereignissen und akuten Fragestellungen als in den 1970er Jahren. ${ }^{98}$ Unter dem allgemeinen Stichwort „Hawaiians“ verzeichnet der Index in den 13 Jahren von 1973 bis 1984 im Durchschnitt 36 Artikel im Jahr, in den zehn Jahren von 1985 bis 1994 betrug der Mittelwert 159 Titel, die sich mit hawaiischen Themen befassten. ${ }^{99}$ Am Ende des Jahrzehnts taucht 1989 dann erstmals das Stichwort „Sovereignty“ auf, das die Berichterstattung zu spezifisch politischen Forderungen der hawaiischen Bewegung zusammenfasste. Waren hier 1989 nur 8 Titel aufgeführt, so steigerte sich die Zahl auf 62 im Jahre 1992 und erreichte im Jahre darauf 204 - die Forderungen des „Hawaiian Sovereignty Movement“ waren in der Öffentlichkeit des Bundesstaates angekommen.

Sowohl lokaler Widerstand hawaiischer Bewohner gegen Änderung von Flächennutzungsplänen, die vor allem den Interessen von Investoren dienten, als auch Landbesetzungen fanden weiterhin statt. Beispiele für diese Proteste, die sich auch zunehmend auf die anderen Inseln des Archipels ausbreiteten, sind die Besetzung von Sand Island, einer dem Hafen von Honolulu vorgelagerten Landfläche, auf der fast 150 hawaiische Familien lebten, als 1980 der Räumungsbefehl erging, oder auch die Okkupation des Strandes von Mākua an der Nordostküste O`ahus im Jahre 1983. Das Beispiel Sand Island zeigt deutlich, wie sehr es neben der konkreten Inbesitznahme eines Ortes auch um eine Manifestation des besonderen hawaiischen Anspruchs auf das Land ging (cf. Milner 2006:170). Fotografien des besetzten Landes zeigen Transparente, auf denen mit „Aloha "Āina“ hawaiische Werte geltend gemacht werden oder die einen besonderen Status der Besetzer proklamieren, wenn es z.B. heißt: „Keiki o ka aina [Kinder des Landes; U.M.] united to regain our lands“. ${ }^{100}$ Auf der Insel Hawai‘i begann 1985 der Widerstand gegen die geothermische Energie-

\footnotetext{
${ }^{98}$ So war das Kalama Tal 1971, dem Jahr der Räumungen, Thema von 60 Artikeln, im Jahr darauf waren es noch zwei; über Kaho'olawe und die Protect Kaho'olawe 'Ohana wurde im Jahr der Gründung der PKO (1976) 87 mal berichtet, 1977 waren es sogar über 250 Artikel - im folgenden Jahr und in den Jahren darauf erschienen jeweils nicht mehr als 20 Artikel zum Thema. Gleiches gilt für hawaiische Gruppierungen: über „The Hawaiians“ und Kōkua Hawai'i berichten die Zeitungen im Umfeld des Kalama Valley-Konflikts (jeweils weniger als 10 Artikel im Jahr), ab 1973 werden beide Gruppen im Index nicht mehr aufgeführt; A.L.O.H.A. findet sich während der Zeit ihres Bestehens kontinuierlich, wenn auch in geringem Umfang, in den Nachrichten, die von A.L.O.H.A. angestoßenen Fragen der Reparationszahlungen hingegen bleiben unter dem Stichwort „Hawaiian Native Claims“ von 1974 an bis in die 1990er Jahre mit durchschnittlich 20-30 Artikeln/Jahr ein Thema der Zeitungen.

${ }^{99} 1978$ waren es 50 Artikel, 1976 lediglich neun - die beiden Jahre markieren das maximale sowie das geringste Ausmaß der Berichterstattung im ersten Zeitraum; für den zweiten Abschnitt lauten die entsprechenden Zahlen 48 für das Jahr 1985 (bis 1990 liegt der Mittelwert bei fast 90 Titeln/Jahr) sowie 419 im Jahre 1994. Hinzu kommt auch hier die Berichterstattung zu jeweils aktuellen Themen.

${ }^{100}$ Beispiele hierfür finden sich unter H.-K. Trask (2008).
} 
gewinnung, wobei der Pele Defense Fund seinen Widerstand u.a. mit den Eingriffen in das Gebiet der Vulkangöttin Pele begründete; die Proteste gegen das Projekt dauerten bis in die 1990er Jahre an. 1988 wehrten sich Hawaiier erfolgreich gegen den Bau einer Ferienanlage durch den Bishop Estate in Makalawena auf der Insel Hawai‘i; auf O`ahu besetzten im gleichen Jahr Hawaiier Land am Makapu'u Point. Von 1990 bis 1992 wehrten sich hawaiische Protestgruppen gegen den Bau der Autobahn H-3 auf O`ahu, die über heilige Stätten geführt werden sollte. ${ }^{101}$

In der zweiten Hälfte der 1980er Jahre entstanden jene politisch aktiven Gruppierungen, die das „Hawaiian Sovereignty Movement“ in den 90er Jahren maßgeblich bestimmten. Sie unterschieden sich in ihren konkreten Zielsetzungen, der Zahl ihrer Mitglieder und ihres Organisationsgrades; gemeinsam war ihnen allen das Verlangen nach Autonomie bzw. Souveränität für den hawaiischen Bevölkerungsteil. Sie alle hingen in ihrer Außenwirkung stark von einzelnen Persönlichkeiten ab, die zumeist auch die Gründer der jeweiligen Organisationen waren - ein Muster, das sich schon bei einzelnen früheren Organisationen wie z.B. A.L.O.H.A. oder Nā 'Ohana O Hawai'i fand. Da die im Folgenden aufgeführten Gruppierungen und Institutionen federführend für die Entwicklungen der 1990er Jahre waren und daher in Kapitel 4 ausführlich vorgestellt werden, beschränke ich mich hier auf einige Grundinformationen.

Schon 1985 gründete der Anwalt Hayden F. Burgess das Institute for the Advancement of Hawaiian Affairs (I.A.H.A.) und forderte die völlige Unabhängigkeit Hawai'is von den Vereinigten Staaten.

Zwei Jahre nach der Gründung des Institute for the Advancement of Hawaiian Affairs verabschiedeten 250 Delegierte die Verfassung von Ka Lāhui Hawai'i (The Nation of Hawai'i), einer Organisation, die sich als unabhängige Nation innerhalb des Bundesstaates versteht.

1989 initiierte Kekuni Blaisdell, Arzt und Professor an der University of Hawai‘ $i$, Ka Pākaukau, einen Zusammenschluss von 12 hawaiischen Gruppierungen, die z.T. schon seit den 1970er Jahren aktiv waren. ${ }^{102}$ Nicht alle Mitgliedsgruppen vertraten einen so konkreten politischen Standpunkt, nämlich die vollständige Unabhängigkeit Hawai'is von den USA, wie ihn Blaisdell für Ka Pākaukau formulierte.

Als letzte der hier zu nennenden Gruppierungen wurde 1992 das 'Ohana Council of the Hawaiian Kingdom gegründet. Der Kopf dieser Gruppierung, die sich später Nation of Hawai'i nannte, ist bis heute Dennis „Bumpy“

\footnotetext{
${ }^{101}$ Weitere Beispiele für hawaiische Protestaktionen bis 1993 finden sich bei Minerbi (1994).

${ }^{102} \mathrm{Zu}$ den Mitgliederorganisationen von Ka Pākaukau gehörten die Protect Kaho'olawe 'Ohana, der Pele Defense Fund, Nā Maka o Ka 'Āina (ein Videoproduzent), die Pro-Hawaiian Sovereignty Working Group (entwickelt Informationsmaterialien über „Hawaiian Sovereignty“) und Nā 'Ōiwi o Hawai'i (v.a. kulturpolitisch engagiert). Die Mitgliederbasis dieser Organisationen überschneidet sich in vielen Fällen (Kame`eleihiwa 1993:67).
} 
Kanahele, der vor der Gründung des 'Ohana Council bereits in der Gruppe La Ea o Hawai'i Nei aktiv war. Beide Organisationen betrieben ihre Forderungen nicht nur durch Lobbyarbeit und Demonstrationen, sondern lehnten auch drastischere Maßnahmen wie illegale Landbesetzungen nicht ab.

Mit einem „Blueprint for Native Hawaiian Entitlements“, der die Rückgabe hawaiischen Landes, Reparationen sowie die eigenständige Verwaltung dieses Landes durch eine gewählte hawaiische Institution verlangte, meldete schließlich auch das quasi-staatliche Office of Hawaiian Affairs (OHA) 1989 seinen Anspruch an, Mitspieler im Kreis der „Sovereignty“Organisationen zu sein (OHA 1989).

Der wachsenden Aufmerksamkeit gegenüber Fragen der hawaiischen Autonomie trägt 1991 die Gründung von Hui Na‘auao („An Association Engaged in Promoting Awareness, Hawaiian Sovereignty and SelfDetermination“ [Hui Na‘auao 1993]) Rechnung. Diese Koalition aus 40 unterschiedlichen hawaiischen Gruppen, gefördert aus Mitteln der U.S. Administration for Native Americans, vermittelte Informationen über „Hawaiian Sovereignty“ in der „Hawaiian Community“, schloss dabei aber NichtHawaiier explizit in seine Programme ein. ${ }^{103}$

\footnotetext{
103 „Hawaiian groups get sovereignty education funds“, Becky Ashizawa , H S-B 5.10.1991:A-4; „Sovereignty education funds top \$ 1 million“, Becky Ashizawa, H S-B 11.1.1993: A-6.
} 



\section{Demographie, Ethnizität und „Race“: Wer sind die Hawaiier?}

Das vorangegangene Kapitel beleuchtete die politische Geschichte Hawai'is und den hawaiischen Widerstand gegen die fundamentalen Veränderungen des 19. Jahrhunderts. In den Diskursen der Autonomiebewegung nehmen diese historischen Brüche sowie die hawaiischen Reaktionen darauf breiten Raum ein; weniger deutlich auszumachen - und doch von großer Bedeutung für das hawaiische Selbstbewusstsein - ist die Geschichte des rapiden Bevölkerungsrückgangs nach dem Kontakt mit den europäischen Entdeckern, Walfängern, Händlern und Siedlern. Noch im 19. Jahrhundert führte darüber hinaus eine forcierte Einwanderung asiatischer Arbeitskräfte zu demographischen Verschiebungen, in deren Folge die Hawaiier zu einer Minderheit im eigenen Land wurden - zu einer auch sozialen Minorität, deren Kennzahlen im 20. Jahrhundert auf eine dramatische Verschlechterung der Lebensverhältnisse des Großteils der Hawaiier hinwiesen. Neben den Politikfeldern des Machtverlustes, der Landrechte und der Rückgewinnung der Deutungshoheit über „hawaiische Kultur" sind eben auch Fragen der sozialen Entwicklung für die Legitimierung politischer Ansprüche der hawaiischen Bewegung und der Begründung ihres politischen Handelns von Belang. Im Folgenden werde ich die für die gegenwärtige demographische Situation sowie die für die soziale Stratifizierung der 
Gesellschaft in Hawai'i verantwortlichen Entwicklungen nachzeichnen. Dazu sei angemerkt, dass diese natürlich eng mit den im vorigen Kapitel aufgezeigten politischen Veränderungen verwoben sind. Entsprechende Zusammenhänge und Bedingungsrelationen lassen sich in großer Zahl aufzeigen - die hier erfolgte Trennung ist ausschließlich der Übersichtlichkeit geschuldet.

Im zweiten Teil dieses Kapitels werde ich auf die Eingrenzung der Bezeichnung „Hawaiian“ im sprachlichen, juristischen und kulturellen Kontext des Bundesstaates eingehen. Im Gefolge politischer und demographischer Verschiebungen sind rechtliche Regelungen und Definitionen entwickelt worden, die das ethnische und kulturelle Selbstverständnis weiter Teile der hawaiischen Bevölkerung überlagert haben. Die Veränderung der Begriffe und Bedeutungen war Bestandteil eines kolonialen Projekts und stellte einen Weg zur Erringung der kulturellen und politischen Hegemonie auf den Inseln dar; doch bleibt, anders als bei den Herkunftszuschreibungen in den übrigen USA, die hawaiische Abstammung bis heute eine notwendige Bedingung, um in Hawai' $i$ als Hawaiier zu gelten - anders als z.B. die Bezeichnungen New Yorker, Texaner oder Kalifornier besteht hier ein klarer ethnischer Bezug.

Eine Besonderheit der Gesellschaft des Staates Hawai‘i, dessen Bürger sich - vielleicht in stärkerem Maße als in den übrigen USA - in ihrer jeweiligen ethnischen Gemeinschaft verorten, stellt die inklusive, sozial wie kulturell begründete Kategorie „Local“ dar. Im Rahmen dieser Arbeit ist sie insofern von Bedeutung, als sie zum einen Menschen hawaiischer Abstammung selbstverständlich einschließt, zum anderen aber auf Seiten der Hawaiier eine zunehmende, politisch begründete Tendenz erkennen lässt, sich dieser Kategorie nicht zugehörig zu fühlen und gegen die „Locals“ abzugrenzen. ${ }^{104}$

Aufgrund ihrer Geschichte verfügen heute fast alle Hawaiier über mehrfache ethnische Identifikationsmöglichkeiten, wobei über die Zeiten hinweg eine deutliche Präferenz für die hawaiische Abstammung auszumachen ist. Die drei Teile dieses Kapitels bieten eine Grundlage für das Verständnis dieser Politik der Identitäten sowie des komplexen Gefüges der multiethnischen und multikulturellen Gesellschaft im gegenwärtigen Hawai‘ $i$.

\footnotetext{
${ }^{104}$ Vergleiche z.B. die Entwicklung einer Organisation wie Kōkua Kalama, in deren Selbstverständnis die Kategorie „Local“ eine zunächst bestimmende Rolle spielte, aber zunehmend durch „Hawaiian“ verdrängt bzw. ersetzt wurde.
} 


\subsection{Demographische Veränderungen}

Der hawaiische Lyriker 'İmaikalani Kalāhele (2002:19) verbindet in seinem Gedicht „Tūtū" die hawaiische Gegenwart mit dem 19. Jahrhundert, einer Zeit des Sterbens unter der hawaiischen Bevölkerung: „Moaning a sad song / a Tūtū / lamenting on a / darkened porch / gently rocking / knowing she's / seeing the end / the only child / of an only child / now sits in / a dark room / dying / Ma'i Haole / sings again“. ${ }^{105}$ In seiner metaphorisch-poetischen Diktion lenkt der Text den Blick auf eine bis in die Gegenwart andauernde Entwicklung, in deren Verlauf die Hawaiier nicht nur zu einer numerischen Minderheit, sondern auch zu einer sozial marginalisierten Gruppe innerhalb der Inselgesellschaft wurden. Ausgenommen hiervon blieb vor allem die schmale Schicht des hochrangigen Adels, der zwar von Krankheiten nicht verschont blieb, aber von den ökonomischen Veränderungen des 19. Jahrhunderts zum Teil in erheblichem Maße profitieren konnte. Zugleich benennt er - wie es auch viele Vertreter der Autonomiebewegung tun - die Schuldigen an dieser Entwicklung: es waren die „Weißen“, mit denen bislang ungekannte Krankheiten auf die Inseln kamen, und es war der Kontakt mit den Fremden, der zu einem dramatischen Bevölkerungsverlust führen sollte.

Um die Größenordnung dieses Sterbens in der ersten Hälfte des 19. Jahrhunderts ermessen zu können, stellt sich die Frage nach der Bevölkerungszahl des Archipels im Jahre 1778, als mit den Mannschaften Kapitän James Cooks die ersten Europäer nach Hawai'i kamen. Es war der Leutnant James King, der einen ersten Versuch zur Ermittlung der Bevölkerungszahl unternahm:

„It may be thought extremely difficult to form any probable conjectures respecting the population of islands, with many parts of which we are but imperfectly acquainted. There are, however, two circumstances, that take away much of this objection; the first is, that the interior parts of the country are entirely uninhabited; so that, if the number of the inhabitants along the coast be known, the whole will be pretty accurately determined. The other is, that there are no towns of any considerable size, the habitations of the natives being pretty equally dispersed in small villages round all their coasts. It is on this ground that I shall ven-

\footnotetext{
${ }^{105} T \bar{u} t \bar{u}(K \bar{u} k \bar{u})$ ist das hawaiische Wort für Großmutter; die Bildung Ma'i Haole setzt sich aus den Wörtern für Krankheit $\left(M a^{\prime} i\right)$ und der Bezeichnung für die Weißen (Haole) zusammen und spielt in ihrer Mehrdeutigkeit auf die negativen Auswirkungen des Kontaktes und des Zusammenlebens mit den euroamerikanischen Zuwanderern für die hawaiische Bevölkerung an [U.M.].
} 
ture at a rough calculation of the number of persons in this group of islands“ (King 1785:127-8).

King, der Hawai'i vom Schiff aus beobachtete, schätzte aufgrund seiner Annahmen zur Siedlungsstruktur eine Bevölkerungszahl von 400.000 für die gesamte Inselkette und lieferte damit eine Ausgangsgröße für spätere Berechnungen. Unter Einbeziehung von Beobachtungen späterer Besucher errechnete der Statistiker Robert Schmitt eine erheblich niedrigere Zahl von 250.000 Bewohnern (Schmitt 1971:240), die eine untere Grenze der weithin akzeptierten Bevölkerungszahlen von 250.000 bis 300.000 darstellt. ${ }^{106}$ Zahlen ganz anderer Größenordnung stellte David Stannard, Professor für American Studies an der University of Hawai'i, 1989 zur Diskussion (Stannard 1989): auf der Grundlage einer Neubewertung der „Carrying Capacity“ ${ }^{107}$ des Archipels kommt er zu einer Bevölkerung von mindestens 800.000 Menschen im voreuropäischen Hawai'i. Diese Hypothese wird allerdings von Eleanor Nordyke und Robert Schmitt - in den Worten Stannards ,currently ... by far the most recognized experts in matters pertaining to Hawai'i's population, past and present" (Stannard 1989:122) - in ihren Kommentaren zu Stannards Buch zurückgewiesen (Nordyke 1989 b:105-13; Schmitt 1989:114-21). Es kann hier nicht um eine wissenschaftliche Bewertung der Hypothesen Stannards gehen; ihre tatsächliche Bedeutung liegt m.E. denn auch weniger in einer Revision der in Archäologie und Demographie verwendeten Zahlen und Methoden, als vielmehr in einer politischen Neubewertung der Entvölkerung Hawai'is oder wie Stannard selbst schreibt:

,...there is no point in denying that this is a subject with powerful social and political ramifications. A people, after all, are what their historical experience - and their knowledge of it - has made them, which is why governments have a meddlesome interest in the history that is written during their watch. To native peoples, who have suffered so terribly and in so many ways as the victims of every sort of Western cultural avarice, knowledge of the size of their populations prior to Western contact is a matter of crucial historical importance [...] because the larger the population on the eve of Western contact, the greater the subsequent horror“ (Stannard 1989:143).

\footnotetext{
106 Siehe hierzu Nordyke (1989:17 f.); vgl. u.a. Lind (1967:7); Goldman (1970:201); Kirch (1985:82-6); Terrell (1986:220); Oliver (1989:883).

${ }^{107}$ Der Begriff stammt aus der Biologie und bemisst die Fähigkeit eines Ökosystems, eine konstante Population dauerhaft erhalten zu können.
} 
Aus diesen Zeilen spricht ein klarer politischer Standpunkt und es wird deutlich, wie sehr historische und archäologische Forschung in den zwischen der „westlichen“ Wissenschaft und den Vertretern der Autonomiebewegung ausgefochtenen Streit um Definitionsmacht hinsichtlich der hawaiischen Vergangenheit involviert ist. Während die etablierte Wissenschaft die Zahlen Stannards ablehnend bis kritisch betrachtete, haben Autoren aus dem Umfeld der Autonomiebewegung in ihren Publikationen die von Stannard ermittelten Zahlen als neuen Standard akzeptiert (siehe u.a. Dudley u. Agard 1993:1; H.-K. Trask 1994a:16; Kame'eleihiwa 1992:20, 81; cf. Osorio 2002:9-10).

Unumstritten sind hingegen die aus dem 19. Jahrhundert vorliegenden Zahlen zur Entvölkerung Hawai'is, die - unabhängig von der Ausgangsgröße von einem großen Sterben der hawaiischen Bevölkerung berichten. Seine Ursachen gleichen denen andernorts im Pazifik: Schon von den Besatzungen der ersten europäischen Schiffe wurden Geschlechtskrankheiten übertragen, die als eine der Ursachen für die bis zu 60\% unter denen vergleichbarer Populationen liegenden Geburtenraten der hawaiischen Bevölkerung gelten. ${ }^{108}$ Und selbst wenn wir die konservative Schätzung von 300.000 Bewohnern im Jahre 1778 akzeptieren, hatte sich die Bevölkerungszahl des Archipels den Berichten des englischen Missionars William Ellis zufolge bis 1823 halbiert - die Landung Kapitän Cooks lag gerade 45 Jahre zurück.

„They [die Hawaiier; U.M.] were estimated by their discoverers at 400,000 . There is reason to believe this was somewhat above the actual population at that time, though traces of deserted villages, and numerous enclosures formerly cultivated, but now abandoned, are every where to be met with. At present it does not exceed 130,000 or 150,000 ...“(Ellis 1827:22-3).

Von amerikanischen Missionaren in den Jahren 1832 und 1836 durchgeführte Zählungen ermittelten 130.300 bzw. 108.600 Einwohner; der erste staatliche Zensus von 1850 nennt nur noch eine Zahl von 84.165 Menschen. Das hier in nüchternen Zahlen erfasste Sterben der hawaiischen Bevölkerung setzte sich in den folgenden Jahren unverändert fort: 1872 registrierte der Zensus eine Gesamtbevölkerung von 57.900 Menschen, für das Jahr 1876 schätzt Schmitt eine Gesamtbevölkerung von nurmehr 53.900 und markiert damit den absoluten Tiefstand der Bevölkerungszahlen nach 1778. ${ }^{109}$ Folgt man diesen Zahlen, so

\footnotetext{
${ }^{108}$ Siehe hierzu Beaglehole (1967:265-6, 575-6); Nordyke (1989 a:20 ff.); Bushnell 1993:133-4, 293); Schmitt (1971:239 f.).

${ }^{109}$ Für alle hier genannten Zahlen siehe Nordyke (1989 a:18) und Schmitt (1977:3, 9); nach Lind (1967:28), der allerdings auf der Grundlage anderer Zahlen gearbeitet hat, stellten die Bewohner hawaiischer Abstammung im Jahre 1872 noch 90,6\% (51.531 Menschen) der Gesamtbevölkerung, 1878 waren es noch 81,9\% bzw. 47.508 Menschen.
} 
hätte sich die hawaiische Bevölkerung in den 100 Jahren nach der europäischen Entdeckung auf ein Sechstel ihrer ursprünglichen Größe verringert - bei $\mathrm{Zu}-$ grundelegung der von Stannard vertretenen Ausgangsgröße wäre sogar nur ein Sechzehntel der Bevölkerung von 1778 gezählt worden.

Markierte die Bevölkerungsschätzung von 1876 den Tiefpunkt der Bevölkerungsentwicklung im Königreich Hawai' $i$, so zeigte bereits der nächste Zensus von 1878 einen Zuwachs von 2.000 Menschen und 1884 lebten bereits wieder 78.500 Menschen auf den Inseln, fast 25.000 mehr als acht Jahre zuvor (Schmitt 1977:9). Doch dürfen diese Zahlen nicht über die Situation der hawaiischen Bevölkerung hinwegtäuschen, deren Sterben sich unvermindert fortsetzte: Ihr Anteil an der Gesamtbevölkerung der Inseln betrug im Jahre 1884 nur noch 54,9\%, das waren 44.232 Menschen, und bis zur Jahrhundertwende sank die Zahl der Hawaiier auf 39.656, die zu diesem Zeitpunkt nur noch $24,4 \%$ der Gesamtbevölkerung stellten (Lind 1967:28). ${ }^{110}$ Die Ursache des verzeichneten Bevölkerungszuwachses findet sich vielmehr in einem Prozess gesteuerter $\mathrm{Zu}-$ wanderung ausländischer Arbeitskräfte für die von den Haole angelegten Pflanzungen - die Situation der Hawaiier in ihrem Land sollte sich durch diese Verschiebungen der Bevölkerungsstruktur in der zweiten Hälfte des 19. Jahrhunderts nachhaltig verändern.

„King Sugar": Plantagenwirtschaft und Einwanderung

Nachdem die Māhele die Landfrage im Sinne der euro-amerikanischen Pflanzer geklärt hatte, stand einem Ausbau von Plantagen zunächst nichts mehr im Wege. Die Auswirkungen des amerikanischen Bürgerkriegs sowie diverse Verträge zwischen dem Königreich Hawai' $i$ und den USA trugen darüber hinaus zu einer beständig zunehmenden Zuckerproduktion bei (Takaki 1983:17-9). Schon 1850 gründete sich mit der „Royal Hawaiian Agricultural Society“ eine Interessenvertretung der ausländischen Plantagenbesitzer, zu deren Zielen u.a. die Erschließung neuer Arbeitskräftereservoirs gehörte:

„Since enslavement of the native population, a practice common in developing plantation frontiers elsewhere, was out of the question under the Hawaiian monarchy, planters fell back on the other alternative of imported labor under contract ..." (Lind 1982:11).

Noch im Gründungsjahr der „Royal Hawaiian Agricultural Society“ verabschiedete die Regierung das Gesetz „For the Government of Masters and Servants“ und damit Regelungen für eine gesteuerte Zuwanderung von Ar-

\footnotetext{
${ }^{110}$ Die Zahlen Linds sind nicht unmittelbar mit den Ergebnissen Schmitts zu vergleichen, da ihre Berechnungsgrundlagen unterschiedlich sind. Allerdings besteht Übereinstimmung in der dargestellten Tendenz der Bevölkerungsentwicklung.
} 
beitskräften aus dem Ausland. ${ }^{111}$ Damit fielen entscheidende Grenzen für die Expansion der Zuckerwirtschaft und Fuchs (1961:19) kann feststellen: „From then until annexation, when nearly two thirds of the workers of Hawaii labored on sugar plantations, sugar was king in the islands“.

1852 gelangten die ersten Arbeiter unter den Bedingungen des „Masters and Servants"-Gesetzes nach Hawai'i: mit dem ersten Schiff kamen 293 Kontraktarbeiter aus China, denen bis zum Jahre 1899 insgesamt 50.000 chinesische Arbeiter folgen sollten (Kreisel 1984:65-6). 1884 lebten bereits mehr als 18.000 Chinesen in Hawai'i, die damit 22\% der Gesamtbevölkerung stellten (Lind 1967:3). Von 1885 bis 1894 kamen 30.000 Japaner im Rahmen der Kontraktarbeit nach Hawai'i und bis zur Jahrhundertwende waren fast $40 \%$ der Bevölkerung Hawai` is japanischer Herkunft - die Hawaiier machten zu diesem Zeitpunkt nicht einmal mehr ein Viertel der Gesamtbevölkerung aus (Kreisel 1984:73; Lind 1967:28). Insgesamt ist der Verlauf der gelenkten Einwanderung in Hawai‘ $i$ von einem Wechsel der jeweiligen Anwerbungsländer geprägt: machten Chinesen und Japaner die Mehrzahl der Einwanderer aus, so kamen daneben fast 25.000 Portugiesen, 8.000 Koreaner, 2.500 Einwanderer aus Ozeanien, einige Deutsche und Skandinavier unter den Bedingungen des „Masters and Servants"-Gesetzes auf die Inseln. ${ }^{112}$ Als letzte große Einwanderergruppe unter der Aufsicht der inzwischen umbenannten „Hawaiian Sugar Planters Association“ kamen bis 1930 fast 100.000 Filipinos nach Hawai‘i, von denen viele allerdings weiter in die USA oder zurück in ihr Heimatland zogen (Nordyke 1989 a:78). Noch 1897, ein Jahr vor der Annexion Hawai' is, stellten US-Amerikaner lediglich 2\% der Bevölkerung im gesamten Territorium - eine Zahl, die in keinem Verhältnis zur wirtschaftlichen Macht und zum politischen Einfluss dieser Bevölkerungsgruppe stand - doch mit der Eingliederung des Archipels in die USA nahm auch die Zuwanderung vom Festland massiv zu. ${ }^{113}$

\footnotetext{
${ }^{111}$ Das Gesetz regelte die Bedingungen, unter denen Arbeitskräfte für die Plantagen angeworben werden konnten: es handelte sich um Kontraktarbeit, d.h. die Arbeiter verdingten sich für eine Zeit von maximal fünf Jahren, bekamen freie Überfahrt, den ausgehandelten Lohn, Kleidung und Unterkunft (Kuykendall 1947:330, 1953:185-6; Nordyke 1989 a:54).

${ }^{112}$ Der Wechsel von einem Herkunftsland zu einem anderen hatte seine Ursache u.a. in dem Bestreben der Plantagenbetreiber, ethnisch einheitliche Arbeiterschaften zu vermeiden und so deren Streikbereitschaft zu vermindern (siehe hierzu Takaki 1983; Beechert 1985). Daneben beendeten US-amerikanische Einwanderungsgesetze ab 1898, dem Jahr der Annexion Hawai'is, z.B. die Einwanderung aus China völlig.

${ }^{113}$ Von erheblichem Einfluss war hierbei auch die große Zahl von Militärangehörigen, die sich von 245 Personen im Jahre 1900 auf mehr als 56.000 im Jahre 1970 steigerte (Lind 1982 a:16). 2005 betrug die Zahl der in Hawai'i lebenden Soldaten und Mitarbeiter des US-Militärs sowie ihrer Angehöriger 102.200 Personen (State of Hawaii 2006:10.4). Nicht ohne soziale Folgen blieb auch die Stationierung von bis zu 300.000 Mann in den Jahren 1944/45 (siehe hierzu Bailey u. Farber 1992).
} 
Während die große Zahl der Einwanderer zu einer Verringerung des hawaiischen Bevölkerungsanteils führte, bewirkte ein sehr hoher Anteil interethnischer Heiraten zugleich deutliche Verschiebungen im numerischen Verhältnis von Hawaiiern ausschließlich hawaiischer Abstammung und jenen, deren Eltern und Vorfahren eine andere ethnische Zugehörigkeit hatten. Anders als in den kontinentalen USA bestanden in Hawai' $i$ zu keiner Zeit rechtliche Vorbehalte gegen Heiraten oder sexuelle Beziehungen zwischen Angehörigen unterschiedlicher, aufgrund von „race“ definierter Gruppen ${ }^{114}$ und so wies bereits die Volkszählung von 1853 neben 70.036 Hawaiians auch 983 als „Hapahaole“, „Halb-Weiße“, bezeichnete Hawaiier gemischter Abstammung aus (Nordyke 1989a:33).

Heute beantworten 21,4\% der Gesamtbevölkerung Hawai'is die Zensus-Frage nach der „race“-Zugehörigkeit mit der „uneindeutigen“ Antwort „two or more races“, mehr als in irgendeinem anderen US-Bundesstaat (U.S. Census Bureau 2000). Zugleich ist Hawai'i einer von nur vier Bundesstaaten, in denen die „,weiße“ Bevölkerung nicht die Bevölkerungsmehrheit bildet. ${ }^{115}$ Nach der Statistik des US-Zensus von 2000 setzt sich die Bevölkerung des Inselstaates von ca. 1,2 Millionen wie folgt zusammen: neben den genannten 21,4\% mit „gemischter Abstammung“ ordnen sich 78,6\% der Bevölkerung einer „,race“ zu, von ihnen sind 24,3\% „White“, 41,6\% „Asian“ und 9,4\% rechnen sich der Kategorie „Native Hawaiian and other Pacific Islander“ zu (U.S. Census Bureau 2000). ${ }^{116}$ Was bedeuten diese Zahlen für die demographische Situation der Hawaiier in der Gegenwart? Der Zensus von 2000 verzeichnet 239.655 „Native Hawaiians“ in Hawai' $i$, also Menschen, die eine zumindest teilweise hawaiische Abstammung angaben (s.a. OHA 2006). ${ }^{117}$ Sie sind eine deutlich anwachsende Bevölkerungsgruppe, die sich zugleich spätestens seit den 1960er Jahren durch eine sehr hohe Rate interethnischer Heiraten auszeichnet (Nordyke 1989:39). Durch den starken Bevölkerungsrückgang, die

\footnotetext{
${ }^{114}$ In den USA erklärte der Oberste Gerichtshof erst 1967 die Gesetze der Bundesstaaten gegen so genannte „,interracial marriages“ für verfassungswidrig. Bis zu diesem Zeitpunkt waren solche Gesetze noch in 16 Bundesstaaten der USA in Kraft (Paddock 1996:358).

${ }^{115}$ Die anderen der so genannten „Minority-Majority States“ sind Kalifornien, New Mexico und Texas, deren Bevölkerung in jüngerer Zeit durch eine starke Zuwanderung aus Lateinamerika geprägt ist.

116 Die anderen „races“ machen lediglich sehr geringe Bevölkerungsanteile aus: als „Black or African American“ bezeichneten sich 1,8\% der Bevölkerung, 0,3\% zählten sich zu den „American Indian or Alaska Native“ und 1,3\% fielen unter die Sammelkategorie „Some other race“ (U.S. Census Bureau 2000).

${ }^{117}$ In der Kategorie „Race alone“ führte der Zensus 80.137 „Native Hawaiians“ auf, allerdings handelt es sich hierbei um eine Selbstzuschreibung und somit nicht notwendig um Menschen ausschließlich hawaiischer Abstammung. Die letzten mir vorliegenden Zahlen des Hawai‘i Health Survey aus dem Jahr 1990 nannten 8.711 „Hawaiians“, die auf eine ausschließlich hawaiische Abstammung verweisen konnten (State of Hawaii 1994:37).
} 
parallele Zuwanderung und die Integration der Neuankömmlinge durch Heiraten haben sich die Hawaiier zu einem Bevölkerungsteil sehr diverser ethnischer und kultureller Herkunft entwickelt. Noch während im Zuge der kulturellen „Hawaiian Renaissance“ und der aufkeimenden politischen Bewegung seit den 1960er Jahren eine demonstrative „Hawaiianness“ an Bedeutung gewann, beschrieb einer der Protagonisten der kulturellen Revitalisierung die zeitgenössischen Hawaiier, die „New Hawaiians“, im Jahre 1982 wie folgt:

„Not only are present-day Hawaiians a different people, they are also a very heterogeneous and amorphous group. While their ancestors once may have been unified politically, religiously, socially, and culturally, contemporary Hawaiians are highly differentiated in religion, education, occupation, politics, and even in their claims to Hawaiian identity. Few commonalities bind them, although there is a continuous quest to find and develop stronger ties. In short, they are as diverse in their individual and collective character as any other ethnic population" (Kanahele 1982:21).

Wie ich im weiteren Verlauf dieser Arbeit zeigen werde, wird eine Analyse, wie sie hier insbesondere aus der abschließenden Feststellung mit ihrer starken Betonung der internen Differenzierung und fehlender gemeinsamer Bindungen ergibt, von den Akteuren der politischen Bewegung abgelehnt. Sie rücken vielmehr gerade die von ihnen gesehenen Aspekte der Gemeinschaft und die auf diesen Gemeinsamkeiten beruhenden kollektiven Rechte und Ansprüche in den Vordergrund. Im Folgenden sollen nun die wesentlichen sozialen Veränderungen dargestellt werden, die zum einen die Aufgliederung der hawaiischen Gesellschaft im Sinne der euro-amerikanischen Ökonomie und Gesellschaftsnormen bewirkten, zum anderen aber auch zu einer Marginalisierung weiter Teile der hawaiischen Bevölkerung und damit zu neuen Gemeinsamkeiten und auch Bindungen führten.

\section{Sozialer Wandel und die hawaiische Bevölkerung}

Betrachtet man neuere Statistiken zur Situation der Hawaiier, so ist eines der augenfälligsten Merkmale die ungleiche Verteilung der hawaiischen Bevölkerung auf den Inseln des Archipels. 2000 lebte der größte Teil der Hawaiier (66\%) auf der Insel O`ahu, während auf den anderen Inseln zwischen 5,2\% (Kaua`i) und 16,8\% (Hawai`i) der Hawaiier wohnten (OHA 2002:9). ${ }^{118}$ Setzt

\footnotetext{
${ }^{118}$ Ich beziehe mich hier auf Zahlen des Hawaiian Health Surveys 2000, der vom Department of Health des Staates Hawai`i durchgeführt wird. Diese Statistik weicht z.T. von den Zahlen des US-Zensus ab, doch können diese Abweichungen für die Darstellung einer prozentualen Bevölkerungsverteilung vernachlässigt werden.
} 
man die Bevölkerungszahlen mit den Gesamtbevölkerungen der jeweiligen Inseln bzw. Verwaltungseinheiten ins Verhältnis, so ergibt sich folgendes Bild: Auf O‘ahu („City and County of Honolulu“ mit 874.854 Einwohnern) stellen die Hawaiier lediglich 19,3\% der Einwohner, während es im „Maui County“ (117.115 Einwohner) fast 26\% und auf der Insel Hawai'i (148.574 Einwohner) sogar $28,8 \%$ sind. ${ }^{119}$ Heute leben also zwei Drittel der hawaiischen Bevölkerung auf O`ahu - dies ist nicht zuletzt Folge einer bereits im frühen 19. Jahrhundert einsetzenden Zuwanderung in die neuen städtischen Zentren Honolulu, Lahaina (Maui) und auch Hilo (Hawai'i):

„The ports kept growing, and so did the white community, but the total population of the kingdom declined tragically. At the turn of the nineteenth century only a few hundred natives lived at Honolulu and Lahaina, and the total native population of the kingdom was more than a quarter of a million. By 1840 the proportion was about eleven thousand natives at the ports in a total population of one hundred thousand; by 1860 about eighteen thousand in less than seventy thousand; and by the early seventies about eighteen thousand in less than sixty thousand“ (Daws 1968:168).

Ab der Mitte des 19. Jahrhunderts verstärkte sich die hawaiische Zuwanderung in die Städte durch die Konkurrenz mit einer wachsenden Zahl von Kontraktarbeitern (Kreisel 1984:53-6, 124-8). ${ }^{120}$ Die euro-amerikanische Plantagenökonomie verdrängte die hawaiische Subsistenzwirtschaft zunehmend und die Hawaiier gerieten ins wirschaftliche Abseits. Zwar gelang es einzelnen Adligen immer wieder, die neuen Möglichkeiten zu ihrem eigenen Vorteil zu nutzen, doch vollzogen sich die von den Haole in Gang gesetzten ökonomischen Veränderungen, wie Kreisel (1984:56) feststellt, im Wesentlichen ohne die aktive Einflussnahme von Hawaiiern und kamen letztlich auch nur den Haole zugute.

Die sich im Laufe des 19. Jahrhunderts herausbildende Vormachtstellung der Haole zeigt sich deutlich, wenn man ihren Anteil am Haus- und Grundbesitz dem der Hawaiier gegenüberstellt: Zwar stellten die Hawaiier im Jahre 1896 noch 74,5\% aller Grund- und 59,3\% aller Hausbesitzer in Hawai‘ $i$ (US-Zensus 1896, zit. nach Kreisel 1984:139), doch lag ihr Anteil am Gesamtwert aller Immobilien in Privatbesitz 1895 lediglich bei 31,4\%, während die

\footnotetext{
${ }^{119}$ Der Verwaltungsbezirk des „Maui County“ umfasst auch die Inseln Moloka‘i, Lana‘i sowie die unbewohnte Insel Kaho‘olawe; im „ Kaua‘i County“ (68.790 Einwohner), das auch die Privatinsel Ni‘ihau mit einer fast ausschließlich hawaiischen Bevölkerung einschließt, beträgt der Anteil der Hawaiier ca. 19\%.

${ }^{120}$ Im Folgenden beziehe ich mich auf die Arbeit von Kreisel (1984), der sich mit den Wechselwirkungen von Immigration bzw. Binnenmigration und Wirtschaftsstruktur sowie mit der kulturräumlichen Gliederung in Hawai' $i$ auseinandersetzt.
} 
Euro-Amerikaner im gleichen Jahr über einen Gesamtanteil von 58,4\% verfügten (Hawaiian Annual 1898, zit. nach Kreisel 1984:139). Die wertvollsten Immobilien und ein großer Teil des nutzbaren Landes befanden sich zu diesem Zeitpunkt nicht in hawaiischer Hand. Ein Vergleich des Besitzes an mobilem Eigentum zeigt darüber hinaus deutlich, dass die Hawaiier an der neuen, das Leben auf den Inseln inzwischen dominierenden Ökonomie nur sehr begrenzt teilhatten: sie verfügten im Jahre 1895 über einen Anteil von 6,5\% des Gesamtwerts alles mobilen Eigentums, während die Haole 12,4\%, die von ihnen kontrollierten Kapitalgesellschaften 53,4\% und andere euro-amerikanische Unternehmen 12,9\% dieser Werte besaßen (Hawaiian Annual 1898, zit. nach Kreisel 1984:139). ${ }^{121}$

Zugewanderte asiatische und europäische Kontraktarbeiter, die nach Beendigung ihrer Verträge in Hawai‘i blieben, stiegen schon bald innerhalb des euro-amerikanisch geprägten sozialen Gefüges auf. Zur Zeit der Annexion (1898) stellten die Haole als Plantagenbesitzer, Viehzüchter, Großhändler und Akademiker unangefochten die ökonomische Oberschicht. In der Mittelschicht, zu der immerhin noch ein großer Teil der Hawaiier zu rechnen war, kam es durch den wirtschaftlichen Aufstieg der Japaner, Chinesen und auch Portugiesen $\mathrm{zu}$ deutlichen Verschiebungen. ${ }^{122}$ Die ökonomische Unterschicht von Kleinbauern, Plantagenarbeitern und Arbeitslosen rekrutierte sich aus den gleichen ethnischen Gruppen, wobei hier die hawaiische Bevölkerung den größten Anteil stellte (siehe hierzu Kreisel 1984:133 ff.).

Eng verbunden mit der Etablierung der Haole als der neuen ökonomischen und politischen Oberschicht war die frühe Einrichtung eines Bildungssystems nach „westlichem“ Muster. Schon die Missionare hatten 1820 erste Schulen eingerichtet, in denen allerdings noch in hawaiischer Sprache ('Ölelo Hawai ${ }^{~} i$ ) unterrichtet wurde. ${ }^{123}$ Im Jahre 1854 existierten bereits 412 dieser so genannten „Common Schools“ mit insgesamt 11.782 Schülern. Zunehmend ersetzten allerdings englischsprachige „Select Schools“ diese frühen Schulen, und 1896 wurden bereits 99,5\% aller Schüler in Hawai' ${ }^{i}$ in Englisch unterrichtet. Zwar waren fast alle Hawaiier zu diesem Zeitpunkt des Lesens und Schreibens kundig, allerdings galt dies für viele ausschließlich für die hawaiische Sprache: Nur 26\% der Hawaiians und 69,3\% der Part Hawaiians ${ }^{124}$ konnten Englisch lesen oder schreiben. Als durch die Regelungen des Organic Act von

\footnotetext{
${ }^{121}$ Die übrigen 14,8\% teilten sich andere Bevölkerungsgruppen, wobei allein 12,6\% zum Besitz von Bürgern chinesischer Abstammung gehörten.

${ }^{122}$ Anzumerken bleibt, dass insbesondere einige der hawaiischen Ali ‘ $i$ durch Grundbesitz, Heiratsbeziehungen und politische Ämter in der neuen Oberschicht vertreten waren.

${ }^{123}$ Ausführlich zur frühen Geschichte des Unterrichtswesens in Hawai'i siehe Kuykendall (1947:104-13, 347-67)

${ }^{124}$ Der Zensus unterschied bis in das 20. Jahrhundert zwischen Hawaiiern ausschließlich hawaiischer Abstammung und jenen mit anderen „races“ zugehörigen Vorfahren.
} 
1900 Englisch endgültig offizielles Idiom Hawai'is wurde, war ein deutlicher Teil der hawaiischen Bevölkerung zunächst effektiv von höheren Posten in Politik und Verwaltung ausgeschlossen (U.S. Zensus 1896, zit. nach Kreisel 1984:131).

Im frühen 20. Jahrhundert konnten insbesondere japanische und chinesische Zuwanderer in der sozialen Hierarchie des Territoriums aufsteigen, während sich die soziale Situation eines Großteils der Hawaiier weiterhin verschlechterte (Kreisel 1984:177). Erst zum Ende des letzten Jahrhunderts begann sich ein Umschwung anzudeuten, doch bestimmen nach wie vor Faktoren der sozialen Benachteiligung das statistische Bild dieser Bevölkerungsgruppe. So bleibt das Einkommen hawaiischer Haushalte weiterhin hinter dem Durchschnittseinkommen zurück: 1989 lag der Anteil hawaiischer Familien, die lediglich über ein Jahreseinkommen unter US \$ 15.000 verfügen konnten, erheblich über dem Durchschnitt (19,49\% gegenüber 14,87\%). Und während 6,03\% aller Familien im Bundesstaat unterhalb der Armutsgrenze leben mussten, waren es bei den hawaiischen Familien mit 14,08\% sogar mehr als doppelt so viele (OHA 1996:472, 486). ${ }^{125}$

In den 1990er Jahren waren Arbeitslosigkeit und Abhängigkeit von staatlicher Unterstützung unter der hawaiischen Bevölkerung signifikant häufiger als in der Gesamtbevölkerung. ${ }^{26}$ Weitere Indikatoren für die anhaltende gesellschaftliche Benachteiligung eines großen Teils der Hawaiier finden sich im Bildungssystem, wo die hawaiische Bevölkerung zwar überproportional viele Schulabgänger mit entsprechendem Abschluss stellt (ebenso überproportional ist allerdings auch die Zahl der Schulabbrecher), im System der tertiären Bildung aber deutlich unterrepräsentiert ist. ${ }^{127}$ In der Kriminalitätsstatistik Hawai‘is sind Hawaiier überdurchschnittlich vertreten und mit 40,4\% stellten sie 1993-94 den größten Anteil an den in den Gefängnissen Hawai'is Inhaftierten (OHA 1996:444). Schließlich weisen die Zahlen zur gesundheitlichen Situation der hawaiischen Bevölkerung ebenfalls auf einen marginalen Status dieser Gruppe hin. Die Autoren des „Native Hawaiian Data Book“ führen hierzu zusammenfassend aus:

\footnotetext{
${ }^{125}$ Innerhalb der hawaiischen Bevölkerung gibt es allerdings sehr große Unterschiede in der Einkommensverteilung: so konnten immerhin 12,29\% der hawaiischen Haushalte über mehr als US \$ 75.000 jährliches Einkommen verfügen (OHA 1996:478).

${ }^{126}$ Von 1991 bis 1995 stieg die Arbeitslosenquote in Hawai'i von 2,7\% auf 5,9\% an, für den hawaiischen Bevölkerungsteil waren die entsprechenden Zahlen 4,0\% bzw. 10,0\%; während im Jahre 1989 6,8\% aller Haushalte auf staatliche Unterstützung angewiesen waren, empfingen 14,5\% der hawaiischen Haushalte entsprechende Leistungen (OHA 1996:244, 540).

${ }^{127}$ Die entsprechenden Zahlen aus dem Jahr 1990 zeigen, dass 15,73\% der hawaiischen Bevölkerung älter als 25 Jahre über irgendeinen Abschluss des tertiären Bildungssystems verfügten, in der Gesamtbevölkerung waren dies 31,22\% (OHA 1996:214).
} 
„Disease incidence and mortality are strongly associated with lifestyle and risk factors. Of all racial groups living in Hawai' $i$, Native Hawaiians are the racial group with the highest proportion of multiple risk factors leading to illness, disability, and premature death. Statistics for 1992 reveal a high risk profile for Native Hawaiians with the bulk of them having one of the following risk factors: sedentary life, obesity, hypertension, smoking, and acute drinking [...] Also the statistics indicate that large segments of the Native Hawaiian population were recipients of state- and federal-sponsored health care services, a clear indication that low income is a barrier to full access to health care systems“ (OHA 1996:409).

Die vorangegangenen Ausführungen zeigten eine Reihe von Faktoren, die den hawaiischen Bevölkerungsteil des Inselstaates als eine sozial marginalisierte Gruppe charakterisieren. Verantwortlich für diese signifikante Häufung von Negativfaktoren sind nicht nur Entwicklungen des 20. Jahrhunderts, auch die frühere hawaiische Geschichte trug einen wesentlichen Teil hierzu bei. Nachdem ich in diesem Abschnitt demographische und ökonomische Faktoren dargestellt habe, die zur Herausbildung einer spezifischen hawaiischen „Minderheit" geführt haben, werde ich im Folgenden auf den Wandel der Eigen- wie auch der Fremdbezeichnungen für die Hawaiier eingehen. Die Auseinandersetzungen um die Namen bzw. Namensgebung stellten nicht nur einen zentralen Aspekt der zwischen Autonomiebewegung und Akteuren der Mehrheitsgesellschaft geführten Kontroverse um kulturelle Hegemonie dar. Die Veränderung und Neufassung der Namen und Bezeichnungen hinsichtlich Sprache, Semantik und kultureller Bezüge war (und ist) auch Teil eines kolonialen Projekts, das Hawai'i und die Hawaiier nicht nur sprachlich und kulturell vereinnahmte, sondern auch politisch und rechtlich neu deutete und den Regelungen der Mehrheitsgesellschaft unterwarf. Zugleich verweisen die Wandlungen von Terminologie und Bedeutung auf Aus- und Abgrenzungen, wie sie von Vertretern der Mehrheitsgesellschaft, aber auch von Seiten der Autonomiebewegung angestrebt wurden und werden.

\section{2 „Hawai'i““ und „Hawaiians“: Aus- und Abgrenzungen}

„Hawaiian Airlines, Royal Hawaiian Hotel, Hawaiian Electric Co., Hawaiian Telephone Co., Hawaiian Sugar Planters Association, Hawaiian Life Insurance, and even the Hawaiian Historical Society are not Hawaiian!“ (Kekuni Blaisdell 1989:77 [Hervorhebung im Original]). 
Die Veränderung der Namen - sei es die terminologische Neuordnung der Geographie, die Umbenennung einheimischer Bevölkerungen oder auch die Neuregelung der persönlichen Namensgebung - gehört zum Machtinventar kolonialer Eroberer und hat zumal in den USA weit reichende spanische, französische und vor allem angelsächsische Spuren hinterlassen. Der US-Bundesstaat Hawai'i scheint hingegen hinsichtlich der geographischen Namen und Bezeichnungen, die der Besucher auf Landkarten, Straßenschildern oder an Hotels findet, nach wie vor sehr „hawaiisch“ zu sein. Tatsächlich haben hier im Gegensatz zu den kontinentalen USA, wo im Allgemeinen die Benennungen der früheren Bewohner ausgelöscht bzw. bedeutungslos wurden, nach wie vor fast 90\% aller Ortsbezeichnungen ihren Ursprung in der hawaiischen Sprache, die dem Neuankömmling allerdings jenseits musikalischer Darbietungen kaum noch (oder aufgrund intensiver Bemühungen um ihre Wiederbelebung schon wieder) als gesprochenes Wort begegnet. Gerade Namensgebungen neueren Datums weisen darüber hinaus häufig auf eine gewisse Nonchalance im Umgang mit der Sprache hin und lassen vermuten, dass es den Namensgebern eher um einen „hawaiischen Klang“ der zu vermarktenden Grundstücke, Hotelzimmer oder auch Privatschulen ging als um die jeweilige Eigenheit des Ortes (cf. Pukui, Elbert u. Mookini 1974:241-4). ${ }^{128}$ Auch folgen die in der Zeit der euroamerikanischen Dominanz vergebenen Namen oft nicht mehr der traditionellen hawaiischen Namensgebung, die den jeweiligen Ort mit seinen spezifischen Eigenheiten bzw. historischen oder legendären Ereignissen verknüpfte (Wood 1999:10f.), sondern einer euro-amerikanischen Tradition, in der Orte nach Personen, aber auch willkürlich gewählten Vögeln, Blumen o. ä. benannt werden.

Wer sich mit dem heutigen Hawai'i und seiner Gesellschaft beschäftigt, sieht sich bald mit Namen und Bezeichnungen für Land und Bevölkerung konfrontiert, deren divergierende Bedeutungen und jeweiligen Verwendungszusammenhänge verwirren können. So begegnet bereits der Name des Bundesstaates - abgesehen davon, dass er sowohl die Gesamtheit der Inseln als auch die größte Landmasse („Big Island“) bezeichnet - in unterschiedlicher Schreibweise: während die regierungsamtliche Schreibung des Staatsnamens nach wie vor auf die schriftliche Umsetzung des Verschlusslauts im Wort „Hawai'i“" verzichtet (wie es in der Schreibung des Hawaiischen bis ins 20. Jh. allgemein üblich war), setzt sich in fast allen übrigen Bereichen die heute als sprachlich korrekt angesehene Schreibweise des Hawaiischen unter Berücksichtigung des 'okina-Zeichens für den Okklusiv (sowie des so genannten kahakō für die Vokaldehnung) immer mehr durch. So stehen „Hawaii“ und

\footnotetext{
${ }^{128}$ Als eher unglücklich gewählte Namensgebungen nennen die Autoren u.a. Le`ale'a Hale, Haus des Genusses, als Name eines Hotels oder Hana hau 'oli, Freudige Arbeit, den Namen einer privaten Grundschule - beide Bezeichnungen besitzen im Hawaiischen von den Namensgebern sicher nicht gewünschte starke sexuelle Konnotationen.
} 
„Hawai'i“ nebeneinander. Welche (auch politische) Bedeutung man einem so klein scheinenden Unterschied in den 1990er Jahren beimaß, zeigten exemplarisch die Auseinandersetzungen um die Schreibweise des Namens der staatlichen Universität, die von Seiten organisierter Studenten mit Unterstützung von Teilen der Autonomiebewegung vehement geführt wurden und zum Ende des Jahrzehnts die Änderung der offiziellen Bezeichnung des Campus in Honolulu („University of Hawai'i at Mānoa“) bewirkten. Im Folgenden gehe ich auf den Wandel der Eigen- und Fremdbezeichnungen für die Inseln sowie für den hawaiischstämmigen Teil der Bevölkerung ein, beleuchten doch die im Lauf der Zeit sich wandelnden Namen, Schreibweisen, Definitionen und Wortverwendungen beispielhaft das im Rahmen dieser Untersuchung zentral stehende Problem der Ab- und Ausgrenzungen der hawaiischen Minderheit.

Die Namen „Hawai' $i$ “ und „Hawaiian Islands“ sind heute (unabhängig von der Schreibweise) sowohl die offiziellen als auch die gebräuchlichsten Bezeichnungen der Inselgruppe im Nordpazifik, die bis in ins 20. Jh. im internationalen Verkehr unter dem Namen „Sandwich Inseln“ bekannt war. Europäischer Namensgeber des Archipels war James Cook, der die Inselkette am 2. Februar 1778 zu Ehren von John Montagu, 4th Earl of Sandwich, benannte:

„These five Islands, Atoui, Eneeheeou, Orrehoua, Otaoora and Wouahoo, names by which they are known to the Natives, I named Sandwich Islands in honour of the Earl of Sandwich“ (Beaglehole 1967:278). ${ }^{129}$

Dieser Name, der im Gegensatz zu den Namen der einzelnen Inseln keiner einheimischen Benennung entsprach und unter der indigenen Bevölkerung des Archipels immer unüblich blieb, hatte im offiziellen Sprachgebrauch lange Bestand. Erst die am 8. Oktober 1840 verabschiedete Verfassung des Königreichs verwendete die offizielle Bezeichnung „Hawaiian Islands“, während die Bill of Rights aus dem vorhergehenden Jahr noch von den „Sandwich Inseln“ sprach. Schon bald nach der Verabschiedung der Verfassung ging James Jarves in seiner 1843 in London erschienenen „History of the Hawaiian or Sandwich Islands“ ebenfalls auf die „korrekte“ Benennung der Inseln ein:

„Their legitimate appellation, and the one by which they still continue to be distinguished by the aboriginal inhabitants, is ,Hawaii nei', a collective term, synonymous with the Hawaiian Islands. They are so

\footnotetext{
${ }^{129}$ Cook führt hier nur die Inseln Kaua‘i, Ni‘ihau, Ka‘ula und O`ahu auf, da er die anderen Inseln des Archipels erst bei seinem zweiten Besuch 1778-1779 sichtete. Der 4th Earl of Sandwich, John Montagu (1718-1792) war Erster Lord der britischen Admiralität während des amerikanischen Unabhängigkeitskrieges.
} 
called from the largest of the group, Hawaii; from which the reigning family originated“ (Jarves 1843:1).

Auch wenn sie der der hawaiischen Bezeichnung der Inseln nahekommt, so steht die Umbenennung von 1840 durchaus in einer Reihe kolonialer Namensänderungen: Wie James Cook die „Sandwich Islands“ in den neuen Kontext eines britischen Pazifik stellte, so entsprach der Wechsel zu „Hawaiian Islands“ den Wünschen der zunehmend einflussreicheren Amerikaner in der Regierung des Königreichs, die einen „britischen“ Namen der Inselgruppe strikt ablehnten (Clement 1980:53-5) - ein erstes Indiz auf die sich anbahnenden radikalen gesellschaftlichen, ökonomischen und politischen Veränderungen des Gemeinwesens.

Stärker noch als die geografische Namensgebung waren Eigen- und vor allem Fremdbezeichnungen der einheimischen Bevölkerung in den vergangenen zwei Jahrhunderten einem Wandel unterworfen. Zu Beginn des Kontaktes mit den europäischen Seefahrern, Missionaren und Kaufleuten existierte keine spezifisch hawaiische Eigenbezeichnung: das hawaiische Wort Kanaka meinte sowohl Hawaiier als auch Menschen schlechthin. So verwendet David Malo in seiner bereits vor 1840 verfassten und 1903 erstmals in englischer Übersetzung erschienenen Geschichte Hawai' is (Malo 1992:4) das Wort in beiden Bedeutungen. In dem bereits 1838 im Druck erschienenen Buch „Ka Mooolelo Hawaii“ (Mo 'o 'ólelo Hawai ' $i$ - Geschichte von Hawai'i) verwenden die Autoren, Schüler der 1831 gegründeten Missionsschule in Lahaina Luna, Maui, Termini wie Kānaka ma Hawai 'i (Menschen von Hawaii) oder Nä Kama ăina (die auf dem Land Geborenen, Landeskinder), um die Hawaiier von den Fremden, den Haole, zu unterscheiden (Kahananui 1984:3,5). Schon früh übernahmen die Haole das hawaiische Wort Kanaka, das durch die stark wachsende Zahl von Hawaiiern, die auf amerikanischen und europäischen Schiffen anheuerten oder Arbeit auf dem nordamerikanischen Kontinent annahmen, weit über Hawai‘i hinaus Bedeutung erlangte. Zunehmend mit rassistischen Konnotationen behaftet, wandelte sich das Wort bald zu einer abwertenden Bezeichnung für alle Bewohner der Südseeinseln (Duncan 1973:94-5). ${ }^{130}$ Noch 1865 verzeichnet Lorrin Andrews in seinem Wörterbuch der Hawaiischen Sprache unter dem Eintrag Kanaka lediglich die Übersetzungen „Mensch, Person“, um dann aber

\footnotetext{
${ }^{130}$ In der zweiten Hälfte des 19. Jahrhunderts und im frühen 20. Jahrhundert wurden vor allem jene Melanesier als Kanaka bezeichnet, die von ihrem Heimatinseln entführt und unter erbärmlichen Bedingungen auf den Plantagen in Queensland, Australien, arbeiten mussten. Ab 1904 durfte keine „Anwerbung“ mehr stattfinden und seit 1906 ist „Kanaka Labour“ in Australien verboten. Besondere Bedeutung gewann das Wort kanaka im 20. Jahrhundert auch in Neukaledonien, wo der in der Kolonialzeit von den französischen Siedlern durchaus abwertend benutzte Begriff Kanak seit den 1960er Jahren von der melanesischen Bevölkerung der Inseln als integrierende Eigenbezeichnung benutzt wird (s. West 2009:360).
} 
hinzuzufügen: „In a vulgar, low sense as sometimes used by foreigners, a Hawaiian, a native, in distinction from a foreigner“ (1865:256). Das „Hawaiian Dictionary“ von Mary Kawena Pukui und Samuel H. Elbert aus dem Jahr 1986 fügt dem Eintrag Kanaka neben den Bedeutungen „Human being, person, individual“ die weitere, weder positiv noch negativ vermerkte Übersetzungsmöglichkeit „Hawaiian“ hinzu. So wird hier, zumindest auf der lexikalischen Ebene, die „Unschuld“ eines Wortes wieder hergestellt, das heute zwar wieder in bestimmten Kontexten zur Eigenbezeichnung genutzt, dessen Verwendung durch Nicht-Hawaiier aber nach wie vor als unangemessen angesehen wird.

Der heute gebräuchlichste Name für die indigene Bevölkerung ist das zunächst relativ unspezifische englische Wort Hawaiian, das sowohl substantivisch als auch adjektivisch gebraucht werden kann - also jemand oder auch etwas, der, die oder das von Hawai'i stammt bzw. in einem mehr oder weniger eindeutigen Bezug zu den Inseln steht. Im offiziellen Sprachgebrauch wie auch in der Umgangssprache des Inselstaates bezeichnet der Terminus Hawaiian in seiner Verwendung für Personen aber eben nicht einen Bürger des Staates Hawai'i bzw. jemanden, der auf eine Abstammung von Bürgern des Staates, des Territoriums oder des Königreichs Hawai'i verweisen kann, sondern ausschließlich die Nachfahren der polynesischen Urbevölkerung des Archipels. Das Hawaiian Dictionary (Pukui u. Elbert 1986) gibt verschiedene Bedeutungen für das Stichwort Hawai ' $i$ : zum einen ist dies die geographische Bedeutung als Name der größten Insel des Archipels als auch der gesamten Inselkette; zum anderen wird Hawai ‘ $i$ übersetzt mit „Hawaiian person, Hawaiian“. Das Wörterbuch von Andrews führt unter „Hawai'i“ noch aus:

„Name of the largest island of the Hawaiian group, and gives name to the group. From time immemorial the people have called themselves 'ko Hawaii', and the islands 'ka pae aina o Hawaii', 'na moku Hawaii', \& c.“ $(1865: 152){ }^{131}$

In eben dieser Doppelbedeutung, der Vermischung des geographischen Bezugs mit der Frage ethnischer Zugehörigkeit, liegt die Problematik, auf die Kekuni Blaisdell in dem am Anfang dieses Kapitels stehenden Zitat anspielt.

Während mit dem Verlust der eigenen Sprache hawaiische Eigenbenennungen außer Gebrauch gerieten und dem englischen Hawaiian Platz machten, blieben die euroamerikanischen Neusiedler für die meisten Hawaiier Haole bzw. Foreigner, Fremde. Bemerkenswert ist eine in den ersten Jahrzehnten des 20. Jahrhunderts einsetzende Umdeutung der hawaiischen Bezeichnung $\mathrm{Ka}$ ma 'äina („Landeskind“) durch die Fremden und damit die eigenmächtige Feststellung der Zugehörigkeit: Nā Kama 'äina waren die an einem bestimmten Ort

${ }^{131}$ „Kō Hawai ' ${ }^{\prime}$ “ bedeutet: „von Hawai‘i stammend, zu Hawai‘i gehörend“ [U.M.]. 
des Archipels Geborenen, die „Kinder des Landes“, doch in der Verwendung des Wortes durch die Siedler schloss der Begriff bald alle „weißen“ euroamerikanischen Bewohner der Inseln ein, ob sie dort geboren oder zugewandert waren. ${ }^{132}$

Angesichts seiner heute üblichen, relativ offenen und alles andere als eindeutigen Verwendung verdient der Begriff Hawaiian hier eine nähere Betrachtung. Die historischen Entwicklungen in Hawai‘i führten dazu, dass die Hawaiier heute eine intern sehr stark differenzierte Minderheit innerhalb der Bevölkerung Hawai' is darstellen. Insbesondere dieser Bevölkerungsteil ist von einer sehr hohen Rate interethnischer Heiraten gekennzeichnet, so dass Howard 1980 im Hinblick auf die Bezeichnung „Hawaiian“ feststellte, dass es einen immer größeren Teil der Hawaiier gäbe, deren ethnische Zugehörigkeit als problematisch bezeichnet werden könne und: „A combination of circumstances has rendered the term ambiguous and variable in current usage ... and it is far from clear who is a Hawaiian in the contemporary world“ (Howard 1980:449). Es bleibt zu fragen, inwieweit eine solche Aussage in den 1990er Jahren und danach aufrecht erhalten werden kann; über den Gebrauch in der Literatur, im Recht und in den offiziellen Statistiken und Volkszählungen des Staates und des Bundes hinaus hat der Begriff zahlreiche weitere Bedeutungen erfahren.

So fragt der US-Zensus, die offizielle Volkszählung der Vereinigten Staaten, u.a. auch nach der „Rasse“ (race) der befragten Personen. ${ }^{133}$ Dem System der Klassifizierung der Bevölkerung nach „Races“ lag in den USA lange Zeit die Vorstellung von großen, durch das Kriterium Hautfarbe voneinander unterscheidbarer Bevölkerungsgruppen zugrunde - eine Klassifizierung, die im Hawai‘i des 19. Jahrhunderts ungebräuchlich war (hier wurde vielmehr auf der Basis von Herkunftsland und -kultur unterschieden) und erst nach der Annexion durch den zunehmenden Einfluss des US-Systems eingeführt wurde (Lind 1967:19 ff.). Seit 1990 beschränkte sich der Zensus auf die Feststellung von vier „basic racial categories“: „American Indian or Alaska Native, Asian or Pacific Islander, Black, and White“ (U.S. Bureau of the Census 1995:4). Der Zensus von 1980 kannte dagegen noch 15 verschiedene Gruppen, die unter dem Stichwort „race“ aufgeführt wurden, u.a. auch die Hawaiier als eigenstän-

\footnotetext{
${ }^{132}$ Im heutigen Hawai ‘i ist der Begriff vor allem durch die Werbung allgegenwärtig: insbesondere Hotels und Reiseunternehmen bieten freie Kapazitäten auf den Nachbarinseln im Rahmen von „Kama‘āina Specials“ zu günstigen Preisen der Wohnbevölkerung an.

${ }_{133}$ Die Zensusbehörde bemerkt zu der Frage nach der „Rassenzugehörigkeit“: „The concept of race the Bureau of the Census uses reflects self-identification by respondents; that is the individuals perception of his/her racial identity. The concept is not intended to reflect any biological or anthropological definition ... Furthermore, the Bureau of the Census recognizes that the categories of the race item include both racial and national origin or socio-cultural groups" (U.S. Bureau of the Census 1995:4); siehe zum US-amerikanischen „race“-Konzept auch Zack (1993:9-18).
} 
dige Kategorie: „White, Black, American Indian, Eskimo, Aleut, Chinese, Filipino, Japanese, Asian Indian, Korean, Vietnamese, Hawaiian, Samoan, Guamanian, and Other“ (U.S. Bureau of the Census 1991:4). Die „racial categories" des Zensus reflektierten immer gesellschaftliche und politische Veränderungen in den USA. So wird z.B. seit 1980 auch nach der neugeschaffenen Kategorie Hispanic (Einwanderer aus Latein- und Mittelamerika) gefragt, zu der allerdings festgestellt wird, dass ,....Hispanic persons may be of any race“ (U.S. Bureau of the Census 1995:5). Bis zur Volkszählung von 1970 differenzierte der US-Zensus darüber hinaus noch zwischen Hawaiians, d.h. hier: Personen mit einer rein hawaiischen Abstammung, und Part Hawaiians, d.h. Personen, unter deren Vorfahren sich nicht nur Angehörige der polynesischen Urbevölkerung finden. Die Klassifikation Part Hawaiian ist von den Zensusbehörden des Bundes sowie des Staates Hawai' $\mathrm{i}$ in den vorhergehenden Jahrzehnten immer wieder geändert und neu bestimmt worden:

„The 1853 census applied the terms 'natives' and 'half-natives' to the people later called Hawaiian and Part Hawaiian. Between 1860 and 1890 the term 'half castes' was used. The U.S. censuses between 1910 and 1930 adopted a special classification system for Hawai' $i$, attempting to differentiate between 'Caucasian-Hawaiians' and 'AsiaticHawaiians'. In 1940, 1950, and 1960 the United States census and the Hawaiian Department of Health reported any Hawaiian admixture as Part Hawaiian, regardless of percentage“ (Nordyke 1989 a:34).

Nach 1970 fällt diese Differenzierung nach Abstammungsgrad innerhalb des US-Zensus fort, da für den gesamten Geltungsbereich die Kategorie „mixed race“ abgeschafft wurde, was insbesondere in Hawai'i zu einem starken Anstieg in der Sammelkategorie „Other“ führte. Bei gleichzeitiger Einführung der Selbstidentifikation der Befragten hinsichtlich der Kategorie „Race“ galt ab 1970 die Gesamtkategorie Hawaiian (Nordyke 1989 a:34-5; Kreisel 1984:301).

Anders als die Bundesverwaltung bezieht sich der Staat Hawai'i in seinen Planungen nicht auf die zehnjährigen Zensusbefragungen, sondern auf jährlich erhobene Daten des Hawaii Health Surveillance Program (HSP) des Hawaii Department of Health, die auf der Befragung repräsentativer Bevölkerungsstichproben beruhen und detaillierte Daten zu Gesundheit und SozioDemographie im Staate Hawai'i liefern (Hawaii Department of Health 1997). ${ }^{134}$ Das HSP hat abweichend von den Statistikern des US-Zensus die Kategorie „mixed race“ beibehalten und trägt damit der großen Bedeutung

\footnotetext{
${ }^{134}$ Aufgrund der voneinander abweichenden Erhebungsmethoden existieren signifikante Unterschiede zwischen den Zahlen des HSP und denen des US-Zensus, so dass eine direkte Vergleichbarkeit der Statistiken nicht immer gegeben ist (Nordyke 1989 a:104-9).
} 
interethnischer Heiraten in Hawai‘i Rechnung, ${ }^{135}$ es klassifiziert so z.B. weiterhin nach Hawaiian (100\% Abstammung) und Part Hawaiian - zwei Kategorien, die für eine Reihe geltender Gesetze des Staates Hawai'i keineswegs unbedeutend sind.

Die Vergabekriterien für öffentliche Hilfen der USA sowie des Staates Hawai'i an Personen hawaiischer Abstammung sind durch verschiedene gesetzliche Regelungen und Bestimmungen des Staates Hawai'i an den Grad der biologischen Abstammung von der polynesischen Urbevölkerung gebunden. Insbesondere das biologistische Konzept des „blood quantum“ ist in diesem Zusammenhang von entscheidender Bedeutung. Im Rahmen dieser Vorstellung wird die Zugehörigkeit zu einer „racial group“ vom Anteil des jeweiligen Blutes abhängig gemacht, so dass der Grad der Abstammung in Quotienten der Blutmenge angegeben werden kann. Diese Art der graduellen Abstammungsbestimmung ist in den USA (außerhalb der Naturwissenschaften) noch immer sehr gebräuchlich und geht zurück auf Vorstellungen des 19. Jahrhunderts, die die Zugehörigkeit zu einer menschlichen Rasse z.B. im Blut verankert sahen. ${ }^{136}$ Es ist in Hawai' $i$ auch heute üblich, den Grad seiner hawaiischen Abstammung in Fraktionen der reinen, 100\%igen Abstammung anzugeben: so ist jemand z.B. 1/4 oder auch nur 1/16 Hawaiisch, und auch das Hawaii State Databook übernahm bis 1994 Tabellen, die mit einer solchen Klassifikation arbeiten (State of Hawaii 1994:40).

Eine konkrete Auswirkung dieser Regelungen findet sich in den Gesetzen des Hawaiian Homes Commission Act (HHCA), der die Vergabe von Land an berechtigte Hawaiier regeln sollte. Im Jahre 1921 legte der US-Kongress den Status Native Hawaiian durch eine blood quantum-Regel fest: Native Hawaiians im Sinne dieses Gesetzes müssen ein blood quantum von mindestens $50 \%$ „hawaiischen Blutes“ nachweisen und sind damit berechtigt, Land nach den Regelungen des HHCA zu erhalten (Murakami 1991:43; Department of Hawaiian Home Lands 1994:6). Die Implikationen dieser Festlegung des Status Native Hawaiian reichen über die reine Landvergabe hinaus: Da das Office of Hawaiian Affairs einen Teil seiner Mittel aus den Erträgen des Public Land Trusts erhält, fallen diese unter die „50\%-blood quantum“-Klausel. Die Förderprogramme und Unterstützungen des Office of Hawaiian Affairs, deren Finanzierung aus dieser Quelle stammt, sind somit ebenfalls ausschließlich

\footnotetext{
${ }^{135}$ Es stellt sich natürlich bei einer kritischen Betrachtung des Konzeptes „,race“ sofort die Frage nach der Sinnhaftigkeit eines Konzeptes „mixed race“, da dieses ja die Vorstellung einer wie auch immer gearteten konstanten „Rasse“ impliziert (Zack 1995 a:Xviii).

136 Es sei hier noch einmal festgestellt, dass diese Vorstellungen jeglicher naturwissenschaftlichen Grundlage entbehren. Zur kulturellen Bewertung des Blutes als Träger unveränderbarer Eigenschaften siehe Hauser-Schäublin (1995).
} 
Native Hawaiians im oben genannten Sinne vorbehalten. ${ }^{137}$ Der Begriff Native Hawaiians hat also im Rahmen der Gesetzgebung eine spezifische Bedeutung, die durch den HHCA von 1921 definiert ist.

Außerhalb des oben umrissenen offiziellen Rahmens wird der Terminus (Native) Hawaiian allerdings nicht nur in dem genannten engen Sinne mit seinen juristischen Implikationen verwendet. Er steht vielmehr zugleich verallgemeinernd für alle Hawaiier, gleich welchen Abstammungsgrades, und ist heute die gewöhnliche Eigenbezeichnung. Die Übernahme des Zusatzes Native (eingeboren) oder auch Indigenous (indigen) durch Vertreter der Autonomiebewegung erfolgt im Kontext der Abgrenzung gegen die übrige Bevölkerung, die als Einwanderer und Kolonialisten gesehen werden (siehe u.a. H.-K. Trask 1993:70; MacKenzie 1991:ix-xi). In diesem Zusammenhang sind auch die Bestrebungen zur Reklassifizierung der Hawaiier hinsichtlich der „racial categories“ des US-Zensus (s.o.) zu sehen (natürlich spielte die deutlichere Repräsentation der Bevölkerungsgruppe in Hinblick auf Bundeshilfen etc. ebenso eine Rolle). Seit 1990 waren die Hawaiier der Zensuskategorie „Asian or Pacific Islander" zugeordnet, waren also als ethnische Kategorie bzw. „Race“ aus den Statistiken des Zensus verschwunden. Nach Ansicht hawaiischer Gegner dieser Änderung wurde diese Zusammenfassung der Hawaiier mit der großen Bevölkerungsgruppe der Asiaten dem besonderen Status der Hawaiier als indigener Bevölkerung nicht gerecht und verzerrte darüber hinaus die wahre soziale und ökonomische Situation der Hawaiier. Die Bestrebungen hawaiischer Organisationen, unterstützt durch die Abgeordneten des Staates Hawai'i im US-Kongress, dem besonderen Status der Hawaiier durch Bildung einer neuen Kategorie American Indian, Alaskan Native or Native Hawaiian bzw. einer eigenständige Kategorie für die Hawaiier gerecht zu werden, führten 1997 schließlich zu einer Änderung. Zwar lehnte das maßgebliche Office of Management and Budget die vorgeschlagene „indigene“ Bevölkerunskategorie $\mathrm{ab}^{138}$, beschloss aber die Einführung einer neuen Kategorie Native Hawaiian or Other Pacific Islander und wurde damit einem Teil der Forderungen gerecht. Die Begründung des Office of Management and Budget für diese Änderung bezieht sich allerdings primär auf die bessere Repräsentation des hawaiischen

\footnotetext{
${ }^{137}$ Das Office of Hawaiian Affairs hat 1984 eine Untersuchung durchgeführt, die ergab, dass im Jahre 1984 39\% der Hawaiier ein „blood quantum“ von 50\% oder mehr besaßen. Noch insgesamt 4\% der Hawaiier besaßen ein „blood quantum“ von 100\%, während die Zahl der Hawaiier mit einem „blood quantum“ unter 50\% ein deutliches Wachstum aufweist (OHA 1996:36-43). Zur Bedeutung des “blood quantum” in Bezug auf „Native Americans“ in den USA siehe u.a. Wilson (1992).

${ }^{138}$ Nicht zuletzt lehnten auch Vertreter der American Indians eine solche Kategorie ab, da die unter der bisherige Zensus-Kategorie American Indian or Alaskan Native gesammelten Daten von zentraler Bedeutung für die Durchführung von Bundesprogrammen für diese Bevölkerungsgruppen seien (OMB 1998).
} 
Bevölkerungsteiles in den Daten des Zensus durch die neue Klassifizierung und nicht auf einen besonderen Status der hawaiischen Minderheit:

„The Native Hawaiians presented compelling arguments that the standards must facilitate the production of data to describe their social and economic situation and to monitor discrimination against Native Hawaiians in housing, education, employment, and other areas. Under the current standards for data on race and ethnicity, Native Hawaiians comprise about three percent of the Asian and Pacific Islander population. By creating separate categories, the data on the Native Hawaiians and other Pacific Islander groups will no longer be overwhelmed by the aggregate data of the much larger Asian groups. Native Hawaiians will comprise about 60 percent of the new category“ (OMB 1998).

Damit wurde, unter Voraussetzung der Annahme, dass eine Bevölkerungsdifferenzierung nach ethnischen und „rassischen“ Gesichtspunkten überhaupt sinnvoll sein kann $^{139}$, anerkannt, dass eine Kategorie wie Asian or Pacific Islander für eine Bevölkerungsstruktur wie die Hawai'is völlig unpraktikabel war und hinsichtlich der großen hawaiischen Diaspora in den kontinentalen USA ${ }^{140}$ das faktische Verschwinden der Minderheit zur Folge hatte (und natürlich meint auch hier "Native Hawaiian" nicht nur Native Hawaiians im Sinne des Hawaiian Homes Commission Act). In der Verwendung oben angesprochener Begriffe und den Änderungsbestrebungen zeigt sich nicht zuletzt auch der deutliche Wunsch nach Abgrenzung von den später eingewanderten Bewohnern der Inseln, nicht nur den Haole sondern insbesondere auch den Nachkommen der vor allem aus Asien stammenden Plantagenarbeiter, den Locals (cf. Fujikane 2000:xv-xxii; H.-K. Trask 2000:1-24). ${ }^{141}$

Der Wandel der Begriffe zur Eigen- bzw. Fremdbezeichnung der indigenen Bevölkerung des hawaiischen Archipels spiegelt die sehr früh beginnenden und sich über zwei Jahrhunderte fortsetzenden Verwerfungen in den kulturellen, ökonomischen und politischen Machtbalancen auf den Inseln. Mit der im 19. Jh. erfolgten Einführung und Durchsetzung des so neutral klingenden „Hawaiian“ durch die euro-amerikanische Minderheit wird die faktische Ausgrenzung der (Noch-) Mehrheit der Hawaiier auch sprachlich dokumentiert, war das englische Wort doch mit überwiegend negativen Vorstellungen von der kulturellen und physischen Andersartigkeit der so bezeichneten Menschen be-

${ }^{139}$ Es lassen sich hier durchaus auch andere Standpunkte beziehen, siehe u.a. Webster (1992).

${ }^{140}$ Der US-Zensus von 2000 weist neben 239.655 Hawaiiern in Hawai'i insgesamt 161.507 Hawaiier in den kontinentalen USA aus (OHA 2006:18-9).

141 Die von zahlreichen Überschneidungen geprägten komplexen Beziehungen zwischen „Locals“ und „Hawaiians“ rechtfertigen eine gesonderte Betrachtung, die im Anschluss an dieses Kapitel erfolgt. 
frachtet. Die spätere Übernahme von Begriffen aus der Sprache der inzwischen zur Minderheit gewordenen Hawaiier durch die Haole, wie das mit positiven Werten (Aloha!) aufgeladene Kama 'äina oder die zahlreichen willkürlichen Ortsnamen, zeigt deutlich, dass die sprachliche Machtpolitik hinsichtlich der nun als unbedrohlich für die eigene Lebensweise wahrgenommenen Minderheit in beide Richtungen arbeitete.

Der von Außen übergestülpte Name „Hawaiians“ und die ihn begleitenden näheren Bestimmungen stehen im Kontext einer „Rassifizierung“ und Ausgrenzung der indigenen Bevölkerung, die durch die „blood-quantum“Bestimmungen des Hawaiian Homes Commission Act noch verstärkt wird. Ein Vergleich der rechtlichen Implikationen des „blood quantum“-Konzepts in den kontinentalen USA zeigt deutlich, wie willkürlich die Bewertung der Blutanteile war und ist. Während schon der geringste Anteil „schwarzen Blutes“ die Zugehörigkeit zur afro-amerikanischen Minderheit bestimmte (,one-drop rule") und so den Fortbestand der Sklaverei sichern half, setzte die Regierung den für die „Rassenzugehörigkeit“ notwendigen Blutanteil bei Native Americans (wie entsprechend auch bei den Native Hawaiians) wesentlich höher an, waren und sind doch mit dem Status als „American Indian“ nicht unerhebliche Ansprüche auf Land und Geldmittel verbunden. Wie Zack (1995 a:xvii) feststellt, bestimmt das Interesse der herrschenden Mehrheit die Definitionen hinsichtlich der Zugehörigkeit zu einer Minderheit aufgrund eines bestimmten Blutanteils: „...ideas of racial purity work against oppressed groups, regardless of whether the purity of the oppressor or the purity of the oppressed is at stake“.

Mit der Übernahme der euro-amerikanischen Begriffe und im Kontext der seit über 100 Jahren bestehenden Minderheitensituation hat sich die ehemals inklusive polynesische Gesellschaft in eine hinsichtlich ihrer eigenen $\mathrm{Zu}$ gehörigkeitskriterien exklusive Gruppe der Hawaiier - Hawaiian, Part Hawaiian oder Native Hawaiian - gewandelt. Für die Zugehörigkeit ist die Abstammung von den voreuropäischen Bewohnern der Inseln notwendig oder wie Mililani Trask, ehemalige Kia 'Āina (Gouverneurin) von Ka Lāhui Hawai $i$, einer der wichtigsten Organisationen der hawaiischen Bewegung, kategorisch feststellt: „To be Hawaiian ... you have to have the koko (blood)“ (1993:113). Dass eine solche Übernahme von Kategorien der weißen Mehrheit durch indigene Minderheiten nicht unproblematisch ist (Howard 1980:449; Zack 1995 a:xvi-xvii), zeigt sich z.B. in einem Verfassungsentwurf Ka Lāhuis, der bestimmte leitende Funktionen innerhalb eines zu schaffenden hawaiischen Gemeinwesens Hawaiiern mit einem „blood quantum“ von über $50 \%$ vorbehält (Ka Lāhui 1993:15). Auch hier erhält die Biologie Vorrang vor kulturell begründeter Ethnizität, was innerhalb der hawaiischen Bevölkerung zu Widerspruch führte, da zugleich massive Bestrebungen bestehen, die hoch problema- 
tischen Regelungen des Hawaiian Homes Commission Act endlich abzuschaffen.

Die Eigendefinition unter Voraussetzung der biologischen Abstammung wendet sich auch gegen das u.a. von der Tourismusindustrie vertretene Konzept eines „Hawaiian at Heart“, das, ähnlich wie die Vorstellung von den euro-amerikanischen Kama 'äina, von einer Zugehörigkeit aufgrund emotionaler Verbundenheit mit „hawaiischen Werten“ ausgeht (Blaisdell 1989:78; H.-K. Trask 1993:3; M. Trask 1993:113). Ein solches Konzept sieht Hawaiians wiederum als eine offene Gruppe, in der zumindest jeder Gutwillige Mitgliedschaft beanspruchen kann. Nicht alle Hawaiier lehnen eine solche Vorstellung rundweg ab; so schreibt z.B. George S. Kanahele:

„... Hawaiians and their Hawaiian-ness give Hawai‘i its strongest sense of identity, to which all Island people ... can relate in a meaningful way ... In a real sense, Hawaiian-ness should belong to anyone who claims or adopts Hawai' $i$ as Home. It is the common resource - mana -- and to the extent that any person draws upon it, that person becomes Hawaiian“ (Kanahele 1982:30).

Wogegen sich hawaiische Aktivisten in den 1990er Jahren wandten, wenn sie die Exklusivität der hawaiischen Zugehörigkeit feststellten, macht ein Zitat der Statistikerin Eleanor Nordyke aus ihrem Standardwerk zur Demographie Hawai' is aus dem Jahr 1989 deutlich:

„By the end of the next century the Hawaiians, the most intermingled of all the races in the Islands, may have merged to fuse biological differences, and education and life in Hawai'i may have reduced cultural dissimilarities. By then it may not be necessary to have some blood of the ancient Polynesians to qualify as a Hawaiian“ (Nordyke 1989 a:41).

Wie Jocelyn Linnekin (1990a:208) feststellte, kann man diese Aussagen, die eine Vereinnahmung und Auflösung der hawaiischen Bevölkerung mit den Mitteln der Statistik betreiben, zu Recht als „naiv und veraltet“ zurückweisen. Die seit den 1980er Jahren angestoßenen Entwicklungen auf kulturellem und politischem Gebiet sprechen fraglos eine gegenteilige Sprache. Im Kontext der Autonomiebewegung und in der Folge ihrer Bemühungen um kulturelle Hegemonie mag da ein Standpunkt, wie ihn Lisa Kahaleole Hall vertritt, allemal repräsentativer sein:

„,Hawaiians at heart' assume that knowing and appreciating Hawaiian culture is enough to transform them into being Hawaiian. Indeed, some have gone so far as to claim that they are more Hawaiian than actual 
Hawaiians, because they have greater cultural or language knowledge“ (Hall 2005:410-1).

Im Untersuchungszeitraum begegneten in den Texten der Autonomiebewegung vornehmlich zwei unterschiedliche Eigenbezeichnungen:

- Auf der einen Seite hat der Rückgriff auf eine voreuropäische Eigenbezeichnung, die Kekuni Blaisdell in einem Artikel von 1989 propagierte, zunehmend an Bedeutung gewonnen. Das hawaiische Kanaka maoli, Pukui und Elbert zufolge die Bezeichnung für „Hawaiian Native“ (1986:240) oder, wie Blaisdell wörtlich übersetzt, „true or real person“ (1989:79), löst in vielen Texten der Bewegung, aber auch der wissenschaftlichen Auseinandersetzung das als problematisch wahrgenommene „Hawaiian“ ab, hat sich aber im allgemeinen Sprachgebrauch nicht durchsetzen können (u.a. Blaisdell u. Mokuau 1994; Prejean 1994; Kamauu 1993; Wood 1999).

- Häufiger wird jedoch auch in den politischen Texten das in der Alltagssprache gebräuchliche „Hawaiian“ beibehalten - in einem Kontext, der sich unter Verwendung der vorherrschenden Sprache gegen die Machtstrukturen der kulturellen und politischen Elite wendet.

Die Widersprüchlichkeit und schier unauflösbare Problematik des Umgangs mit den kolonialen Namen wird nicht zuletzt in diesen „autoethnographischen“ Texten (Pratt 1991,1992 [nach Wood 1999:15]) offenbar, wie das Beispiel aus einem Leserbrief von Kekuni Blaisdell aus dem Jahr 1998 zeigt, in dem er die spezifische Bindung an das Land und die zentrale Bedeutung des Landes für die Existenz der Hawaiier anspricht:

„Kalo is our hiapo (elder sibling), Haloa, who feeds us. Without wai (water) and 'āina (land), we have no kalo, and therefore, we kanaka maoli (Native Hawaiians) perish as a distinct people and nation“ (Blaisdell 1998, zit. nach Wood 1999:13).

Beharrlich färbt die koloniale Sprachregelung als eine für den Großteil der Hawaiier und anderer Leser heute notwendige Übersetzung der Gegenbezeichnungen auch hier durch.

\section{3 „Local“ - eine übergreifende ethnische/kulturelle Ka- tegorie}

Der Begriff „Local“ begegnet dem Besucher des heutigen Hawai'i in unterschiedlicher Bedeutung. Seit den 1990er Jahren als modisches Motto (z.B. „Local Boy“) von einheimischen Jugendlichen getragen, die sich damit gegen 
Zuwanderer und Touristen abzugrenzen suchten (cf. Pratt u. Smith 2000:57), findet sich der Begriff inzwischen auf von Touristen wie Einheimischen gekauften und viel getragenen T-Shirts, Badeshorts und Surfbrettern (wie z.B. der Marke „Local Motion“). Mit diesen Konsumartikeln wird ein als charakteristisch für die Inseln empfundener lässiger Lebensstil verbunden, der sich idealtypisch in der Welt der Surfer zu manifestieren scheint und dessen Bindung an den Ort Hawai'i durch Kauf und Mitnahme dieser Artikel scheinbar gelöst werden kann.

Doch der Begriff „local“ markiert nicht nur eine gefühlte lokale Kultur gegenüber einer zunehmend globalisierten Welt, die Unterscheidung „local“ zog auch eine tief gehende soziale und kulturelle Trennlinie durch die multiethnische und multikulturelle Inselgesellschaft. Schon um 1900 gebräuchlich, gewann das Wort seine eigentliche und engere Bedeutung im Zusammenhang mit dem so genannten Massie-Prozess von 1931. In diesem Verfahren standen mehrere asiatisch- bzw. hawaiisch-stämmige Männer wegen der angeblichen Vergewaltigung der Ehefrau eines Angehörigen der US-Marine vor Gericht und in der von den Angehörigen des vermeintlichen Opfers angeheizten Stimmung wurde ein Hawaiier gelyncht. ${ }^{142}$ In dieser Atmosphäre des „Wir gegen die Anderen“ meinte „local“ die Gemeinschaft der Unterlegenen oder wie Candace Fujikane schreibt:

„The term was particularly significant in that it gave shape to and set the stakes for an imagined community that unified Native Hawaiians and non-Native Hawaiians in defense against continental imperial power“ (Fujikane 1996:66).

Der Begriff grenzte also nicht nur die vorgestellte Gemeinschaft der in Hawai‘ $\mathrm{i}$ beheimateten Menschen und die ihnen zugeschriebenen kulturellen Aspekte gegen von außen kommende Machtfaktoren ab: er war zugleich auch ein Zeichen für die komplexe interne Differenzierung der ansässigen Bevölkerung entlang nicht kongruent verlaufender ethnischer wie sozialer Grenzen. Auch in diesem Fall war es der Kontrast zu einer als überlegen empfundenen Gruppe bzw. der Zwang, sich den von ihr vorgegebenen soziokulturellen Mustern anzupassen, der die opponierende Grenzziehung motivierte und begründete. Jonathan Okamura (1980:126) nennt verschiedene Kriterien für die Zugehörigkeit zu den „locals“, wobei er die Anpassung an neue Gesellschaftsnormen (,accommodation“) als wesentlichen Faktor benennt. Hinzu kommen Okamura zufolge eine gemeinsame Vergangenheit als Plantagenarbeiter sowie die Herausbildung und der Gebrauch einer gemeinsamen, bis heute allgemein als

${ }^{142} \mathrm{Zu}$ dem Prozess und seinen Auswirkungen auf die Gesellschaft des US-Territoriums Hawai`i siehe Stannard (2005). 
Pidgin bezeichneten Sprache, des Hawai'i Creole English (HCE). Menschen euro-amerikanischer Abstammung werden in aller Regel nicht als „local“ bezeichnet, waren es doch gerade die Haole, die den übrigen Bevölkerungsgruppen Hawai'is neue Normen vorgaben und selbst keinem Anpassungsdruck unterlagen:

„... Haoles are often not identified as local people, despite the fact that they have been in Hawaii longer than any of the immigrant plantation groups and have been influential in shaping the collective institutions of the public domain, that is, government, economy, law, education and language, along American lines. [...]

... viewed from the accommodation perspective, the former subordinate status of Hawaiians and the immigrant peoples to the dominant Haole group is the basis of their present local identity“ (Okamura 1980:126).

Welch große und komplexe Rolle kulturelle bzw. soziale Faktoren für das Verhältnis und die Beziehungen zwischen ethnischen Gruppen in Hawai'i spielten und spielen, wird einmal mehr am Beispiel einer Einwanderergruppe wie den Portugiesen deutlich - der „race“-Diskurs des US-amerikanischen Festlandes hat demgegenüber wenig Einfluss auf den Inseln gewinnen können: Obwohl die Einwanderer aus Portugal aufgrund ihrer Erscheinung und ihrer Herkunft eindeutig zum Bevölkerungsteil europäischer bzw. amerikanischer Abstammung gehörten, wurden sie im Allgemeinen aufgrund ihrer Vergangenheit als Plantagenarbeiter nicht den Haole zugerechnet, sondern zählten in vielerlei Hinsicht zu den „locals“. Okamura (1980:129) benennt als ein weiteres Beispiel die Differenzierung zwischen „local Pilipino“ und „immigrant Pilipino“, wobei angenommen wird, dass es signifikante Unterschiede z.B. im kulturellen Verhalten der den jeweiligen Kategorien zugeordneten Personen gibt. Bei aller Kritik, die Okamura am Konzept „local“ übt - so sieht er die daraus erwachsende und zum Teil auch gewollte Abgrenzung zwischen Alteingesessenen und Neuzuwanderern als problematisch an - stellt er zugleich das integrative, ethnische Grenzen durchkreuzende und überwindende Potenzial einer solchen Kategorie heraus und endet seinen grundlegenden Artikel mit den Sätzen:

„,... it must not be forgotten that local also represents a coalescence of ethnic groups, that it can transcend ethnic differences, and that it has the potential to change its meaning over time. Thus, the possibility exists that the concept of local may in the future come to represent all of the people of Hawaii“ (Okamura 1980:136).

Wie die von Eleanor Nordyke am Ende der 1980er Jahre propagierte Ausweitung der Bezeichnung „Hawaiian“ auf alle in Hawai‘ $\mathrm{i}$ ansässigen Bevölke- 
rungsgruppen stößt auch die von Okamura herausgestellte integrative Wirkung einer Bevölkerungskategorie „local“ auf Widerspruch vonseiten der hawaiischen Autonomiebewegung. Das Konzept einer „lokalen Identität“, die Inselbewohner hawaiischer wie asiatischer Abstammung verbindet, hat beim hawaiischen Bevölkerungsteil deutlich an Akzeptanz eingebüßt. Eine hawaiische Neubewertung der Bezeichnung „local“ lehnt die Verwendung als Eigenbezeichnung $\mathrm{ab}$ - und missbilligt zugleich die Aneignung dieses Attributs durch die Bevölkerung asiatischer Herkunft, deren größerer Teil heute längst nicht mehr zu den Unterprivilegierten in der Gesellschaft des Staates Hawai`i zählt.

Haunani-Kay Trask spitzt die Kritik an der Verwendung der Bezeichnung „local“ zu, indem sie den seit Generationen in Hawai‘i ansässigen Nachfahren asiatischer Einwanderer jeglichen Anspruch auf die begriffliche Aneignung ihres Geburtslandes abspricht:

„Today, modern Hawai'i, like its colonial parent the United States, is a settler society. Our Native people and territories have been overrun by non-Natives, including Asians. Calling themselves „local,“ the children of Asian settlers greatly outnumber us. They claim Hawai'i as their own, denying indigenous history, their long collaboration in our continued dispossession, and the benefits therefrom“ (H.-K. Trask 2000:2).

Zur Begründung ihrer Ablehnung der Bezeichnung „local“ führt Trask weiterhin aus, dass die „, local' ideology“ dazu benutzt würde, Geschichte und Ansprüche der indigenen Bevölkerung auszublenden. Zugleich koppele sie die Einwanderungshistorie der Asiaten (die in der ersten Generation fast ausschließlich als mehr oder weniger rechtlose Plantagenarbeiter nach Hawai`i kamen) von jener der Haole ab, denen so die alleinige Verantwortung für die Unterdrückung der Hawaiier zugeschrieben werden könne:

„... the responsibility for continued Hawaiian dispossession falls to imperialist haole and incapacitated Natives, that is, not to Asians. Thus do these settlers deny their ascendancy was made possible by the continued national oppression of Hawaiians, particularly the theft of our lands and the crushing of our independence“ (H.-K. Trask 2000:4).

Für Haunani-Kay Trask, die ihre aggressiv formulierte Analyse des „being local“ in den politischen Kontext der hawaiischen Autonomie- und Souveränitätsforderungen stellt, ist die Aneignung einer lokalen Zugehörigkeit durch die asiatischen Mittelschichten nicht zuletzt Teil einer Strategie, die Hawaiische Bewegung zu unterwandern und schließlich zu destabilisieren. Aber auch weniger deutlich politisch motivierte Hawaiier gehen kritisch mit der Bezeichnung „local“ um (cf. Halualani 2002:3-4; Hall 2005:406-7; Kauanui 2007:151). 
So stellt z.B. die hawaiische Schriftstellerin Georgia Ka‘apuni McMillen dazu in einem Interview fest:

„As to the ,local' style/culture notion, there's an attractive myth that people in Hawai'i have shared cultures and behaviors. Sure, I suppose everyone has a favorite flavor of shave ice, a favorite Kalapana ${ }^{143}$ hit, a favorite beach, etc. But overall, the notion of ,local' history/culture buys into the historical whitewashing of Hawaiian history, and the marketing campaigns of the tourism industry - that we're all one big

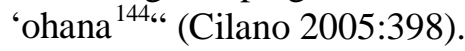

Der Literaturwissenschaftler Stephen Sumida konstatierte zu Beginn der 1990er Jahre: „A Hawaiian is quintessentially a local, but a local is not necessarily a Hawaiian“ (Sumida 1991:xv). Insgesamt lässt sich heute aber feststellen, dass „local“ im Kontext der hawaiischen Bewegung so gut wie keine Rolle mehr spielt. „Local“ bleibt jedoch Ausdruck der Eigenwahrnehmung eines bedeutenden Teils der Bevölkerung des Bundesstaates - bedeutend nicht nur hinsichtlich seiner numerischen Größe, sondern spätestens seit den 1960er Jahren auch was seinen politischen und ökonomischen Einfluss anbelangt. Diese seit mehreren Generationen ansässigen Einwohner asiatischer Herkunft empfinden sich nicht als „Asian Americans“, im Sinne der in den kontinentalen USA üblichen Bezeichnung. Ihre spezifischen historischen und sozialen Erfahrungen in Hawai'i sind seit Jahrzehnten Gegenstand einer ausdifferenzierten „lokalen“ Literatur, aber auch wissenschaftlicher Analyse. Auf hawaiischer Seite haben die Eigenbenennungen „Hawaiian“, „Native Hawaiian“ oder „Kanaka Maoli“ seit den 1970er Jahren an Bedeutung gewonnen - also im Zuge der kulturellen Revitalisierung wie der politischen Bewegung, aber auch angesichts der Etablierung weiter Kreise der asiatischen „Locals“ als neuer Elite im Bundesstaat. Die hier soweit nachgezeichnete Entwicklung der Verwendung des Wortes „local“, die den zugehörigen Realitätshintergrund in seiner Vielschichtigkeit aufscheinen lässt, kann als weiteres Indiz eines gewachsenen hawaiischen Selbstbewusstseins und einer damit einhergehenden begrifflichen Ausgrenzung aller als allochthon wahrgenommenen Bevölkerungsgruppen durch die autochthonen Hawaiier gesehen werden.

In der vorausgegangenen Diskussion der Ein-, Aus- und Abgrenzungen blieben jene Grenzziehungen innerhalb der hawaiischen Bevölkerung ausgeblendet, die nicht durch Gesetze und andere von außen kommende juristische Regelungen begründet sind. Natürlich sind die Hawaiier auch heute keine homogene Bevölkerungsgruppe - Alter, soziale Herkunft, Bildung und ökonomi-

\footnotetext{
${ }^{143}$ Kalapana ist eine vor allem in den 1980er Jahren bekannte Musikgruppe in Hawai‘i. 144 'Ohana: Familie, Verwandschaftsgruppe [U.M.]
} 
sche Position sorgen für bisweilen erhebliche Differenzierungen. Auch in persönlichen Gesprächen mit Hawaiiern begegneten mir immer wieder Unterscheidungen zwischen der jeweils eigenen sozioökonomischen Position innerhalb der hawaiischen Bevölkerung und derjenigen anderer Hawaiier. Die Feststellung solcher Unterschiede ging teilweise mit deutlicher Ablehnung der Strategien einzelner Gruppierungen innerhalb der hawaiischen Bewegung oder gar der gesamten Autonomiebestrebungen einher. Dass angesichts der multiethnischen Zusammensetzung der hawaiischen Bevölkerung und der komplexen sozialen Strukturen einer modernen Gesellschaft wie der des Bundesstaates Hawai`i persönliche Interessen Einzelner den allgemeinen Zielen der Autonomiebewegung entgegenstehen können, ist allerdings nicht weiter verwunderlich. So legten Vertreter der Bewegung immer wieder Wert auf die Feststellung, dass die politische Realisierung einer „Hawaiian Sovereignty“ z.B. staatliche Pensionszahlungen an ehemalige Mitglieder der US-Armee oder persönlichen Besitz unangetastet ließe.

In gewissem Maße haben auch die überkommenen Standesunterschiede der traditionellen hawaiischen Gesellschaft bis heute Bestand. Dass allerdings den heute lebenden Vertretern des hawaiischen Adels nicht nur Zustimmung zuteil wird, lässt sich einem Gedicht des bekannten hawaiischen Künstlers und Lyrikers 'Īmaikalani Kalāhele mit dem Titel „Nā Ali‘i - Now Kine“،145 entnehmen:

„Over the backs / of Men / they launched / their canoes. / Over the will / of the People / they pass / their laws. / Once you walked / proud in your malo ${ }^{146}$ / now Reyn's shirts / cover your shoulders / Liberty House Dockers ${ }^{147}$ / cover your legs / and Gucchi shoes / separate you from / your source“ (Kalahele 2002:67).

In seinem Gedicht, das auch nicht mit Kritik an den traditionellen gesellschaftlichen Verhältnissen Hawai'is im 18./19. Jahrhunderts spart, ordnet Kalahele den heute in sozialer und ökonomischer Hinsicht sehr gut gestellten kleinen Teil der hawaiischen Bevölkerung der allgemeinen Oberschicht im Staat Hawai‘i zu. Im Gegensatz zum Großteil der Mitglieder dieser Oberschicht, die sich vor allem aus der Bevölkerung euro-amerikanischer bzw. asiatischer Abstammung rekrutiert, gesteht er den Hawaiiern unter ihnen aber grundsätzlich

\footnotetext{
${ }^{145}$ Der Titel des Gedichts verbindet Hawaiisch mit Hawaiian Pidgin und kann als „Die Ali ‘ $i$ wie sie heute sind“ übersetzt werden.

${ }^{146}$ Malo ist die Bezeichnung für die traditionell getragenen Lendentücher der Männer.

${ }^{147}$ Die Namen „Reyn’s“ und „Liberty House“ bezeichnen eine eher hochpreisige Kleidungsmarke bzw. ein bis 1998 bestehendes Kaufhaus für den gehobenen Bedarf. Wie die Marke Gucchi verweisen diese Markennamen auf die wirtschaftliche Position der angesprochenen Ali ‘ $i$ und die nach Ansicht Kalāheles daraus resultierende Entfermdung von den kulturellen Wurzeln.
} 
zu, den Weg zu ihren Wurzeln und damit einer übergreifenden hawaiischen Gemeinschaft zu suchen und wiederzufinden. So manifestiert sich in diesem Gedicht auch wieder die von der Autonomiebewegung progagierte übergeordnete und prinzipiell verbindende Bedeutung der hawaiischen Abstammung. 



\section{Politische Gruppierungen und staatliche Institu- tionen}

Die vorausgegangenen Kapitel haben ein schärferes Bild der hawaiischen Geschichte und der gegenwärtigen Bevölkerungssituation mit ihren oftmals diffusen sozialen, kulturellen und ethnischen Konturen zu zeichnen versucht. Vor diesem Hintergrund schließt sich eine nähere Betrachtung politischer Akteure und Repräsentanten der hawaiischen Bevölkerung, die den Autonomiediskurs der 1990er Jahre bestimmten, an.

Die Autonomiebewegung, das „Hawaiian Sovereignty Movement“, setzte sich in diesem Jahrzehnt aus einer Vielzahl von Gruppierungen und Individuen zusammen, die unterschiedliche politische Ziele (,models of sovereignty“) verfolgten und in der Öffentlichkeit vertraten. In 4.1 werden vier maßgebliche Organisationen der Bewegung im Vordergrund stehen, die sich u.a. durch ihre dauerhafte Medienpräsenz und kontinuierliche Arbeit über einen langen Zeitraum auszeichneten. ${ }^{148}$ Die Zahl der in den einzelnen Initiativen vertretenen Mitglieder blieb bei dieser Auswahl im Wesentlichen unbeachtet, da zum einen keinerlei verlässliche Zahlen über die organisierte Anhängerschaft vorliegen, zum anderen aber vor allem die Mobilisierungsfähigkeit ein-

\footnotetext{
${ }^{148}$ Neben den in 4.1 genannten Gruppierungen existierten in den 1990er Jahren und auch in den folgenden Jahren zahlreiche kurzfristig sichtbare Initiativen, die mit einzelnen Forderungen an die Öffentlichkeit traten, vielleicht eine Flugschrift herausbrachten und dann wieder aus dem Blick verschwanden. Es bleibt darüber hinaus völlig unklar, ob diese sich teilweise mit erheblichem Aufwand präsentierenden „Gruppen“ über eine Anhängerschaft verfügten, die über die jeweiligen Gründer bzw. Sprecher hinausreichte. Nicht weiter betrachtet werden hier auch die in erheblicher Zahl bestehenden lokalen Unterstützergruppen (oft im Rahmen von Hula Hālau, Kulturvereinen oder Kirchengruppen organisiert), die im Allgemeinen keine eigenen politischen Forderungen erhoben und im Rahmen von Auseinandersetzungen und Diskussionen nicht als eigene Akteure in Erscheinung traten. Beispiele für beide Kategorien, kurzlebige Initiativen und lokale Unterstützer, werden jedoch, soweit dies in konkreten Zusammenhängen angebracht ist, im weiteren Verlauf der Arbeit eingeführt.
} 
zelner Gruppierungen über den Kreis der unmittelbaren Anhängerschaft hinaus sowie die von ihnen jeweils angeregte und aufrechterhaltene Resonanz in Medien und öffentlicher politischer Diskussion ins Gewicht fallen.

Neben den unabhängigen Initiativen, die unmittelbar der Autonomiebewegung zugeordnet werden können, stehen in diesem Kapitel auch verschiedene staatliche Institutionen im Fokus der Betrachtung. Insbesondere das in 4.2 dargestellte Office of Hawaiian Affairs nimmt eine herausragende Sonderstellung ein: Als eine in der Staatsverfassung verankerte Institution versteht sich das Office of Hawaiian Affairs als Interessensvertretung par excellence aller Hawaiier und hat sich schon sehr früh als maßgeblicher Teilnehmer an der Autonomie-Diskussion beteiligt.

In 4.3 werde ich schließlich auf die Rolle des Bundesstaates Hawai' $i$, der USA und ihrer diversen Institutionen eingehen, die als Urheber und Vollstrecker juristischer Rahmenbedingungen Hauptangriffsziele der Autonomiebewegung darstellen. Wie sich zeigen wird, ist zumal die bundesstaatliche Seite nicht als klarer Gegner einer hawaiischen Autonomie auszumachen - vielmehr verschwimmen oftmals die Grenzen zwischen Gegnerschaft und Zusammenarbeit, was u.a. durch die wechselnden Rollen von Akteuren in verschiedenen institutionellen und diskursiven Zusammenhängen bedingt sein dürfte.

\subsection{Die Hawaiische Autonomiebewegung}

Institute for the Advancement of Hawaiian Affairs (I.A.H.A.) ${ }^{149}$

Das Institute for the Advancement of Hawaiian Affairs (I.A.H.A.) ${ }^{150}$ wurde bereits 1985 von dem hawaiischen Anwalt Hayden F. Burgess, der vor allem den hawaiischen Namen Pōkā Laenui verwendet, gegründet. I.A.H.A. ist eine jener Bewegungsgruppierungen, deren Mitgliederzahl nicht bekannt und im Zweifel eher als gering einzuschätzen ist: ${ }^{151} 1994$ wurde das Institut in einer Aufstellung unterschiedlicher Organisationen der „Sovereignty“-Bewegung als „a group consisting of Burgess and a handful of followers“ charakterisiert (Tachihata 1994:204) und in einem Artikel des Honolulu Star-Bulletin hieß es: „Burgess has not organized a group or following around his proposal. He says he shares his approach with others in hopes that if they agree, they too will pass it on. “152 Pōkā Laenui tritt als einziger Vertreter des Instituts in der Öffentlichkeit auf und zeichnet für sämtliche Publikationen des I.A.H.A. verantwortlich.

\footnotetext{
${ }^{149}$ Die Reihenfolge der hier besprochenen Gruppierungen stellt keine Wertung oder Gewichtung meinerseits dar: die vier nach den o.g. Kriterien ausgewählten Initiativen werden in alphabetischer Reihenfolge behandelt.

${ }^{150}$ Das Akronym I.A.H.A. kann als iaha oder 'aha gelesen werden; die beiden hawaiischen Synonyme bedeuten Versammlung, Zusammenkunft oder auch Partei [U.M.].

${ }^{151}$ „Groups promoting sovereignty“, Kevin Hand (H S-B 24.8.1995:A-8).

152 „Burgess wants choice of total independence“ (H S-B 12.1.1993:A-6).
} 
Durch seine dauerhafte Medienpräsenz und Teilnahme an den öffentlichen Diskussionen der 1990er Jahre genoss das Institute for the Advancement of Hawaiian Affairs eine gewiss nicht geringe Aufmerksamkeit und konnte seine Vorstellungen zur Selbstbestimmung und Souveränität der Hawaiier publik machen:

„,Self-Determination“ is the deciding by the people of a nation what form of government they shall have, without reference to the wishes of any other nation. In making that decision, the people have a range of choices from total assimilation within another nation, territory status, autonomy (some erroneously call this ,a nation within a nation'), statehood, free association, commonwealth, to total independence and sovereignty.

,Sovereignty، is to be free from any other nation's control - to have no higher legislature but $\operatorname{God}(\mathrm{s})$. Sovereignty is not a privilege to be granted by another nation but a right inherent in a people.

[...] Colonizing nations have used these same words to carry quite different meanings in order to deny basic human rights. Let's not adopt their definitions and fall victims to their abuses“ (I.A.H.A. 1992:23).

Laenui propagiert also ein eigenstaatliches, von den USA unabhängiges und vollständig souveränes Hawai'i, dessen Staatsgebiet den gesamten Archipel einschließlich der 200-Meilen Zone umfassen würde (Laenui 1992:19). Vorstellungen einer Autonomie, wie sie von anderen Organisationen - so u.a. von Ka Lāhui oder auch dem Office of Hawaiian Affairs vertreten werden (s.u.) erteilt er eine klare Absage. Die Errichtung einer autonomen Region Hawai‘ ${ }^{i}$ innerhalb der USA und damit der Verzicht auf die politische Souveränität eines Staates entspräche den bestehenden Indianernationen in den kontinentalen USA - hierzu Laenui:

„Our history and the American Indian experience are completely separate, although the U.S. government would like to have us see ourselves as the same. We come from a different national genealogy. We were an independent nation recognized throughout the world as an equal ... Our history is one of clear invasion of an internationally recognized independent nation by another nation which has never really withdrawn from that original occupation. To apply the U.S. solution to the American indians for us would be inappropriate, we would be selling ourselves short“" (I.A.H.A. 1987:14).

Im Einklang mit der staatsrechtlichen Argumentation Laenuis stehen auch die Vorstellungen des I.A.H.A. hinsichtlich der zukünftigen Staatsbürgerschaft, die 
ihm zufolge nicht von der biologischen Abstammung des Einzelnen abhängen könne. Vielmehr soll die Loyalität gegenüber dem neuen Staatswesen und seiner Ordnung - in der durchaus Sonderrechte für die hawaiisch-stämmige Bevölkerung, wie z.B. ein gewichtetes Wahlrecht, vorgesehen sind ${ }^{153}$ - die Zugehörigkeit zur hawaiischen Nation begründen:

„If the objective is truly to live up to the internationally recognized right of self-determination, the program scope for Hawaiian sovereignty and self-determination must be expanded to include a wider base of people as Hawaiian citizens. That base should be established on the grounds of how one relates to Hawai'i, not on one's racial extraction“ (Laenui 1994:13).

Laenui und das I.A.H.A. nennen vor allem zwei Bereiche der internationalen Politik, in denen Hawaiier tätig werden müssten. Zum einen sei dies die Mitarbeit in der 1982 gegründeten UN-Working Group on Indigenous Populations (WGIP), um so den Status der Hawaiier als indigene Bevölkerung Hawai'is zu dokumentieren sowie die daraus ableitbaren Rechte einzufordern. Zum anderen sollten die Bemühungen um die Wiederaufnahme Hawai' is in die UN-Liste der „Non-Self-Governing Territories“ intensiviert werden, damit eine Dekolonisation des Archipels unter Aufsicht der Vereinten Nationen möglich wird (Laenui 1993).

Allerdings fordert Laenui nicht nur internationales Engagement; auch im Verhalten gegenüber dem US-Bundesstaat Hawai'i und den USA sieht er konkrete Möglichkeiten, die seiner Meinung nach weiter bestehende Souveränität Hawai'is zu demonstrieren und Forderungen nach ihrer Anerkennung Nachdruck zu verleihen:

„We must begin to live our Hawai'i as we see Hawai'i must be. I see Hawai' $\mathrm{i}$ as an occupied nation. I see Hawai' $\mathrm{i}$ as an independent nation. So I must conduct my response accordingly. I refuse to recognize the United States' jurisdiction over me. I therefore refuse to file an income tax return. I refuse to pay any taxes to a foreign government which is essentially attempting to hijack me, forcing me to finance its occupation of my country“ (Laenui, in: Mast u. Mast 1996:416).

Laenui vertritt darüber hinaus den Standpunkt, dass Gesetze der USA wie des Bundesstaates Hawai' $i$ auf die souveränen hawaiischen Bürger nicht anzuwenden wären - eine Ansicht, die er als Anwalt hawaiischer Mandanten immer wieder vor Gericht vertreten hat (Laenui, in: Mast u. Mast 1996:410-1). Weni-

153 Siehe Laenui 1992:19-20; 1994:46-9. 
ger spektakulär sind seine Aufrufe zu Gedenkveranstaltungen, wie sie z.B. aus Anlass der einhundertjährigen Wiederkehr der Annexion im Oktober 1997 stattfanden; sie bieten Möglichkeiten, allgemeine Aufmerksamkeit für die Sache der „Hawaiian Sovereignty“ zu erzielen und zugleich die Ansichten des I.A.H.A. und seines Direktors zu propagieren (Laenui 1997a; 1997b). ${ }^{154}$

Im deutlichen Unterschied zu anderen Gruppierungen der Autonomiebewegung nimmt das I.A.H.A. eine eher positive Haltung gegenüber bundesstaatlichen Initiativen ein, wie z.B. der vom Staat Hawai‘i initiierten „Native Hawaiian Vote“. Anders als fast die gesamte Autonomiebewegung sieht Laenui in der Mitwirkung des Staates am Prozess der „Hawaiian Sovereignty“ auch keine widerrechtliche staatliche Einmischung oder gar den Versuch, die Autonomiebewegung zu schwächen, sondern nichts weniger als die Pflicht des Kolonialstaates, der für eine Dekolonisation im Einklang mit dem Völkerrecht Sorge zu tragen hat (Laenui 1996:pt. II). Sowohl die Gründung des Office of Hawaiian Affairs, die Entschuldigungsresolution des US-Kongresses von 1993 als auch die Vorbereitungen zur umstrittenen „Native Hawaiian Vote“ von 1996 sind für ihn wichtige Schritte auf dem Weg zu mehr hawaiischer Eigenständigkeit:

„The process in which the Native Hawaiian Vote was taken, the acknowledgement by the United States of its violation of the sovereign integrity of the Kingdom of Hawai'i and the Office of Hawaiian Affairs are significant in domestic as well as international law for it reflects a positive step forward in the practical development of indigenous people‘s rights“ (Laenui 1996:pt. I).

Das Institute for the Advancement of Hawaiian Affairs stellt nicht nur hinsichtlich der kaum nachvollziehbaren bzw. fehlenden Mitgliederbasis einen Sonderfall unter den am Autonomiediskurs der 1990er Jahre beteiligten Initiativen und Institutionen dar, sondern unterscheidet sich von den übrigen Gruppierungen auch durch seine dezidiert am Völkerrecht und den allgemeinen Menschenrechten orientierten Argumentationen.

The Sovereign Nation of Hawai ' $i$ - Ka Lāhui Hawai ' $i$

Mit Ka Lāhui Hawai'i wende ich mich einer politischen Gruppierung der hawaiischen Autonomiebewegung zu, bei der es sich in vielerlei Hinsicht um das Gegenteil des zuvor dargestellten I.A.H.A. handelt. Ka Lāhui Hawai'i (im weiteren Text Ka Lāhui genannt) lehnt nicht nur jegliche Einmischung staatlicher Stellen in den Autonomieprozess ab, in den 1990er Jahren verfügte die Organi-

${ }^{154}$ Die Aufrufe sind von einer Organisation mit dem Namen „Of Sacred Times \& Sacred Places“ gezeichnet, die allerdings die Adresse des I.A.H.A. angibt. 
sation auch über die unbestritten größte Anhängerschaft unter der hawaiischen Bevölkerung sowie das bedeutendste, in zahlreichen Aktionen demonstrierte Mobilisierungspotential aller Bewegungsgruppierungen.

Ka Lāhui wurde 1987 in Keaukaha, einer hawaiischen Siedlung gegründet und versteht sich ausdrücklich als „,a native initiative for Hawaiian self-governance, formed by and for native Hawaiians, without the interference of State or Federal agencies“ (Ka Lāhui 1993:2). Ka Lāhui erhebt den Anspruch, die bereits existierende „Sovereign Nation of Hawai $\mathrm{i}^{\prime}$ “ zu sein - dies zeigt sich sowohl hinsichtlich der Organisationsstruktur der Gruppierung wie auch bei der Benennung verschiedener Funktionsträger, die sich beide an nationalstaatlichen Mustern orientieren.

Darüber hinaus verfügt Ka Lāhui über eine „Verfassung“, deren erster Entwurf auf der Gründungsversammlung der Organisation gebilligt worden war. ${ }^{155}$ So genannte „Constitutional Conventions“ in den Jahren 1989 und 1992 änderten und erweiterten diese Verfassung, wobei wesentliche Änderungen die Rolle von Native Hawaiians im Sinne des Hawaiian Homes Commission Act von 1921 betrafen. Sah die ursprüngliche Verfassung Ka Lāhuis (1991:4,7) noch vor, dass ausschließlich Native Hawaiians über alle Fragen in Zusammenhang mit den Hawaiian Home Lands befinden dürfen und dass der als Gouverneur (kia 'äina) bezeichnete Vorsitzende ebenfalls aus den Reihen der Native Hawaiians zu wählen ist, so sind diese Passagen seit 1992 ersatzlos gestrichen. Die Betonung der hawaiischen Abstammung blieb aber in einem gewichteten Wahlrecht erhalten, das den „hawaiischen“ Stimmen mehr Gewicht gibt und wonach die Hälfte aller Abgeordneten der Legislative Ka Lāhuis aus Native Hawaiians bestehen muss, die wiederum ausschließlich von Native Hawaiians gewählt werden können (Ka Lāhui 1991:5; 1993:15).

Eine weitere Besonderheit der Verfassung Ka Lāhuis, in der der Grundrechtskatalog gesichert, die zukünftige Staatsbürgerschaft geregelt und die Regierungsform der Nation bestimmt wird, ist die dem hawaiischen Adel zugewiesene Rolle. So ist neben den an der klassischen Gewaltenteilung orientierten Staatsfunktionen der Legislative, der Exekutive und der Judikative auch ein mit Abkömmlingen hawaiischer Adliger (ali $i$ ) besetztes „Oberhaus“ unter Leitung eines „ali ‘i nui““156 vorgesehen, das Ka Lāhui wie folgt beschreibt:

\footnotetext{
${ }^{155}$ In ihrer Eigendarstellung weist Ka Lāhui ausdrücklich auch darauf hin, dass sowohl die juristische Seite wie auch die Bedürfnisse und Vorstellungen der hawaiischen Bevölkerung in diese „Verfassung“ eingeflossen wären: „Ten years of legal and historical research, and many community meetings, were conducted to identify the best way for Hawaiians to reinstate their sovereign nation" (Ka Lāhui 1993:2).

${ }^{156}$ Ali ‘ $i$ nui (großer, hoher Adliger [U.M.]) bezeichnete in der traditionellen hawaiischen Gesellschaft die jeweils höchstrangigen Oberhäupter.
} 
„The Ali‘i Nui Branch is responsible for matters relating to culture, tradition, and protocol. The Ali'i Nui has no voting or veto power. The Ali'i Nui branch includes two councils: the 'Aha Ali‘i, which is comprised of individuals of chiefly clans who have produced genealogies proving their Ali'i lineage; and the 'Aha Kūkā o Ke Ali'i Nui, comprised of specialists who advise the Ali'i Nui on matters of history, genealogy, language, and cultural traditions" (Ka Lāhui 1993:5). ${ }^{157}$

Hier sieht also die Verfassung der zukünftigen autonomen Nation die Wiederbelebung und Integration eines auch unter Hawaiiern umstrittenen Aspektes der traditionellen hawaiischen Gesellschaftsstruktur vor: zwar spricht man in Texten, Diskussionen und Gesprächen immer wieder mit großer Achtung von den Ali ${ }^{\top} i$, sieht aber ansonsten die Stratifizierung der traditionellen Gesellschaft und insbesondere die Rolle des hawaiischen Adels im 19. Jahrhundert durchaus kritisch. ${ }^{158}$ Was die politische Bedeutung einer hochrangigen Herkunft im Kontext der Autonomiebestrebungen anlangt, so lehnt der Großteil der Hawaiier die Zuweisung einflussreicher Positionen auf der Basis eines Geburtsranges ab. ${ }^{159}$

Trotz einer offenbar sehr straffen Organisationsstruktur bleiben die Angaben zu den Mitgliederzahlen Ka Lāhuis eher vage: nannte die Organisation für 1989 noch eine Zahl von 5.000 eingeschriebenen Mitgliedern, die dem Selbstverständnis der Organisation entsprechend als „Bürger“ („citizens“) bezeichnet werden, so wurden für 1992 bereits 10.000 und für 1993 schließlich 16.000 Bürger der souveränen Nation angegeben (Ka Lāhui 1993:6). Im Jahre 1995 nannte Mililani Trask, zu der Zeit gewählte Gouverneurin oder kia ‘äina Ka Lāhuis, schließlich eine Gesamtzahl von fast 25.000 Mitgliedern. ${ }^{160}$ Diese hohen Zahlen zweifelten Kritiker wiederholt an, da Ka Lāhui keine nachprüfbaren Mitgliederzahlen veröffentlicht und $\mathrm{M}$. Trask in diesem Zusammenhang

\footnotetext{
${ }^{157}$ Es gab Berichte, dass ein noch minderjähriger Ali‘i Nui bereits 1988 auf seine mögliche zukünftige Rolle vorbereitet wurde, wobei mir keine späteren Nachrichten zu diesem Thema bekannt sind („Noa de Guiar, little boy who would be ali‘i nui of Hawaiian nation“ [H S-B 1.11.1988:B-1]). Auffällig ist jedoch, dass die ja weiterhin bestehende monarchische Linie der Kawananakoas, den ali ‘i nui des ausgehenden 19. Jahrhunderts, die in der Nachfolge Kalākauas auch Anspruch auf den hawaiischen Thron erheben könnten, von Ka Lāhui nicht vorgesehen wurde. Hierbei mag die Zugehörigkeit der Familie Kawananakoa zum gesellschaftlichen und politischen Establishment des Staates Hawai'i eine gewichtige Rolle gespielt haben.

${ }_{158}$ Ausnahmen sind sicherlich die herausragenden, am Beginn und am Ende des hawaiischen Königreichs stehenden Monarchen Kamehameha I. und Lili‘uokalani.

${ }^{159}$ Diese Ablehnung lässt sich nicht durch Zahlen belegen, wird jedoch nach einer Wiedereinführung der Monarchie in Hawai'i gefragt, so lehnten dies im Jahre 1995 61\% der befragten Hawaiier ab, während die Monarchie von $20 \%$ befürwortet wurde (repräsentative Umfrage im Auftrag des Honolulu Advertiser: „Most Hawaiians say: no kings need apply“, Mark Matsunaga [HA 20.11.1995:A-1]).

160 „Ka Lahui takes ,realistic‘ path to self-rule“, Mililani Trask (HA 13.8.1995:B-3).
} 
lediglich auf die Kontrolle der mehrfach durchgeführten Wahlen zur Leitungsebene der Organisation durch die renommierte „League of Women Voters“ hinweist (Honolulu 1993:70). ${ }^{161}$

Ka Lāhui benannte zwar auf allen Inseln Sprecher der jeweiligen so genannten „District Councils“, jedoch konzentrierte sich die öffentliche Aufmerksamkeit in den 1990er Jahren vor allem auf die Rechtsanwältin Mililani Trask. Daneben traten ihre Schwester, die Autorin und damalige Direktorin des Center for Hawaiian Studies der University of Hawai'i at Mānoa, Haunani-Kay Trask und die Historikerin Lilikalā Kame'eleihiwa, ebenfalls Professorin am Center for Hawaiian Studies, als Sprecherinnen für Ka Lāhui in Erscheinung. Ähnlich wie bei I.A.H.A. und ungeachtet der sicher größeren Anhängerschaft Ka Lāhuis verband die weitere Öffentlichkeit auch diese Organisation mit wenigen Einzelpersonen und deren Aussagen und Auftreten - die drei genannten Frauen gehörten auch nach 2000 zu den bekanntesten Befürwortern einer hawaiischen Autonomie.

Anders als das Institute for the Advancement of Hawaiian Affairs strebt Ka Lāhui allerdings keine nationalstaatliche Souveränität an. Das politische Ziel der Gruppierung ist vielmehr das Modell einer „nation within a nation“ nach dem Muster der „Indian Nations“ in den kontinentalen USA:

„Ka Lāhui’s approach to gain sovereignty is simple. Ka Lāhui seeks inclusion of the Hawaiian people in the existing U.S. federal policy which affords all Native Americans the right to be self-governing and provides access [to] federal courts for judicial review ... Once this is achieved, the sovereign nation can relate „Nation to Nation“ with the United States and would be in a position of standing and authority to effectively advance and resolve Hawaiian claims to native trusts and other entitlements“" Ka Lāhui 1993:3).

Neben der Staatsnähe des Office of Hawaiian Affairs ist seine dezidierte Ablehnung durch Ka Lāhui ${ }^{162}$ auch darin zu suchen, dass Ka Lāhui sich selbst in der Rolle als Verhandlungspartner des Staates sowie als Adressat und Administrator zukünftiger Landübertragungen und Ausgleichszahlungen sieht und insoweit mit dem OHA konkurriert.

\footnotetext{
${ }^{161}$ Das Fehlen verlässlicher Zahlen lässt sich unter Umständen auch auf die doch eher informellen Strukturen der Mitgliedschaft zurückführen: es wurden keine Beiträge erhoben und offenbar wurde auch kein Mitgliederverzeichnis geführt - wie bei den US-amerikanischen Parteien offenbart sich das Ausmaß der Anhängerschaft also vor allem bei Wahlen und konkreten Anlässen wie Demonstrationen o.ä.

162 Die immer wieder angeführte „offizielle“ Begründung für die ablehnende Haltung gegenüber dem Office of Hawaiian Affairs bezieht sich auf die Staatsnähe der Institution.
} 
Ka Lāhui benennt verschiedene Faktoren, die von der Organisation als Grundvoraussetzung für die Schaffung der anvisierten „Nation in der Nation“ gesehen werden. Sie sollen hier vollständig zitiert werden, da sie noch einmal Grundvorstellungen und Ziele Ka Lāhuis zusammenfassen und verdeutlichen.

„1. A STRONG AND ABIDING FAITH IN THE AKUA because a spiritually empty people do not make a strong nation.

2. A PEOPLE WITH A COMMON CULTURE, language, tradition, and history. For example: Po'e Hawai‘i (Hawaiian people).

3. A LAND BASE so that Hawaiians are able to live and practice their cultural traditions. Ka Lāhui is working to secure the Hawaiian Home Lands Trust, which includes approximately 200,000 acres, and the 5(f) Ceded Lands Trust, which includes approximately 1.4 million acres.

4. A GOVERNMENTAL STRUCTURE to enable Hawaiians to be self-determining. Self-determination is the ability of a Nation of people, through their government structure, to manage their lands and natural resources and to create a plan for their future.

5. AN ECONOMIC BASE that will enable Hawaiians to be selfsufficient. Economic self-sufficiency is the goal of nationhood“ (Ka Lāhui 1993:2 [Hervorhebungen im Original]).

Ka Lāhui stellt in den Punkten 1 bis 3 zunächst kulturelle Faktoren in den Vordergrund, wobei insbesondere der zweite Punkt eher den Charakter einer politisch nutzbaren Fiktion hat, da zur Zeit der Programmabfassung Hawaiisch nur wenig gesprochen wurde und die einstige Basis einer gemeinsam gelebten hawaiischen Kultur und Tradition so nicht mehr vorhanden war. Punkt 3 verknüpft die vorausgesetzten gemeinsamen hawaiischen Traditionen mit konkreten Forderungen nach Rückgabe von Landflächen, die einen großen Teil des Staatsgebietes umfassen, während die beiden letzten Punkte noch einmal die angestrebte politische Autonomie und die ökonomischen Eigenständigkeit eines hawaiischen Gebietes hervorheben.

Im Jahre 1995 legte Ka Lāhui mit dem so genannten „Master Plan“ dann ein Programm vor, das eine konkrete Strategie für die Erlangung von Selbstbestimmung im Sinne Ka Lāhuis formulierte (Ka Lāhui 1995:12-3). Neben der Information der hawaiischen Bevölkerung und dem Aufbau einer de facto-Regierungsstruktur stand dabei die Änderung des rechtlichen Verhältnisses zwischen USA, Bundesstaat und Hawaiiern im Vordergrund, das nach wie vor einer formalen Vormundschaft z.B. hinsichtlich der Landrechte gleicht; wie bei I.A.H.A. steht auch für Ka Lāhui die neuerliche Einschreibung Hawai' is in die UN-Liste der „Non-Self Governing Territories“ obenan auf der Agenda. Des weiteren soll durch die Intensivierung der Beziehungen zu anderen indigenen Nationen sowie eine stärkere Vernetzung untereinander die internationale 
Unterstützung für den Schutz der Territorial- und Menschenrechte indigener Völker gefördert werden.

Ka Lāhui hat sich zur Gewaltlosigkeit verpflichtet, steht Aktionen des zivilen Ungehorsams aber ausdrücklich positiv gegenüber (Ka Lāhui 1995:3). Die Organisation steht für eine strikte Staatsferne, die auch schon die Verfassung von 1993 reflektierte, deren Artikel II (C) die Übernahme eines Amtes durch gewählte Funktionsträger des Staates verbietet (Ka Lāhui 1993:12).

Die oben genannten „Arbeitsbereiche“ drückten sich auch im konkreten Vorgehen der Gruppierung aus: hierzu zählten die Teilnahme am bundesstaatlichen Gesetzgebungsverfahren ${ }^{163}$, die Partizipation an allen wesentlichen Anhörungen des Parlaments, die Durchführung von Bildungsmaßnahmen unter der hawaiischen Bevölkerung ${ }^{164}$, die Organisation und Unterstützung von Demonstrationen und Protesten gegen Einschränkungen hawaiischer Rechte, die Präsenz der Organisation auf nationalen und internationalen Tagungen, Seminaren und Konferenzen (wobei vor allem Arbeitsgruppen der Vereinten Nationen zu nennen sind) und schließlich die Bildung eines politischen Netzwerkes durch Vertragsschließungen mit anderen indigenen Nationen in Amerika.

Trotz der eher gemäßigten Positionen Ka Lāhuis in Hinblick auf Forderungen nach Land und Wiedergutmachung blieb die Organisation sowohl innerhalb wie außerhalb der hawaiischen Bevölkerung heftig umstritten. Dies lag nicht zuletzt an der konsequenten Ablehnung der Zusammenarbeit mit staatlichen Institutionen, die ja ebenfalls eine große Zahl von Hawaiiern vertreten (und durch finanzielle Leistungen unterstützen). Die häufig sehr aggressiv formulierten Verlautbarungen aus dem direkten Umfeld der Gruppierung (so z.B. von Haunani-Kay Trask) taten ein Übriges, um nicht nur Gegner der hawaiischen Autonomie zu bestärken, sondern auch bei Befürwortern für Irritationen zu sorgen.

Der „Runde Tisch“-Ka Pākaukau

Ka Pākaukau konstituierte sich im Jahre 1989 als Allianz unterschiedlich operierender Einzelorganisationen der hawaiischen Autonomiebewegung. Bis weit in die 1990er Jahre setzte sich Ka Pākaukau ${ }^{165}$ aus 12 verschiedenen Gruppierungen zusammen, die in monatlichen Treffen ihre Arbeit koordinierten (Blaisdell, in: Mast u. Mast 1996:363). Bei den beteiligten Gruppen handelte es sich u.a. um Nuclear Free and Independent Pacific, Na 'Ōiwi o Hawai'i (för-

\footnotetext{
${ }^{163}$ Ka Lāhui partizipiert an den Anhörungen des Parlaments, bringt aber auch selbst, so wie in Hawai'i vorgesehen, Gesetzesinitiativen in die Legislative ein.

${ }^{164}$ Zur Durchführung von Bildungsmaßnahmen zur „Hawaiian Sovereignty“ erhielt Ka Lāhui Zuschüsse u.a. von der katholischen Kirche Hawai'i (US\$ 12.000,-) und der Administration for Native Americans (US\$ 172.000,-) (Ka Lāhui 1993:6).

${ }^{165}$ Ka Pākaukau bedeutet „Tisch“ bzw. wird im übertragenen Sinne auch verstanden als „Der runde Tisch“ oder „Der Konferenztisch“.
} 
dert den Erhalt traditioneller Kultur), Na Maka o Ka 'Āina (produziert Videos zu Kultur und Geschichte Hawai'is), Pro-Kanaka Maoli Independence Working Group (entwickelt Unterrichtsmaterialien zu dem Thema „Hawaiian Sovereignty"), Protect Kaho'olawe 'Ohana (bemüht sich vornehmlich um die Rückgewinnung der Insel Kaho'olawe) sowie der Pele Defense Fund (wendet sich gegen den Ausbau von Thermalenergie in den vulkanischen Regionen der Insel Hawai'i). Die Mitgliederzahl Ka Pākaukaus wurde für 1993 mit ca. 100 Personen angegeben, die jeweils verschiedenen Gruppierungen zugleich innerhalb des Zusammenschlusses angehören konnten (Kame'eleihiwa 1993:67). ${ }^{166}$

Die Gruppierung wird seit ihrer Gründung durch den Arzt und Professor an der University of Hawai'i at Mānoa Richard Kekuni Blaisdell vertreten, der als Sprecher der Allianz zugleich für einen großen Teil der von ihr öffentlich vertretenen Inhalte verantwortlich zeichnet. ${ }^{167}$ So ist Blaisdell auch der Verfasser von so genannten „Position Statements“ (Ka Pākaukau 1993a), in denen die grundsätzlichen politischen Standpunkte der Allianz niedergelegt sind. Demnach ist das primäre Ziel der Organisation wie bei I.A.H.A. die vollkommene staatliche Unabhängigkeit Hawai'is. Zwischenstufen, wie z.B. die von Ka Lāhui propagierte „Nation in der Nation“, werden nur insoweit unterstützt, solange sie den Weg hin zu einer staatlichen Souveränität der Inseln nicht verstellen (Blaisdell, in: Mast u. Mast 1996:373). Souveränität, so wie Blaisdell und Ka Pākaukau sie verstehen, meint dabei mehr als nur die völkerrechtliche Unabhängigkeit Hawai' is - es ist gleichsam eine innere Grundhaltung, deren politische Manifestation im Sinne des internationalen Rechts ausschließlich Sache der Hawaiier selbst sein kann:

„Sovereignty means that we kānaka maoli accept no higher human authority over our lives, our lands, our ocean resources and our future than ourselves. That is, we kānaka maoli make the decisions in our homeland, not foreigners, unless they meet our laws for their participation in our government ... Our sovereignty is inherent ... It is not something that can be given to us. We cannot receive or achieve sovereignty. Sovereignty is ours to assert or give up" (Ka Pākaukau 1993a:1 [Hervorhebungen im Original]).

\footnotetext{
${ }^{166}$ Bei dieser Zahl handelt es sich um eine Angabe der genannten Autorin - sie scheinen aber durchaus realistisch zu sein; von Ka Pākaukau selbst liegen keine Angaben zur Mitgliedschaft vor.

${ }^{167}$ Blaisdell ist zugleich „Convener“ der Pro-Kanaka-Maoli Independence Working Group (ebenfalls Mitglied Ka Pākaukaus) und der Indigenous Rights Working Group (IRWG), die 1997 als Untergruppe der Pacific Islands Association of Non-Governmental Organizations (PIANGO) gegründet wurde (Blaisdell 1998; PIANGO 1998)
} 
Anders als Ka Lāhui bietet Ka Pākaukau kein bereits ausgearbeitetes Modell einer Regierungs- und Verwaltungsstruktur des unabhängigen Hawai' $i$ an. Die „Position Statements“ stellen aber klar, dass es zu gegebener Zeit ausschließlich die Angelegenheit der Hawaiier selbst sein wird, entsprechende Regeln und Strukturen zu entwickeln - wobei zugleich aber deutlich gemacht wird, dass die zukünftige Nation auf der Basis „hawaiischer Kultur“ organisiert sein soll: „,... a necessary prerequisite to designing our government structure is revitalization of our traditional culture adapted to modern times" (Ka Pākaukau 1993 a:2 [Hervorhebungen im Original]). Und in einem Zeitungsartikel stellt Blaisdell darüber hinaus fest: „We favor governing structures based on our 'ohana-ahupua' $a$ taro-roots traditions in which all adults in each local community provide input in communal decisions by consensus. “168 Blaisdell greift hier auf ein idealisiertes Bild der ferneren hawaiischen Vergangenheit zurück: die offene Verwandtschaftsgruppe ('ohana), die gemeinsam einen ahupua'a, eine im Idealfall vom Meer bis zu den Bergen reichende Grundeinheit des traditionellen hawaiischen Landrechts, bewohnt und wirtschaftlich nutzt - blendet dabei aber die übergeordneten, ebenfalls traditionellen Kontrollund Verwaltungsebenen des Adels aus. Er benutzt vielmehr mit „taro-roots traditions“ eine „hawaiisierte“ Version des amerikanischen „grass-roots“Begriffs und impliziert damit eine basisdemokratische „Herrschaft von unten“ als vorherrschendes Element der Gesellschaft des alten wie des neuen Hawai` $i$.

Hinsichtlich des von der souveränen Nation Hawai'i beanspruchten Territoriums geht Ka Pākaukau keine Kompromisse ein:

„Our territory includes all of the lands, including submerged lands, waters, atmosphere and natural resources of Ka Pae aina. ${ }^{169}$ That is, all of these will return to our jurisdiction as in the time of our pre-Western Kanaka Maoli ancestors.“" ${ }^{170}$

Den besonderen rechtlichen Status der großen Treuhandgebiete (Hawaiian Home Lands und Ceded Lands) erkennt Ka Pākaukau nicht an, sondern bezeichnet sie lediglich als „stolen kanaka maoli lands“ - die sich aus der unrechtmäßigen Aneignung dieser Landflächen ergebenden Verpflichtungen des

168 „Kanaka Maoli seek total control of land, sea“, Kekuni Blaisdell (HA 13.8.1995:B-1).

169 Ka Pae 'àina ist die hawaiische Bezeichnung für den gesamten hawaiischen Archipel; die Angabe bleibt insofern unpräzise, als das heutige Gebiet des Bundesstaates sich bis zum fast 1.000 km nordwestlich Kaua'is gelegenen Kure-Atoll erstreckt, wobei die dazwischen liegenden Midway-Inseln unter der Administration der US-Navy stehen. Spuren früherer menschlicher Anwesenheit auf den nordwestlichen Inseln finden sich lediglich auf den Kaua‘i am nächsten gelegenen Inseln Nihoa und Necker.

${ }^{170}$ „Kanaka Maoli seek total control of land, sea“, Kekuni Blaisdell (HA 13.8.1995:B-1). 
Bundesstaates wie der USA gegenüber den Hawaiiern müssten jedoch endlich erfüllt werden (Ka Pākaukau 1993 a:5).

Ein zentrales Ziel der Politik Ka Pākaukaus, wie ja auch der beiden anderen zuvor dargestellten Gruppierungen, ist die erneute Aufnahme Hawai'is in die Liste der „Non-Self-Governing Territories“ der Vereinten Nationen, von der Hawai'i nach der Aufnahme in die Vereinigten Staaten im Jahre 1959 gestrichen wurde (Ka Pākaukau 1993 a:6). ${ }^{171}$ Die Mitarbeit in internationalen Organisationen wie der United Nations Working Group on Indigenous People oder der 1990 gegründeten Pacific Islands Association of Non-Governmental Organizations (PIANGO) soll u.a. diesem Ziel dienen (Blaisdell 1997). Verträge mit den USA ermöglichen nach den Vorstellungen Ka Pākaukaus in aufeinander folgenden Schritten die Souveränität Hawai'is und den vollständigen Rückzug der USA von den Inseln. In diesem Zusammenhang will die Organisation Verhandlungen ausschließlich auf der Regierungsebene führen, eine Vermittlung des US-Kongresses oder des Bundesstaates Hawai'i lehnt Ka Pākaukau grundsätzlich ab (Ka Pākaukau 1993 a:7; s.a. Blaisdell, in: Mast u. Mast 1996: 373).

Konkrete Aktionen der Allianz in Hawai` $\mathrm{i}$ schließen die Unterstützung hawaiischer Proteste gegen die missbräuchliche Nutzung von Land bzw. die Einschränkung hawaiischer Landnutzungsrechte ein (Da Skupes 1994:1). Daneben ist Ka Pākaukau Ausrichter des alljährlichen Sovereignty Restoration Day (Ka Lā Ho'iho'i Ea), einer feierlichen Gedenkzeremonie aus Anlass der Rückübertragung der Souveränität des Königreichs Hawai`i an König Kamehameha III. durch den englischen Admiral Richard Thomas im Jahre 1843. ${ }^{172}$ Der Einladungstext zur Gedenkveranstaltung 1993 stellte nochmals die Bedeutung des Tages für die heutigen Hawaiier aus der Sicht Ka Pākaukaus dar:

\footnotetext{
${ }^{171}$ Mit der Verwaltung von Territorien, die über keine politische Autonomie oder Souveränität verfügen, gehen laut Charta der Vereinten Nationen umfangreiche Verpflichtungen einher. In Kapitel XI heißt es hierzu u.a.: , Members of the United Nations which have or assume responsibilities for the administration of territories whose peoples have not yet attained a full measure of self-government recognize the principle that the interests of the inhabitants of these territories are paramount, and accept as a sacred trust the obligation to promote to the utmost, within the system of international peace and security established by the present Charter, the well-being of the inhabitants of these territories, and, to this end:

1. to ensure, with due respect for the culture of the peoples concerned, their political, economic, social, and educational advancement, their just treatment, and their protection against abuses;

2. to develop self-government, to take due account of the political aspirations of the peoples, and to assist them in the progressive development of their free political institutions, according to the particular circumstances of each territory and its peoples and their varying stages of advancement [...]“ (United Nations 1973).

${ }^{172}$ Zu den Begründern dieser Gedenkfeier, die seit 1985 jährlich am 31. Juli stattfindet, gehörte auch Kekuni Blaisdell („Leader to miss Restoration Day“, Mark Matsunaga [HA 17.7.94:A-7]).
} 
„....all kānaka maoli (indigenous Hawaiians) and kāko'o (supporters) are invited to redeclare our inherent sovereignty ... Only with ku'oko'a (independence), reparations for the grave injury to our kānaka maoli who suffer the worst conditions in our homeland, payment of back rent and return of our lands and ocean resources, can we become selfsufficient. Only then will we be a whole people again in harmony with the great spiritual forces responsible for our origin and necessary for our survival as a distinct people“ (Ka Pākaukau 1993b).

Darüber hinaus war Ka Pākaukau auch einer der Hauptorganisatoren des Ho'okolokolonui Kānaka Maoli (The People‘s International Tribunal) im Jahre 1993, vor dem die Vereinigten Staaten der Beteiligung am illegalen Sturz der Königin Lili‘uokalani, des Diebstahls hawaiischen Landes und der Unterdrückung der hawaiischen Bevölkerung angeklagt wurden (Kame‘eleihiwa 1993:70-1; siehe 6.2).

Insgesamt stehen die Bemühungen $\mathrm{Ka}$ Pākaukaus in einem Zusammenhang, der in den „Position Statements“ so formuliert wurde: „....we must educate our po'e kanaka moali [sic!], the U.S. and the world of these offenses against our people that have been intentionally hidden or distorted as part of the official U.S. policy of colonialism, exploitation, coercive assimilation, oppression of us kanaka maoli and suppression of the truth of these wrongs“ (Ka Pākaukau 1993 a:6 [Hervorhebungen im Original]). Da Ka Pākaukau eine Zusammenarbeit mit staatlichen Institutionen in politischen Fragen ablehnt und darüber hinaus von einer bestehenden Souveränität der Hawaiier ausgeht, die letztlich nur noch der Anerkennung durch die USA bedarf, steht die Organisation auch allen Autonomie-Initiativen des Staates Hawai`i grundsätzlich ablehnend gegenüber.

Nation of Hawai ' $i$ - 'Ohana Council of the Hawaiian Kingdom

Als jüngste der hier betrachteten Gruppierungen wurde die Nation of Hawai'i von Dennis „Bumpy“ Kanahele im Jahre 1992 gegründet. Zunächst unter dem Namen „'Ohana Council of the Hawaiian Kingdom“ bekannt, hat sie ihren Namen später in „Sovereign Nation-State of Hawai ‘ $i$ “ geändert und läuft heute unter der Bezeichnung Nation of Hawai'i. Die Gruppe verfügt nach eigenen Angaben über „Tausende“ von Mitgliedern, stellt allerdings keinerlei konkrete Nachweise für diese Zahl bereit. Eine Schätzung von 1993 nennt hingegen lediglich ca. 150 in der Gruppierung aktive Mitglieder (Kame'eleihiwa 1993:67). Wie bei I.A.H.A., Ka Lāhui oder Ka Pākaukau, deren jeweilige Außenwirkung auf wenigen exponierten Persönlichkeiten ruht, bestimmt auch der Sprecher der Nation of Hawai'i, Dennis Kanahele, die Außenwirkung der Gruppierung. 
Dennis „Bumpy“ Kanahele ist eine der schillerndsten Figuren der „Sovereignty“-Bewegung und gehört als einziger der hier vorgestellten Protagonisten nicht dem akademischen Umfeld an. Kanahele kam verschiedene Male mit den Gesetzen des Bundesstaates in Konflikt: 1983 wurde er wegen der Mitwirkung bei Autodiebstählen zu einer Bewährungsstrafe verurteilt, ein Jahr später folgte eine Verurteilung wegen Körperverletzung. 1987 wurde Kanahele wegen des unerlaubten Tragens einer Schusswaffe und Bedrohung eines Polizisten zu einer einjährigen Gefängnisstrafe verurteilt. ${ }^{173} 1992$ schließlich, im Gründungsjahr des 'Ohana Councils, wurde er zusammen mit 31 weiteren Demonstranten verhaftet und wegen Hausfriedensbruchs angeklagt. ${ }^{174}$ Es ist nicht zuletzt dieses Vorleben, das Kanahele auch innerhalb der hawaiischen Bewegung sehr umstritten macht.

Primäres politisches Ziel der Nation of Hawai' $i$ ist die uneingeschränkte Souveränität Hawai‘ is, wobei sie allerdings davon ausgeht, dass die souveräne Nation Hawai'i niemals aufgehört hat zu existieren. Dies leitet sie unter anderem aus der „Apology Bill“ von 1993 her und stützt sich dabei vor allem auf die Interpretation des Resolutionstextes durch Francis A. Boyle ${ }^{175}$ :

„... now the United States government, after one hundred years, has finally and officially conceded, as a matter of United States law, that Native Hawaiian people have the right to restore the Independent Nation State that you had in 1893 when the United States government came and destroyed it. And also then that as a matter of international law the Native Hawaiian people have the right to go out now and certainly proclaim the restoration of that state“ (Boyle 1993).

Unter Berufung auf die Entschuldigung der Vereinigten Staaten bei den Hawaiiern und der Interpretation dieses Gesetzes durch Boyle erklärte die Organisation am 16.1.1994, 101 Jahre nach dem Sturz der Monarchie, die Unabhängigkeit Hawai’is. In der Erklärung mischen sich Verweise auf das Internationa-

173 „Activist had a role in ruckus at Makapuu“, Devi Sen Laskar (S-B 14.5.1993:A-6).

174 „Hawaiian arrests at palace: 32 activists defy order to stay“, Thomas Kaser (HA 12.6.1992:A1). Der Polizeieinsatz wurde mit einer drohenden Besetzung des Palastes durch die Demonstranten und einer Überschreitung der genehmigten Aufenthaltsdauer im Park des Palastes begründet („Feared Palace would be stormed, official says“, Kevin Dayton [HA 13.6.1992:A-1]), seine Härte sowohl von hawaiischer als auch nicht-hawaiischer Seite kritisiert (siehe die Berichterstattung und Kommentare der Tageszeitungen, u.a. „State sensitivity needed in future demonstrations“ [H S-B 13.6.1992]). Einen Monat nach den Verhaftungen ließ der Staat die Anschuldigungen fallen (,State drops charges in palace case“, David Waite, HA 31.7.1992:A-1)

${ }^{175}$ Francis A. Boyle, Professor für Völkerrecht an der University of Illinois, „,berät“ die Nation of Hawai‘i in Fragen des Völkerrechts und seiner Auswirkungen auf die Politk der Nation of Hawai'i im Bundesstaat Hawai'i. 
le Recht, die Charta der Vereinten Nationen und eine Eigeninterpretation des traditionellen hawaiischen Landrechtes vor 1848:

„Today, We, the Kanaka Maoli, proclaim our Right of selfdetermination as a people in accordance with Article 1(2) of the United Nations Charter, and join the World Community of States as an Independent and Sovereign Nation. We hereby reestablish our Independent and Sovereign Nation of Hawai'i, that was illegally taken from the Kanaka Maoli on January 17, 1893.

By virtue of our Right to self-determination, the Kanaka Maoli claim this right to freely determine our political status and freely pursue our economic, social and cultural development ...

We, the Kanaka Maoli, claim our Right for our own ends, to freely control and dispose of our natural wealth and resources, including our lands and our waters ...

We, the Kanaka Maoli, claim all our Land, Natural Wealth, Resources, Minerals, and Waters, which have always resided and will always reside within the hands of the Kanaka Maoli, to be ours forever, originally under communal land tenure“ (Nation of Hawai‘i 1994).

Vertreter anderer Gruppierungen (so z.B. Kekuni Blaisdell und Mililani Trask) lehnten dieses Vorgehen der Nation of Hawai'i ab, da ihm jede demokratische Legitimation von außerhalb der Mitgliedschaft der relativ kleinen Gruppierung fehlte. Zudem griff diese eigenmächtige Proklamation, die gegen Internationales Recht und ohne Abklärung etwaiger diplomatischer Anerkennung durch andere Nationen erfolgte, ${ }^{176}$ den jeweils eigenen Autonomiebestrebungen und den hierfür vorgesehenen Schritten voraus. ${ }^{17}$

Die Nation of Hawai'i ließ sich hiervon nicht beirren und stellte sich auf ihrer Internetseite unter der Rubrik „Present Status of Hawai'i“ den eigenen Vorstellungen gemäß dar: „Hawaii is an independent nation state undergoing a process of political, economic, social and cultural restoration and transition from a status of imposed involuntary colonial integration with the United States“ (Nation of Hawai'i 1998 a). Im Einklang mit der einseitigen Unabhängigkeitserklärung bildete die Nation of Hawai‘i noch 1994 eine „provisorische

\footnotetext{
${ }^{176}$ Vergleiche z.B. die aktuelle Unabhängigkeitserklärung des Kosovo im Jahre 2008: Das Völkerrecht erlaubt die Sezession eines Landesteiles aufgrund einer einseitigen Erklärung nicht jedoch wurde die unahängige Republik sofort von maßgeblichen Vertretern der Staatengemeinschaft (darunter Deutschland und die USA) anerkannt, was dem Vorgang eine de factoLegitimität verlieh.

177 „Group declares Hawaiian independence“, Jon Yoshishige (HA 17.1.1994:A-1); Rees (1994:4)
} 
Regierung", den 'Aha Kūpuna (Ältestenrat), und verabschiedete 1995 ihre „Verfassung“ (Nation of Hawai'i 1995; Nation of Hawai'i 1998b).

Diese Verfassung gesteht die Staatsbürgerschaft in der souveränen Nation Hawai'i allen Menschen hawaiischer Abstammung zu, Nicht-Hawaiier können eingebürgert werden soweit sie „...qualify and choose to become citizens of the Nation“. Allerdings würden sie hinsichtlich des Wahlrechts benachteiligt, steht doch der so genannten „Bürgerversammlung“eine gewählte Zweite Kammer, das Kūpuna Council (Ältestenrat) zu Seite, dessen Mitglieder hawaiischer Abstammung sein müssen und anerkannte Kenntnisse der hawaiischen Kultur und Tradition haben sollen. Anders als das in dem Verfassungsentwurf Ka Lāhuis vorgesehene beratende Oberhaus würde dieser Rat erhebliche Befugnisse haben, wozu z.B. ein generelles Vetorecht hinsichtlich aller Beschlüsse der Bürgerversammlung gehört (Nation of Hawai'i 1995:Art. VI (1); Art. VII $(4,5)$.

Für die Nation of Hawai'i steht weiterhin fest, dass „the illegal occupying foreign regime of the State of Hawai' $i$ and the United States of America may continue to provide services to such extent necessary as the government of the Nation of Hawai'i shall deem proper and expedient for a peaceful transition towards restoration“ (Art. XVII [5]), dass alle Funktionsträger des Staates oder des Bundes nach den Regelungen der Verfassung für ihre Handlungen verantwortlich gemacht werden sollen (Art. XVII [2]) und dass, last but not least, alle Gesetze des Staates Hawai'i und der Vereinigten Staaten, die nicht in Übereinstimmung mit dieser Verfassung stehen, mit ihrer Ratifizierung am 16.1.1995 ihre Gültigkeit verlieren (Art. XVII [6]).

Auch schon vor der Verabschiedung dieser Verfassung konzentrierten sich die Nation of Hawai' $i$ und ihre Mitglieder weniger auf den Bereich theoretischer Auseinandersetzungen mit nationalen und internationalen Institutionen sowie deren rechtlichen Standards, sondern bemühten sich eher um die konkrete Umsetzung der sich selbst eigenmächtig zuerkannten Rechte als Bürger des unabhängigen Hawai'i. In der Praxis bedeutete dies, dass Kanahele und die Nation of Hawai‘i die Gesetze des Staates Hawai‘i bzw. der USA nicht als bindend für Mitglieder oder „Bürger“ der Nation akzeptierten. So weigerten sich z.B. viele Nation of Hawai'i-Mitglieder, reguläre (staatliche) Nummernschilder an ihren Autos zu führen und machten sich damit strafbar. ${ }^{178}$ Diese Taktik brachte durchaus Erfolge: Nach einer Landbesetzung im Jahre 1993 sprachen staatliche Stellen den Besetzern Land aus dem Bestand der Hawaiian

178 „Kings of the road? Ohana drives law to distraction“, Mark Matsunaga (HA 3.5.1994). 
Home Lands zu. Als Pu'uhonua ${ }^{179}$ o Waimānalo gilt dieses Land der Nation of Hawai`i seither als Keimzelle ihrer neuen hawaiischen Nation. ${ }^{180}$

Dennis Kanahele, die Nation of Hawai'i und ihr „Zufluchtsort“ spielten schließlich 1995 die Hauptrollen in einem juristischen Schauspiel, das über längere Zeit die Öffentlichkeit beschäftigte. Die Nation gewährte dem wegen Steuerhinterziehung rechtskräftig zu mehreren Jahren Zuchthaus und einer hohen Geldstrafe verurteilten Jack Gonzales Asyl auf ihrem Gelände in Waimānalo. Ihr „Oberster Gerichtshof“ hatte zuvor verkündet, Gonzales bzw. Keaka M. Kamekona Makuaole sei Generalkonsul und Außenminister der Nation of Hawai'i und genieße diplomatische Immunität - die amerikanische Gerichtsbarkeit sei also auf keinen Fall zuständig. ${ }^{181}$ Ein Schreiben des „Obersten Gerichtshofes“ ging kurz darauf an Staats- und Bundesrichter sowie hohe Beamte der Staatsanwaltschaft, des FBI sowie der Steuerbehörden und wies sie darauf hin, dass sie sich Menschenrechtsverletzungen gegenüber den Kānaka Maoli schuldig gemacht hätten und hierfür zur Rechenschaft gezogen würden eine solche Bedrohung von Richtern gilt nach amerikanischen Recht als strafbare Handlung. ${ }^{182}$ Am 8.3.1995 verhaftete die Polizei Kanahele, der inzwischen den flüchtigen und ebenfalls wegen Steuerhinterziehung verurteilten Nathan Brown (ebenfalls Mitglied der Nation) dem Zugriff der staatlichen Strafverfolgung entzogen hatte. Kanahele, der sich letztlich schuldig bekannte, war vier Monate inhaftiert - seine Anhänger werteten dies als ausschließlich politisch motivierten Vorgang und als einen Schlag des Staates gegen die gesamte „Sovereignty“-Bewegung. ${ }^{183}$

Die Haltung Kanaheles gegenüber staatlichen Autonomie-Initiativen blieb ambivalent. So war er zunächst zwar Mitglied der vom Staat Hawai‘i eingesetzten Sovereignty Advisory Commission, der Vorläuferin des Hawaiian Sovereignty Elections Council (HSEC), trat dann 1994 aus dem HSEC aus und bezeichnete die vom Bundesstaat unterstützte Native Hawaiian Vote als illegal. Nach Verabschiedung der Nation of Hawai‘i-Verfassung äußerte er sich wiederum positiv über die bevorstehende Wahl. ${ }^{184}$

Für die Nation of Hawai‘i zahlte sich die Politik am Rande bzw. jenseits der Legalität in den 1990er Jahren durchaus aus - zum einen sicherte sich

\footnotetext{
${ }^{179} \mathrm{Pu}$ 'uhonua: Zufluchtsort, Asyl [U.M.].

180 „A year of controversy at beach park“, Christopher Neal (HA 21.5.1994:A-2); „Rebirth of a nation“, Bob Krauss (HA 26.2.1995:D-1,3).

181 „Gonzales a fugitive, claims immunity“, Darren Pai u. James Dooley (HA 10.6.1995:A-1).

182 „Nation of Hawaii warns U.S., state judges: Kanahele group cites , war crimes““, Mark Matsunaga (HA 28.6.1995:A-1,2).

183 „Kanahele arrested at airport“ (S-B 3.8.1995:A-3); „Kanahele‘s supporters plan protest“ (HA 4.8.1995:A-5); „Activist pleads guilty in `94 U.S. case“, Ken Kobayashi (HA 13.9.1997:B-1).

${ }^{184}$ „Kanahele quits advisory panel on sovereignty“, Kris M. Tanahara (HA 9.1.1994:A-3); „Kanahele now finds usefulness in Hawaiian sovereignty council“, Paula Gillingham (HA 26.2.1995:A-2).
} 
die Nation dauerhaft Land, auf dem sie eigene Vorstellungen umsetzen und ihren Anhängern konkrete Perspektiven bieten konnte (Nation of Hawai‘i 1998c). Zum anderen erhielt die Gruppierung dadurch vor allem in der ersten Hälfte des Jahrzehnts ein Medienecho, das die Bemühungen anderer Organisationen der hawaiischen Bewegung zeitweise in den Schatten zu stellen und auch zu diskreditieren drohte, weshalb Miliani Trask (Ka Lāhui) 1995 in einem Zeitungsartikel forderte: „Take the focus off Kanahele!“‘185

Gemeinsamkeiten und Trennendes - die vier Gruppierungen im Überblick Die vier betrachteten Gruppierungen, die Vorstellungen der hawaiischen Autonomie maßgeblich gestalteten und einer hawaiischen wie auch nichthawaiischen Öffentlichkeit nahebrachten, weisen hinsichtlich ihrer Ziele und Strategien zahlreiche Gemeinsamkeiten auf. Jedoch findet sich auch genug Gegensätzliches, weshalb der Eindruck der „Zerstrittenheit“ der Bewegung immer wieder Nahrung erhielt. Nachdem Protagonisten, politische Ziele und Strategien soweit vorgestellt wurden, fasse ich die Übereinstimmungen wie auch das Trennende zwischen den hawaiischen Gruppen in einem abschließenden Überblick zusammen.

Gründung und Mitgliedschaft: Nur bei 'Ohana Council-Nation of Hawai‘i liegt das Gründungsdatum in den frühen 1990er Jahren, alle anderen der hier genannten Gruppen entstanden bereits in der zweiten Hälfte der 1980er Jahre, haben die sich herausbildende politische Bewegung also von Anbeginn mitbestimmt und geprägt. Keine der besprochenen Gruppierungen veröffentlichte verlässliche Zahlen über die Größe ihrer jeweiligen organisierten Anhängerschaft und bei allen stehen im Verlauf der 1990er Jahre ihre jeweiligen Sprecher, die in jedem Fall auch die Gründer der jeweiligen Organisation waren, im Vordergrund des öffentlichen Interesses.

Politische Ziele: Am deutlichsten fällt die Nähe von Gründerpersönlichkeit und Organisation beim I.A.H.A. ins Auge. Aber auch bei Ka Pākaukau und bei Ka Lāhui steht zu vermuten, dass die jeweiligen Gründer, Kekuni Blaisdell und Miliani Trask, die Leitlinien der Politik vorgaben (im Falle Ka Lāhuis spricht hierfür auch, dass es nach dem Ausscheiden Mililani Trasks als kia 'äina merklich ruhiger um die Organisation wurde). Die Verweise auf Abstimmungen in Gremien der jeweiligen Gruppierungen wie bei Ka Lāhui und der Nation of Hawai“i („Constitutional Conventions“, „Ältestenräte“ o.ä.), bei denen es sich ausschließlich um Eigenangaben handelt, bleiben vage und waren für mich in keinem Fall nachprüfbar.

Drei der genannten Gruppen (I.A.H.A., Ka Pākaukau und Nation of Hawai' $i$ ) streben eine nationalstaatliche Souveränität in den Grenzen des heutigen Bundesstaates an, wobei für I.A.H.A. und Ka Pākaukau die Wiederauf-

185 „Take the focus off Kanahele“, Mililani Trask (HA 21.9.1995:A-16). 
nahme Hawai' is in die UN-Liste der zur Dekolonisation vorgesehenen Territorien wichtigstes Nahziel ist. Auch Ka Lāhui strebt die erneute Anerkennung als „non-self governing territory“ durch die Vereinten Nationen an, verfolgt aber das Ziel einer hawaiischen Autonomie im Rahmen bestehender USamerikanischer Strukturen („Indian Nations“) und begrenzt den hawaiischen Gebietsanspruch auf die zur Zeit staatlich verwalteten Treuhandgebiete. ${ }^{186}$

Politische Strategien: Bis auf das I.A.H.A. lehnen alle hier genannten Gruppierungen eine konkrete Zusammenarbeit mit staatlichen Stellen grundsätzlich ab - wobei sich die Haltung der Nation of Hawai' $i$ in diesem Punkt als ambivalent erweist (siehe z.B. die Mitarbeit bei Vorbereitung und Durchführung der vom Staat initiierten so genannten „Native Hawaiian Vote“). Allen vier Organisationen gemein ist eine deutliche Abgrenzung gegenüber dem Bundesstaat, die zum einen dem jeweiligen Selbstverständnis als bereits existierende souveräne hawaiische Nation geschuldet ist, sicherlich aber auch der Stärkung des Gruppengefühls der umworbenen hawaiischen Klientel dienen dürfte.

Wesentliche Unterschiede zwischen den maßgeblichen Autonomieinitiativen offenbaren sich hinsichtlich der verfolgten Strategien zur Durchsetzung ihrer jeweiligen politischen Ziele. Während I.A.H.A., Ka Pākaukau und Ka Lāhui hierbei primär auf legale Demonstrationen und andere Events (wie Konferenzen, Kulturveranstaltungen u.ä.) sowie auf Medienpräsenz ihrer Sprecher setzten, stellten gezielte Übertretungen geltender Gesetze für die Nation of Hawai`i ein probates Mittel zur Manifestation ihres Souveränitätsanspruches dar.

Während I.A.H.A. als einzige Gruppierung die mögliche Zusammenarbeit mit dem Staat für sinnvoll erachtet, steht für Ka Lāhui die Lobbyarbeit und Einflussnahme auf Gesetzesvorhaben zu hawaiischen Themen im Vordergrund; Ka Pākaukau schließlich grenzt eine potenzielle Zusammenarbeit von vornherein auf die Ebene der Bundesregierung und auf Verhandlungen zur Durchführung der Dekolonisation ein. Zentrale Bedeutung für die drei Gruppen hat jedoch die Arbeit und Vernetzung auf internationaler Ebene - handle es sich nun um die Vereinten Nationen und ihre Arbeitsgruppen, im Pazifik tätige NGOs oder unabhängige Vereinigungen indigener Bevölkerungen. Die Nation of

\footnotetext{
${ }^{186}$ Andere in den 1990er Jahren diskutierte und von verschiedenen Gruppierungen propagierte Autonomiemodelle waren zum einen Vorstellungen eines „Staates im Staate“, bei dem (anders als beim „Nation in der Nation“-Modell Ka Lāhuis) nicht die USA, sondern der Bundesstaat Garant der Autonomie wäre; zum anderen gab es den Vorschlag einer „freien Assoziierung“ des unabhängigen Hawai'i mit den USA - ein Modell, das sich an die in Mikronesien (Palau, Federated States of Micronesia, Marshall Islands) verwirklichten Staatsmodelle („Compact of Free Association“) anlehnte. Insgesamt wurde diesen Vorschlägen außerhalb organisierter Diskussionsrunden allerdings wenig Aufmerksamkeit zuteil.
} 
Hawai'i stand in diesen Fragen deutlich abseits - setzte sie doch vor allem auf Konfrontation und die einseitige Inanspruchnahme selbsterklärter Rechte.

Politische Nutzung kultureller Faktoren: Alle vier Initiativen verweisen auf ihre besondere Stellung als Vertreter der indigenen hawaiischen Bevölkerung, der besondere Rechte in ihrem Heimatland zustehen, sie alle sehen in ihren Entwürfen zukünftiger Verfassungen oder in den Erklärungen zur angestrebten Souveränität eine, wenn auch unterschiedlich ausgeprägte, rechtlich herausgehobene Stellung des hawaiischen Bevölkerungsteiles vor. Allerdings bleibt I.A.H.A. hierbei im Rahmen einer vor allem legalistischen Argumentation, während bei den anderen Gruppen unterschiedliche Vorstellungen hawaiischer Kultur und Traditionen in die Rechtsvorstellungen integriert werden (z.B. „ali'i nui“" bei Ka Lāhui, „Ältestenrat" bei der Nation of Hawai'i oder die neu bewerteten „'ohana“-Strukturen bei Ka Pākaukau). Eine Außenabgrenzung und Manifestation kultureller und ethnischer Eigenständigkeit erfolgt schließlich auch durch die Namensgebung der genannten Gruppierungen, die sich dabei entweder der hawaiischen Sprache bedienen oder aber, wie bei der Nation of Hawai'i (ehemals auch 'Ohana Council), in ihrem Namen die hawaiische Souveränität vorwegnehmen.

\subsection{Office of Hawaiian Affairs (OHA)}

Auch wenn das 1978 im Rahmen einer Verfassungsänderung gegründete und vom Staat Hawai'i finanzierte Office of Hawaiian Affairs (OHA ${ }^{187}$ nicht den unabhängigen Gruppierungen der hawaiischen Autonomiebewegung zuzurechnen ist, war es doch während der gesamten 1990er Jahre und darüber hinaus einer der einflussreichsten Akteure in den politischen Auseinandersetzungen um eine hawaiische Autonomie. Im Artikel XII der Verfassung Hawai'is finden sich die rechtlichen Grundlagen des Office of Hawaiian Affairs - ebenso wird OHA hier auch als potenzieller Empfänger von Landübertragungen benannt:

„There is hereby established an Office of Hawaiian Affairs. The Office of Hawaiian Affairs shall hold title to all the real and personal property now or hereafter set aside or conveyed to it which shall be held in trust for native Hawaiians and Hawaiians. There shall be a board of trustees for the Office of Hawaiian Affairs elected by qualified voters who are

\footnotetext{
${ }^{187}$ Das Akronym „OHA“ bildet das hawaiische Wort für den Taro-Schössling - die Institution nutzt so, neben ihrem englischsprachigen Namen, eines der hawaiischen Kernsymbole zur Eigenbezeichnung. Die Vorstellung eines Schösslings korrespondiert zudem mit der vom Office of Hawaiian Affairs propagierten Darstellung des OHA als Keimzelle einer hawaiischen Nation.
} 
Hawaiians, as provided by law. The board members shall be Hawaiian. [...]“ (Hawaii Constitution 1997:Art. XII [5]).

Die Verfassungsversammlung von 1978 sah ursprünglich sogar vor, die Verwaltung und Nutzung der Hawaiian Home Lands an das Office of Hawaiian Affairs zu übertragen und so eine staatliche Behörde, das Department of Hawaiian Home Lands, entbehrlich zu machen (cf. McKenzie 1991:88-9; Hawaiian Affairs 1978:5, 7). Allerdings folgte das Parlament diesem Vorschlag nicht und nahm die Hawaiian Home Lands in den entsprechenden Gesetzen ausdrücklich von etwaigen zukünftigen Landübertragungen aus (Hawaii Revised Statutes o.J.:§10-3 [1,3]). Dies ist insofern von Bedeutung, als OHA ansonsten schon seit seiner Gründung über eine eigene Landbasis hätte verfügen können, was seine Ansprüche als Vorläufer einer autonomen hawaiischen Einheit noch untermauert hätte.

Zwar stehen OHA immerhin 20\% aller Einnahmen aus dem „Public Land Trust“ zur Verfügung, doch dürfen diese Einkünfte gemäß den Bestimmungen des Aufnahmevertrages von 1959 nur für Native Hawaiians im Sinne des Hawaiian Homes Commission Act, d.h. für Hawaiier mit einer mindestens zu 50\% hawaiischen Abstammung verwendet werden. Darüber hinaus ist das Office of Hawaiian Affairs zu einem guten Teil auf finanzielle Zuweisungen des Parlaments angewiesen, über deren Verwendung es Rechenschaft ablegen muss - es verfügt also nur über eine eingeschränkte Unabhängigkeit (cf. MacKenzie 1991 b:89). ${ }^{188}$

In seiner Selbstdarstellung betont OHA jedoch seine Unabhängigkeit vom US-Staat Hawai'i und verweist vor allem auf hawaiische Wurzeln seiner Entstehung:

„In 1977, an unprecedented series of ,Puwalu Seminars“ provided representatives from 28 organizations and many individual Hawaiians with an opportunity to discuss a wide range of Hawaiian issues. [...] In later sessions, island representatives were elected to serve as members of a mini-legislature, which prepared a set of legislative proposals called the Native Hawaiian Legislative Package“ (OHA 1998).

Die Aufgabenstellung des Office of Hawaiian Affairs sind in den Hawaii Revised Statutes festgelegt: OHA soll die allgemeinen Lebensbedingungen der

1881996 betrugen die Mittel aus dem Public Land Trust und anderen Quellen, die nach dem Aufnahmevertrag von 1959 für Native Hawaiians verwendet werden müssen, US \$ 22.434.240. Sie wurden ergänzt durch allgemein verwendbare Mittel in Höhe von US \$ 3.901.563. Um seinen Aufgaben gerecht werden zu können, verrechnet OHA unterschiedliche Posten. Das Gesamtvermögen des Office of Hawaiian Affairs belief sich im Juni 1996 auf US\$ 217.347.438 (OHA 1997:43, 47). 
Hawaiier verbessern, indem es Förderprogramme für die hawaiische Bevölkerung entwirft und durchführt sowie Programme anderer Behörden bewertet und koordiniert; es soll sich um finanzielle Mittel für diese Programme bei allen möglichen Quellen bemühen. Und schließlich soll OHA auch der Empfänger von etwaigen zukünftigen Reparationszahlungen staatlicher Stellen an die hawaiische Bevölkerung sein (Hawaii Rev. Statutes o.J.:§10-3 [1-6]). Diese Aufgaben und damit sein Selbstverständnis als Interessenvertretung der gesamten hawaiischen Bevölkerung hebt das Office of Hawaiian Affairs in seiner Eigendarstellung ausdrücklich hervor:

„OHA then, is an agency, a trust and a government all at once. Its mission is the betterment of conditions of all Hawaiians and to provide Hawaiians the opportunity for a better life and future“ (OHA 1998).

Zur Erfüllung seiner Aufgaben verfügt $\mathrm{OHA}$ über einen umfangreichen Stab von Mitarbeitern, der sich auf zehn Abteilungen verteilt, die gewissermaßen Regierungsstrukturen nachbilden (so u.a. Government Affairs Office, Culture Office, Economic Development Division und Land and Natural Resources Division). Nach den Vorstellungen der ursprünglichen Satzung sollte die Institution von einem Vorstand aus neun gewählten hawaiischen Treuhändern, dem „Board of Trustees“, geleitet werden (Hawaii Constitution 1997:Art. XII [5]). In den Hawaii Revised Statutes (o.J.:§13 D-3, 4) war darüber hinaus festgelegt worden, dass ausschließlich wahlberechtigte Bürger hawaiischer Abstammung an den alle vier Jahre stattfindenden Wahlen teilnehmen konnten - ein Urteil des Obersten Gerichtshofes der USA änderte diese Regel im Jahr 2000, so dass nun auch Nichthawaiier über den OHA-Vorstand entscheiden können. Die Zahl der registrierten Wähler zu den OHA-Wahlen stieg seit 1990 beständig an waren es 1990 noch 63.432 Personen (MacKenzie 1991 b:89), so stieg die Zahl auf 75.766 im Jahre 1994 und 87.298 im Jahre 1996 an. ${ }^{189}$ Diese im Gegensatz zu den Mitgliedszahlen der unabhängigen Initiativen nachprüfbaren Zahlen machen das Office of Hawaiian Affairs zumindest hinsichtlich des mittelbar involvierten Bevölkerungsteiles zum bedeutendsten Repräsentanten der hawaiischen Bevölkerung.

1989 trat OHA mit einem so genannten „Blueprint for Native Hawaiian Entitlements“ an die Öffentlichkeit, einem als Diskussionsgrundlage gedachten Plan zur Übertragung von Land an eine hawaiische Verwaltungseinheit und die Gewährung politischer Unabhängigkeit (OHA 1989). ${ }^{190}$ In der Zusammenfassung des „Blueprints“ heißt es:

\footnotetext{
189 „OHA voters active, growing“, Richard Borreca (H S-B online 27.5.97: starbulletin.com/ 97/05/27/news/story3.html [23.9.1998]).

${ }^{190}$ Siehe ausführlich zu dem Dokument MacKenzie (1991b:91-2).
} 
„Native Hawaiians have the right to pursue their own culture, traditions and goals. To achieve this right, this blueprint proposes a gathering of elected Native Hawaiian representatives who will draft a governing document (such as a constitution). This document should provide for a process of ratification by Native Hawaiian voters. If adopted, it should provide for establishment and powers of such an entity" (OHA 1989:1).

Hier muss zunächst angemerkt werden, dass das Office of Hawaiian Affairs trotz der besonderen Rolle, die es für die Hawaiier im Sinne des Hawaiian Homes Commission Act spielt, die Bezeichnung „Native Hawaiians“ im oben zitierten Passus wie auch in seinen sonstigen Selbstäußerungen auf alle Hawaiier, gleich welchen Abstammungsgrades anwendet (OHA 1989:1). So vermeidet es zumindest vordergründig eine Trennung seiner Wählerbasis in Gruppen mit unterschiedlichem Rechtsstatus. Des Weiteren konstatiert OHA mehr oder weniger deutlich, dass es sich als weitgehend selbst verwaltete hawaiische Institution bereits als oberste Instanz der im „Blueprint“ genannten autonomen Gebietseinheit („Entity“) sieht (OHA 1989:3 [10. a,b]). Dieses durchgängig geäußerte Selbstverständnis klingt auch in der Aufzählung der vorgesehenen Befugnisse bzw. Aufgaben der o.a. autonomen Institution deutlich an:

„The document drafted may give OHA, or another entity chosen by Native Hawaiians, the power to adopt ordinances for the health, safety, and welfare of Native Hawaiians; the power to levy taxes, zone trust lands, produce income from such lands, and regulate conduct on trust lands; the power to receive and manage any lands, resources or funds; the power to allot lands to individuals; and the power to regulate hunting, fishing, gathering, access and other traditional rights and practices of Native Hawaiians“ (OHA 1989:3 [10. a]).

Einige Jahre später, im Gedenkjahr 1993, hatten sich die Vorstellungen des Office of Hawaiian Affairs erheblich konkretisiert: die Behörde vertrat nun unmissverständlich das Autonomiemodell einer hawaiischen Nation nach dem Vorbild der „Indian Nations“ in den kontinentalen USA - wie es auch von Ka Lāhui favorisiert wird. Diese Vorstellungen finden sich in dem Entwurf eines „Native Hawaiian Recognition and Restoration Act“, der in den US-Kongress eingebracht werden sollte. Hier heißt es u.a.:

„The form and structure of the Native Hawaiian government is for Native Hawaiians to decide... [...] (1) Native Hawaiians are indigenous people with a long historical and political relationship with the United States. (2) ...Congress has the constitutional authority to legislate in 
matters affecting indigenous people, including Native Hawaiians. (3) A re-established Native Hawaiian Government would have a status and relationship with the United States similar to that which federally recognized Indian tribes now possess. [...]“ (OHA 1993a:3). ${ }^{191}$

Auch OHA sieht also die USA in der Verantwortung, durch Gesetze wie den „Recognition Act“ die politische und rechtliche Situation der hawaiischen Bevölkerung grundlegend zu ändern. Hinsichtlich seiner eigenen Rolle in diesem Prozess versteht sich das Office of Hawaiian Affairs als treibende Kraft und als wichtigster Ansprechpartner der entscheidenden Regierungsinstanzen:

„OHA would be designated as the interim organization to manage the process and petition the President and Congress with the results“ (OHA 1993a:3).

Soweit die Äußerungen des Office of Hawaiian Affairs zu Autonomiemodellen und Vorstellungen der eigenen Rolle bei diesen Projektionen, die in den 1990er Jahren einen wesentlichen Aspekt des hawaiischen Autonomiediskurses bildeten.

Das Office of Hawaiian Affairs hat die vom Staat angeregte und geförderte „Native Hawaiian Vote“ von 1996 nachdrücklich unterstützt und eine zentrale Rolle bei Planung und Durchführung dieser Wahl gespielt. OHA hatte nicht nur einen festen Sitz sowohl in der Hawaiian Sovereignty Advisory Commission als auch im Hawaiian Sovereignty Election Council (HSEC) - es finanzierte die zuletzt genannte Institution darüber hinaus zu 50\% aus seinem Haushalt. Eine der dem Office of Hawaiian Affairs im Zuge der Wahlvorbereitungen zufallenden Aufgaben war die Information der wahlberechtigten Bevölkerung über die unterschiedlichen politischen Modelle hawaiischer Selbstbestimmung, wobei dem von ihm selbst favorisierten Modell einer „Nation within a Nation“ keine offensichtliche Vorrangstellung eingeräumt wurde (siehe z.B. OHA 1994).

Allerdings stand der Vorstand des Office of Hawaiian Affairs nicht einheitlich hinter der Idee einer hawaiischen Wahl zur Festlegung des weiteren Weges zur Autonomie. So machte zumindest ein Mitglied des Vorstandes, Samuel L. Kealoha, aus seiner Ablehnung des Wahlganges keinen Hehl und schrieb in der OHA-Zeitung Ka Wai Ola:

„HSEC [Hawaiian Sovereignty Election Council] does not represent self-determination for the Hawaiian people. HSEC represents a state-

${ }^{191}$ Man vergleiche die hier vom Office of Hawaiian Affairs formulierten Zielvorstellungen mit jenen der „Akaka-Bill“; siehe hierzu 8.3. 
controlled process for achieving a state-controlled outcome - an outcome that protects state interests, not the interests of the Hawaiian people. “192

Diese Vorbehalte hinsichtlich einer Beteiligung des OHA an der „Native Hawaiian Vote“ wurden von vielen Hawaiiern und einem großen Teil der organisierten Autonomiebewegung geteilt. Eine ganz ähnliche Kritik galt zudem überhaupt jeglicher Beteiligung des Office of Hawaiian Affairs am Autonomieprozess. Insbesondere Ka Lāhui Hawai'i wandte sich bei jeder Gelegenheit gegen OHA als Mitstreiter für die hawaiische Selbstbestimmung. Gerade diese beiden so unterschiedlichen Organisationen zeichneten sich durch ein ausgeprägtes Konkurrenzverhältnis aus, das u.a. durch eine erhebliche gemeinsame Schnittmenge ihrer politischen Zielvorstellungen und durch den beiderseits erhobenen Anspruch, bereits eine jederzeit handlungsfähige hawaiische „Regierung“ zu bilden, begründet war.

Neben dem Office of Hawaiian Affairs als einer hawaiischen Institution mit Verfassungsrang waren aber auch andere staatliche Stellen und Institutionen in die Auseinandersetzungen um die „Hawaiian Sovereignty“ involviert auf ihre Rolle und Bedeutung im Autonomiediskurs der 1990er Jahre werde ich im Folgenden eingehen.

192 „HSEC plebiscite is not Hawaiians“ choice“, Samuel L. Kealoha (Ka Wai Ola o OHA, May 1995, 12 [5]:15) 


\subsection{Hawaiian Sovereignty Elections Council (HSEC)}

Das Hawaiian Sovereignty Elections Council (HSEC) war neben dem Office of Hawaiian Affairs die zweite staatsnahe Institution, die in der politischen Diskussion um die „Hawaiian Sovereignty“ und für die Entwicklungen der Jahre 1995-96 eine entscheidende Rolle spielte. HSEC wurde vom Staat Hawai`i 1994 ins Leben gerufen, um eine Abstimmung unter der hawaiischen Bevölkerung zu organisieren und durchzuführen. Sie sollte die Frage entscheiden, ob die Hawaiier eine weitergehende Unabhängigkeit vom Staat wünschten. Nachdem diese Befragung schließlich 1996 stattgefunden hatte, wurde das Hawaiian Sovereignty Elections Council noch im gleichen Jahr durch Parlamentsbeschluss aufgelöst.

Die Geschichte des HSEC beginnt mit seinen Vorläufern, dem Sovereignty Advisory Council (SAC) und der Hawaiian Sovereignty Advisory Commission (HSAC). Ersteres wurde bereits 1991 vom Parlament durch Gesetz eingerichtet: „The council shall develop a plan to discuss and study the sovereignty issue“ (Hawaii Session Laws 1991:909-10,Act 301[11,12]). Dieses Gesetz nannte 12 zumeist nicht-staatliche Organisationen, die in dem Gremium vertreten sein sollten und deren Vertreter im September 1991 die Arbeit aufnahmen. Unter den genannten Gruppierungen und Institutionen befanden sich das Office of Hawaiian Affairs, das Department of Hawaiian Home Lands, die Association of Hawaiian Civic Clubs, Ka Lāhui, Ka Pākaukau, IAHA, das State Council of Hawaiian Homestead Associations und 'Ōhana O Hawai' $i$, die eine große Bandbreite der hawaiischen Repräsentanten vertraten.

Ein 1992 veröffentlichter Abschlussbericht des SAC enthielt einen Plan für das weitere Vorgehen und eine Liste mit Empfehlungen an den Gesetzgeber (SAC 1992). Die Autoren benennen in diesem Zusammenhang zwei Alternativen: zum einen das Modell einer autonomen hawaiischen Nation innerhalb der Grenzen der Vereinigten Staaten, zum anderen ein nach außen und innen souveräner Staat Hawai`i (SAC 1992:xx-xxi). Es scheint, dass die Legislatur während der Sitzungsperiode 1992 nicht auf die Empfehlungen des SAC reagierte und auch sonst keine Maßnahmen hinsichtlich der „Hawaiian Sovereignty“ ergriff (siehe Mardfin 1994:33).

Im folgenden Jahr schuf das Parlament allerdings eine Institution, deren Vollmachten weit über jene des SAC hinausgingen. Mit dem Gesetz 359 (Hawaii Session Laws 1993:1009-13) wurde die Hawaiian Sovereignty Advisory Commission (HSAC) etabliert:

„The purpose of this Act is to acknowledge and recognize the unique status the native Hawaiian people bear to the State of Hawaii and to the 
United States and to facilitate the efforts of native Hawaiians to be governed by an indigenous sovereign nation of their own choosing. [...] There is established within the office of state planning for administrative purposes the Hawaiian sovereignty advisory commission, to advise the legislature in carrying out the purposes of this act" (Hawaii Session Laws 1993:1010-11, Act 359[2,4]).

Dieses Gremium sollte den Gesetzgeber in der Frage eines hawaiischen Referendums über die Bildung einer hawaiischen Verfassungsversammlung beraten und die Modalitäten dieser Abstimmung sowie der Einberufung einer solchen Versammlung erarbeiten. Das Gesetz schrieb 19 vom Gouverneur zu ernennende Kommissionsmitglieder vor, wobei für mindestens 12 der Mitglieder ein Vorschlagsrecht durch hawaiische Organisationen vorgesehen war. Den vier mitgliederstärksten hawaiischen Organisationen (das Gesetz nennt namentlich das Office of Hawaiian Affairs, Ka Lāhui Hawai'i, State Council of Hawaiian Homestead Associations, Association of Hawaiian Civic Clubs) wurde ein Platz garantiert (Hawaii Session Laws 1993:1011, Act 359[4]). Insgesamt nominierten 50 Organisationen über 100 Kandidaten, von denen schließlich 20 durch Gouverneur John Waihe‘e ernannt wurden (HSAC 1994:15).

Ka Lāhui Hawai'i lehnte bereits im Vorfeld der Entscheidung über die Zusammensetzung der Hawaiian Sovereignty Advisory Commission jegliche Mitarbeit ab und begründete dies mit der mangelnden Staatsferne der Institution (HSAC 1994:9). In diesem Zusammenhang ist ein Blick auf die Geschichte des Gesetzes angebracht, das auf einen Vorschlag des Office of Hawaiian Affairs zurückgeht. Da der Entwurf zeitgleich mit einem Gesetzesvorschlag Ka Lāhuis, der die Übereignung sämtlicher Treuhandgebiete beinhaltete, beraten wurde, kam es zu heftigen Kontroversen (Kame'eleihiwa 1993:69). ${ }^{193}$ Der zuständige Ausschuss lehnte schließlich den Ka Lāhui-Vorschlag ab, während das Parlament eine überarbeitete Version des OHA-Gesetzentwurfs verabschiedete. ${ }^{194}$ Dieses Gesetz sowie das Projekt eines hawaiischen Referendums wurde von Ka Lāhui, die ja noch intensiv im Sovereignty Advisory Council mitgearbeitet hatte, sowie anderen hawaiischen Gruppierungen konsequent abgelehnt und bekämpft.

Die Hawaiian Sovereignty Advisory Commission nahm im August 1993 ihre Arbeit auf; ihre Finanzierung teilten sich je zur Hälfte Staat und Office of Hawaiian Affairs (Hawaii Session Laws 1993:1012, Act 359[9]; HSAC 1994:12). ${ }^{195}$ Die von Arbeitsgruppen ausgearbeiteten Vorschläge wurden durch

\footnotetext{
${ }^{193}$ Siehe hierzu auch 6.3.

194 „A new plan for sovereignty referendum“, Kevin Dayton (HA 29.4.1993:A-3); „Sovereignty

bill alive“, Darren Pai (HA 25.6.1993:A-7)

${ }^{195}$ Insgesamt stand der Kommission ein Etat in Höhe von US\$ 420.000 zur Verfügung.
} 
Anregungen aus der „Hawaiian Community“ ergänzt, die in 16 öffentlichen Informationsveranstaltungen auf allen Inseln sowie in den kontinentalen USA eingebracht werden konnten. Auf dieser Grundlage legte die Kommission einen Entwurf ihrer Empfehlungen an die Legislatur vor, der wiederum in 20 öffentlichen Veranstaltungen diskutiert werden konnte (HSAC 1994:16-8). Die von den Besuchern dieser Veranstaltungen geäußerten Anregungen fasste die Hawaiian Sovereignty Advisory Commission wie folgt zusammen:

„(1) Provide more information and education on sovereignty before the plebiscite is held.

(2) Hawaiian groups need to work together and provide better leadership.

(3) Set up a process that is independent of the State.

(4) Stop State actions that decrease or mis-use Hawaiian national trust lands (Hawaiian Homelands, ceded public land trusts)“ (HSAC 1994:19).

Diese Bedenken griff die HSAC in ihren der Legislatur unterbreiteten Vorschlägen sowie in zwei Gesetzentwürfen auf: So sollte das Parlament zum einen ein von Hawaiiern gewähltes Hawaiian Sovereignty Elections Board schaffen und zum anderen ein Moratorium über den Verkauf von Ceded und Hawaiian Home Lands verhängen. ${ }^{196}$ Die Hawaiian Sovereignty Advisory Commission schlug darüber hinaus die Durchführung eines „Hawaiian Sovereignty Plebiscite“ für das Jahr 1995 vor, das den Hawaiiern die Frage vorlegen sollte: „,E ho'omaka 'anei kākou e ho'okō i na kuleana o ka ho'iho'i ea o kō Hawai'i aupuni' "Shall a process begin to restore the Sovereign Hawaiian Nation?““ (HSAC 1994:20-3).

1994 wurde schließlich mit dem Hawaiian Sovereignty Elections Council (HSEC) die Nachfolgeorganisation der lediglich beratenden Hawaiian Sovereignty Advisory Commission (HSAC) eingerichtet, um notwendige Maßnahmen zur Durchführung eines Referendums einzuleiten (Hawaii Session Laws 1994:479-83, Act 200). ${ }^{197}$

\footnotetext{
${ }^{196}$ Ein Moratorium über die Veräußerung von genannten Landflächen zog die Legislatur allerdings nicht in Betracht (Mardfin 1994:34).

${ }^{197}$ Das Gesetz sah die Übernahme aller Mitglieder der HSAC in die neue Institution vor, ansonsten ernannte der Gouverneur neue Mitglieder. Insgesamt sechs Mitglieder werden aufgrund von Vorschlägen hawaiischer Organisationen neu aufgenommen, da alte Mitglieder zurücktraten. Ka Lāhui Hawai'i lehnte den ihr zustehenden Sitz im HSEC wiederum ab (HSEC 1995:15). Dem Hawaiian Sovereignty Elections Council stand für das Jahr 1994/95 ein Budget aus Mitteln des Staates sowie des Office of Hawaiian Affairs in Höhe von US\$ 1,8 Mio. zur Verfügung (HSEC 1996:11-2).
} 
Die Registrierung der potenziellen Wähler begann am 17. Januar 1995 und wurde noch im Juli desselben Jahres abgeschlossen; allerdings musste die Abstimmung, ursprünglich für November 1995 vorgesehen, wegen finanzieller Probleme auf den Juli/August des folgenden Jahres verschoben werden (HSEC 1996:11). ${ }^{198}$ Die Frage, die das Hawaiian Sovereignty Elections Council schließlich den wahlberechtigten Hawaiiern in Englisch und Hawaiisch vorlegte, unterschied sich in einem wesentlichen Punkt vom früheren Vorschlag der Hawaiian Sovereignty Advisory Commission und lautete: „,Shall the Hawaiian people elect delegates to propose a native Hawaiian government? ,He pono inā koho nā Hawai'i i nā 'elele no ka noi 'ana i kekahi aupuni Hawai‘i maoli،“ (HSEC 1995:5). Im Gegensatz zum früheren Vorschlag, der ja die grundsätzliche Frage nach dem Beginn eines Autonomieprozesses stellte, wird nun nach der Wahl von Delegierten gefragt, die wiederum die Bildung einer hawaiischen Regierung anregen sollen - wobei die Begriffe "sovereign" und "nation" im Gegensatz zur früheren Fragestellung ausgeklammert blieben.

Allerdings wies das Hawaiian Sovereignty Elections Council darauf hin, dass die Abstimmung nur ein erster Schritt auf dem Weg zur hawaiischen Autonomie sein könne: „True self-determination appropriate to the historical experience and contemporary circumstance of a sovereign nation of Hawai' $i$ will not be fully accomplished under the present limitations of the states's legislation“ (HSEC 1995:Anh. A). Es handele sich bei der „Native Hawaiian Vote“ schließlich um eine Willensbekundung der hawaiischen Bevölkerung, die den Staat in seinen Entscheidungen nicht binde, wie eine der Schlussbestimmungen des grundlegenden Gesetzes sehr deutlich festlegte:

„Nothing arising out of the Hawaiian convention provided for in this Act, or any results of the ratification vote on proposals from the Hawaiian convention, shall be applied or interpreted to supersede, conflict, waive, alter, or affect the constitution, charters, statutes, laws, rules, regulations, or ordinances of the State of Hawaii or its political subdivisions, including its respective departments, agencies, boards, and commissions“ (Hawaii Session Laws 1994:483, Act 200[14]).

So fügte das Hawaiian Sovereignty Elections Council den Wahlscheinen eine Erklärung bei, die klarstellte, ,that the plebiscite is not an election by Hawaiians to relinquish their inherent sovereignty as a people or over their national lands to the United States or the State of Hawai 'i“ (HSEC 1995:6).

Nach intensiven Informations- und Werbekampagnen, die Werbung in Radio, TV und Printmedien sowie Informationsveranstaltungen auf allen Inseln einschlossen, begann im Juli 1996 unter scharfem Protest hawaiischer Organi-

198 „Sovereignty vote jeopardized?“, Mark Matsunaga (HA 1.3.1995:A-7). 
sationen ${ }^{199}$ der Versand von insgesamt 81.598 Wahlscheinen an die registrierten Wähler (HSEC 1996:12, 37-40). Bis zum 15. August 1996 wurden 30.783 gültige Stimmzettel zurückgeschickt, was einer Wahlbeteiligung von 37,77\% entsprach. Der Anteil der Jastimmen lag bei 73,28\% (22.294), mit Nein stimmten 26,72\% (8.129) (HSEC 1996:28). Trotz der sehr niedrigen Wahlbeteiligung begannen nun die Vorbereitungen $\mathrm{zu}$ einer verfassungsgebenden Versammlung. Da das Hawaiian Sovereignty Elections Council dem entsprechenden Gesetz zufolge zum Ende des Jahres 1996 aufgelöst wurde und der Staat keine weiteren Mittel zur Verfügung stellte, übertrugen die Mitglieder des HSEC die weiteren Vorbereitungen an eine gemeinnützige Organisation - Hā Hawai'i. ${ }^{200}$

„Hā Hawai'i's sole objective is to carry out the Hawaiian Vote Mandate to propose a Hawaiian government" (Hā Hawai'i 1998a) - zu diesem Zweck hatte die Organisation, die von zehn hawaiischen Organisationen unterstützt wurde und keine staatliche Unterstützung erhielt, im April 1998 mit der Registrierung von Wählern und Kandidaten begonnen; die Wahl einer „Native Hawaiian Convention“ sollte am 17. Januar 1999 stattfinden (1998b) ${ }^{201}$ Hā Hawai‘i führte diese Delegiertenwahl unter dem Protest des größten Teils der Bewegung und von Vertretern des Office of Hawaiian Affairs durch, allerdings gibt es keine weiteren Nachrichten über eine tatsächlich abgehaltene „Constitutional Convention“. Aus der von hawaiischen Organisationen angeregten, quasi-staatlichen Initiative, die allerdings nur unzureichend gefördert und finanziell ausgestattet war, wurde schließlich eine der bis heute immer wieder auftretenden Splittergruppen. Diese Gruppierungen nehmen zwar für sich in Anspruch, alle Hawaiier zu repräsentieren, verfügen aber über keinen nennenswerten Rückhalt in breiteren Kreisen der hawaiischen Bevölkerung.

\footnotetext{
${ }^{199}$ Der Protest gegen die „Native Hawaiian Vote“ ging vornehmlich von Ka Lāhui Hawai‘i und Ka Pākaukau aus. Letzterer hatte sich mit anderen Gruppierungen in einer „Coalition to stop the state-sponsored plebiscite" zusammengeschlossen.

${ }^{200}$ Aufgrund von Budgetkürzungen hatte das Hawaiian Sovereignty Elections Council die gemeinnützige Organisation Hā Hawai'i bereits 1995 als „Spendensammler“ gegründet (HSEC 1996:57).

${ }^{201}$ Die Unterstützer von Hā Hawai'i waren: State Council of Hawaiian Homestead Associations, Association of Hawaiian Civic Clubs, Native Hawaiian Chamber of Commerce, Native Hawaiian Bar Association, Native Hawaiian Legal Corporation, Nation of Hawai 'i, Hui Kalai‘āina, Pa Kui a Holo, Nation of Kū, Council of Hawaiian Organizations (Hā Hawai'i 1998b). Außer der Nation of Hawai`i befanden sich unter diesen Unterstützergruppen keine maßgeblichen Vertreter der „Sovereignty-Bewegung“.
} 


\subsection{Der Bundesstaat Hawai‘i und die „Hawaiian Sovereignty“}

Dem Bundesstaat Hawai‘i und seinen Institutionen kommt über direkte bzw. indirekte Einflussnahmen eine zentrale Rolle für die hawaiischen Bemühungen um Selbstbestimmung zu. Der Bundesstaat kontrolliert nicht nur den größten Teil der von den Vertretern der „Sovereignty“-Bewegung für die Hawaiier reklamierten Ressourcen, seine vielfältigen Institutionen und die staatliche Gesetzgebung bestimmen unmittelbar viele Lebensaspekte der hawaiischen Bevölkerung und grenzen auch den legalen Aktionsradius der politischen Bewegung ein.

Es kann nicht verwundern, wenn der größte Teil der organisierten Autonomiebewegung den Bundesstaat Hawai'i immer wieder auf seine „illegale“ Herkunft hinweist und ihm das Recht der Mitwirkung an Organisation und Planung hawaiischer Angelegenheiten abspricht. Doch teilen bei weitem nicht alle Hawaiier diese ablehnende Haltung dem Staat und seinen Institutionen gegenüber. Schließlich arbeiten Hawaiier in sämtlichen Bereichen und auf allen Ebenen der staatlichen Verwaltung und mit John Waihe'e stand von 1986 bis 1994 erstmals ein Gouverneur hawaiischer Herkunft an der Spitze des Bundesstaates. Seine Verfassung verpflichtet den Bundesstaat gegenüber allen Staatsbürgern gleichermaßen - eine Trennung zwischen hawaiischen Angelegenheiten und allgemeinen staatlichen Angelegenheiten, was faktisch eine Autonomie des hawaiischen Bevölkerungsteiles bedeuten könnte, ist weder von den Gesetzen des Bundesstaates noch jenen der USA vorgesehen ${ }^{202}$ Es ist vielmehr eine integrativ ausgerichtete, auf die formale Gleichberechtigung und letztlich die Assimilation aller ethnischen Gruppen in die vorherrschende USamerikanische Gesellschaft abzielende Philosophie, die zumindest vordergründig die bundesstaatliche Politik bestimmt. Eine Werbebroschüre der staatlichen Tourismusbehörde „Hawaii Visitors Bureau“ formuliert das verbreitete Selbstbild des Staates wie folgt: „Hawaii's history has resulted in a multi-racial society admired for its high degree of harmony and assimilation“ (Hawaii Visitors Bureau 1992). Allerdings wird der hohe Grad an „Harmonie und Assimilation“ von vielen Vertretern der Hawaiier als Unterdrückung der hawaiischen Minderheit durch die Mehrheit und den Staat gebrandmarkt, und auch die großen sozialen Unterschiede zwischen den ethnischen Gruppen in Hawai‘i zeigen, wie weit Anspruch und Wirklichkeit hier auseinander klaffen.

\footnotetext{
${ }^{202}$ So ist das Office of Hawaiian Affairs, in dem diese Autonomie der hawaiischen Angelegenheiten im Keim angelegt ist und von den Verfassern der Erläuterungen zu den Verfassungsänderungen von 1978 in Ansätzen auch beabsichtigt war, durch die Ausführungsgesetze des Staates nach wie vor abhängig von den Entscheidungen staatlicher Stellen.
} 
Der Staat ist gegenüber der hawaiischen Bevölkerungsminderheit durch verschiedene Gesetze und vertragliche Vereinbarungen besonders verpflichtet: So müssen die staatlichen Institutionen zum einen die Gleichheit aller Bürger vor dem Gesetz (,equal protection“) garantieren, zum anderen aber auch den besonderen Rechten („,special rights“) der hawaiischen Bevölkerung Rechnung tragen. Letzteres stimmt nicht unbedingt mit ersterem überein, so dass der Bundesstaat in einen Grundsatzkonflikt gerät, auf den schließlich viele Auseinandersetzungen zwischen Hawaiiern und staatlichen Stellen zurückgeführt werden können: „The core problem is the impossibility of a state ministering to two parties with conflicting interests“ (L. K. Friedman 1992:550). Beispiele für entgegenstehende Interessen zeigen sich u.a. in Hinblick auf Fragen der Landund Wasserrechte, bei Konflikten um die traditionellen Sammel-, Jagd- und Fischrechte der Hawaiier oder auch in den Auseinandersetzungen um das $\mathrm{Zu}-$ gangsrecht zu Küsten und Stränden. ${ }^{203}$

Ein Erbe der Monarchie ist auch die zentralistische Verwaltungsstruktur Hawai'is, die unterhalb der bundesstaatlichen Administration nur noch die Verwaltungsebene der „Counties“ kennt. ${ }^{204}$ Kommunalverwaltungen fehlen in dieser Struktur völlig, was Hawai‘i zu dem am stärksten zentralisierten Staat der USA macht (Meller 1992:13). Aufgrund fehlender unterer Verwaltungsebenen stellt die bundesstaatliche Administration in vielen hawaiischen Belangen den ersten Ansprechpartner der Bürger dar: So konzentriert sich u.a. die Verwaltung von Land, dessen Nutznießer die Hawaiier sein sollten, beim Staat und seinen Behörden.

Land war seit jeher eine der wichtigsten und am meisten umkämpften Ressourcen auf den Inseln. Die Aufnahme des Hawaiian Homes Commission Acts in die Staatsverfassung stellte eine Bedingung des US-Kongresses für die Aufnahme Hawai'is in die Union dar (Lee 1993:175); hingegen wurde die treuhänderische Bestimmung des „PublicLand Trusts“ erst 1978 in der Verfassung festgeschrieben.

Am Beispiel dieser „Trust Lands“ verdeutlicht sich der staatliche Konflikt zwischen „equal protection“ und „special rights“ in dem Bemühen, die

\footnotetext{
203 Die besonderen Rechte der Bevölkerung hawaiischer Abstammung leiten sich z.T. aus der Zeit der Monarchie her. Das Fortbestehen solcher Sonderrechte wurde in der bundesstaatlichen Verfassung abgesichert (Hawaii Constitution 1997:Art. XII [7]), wobei aber schon die verfassungsgebende Versammlung von 1978 Bedenken dahingehend äußerte, dass diese Bestimmungen auf der Basis der „equal protection“ aller Bürger anfechtbar seien (Lee 1993:179). Siehe allgemein zur Frage des Konfliktes zwischen traditionellem und modernem Recht in Hawai'i Matsuda (1988).

${ }^{204}$ Die vier Verwaltungsbezirke des Staates Hawai`i sind Kaua‘i County (Kaua‘i und Ni`ihau), City and County of Honolulu ( $\mathrm{O}^{`}$ ahu und die unbesiedelten Northwestern Hawaiian Islands), Maui (Maui, Moloka'i, Lāna'i und Kaho'olawe) sowie Hawai'i County. Hinzu kommt die frühere Leprakolonie von Kalawao auf Moloka‘i, die offiziell ebenfalls als ein eigener Verwaltungsbezirk gilt (State of Hawaii 1996:6-7).
} 
Vorschriften der Treuhandbestimmungen (weitgehend) formal einzuhalten und zugleich die aus diesen Bestimmungen sich ergebenden finanziellen Belastungen für den Staatshaushalt möglichst gering zu halten. Als weitere Beispiele können hier u.a. andauernde Auseinandersetzungen um die Ausgleichszahlungen für die missbräuchliche Nutzung von Hawaiian Home Lands durch den Staat oder auch der Dauerkonflikt um die Höhe der dem Office of Hawaiian Affairs zustehenden Einkünfte aus dem Public Land Trust. Eine (endgültige) Lösung dieser Konflikte durch die Übergabe der betreffenden Landflächen an eine autonome hawaiische Instanz wird von den maßgeblichen staatlichen Stellen nicht offensiv vertreten oder gefördert, auch wenn die Verfassung durchaus die Landübertragung an das Office of Hawaiian Affairs vorsieht (Hawaii Constitution 1997:Art. XII [6]) und die unabhängige hawaiische Initiative Ka Lāhui Hawai'i in den „Treuhandgebieten“ die Grundlage der souveränen hawaiischen Nation sieht.

Die im Kontext der Landpolitik aufscheinende, bestenfalls als ambivalent zu bezeichnende Position des Staates zeigt sich ähnlich in den Bemühungen um die hawaiische Autonomie oder „Hawaiian sovereignty“. Am 26. Januar 1998 stellte Gouverneur Ben Cayetano in seiner Grundsatzrede zur Lage des Bundesstaates zu diesem Thema fest:

„Broadly based efforts are now under way within the Hawaiian community to develop a model for Hawaiian sovereignty. Today I urge the full spectrum of the Hawaiian community to join in this unique and historic undertaking“ (Cayetano 1998).

Die Unverbindlichkeit dieser Aussage ist charakteristisch für das mehrdeutige Gebaren staatlicher Stellen in Bezug auf eine wie auch immer ausgestaltete hawaiische Autonomie. Den beiden am offensichtlichsten in den Autonomieprozess der 1990er Jahre involvierten (quasi-) staatlichen Institutionen, dem Office of Hawaiian Affairs (OHA) und dem Hawaiian Sovereignty Election Council (HSEC), wurde und wird durch die zugrunde liegende Gesetzgebung eine wirkliche Unabhängigkeit versagt - nicht zuletzt auch als Folge einer derart uneindeutigen Politik des Bundesstaates lehnten viele Hawaiier diese Einrichtungen als Wortführer im Autonomiediskurs ab.

Über diese institutionalisierten Ergebnisse bundesstaatlicher Politik hinaus fehlen weitere konkrete staatliche Maßnahmen zur Förderung einer umfassenden hawaiischen Selbstverwaltung. Allerdings finden sich Aussagen und Formulierungen in offiziellen parlamentarischen Verlautbarungen und sogar in einigen Gesetzen, die eine grundsätzliche, immer aber unverbindliche Akzeptanz einer hawaiischen Autonomie bekunden. So verabschiedeten z.B. 1992 beide Häuser des Parlaments eine Resolution, mit der sich der Staat bei den Hawaiiern für erlittenes Unrecht entschuldigte, die andauernde Verletzung der 
Treuhandverpflichtungen durch den Staat sowie das hawaiische Recht auf Selbstbestimmung anerkannte und die US-Regierung aufforderte, eine unabhängige Regierung der Hawaiier anzuerkennen:

„[...] BE IT RESOLVED by the House of Representatives of the Sixteenth Legislature of the State of Hawai'i, Regular Session of 1992, the Senate concurring, that the Legislature recognizes the breaches of trust responsibility between the State of Hawai'i and the Hawaiian people ...

BE IT FURTHER RESOLVED that the Legislature hereby extends a formal and sincere apology to the Hawaiian people for the subsequent suffering and pain of all Hawaiians adversely affected by such breaches of trust responsibility; and

BE IT FURTHER RESOLVED that the citizens of the State of Hawai' $i$ recognize the inherent right of the indigenous Hawaiian people to sovereignty and self-determination; and

BE IT FURTHER RESOLVED that the citizens of the state of Hawai' $i$ call upon the President and the Congress of the United States of America to renew the recognition of and assist the re-establishment of a sovereign indigenous Hawaiian government, as requested by the Hawaiian people ... Such renewed recognition, re-establishment, and trust obligation confirmation shall be without prejudice to the indigenous Hawaiian people's inherent right to the full exercise of sovereignty, which they have never surrendered [...]“ (House Concurrent Resolution 1992).

Eine solche Resolution hat allerdings keine Gesetzeskraft, sondern stellt lediglich eine Absichtserklärung der jeweiligen parlamentarischen Kammer dar generell sind sie ein beliebtes Mittel zur Bekundung des politischen Willens, ohne unter Umständen weit reichende Gesetzesänderungen vornehmen zu müssen. ${ }^{205}$

Auch 1993, dem Jahr der formalen Entschuldigung des US-Kongresses für die amerikanische Beteiligung am Umsturz von 1893, verabschiedeten beide Häuser der Legislatur jeweils eine Resolution, die zum einen Forderungen nach öffentlicher Diskussion der hawaiischen Selbstbestimmung unterstützten, zum anderen aber auch auf die Verpflichtungen der USA gegenüber den Ha-

\footnotetext{
205 „Resolutions adopted by the Legislature do not have the force and effect of law as do bills that are enacted. Nevertheless, they are important in that they are vehicles for conveying legislative policy and intent [...] Concurrent resolutions are used to state officially the position of the legislature on an issue or to request action formally without having to mandate it by law [...] Single house resolutions are used to state the position of the Senate or House ... or to request some action, such as a study by an agency“ (State of Hawaii 1997:43-4).
} 
waiiern hinwiesen (State of Hawaii 1993 a, b). So schließt die Resolution des Senats mit den Worten: ${ }^{206}$

„[...] BE IT FURTHER RESOLVED that this body believes that the process of gaining sovereignty is a matter of federal jurisdiction and obligation premised on the status of native Hawaiians as a native American people and a trust relationship initiated by the federal government with the 1893 overthrow that remains intact and in equity and justice must be honored“ (State of Hawaii 1993a).

Ein Jahr darauf forderte eine Resolution des Senats (State of Hawaii 1994) die Erstellung einer Analyse der rechtlichen Grundlagen und der juristischen Prozesse, die eine Anerkennung der Souveränität indigener Nationen durch die Bundesregierung der USA ermöglichten. In der Resolution heißt es u.a.:

„[...] WHEREAS, the State of Hawai'i encourages a process by which indigenous Hawaiian people could achieve self-determination and selfgovernance in a manner which the Hawaiian people deem appropriate [...] WHEREAS, throughout the United States, native governments and states govern side-by-side, but too often these native governments were imposed upon the states by the federal government, causing years of mistrust and separation [...] WHEREAS, the Legislature understands that the State of Hawai'i cannot confer sovereignty, and can best support the process of self-determination if it follows the protocols and provides the documentation recognized by the international community [...] (State of Hawaii 1994).

In der resultierenden Untersuchung des Legislative Reference Bureaus, einer beratenden Einrichtung des Parlaments, wird denn auch darauf hingewiesen, dass die Rolle des Bundesstaates zwiespältig ist (Mardfin 1994:35-7): Zum einen kann er zwar keine Souveränität übertragen - dies kommt dem amerikanischen Kongress zu - und es erscheint daher unerheblich, ob der Bundesstaat den in der Resolution angesprochenen international anerkannten Verfahrensweisen zur Ermöglichung politischer Selbstbestimmung folgt. Zum anderen sind aber konkret mögliche Schritte bisher nicht in die Wege geleitet worden so gibt es z.B. bis heute kein Moratorium für den Verkauf bzw. Austausch von Landflächen aus dem Public Land Trust, wie es ebenfalls bereits 1994 von der Hawaiian Sovereignty Advisory Commission gefordert wurde, obwohl so die territoriale Basis einer hawaiischen Nation gefährdet wird (HSAC 1994:26).

${ }^{206}$ Die Resolution des Repräsentantenhauses ist im wesentlichen gleichen Inhalts (State of Hawaii 1993 b). 
Dass auf der anderen Seite die Annahme einer selbst verwalteten hawaiischen Nation durchaus auch in der regulären Gesetzgebung (und nicht nur in der Sprache der parlamentarischen Resolutionen) auftaucht, zeigen die Gesetze, mit denen die Insel Kaho'olawe aus der Verwaltung des US-Kongresses bzw. der US-Luftwaffe am 7. Mai 1994 an den Bundesstaat Hawai‘i übertragen wurde. In der entsprechenden Gesetzgebung heißt es:

„Upon its return to the State, the resources and waters of Kaho'olawe shall be held in trust as part of the public land trust; provided that the State shall transfer management and control of the island and its waters to the sovereign native Hawaiian entity upon its recognition by the United States and the State of Hawaii“ (Haw. Rev. Stat. o.J.:§ 6K-9).

Hier wird ohne weiteres von der Existenz einer hawaiischen „sovereign entity“ ausgegangen, an die sämtliche Rechte über die Insel Kaho'olawe zu übertragen wären, nachdem ihre Anerkennung durch die US- und Staatsregierung erfolgt sei. Insgesamt bleibt die Rolle des Staates im Verlauf der 1990er Jahre aber kritisch: Zwar stellte er finanzielle Mittel für die Unterrichtung der Bevölkerung über die hawaiische Autonomie zur Verfügung und mit dem Hawaiian Sovereignty Elections Council und der „Native Hawaiian Vote“ schuf er ein Forum für die hawaiische Meinungsäußerung zur „Sovereignty“. Dem gegenüber steht aber die ambivalente Haltung des Bundesstaates in Fragen der Landrechte sowie die zögerliche bis abwehrende Grundhaltung staatlicher Stellen hinsichtlich konkreter Schritte zu mehr Selbstverwaltung. Darüber hinaus hat die Beteiligung des Staates am Autonomieprozess zu einer Aufspaltung der hawaiischen Unterstützer dieses Ziels geführt, die die Effektivität der Bewegung nicht unerheblich einschränken könnte. 



\section{Politisierung des Kulturellen - Kulturalisierung des Politischen}

Schon seit den 1960er Jahren war unter der hawaiischen Bevölkerung eine wachsende Rückbesinnung auf die seit fast 200 Jahren ins Abseits gedrängten hawaiischen Traditionen und kulturellen Praktiken - Sprache, Tanz und Musik, traditionelle Techniken und Kunstformen - zu beobachten. Damit einher gingen Beschäftigung und Auseinandersetzung mit der eigenen Geschichte, deren kritische Neubewertung zunehmend auch in Differenz zur „offiziellen“ Historiographie Hawai' is gesehen wurde.

In einer Zeit, die geprägt war von der US-amerikanischen Bürgerrechtsbewegung der 1950er und 1960er Jahre, der Studentenbewegung und nicht zuletzt den radikalen Forderungen der „Black Power“-Partei der Afroamerikaner (sowie der „Red Power“-Fraktion unter den amerikanischen Indianern), blieb es nur eine Frage der Zeit, bis sich zu den kulturell ausgerichteten Initiativen der so genannten „Hawaiian Renaissance“ auch Gruppierungen mit eindeutig politischen Forderungen gesellten. Der hawaiische Autor John Dominis Holt schrieb in der Einleitung zu seinem erstmals 1964 erschienenen Text „On being Hawaiian“:

„We have been wronged cruelly and now we ask that we be given back what is ours: our self-respect, the right to be ourselves as Polynesians of today, as our black brothers and sisters have fought so hard to regain a respect for blackness, for Africanness in the turbulence of recent years. We assume the right to win back our lands, those belonging to us, as ,reservations“ ,belong' to our Indian brothers and sisters“ (Holt 1995:9).

Nachdem die USA Hawai' $i$ als neuen Bundesstaat integriert hatten, schien eine eigenständige „hawaiische“ Politik zunächst allerdings nicht weiter auf der 
Tagesordnung zu stehen. Die Idee des „New Hawai ‘ $i$ “ (Kent 1983:3), eines von ethnischer Harmonie und politischem Konsens geprägten Gemeinwesens, dessen Wirtschaftswachstum allen Bevölkerungsgruppen den sozialen Aufstieg ermöglichen sollte, versprach erfolgreich zu werden. Allerdings blieb gerade die hawaiische Minderheit auffallend hinter den ökonomischen und sozialen Entwicklungen der 1960er und 70er Jahre zurück.

Eine Grundlage des wirtschaftlichen Aufschwungs war das rapide Wachstum der Tourismusindustrie, die um potentielle Besucher des Inselstaates nicht nur mit der grandiosen Landschaft und dem milden Klima warb, sondern auch mit jenem Mythos der Südsee, der sich vor allem aus den Vorstellungen einer lasziven Kultur voll exotischer Erotik speiste. In den von dieser „Aloha Industrie“ (Trask u. Trask 1992:50) produzierten Bildern war die Gesamtheit einer traditionellen hawaiischen Kultur auf wenige, erfolgreich zu vermarktende Versatzstücke reduziert - eine Darstellungsweise, die eine wachsende Zahl von Hawaiiern als verzerrend und herabsetzend empfand (siehe z.B. Trask u. Trask 1992).

Die politische Mobilisierung eines kleinen Teils der hawaiischen Bevölkerung ging zunächst auf eine unmittelbare Erfahrung sozialer Benachteiligung - letztlich aufgrund der Abstammung - zurück. Hinzu kam aber schon bald ein anwachsendes - von der gemeinsamen Abstammung gespeistes und kulturell bekräftigtes - „Wir-Gefühl“, wie es auch John Dominis Holt in seinem eingangs zitierten Text anspricht. Angesichts der sehr heterogenen Abstammungsgeschichte der Hawaiier im 19. und 20. Jahrhundert sowie den daraus resultierenden, zumindest potentiell vielfältigen subjektiven Identifikationsmöglichkeiten, stellt sich die Frage nach den verbindenden Faktoren einer aktuellen - und auch politisch nutzbaren - „hawaiischen“ Identität. In diesem Kapitel möchte ich drei „Kernbestandteile“ hawaiischer kultureller Praxis ausführlicher betrachten, denen eine besondere Bedeutung im Zusammenhang mit der (Re-) Ethnisierungsphase der zweiten Hälfte des 20. Jahrhunderts zukommt. Gemeinsam ist diesen Kernbeständen oder „summarizing symbols“ ${ }^{207}$ sowohl ihre feste Verankerung in der Vergangenheit als auch eine starke Gegenwartsbezogenheit. Die hier betrachteten „Kernsymbole“ sind nicht nur wichtige Ausgangs- bzw. Angelpunkte der heutigen Identitätsdiskussion, sie traten darüber hinaus schon in der kulturellen Bewegung der „Hawaiian Renaissance“ der 1960er und 1970er Jahre in Erscheinung.

- Hierher gehört die weitestgehend außer Gebrauch geratene hawaiische Sprache ('Ōlelo Hawai ‘i). Sie gewann seit den 1970er Jahren immens

\footnotetext{
${ }^{207}$ Zum Konzept derartiger „summarizing symbols“ siehe Ortner 1973:1344. Die Nutzung von Traditionen zur Festigung einer politisch nutzbaren ethnischen Identität ist für Hawai‘i u.a. von Linnekin (1983, 1990b, 1992) und J. Friedman (1992) untersucht worden.
} 
an Bedeutung - zwar bleibt die Zahl der Muttersprachler nach wie vor sehr klein, aber durch eine Ausweitung des Sprachunterrichts und den Gebrauch der Sprache in Musik, Literatur und nicht zuletzt den politischen Parolen der Autonomiebewegung wuchs der hawaiischen Sprache eine erhebliche Bedeutung als Identifikationsfaktor zu.

- Ebenso erlernten und praktizierten vor allem Hawaiierinnen den Hula, einen von der Tourismusindustrie zum Inbegriff hawaiischer Kultur stilisierten Tanz, der im alten Hawai'i eine Schnittstelle zwischen religiösen und weltlichen Lebensbereichen darstellte und durch den eine wachsende Zahl von Hawaiiern Zugang zu den spirituellen Werten ihrer Kultur suchte und sucht.

- Schließlich projiziert ein den traditionellen Baumustern nachempfundenes hawaiisches Doppelrumpfboot, die Hōkūle‘a, seit seiner Kiellegung 1975 eine heroische Vergangenheit der großen Entdeckungsfahrten in die nüchternere Prosa der hawaiischen Gegenwart. ${ }^{208}$

Die genannten Beispiele stehen stellvertretend für das weite Feld aktueller hawaiischer kultureller Praktiken, die in ihrer vordergründigen Widersprüchlichkeit die historischen, demographischen und sozialen Umbrüche der vergangenen 200 Jahre reflektieren. ${ }^{209}$ Kulturelle Revitalisierung einerseits und politischer Protest andererseits bieten jeweils einen gleichermaßen eingeschränkten Blick auf die vielfältigen Entwicklungen und Verflechtungen, die hawaiisches Leben im ausgehenden 20. Jahrhundert ausmachten. Sprache, Tanz, Seefahrt und das „hawaiische“ Land stellen gewissermaßen ,umkämpfte Territorien’ dar - grundsätzliche Konflikte werden in ihnen nicht nur reflektiert, sie können auch selbst potenzielle Auslöser ethnisch begründeter Konflikte sein. Es handelt sich bei ihnen um „spezifische Ressourcen“, deren „Wert“ an die Existenz der sie tragenden Gruppe gebunden ist; von außen wirkende Einflüsse können diesen Wert gefährden (Esser 1996:68). Alle genannten Bereiche (Land, Sprache, Seefahrt, Tanz) sind im heutigen Hawai`i einer solchen Gefährdung durch Institutionen und Individuen der Mehrheitsgesellschaft ausgesetzt; am offensichtlichsten ist dies bei Fragen der Landrechte und Landnutzung der Fall, doch auch die weiteren Identitätssymbole waren und sind einer Vereinahmung durch nicht-hawaiische Interessen ausgesetzt. Ein zentraler Aspekt der in diesen Jahrzehnten zu registrierenden Ethnisierung und Mobilisierung eines immer größeren und heterogeneren Teils der hawaiischen Minderheit war denn auch der

\footnotetext{
${ }^{208}$ Weitere bedeutende Symbole des „Hawaiischen“ im heutigen Hawai‘i sind die Insel Kaho'olawe, Sinnbild der 'Āina, des hawaiischen Landes, und die als Sinnbild für den traditionellen hawaiischen Lebensstil stehende Taropflanze; siehe Linnekin (1983:282-3).

${ }^{209}$ Einen Darstellungsversuch der aktuellen Vielschichtigkeit hawaiischer Kultur in der Gegenwart unternimmt Hartwell (1996).
} 
Diskurs über die Definitionsmacht hinsichtlich dessen, was „Hawaiische Kultur“ ausmacht - die hawaiische Gegenwartskultur und ihre Verwurzelung in Traditionen und Geschichte(n) bildeten also nicht nur den Hintergrund für politische Entwicklungen, sondern sie standen auch immer wieder im Zentrum politischer Strategien und Forderungen.

\section{1 'Ōlelo Hawai' $i$, die hawaiische Sprache}

Dem heutigen Besucher Hawai'is begegnet die hawaiische Sprache in den Ortsbezeichnungen und Straßennamen, in zahlreichen Wörtern und Wendungen, die Eingang in die englische Umgangssprache auf den Inseln gefunden haben, und nicht zuletzt in den Texten der allgegenwärtigen hawaiischen Unterhaltungsmusik. Diese Präsenz des Hawaiischen bzw. einiger Fragmente der Inselsprache mag den Touristen zunächst darüber hinwegtäuschen, dass 'Ōlelo Hawai $i$, die Sprache der Hawaiier, für die alltägliche Kommunikation der Menschen seit langem kaum noch Bedeutung hat. Verkehrssprache ist Hawaiisch gegenwärtig nur noch auf der kleinen, vom Betrieb der anderen Inseln abgeschotteten Privatinsel Ni'ihau, und der US-Zensus von 1990 verzeichnete lediglich 8.872 Personen älter als 5 Jahre, für die nach eigenen Angaben das Hawaiische die Umgangssprache in der Familie ist (OHA 1996:220). ${ }^{210}$

Ohne dass sich dies zunächst auf die Gesamtzahl der Sprecher auswirkte, hat 'Ōlelo Hawai ' $i$ durch die kulturellen Revitalisierungsbestrebungen seit den 1970er Jahren einen merklichen Bedeutungszuwachs erfahren und ist heute trotz des weiterhin geringen Grades tatsächlicher Sprachbeherrschung in der Bevölkerung zweifelsfrei eines der wichtigsten Identifikationssymbole der Hawaiier. Die eigene bzw. erneut angeeignete Sprache ist nicht nur ein zentraler Aspekt des ethnischen Selbstverständnisses, sie bündelt in ihrer Geschichte auch die kollektiven Erinnerungen an den Verlust politischer Macht und kultureller Selbstbestimmung. Die Entwicklung der hawaiischen Sprache zur Minderheitensprache gibt somit zugleich Zeugnis von den mannigfachen Verflechtungen unterschiedlichster Interessen sowohl hawaiischer als auch nichthawaiischer Protagonisten, die in ihrer Gesamtheit den Niedergang der Sprache beförderten.

Kolonialisierung der Sprache - Übergänge vom Hawaiischen zum Englischen In der Zeit zwischen 1820, dem Beginn der christlichen Missionierung durch amerikanische Missionare, und 1898, der Annexion Hawai`is durch die USA,

\footnotetext{
${ }^{210}$ Der Sprachhistoriker Schütz (1994:380, Anm. 41) fragt sich angesichts dieser doch recht hohen Zahl, inwieweit diese Befragten tatsächlich „Native Speakers“ repräsentierten, da es sich um Eigenangaben der Befragten handelte. In seiner Arbeit verwendet er denn auch eine wesentlich niedrigere Schätzung von ca. 2.000 Muttersprachlern (1994:365).
} 
verlor 'Ōlelo Hawai 'i kontinuierlich an Bedeutung. Insbesondere in der zweiten Hälfte des 19. Jahrhunderts lief das Zusammenwirken verschiedener, ursprünglich nicht aufeinander bezogener Gesetze, Bestimmungen und Regelungen mehr oder weniger zielgerichtet auf die Übernahme US-amerikanischer Gesellschaftsvorstellungen durch die hawaiische Bevölkerung hinaus, was die Vorherrschaft des Englischen einschloss. Es kann zwar, wie Day (1985:168) anmerkte, keine dezidierte Politik zur Abschaffung des Hawaiischen festgestellt werden:

„There is no one turning point, no one piece of legislation, no royal decree that we can point to and say with confidence that it marked the time when Hawaiian lost to English. There was no policy which had as its stated goal the death of Hawaiian.“

Allerdings blieben Bemühungen staatlicherseits zur Abschwächung oder gar Umkehrung des Trends ebenso aus - unter anderem wohl auch, weil die Sprachverschiebung den Bestrebungen politisch einflussreicher Haole und damit einer immer engeren Anlehnung Hawai'is an die USA sehr entgegenkam (cf. Schütz 1994:350-1). Wenn also im Kontext der politischen Bewegung die Haole für das Verschwinden des Hawaiischen verantwortlich gemacht werden, so geschieht dies angesichts des Zusammenwirkens von politischen und gesellschaftlichen Faktoren im ausgehenden 19. Jahrhundert mit einigem Recht - so wie der Dozent für Hawaiian Studies an der University of Hawai'i in Hilo, Larry Kimura, konstatierte: „Hawaiians didn`t lose their language. It was taken away from them deliberately“. ${ }^{211}$

Allerdings lassen sich, wie so häufig in der neueren hawaiischen Geschichte, die beteiligten Gegenspieler in diesem schleichenden Prozess des Wechsels vom Hawaiischen zum Englischen nicht klar voneinander trennen. Waren es doch gerade die neuenglischen Missionare, viel zitierte Agenten des kulturellen Wandels und verantwortlich für bedeutende Kulturbrüche im 19. Jahrhundert, die wichtige Voraussetzungen für den dauerhaften Erhalt des Hawaiischen in der sich rapide wandelnden Inselgesellschaft schufen. Mit der Entwicklung eines Alphabets für 'Ōlelo Hawai ' $i$ legten sie die Grundlagen für die Fixierung der bis zu diesem Zeitpunkt schriftlosen Sprache. Bereits ab 1822 produzierten die Druckerpressen der Mission religiöses Schriftgut in hawaiischer Sprache und schon bald war ein Großteil der hawaiischen Bevölkerung des Lesens und Schreibens in ihrer eigenen Sprache mächtig. Ab 1852 richtete die Regierung des Königreichs dann englischsprachige Schulen ein - zunächst für die Kinder der Ausländer bestimmt, besuchten bald auch immer mehr ha-

${ }^{211}$ Kimura in einem Artikel der Zeitschrift „Honolulu“ 1987, zit. nach Schütz (1994:353). 
waiische Kinder diese fremdsprachigen Schulen. ${ }^{212}$ War der Besuch dieser Schulen durch Hawaiier noch freiwillig, so bedeuteten Regelungen und Bestimmungen des ausgehenden 19. Jahrhunderts das faktische Ende des Hawaiischen als Unterrichtssprache: An einer der bekanntesten hawaiischen Lehranstalten, den 1887 gegründeten und so gut wie ausschließlich von hawaiischen Schülern besuchten Kamehameha-Schulen, untersagte die Schulleitung (ausschließlich Haole) von Anfang an jeglichen Gebrauch der 'Ōlelo Hawai'i. Drei Jahre nach dem Sturz der Monarchie erlangten dann auch im Bereich der staatlichen Schulen Gesetze Geltung, die das Hawaiische als Unterrichtssprache abschafften und verboten. Als in den Jahren 1921-22 das Unterrichtsfach „Hawaiisch" an den staatlichen Schulen und der Universität von Hawai'i eingeführt wurde, dokumentierte dies auch den tief greifenden Wandel in der Sprachgeschichte: 'Ōlelo Hawai ' $i$ war nun mitnichten Unterrichtssprache, sondern lediglich Gegenstand eines nicht mehr unbedingt ernst genommenen Faches (Schütz 1994:357-61). ${ }^{213}$

Ebenso reflektieren Aufschwung und Niedergang der hawaiischsprachigen Presse die Veränderungen in Gebrauch und Verbreitung des Hawaiischen. Ausgehend vom ersten Erscheinen der Missionszeitung „Ka Lama“ (Die Fackel) im Jahre 1834 entwickelte sich bis zur Mitte des Jahrhunderts eine bedeutende und vielfältige hawaiische Presselandschaft, die im Wesentlichen bis zur Annexion des Archipels Bestand hatte:

„Without a doubt, the Hawaiian language newspapers had the largest readership of any papers in the islands ... A highly literate Hawaiian population read the output of Native Hawaiians and their Caucasian allies who from the 1860s to 1900 produced almost 70 newspapers...“(Chapin 1984:67).

Nach der Annexion nahm die Bedeutung der hawaiischen Presse dann sehr schnell ab - von nur noch 12 Titeln im Jahre 1910 (davon waren drei religiös gebunden) ging die Zahl bis 1930 auf drei Zeitungen zurück, von denen eine einen religiösen Hintergrund hatte; weniger als 20 Jahre darauf zählte man 1948 gerade noch ein religiöses Periodikum in hawaiischer Sprache (Schütz 1994:362-3).

\footnotetext{
${ }^{212}$ Einen kurzen Überblick über die Verschiebungen zugunsten des Englischen im Schulwesen bietet Schütz (1994:351-4).

${ }^{213}$ Bereits seit 1870 war Hawaiisch auch nicht mehr die Sprache amtlicher Regierungsdokumente, deren Originale von nun an in Englisch vorlagen, und der Organic Act von 1900, die gesetzliche Grundlage der Eingliederung des Territoriums in die USA, schaffte Hawaiisch auch als Amtssprache des Territoriums ab (Day 1985:167, 169).
} 
Neue Anfänge

Das Aufkommen der „Hawaiian Renaissance“ in den 1970er Jahren stellte einen entscheidenden Wendepunkt für 'Ōlelo Hawai ' $i$ dar: im Zuge der Bemühungen um kulturelle Revitalisierung setzten sich immer mehr Hawaiier mit der „eigenen“ Sprache auseinander, die für viele von ihnen, zumindest hinsichtlich der eigenen Sprachkenntnisse, zu einer „Fremd“-Sprache geworden war. Ein Indiz für das wachsende Interesse waren die stark ansteigenden Studentenzahlen in den Hawaiisch-Kursen der University of Hawai'i in Mānoa: Dort waren im Studienjahr 1961/62 lediglich 27 Studenten für einen solchen Kurs eingeschrieben, im Studienjahr 1991/92 besuchten hingegen bereits 1.277 Studenten den Sprachunterricht am größten Standort der Universität (Schütz 1994:362). ${ }^{214}$

Von den umfangreichen Verfassungsänderungen des Jahres 1978 beschäftigten sich zwei Artikel mit der hawaiischen Sprache. So erhielt 'Ōlelo Hawai ‘ $i$ den Rang einer offiziellen Sprache des Bundesstaates:

„English and Hawaiian shall be the official languages of Hawaii, except that Hawaiian shall be required for public acts and transactions only as provided by law“ (Hawaii Constitution 1997:Art. XV, Section 4).

Hierbei handelte es sich allerdings um Symbolpolitik des Staates, da ein fast zeitgleich verabschiedetes Gesetz (Hawaii Session Laws 1979:189, Act 97) den Gebrauch des Hawaiischen in der öffentlichen Verwaltung ausdrücklich nicht vorsah.

Hinsichtlich der Förderung des hawaiischen Sprachunterrichts, auch dies eine 1978 in die Verfassung aufgenommene Verpflichtung (Hawaii Constitution 1997:Art. X, Section 4), blieb der Bundesstaat lange Zeit untätig. Mit der Zulassung des Hawaiischen als Unterrichtssprache an staatlichen Schulen im Jahre 1986 - 90 Jahre nach den ersten staatlichen Verboten des Gebrauchs an Schulen - reagierte das Department of Education nicht zuletzt auf private Initiativen zur Förderung des Spracherwerbs. Bereits 1984 gründeten Hawaiier die ersten privaten Vorschulen nach dem Vorbild der neuseeländischen Ngā Kōhanga Reo ${ }^{215}$, in denen das Hawaiische die ausschließlich verwendete Sprache zwischen Lehrern und Schülern war (Pūnana Leo 1998;

\footnotetext{
${ }^{214}$ Zum Vergleich noch die entsprechenden Zahlen der University of Hawai'i in Hilo auf der Insel Hawai` 'i: hier waren 1995, bei einer erheblich geringeren Gesamtzahl aller Studenten, 600 Studierende für das Hawaiian Studies Programm der Universität eingeschrieben („No cuts at UH Hilo Hawaiian studies“; H S-B 2.5.1995:A-4).

${ }^{215} \mathrm{Zu}$ den neuseeländischen Māori-Schulprogrammen Kōhanga Reo und Kura Kaupapa Māori siehe Durie (1998:63-66); anzumerken bleibt hier auch, dass es im US-amerikanischen Schulwesen leichter ist als zum Beispiel in der Bundesrepublik, private Unterrichtsinitiativen in konkrete privat organisierte Lehranstalten umzusetzen.
} 
Schütz 1994:365-7; Hartwell 1996:69-71). Diese hawaiischen Pūnana Leo (,Sprachnester“) förderten das Erlernen von 'Ōlelo Hawai ' $i$ durch das „Eintauchen“ der Kinder in die ihnen noch fremde Sprache („language immersion“). Ab 1987 ergänzten dann auch staatliche Schulen das Angebot der privat organisierten Pūnana Leo: Mit der Kula Kaiapuni Hawai'i bot das Department of Education an wenigen Standorten ein „Language Immersion Program“ an, das sich zunächst auf die Grundschule beschränkte, ab 1991 aber auch die Highschool einbezog (Pūnana Leo 1998; Schütz 1994:367-9). 1995 unterrichteten schon 82 Lehrer 1.000 Schüler an 13 Standorten, teilweise bis zur neunten Klasse der Highschool (OHA 1996:222), und 1998 gab es die ersten Schulabgänger, deren Schulausbildung ausschließlich in hawaiischer Sprache erfolgte. Es ist bisher nicht absehbar, ob die nunmehr seit fast 35 Jahren andauernden Bemühungen um die Revitalisierung der hawaiischen Sprache Erfolg haben werden: außerhalb des Bildungsbereichs und des Familienkreises ist 'Ōlelo Hawai' $i$ als Arbeits- oder Umgangssprache weiterhin so gut wie nicht präsent auch wenn das Lexicon Committee (Kōmike Hua'ōlelo) an der University of Hawai'i in Hilo seit 1987 den Wortschatz laufend an den aktuellen Sprachgebrauch und die Anforderungen von Technologie und Wissenschaft anzupassen sucht (siehe z.B. Hale Kuamo‘o 1996).

Ein Bereich des kulturellen Lebens, in dem die hawaiische Sprache durchgängig präsent blieb, ist die populäre Musik. Versatzstücke der Inselsprache gehörten neben spezifischer Rhythmik und Instrumentierung zur „typischen Hawaiimusik“, wie sie u.a. die Tourismusindustrie in den USA und anderswo propagierte. Im Zuge der „Hawaiian Renaissance“ und der aufkeimenden politischen Bewegung erhielt die moderne hawaiischsprachige Musik einen Bedeutungszuwachs, der mit seiner dezidierten Abgrenzung gegen die dominierende angloamerikanische Musikkultur an die Rolle der Musik im ausgehenden 19. Jahrhundert erinnert. In der Musik äußerte sich nicht nur sozialer und politischer Protest, sie bot darüber hinaus ein erhebliches Identifikationspotential, das hawaiischen Hörern die Abgrenzung gegen Nicht-Hawaiier ermöglichte, ohne diese völlig auszugrenzen. ${ }^{216}$ Die Alben eines Keali‘i Reichel, des 1997 verstorbenen Israel Kamakawiwo'ole oder auch der Sängerin Robi Kahakalau sind allesamt kommerziell sehr erfolgreiche Produktionen, in denen aber, neben den üblichen Themen der Popmusik, das „hawaiische“ Hawai'i und das „Hawaiisch-Sein“ im Vordergrund stehen.

Die Bedeutung der 'Ólelo Hawai' $i$ als ein potentiell einigendes Band der „hawaiischen Gemeinschaft“ wurde auch von der Autonomiebewegung früh als „ethnisches Kennzeichen“ genutzt. Nicht nur die Namen der politischen Gruppierungen sind seit den 1980er Jahren fast ausschließlich dem Hawaiischen verpflichtet, in Aufrufen und Texten der Bewegung werden be-

${ }^{216}$ Zur Rolle von Musik und Musikern in der hawaiischen Protestbewegung siehe Lewis 1992. 
stimmte Schlüsselwörter im hawaiischen Original verwendet und auch längere Passagen konfrontieren die Leser mit „ihrer“ Sprache - allerdings wird dann dem Text die englische Übersetzung an die Seite gestellt. Haunani-Kay Trask fasst ihr Verständnis von der Bedeutung der hawaiischen Sprache für die politische Auseinandersetzung wie folgt zusammen:

„Language is a critical de-colonizer. Thinking in Hawaiian means, at the least, thinking in the language of the land and the culture. Even without political analysis, Hawaiians who are familiar with their Native tongue are already thinking about things Hawaiian. They are more receptive to a Native nationalist argument because the language explains our commonalities as a people, that is, as a nation" (H.-K. Trask 1993:274).

Für sie steht also der Erwerb von Sprachkenntnissen in einem direkten $\mathrm{Zu}$ sammenhang mit der politischen Mobilisierung, Kenntnis des Hawaiischen erhöht ihrer Argumentation zufolge die Disposition der Menschen, sich den Vorstellungen der Autonomiebewegung anzuschließen. Nicht alle Anhänger der hawaiischen Autonomiebewegung würden so weit gehen, der hawaiischen Sprache, wie Trask hier, die Funktion eines Katalysators für den politischen Kampf zuzuschreiben. Jedoch steht m.E. auch die fragmentarische Verwendung der als „eigen“ empfundenen Sprache, die bei anhaltend niedriger Zahl aktiver Sprecher häufig eine „Fremd“-Sprache bleibt, für die Zugehörigkeit zu einer vorgestellten hawaiischen Wertegemeinschaft. Zudem stellt selbst ihr eingeschränkter Gebrauch im Kontext der politischen Bewegung mit ihren z.T. harten Auseinandersetzungen eine gewisse verbindende Nähe unter den hawaiischen Teilnehmern am Diskurs über „Sovereignty“ her.

Aber auch auf der Ebene der politischen Praxis spielt 'Ōlelo Hawai ' $i$ spätestens seit den 1990er Jahren eine nicht zu unterschätzende Rolle - kann das Thema der staatlichen Sprach- und Unterrichtspolitik doch eine große Zahl von Hawaiiern mobilisieren. So protestierten im Jahr 1995 Eltern gegen die zu geringe Kapazität des staatlichen „Hawaiian Language Immersion“-Programms in Kāne'ohe, O'ahu. Das Vorhaben, die vorhandenen Plätze durch eine Lotterie zu vergeben, führte zu erbittertem Widerstand von Eltern, die Unterstützung beim Office of Hawaiian Affairs fanden. ${ }^{217}$

Noch weitaus heftigeren Widerstand riefen Kürzungsabsichten im Bereich der universitären Sprachausbildung hervor, als ebenfalls 1995 an den Standorten Mānoa und Hilo insgesamt 19 Hawaiischkurse, davon allein 14 in Mānoa, gestrichen werden sollten. Nachdem über 300 Demonstranten, Studen-

${ }^{217}$ „In any language, parents angry over school lottery“, Mary Adamski (H S-B 8.4.1995:A-3); „OHA plans Hawaiian immersion suit“, Mark Matsunaga (HA 27.4.1995:A-5). 
ten wie auch Vertreter der Autonomiebewegung, das Verwaltungsgebäude der Universität in Mānoa besetzt hielten, nahm die Universität die Kürzungen zurück. In Hilo besetzten ca. 150 hawaiische Studenten das Büro des Kanzlers und forderten auch hier erfolgreich die Rücknahme der Kürzungen. ${ }^{218}$ Der Protestzug in Hilo, an dem ich am 1. Mai 1995 teilnehmen konnte, verband mit dem aktuellen Protest auch eine Demonstration einzelner Aspekte „erneuerter“ hawaiischer Tradition: Viele Studenten trugen in Anlehnung an traditionelle hawaiische Kleidung ein über der Schulter geknotetes Baumwolltuch, als sich der Zug unter Rufen von „,E kiu- $\bar{e}$ kümaumau!“ („Steht immer zusammen!“ [U.M.]) über den Campus bewegte. An der Spitze des Zuges ging die mit Ketten aus maile-Ranken ${ }^{219}$ besonders geschmückte Sprecherin, begleitet von Rufern (Chanters) und Läufern, die den Weg mit Kokosbündeln fegten. ${ }^{220}$ Der Sprecherin folgte schließlich ein Fahnenträger mit der Flagge des ehemaligen Königreichs Hawai' $i$, die heute auch die Flagge des Bundesstaates ist - allerdings wird sie im Kontext hawaiischer Proteste gemeinhin als Zeichen der hawaiischen Nation verstanden und verwendet. Politische Forderungen, Folklore und das Bemühen um den Erhalt der hawaiischen Sprache verbanden sich hier zu einer Manifestation von „Hawaiisch-Sein“, die das konkrete Anliegen der Studenten untermauern sollte. Auch die Schlagzeile einer der großen Tageszeitungen aus Honolulu, des Honolulu Advertisers, verwies auf die Verknüpfung zwischen Unterrichtspolitik und der an den Rand gedrängten indigenen Kultur des Archipels. Sie zitierte den Dozenten Kaleikoa Kaeo mit den Worten: „No Hawaiian language student should be turned away. This is Hawaii, for God's sake.“221 Ein Leserbrief in der gleichen Tageszeitung nahm dieses Thema auf und hob zugleich den politischen Kontext, den Kampf um die kulturelle Definitionsmacht im Staat Hawai' $i$, deutlich hervor:

„The recent 'Olelo Hawaii protest is evidence of the trend toward Hawaiian unification. 'Olelo Hawaii is one means by which Hawaiians are attempting to perpetuate their culture and regain their identity ... Keep in mind that the kanaka maoli will no longer take a back seat to anyone in their own aina. I am glad the protest occurred because there should be a continuing effort to support the restoration and perpetuation of the native Hawaiian culture“. ${ }^{222}$

\footnotetext{
218 „Students march on offices“, Eric Lee (Ke Kalahea Extra 2. 5.1995).

219 "Die Blätter des rankenden Maile-Strauchs (Alyxia olivaeformis) kennzeichnen sowohl im traditionellen als auch im zeitgenössischen Kontext besonders herausgestellte bzw. geehrte Personen.

${ }^{220}$ Die Bedeutung sowie die kulturelle Herleitung des „Fegens“ sind mir nicht bekannt.

221 „UH relents, rescinds Hawaiian cuts“, Mark Matsunaga (HA 29.4.95:A-1, A-2).

222 „UH protest a sign of Hawaiian unification“, Denise K. Canon (HA 6.5.1995:A-6).
} 
Um die sehr dichte Verzahnung von hawaiischer Sprache, Geschichte, kollektivem Gedächtnis und Kultur- sowie Identitätspolitik zu verdeutlichen, möchte ich abschließend Lisa Kalaheole Halls politische Analyse der Aneignungsweisen „hawaiischer“ Identität durch Nicht-Hawaiier zitieren. In einer dezidiert hawaiischen Sichtweise geht sie auch auf die Bewertung der Sprache ein und stellt diese in den weiteren Kontext problematischer hawaiischer Gegenwartsidentitäten:

„,... some [Nicht-Hawaiier; U.M.] have gone so far as to claim that they are more Hawaiian than actual Hawaiians because they have greater cultural or language knowledge. A bitter irony exists in the psychological ease with which non-Hawaiians study Hawaiian language and culture. Often Hawaiians are misread as uninterested or resistant to learning when they do not fully participate in Hawaiian education, but the reality is that all contemporary Hawaiians come from a past where our parents', grandparents', or great-grandparents' use of Hawaiian language and culture was forbidden, legislated against, brutally punished, or a combination of these. Non-Hawaiians without this history do not carry a history of internalized fear, shame, and anger to impede their study, nor do they feel guilt about this history. The anger and shame that Hawaiians cut off from their cultural history feel toward nonHawaiians' attempted cultural one-upmanship is very real, though rarely articulated“ (Hall 2005:410-1).

So steht 'Ōlelo Hawai' $i$, die noch vor einem halben Jahrhundert außerhalb der Bereiche akademischer Forschung und musikalischer Traditionspflege fast bedeutungslose Sprache, mittlerweile im Schnittpunkt vielerlei Interessen. Im Kontext der politischen Bewegung kommen hierbei positive wie negative Aspekte zum Tragen. Exemplarisch bündeln sich im Symbol der 'Ōlelo Hawai ' $i$ Niedergang und Beharrungsvermögen, Repression, Widerstand und Neubeginn - all dies schwingt in der politisch motivierten Verwendung des Hawaiischen mit und macht diese eigene „fremde“ Sprache zu einem der wirkungsvollsten ethnischen Kennzeichen im Repertoire der hawaiischen Autonomiebewegung.

\subsection{Hula: Kommerz, Spiritualität und Widerstand}

Kaum etwas wird bis heute so sehr mit dem Bild eines „Südseeparadieses Hawai ${ }^{\prime} i “$ verbunden wie das Klischee exotisch gewandeter Hawaiierinnen, die zu melodischen Klängen die fließenden Bewegungen eines Tanzes, des Hula, vollführen. Das hawaiische Wort Hula ist der Oberbegriff für mehrere, je nach Instrumentierung, Gesang, Aufführungskontext usw. unterschiedene Tänze, 
denen jedoch das gleiche Regelwerk der Verbindung von Text, Musik und Bewegung zugrunde liegt. In organisierten Darbietungen ist Hula in seinen unterschiedlichsten und auch merkwürdigsten Formen heute für den Hawai'iTouristen allgegenwärtig, aber auch jenseits dieser touristischen Aufführungen hat Hula für das hawaiische Kulturleben einen nicht zu unterschätzenden Stellenwert. Hula ist nicht nur touristisches Klischee, er ist in bestem Sinne „ortsgebunden“: In den Vorbereitungen der Aufführung, in den Bewegungen des Tanzes und in der ihn begleitenden Musik fließen überlieferte Kenntnisse hawaiischer Kultur und Geschichte sowie das Wissen um die Natur der Inseln zusammen, so dass seine Bedeutung für die kulturelle Revitalisierung seit den 1970er Jahren kaum verwundern kann.

Neben seiner Bedeutung für die politische Bewegung gingen dem Hula mehrere Bedeutungszuschreibungen voraus, die im Laufe des 19. Jahrhunderts je nach zeitlichem, ethnischem oder politischem Kontext einem Wandel unterlagen. So war „Hula“ bis zur Aufgabe der offiziellen hawaiischen Religion 1819 (und im privaten Umfeld wohl auch darüber hinaus) der variantenreiche, zu Ehren der Götter und des Adels aufgeführte und auf sakrale Wurzeln zurückzuführende Tanz, den Hi‘iaka, die Schwester der Vulkangöttin Pele, den Menschen gebracht haben soll und der unter dem Schutz von Laka, der Göttin der Fruchtbarkeit, stand (Beckwith 1970:180-6). Im Hula kahiko, dem an die alten Formen angelehnten Hula, leben diese Vorstellungen bis heute fort. Protestantischen Beobachtern aus der ersten Hälfte des 19. Jahrhunderts galt „Hula“ hingegen als der Tanz gewordene Ausdruck jener moralischen Verworfenheit, die sie unter den Hawaiiern zu sehen glaubten und deren Bekämpfung die amerikanischen Missionare auf ihre Fahnen geschrieben hatten (Buck 1993:154-5). In der Gegenwart spielen viele touristische Tanzdarbietungen, in denen häufig Elemente der schnelleren und freizügigeren tahitischen Tanzformen den Hula überlagern, mit diesem erotischen Klischee missionarischer Prüderie von ehedem. Und in den letzten Jahrzehnten des 19. Jahrhunderts, unter dem Eindruck des immer weiter wachsenden Einflusses der Haole sowie der drohenden Annexion durch die USA, gewann „Hula“ unter den Hawaiiern schließlich Bedeutung als getanzter Ausdruck von Eigenständigkeit und politischer Souveränität, eine Bedeutung, die dem hawaiischen Tanz im Kontext und unter den Bedingungen der Autonomiebewegung am Ende des 20. Jahrhunderts in ähnlicher Weise zukam. ${ }^{223}$

Wie die hawaiische Sprache war auch der Hula zu verschiedenen Zeiten immer wieder Restriktionen unterworfen (sowohl seitens missionarischer Missbilligung als auch durch staatliche Gesetze), die jedoch ein Abreißen der

${ }^{223}$ Zur Geschichte des Hula siehe Barrère 1980, Stagner 1985:9 ff. 
Überlieferungsstränge letztlich nicht bewirken konnten. ${ }^{224}$ Auch als Geste des Widerstands gegen die zunehmende Vereinnahmung durch euro-amerikanische Wertevorstellungen setzte sich der Hula im 19. Jahrhundert immer wieder durch: Heimliche Aufführungen der maka 'äinana, die sich so gegen den Eifer der Missionare auflehnten, oder König Kamehamehas III. (1813-1854) Aufhebung von Restriktionen im Jahre 1833, wodurch er ein deutliches Zeichen der Ablehnung gegen die Missionarsherrschaft setzte, sind Beispiele einer Praxis, die unter den Monarchen Kalākaua, der den Hula explizit förderte, und Lili ‘uokalani weiterhin an Boden gewann.

Nach der erfolgten Annexion durch die USA bemächtigten sich immer weitere Kreise der in Hawai'i ansässigen Haole des Hulas. Fragen der christlichen Moral traten in den Hintergrund und in der neuen, bis auf weiteres gesichert erscheinenden politischen Situation meinte man das Widerstandspotential eines hawaiischen Tanzes vernachlässigen zu können. Stattdessen schob sich die Diskussion um wirtschaftlichen Fortschritt auf den Inseln und die Annäherung an die USA immer stärker in den Vordergrund - und im Zuge des sich bereits früh ankündigenden Aufschwungs der Tourismusindustrie änderte sich, anders als hinsichtlich der hawaiischen Sprache, die grundsätzliche Einstellung gegenüber dem Hula und der ihn begleitenden Musik:

„... hula was seen as pleasant and harmless entertainment and a possible cultural commodity that could be economically exploited to attract and amuse the growing number of visitors to Hawai' $\mathrm{i}^{\text {“ }}$ (Buck 1993:156).

Nicht mehr die Verdrängung bzw. Bekämpfung des hawaiischen Tanzes stand nun im Vordergrund staatlicher Kulturpolitik, sondern vielmehr dessen wirtschaftliches Potenzial. Öffentliche Hula-Aufführungen verlagerten sich immer mehr in das zum Tourismusbezirk sich entwickelnde Waikīkī, und in der Musik gewannen die englische Sprache sowie Rhythmen und Instrumentierungen der zeitgenössischen amerikanischen Unterhaltungsmusik an Bedeutung (so genannte hapa-haole [,,halb-Haole“; U.M.] Musik). In den Jahren nach 1910 griffen US-amerikanische Musikproduzenten das marktgängige Klischee vom Inselparadies auf, als dessen Sinnbild gemeinhin der Hula bzw. die Hulatänzerin galt. Titel-Highlights wie „Oh how she could yacki hacki wicki wacki woo“ oder „Yacka hula hicky dula“ zierten Musikstücke, die zwar nichts mehr mit „hawaiischer“ Musik zu tun hatten, dafür aber umso erfolgreicher mit den Ver-

\footnotetext{
${ }^{224}$ So die hawaiische Musikwissenschaftlerin Amy Ku'uleialoha Stillman in einem unveröffentlichten Vortrag auf der Tagung „Changing contexts, shifting meanings“ (Honolulu Academy of Arts, 23.-26. 2. 2006). Sie stellte fest, dass der mündlich überlieferte „skill set“, der das für die Fortführung der Hula-Traditionen notwendige Wissen umfasste, trotz aller Restriktionen bis in die Gegenwart Bestand hatte.
} 
satzstücken des touristischen Mythos vom Paradies spielten. Sie gaben damit einen Trend vor, der sich bis in die 1950er und 1960er Jahre in den USA, aber auch in Europa fortsetzte. 225

Revitalisierung und Traditionalisierung des Hula im 20. Jahrhundert Gegen das von einer touristischen Wahrnehmung geprägte Hula-Bild wandten sich seit den späten 1960er Jahren vermehrt hawaiische Hulameister und -meisterinnen, Kumu hula. In zahlreichen neu gegründeten Hālau hula, Hulaschulen, begannen sie, eine an traditionellen Vorbildern orientierte Version des Hula zu lehren - verbunden mit der Vermittlung religiöser Traditionen und unterschiedlicher hawaiischer Kulturtechniken, wie z.B. der Herstellung von Lei und traditionellen Begleitinstrumenten.

Aufbauend auf diesem Erstarken alter und erneuerter Hulatraditionen entstanden bereits in den 1970er Jahren große Hula-Wettbewerbe und Festivals, auf denen die verschiedenen Hālau hula gegeneinander antraten. Als erster und bis heute bedeutendster dieser Hula-Wettbewerbe wurde 1971 das „Merrie Monarch Festival“ in Hilo auf der Insel Hawai'`i begründet - ein heute in fast jeder Hinsicht hawaiisches Festival, das allerdings aus einer bereits 1964 gegründeten touristischen Veranstaltung (noch ohne Wettbewerb) hervorging (Stillmann 1996:361). ${ }^{226}$

Diese Wettbewerbe beförderten auch die zunehmende Unterteilung des Hula-Repertoires in zwei grundlegende Kategorien: den so genannten hula kahiko oder „alten“ Hula - begleitet von traditionellem Sprechgesang („chant“) und indigenen Schlaginstrumenten (Trommeln etc.) - , auf der anderen Seite den so genannten hula 'auana, den modernen (,geschrittenen“) Hula - getanzt nach den von europäischen Musikstilen beeinflussten Melodien der neueren hawaiischen Musik seit König Kalākaua. ${ }^{227}$

Insgesamt blieben die Vertreter der organisierten Hula-Bewegung aber überraschend unpolitisch, direkte Verbindungslinien zwischen dem HālauHula sowie seinen Protagonisten und der aufkeimenden politischen Bewegung in den 1970er Jahren fehlen. Allerdings trug das Aufleben und die Propagierung des hula kahiko ein deutliches Ideologisierungspotential in sich, wurde doch gerade der „alte“ Hula als Gegenbild zu den vermeintlichen Inhalten kommerzialisierter Tanz-Darbietungen aufgebaut. Ein besonders deutliches Beispiel für die politische Instrumentalisierung des „traditionellen“ Hulabildes

\footnotetext{
${ }^{225}$ Hierzu und allgemein zur Entwicklung von Tanz und Musik im Hawai'i des späten 19. und des 20. Jahrhunderts siehe Tatar 1987. Zur Rezeption hawaiischer Musik in den kontinentalen USA und darüber hinaus siehe Lewis 1996.

${ }^{226}$ Der Name bezieht sich auf den „Merrie Monarch“ Kalākaua, der den Hula und die hawaiischen Künste förderte.

${ }^{227}$ Siehe für eine Analyse der Hulawettbewerbe in Hawai'i sowie ihrer Rolle bei der Veränderung des Hularepertoires seit Beginn der 1970er Jahre Stillmann (1996).
} 
findet sich bei Haunani-Kay und Mililani Trask, die sehr kritische Worte für die von den Tourismusmanagern propagierte „Hotel-Version“ des Tanzes finden:

„Hula dancers wear clown-like makeup, don costumes from a mix of Polynesian cultures, and behave in a smutty manner, rather than in a powerful erotic manner. The hula is erotic because it depicts the energy of life force - in the earth and among the people of the earth. This life force or mana (energy) is sensual and fecund. In the hotel version of the hula, the sacredness of the dance has disappeared and been replaced with an ornamental hoax" (Trask u. Trask 1992:51).

In einem späteren Text verdeutlichte Haunani-Kay Trask noch einmal die Stoßrichtung ihrer Kritik, wenn sie über den kommerzialisierten Hula schreibt:

„The purpose is entertainment for profit rather than a joyful and truly Hawaiian celebration of human and divine nature. The point, of course, is that everything in Hawai' $i$ can be yours, that is, you the tourists', the non-Natives', the visitors'. The place, the people, the culture, even our identity as a ,Native’ people is for sale“ (H.-K. Trask 1999:144).

Trask konfrontiert ihre Leser hier mit idealen hawaiischen Werten, die sie denen der „Außenwelt“ gegenüberstellt, die nicht nur die Existenz des Hula, sondern hawaiische Menschen, hawaiisches Land und hawaiische Kultur bedrohen. Doch war die Wahrnehmung des Hula im Laufe des 20. Jahrhunderts zu keiner Zeit von einfachen Dichotomien bestimmt - hier die Hawaiier und der „richtige“ Hula, dort die Tourismusindustrie mit ihrer verfälschten Kommerzversion des Tanzes - vielmehr prägten Hawaiier als Tänzer, Musiker, Komponisten und Choreographen letztlich die Gesamtheit der jeweiligen „Hula-Szenen" und traten als kundiges Publikum durchaus auch bei touristischen Veranstaltungen in Erscheinung. Als ich 1998 die „Kodak Hula Show“ in Waikīkī besuchte, seit ihrer Gründung im Jahre 1937 eine „Pflichtübung“ für Touristen auf $\mathrm{O}^{`} \mathrm{ahu},{ }^{228}$ fand ich mich auf der Tribüne inmitten einer Frauengruppe aus Wai'anae, die sich durch ihre bedruckten Baseballkappen als „Hawaiian Women’s Club“ auswiesen und die unterschiedlichen Tanzdarbietungen begeistert, ernsthaft und lautstark kommentierten.

\footnotetext{
${ }^{228}$ Als „nicht mehr zeitgemäß“ wurde die „Kodak Hula Show“ schließlich 2002 endgültig eingestellt; „Waikiki hula show ends run“, Tim Ruel (H S-B 26.09.2002, starbulletin.com/2002/09/26/news/ story1/html [17.10.2004]).
} 
Eine weitere Grundlage für die politisch motivierte Polarisierung zwischen „Touristenversion“ und „echtem“ Hula ist die Festschreibung des Hula kahiko als authentische also „richtige“ Form des Hula - wobei die Authentizität der Traditionen, sonst eher ein Thema der nicht-hawaiischen Wissenschaft, unter den Kumu hula intensiv und kontrovers geführt wurde und wird. ${ }^{229}$ Für viele Hulameister stellt das überlieferte Korpus von Regeln und Inhalten einen unveränderbaren Schatz dar, der ein quasi zeitloses „Urbild“ hawaiischer Kultur greif- und erfahrbar macht. Dabei haben aber gerade die großen Wettbewerbe zu Veränderungen der Aufführungspraxis dieses ,alten“ Hula geführt: So veränderten sich die kahiko-Tanzstile seit den 1970er Jahren hinsichtlich Technik und Tempo erheblich, und auch die zugehörigen Gesänge, die „chants“, wurden zunehmend neu verfasst und komponiert und blieben so nicht nur auf die Überlieferungen beschränkt (Stillman 1996:372-4). ${ }^{230}$

Wie fest gefügt aber dennoch die Bilder eines „authentischen“ Hula sind, wird am Beispiel der Tanzbekleidung bei Aufführungen des Hula kahiko recht augenfällig: So gelten die knielangen weiten Baumwollröcke und die weißen Blusen der Tänzerinnen bis heute in hawaiischen Hulakreisen (und darüber hinaus) allgemein als „authentische“ Kleidung für den „alten“ Hula, obwohl dieser Kleidungsstil auf das Wirken der neuenglischen Missionare im 19. Jahrhundert zurückzuführen ist. Beim Merrie Monarch Festival 1995 riskierte der Hālau hula Ke 'ala o ka Laua'e einen kleinen Schritt zurück in die Vergangenheit vor der Übernahme euro-amerikanischer Moralvorstellungen durch die Hawaiier: Die Tänzerinnen des Hālau trugen bei ihrem Auftritt um die Hüften geschlungene Stoffbahnen, die an die Bekleidung aus Rindenbaststoff (kapa) erinnern sollten, wie sie auf europäischen Abbildungen aus dem frühen 19. Jahrhundert noch zu sehen ist. ${ }^{231}$ Doch stieß diese Hula-Schule nicht etwa auf Zustimmung, sondern erntete vielmehr deutliche Ablehnung von Seiten der über die Einhaltung der Traditionen wachenden Kumu hula. Der Auftritt in diesen „daring costumes“ blieb natürlich auch von der Presse nicht unbeachtet:

„In a daring innovation, a women's hula halau took the stage last night wearing abbreviated, sarong-like costumes reminiscent of the men's styles at the 32nd Merrie Monarch Festival ... , It shook up the tradi-

\footnotetext{
${ }^{229}$ Buck (1993:6-8) führt unterschiedliche Beispiele für die Einstellung von Hulalehrern zur Traditionspflege an, auf die ich mich im Folgenden u.a. beziehe.

${ }^{230}$ Siehe hierzu auch Kīhei deSilva (in: Hartwell (1996:46-7).

${ }^{231}$ Ein Bildbeispiel von Louis Choris („Danse des femmes dans les îles Sandwich“, 1822) findet sich in Barrère (1980:25).
} 
tional hula teachers,' said Kamaki Kanahele, president of the State Council of Hawaiian Homestead Associations.“ ${ }^{232}$

Das Beispiel macht erneut deutlich, wie stark und zugleich brüchig die Traditionsbezüge beim Hula und darüber hinaus im weiteren Kontext der hawaiischen Revitalisierungsbewegung sind. Wenn der Rückgriff auf „noch traditionellere“ Formen in der öffentlichen Wahrnehmung als „daring innovation“ dargestellt werden kann, wirft dies ein Schlaglicht auf einen verbreiteten Traditionalismus, der zumal in der Anfangsphase der „Hawaiian Renaissance“ und eben auch bei der politischen Aneignung des Hula eine bedeutende Rolle spielte und spielt.

\section{Hula, „Hawaiian Values“ und Religionen}

Im Spannungsfeld von Traditionsbewahrung einerseits und den Anforderungen einer dynamischen hawaiischen Gegenwart kommt einem Aspekt des Hula besondere Bedeutung zu: Für viele Kumu hula stellt die Vermittlung überlieferter hawaiischer kultureller Werte und Normen einen integralen Bestandteil der von ihnen gebotenen Hula-Ausbildung dar. ${ }^{233}$ Politische Fragen treten hierbei in den Hintergrund - in der Ausübung des Tanzes heben sich für viele HulaLehrer und Schüler die Gegensätze zwischen gesellschaftlicher Realität und Idealvorstellungen einer hawaiischen Gemeinschaft auf; eine gewisse Romantisierung des Hula und seiner Bedeutung für das alltägliche Leben der Tänzer ist die Folge (cf. Kamahele 2000:53, 65). So sagt z.B. Māpuana deSilva, Kumu hula des Hālau Mōhala 'Ilima, über die Regeln, die sie ihren HulaSchülerinnen vermittelt:

„In the beginning they're rules ... but after a while they just become choices for how the dancers choose to be, not just when they come to hula. ... The values that we teach them here become the values that they live by every day, wether they are in hula or in school or at work or with their families ... through hula you can learn all good values“ (Hartwell 1996:50-1).

Zu diesen „guten Werten“ gehören auch so ambivalente und schwer zu fassende Aspekte der kulturellen Revitalisierung wie Spiritualität und Religion, die im Kontext des Hula jedoch besondere Bedeutung erlangen - feiern doch die Gesänge und Tänze des Hula kahiko oft die hawaiischen Götter und ihre Taten. Für viele Kumu hula und ihre Hālau sind Gebete an Laka, Göttin des Hula und

232 „Women's halau shakes 'em up at hula festival“, Catherine Kekoa Enomoto (H S-B 22.4.1995:A-3).

${ }^{233}$ Die Bedeutung der Hālau hula für die Verbreitung kultureller Vorstellungen und auch politischer Inhalte darf wohl nicht unterschätzt werden, war aber bisher meines Wissens noch nicht Gegenstand wissenschaftlicher Untersuchungen (cf. Stillman 1996:376, n. 9). 
des Waldes, beim Sammeln der Pflanzen für Lei (Farn- und Blütenkränze) sowie Tanzkleidung selbstverständlicher Teil der Hulapraxis. ${ }^{234}$ So besteht im Umfeld der Hālau hula durchaus eine gewisse Affinität zu Vorstellungen der alten hawaiischen Religion, die von der übrigen, größtenteils christlichen hawaiischen Bevölkerung nicht unbedingt geteilt wird.

Ein wiederholt zitiertes Beispiel ${ }^{235}$ für den möglichen Konflikt zwischen traditionellen religiösen Vorstellungen und der modernen Gesellschaft überwiegend christlichen Glaubens ist der Rücktritt des Hālau Mōhala 'Ilima vom Wettbewerb des Merrie Monarch Festivals im Jahre 1986 - die Mitglieder des Hālau hatten Wolkenbrüche und Gewitter als Zornesäußerung der Göttin Hina gedeutet. Solche religiösen Bezüge werden sehr kontrovers beurteilt: Viele christliche Hawaiier lehnen jede Wiederbelebung der alten Glaubensinhalte strikt ab, für andere ist der christliche Glaube aber durchaus mit dem Respekt gegenüber den hawaiischen Akua (Göttern) vereinbar. Wie gegensätzlich diese Standpunkte sein konnten, erlebte ich 1995 u.a. beim Besuch eines Seminars des Hui Na‘auao zum Thema „Models of Sovereignty“ in Kahului, Maui. Gastgeber war die der fundamentalistischen Church of Christ nahe stehende Organisation 'Oihana o Keopuolani: angesichts ihres radikalprotestantischen Hintergrunds vielleicht nicht verwunderlich, waren die hawaiischen Teilnehmer der Informationsveranstaltung jeglicher Hinwendung zur alten Religion durchweg abgeneigt. Hingegen äußerte ein hawaiischer katholischer Seelsorger in Hilo mir gegenüber die Ansicht, dass hawaiische Akua (Götter) und 'Aumakua (persönliche Ahnengötter) auch Erscheinungsformen des einen Gottes (im Hawaiischen ebenfalls mit dem Namen Akua bezeichnet) seien. Die Bezugnahme auf die alten Götter, so wie sie im Diskurs der politischen und kulturellen Bewegung begegnet, sei für ihn daher unproblematisch. ${ }^{236}$

Allerdings sind Reverenzen an die hawaiische Religion vonseiten der Hulameister und -tänzer (und auch politischer Aktivisten) in der nichthawaiischen Gesellschaft alles andere als unverfänglich. Buck kommentiert die mögliche Reaktion der „Außenwelt“ wie folgt:

„....when they talk about chant and hula as religious rites ... Hawaiians move outside the boundaries of Western rational discourse, and this discourse of religion makes the majority of listeners uncomfortable. Such social institutions as the legislature, government agencies, and the courts are willing to make some concessions on the basis of Hawaiian culture - its protection and maintenance - but not on the basis of Hawaiian religion“ (Buck 1993:188).

\footnotetext{
${ }^{234}$ Siehe z.B. „,The Lei of the land“, Vicki Viotti (HA 16.4.95:F-1, F-14).

${ }^{235}$ So z.B. bei Buck (1993:164); Hartwell (1996:41-2).

${ }^{236}$ Siehe auch Kanahele (1986:34-5).
} 
So bleiben Verweise auf die alte hawaiische Religion im politischen Diskurs zwar ein Randphänomen, doch mögen „traditionelle“ religiöse Vorstellungen inzwischen auch außerhalb des Hula-Kontextes einen kleinen Kreis von Anhängern gefunden haben. Seien es die Rituale beim Auslaufen oder der Ankunft des hawaiischen Doppelrumpfbootes Hōkule'a und ihrer Schwesterschiffe, die Anrufung hawaiischer Götter im Rahmen von Begrüßungszeremonien nach den Regeln eines „Hawaiian Protocol“, religiös/kulturell begründete Proteste wie z.B. gegen den Neubau der Autobahn „H 3“ von Honolulu nach Kāne“ohe, die über archäologische Stätten alter Heiau (Tempel) führen sollte oder auch die (seltene) Einbindung religiöser Zeremonien in Demonstrationen des „Hawaiian Movement“: Zumindest die mit den alten religiösen Vorstellungen verbundenen äußeren Formen der Verehrung und des Gottesdienstes haben heute einen sichtbaren Platz - über die tatsächliche Verbreitung von Glaubensinhalten lassen sich jedoch kaum Aussagen treffen.

Auch von staatlichen Stellen wird das Recht der Hawaiier auf Ausübung ihrer Religion in Einzelfällen anerkannt: Hawaiier sind z.B. berechtigt, die Nationalparks zum Zwecke religiöser Verehrung kostenfrei zu betreten, und verschiedene historische Sehenswürdigkeiten wurden unter Verweis auf ihre religiöse Bedeutung („Kapu!“) für nicht-hawaiische Besucher offiziell gesperrt - im Allgemeinen jedoch, und hier zeigt die oben zitierte Aussage Bucks ihre Gültigkeit, legen die Gerichte das hawaiische Recht auf „free exercise of religion“ sehr eng aus (Kau u. MacKenzie 1991:238-40).

Das im Hula auch jenseits der traditionellen Religionsvorstellungen angelegte Konfliktpotential machte eine kurze Zeitungsnotiz deutlich, die 1998 in deutschen Tageszeitungen auf die Seite „Vermischtes“ gelangte:

„Mit einer ungewöhnlichen Bitte will der katholische Bischof von Hawaii, Francis DiLorenzo, an den Papst herantreten: Der Vatikan soll Hula-Tänze im Gottesdienst erlauben. [...] Nach der Beschwerde eines Hawaiianers hatte der Vatikan die polynesischen Hula-Tänze aus Gottesdiensten verbannt. DiLorenzo vermute, daß Rom die kulturelle Bedeutung des Hula in Hawaii nicht verstehe, sagte Downes [Patrick Downes, Sprecher der Diözese von Honolulu; U.M.].“ ${ }^{237}$

Vor dem Hintergrund der gängigen Klischees über den Hula - Gitarrenklänge, Baströcke, Blütenkränze und natürlich „Hulamädchen“ - konnte diese Agenturmeldung (AP) den Leser wohl tatsächlich verwundern und amüsieren. Und es steht zu vermuten, dass es das Spiel mit diesen Klischees war, das der Nachricht den Weg in die Zeitungen ebnete (eine weiterführende Berichterstattung fand nicht statt). Wie auch immer, die Meldung verweist tatsächlich auf einen

${ }^{237}$ „Hula-Tänze im Gottesdienst“ (Göttinger Tageblatt 6.7.1998:6). 
ernsten Konflikt um den Hula, seine inner-hawaiische kulturelle Bedeutung und Bewertung sowie seine Verwendung in der katholischen Liturgie. Untersagen die katholischen Regeln im Allgemeinen die Aufführung von Tänzen im Gottesdienst, so war doch der Hula in Hawai' $i$, wie z.B. auch Tanzformen in anderen nicht-westlichen Liturgien, in vielen Kirchen des Archipels in den Ablauf der Messe integriert. Der Erlass des Vatikans erging auf die Beschwerde eines Gemeindemitglieds hin, ob dieses Mitglied tatsächlich hawaiischer Abstammung ist (wie die zitierte Nachricht suggeriert) oder nicht, bleibt der differenzierteren Berichterstattung in Hawai'i zufolge unklar - sie spricht von einem ,island resident“ (d.h. einem Bürger unbestimmter ethnischer Zugehörigkeit). ${ }^{238}$ Letztlich wurde der Hula als Ausdruck christlicher Gottesverehrung wieder zugelassen - unter der Voraussetzung, dass alle Gottesdienstbesucher Gelegenheit zum Verlassen der Messe vor Beginn des Tanzes bekommen - und damit als kulturell verankertes Medium des Gottesdienstes, des Gebets oder der Besinnung vom Vatikan anerkannt. ${ }^{239}$

\section{Hula als Medium hawaiischer Politik}

Durch staatliche Gesetzgebungen drohten im Jahre 1997 wichtigen Aspekten der Hula-Praxis, wie dem Sammeln von Pflanzen und Früchten sowie der Durchführung religiöser Zeremonien an besonderen Orten (wie z.B. den Vulkankratern auf der Insel Hawai ‘i) massive Einschränkungen, wenn nicht gar Verbote. ${ }^{240}$ Diese unmittelbare Bedrohung ihrer überlieferten Rechte und eine damit einhergehende „Entwertung“ der kulturellen Ressource „Hula“ durch Eingriffe des Staates erregte schließlich auch unter den Vertretern des HālauHula Widerstand, der sich zunächst in der Gründung einer Protestorganisation mit dem Namen 'Īlio'ulaokalani („Roter Hund des Himmels“; U.M.) äußerte. Eine Mitorganisatorin von 'Îlio'ulaokalani, Momiala Kamahele, beschreibt das eher unfreiwillige Erscheinen der Hulalehrer auf der politischen Bühne:

\footnotetext{
${ }^{238}$ U.a. „Bishop plans to appeal Vatican ban on dance: catholics find themselves in a spirited debate over the liturgical use of Hula“, Mary Adamski, (H S-B 9.7.1998 [starbulletin.com/98/07/09/news/story6.html 27.8.1998]); „Vatican to send rules on hula: guidelines for pastors will clarify a June order banning hula in services“, (H S-B 19.12.1998 [starbulletin.com/98/12/19/news/story2.html 12.10.2004]).

${ }^{239}$ Weniger positiv gesehen, erscheint der ehemals sakrale Charakter des Hula der katholischen Kirche heute als so „ungefährlich“, dass er gestattet und für den liturgischen Gebrauch vereinnahmt werden kann. Reaktionen der politischen Bewegung auf den katholischen „Hula-Streit“ sind mir nicht bekannt.

${ }^{240}$ Die Entscheidung des Obersten Gerichthofes von Hawai‘i im Fall „Property Access Shoreline Hawaii (PASH) vs. Hawaii County Planning Commission“ von 1995 gestand allen Hawaiiern das Recht zu, traditionelle religiöse, kulturelle sowie der Subsistenz dienende Praktiken auf allen ungenutzten Landflächen im Staat Hawai'i auszuüben. Das hier in Rede stehende Gesetz (Senate Bill 8; House Bill 1920) wollte diese Rechte nur jenen zugestehen, deren Vorfahren diese Praktiken bereits vor 1893 auf einer spezifischen Landfläche ausgeübt hatten.
} 
„Kumu hula throughout the Hawaiian Islands mobilized hundreds of their hula students in an extraordinary feat of grace and power never seen in modern colonial times. Essentially a conservative element of the Native Hawaiian community, practitioners unwittingly participated in the politicization of hula. Thus, in the very act of organizing to defend hula was born the union of culture and politics..." (Kamahele 2000:52-3).

Verbindungen von Kultur und Politik (wie z.B. im Bereich der Sprachpolitik) gab es natürlich auch schon vor der Gründung von 'Îlio'ulaokalani. Doch als am 25. Februar 1997 im Innenhof des Parlamentsgebäudes von Honolulu mehr als 250 Mitglieder verschiedener Hälau hula zusammenkamen, war dies ein erstes und deutliches Beispiel für die direkte Nutzung des Hula zur Durchsetzung politischer Ziele. Der von 'Īlio'ulaokalani organisierte 24-stündige Protest mit traditionellem Gesang und Tanz war schließlich erfolgreich, die Gesetzesvorlage zur Einschränkung des Rechts auf kulturelle Nutzung des Landes wurde nicht an das Parlament weitergeleitet. ${ }^{241}$

Mit 'Īlio'ulaokalani gründete sich eine Organisation, für die die Verteidigung so genannter kultureller Werte im Vordergrund stand, die aber in den Jahren nach ihrer Gründung auch explizit Standpunkte der Autonomiebewegung übernahm. So stellte Victoria Holt Takamine, Mitbegründerin und derzeitige Präsidentin von 'Īlio'ulaokalani, noch im Jahr der Gründung fest: „This [die Gründung der Organisation; U.M.] is very much a move toward sovereignty [...] The sovereignty movement in 'Tेlio'ulaokalani is the preservation and perpetuation of Hawaiian culture. “242

Die Organisation beschreibt ihre Ziele wie folgt: „'̄lio'ulaokalani is committed to preserving and protecting Hawaiian's traditional way of life and ancestral rights. Our mission is to protect and preserve the integrity of Hawai'i's cultural environment" ('Îlio'ulaokalani 2004). Im Einklang mit dieser Aufgabenbeschreibung organisierte die Hula-Koalition von 2003 bis 2007 unter dem Titel „Ka “Aha Pono“ (Native Hawaiian Intellectual Property Rights) jährliche Konferenzen zu Fragen des kulturellen und intellektuellen Copyrights. In der Abschlusserklärung der Tagung von 2003 heißt es u.a.:

„Kanaka Maoli have the right of self-determination. By virtue of that right we freely determine our political status and freely pursue economic, social, and cultural development, which includes determining ap-

\footnotetext{
241 „Bill to limit gathering rights drummed away during Hawaiian protest vigil at capitol“, Kelli Meskin (KWO ‘Apelila [April] 1997:A4-A5).

${ }^{242}$ „Dance of the red dog: kumu hula unite and realize their power in the push for sovereignty and preservation of culture“, Catherine Kekoa Enomoto (H S-B 29.12.1997 [starbulletin.com/97/12/29/features/story1.html 12.10.2004]).
} 
propriate use of our traditional knowledge, cultural expressions and artforms, and natural and biological resources [...] We maintain our inherent right of self-determination, despite the oppression of colonization and illegal occupation of our land base since January 17, 1893 when our sovereign Kingdom of Hawai'i was overthrown by the military force of the United States" ('Īlio'ulaokalani 2003).

Die in diesem Statement enthaltenen Aussagen und Formulierungen zeigen, dass hier Vorstellungen der Autonomiebewegung in wesentlichen Zügen übernommen wurden; und auch wenn es sich hier nicht um einen von 'Īlio'ulaokalani verfassten Text handelt, lässt doch die Tatsache seiner Verbreitung über die offizielle Website der Koalition grundsätzliche Zustimmung vermuten. Die Hula-Koalition, die weiterhin fest in den Werten und der Ausübung des Hula gründet, überwand mit der Verknüpfung von kultureller Praxis, politischen Forderungen und Protest-Aktionen eine Trennlinie zwischen kultureller und politischer Bewegung. Zwar waren auch vor 1997 einzelne Personen auf beiden Feldern aktiv, bei verschiedenen Themenkomplexen flossen Politik und Kultur zusammen und politische Gruppierungen nutzten kulturelle Elemente zur Unterstützung und Untermauerung ihrer politischen Ziele - doch fehlte es an Gruppierungen und Organisationen, deren politische Agenda explizit durch kulturell begründete Zielsetzungen bestimmt war.

Seit den 1970er Jahren entwickelte sich Hula von einer nur noch wenigen Eingeweihten in ihrer ganzen Tiefe und Weite bekannten Kunst, die zunehmend hinter den von der Tourismusindustrie propagierten eingängigeren Tanzdarbietungen unterschiedlicher Provenienz zurückstand, zu einer eigenständigen kulturellen Bewegung. Der Hula und sein gesamtes kulturelles wie soziales Umfeld befinden sich im Zentrum des Diskurses über die kulturelle, und wie das Beispiel 'Îlio'ulaokalani zeigt, zuweilen auch die politische Autonomie der Hawaiier im Hawai'i der Gegenwart. So konnte Buck (1993:6) in ihrer Analyse der kulturpolitischen Bedeutung des Hula feststellen: „Over the past two decades, hula and chant have been a microcosm of the cultural politics of the islands“.

Hula und die ihn begleitende Musik sind „...vivid public markers of Hawaiian difference and identity“ (Buck 1993:9). Nicht zuletzt die Wandlung von einer eher defensiv ausgerichteten Bewegung, die ihr Ziel in der Rückbesinnung auf Traditionen und in deren Bewahrung sah, hin zu einer aktiven Protestbewegung, die eben diese traditionellen Formen zur Durchsetzung aktueller politischer Zielvorstellungen einsetzt, hat dem Hula eines der stärksten Identifikationspotentiale im Kontext einer dynamischen hawaiischen Gegenwartskultur verliehen. Und dies gilt weit über Hawai'i hinaus - gerade für die hawaiischen Gemeinden auf dem amerikanischen Festland ist Hula einer der wich- 
tigsten ethnisch-kulturellen Marker und erhält die Bindung an Kultur (und Politik) der weit entfernten Inseln aufrecht (Halualani 2002:216-8).

\subsection{Hawaiische Hochseefahrt: Hōkūle'a und Hawai'iloa}

Wie die hawaiische Sprache und der Tanz Hula, bleibt auch das hochseetüchtige Segelboot Hōkūle'a ${ }^{243}$ bis heute ein herausragendes Beispiel kultureller Revitalisierung und Kernsymbol hawaiischer Kultur. Noch immer ziehen das 1975 gebaute Doppelrumpfboot und die Reihe späterer Nachbauten der großen polynesischen Fernreiseboote die Menschen in ihren Bann, sei es bei der Besichtigung der inzwischen an der Kaimauer des Hawai'i Maritime Museum in Honolulu liegenden Hōkūle'a oder bei Aufbruch oder Heimkehr ihrer Schwesterschiffe Makali‘i und Hawai'iloa. Historische Abbildungen von Doppelrumpfbooten oder zum Logo stilisierte Formen werden aber auch von Institutionen und Firmen genutzt, ${ }^{24}$ um ihre jeweils spezifische Bindung an den Archipel herauszustreichen - mehr als irgendeines der anderen kulturellen Kernsymbole wirken die traditionellen Doppelrumpfboote auch über den Kreis der hawaiischstämmigen Inselbevölkerung hinaus. Die Fahrt der Hōkūle'a nach Tahiti im Jahre 1976 gab den Anstoß für eine erneute, intensive Auseinandersetzung mit traditionellen Techniken sowie kulturellen Vorstellungen, und George Kanahele schreibt der Hōkūle'a sogar eine Katalysatorfunktion für die gesamte spätere Entwicklung der „Hawaiian Renaissance“ zu (Finney 1994:75; [Kanahele 1982]).

Kulturelle und politische Aspekte wie eine pan-polynesische Zusammengehörigkeit, die „uralte“ polynesische Tradition der Langstreckenreisen und eine bestimmte Vorstellung der 'Ohana, des hawaiischen Familienverbandes, wurden durch die Fahrten der Hōkūle'a popularisiert und in Liedern gefeiert. Heute ist die hawaiische Seefahrt innerhalb der politischen Bewegung (nach den bewegten 1970er Jahren) von eher marginaler Bedeutung; doch haben ritualisierte Verehrung, Poetisierung sowie alte und neue hawaiische Vergangenheitsdiskurse den Booten auf ihrem Weg vom konfliktträchtigen Experiment zum gefeierten „Mainstream-Event“ einen bleibenden Ehrenplatz gesichert.

\footnotetext{
${ }^{243}$ Die Hōkūle‘a („Stern der Freude“) ist nach einem polynesischen Navigationsstern benannt; wahrscheinlich handelt es sich dabei um den Fixstern Arcturus (Pukui u. Elbert 1986).

${ }^{244}$ Beispiele hierfür sind u.a. das Signet der Bank of Hawai`i oder das Logo des Online-Auftritts der University of Hawai‘i - „Hawai‘i Voyager“.
} 
Hōkūle 'a: wissenschaftliches Projekt und kulturelle Revitalisierung

Während hawaiische Initiativen zur Förderung der Sprache und des Hula auf durchgängige, über die Zeit der großen gesellschaftlichen und politischen Umbrüche hinaus fortbestehende Traditionen zurückgreifen konnten, waren die Überlieferungsstränge des hawaiischen Bootsbaus und der Navigation kaum noch nachvollziehbar oder gar abgerissen. Anders als die Revitalisierung der hawaiischen Sprache oder des spirituell geprägten Hula begann die Erneuerung der hawaiischen Hochseefahrt - und damit die Schaffung eines der zentralen hawaiischen Identifikationssymbole - als ein zunächst überwiegend nichthawaiisches Wissenschaftsprojekt: Für den Ethnologen Ben Finney, der sich in den 1960er Jahren mit der Besiedlungsgeschichte des hawaiischen Archipels beschäftigte, stand die Frage nach der Möglichkeit gezielt navigierter Seefahrten zwischen Hawai'i und Tahiti (Kahiki), von denen hawaiische Legenden für die Zeit vom 12. bis 14. Jahrhundert berichten, im Vordergrund seiner Untersuchungen. ${ }^{245}$

Als 1778 die ersten Europäer den abgelegenen Archipel erreichten, kamen ihnen Hawaiier in Doppelrumpfbooten (wa'a kaulua) entgegen, die jedoch bereits zu dieser Zeit nur mehr dem interinsularen Verkehr sowie Fahrten entlang den Küsten dienten - die Zeit der legendären Fernfahrten war vorbei und auch die notwendigen Navigationskenntnisse waren in Vergessenheit geraten. ${ }^{246}$ Finney wollte nun im Rahmen einer ,experimentellen Archäologie“ herausfinden, ob das traditionelle wa'a kaulua am Wind (und somit gegen die jeweils herrschenden Passate) gesegelt werden konnte - eine der Grundvoraussetzungen für eine Fahrt von Hawai'i nach Tahiti mit der den alten Hawaiiern bekannten Technologie. Nach gelungenen Probefahrten eines ersten Bootsnachbaus vor der kalifornischen Küste machte Finney es sich zur Aufgabe, den Nachweis zu erbringen, dass ein mit traditionellen polynesischen Navigationstechniken gesteuerten wa'a kaulua in der Lage wäre, die Distanz von fast $5000 \mathrm{~km}$ nach Tahiti und zurück zu überwinden.

1973 gründete Ben Finney gemeinsam mit Charles T. Holmes und Herb Kawainui Kāne die „Polynesian Voyaging Society“ (PVS), deren Ziel der

\footnotetext{
${ }^{245}$ Siehe zusammenfassend zu wissenschaftlichen Besiedlungstheorien und der Frage möglicher „return voyages“ Bellwood (1978:301-3) und Finney (1994:13-29). Während die Herkunft der polynesischen Entdecker der hawaiischen Inseln nach den Ergebnissen der Linguistik und Archäologie allgemeine Akzeptanz findet, werden die Fragen der technischen und navigatorischen Voraussetzungen jener Seefahrer, d.h. ob die Entdeckungsfahrten planmäßig durchgeführt wurden und ob z.B. navigierte Fahrten zwischen Tahiti und Hawai ‘ $i$ in beide Richtungen möglich waren, weiterhin diskutiert.

${ }^{246}$ Die Zahl dieser Boote muss beeindruckend gewesen sein: allein die von Kamehameha I. zur Eroberung Kaua'is ausgerüstete Flotte soll bis zu 800 Doppelrumpfboote umfasst haben (Lal u. Fortune 2000:80). Das Ende der polynesischen Fernfahrten wird von Finney (1994:292-304) diskutiert. Zur Zeit der europäischen Entdeckung bestanden aber noch Seefahrtsverbindungen zwischen Sāmoa, Fiji und Tonga.
} 
Nachbau eines hawaiischen Doppelrumpfbootes sowie die Durchführung einer Fahrt von Hawai‘i nach Tahiti und zurück war (Finney 1979:20). Neben der Verfolgung des wissenschaftlichen Programms beabsichtigten die Initiatoren zugleich, mit dem Bau der 1975 zu Wasser gelassenen 19 Meter langen Hōkūle'a einen Beitrag zur kulturellen Revitalisierung in Hawai' $i$ und anderen pazifischen Inselstaaten zu leisten:

„We hoped that our effort to reconstruct a voyaging canoe, and then sail it over a traditional route celebrated in chant and legend, would also serve the cause of Polynesian cultural revival - would make Hawaiians, and other Polynesians whose cultural identity has become blurred in modern times, know and better appreciate the great maritime achievements of their stone age ancestors“" (Finney 1979:6).

Vor allem der Konstrukteur der Hōkūle‘a, Herb Kāne, trieb diesen Aspekt des Projektes aktiv voran. Für Kāne - von hawaiischer Abstammung, doch fern den Inseln im Mittleren Westen der USA aufgewachsen und ausgebildet - war das Projekt auch Teil eigener Identitätssuche (Finney 1979:21). ${ }^{247}$ Kurz vor dem Aufbruch zur ersten Fahrt nach Tahiti beschreibt er in einem Artikel mit dem Titel „A canoe helps Hawaii recapture her past“ das historische Doppelrumpfboot als „Raumschiff“ („,spaceship“ [Kane 1976:471]) der hawaiischen Vorfahren, das einst überall auf den Inseln die ehrfürchtige Bewunderung der hawaiischen Bevölkerung hervorrief: „The voyaging canoe! It lay at the very heart of Polynesian culture. Without it there would be no Polynesia“ (Kane 1976:475) eine emphatische Feststellung, die weit über den Rahmen kultureller Revitalisierung in Hawai'i selbst hinausgreift und die Inseln sowie ihre indigenen Bewohner in einem pan-polynesischen kulturellen Kontext lokalisiert und verankert.

Für die sich entwickelnde hawaiische Bewegung war der Nachbau eines traditionellen hawaiischen Doppelrumpfbootes bald eine ideale Projektionsfläche für zunehmend vehement artikulierte kulturelle Vorstellungen und auch politische Forderungen. Das Boot selbst sowie die später mit ihm verbundenen kulturellen Aktivitäten waren offenbar ein ideales „Bindeglied“ zwischen der US-amerikanisch geprägten Gegenwart des größten Teils der überwiegend urbanen Hawaiier und einer bedeutenden voreuropäisch-hawaiischen Kultur und Geschichte, zu der viele von ihnen jeglichen Kontakt verloren hatten.

Zugleich zeigten sich aber auch gerade zu Beginn des Projektes sehr deutlich widerstreitende Interessen der beteiligten Parteien: Während die Initia-

\footnotetext{
${ }^{247}$ Herb Kawainui Kāne ist heute unter der hawaiischen Künstlerschaft sehr anerkannt: im Frühjahr 2006 wurde ihm der Kū i ka Ni`o-Award für sein Lebenswerk verliehen.
} 
toren des Projektes eine möglichst genaue historische Rekonstruktion verfolgten, sahen viele Hawaiier die Hōkūle'a vielmehr als Symbol einer auch politisch motivierten hawaiischen Identität. ${ }^{248}$ Anders als bei der im vorangegangenen Kapitel aufgezeigten Traditionalisierung und „Festschreibung“ des Hula bot der Rekurs auf die traditionelle Seefahrt zahlreiche „Leerstellen“ hinsichtlich „hawaiischer“ Praktiken, die im Sinne einer gegenwärtigen hawaiischen Kultur- und Identitätspolitik von beteiligten Hawaiiern mit (Neo-) Traditionen gefüllt werden konnten. Finney selbst merkte hierzu kritisch an: „We were not prepared for the degree to which some Hawaiians who became associated with the project after its inception freely began to innovate culturally“ (1991:398). Als Beispiel nennt er die Neuschöpfung einer 'Awa- (Kawa-) Zeremonie durch Mitglieder der Crew, die sich hierbei nicht an hawaiischen Überlieferungen, sondern vielmehr an zentralpolynesischen Vorbildern orientierten (anders als z.B. in Sāmoa oder Tonga war der Konsum von 'Awa in Hawai'i den Überlieferungen zufolge nicht mit komplexen Zeremonien verbunden).

Jocelyn Linnekin (1983:244-6) stellte die Hōkūle‘a in eine Reihe ,nationalistischer Symbole“ der hawaiischen Bewegung, wobei sie die Frage der Authentizität in den Vordergrund rückte (1983:245): „In the cultural revival, isolated facts have been transformed into symbols of Hawaiianness and accorded a significance without precedent in aboriginal Hawaiian society“. Hinsichtlich des neu erbauten Doppelrumpfbootes heißt es bei ihr weiter: „Arguments ensued over the authenticity of the Hokule'a's construction; the purists in the dispute were the haole academicians. The Hawaiian crewmen, although fiercely anti-haole, felt that modern improvements would not tarnish the canoe’s significance for their cause““. ${ }^{249}$ Kritikpunkte waren die Verwendung moderner Techniken und Materialien für die Konstruktion der Hōkūle‘a (deren Rümpfe aus Fiberglas und Holz bestanden) sowie der Verzicht der hawaiischen Crew auf den traditionellen Proviant. ${ }^{250}$ Eine sehr weitgehende Neuinterpretation hawaiischer Traditionen war die Forderung der hawaiischen Mannschaft nach Aufgabe hierarchischer Entscheidungsstrukturen. Eine solche Rangordnung widerspräche der hawaiischen 'Ohana-Struktur, ${ }^{251}$ unter der sie eine egalitäre, gleichberechtigte Gemeinschaft verstanden - wobei sie den Hintergrund der in allen Lebensbereichen hierarchisch nach Abstammung, Ge-

\footnotetext{
${ }^{248}$ Siehe z.B. Linnekin (1983:244-6).

249 Mehr als ein Jahrzehnt nachdem Linnekin dies schrieb, fand vor dem neuen Hintergrund inzwischen existierender unterschiedlicher Modelle hochseetüchtiger Doppelrumpfboote diese Debatte auch unter den indigenen Mannschaften und Bootsbauern statt (s.u.).

250 Überlieferte Konservierungstechniken wurden im Vorfeld der ersten Reise zu Herstellung des Proviants benutzt. Allerdings lehnten gerade die Hawaiier den Verzehr von Tarobrei (poi) und anderen mehr oder weniger gut konservierten Nahrungsmitteln ab (Lewis 1976:518; Kane 1976:477 f.).

${ }^{251}$ Die 'Ohana ist der traditionelle erweiterte Familienverband.
} 
schlecht oder Alter organisierten Gesellschaft der voreuropäischen Zeit offenbar ausblendeten. Auch gab es deutliche Kritik an der Führung des Bootes durch einen Navigator aus Mikronesien, Mau Piailug von der Insel Satawal (Yap), der die traditionellen (mikronesischen) Methoden der Sternennavigation noch beherrschte. In Hawai' $i$ fand sich niemand, der über solche überlieferten seefahrerischen Kenntnisse verfügte. Zwischen dem alten Navigator und der jungen hawaiischen Mannschaft brachen darüber hinaus so deutliche Konflikte hinsichtlich der Autoritätsstrukturen auf, dass Piailug sich weigerte, die Hōkūle'a auch bei ihrer Rückfahrt zu steuern, und direkt von Tahiti nach Yap zurückkehrte.

Ben Finney, der Initiator des Projekts, musste schließlich feststellen, dass sich die beabsichtigte Zusammenführung von Wissenschaft und kultureller Revitalisierung vor dem Hintergrund der politischen Situation im Hawai'i der 1970er Jahre als ausgesprochen schwierig erwies: „Little did we know that, in the context of modern Hawaii, to join cultural revival with experimental voyaging was to create an explosive mixture...“ (1979:6).

Die kulturelle Aneignung der modernen Doppelrumpfboote: hawaiische Stimmen

Die Jungfernfahrt der Hōkūle'a von Hawai'i nach Tahiti galt zunächst dem Nachweis, dass die Bewältigung der Distanz zwischen Hawai'i und den Inseln Ostpolynesiens in beiden Richtungen für die Hawaiier der voreuropäischen Zeit technologisch und navigatorisch möglich war. Sie sollte zugleich zeigen, dass Hawaiier der Gegenwart die Taten ihrer Vorfahren wiederholen konnten und bot damit die außergewöhnliche Möglichkeit, eine ganze Bevölkerungsgruppe in die unumstritten große seefahrerische Vergangenheit der Polynesier zu stellen. Die großen Erwartungen und das emotionale Potential dieser Fahrt und des Bootes begegnen uns z.B. im Text eines Liedes, das aus Anlass der Jungfernfahrt für die damals einzige hawaiischsprachige Radiosendung in Hawai'`i geschrieben wurde und dessen englische Übersetzung lautet: „We have seen for ourselves / Hōkūle'a / The beautiful double hull canoe / Like a bird sailing at sea / [...] / This is our song / For the Hōkūle'a canoe / We will be with you / On your trip to and from Tahiti“ (Übersetzung bei Kimura 1976). ${ }^{252}$

War die erste Reise der Hōkūle'a noch von Kontroversen zwischen Projektleitung und großen Teilen der Mannschaft geprägt (hawaiische Mitglieder verlangten den Ausschluss aller „Haole“ von dem Projekt sowie den Einsatz des Doppelrumpfbootes im politischen Protest gegen die Besetzung der

\footnotetext{
${ }^{252}$ „Ua 'ike maka mākou / Iā Hōkūle’a / Wa'a kaulua nani / Mehe manu a ka holo kai [...] / Puana 'ia mai / Wa'a Hōkūle'a / 'O mākou pū me 'oe / I ka holo a ho'i mai“" (Kimura 1976).
} 
Insel Kaho'olawe durch die US-Navy ${ }^{253}$ ), so stand die zweite große Fahrt des Bootes im Jahre 1980 bereits unter einem anderen, überwiegend hawaiischen Vorzeichen. Inzwischen war der Hawaiier Nainoa Thompson vom Meister Piailug in der Kunst der Sternennavigation unterwiesen worden, und es war Thompson, der die Hōkūle'a von Hawai'i nach Tahiti und zurück führte. 1985 brach die Hōkūle‘a zur so genannten „Voyage of Rediscovery“ auf, die über Tahiti, Rarotonga und Sāmoa nach Aotearoa/Neuseeland führte und auch auf diesen Reisestationen zu einer Popularisierung der polynesischen Seefahrttechniken beitrug. In einem später geschriebenen Mele Oli, einem an alt-hawaiische Vorbilder angelehnten Sprechgesang, erinnerte der Hawaiischlehrer K. Kalani Akana an die Rückkehr der Hōkūle‘a im Mai 1987. Die englische Übersetzung des Textes lautet: ,[...] Here comes the worthy canoe / A vessel that seeks knowledge / A canoe that awakens us at the breaking of dawn / It is best that you paddle, warriors, who champion us / For Hawai'i, for the nation / Here is the sustaining water, an immense cloud / This is the sign that you look for" ${ }^{254}$ Wenn der Autor die „Krieger, die für Hawai‘ ${ }^{i}$ und die Nation eintreten“ (,nā koa ...") anruft, treten in diesem späteren Text die politischen Gegenwartsbezüge sehr deutlich neben den Aspekt des Stolzes auf die Vergangenheit.

Die Hōkūle'a blieb nicht allein: Fast 20 Jahre nach ihrem Stapellauf segelten 1995 bereits drei Doppelrumpfboote von Hawai'i nach Tahiti: außer der Hōkūle'a die nach dem Gestirn der Pleiaden benannte Makali'i (ein kleineres, aus Fiberglas hergestelltes Doppelrumpfboot von der Insel Hawai'i) sowie die Hawai'iloa, benannt nach jenem legendären Seefahrer, dem die hawaiischen Legenden die Entdeckung der Inseln zuschreiben (siehe Beckwith 1970:363-4). ${ }^{255}$ Der Bau der Hawai'iloa war ein gemeinsames Projekt der Polynesian Voyaging Society und des Native Hawaiian Culture and Arts Program/Bishop Museum (NHCAP), das seine Mission ganz im Sinne der kulturellen Revitalisierung beschreibt und mit diesem „Mission Statement“ zugleich den Blick auf eine als bedeutend wahrgenommene hawaiische Vergangenheit richtet:

„We pay our highest tribute to our native Hawaiian predecessors and their unique and mighty civilization ... From the antagonisms that first beckoned ancient Polynesian explorers across vast unknown regions of

\footnotetext{
${ }^{253}$ Finney sah diese Forderungen durchaus als Bedrohung des gesamten vorgesehenen wissenschaftlichen Programms an (1979:76-91).

${ }^{254}$, „[...] Eia a'e ka wa'a holo / He wa'a 'imi ola / He ho'ālahia ka wa'a i ke kakahiaka / O kā i ka hoe e nā koa e paio ana / No Hawai'i, no ke aupuni / Eia ka lawe wai, he ka'apeha / 'O ia ka hō‘ailona $\bar{e}^{\prime \prime}$ (zit. nach Hartwell 1996:86; Übersetzung ebd.).

${ }^{255}$ Der Bau der auf Big Island beheimateten Makali'i wurde durch private Spenden und Zuwendungen des Office of Hawaiian Affairs finanziert, während an der Finanzierung des Hawai'iloa Projektes der US National Park Service beteiligt war.
} 
the Pacific to the horrific calamities that befell his [sic!] innocent yet wise Hawaiian descendants, grew an unyielding will to survive. It is from this struggle of our forebears, our Kupuna, that came the rich traditions and customs ... that sustained a highly sufficient society in total isolation. We hope to imbue all who follow with the pride that comes from understanding and appreciating these cherished ancestral gifts, to help them become keepers of their culture and environment and to improve the quality of life in Hawai'i. Our commitment...our mission is the perpetuation of our Hawaiian culture in the future for all generations“ (NHCAP 1991:1).

Die Hawai'iloa ist ein fast vollständig aus Holz und anderen traditionellen Materialien hergestelltes Doppelrumpfboot. Das für den Bau der beiden Rümpfe (bei denen es sich traditionell um Einbäume handelte) benötigte Holz konnte indes nicht auf den Inseln gewonnen werden - die von den frühen hawaiischen Bootsbauern verwendeten gewaltigen Stämme des Koa-Baumes (Acacia koa) sind heute auf den Inseln in der benötigten Größe nicht mehr zu finden. Das benötigte Baumaterial wurde nun nicht einfach importiert, sondern im Rahmen einer stark traditionalistisch betonten „indigenen Kooperation“ der Natur entnommen und nach Hawai'i überführt.

Unter Leitung des zukünftigen Navigators der Hawai'iloa, Nainoa Thompson, verhandelte eine hawaiische Delegation mit Vertretern der an der Westküste Alaskas lebenden Haida und Tlingit (SeAlaska Corporation) über die Überlassung von zwei Baumstämmen. Bei der Außendarstellung des Vorganges wurde der Umstand hervorgehoben, dass auch die Haida und Tlingit einem stark verändernden Druck der amerikanischen Mehrheitsgesellschaft ausgesetzt wären und wie die Hawaiier für den Erhalt ihrer gefährdeten Traditionen und Kultur Sorge zu tragen hätten (Simpson 1995:8). In einer feierlich inszenierten gemeinsamen Übergabezeremonie fand der Beschaffungsvorgang seinen Abschluss: ein Oberhaupt der Tlingit segnete die bereits gefällten Bäume und die in neo-traditionelle Lendentücher (Malo) gekleideten Leiter der hawaiischen Delegation baten die höheren Mächte um Verzeihung für den Eingriff in die Natur. ${ }^{256}$

Neben der Förderung traditioneller hawaiischer Seefahrtechniken sollte die Fahrt nach Tahiti auch eine Manifestation polynesischer Zusammengehörigkeit sein, was sich im Motto der Reise widerspiegelte: „Nā 'Ohana Holo Moana - Voyaging Families of the Vast Ocean“ (Bishop Museum 1995a). Durch den Gebrauch des Bildes der Familie ('Ohana) findet das Gefühl der Zusammengehörigkeit und verwandtschaftlichen Nähe der polynesischen

${ }^{256}$ Videopräsentation in der Ausstellung „Hawai'iloa: Ka 'Imi 'Ike - Seeker of Knowledge“ (Bishop Museum 14.1.-4.6.1995); siehe auch Finney 2006:308; NHCAP 1991. 
Nachbarn seinen knappen und zugleich schlagenden Ausdruck, verstärkt noch durch den Verweis auf die Weite des Ozeans (Holo Moana), der für Gemeinsamkeit, gemeinsame Heimat steht. - Poetischer Ausdruck und politischstrategische Absicht gehen hier eine (gelungene) Verbindung ein. Besonderen Nachdruck erhielt dieser politische Aspekt des Projekts, als auf der Rückfahrt von den Marquesas nach Hawai'i drei Doppelrumpfboote von anderen polynesischen Inseln die hawaiischen Boote begleiteten. ${ }^{257}$

Bei Ankunft der Boote in Hawai'i fanden in Hilo, Kualoa (O‘ahu) und Ke'ehi (Honolulu) feierliche Empfangszeremonien statt. In Kualoa an der Nordostküste der Insel O`ahu, in vergangenen Zeiten als einer der heiligsten Orte auf der Insel angesehen (cf. Pukui, Elbert u. Mookini 1974:119), vollzogen die Seefahrer ein Ritual, welches im Umfeld des „Polynesian Voyaging“ neu geschaffen und über die Jahre modifiziert wurde. Wie zuvor schon im Marae von Taputapuātea auf Ra 'iatea und in Taiohae auf Nuku Hiva legte jede der Mannschaften einen Stein von ihren Heimatinseln auf einen $A h u^{258}$, der aus Steinen der jeweils erreichten Insel aufgeschichtet worden war. So „untermauerten" die Seefahrer auch symbolisch die mit der Reise gefeierte gemeinsame Herkunft der heutigen Polynesier. Roland Maiola Logan, einer der Erbauer des $A h u$, wurde hierzu wie folgt zitiert:

„We'd like to look at it as another tree that grows ... The rocks on the ahu have brought spiritual blessings from all over Polynesia. We hope Kualoa can continue to be a meeting place for canoes and navigators. “ ${ }^{259}$

Gleichwohl blieb auch die hawaiische Gegenwart nicht außen vor: Der eben zitierte Erbauer des Ahu äußerte im gleichen Zusammenhang die Sorge, dass die staatliche Verwaltung die Zerstörung des (immerhin ungenehmigt errichteten) Ahu im „Kualoa Beach Park“ (so die offizielle Bezeichnung des „heiligen“ Ortes) anordnen könne. Auch die so sehr verehrten Hochseeboote und das sie umgebende hawaiische Zeremoniell blieben letztlich eingebunden in die (profanen) Regeln und Gesetze des Staates und waren, über den hawaiischen Kontext hinaus, immer auch Gegenstand medialer oder touristischer Vermarktung.

257 Bei diesen traditionellen Doppelrumpfbooten handelte es sich um „Te“Aurere“ (Aotearoa/Neuseeland) sowie „Te‘au o Tonga“ und „Takitumu“( beide von den Cook Islands).

${ }^{258}$ Das Wort Ahu hat vielerlei Bedeutungen: es kann sich um einen neutralen Steinhaufen, eine Erinnerungs- bzw. Gedenkstätte, allerdings auch um einen Altar handeln und wird in Hawai'‘ im Allgemeinen nicht übersetzt.

259 „Crews take stones to altar in Kualoa“, Bob Krauss (HA 12.5.95:A-3). 
Hawai 'iloa: Doppelrumpfboote in Medien und Öffentlichkeit

Das Bishop Museum in Honolulu, einer der Sponsoren des Bootes, begleitete die am 6. Februar 1995 begonnene Fahrt der Hawai‘iloa mit der Ausstellung „Hawai'iloa: Ka 'Imi 'Ike - Seeker of Knowledge“. Diese Schau zeigte die mit dem Bootsbau verbundenen Techniken und die traditionellen bzw. neotraditionellen zeremoniellen Aspekte der hawaiischen Seefahrt. Darüber hinaus stellte sie die persönlichen Erfahrungen einzelner am Bau und an der Ausrüstung des Schiffes beteiligter Hawaiier in den Mittelpunkt. Das Werbeblatt für die Ausstellung forderte die Besucher auf: „Celebrate the proud heritage and share the triumphs of Native Hawaiian voyagers, past and present.“ “260

Bereits die Ausstellungseröffnung am 14. Januar 1995 stand ganz im Zeichen des „Hawaiian Protocol“, das heute bei vielen mehr oder weniger offiziellen hawaiischen Veranstaltungen selbstverständlich zur Anwendung kommt und bestimmte (in aller Regel hawaiischsprachige) Formen der Begrüßung, des Dankes und der Verabschiedung einschließt. Dieses hawaiische Protokoll orientiert sich an überkommenen sowie neo-traditionellen Formen und Regeln der formalen hawaiischen Etikette. Bei der Eröffnung wurde es zudem ergänzt durch eine Ho'ola' $a$ - (Weihe-) Zeremonie, mit der die „Hawaiian Hall“, das älteste Gebäude des Museums erneut in den Dienst der Bewahrung und zugleich Förderung des hawaiischen kulturellen Erbes gestellt wurde.

„Prayers in Castle Building [ein neuerbautes Ausstellungsgebäude; U.M.] dedicated the voyaging exhibit as a proud testament to the perpetuation of Hawaiian culture for many generations to come. Ho 'okupu [zeremonielle Gaben; U.M.] and prayers of blessing, gratitude and inspiration were presented in honor of the new exhibit and were carried in ceremonial procession from Hawaiian Hall“ (Bishop Museum 1995b).

Ein wichtiger Bestandteil der Zeremonie war eine Prozession vom alten Museum zur neuen Ausstellungshalle, die Wright Bowman, Jr., der Erbauer der Hawai'iloa, und Gordon Pi'ianaia, ein ehemaliges Besatzungsmitglied der Hōkūle‘a, anführten. Beide trugen Paddel aus dem Besitz Kamehamehas IV. in die neue Ausstellung - wodurch die neu erbaute Hawai'iloa zugleich in die Entwicklungsgeschichte der zeitgenössischen hawaiischen Seefahrt und den weiteren Kontext der historischen Epoche vor der Entdeckung Hawai'is durch die Europäer eingereiht wurde. (Allerdings verschwiegen die Organisatoren der Eröffnungsfeier auch nicht, dass die Ausstellung maßgeblich von den Touris-

${ }^{260}$ „Bishop Museum Presents: Hawai‘iloa - Ka 'Imi 'Ike ...“, Informationsblatt zur Ausstellung (Bishop Museum 1995). 
musunternehmen „American Hawaii Cruises“ und „Outrigger Hotels Hawaii“ gesponsert wurde.)

Die beiden großen Tageszeitungen Honolulus (Honolulu Advertiser und Honolulu Star-Bulletin) verfolgten die Reise der Schiffe mit großem Interesse und hielten die Öffentlichkeit mit täglichen Positionsmeldungen und Berichten über besondere Ereignisse auf dem Laufenden. Die Tagespresse war dabei vor allem am Event-Charakter der Reise interessiert, der über unmittelbar hawaiische Interessen (wie z.B. pan-polynesische Gemeinschaft, [quasi-] religiöse Erneuerung, „Ethnic Pride“) hinausreichte. Insbesondere die Landung der Boote in Hawai'i (der erste Ankerplatz war Hilo) am 6. bzw. 7. Mai 1995 hatte großen Nachrichtenwert, wobei allerdings die Nachricht von der drohenden Einschleppung beißender Sandfliegen von den Marquesas und die damit möglicherweise verbundene massive Bedrohung des Fremdenverkehrs viele andere Ereignisse in den Schatten stellte. ${ }^{261}$ Die Berichterstattung hob besonders hervor, dass es die Seefahrer selbst waren, die staatliche Stellen auf die in den Bootsrümpfen brütende Gefahr hingewiesen hatten (als ob das Gegenteil zu erwarten gewesen wäre...). Die Reisenden selbst nutzten wiederum die durch die Bekämpfung der Fliegen entstandene erhebliche Verzögerung sogleich, um auf die großen ökologischen Gefahren hinzuweisen, die der hawaiischen Heimat durch Einschleppung fremder Tier- und Pflanzenarten drohe...

Im Verlauf der Reise räumte der Honolulu Advertiser der Diskussion über die mögliche Herkunft der ersten Hawaiier Priorität ein ${ }^{262}$ und dieselbe Zeitung gab im Vorfeld der Rückkehr einer ,inner-indigenen“ Debatte über die „Authentizität“ der Bootsbaupläne Raum, als deren Wortführer vor allem Herb Kāne (Baumeister der Hōkūle'a), der hawaiische Navigator Nainoa Thompson sowie der Kapitän und Konstrukteur der „Te‘au o Tonga“, Thomas Davis, auftraten. Der Zeitung zufolge bezog Nainoa Thompson einen durchaus interessanten (und diplomatischen) Standpunkt in Hinblick auf die Entwicklung traditioneller Bauweisen von Doppelrumpfbooten im Pazifik, ohne die offenbar von weniger Skrupeln geplagten Konstrukteure von den anderen Inseln herabzusetzen:

„Master navigator Nainoa Thompson said that ancient Polynesian voyaging canoe design developed by trial and error, and modern voyagers are now going through the same process. In the first open ocean trial of

\footnotetext{
261 So lauteten die Schlagzeilen uunter anderem: „War waged on canoe’s biting flies” (HA 5.5.1995:A-1); „Epic voyagers arrive to dubious aloha of bug spray“ (HA 8.5.1995:A-3); „Canoes were insect arks: state discovers 14 kinds after sea de-bugging” (HA 10.5.1995:A-3) oder „Munching midges put brake on canoes” (H S-B 4.5.1995:A-1).

${ }^{262}$ „Sunday Special: Did first Hawaiians come from Marquesas?“ (HA 16.4.1995:A-1, 9).
} 
its kind in modern times the six canoes ... left the Marquesas earlier this month on a historic voyage to Hawaii.“ ${ }^{263}$

Insgesamt ist die Berichterstattung in der Presse Hawai'is über diesen vielschichtigen „Event“ von positiven Bildern geprägt. Euphorisch schreibt z.B. der Honolulu Star-Bulletin zur Rückkehr der Segler: „For the first time in centuries, voyaging canoes from New Zealand and the Cook Islands have sailed to Hawaii...“ - und greift dabei weit über das Ziel hinaus: Denn auch nach den gelungenen Fahrten der modernen polynesischen Fernreiseboote gibt es keinerlei Hinweise darauf, dass in der Vergangenheit tatsächlich einmal Doppelrumpfboote von Aotearoa/Neuseeland nach Hawai‘i segelten. ${ }^{264}$

Auch wenn die Doppelrumpfboote inzwischen ein fast selbstverständlicher Teil hawaiischer Gegenwartskultur geworden sind, bleiben sie für viele Hawaiier in einem sehr positiven Sinne emotional besetzt. Neben ihren noch immer durchgeführten, mehr oder weniger spektakulären Reisen, sind die Boote heute eingebunden in Unterrichtsprogramme für Schüler unterschiedlicher Schulen (so z.B. „Mālama Hawai' $i$ / Caring for Hawai ${ }^{\prime} i^{\prime c}{ }^{265}$, in denen der Stolz auf die eigene hawaiische Herkunft, die Leistungen und das Wissen der Küpuna, der Vorfahren und Alten, im Vordergrund stehen. Clay Bertelman, früher einer der Kapitäne der Hōkūle'a und heute zur Mannschaft der Makali'i gehörig, sagte in diesem Zusammenhang: „We're trying to expose them to something positive ... something our ancestors did through thousands of years of migrations. And make them realize they should be proud of who they are.“ Und der Lehrer einer der betreuten Schülergruppen stellt fest: „It’s a collective dream of the Hawaiian people. The whole idea of way-finding and navigation is a great metaphor for us as we struggle to revive our culture“ (in: Theroux 2002:14).

\footnotetext{
263 „Three canoe designs sailing into dispute over history’s shape“, Bob Krauss (HA 2.5.1995:A-1,2).

${ }^{264}$ „Canoe feasts: returning the favors“, Greg Ambrose (H S-B 11.5.95:A-1).

265 „Program teaches community, promotes well-being“ (KWO 1997, Iune [June]:10); siehe auch Polynesian Voyaging Society (1997); das Programm wird unterstützt von der Polynesian Voyaging Society und dem Queen Lili‘uokalani Children’s Center, das als Teil der Stiftung Königin Lili‘uokalanis hawaiischen Kindern und Jugendlichen Betreuung und Unterstützung anbietet.
} 



\section{Das Gedenkjahr 1993: Brennpunkte symboli- scher Politik}

Im Jahre 1993 jährte sich der Sturz Königin Lili‘uokalanis (1838-1917) und damit das Ende des Königreichs Hawai‘i zum 100. Male. Fast alle Gruppierungen der hawaiischen Autonomiebewegung, aber auch staatliche Institutionen, öffentliche Einrichtungen, Kirchen sowie die Medien im Bundesstaat Hawai`i nutzten dieses Datum, um an die Vorgänge von 1893 zu erinnern. Es waren nicht nur unterschiedliche Formen des Gedenkens und des Erinnerns an die Vergangenheit, die verschiedene Ereignisse des Gedenkjahres charakterisierten. Mit ihnen verbanden sich auch sehr unterschiedliche Interessen der jeweiligen Veranstalter und Initiatoren.

Die Einhundertjahrfeier bildete den Hintergrund für zahlreiche Manifestationen symbolischer Politik sowohl vonseiten des Staates als auch der Autonomiebewegung und ihrer Vertreter. - Wobei hier der politikwissenschaftliche Begriff „symbolische Politik“ oder „Symbolpolitik“ verstanden werden soll als eine Politik, die „reale“ Politik oder Sachpolitik ersetzt und lediglich zu einer Verbesserung subjektiver Befindlichkeiten beiträgt, ohne objektiv Veränderungen herbeizuführen. Symbolpolitik meint hier aber auch die Verdeutlichung oder gar Überhöhung historischer Ereignisse durch den strategischen Einsatz von Symbolen (BPB 2011). Dörner (1996:26) benennt drei Ziele einer solchen Politik: Zum einen sei dies die Stärkung der eigenen Definitionsmacht; zum anderen können so Bedürfnisse nach „Orientierung, Sinn und Identität“ innerhalb des Gemeinwesens bzw. eines seiner Teile befriedigt werden; und schließlich bedeutet Symbolpolitik einen Zugewinn an politischer oder realer Macht, um den jeweiligen Status quo zu legitimieren oder in Frage zu stellen und eine Mobilisierung für oder gegen eine übergeordnete Einheit zu erreichen.

Der Sturz Lili‘uokalanis am 17. Januar 1893 ist der Dreh- und Angelpunkt der hawaiischen Autonomiepolitik, liefert er doch die völkerrechtliche Grundlage für die Bemühungen um Wiedererlangung einer hawaiischen Eigen- 
ständigkeit. Hinzu kommt eine sehr emotionale Verehrung, die gerade viele ältere Hawaiier der Person Liliu'okalanis entgegenbringen, und so verwundert es kaum, wenn das Jahr 1993 mit der Fülle seiner Demonstrationen, Gedenkund Informationsveranstaltungen sowie einer Vielzahl politischer Wortmeldungen zu einem Medienevent wurde. Das Gedenkjahr bildete darüber hinaus den Hintergrund für ein deutliches Anwachsen der öffentlichen Anteilnahme an der hawaiischen Geschichte sowie den sozialen Problemen und politischen Forderungen der heutigen hawaiischen Bevölkerung.

Dieses gesteigerte Interesse an den Fragen hawaiischer Selbstbestimmung zeigte sich u.a. in der Zahl jener Artikel, die in den beiden großen Tageszeitungen O‘ahus, dem Honolulu Advertiser und dem Honolulu Star-Bulletin, zum Thema erschienen: Waren es 1991 nicht mehr als 18 Texte, so wuchs diese Zahl bis 1993 um mehr als das elffache auf 201 Artikel an. ${ }^{266}$ Wie die folgenden Beispiele zeigen, trugen auch Zeitschriften und Wochenblätter dem vom Gedenkjahr hervorgerufenen Interesse Rechnung.

Schon im Juni des Vorjahres berichtete die Monatszeitschrift Honolulu über die Planungen für die Feierlichkeiten im Januar 1993. Der Artikel fasste dabei prägnant zusammen, welche Bedeutung dem Zusammenfall des Gedenkens an den Umsturz und der Existenz einer aktiven Bewegung sowie dem gewachsenen Bewusstsein für hawaiische Belange zukam. Die Autorin beschließt ihren Artikel mit den Sätzen:

„Next to Jan. 17, 1893, Jan. 17, 1993, could be the most important day in Hawaiian history. Hawaiian concerns may never again have as prominent a platform for rallying support for their cause“ (Otaguro 1992:52).

Die unabhängige Wochenzeitung Honolulu Weekly berichtete am 13. Januar 1993 unter dem Titel „100 Years of Subjugation“ (Ferrar u. Steel 1993) über die geplanten Feierlichkeiten. Die Zeitschrift nutzte diesen Artikel auch für die Vorstellung der unterschiedlichen, von hawaiischer Seite in die Diskussion eingebrachten Autonomiemodelle. Ein wöchentlich auf O‘ahu erscheinendes Anzeigenblatt MidWeek widmete seine Titelgeschichte vom 13. Januar ebenfalls der 100. Wiederkehr des Umsturzes von 1893 sowie einer Würdigung der hawaiischen Professorin und Ka Lāhui Hawai'i-Aktivistin Lilikalā Kame'eleihiwa (Sinnex 1993). Eine umfassende Literaturliste zum Thema fanden die Leser in der Kundenzeitschrift der Fluggesellschaft Aloha Airlines, die

\footnotetext{
${ }^{266}$ Die genannten Zahlen beruhen auf dem „Index to the Honolulu Advertiser and the Honolulu Star-Bulletin 1991-1992, 1993-1994“; sie schließen sowohl redaktionelle Artikel als auch Kommentare, Leitartikel sowie veröffentlichte Leserbriefe ein.
} 
mit einem ausführlichen Artikel über die hawaiische „Sovereignty“-Bewegung berichtete (Fullard-Leo 1993).

In den kontinentalen USA blieb das Medienecho auf die vorangegangenen wie auch die aktuellen Ereignisse im Inselstaat verhalten: Zwar berichteten große überregionale Zeitungen wie die New York Times über die Ziele der hawaiischen Autonomiebewegung, und auch die Tageszeitungen an der amerikanischen Westküste brachten anlässlich der Hundertjahrfeier einzelne Artikel. ${ }^{267}$ Abgesehen hiervon muss aber festgestellt werden, dass die USamerikanische Presse außerhalb Hawai' is kaum Notiz von den Entwicklungen im Inselstaat nahm. Blickt man auf den weiteren pazifischen Raum, so fällt die Berichterstattung der Zeitschrift Pacific Islands Monthly auf, die in der Novemberausgabe 1992 (Rampell 1992 a, b) mit einem Bericht über die bevorstehenden Gedenkveranstaltungen eine über die nächsten Jahre anhaltende Folge von Beiträgen zum Thema einleitete. ${ }^{268}$

In diesem Kapitel stehen zwei Ereignisse im Vordergrund, die das Geschehen im Gedenkjahr 1993 bestimmten: Zunächst richtet sich der Blick auf die Großveranstaltung „'Onipa‘a“, an der sich ein breites Spektrum von öffentlichen und privaten Institutionen sowie kulturellen und politischen Gruppierungen beteiligte. Der darauf folgende Abschnitt beschäftigt sich mit „Ka Ho'okolokolonui Kānaka Maoli“, der Inszenierung eines Tribunals gegen die USA, die mehrere Gruppierungen der Autonomiebewegung gemeinsam auf allen Inseln des Archipels durchführten. Aufgrund ihrer zeitlichen und auch personellen Nähe zu den Veranstaltungen des Jahres 1993 bezieht dieses Kapitel darüber hinaus die kontroversen Vorbereitungen zur Native Hawaiian Vote von 1996, einer staatlicherseits initiierten Abstimmung, mit der die Grundlagen für eine gelenkte Weiterentwicklung hawaiischer Autonomie gelegt werden sollten, sowie die hawaiischen Forderungen nach Rückgabe der Insel Kaho'olawe mit ein. So bot das Gedenkjahr den Akteuren und Protagonisten im Autonomiediskurs - neben der Propagierung sachlicher Forderungen - immer wieder Gelegenheiten zur Selbstdarstellung und Abgrenzung gegeneinander.

Während also in den vorausgegangenen Kapiteln verschiedene Aspekte historischer Entwicklungen im Vordergrund der Betrachtung standen, liegt das Augenmerk nun auf mehr oder weniger parallel stattfindenden Ereignissen, die sich vor dem sozialen und politischen Hintergrund der frühen 1990er Jahre

\footnotetext{
267 „A century after Queen’s overthrow, talk of sovereignty shakes Hawaii“, Robert Reinhold (New York Times 8.11.1992:14 y); „Anniversary stirs Hawaii sovereignty movement“ (New York Times 18.1.1993); „Native Hawaiian groups unite to seek sovereignty“, Bruce Dunford (Seattle Post-Intelligencer 27.4.1992); „Native Hawaiians seek sovereignty“, Annie Nakao (San Francisco Examiner 17.1.1993); „Invisible Hawaii emerges“, Annie Nakao (San Francisco Examiner 24.1.1993).

${ }^{268}$ Kernahan 1993; Tiffany 1993; Rampell 1993, 1994 a, b, 1995.
} 
entfalten. Die einmalige Bündelung von Ereignissen, wie sie das Jahr 1993 in Hawai‘i kennzeichnete, ermöglicht eine umfassende synchrone Sicht auf Politik und Dynamik der Bewegung, auf die Beziehungen zwischen ihren Einzelgruppierungen und nicht zuletzt auf die Rezeption ihrer politischen Zielsetzungen in der hawaiischen und auch der weiteren Öffentlichkeit des Bundesstaates Hawai‘i.

\subsection{Die Gedenkfeiern an den Umsturz von 1893: 'Onipa'a}

Der persönliche Wahlspruch Lili‘uokalanis „'Onipa‘a!“ - „Standhaftigkeit!“ wurde zum Motto einer Gedenkveranstaltung in Honolulu, die vom 13. bis zum 17. Januar 1993 große öffentliche Beachtung fand. Diese Aufmerksamkeit war dem Ereignis nicht nur wegen der gewählten Veranstaltungsformen gewiss - so bildete z.B. eine dramatische, vom Fernsehen übertragene Inszenierung der Vorgänge von 1893 an den Originalschauplätzen gewissermaßen die Klammer der gesamten Veranstaltung. Darüber hinaus trug auch die Vielzahl der beteiligten hawaiischen Organisationen sowie das starke Engagement des Bundesstaates und der Stadt Honolulu das Seine sehr dazu bei. Darstellung und Betrachtung dieses Abschnitts umfassen gleichermaßen die der „'Onipa“a!“Veranstaltung vorausgegangenen politischen Absichtsbekundungen und Planungen und darüber hinaus die unmittelbar auf die Veranstaltung folgenden Reaktionen, um so das in die Öffentlichkeit transportierte Bild hawaiischer Geschichte und gegenwärtiger Autonomievorstellungen ein Stück weit abzurunden und plastischer zu profilieren.

Die Planungen für 'Onipa'a und das Medienecho im Vorfeld der Gedenkfeier Die 'Onipa 'a-veranstaltung des Jahres 1993 ist ein erhellendes Beispiel für das oft nicht deutlich erkennbare Nebeneinander, die enge Vermengung von staatlichen Initiativen und Interessen einerseits und den Aktivitäten und Bestrebungen der Autonomiebewegung andererseits. Nicht zuletzt deshalb ist sie auch Gegenstand verschiedener Publikationen, die das Ereignis aus jeweils unterschiedlichen Gesichtspunkten betrachten und auf die ich mich im Folgenden u.a. beziehen werde. ${ }^{269}$

Mit einem Gesetz (Hawaii Session Laws 1992:738-40) legte das Parlament die rechtlichen und finanziellen Grundlagen für eine zentrale und offizielle Veranstaltung zur Erinnerung an den Sturz der letzten hawaiischen Monarchin und erklärte den 17. Januar 1993 zu einem ,day of commemoration for the people of the State to recognize and observe the life of Queen Liliuokalani“. Im Einzelnen sah das Gesetz die Übertragung der administrativen Aufgaben an das Office of Hawaiian Affairs (OHA) vor, dem zu diesem Zweck Geldmittel in

${ }^{269}$ Zu nennen sind neben anderen OHA 1994 b; Baker 1997; Schweizer 2005:423-46. 
Höhe von US\$ 180.000 aus dem Staatshaushalt zur Verfügung gestellt wurden, die durch Eigenmittel in gleicher Höhe zu ergänzen waren. Die eigentliche Planung des „Events“ oblag einem Organisationsgremium (,'Onipa‘a Centennial Committee") von 24 ehrenamtlichen Mitgliedern, deren Mehrheit hawaiischer Abstammung sein sollte. Des Weiteren bestimmte das Gesetz, dass Beiträge und Vorschläge hawaiischer Gruppen bei den Planungen zu berücksichtigen seien und dass die eigentlichen Feierlichkeiten auf das Wochenende des 17. Januar 1993 begrenzt sein sollten.

Im Unterschied zu anderen staatlicherseits eingerichteten Gremien, die sich mit hawaiischen Angelegenheiten befassten, spielten die einflussreichen Gruppierungen der Autonomiebewegung bei der Benennung der Mitglieder keine Rolle: Bei den meisten von ihnen handelte es sich um mehr oder weniger bekannte Namen der hawaiischen Kulturszene sowie staatlicher und nichtstaatlicher Institutionen (cf. Baker 1997:647). Neben der Sponsorensuche ${ }^{270}$ sowie der Organisation der zentralen Feierlichkeiten stand die Einbindung der verschiedenen von Einzelpersonen und hawaiischen Organisationen (unter ihnen auch Ka Lāhui Hawai'i) in die Gesamtplanung eingebrachten 'Onipa'aProjekte.

Als Veranstaltungsort sahen die Organisatoren das Areal des 'Iolani Palastes vor - eine nahe liegende, jedoch zunächst nicht unproblematische Wahl: Hatte doch im Juni 1992 eine Eingreiftruppe der Polizei eine unangemeldete hawaiische Kundgebung beendet und insgesamt 32 Personen verhaftet. ${ }^{271}$ Dies gab Befürchtungen gewaltsamer Auseinandersetzungen im eigentlichen Gedenkjahr Nahrung und warf Fragen nach der Nutzung des symbolträchtigen Areals durch Hawaiier auf, die in der Presse ausgiebig diskutiert wurden. ${ }^{272}$ So scheint ein Leitartikel des Honolulu Star-Bulletin bereits von mehr oder weniger gewalttätigen Ausschreitungen im folgenden Jahr auszugehen:

„It [die Aufhebung der Klagen gegen die Demonstranten; U.M.] provides one less issue to fuel the militancy of celebrants of next year's $100^{\text {th }}$ anniversary of the overthrow of the monarchy. Meetings at

\footnotetext{
${ }^{270}$ Neben Institutionen des Bundesstaates und der Stadt Honolulu sind auch zahlreiche bedeutende Unternehmen der Finanz- und Tourismusindustrie auf der Sponsorenliste zu finden (so u.a. Hawaiian Airlines, Aloha Airlines, Bank of Hawai'i, Hawai‘i National Bank, Aloha Petroleum und Outrigger Hotels; siehe OHA 1997:X).

${ }^{271} \mathrm{Zu}$ den Zielen dieser Aktion vor dem 'Iolani Palast siehe Kawehi Kanui Gill (1992).

${ }^{272}$ Siehe z.B. „Arrests anger Hawaiians“, David Oshiro (H S-B 12.6.1992:A1, A4); „Protest worries Iolani friends”, Pat Omandam (H S-B 13.6.1992:A3); "Iolani Palace: learning from a protest”, (HA 13.6.1992); "Feared palace would be stormed, official says”, Kevin Dayton (HA 13.6.1992:A1-2); “28 protesters plead not guilty”, Shannon Tangonan (HA 20.6.1992:A1); "State drops charges in palace case” (HA 31.7.1992:A1); „Hawaiians urged to find palace protocol accord“, Stu Glauberman (HA 2.7.1992:A1-2); „Hawaiians keep gathering low-key, hopes high“, Kris Tanahara u. William Kresnak (HA 5.7.1992:A1-2).
} 
Kawaiahao Church between state officials and leaders of Hawaiian religious and community groups resulted in guidelines to govern activities at the palace, and a July $4^{\text {th }}$ program commemorating the 1894 start of the short-lived Hawaiian republic took place without incident.

The July $4^{\text {th }}$ event and now the dismissal of the June 11 trespassing charges offer fresh hope that 1993's centennial can be observed in an atmosphere of civility and respect”. ${ }^{273}$

In den Wochen vor dem Jahrestag erschien eine Vielzahl von Veröffentlichungen in der lokalen Presse Hawai'is: historische Darstellungen fanden sich darunter ebenso wie aktuelle Kommentare zu den 100 Jahre zurückliegenden Ereignissen sowie deren Folgen, und auch die Forderungen der Autonomiebewegung wurden veröffentlicht und (kritisch) beleuchtet. Eine der umfangreichsten Veröffentlichungen war „Overthrow: a day-by-day account of the overthrow of Hawaii's monarchy 100 years ago“ - eine 16 Druckseiten umfassende Beilage des Honolulu Advertiser. ${ }^{274}$ Auf diese Beschreibung des Umsturzes (der Honolulu Advertiser kündigte sie als „objective look at the events and issues involved“ an) folgte eine Serie von Artikeln, die „subjektiven“ Ansichten zum Thema gewidmet waren.

Das multikulturelle Hawai 'i und die „Gefahr“ der Autonomie

Als letzter Autor dieser kurzen Serie kam der damalige Gouverneur John Waihe‘e zu Wort. ${ }^{275}$ Waihe‘e, erster und bislang einziger hawaiischstämmiger Gouverneur des Inselstaates, bezog in seinem Textbeitrag keinen klaren Standpunkt hinsichtlich hawaiischer Autonomieentwürfe. Nachdem der Gouverneur den Umsturz als „internationale Aggression“ und „Unrecht“ charakterisiert und die seines Erachtens wesentlichen Punkte für eine weitergehende Besserstellung der Hawaiier benannt hatte, schloss er den Artikel mit den Worten:

„Finally, as governor of all the people of Hawaii, I have strong concerns that, in the process of returning self-determination to Hawaiians, we may 1) ultimately fail to improve the lot of all Hawaiians and 2) inadvertently undermine the multi-cultural community that has taken generations to work out. We must not do either. [...]

Ours is a special place, created out of a unique history. The cultural experiment that was thrust upon us through coincidences was ours to

\footnotetext{
273 „Dropping the charges“, (H S-B 31.7.1992:A3); siehe auch „State sensitivity needed in future demonstrations“, (H S-B 13.6.1992:A3).

274 „Overthrow: a day-by day account of the overthrow of Hawaii’s monarchy 100 years ago“, (HA 19.11.1992); diese Darstellung des Umsturzes wurde u.a. auch von Hui Na'auao in die von dieser Organisation verteilten Informationsmaterialien aufgenommen.

${ }^{275}$ Waihe'e war von 1986 bis 1994 Gouverneur des Bundesstaates Hawai ‘i.
} 
fashion. It could have divided us, as it has other communities in other parts of the world faced with a diversity of members. Or it could define us. Fortunately, we collectively chose to let our diversity define who we are. In striving to right the grievous wrong done to Hawaiians as a people and as a nation, we also need to keep that in mind.“ ${ }^{276}$

Waihe'e beschrieb die Entwicklung der multikulturellen Gesellschaft Hawai'is als Erfolgsgeschichte, die offenkundige und vielfach untersuchte andauernde Benachteiligung großer Teile gerade der hawaiischen Bevölkerung findet sich verklausuliert am Anfang des Zitats. Ein eher symbolischer Akt des Gouverneurs sollte später darauf verweisen, wie groß das Konfliktpotenzial einer eindeutigeren Positionierung hinsichtlich des Umsturzes und seiner Auswirkungen offenbar hätte sein können: Für die Dauer der 'Onipa'a-Feierlichkeiten ordnete Waihe'e das Einholen der amerikanischen Flagge an und ließ sie durch die Flagge des Königreichs Hawai'i ersetzen, die allerdings in unveränderter Form bis heute die Flagge des Bundesstaates geblieben ist. Eine heftige Kontroverse, die sich auch in den Leserbriefspalten der Tageszeitungen niederschlug, war die Folge. ${ }^{277}$

Die Wochen vor der eigentlichen Gedenkfeier boten den Medien aber nicht nur Anlass zur Auseinandersetzung mit der Geschichte des Bundesstaates, auch die hawaiische Autonomiebewegung, ihre Vertreter und Ziele waren Gegenstand zahlreicher Artikel und Kommentare (so etwa in der Serie „Strength from the past“ im Honolulu Star-Bulletin). Unter ihnen fanden sich Darstellungen der einflussreichsten Gruppierungen innerhalb der Bewegung ${ }^{278}$, Berichte über Beispiele autonomer Regionen in den kontinentalen USA und im Pazifik, die Vorbilder für die Entwicklungen in Hawai'i sein könnten, ${ }^{279}$ und schließlich verschiedene Umfragen, die das Meinungsklima in der hawaiischen Bevölkerung zum Gegenstand hatten. ${ }^{280}$ Die Vielzahl der angesprochenen

276 „Sovereignty: Hawaiians must find its meaning for $21^{\text {st }}$ century”, John Waihe‘e (HA 3.1.1993:B1).

${ }^{277}$ Schweizer (2005:443-5) widmet diesem Vorgang eingehend Aufmerksamkeit. Waihe'e war letztlich Repräsentant der Regierung eines in seiner Mehrheit nicht-hawaiischen Gemeinwesens, die antagonistische Positionen bestenfalls zusammenführen konnte.

${ }^{278}$ So z.B. „Sovereignty: a dormant nation stirs“ / „New leaders are mapping the path to sovereignty“, Becky Ashizawa (H S-B 11.1.1993:A1, A6); „The making of a nation” / „Sovereignty could mean total independence or U.S. ties“, Becky Ashizawa (H S-B 12.1.1993:A1, A6).

279 „Native Americans provide sovereign clues“ / „Hawaiians told to plan carefully“ / „Autonomy in Pacific Isles a primer for Hawaiians“, Tino Ramirez (H S-B 13.1.1993:A1, A7).

${ }^{280}$ So waren einer solchen Umfrage des Honolulu Star-Bulletin zufolge 73\% der Hawaiier für eine direkte Kontrolle bestimmter Landflächen durch Hawaiier und nur 26\% lehnten die Idee der „Native Hawaiian Sovereignty“ ab. Weitere veröffentlichte Ergebnisse waren u.a. die Befürwortung der Einbeziehung von Nicht-Hawaiiern in den Entwicklungsprozess einer hawaiischen Nation durch 54\% und dass immerhin 52\% der Hawaiier meinten, die Bevölkerung Hawai'is stünde der hawaiischen Kultur aufgeschlossen gegenüber; „Hawaiians serious on sovereignty“, 
Themen sowie der in Artikeln und Interviews von Bewegungsvertretern geäußerten Meinungen wies aber auch auf eine Problematik hin, die von dem „Trommelfeuer“ der Berichterstattung nicht nur nicht gelöst, sondern fast noch verstärkt wurde: Für viele „Außenstehende“, zu denen 1993 der größte Teil der Bevölkerung zu zählen war, und wohl auch für die meisten Hawaiier selbst stellten die unterschiedlichen Ziele und Herangehensweisen der verschiedenen Gruppierungen ein zunächst verwirrendes Spektrum politischer, ökonomischer und sozialer Perspektiven dar. ${ }^{281}$ Ein Leitartikel des Honolulu Star-Bulletin kommentiert drei Tage vor dem Beginn der 'Onipa'a-Feier diese Vielfalt von Äußerungen hawaiischen Strebens nach Autonomie:

„[...] Does sovereignty mean secession from the United States? A selective immigration policy for mainlanders and foreigners? The vagueness of response on these points among sovereignty advocates is confusing and disturbing.

Does sovereignty mean a designated area for native Hawaiian residency, much like Navajo reservations in Arizona and New Mexico? If so, which land would comprise it and how many islands be part of it? [...] Since most people here agree the events of 1893 were unjust and deserve apologies and some kind of repayment, the question is what should be done about it. If sovereignty is to be part of a federal settlement for past debts and injustices, the burden is on those pursuing the goal to define its boundaries."282

Es fällt auf, dass der Autor seine Aufzählung mit einigen für die meisten NichtHawaiier bedrohlich wirkenden Szenarien begann: einer Sezession Hawai'is, der Begrenzung und Regelung der Zuwanderung von US-Bürgern sowie eingeschränkter Bewegungs- und Niederlassungsfreiheit auf den Inseln für Bürger nicht-hawaiischer Abstammung. Nun besteht zwar hinsichtlich vieler Fragen Uneinigkeit innerhalb der Autonomiebewegung, doch fällt es schwer, dem Autor keine tendenziösen Absichten zu unterstellen: hätte doch das aufmerksame Lesen der eigenen Zeitung deutlich machen müssen, dass es zwar Meinungsverschiedenheiten zwischen den hawaiischen Organisationen und Gruppierungen gab (und gibt), jedoch jede einzelne von ihnen, und zumal Ka Lāhui Hawai`i, Ka Pākaukau oder das I.A.H.A., sehr genaue Vorstellungen von ihren

Becky Ashizawa (H S-B 6.1.1993:A1, A8); „Non-Hawaiians role for nation backed“, Becky Ashizawa (H S-B 15.1.1993:A6).

${ }^{281}$ Um den Informationsstand der (hawaiischen) Bevölkerung hinsichtlich der „Hawaiian Sovereignty“ zu heben, wurde bereits im September 1991 Hui Na'auao, ein Zusammenschluss von ca. 40 hawaiischen Organisationen, gegründet. Hui Na'auao führte Informationsveranstaltungen durch und gehörte zu den Hauptsponsoren des mehrtägigen 'Onipa'a-Schauspiels.

282 „Sovereignty terms could use clearer definition“, (H S-B 12.1.1993:A16). 
jeweiligen Zielen hatte und diese der Öffentlichkeit auch keinesfalls vorenthielt. Allerdings weist der Kommentar auch darauf hin, dass es den verschiedenen Trägern der hawaiischen Bewegung bis zu diesem Zeitpunkt nicht gelungen war - trotz der Bemühungen von Hui Na'auao, dem Office of Hawaiian Affairs und anderen - der Öffentlichkeit die Notwendigkeit einer andauernden innerhawaiischen Diskussion und Meinungsfindung hinsichtlich Autonomie und Sovereignty nahe zu bringen.

„'Onipa'a - Five Days in the History of the Hawaiian Nation"

„Fünf Tage in der Geschichte der hawaiischen Nation“ - so lautet der Titel einer vom Office of Hawaiian Affairs herausgegebenen aufwendig gestalteten Publikation über den Ablauf der 'Onipa'a-Feierlichkeiten (OHA 1994b). Die Herausgeber waren sich dabei der Mehrdeutigkeit des Titels (der zum einen den Rückblick auf fünf Tage im Januar 1893 beschreibt und zum anderen und zugleich einen Bericht über die Gegenwart der hawaiischen Nation während fünf Tagen im Januar 1993 ankündigt) durchaus bewusst. Im Klappentext des Buches heißt es:

the descendants of the ancient civilization that once thrived in these Pacific islands united to express their love for the memory of their Queen Lili'uokalani, to mourn the overthrow of the Hawaiian Kingdom on January 17, 1893, and to call out to the world that the right of selfdetermination must be restored to their sovereign nation" (OHA 1994b:Klappentext vorn).

Die fünf Tage dauernden Festlichkeiten präsentierten sich als breites Spektrum aktueller hawaiischer Formen kultureller und politischer Selbstvergewisserung: Neben der Nachstellung historischer Ereignisse, der Aufführung von hawaiischer Musik und traditionellem Tanz gehörten hierzu auch historisierende Traditionspflege und revitalisierte kulturelle Praktiken. Bei Demonstrationszügen, die ebenfalls verschiedene der genannten Elemente einbezogen, untermauerte der Bezug auf den Umsturz von 1893 die politischen Forderungen der Gegenwart.

Zwischen Parlamentsgebäude und 'Iolani Palast steht eine Statue der Königin Lili‘uokalani, die Besucher nach wie vor regelmäßig mit frischen Blumen schmücken - hier fand am Mittag des 13. Januar 1993 die offizielle Eröffnung der „'Onipa‘a Centennial Observance“ statt. ${ }^{283} \mathrm{Zu}$ den geladenen Gästen zählten zahlreiche Offizielle aus Staats- und Stadtverwaltung, Mitglieder des Office of Hawaiian Affairs sowie internationale Würdenträger. Unter

${ }^{283}$ Siehe zu Eröffnung und weiterem Verlauf der Feier OHA (1993a:10), OHA (1994b:23 ff.), Schweizer (2005:423 ff.). 
den Rednern waren die Vorsitzende des Planungsgremiums Senatorin Tungpalan, Gouverneur Waihe'e, der hier unter großem Applaus seine Entscheidung verkündete, die US-Flagge im historischen Regierungsbezirk von Honolulu einzuholen, und der damalige Vorsitzende des Office of Hawaiian Affairs, Clayton Hee. Grußbotschaften der beiden Senatoren im US-Senat sowie der US-Kongressabgeordneten des Staates Hawai'i wurden verlesen. Am Nachmittag fand dann mit einer Mahnwache des Councils of Hawaiian Organizations die erste symbolträchtige Veranstaltung im Rahmen der Gedenkfeiern statt: Vertreter der Organisation entzündeten 100 Fackeln und begannen eine 100-stündige Wache zur Erinnerung an den Sturz der Königin. Bereits am ersten Veranstaltungstag zeichneten sich deutliche politische Bruchlinien ab: So richtete Ka Lāhui Hawai'i als bedeutendste der staatsfernen politischen Gruppierungen eine eigenständige Parallelveranstaltung aus: eine als „Kūpuna ${ }^{284}$ Vigil“ bezeichnete vierstündige Mahnwache, die auch an den folgenden Tagen wiederholt wurde.

Jeder der weiteren Veranstaltungstage begann mit einer Andacht, von den Veranstaltern als „Spiritual Ho'okupu ${ }^{285}$ to Queen Lili‘uokalani“ angekündigt, an der Gouverneur Waihe'e, Mitglieder der „Royal Societies“ (Ali ‘iGesellschaften, wie sie bis heute in Hawai'i bestehen) sowie geladene Vertreter zahlreicher hawaiischer Organisationen teilnahmen. Bestimmendes Element eines jeden Tages waren aber die Episoden des Schauspiels „January 1893“, das an den Originalschauplätzen der Ereignisse aufgeführt wurde. ${ }^{286}$ Daneben fanden Konzerte der Royal Hawaiian Band mit zeitgenössischer Musik statt und verschiedene Präsentationen machten mit traditioneller hawaiischer Handwerkskunst bekannt. Ein neo-traditionelles Element zahlreicher Einzelveranstaltungen im Rahmen der Gedenktage war die Einleitung durch Kantoren, die überlieferte Trauergesänge und Gebete anstimmten, sowie die begleitende Aufführung von Tänzen im Stil des ausgehenden 19. Jahrhunderts.

Der 17. Januar 1993, der eigentliche Gedenktag an den erzwungenen Thronverzicht Lili‘uokalanis, begann mit einer Gedächtniszeremonie der „Royal Societies“ in Mauna 'Ala, der Begräbnisstätte aller hawaiischer Monarchen seit Kamehameha III. Fast zur gleichen Zeit formierte sich in der Innenstadt von Honolulu eine sehr gegensätzliche und auf die Gegenwart bezogene Veranstaltung: Unter der Leitung von Ka Lāhui Hawai'i versammelten sich Tausende von Hawaiiern und zogen in einem Demonstrationszug zum 'Iolani

\footnotetext{
${ }^{284}$ Kupuna (Plural: Kūpuna) bezeichnet Mitglieder der Großelterngeneration bzw. Ahnen und Vorfahren.

${ }^{285}$ Das Wort Ho'okupu bezeichnete in der traditionellen hawaiischen Gesellschaft die als Zeichen des Respekts an ein Oberhaupt gegebenen zeremoniellen Geschenke.

${ }^{286}$ Das mehrtägige Schauspiel, in das mehr als einhundert Schauspieler und Statisten eingebunden waren, wurde von Hui Na'auao zur Information der Öffentlichkeit gesponsert. Siehe zum Ablauf ausführlich OHA (1994b:53 ff.), Schweizer (2005:425-33).
} 
Palast. ${ }^{287}$ Lilikalā Kame'eleihiwa, selbst Sprecherin auf der Schlusskundgebung, zählte in einem Artikel des Journal of Pacific History zahlreiche Redner und ihre jeweiligen Themen auf. Indem sie zu den Redebeiträgen von Ka Lāhui-Vertretern sehr spezifische Angaben machte, die Beiträge politischer Rivalen eher pauschalisierte und beim Office of Hawaiian Affairs gar auf die namentliche Nennung der Redner verzichtete, ging sie dabei sehr wertend vor dies wirft ein Schlaglicht auf die trotz des gemeinsamen Gedenkens fortbestehende und ausgeprägte Konkurrenz zwischen den einzelnen Autonomiegruppierungen und ihren Vertretern:

„The topics included events of Hawaiian history (by Terry Kanalu Young), discussion of an appropriate land base (Lilikalā Kame'eleihiwa [Ka Lāhui Hawai'i; U.M.]), analysis of American and Democratic Party opposition to the sovereignty movement (HaunaniKay Trask [Ka Lāhui Hawai‘i; U.M.]), a detailed description of how the nation would work (Mililani Trask [Ka Lāhui Hawai'i; U.M.]), a call for the entire island chain to be returned (Kekuni Blaisdell/Hayden Burgess [Ka Pākaukau, IAHA; U.M.]), a plea to regard all Hawaiians, regardless of political chicanery, as potential nice-guy Hawaiians (Dennis 'Bumpy' Kanahele [Nation of Hawai'i; U.M.]), and a suggestion that all Hawaiians should work with the appropriate state agency to achieve sovereignty (Office of Hawaiian Affairs)“ (Kameeleihiwa 1993:65).

Ungenannt bleibt in Kame'eleihiwas Auflistung der Präsident der Generalsynode der United Church of Christ (USA), Paul H. Sherry: Er verlas auf dieser Kundgebung - bereits vor der offiziellen Verkündung am Nachmittag - eine „Apology to Nā Kānaka Maoli““. ${ }^{288}$ Mit dieser nicht unumstrittenen Erklärung

\footnotetext{
${ }^{287}$ Über die Zahl der Teilnehmer liegen unterschiedliche Angaben vor: Während die Polizei eine Zahl von 10.000 schätzte („10.000 march to sounds of sovereignty“, Shannon Tangonan [HA 18.1.1993:A3]), gingen die Organisatoren von 15.000 (Kame'eleihiwa 1993:63) oder sogar 16.000 Teilnehmern aus (OHA 1994b:106). Ich wähle hier die am häufigsten genannte Teilnehmerzahl von 15.000 (vgl. Schweizer 2005:439). Bleibt anzumerken, dass die Gewaltlosigkeit des Demonstrationszuges sowie das Fehlen von Ausschreitungen während der Kundgebung der Presse durchaus eine Notiz wert war („10.000 march to sounds of sovereignty“, Shannon Tangonan (HA 18.1.1993:A3).

${ }^{288}$ Die United Church of Christ (UCC) ist die Rechtsnachfolgerin des American Board of Commissioners for Foreign Missions und vertritt heute 110 Kirchengemeinden in Hawai ${ }^{\wedge}$, die sich zum Teil noch auf Gründungen der Missionare zurückführen lassen. Der Text der Entschuldigung ist vollständig abgedruckt bei Schweizer (2005:436-8). Die Entschuldigung der Kirche geht zurück auf einen Grundsatzentscheid der Generalsynode der UCC im Jahr 1991 („Missionaries' church backs sovereignty: an official apology to native Hawaiians is in order, it says“, Becky Ashizawa [H S-B 6.7.91:A3]).
} 
akzeptierte die Nachfolgerin der früheren Missionarskirche deren Verstrickungen in den Sturz der hawaiischen Monarchie und sicherte den Hawaiiern zugleich Unterstützung bei ihrem Streben nach Wiedergutmachung zu:

„[...] We formally apologize to you for 'our denomination's historical complicities in the illegal overthrow of the Hawaiian Monarchy in 1893,' by unduly identifying the ways of the West with the ways of the Christ, and thereby, undervaluing the strengths of the mature society that was native Hawaii. [...]

Our General Synod resolution promises advocacy for state and national legislation in support of 'grass roots initiatives toward selfgovernment.' We commit ourselves this day to establish a task force to work in partnership with you and the Hawaii Conference as you seek self-determination and justice. We make these promises in the hope that redress may be achieved. [...]“ (Hervorhebungen im Original; zitiert nach Schweizer 2005:438).

Wie die Autoren des vom Office of Hawaiian Affairs herausgegebenen 'Onipa'a-Bildbandes herausstellten, darf die Bedeutung einer solchen Erklärung für die große Zahl gläubiger hawaiischer Christen - und unter ihnen insbesondere die Mitglieder der UCC - wohl nicht unterschätzt werden: „For many Hawaiians who are devout Christians, the apology of the United Church of Christ helped reconcile their personal faith with what they had long known of their history“ (OHA 1994:112). Ebenfalls festzuhalten bleibt aber auch, dass sich die durchaus vorhandene innerkirchliche Ablehnung der Erklärung auch aus Stimmen der Gemeinden mit überwiegend hawaiischer Mitgliedschaft speiste. Eine eindeutige hawaiische Haltung hinsichtlich der historischen Verantwortung ihrer eigenen Kirche war also nicht gegeben. ${ }^{289}$

$\mathrm{Zu}$ den Abschlussinszenierungen 'Onipa'as zählte schließlich eine 'Awa-Zeremonie (Kawa-Zeremonie) auf dem Gelände des 'Iolani Palastes eine Veranstaltung, in der sich Aspekte kultureller Revitalisierung, die medienwirksame Inszenierung hawaiischer Vergangenheitsvorstellungen und politische Forderungen der Gegenwart auf exemplarische Weise verbanden. Während der Zeremonie, organisiert und durchgeführt von Ka Pākaukau und dem Council of Hawaiian Organizations (COHO), reichten Männer, in neotraditionelle Lendentücher gekleidet, Trinkschalen mit 'Awa an die im Kreis sitzenden Teilnehmer, die sich im Gegenzug zu den gemeinsamen Zielen der

\footnotetext{
${ }^{289}$ Siehe hierzu auch „Local rifts hamper church’s move to aid sovereignty“, Kris Tanahara (HA 30.1.1993:A5). Es waren nicht nur einige Haole Gemeinden der UCC, sondern auch Vertreter hawaiischer Gemeinden (ca. 50 Gemeinden haben eine überwiegend hawaiische Mitgliedschaft), die sich gegen den Text der Entschuldigung aussprachen bzw. diese generell für unnötig hielten.
} 
hawaiischen Selbstbestimmung und Unabhängigkeit bekannten (OHA 1994b:108). Diese Zeremonie verknüpfte die mit dem Konsum von 'Awa verbundenen Vorstellungen der alten hawaiischen Gesellschaft und Religion ${ }^{290}$ mit den politischen Forderungen der Gegenwart - ein Aspekt, der während der mehrtägigen Gedenkfeier immer wieder herausgestellt wurde.

Nachdem die letzten Akte des Schauspiels „January 1893“ das Ende der hawaiischen Monarchie nachgestellt hatten, klang der Tag mit Fackelmärschen und Gesang sowie der feierlichen Beendigung der 100-stündigen Mahnwache des Council of Hawaiian Organizations aus. Insgesamt stand bei den 'Onipa'a-Feierlichkeiten das stille Gedenken an die ehemalige Königin Lili‘uokalani und ihr Schicksal im Vordergrund. So reflektierte das Gesamtprojekt, aber auch der größte Teil der in ihm gebündelten Einzelveranstaltungen einen der zentralen Momente hawaiischer Geschichte. Ho'okupu, Mahnwachen, die mehrtägige Aufführung des Schauspiels und Fackelmärsche im Gedenken an den Umsturz waren Elemente einer Rückbesinnung, die in konkrete und direkte politische Forderungen und Aktionen hätten umgesetzt werden können - im Rahmen von 'Onipa'a 1993 fand dies mit Ausnahme der Demonstration und Kundgebung Ka Lāhuis nur sehr begrenzt statt.

'Onipa' $a$ - politische Wirkungen über den Tag hinaus?

Schweizer bewertete die Wirkung der 'Onipa'a-Gedenkveranstaltung in seiner Untersuchung zur hawaiischen Nationalstaatlichkeit durchaus sehr positiv: „In Hawai ' $i$,ONIPA 'A' changed the minds of many, and there is reason to believe that future historians will recall the Centennial Observance of the Overthrow as a major turning point in the history of the islands" (2005:461). Es ist unstrittig, dass die mehrtägige Gedenkfeier zahlreiche Hawaiier erstmals anregte, sich überhaupt mit hawaiischer Geschichte, dem Ende des Königreichs und staatlicher Eigenständigkeit auseinander zu setzen. Ebenso machte die deutliche Betroffenheit einer so großen Zahl von Hawaiiern auch vielen Nicht-Hawaiiern wohl zum ersten Mal deutlich, wie legitim der hawaiische Anspruch auf Wiedergutmachung oder gar „Sovereignty“ womöglich doch ist. Insgesamt aber blieben die von vielen in dieses Ereignis gesetzten Erwartungen unerfüllt - ein

\footnotetext{
${ }^{290}$ Dem gemeinsamen Trinken der 'Awa oder Kawa könnten durchaus auch Aspekte christlicher Rituale zugeschrieben werden - was ihr im Kontext der Gegenwart besondere Bedeutung zukommen ließe. Der christliche hawaiische Autor Samuel Mānaiakalani Kamakau (1815-1876) schreibt über die Zeremonie: „Over the 'awa cup were handed down the tabus and laws of the chiefs, the tabus of the gods, and the laws of the gods governing solemn vows and here the wrongdoer received absolution of his wrongdoing. That was the way, and the priestly practice, of ka po'e kahiko. This was how it was recognized that the gods heeded the repentance of the people: they granted blessings to the race; they increased 'food' and 'fish' and mankind; and they warded off misfortunes that might come, such as diseases, epidemics, contagious diseases, and sudden disaster“ (1992a:43-4).
} 
gemeinsames Vorgehen hawaiischer Gruppierungen und eine Veränderung des Status quo blieben aus. Im Folgenden werde ich Kommentare und Leitartikel des 17. Januars 1993 betrachten und so den Blick nochmals auf widerstreitende Meinungen zu „’Onipa‘a 1993“ sowie zu den Vorgängen von 1893 richten, die sich bei aller Einhelligkeit über die Eindrücklichkeit der Veranstaltung doch nicht annäherten. Abschließend werde ich kurz der Frage nachgehen, warum „'Onipa“a 1993“ außerhalb Hawai' is eine ausgesprochen geringe Aufmerksamkeit zuteil wurde - auch dies m.E. ein Faktor für die weitgehende politische Wirkungslosigkeit der Großveranstaltung.

Am 17. Januar 1993, dem Höhepunkt und Abschluss der Gedenktage, erschienen in der gemeinsamen Sonntagsausgabe der beiden großen Tageszeitungen Honolulus (Honolulu Advertiser und Honolulu Star-Bulletin) vier Artikel, deren Autoren bzw. Inhalt sehr gegensätzliche Einstellungen zu Vorgehensweisen und Zielen der Hawaiier, ja zur grundsätzlichen Existenzberechtigung einer hawaiischen Autonomiebewegung vertraten bzw. aufzeigten. Je einem Gastkommentar von Clayton Hee, dem damaligen Vorsitzenden des Office of Hawaiian Affairs, und von Haunani-Kay Trask, damalige Direktorin des Center for Hawaiian Studies an der University of Hawai'i at Mānoa und erklärte Gegnerin des Office of Hawaiian Affairs, stellte die Ausgabe Auszüge aus der Proklamation des „Provisional Governments“ vom 17.1.1893 gegenüber. ${ }^{291}$ Abgesetzt von diesen Artikeln erschien ein offener Brief des USSenators Daniel Akaka an den neu gewählten US-Präsidenten Bill Clinton ${ }^{292}$ sowie ein Leitartikel, der - wie üblich - namentlich nicht gekennzeichnet war und auf den noch gesondert einzugehen sein wird.

Haunani-Kay Trask verfasste ihren Kommentar in der ihr eigenen „scharfen“ und polarisierenden Sprache, für die sie oft kritisiert und angegriffen wurde, die jedoch Dinge auch auf den Punkt zu bringen vermag: Nach einer kurzen Einleitung stellt sie die Vereinnahmung der Bewegung durch den Staat und seine „Kollaborateure“ ${ }^{293}$ fest, so dass grundlegende Veränderungen unter-

\footnotetext{
291 „Sovereignty: out of pride, not prejudice“, Clayton Hee (HA/H S-B 17.1.1993:B1, B3); „Money cannot substitute for Hawaiian land base“, Haunani-Kay Trask (HA/H S-B 17.1.1993:B1, B3); „Why committee moved to end ,monarchial' rule“ (HA/H S-B 17.1.1993:B3).

${ }^{292}$ „Will Clinton help right these wrongs?: Hawaiians are entitled to an apology, recognition, self-determination”, Daniel K. Akaka (HA/H S-B 17.1.1993:B3). Am Ende der Liste Akakas mit Wünschen an den neuen Präsidenten (formale Entschuldigung der Bundesregierung, die Rückgabe Kaho'olawes, Wahrnehmung der Verpflichtungen aus dem Hawaiian Homes Commission Act, Aufhebung der Ungleichbehandlung der Hawaiier und der Native Americans hinsichtlich der Bemühungen um Wiedergutmachung sowie die formale Abweisung der Ergebnisse der Native Hawaiians Study Commission von 1983) steht die Aufforderung an die Bundesregierung, einen von ihr initiierten Prozess hin zu mehr Selbstbestimmung für die Hawaiier einzuleiten.

${ }^{293}$ Es sei hier darauf hingewiesen, dass das von Trask verwendete englische Wort „collaborator“ nicht notwendigerweise jene „kriegerischen/verräterischen“ Konnotationen besitzt, die dem
} 
blieben und der Status quo im Wesentlichen festgeschrieben wurde. Zu den „Kollaborateuren“ oder „Zuarbeitern“ zählt sie hawaiische Institutionen und Organisationen wie das das Office of Hawaiian Affairs, die Protect Kaho'olawe 'Ohana (eine Gruppierung der Autonomiebewegung und Mitglied von Ka Pākaukau) sowie die Native Hawaiian Legal Corporation. ${ }^{294}$ „Größter Herausforderer“ des Bundesstaates und seiner Politik der „Umarmungen“ sei dagegen ihre eigene Gruppierung Ka Lāhui Hawai'i, deren Ziele sie im Anschluss kurz vorstellt. Sie schließt ihren Artikel mit einem deutlichen, aber auch sehr rigorosen Standpunkt hinsichtlich der Beteiligung von NichtHawaiiern (und somit dem überwiegenden Teil der Bürger Hawai'is) an den Bestrebungen der Autonomiebewegung:

„Both the native and the general public need to understand the effort by the Democratic Party, especially Dan Inouye [US-Senator Hawai'is; U.M.] and John Waihee, to short-circuit Hawaiian self-determination. [...] The role of non-natives is to support our efforts, not to overtake or obstruct them. Everyone who lives in Hawai'i must learn that Hawaiians are the first people of the land and thus deserve to reclaim their nationhood.“

Clayton Hee hingegen versieht seinen Beitrag, der im Wesentlichen die Pläne des Office of Hawaiian Affairs vorstellt, mit einem weitaus versöhnlicheren Schluss. Er verweist auf die vielfältigen verwandtschaftlichen Beziehungen zwischen Hawaiiern und Angehörigen anderer ethnischer Gruppen in Hawai ‘i - die Gründung einer neuen hawaiischen Nation kann ihm zufolge, und dies steht in krassem Gegensatz zu den Äußerungen Trasks, nur durch die gleichberechtigte Zusammenarbeit aller in Hawai'i lebenden Menschen geschehen.

Auf diese beiden Artikel folgten Textauszüge aus der Proklamation der provisorischen Regierung von 1893: Unter der Überschrift: „Why committee moved to end ,monarchial' rule“ wies die Zeitung in einer kurzen Einleitung lediglich darauf hin, dass im weiteren Text die Gründe für das „Eingreifen“ des „Committee of Safety“ zu finden wären. Weitgehend unkommentiert standen

deutschen „Kollaborateur“ eigen sind. Neben dieser Bedeutung stehen im Englischen gleichberechtigt „Mitarbeiter“ bzw. „Zuarbeiter“.

${ }^{294}$ Gerade den beiden letztgenannten wirft sie die Verhinderung der hawaiischen Eigenverwaltung von Landflächen vor: die Protect Kaho'olawe 'Ohana arbeitete vor allem für die Übertragung der Insel Kaho'olawe, die der Administration der US Navy unterstand, an eine zivile (möglichst hawaiische) Institution und war durchaus bereit, auf den Staat Hawai'i und die Bundesregierung zuzugehen. 
sie hier gewissermaßen als weitere Meinung gleichberechtigt neben den Kommentaren der beiden hawaiischen Autoren. ${ }^{29}$

Völlig unbeeindruckt von den Stellungnahmen Clayton Hees und Haunani-Kay Trasks zeigte sich der Autor des bereits erwähnten Leitartikels des Sunday Star-Bulletin \& Advertiser vom 17.1.1993. ${ }^{296}$ Auch wenn der Vorsitzende der Association of Hawaiian Civic Clubs, Bruss Keppeler, allen Lesern in einem Leserbrief nachträglich riet, den Artikel zu ignorieren, ${ }^{297}$ rechtfertigen die hier an prominenter Stelle und zu einem besonderen Zeitpunkt offenbar werdende Sicht auf die Geschichte Hawai'is sowie der lockere Umgang mit historischen Fakten eine ausführlichere Betrachtung des Textes. Nach einigen verständnisvollen Sätzen zur gegenwärtigen Diskussion der Ereignisse vom 17. Januar 1893 stellt der Autor fest:

„Fixing blame for the overthrow has become a rallying point for sovereignty efforts. If the overthrow is deemed an illegal act of the United States, for instance, a demand for reparations from Washington and recognition of a sovereign Hawaiian nation would be more likely to succeed. Without going into the welter of facts and opinions that surround the event, it seems to us not unreasonable to argue that, in the context of $19^{\text {th }}$ century history, the 'legality' of any revolution depended on which side you were on.

\footnotetext{
295 Angesichts einer über die historischen Ereignisse von 1893 noch immer mehr oder weniger uninformierten Öffentlichkeit hätten historische Aussagen, wie die im Folgenden zitierten, durchaus eines Kommentars bedurft, wenn die Absicht nicht darin bestand, einen allzu günstigen Eindruck von den Umstürzlern zu zeichnen: „This [die Regierung Kalākauas; U.M.] finally resulted in the revulsion of feeling and popular uprising of 1887 which wrested from the King a large portion of his ill-gotten powers“ [gemeint ist hier die erzwungene Unterzeichnung der ,Bajonett-Verfassung’ durch Kalākaua im Jahre 1887; U.M.]; oder: „Upon the accession of Her Majesty Liliuokalani, for a brief period the hope prevailed that a new policy would be adopted. This hope was soon blasted by her immediately entering into conflict with the existing cabinet, who held office with the approval of a large majority of the Legislature, resulting in the triumph of the Queen and the removal of the Cabinet. [...] The recent history of that session [gemeint ist die Legislatur der Sitzungsperioden 1892-93; U.M.] has shown a stubborn determination on the part of Her Majesty to follow the tactics of her late brother, and in all possible ways to secure an extension of the royal prerogatives and an abridgement of popular rights“. Dass es hier vor allem um die „popular rights“ einer Haole-Minderheit ging, deren Angehörige zu einem großen Teil nicht einmal über die Staatsbürgerschaft des Königreiches verfügten, hätte in dem hier gegebenen Zusammenhang doch einer Kommentierung bedurft (alle Zitate aus „Why committee moved to end ,monarchial’ rule“, HA/H S-B 17.1.1993:B3).

296 „Overthrow centennial: focus now on Hawaii's, and Hawaiians', needs for $21^{\text {st }}$ century”, (HA/H S-B 17.1.1993:B2); zu diesem Leitartikel und Kritik daran siehe auch Schweizer (2005:440-1).

297 ) „Your editorial ... is so full of misstatements and untruths as to render all of it suspect. I would advise your readers to simply ignore it“ (zitiert bei Schweizer 2005:441).
} 
Revolutions tend to be called 'illegal' by the people being overthrown, but Americans and French look back at their own anti-monarch revolutions a century before Hawaii's as glorious episodes of their history that brought new rights to the people.

We do not feel the facts support the allegation that the overthrow here was a premeditated act by the highest levels of the U.S. government against the Hawaiian people. [...] The overthrow of the Hawaii monarchy did not occur in a vacuum of time and place. As a commentary in last Sunday's paper pointed out, monarchies all over the world fell during the $18^{\text {th }}$ and $19^{\text {th }}$ centuries. By the late 1800 s, only five remained: Japan, Thailand, Ethiopia, China, and Hawaii“ ${ }^{298}$

Diese offensichtliche Rechtfertigung des Umsturzes (und seine Gleichsetzung mit der Erringung der US-amerikanischen Unabhängigkeit von Großbritannien) spricht den Hawaiiern eine privilegierte Stellung im Gefüge der Einwohnerschaft Hawai'is mehr oder weniger ab. Im weiteren Verlauf des Textes machte der Autor seine Sicht auf die historische Entwicklung Hawai' is, die er als stetige Verbesserung der allgemeinen Lebensumstände darstellt, erneut deutlich:

„When Queen Lili‘uokalani assumed the throne and wanted to move to restore the powers of the monarch, rejecting the 1887 constitution, overthrow of that government followed.

Leaders of the business community and the community at large acted to make sure Hawaii remained viable economically and became a place where all citizens were secure and had a voice in their wellbeing. [...] Subsequent changes in the government, from the Provisional to a Republic, to a territory of the United States and finally to a state, all broadened personal freedoms and the role and responsibilities of average citizens in government.“

\footnotetext{
${ }^{298}$ Es steht außer Frage, dass die Beteiligung von US-Truppen am Sturz der hawaiischen Monarchie weder durch das nationales amerikanisches Recht noch durch das Völkerrecht gedeckt war; der Begriff der „Revolution“, zumal in der Gleichsetzung mit der Französischen Revolution, wurde hier sehr „frei“ verwendet - bei den Vorgängen vom Januar 1893 handelte es sich vielmehr um einen von einflussreichen Geschäftsleuten initiierten Staatsputsch zur Sicherung ihrer Vorteile. Auch die im Zitat aufgeworfene Frage, ob es sich bei dem Umsturz um eine von der US-Regierung von langer Hand geplante Aktion handelte, erscheint gegenüber der Tatsache der schließlich gegen das Völkerrecht erfolgten Annexion doch eher unerheblich. Vollkommen den Boden der Tatsachen verlässt der Autor schließlich mit seiner Feststellung, im ausgehenden 19. Jahrhundert hätten nur noch fünf Monarchien auf der Welt bestanden - die konstitutionelle Monarchie Hawai ‘ $i$ befand sich vielmehr nach wie vor in der Gesellschaft eines großen Teils der europäischen Nationen.
} 
Der Leitartikel endet mit einer kurzen Liste von Maßnahmen, die im Wesentlichen eine Fortsetzung und Intensivierung bestehender Programme und Verpflichtungen nennt, und sich entschieden gegen eine Fortdauer bestehender Sonderrechte der Hawaiier ausspricht: „And, ultimately, a strong Hawaii economy with a united population will provide a better life for Hawaiians and everyone else ... Even as we recognize the special place Hawaiians and their culture have in the history of this land, we must not divide the community." Die implizite Gleichsetzung etwaiger zukünftiger hawaiischer Sonderrechte mit der ethnischen Spaltung der Bevölkerung kann Ängste schüren und die $\mathrm{Zu}$ stimmung der Bevölkerungsmehrheit gefährden. Dass es sich hier um eine zwar sehr deutliche, aber nicht um eine allein stehende Meinung handelte, zeigten auch Aussagen des damaligen Gouverneurs John Waihe'e, der - wenn auch in einem weniger polemischen Kontext - ganz ähnliche Äußerungen hinsichtlich des Zusammenhalts der Bevölkerungsgruppen in Hawai' $i$ veröffentlicht hatte (siehe oben). ${ }^{299}$

„... could it be that the interest is only designed to allay Hawaiian passion as this emotional day is recognized for the $100^{\text {th }}$ time?"300 Es war ausgerechnet der Vorsitzende des Office of Hawaiian Affairs, einer der Veranstalter der Gedenkfeiern, der mit dieser wohl eher rhetorisch eingesetzten Bemerkung eine Frage aufwarf, die sich hinsichtlich der Resonanz 'Onipa 'as durchaus stellen konnte: Denn die seit dem Ende des 19. Jahrhunderts mächtigste Demonstration für politische Selbstbestimmung der hawaiischen Bevölkerung blieb jenseits der Grenzen Hawai'is weitgehend unbeachtet.

Lag das fehlende Interesse an den Ereignissen im Inselstaat an der vieles überlagernden Berichterstattung über die Vorbereitungen zur Amtseinführung des neuen Präsidenten Bill Clinton am 21.1.1993, wie Schweizer (2005:445) meinte, oder waren es die am 17.1.1993 ausgeführten amerikanischen Bombenangriffe auf eine Nuklearfabrik vor Bagdad, die eine Berichterstattung über die hawaiischen Proteste gegen „historical incursions of American imperialism“ ungelegen erscheinen ließen, wie Baker (1997:646) vermutet? In einem Artikel des unabhängigen Wochenblattes „Honolulu Weekly“ ging der Journalist Curt Sanburn (1993) der Frage nach und stellte fest, dass eine Benachrichtigung der großen nationalen und internationalen Presseagenturen, Zeitungen und Fernsehstationen von den Organisatoren der Gedenktage bewusst unterlassen worden war. ${ }^{301}$ Auf die Frage, ob die außerhalb Hawai' is ansässigen Medien informiert wurden, antwortete die Öffentlichkeitsreferentin des Office of Hawaiian Affairs: „No, we didn’t. We weren’t holding this event

\footnotetext{
299 „Sovereignty: Hawaiians must find its meaning for $21^{\text {st }}$ century”, John Waihe'e (HA 3.1.1993:B1).

300 „Sovereignty: out of pride, not prejudice“, Clayton Hee (HA/H S-B 17.1.1993:B1, B3).

${ }^{301}$ Vgl. hierzu auch Baker (1997:646-8).
} 
for the media“; und Wendy Hee, zuständig für die Öffentlichkeitsarbeit des 'Onipa'a Komitees, erwiderte auf die gleiche Frage: „The committee did not do anything to draw national attention to the event ... Our attitude was to accommodate [the national press] if they appeared, but it was not a high priority“. Auch die PR-Beraterin des Office of Hawaiian Affairs, Barbara Tanabe, äußerte sich ähnlich: „,... we didn’t want to make this a commercial event““ ${ }^{302}$ Die über das mehrtägige Gedenken hinausweisende politische Dimension der Gesamtveranstaltung blieb bei diesen Überlegungen der (hawaiischen!) Veranstalter ganz offensichtlich unberücksichtigt. Im Gegensatz dazu steht eine Stimme aus der Autonomiebewegung selbst - Kekuni Blaisdell (Ka Pākaukau) stellte gegenüber Sanburn fest:

„... we're not going to get anywhere until we take our appeal beyond Hawaii's shores. We must have U.S. attention - global attention! We have to get on CNN. It’s essential“ (Blaisdell zit. nach Sanburn 1993).

Auch trugen wohl wirtschaftliche Erwägungen zur Einschränkung der Pressearbeit bei (Baker 1997:646-7): Gefragt, ob in frühen Sitzungen des Vorbereitungskomitees Vorbehalte gegen eine umfangreichere Berichterstattung geäußert worden wären, antwortete Mark Segami, ehemaliger Pressereferent des 'Onipa'a Komitees: „Sure, ... there was concern in the visitor industry that it might give people a reason not to come to Hawaii“ (zit. nach Sanburn 1993). Auch bereits anwesende Touristen sollten nicht auf die politische Bedeutung der Veranstaltungen aufmerksam gemacht werden. Bereits am 7.1.1993 versandte das staatliche Hawaii Visitors Bureau (HVB) eine offizielle Stellungnahme seines Direktoriums, das die Tourismusbetriebe zu einem zurückhaltenden Umgang mit den Feierlichkeiten aufforderte und diese implizit zu einer alleinigen Angelegenheit der Hawaiier erklärte. So schrieben sie u.a.: „There will be many observances throughout the islands to mark this centennial and we will ask our visitors to approach such events with respect and dignity. The ceremonies are of a very personal nature to the Hawaiian community and are not intended as visitor attractions.“ (HVB, Fotokopie in der Hawaiian Collection, UH Mānoa).

Mililani Trask, damalige Vorsitzende von Ka Lāhui Hawai'i, die bereits im Oktober 1992 mit der Versendung eigener Presseankündigungen begonnen hatte, stellte in diesem Zusammenhang kategorisch fest:

„They [der Staat; U.M.] didn't want to publicize their own negligence. State policy tries to paint Hawaii as a multi-ethnic paradise, twisting the truth to suit the needs of the Hawaii Visitors Bureau. Our whole

${ }^{302}$ Alle Zitate nach Sanburn (1993) 
culture is grossly commercialized for the visitor industry and this kind of intentional inaction, this black-out, is part of it“" (M. Trask zit. nach Sanburn 1993).

Auch wenn sich dieser Vorwurf einer „Verschwörung“ des Staates, der Tourismusindustrie und des Organisationskomitees kaum nachweisen lässt, spricht vieles für die Analyse Bakers (1993:648): Er sieht die fehlende Berichterstattung über Demonstration und Kundgebung vom 17.1.1993 als Resultat eines vorsätzlich konstruierten „Unwissenheitseffekts“, der die Autonomiebewegung und damit die Idee einer souveränen hawaiischen Nation aus dem Informationskreislauf heraushalten sollte - um so, wie auch schon M. Trask konstatierte, das „Paradies“ Hawai'i und seine wichtigste Einnahmequelle, den Tourismus, „unbeschädigt“ zu lassen.

Vor diesem Hintergrund erscheint die rhetorische Frage des OHA Vorsitzenden Clayton Hee zur eigentlichen Funktion der 'Onipa'a Feier im Kontext der staatlichen Politik ganz und gar nicht mehr abwegig. Die Eingrenzung der Berichterstattung auf den lokalen Rahmen und die Darstellung der Feierlichkeiten als „hawaiische Angelegenheit“ haben der Großveranstaltung einen Teil ihrer Wirkungsmöglichkeiten genommen. Gerade die politischen, explizit auf zukünftige Entwicklungen des gesamten Bundesstaates zielenden Forderungen $\mathrm{Ka}$ Lāhuis und anderer Autonomiegruppierungen gerieten so in den Kontext eines lokalen, darüber hinaus mit einer folkloristischen Note versehenen Ereignisses, das zwar eine gewisse Stärkung des Problembewusstseins im Staat Hawai'i bewirkte, dem aber zugleich eine „Ventilfunktion“ hinsichtlich der politischen Mobilisierung der hawaiischen Bevölkerung nicht abgesprochen werden kann. Im nationalen oder gar internationalen Rahmen (beide von großer Bedeutung für die Durchsetzung politischer Forderungen nach Autonomie und Wiedergutmachung) konnte 'Onipa'a, die bis heute größte hawaiische Gedenk- und Protestveranstaltung, keine nachhaltigen Reaktionen hervorrufen.

Abschließend soll das Augenmerk noch auf ein zufällig ausgewähltes deutsches Beispiel zur Rezeption des Ereignisses im fernen Hawai'i gerichtet werden. Unter der Überschrift „Ureinwohner Hawaiis attackieren die USA“ veröffentlichte die Süddeutsche Zeitung am 19.1.1993 eine Agenturmeldung der Presseagentur Associated Press:

„Etwa 10.000 Eingeborene haben in Honolulu gegen die Regierung der USA und deren Rolle beim Sturz der letzten hawaiianischen Königin Lilioukalani [sic! Liliuokalani; U.M.] vor 100 Jahren demonstriert. Die Menge zog singend und lärmend zum ehemaligen königlichen Palast, wo Haunani Trask, eine Professorin der Universität von Hawaii ausrief: ,Sie (die US-Amerikaner) sind unsere Feinde, sie nahmen unser Land weg, sie haben unsere Königin eingesperrt, sie haben unsere Sprache 
verboten, sie haben uns gewaltsam zu einer Kolonie der Vereinigten Staaten gemacht.' Bei der Demonstration waren Gruppen mit unterschiedlichen Zielsetzungen vertreten, die von Autonomie für Eingeborene bis zur Gründung einer unabhängigen Republik reichen. 1898 wurde Hawaii von den USA annektiert.“303

In diesem kurzen Text, der mit der Verwendung der Bezeichnungen „Ureinwohner“ oder „Eingeborene“ zugleich exotistische Vorstellungen der Leserschaft bedient, ist von der Vielschichtigkeit der Ereignisse nur noch wenig zu spüren. Die Einmaligkeit der Meldung (es gab keine Folgeberichterstattung), die für die als Zitat gekennzeichnete „Zusammenfassung“ der Aussagen Trasks (einer von zahlreichen Rednerinnen und Rednern) gewählte „einfache“ Sprache und die Einordnung der Meldung in die Rubrik „Panorama“ werfen darüber hinaus ein Schlaglicht auf das geringe Interesse und die mangelnde Ernsthaftigkeit, mit denen Meldungen zur hawaiischen Autonomiebewegung auch in einer der renommierten überregionalen Zeitungen Deutschlands behandelt wurden.

303 „Ureinwohner Hawaiis attackieren die USA“, Süddeutsche Zeitung (19.1.93:8). 


\subsection{The People's International Tribunal Hawai' $i$}

War 'Onipa'a eine maßgeblich von staatlichen Stellen initiierte Großveranstaltung, an der zwar zahlreiche hawaiische Gruppen und Organisationen mitwirkten, so ging Ka Ho'okolokolonui Kānaka Maoli ${ }^{304}$ („,The People’s International Tribunal Hawai`i) auf eine ausschließlich hawaiische Initiative im Gedenkjahr 1993 zurück. Im Rahmen dieser Veranstaltung wurde auf fünf Hauptinseln des Archipels gegen die USA und ihre Vertreter „,verhandelt“: zu den Anklagepunkten zählten Vergehen im Zusammenhang mit dem Umsturz 1893, der Annexion 1898 sowie der Aufnahme Hawai' is in die Union im Jahre 1959. Maßgeblicher Initiator dieser Inszenierung eines „Tribunals“, das keinerlei offiziellen Status besaß, war die Gruppierung Ka Pākaukau. Zu den Unterstützern zählte eine Vielzahl unterschiedlicher Gruppierungen und Organisationen; alle staatlichen Stellen (mit Ausnahme einiger Universitätsinstitute) sowie das Office of Hawaiian Affairs trugen - aus nachvollziehbaren Gründen - nicht zur Durchführung bei. ${ }^{305}$ Die Veranstalter hatten die Daten für Eröffnung und Schlussverhandlung (das Tribunal dauerte vom 12. bis zum 21. August 1993) mit Bedacht gewählt: am 12. August 1898 annektierten die USA das Territorium Hawai'i, am 21. August 1959 wurde Hawai‘i zum 50. Staat der Vereinigten Staaten, die Jahreszahl 1993 stand schließlich für den Umsturz und das Ende des formal unabhängigen Königreiches.

Der Ablauf des „Tribunals“ folgte dem Vorbild anderer „inoffizieller“ Tribunale wie z.B. dem Russel-Tribunal von 1967, ${ }^{306}$ auf das auch im „Opening Statement“ verwiesen wurde (Ka Ho'okolokolonui 1993:2). Das beteiligte Personal ${ }^{307}$ setzte sich aus internationalen Teilnehmern zusammen: Von den neun „Richtern“ stammten sechs aus den USA (unter ihnen drei Angehörige so genannter „ethnic minorities“ - Afro-Amerikaner, Cree und

\footnotetext{
${ }^{304}$ Das Große Gericht der Hawaiier; U.M.

${ }^{305}$ Unter den mehr als 30 Unterstützern waren verschiedene Gruppen der Autonomiebewegung vertreten (u.a. Ka Lāhui, Protect Kaho‘olawe ‘Ohana), hawaiische Organisationen wie die Native Hawaiian Legal Corporation oder das Native Hawaiian Advisory Council, kirchliche Organisationen, Institute der University of Hawai'i (u.a. Center for Hawaiian Studies UH Mānoa, Ethnic Studies Program UH Mānoa, Department of Hawaiian Studies UH Hilo), nationale (wie das American Indian Movement AIM) und internationale Organisationen (u.a. Nuclear Free and Independent Pacific NFIP, International Work Group for Idigenous Affairs IWGIA) (Blaisdell u. Kelly 1993:15; Blaisdell et al. 1994:2).

${ }^{306}$ Gegenstand des von dem Philosophen Bertrand Russel initiierten, im Jahre 1967 in London, Stockholm und Kopenhagen tagenden Tribunals waren Kriegsverbrechen der USA in Vietnam das Russel-Tribunal diente späteren Menschenrechtstribunalen als Vorbild.

${ }^{307}$ Die Bezeichnungen der geladenen Teilnehmer orientierten sich an den im US-amerikanischen Gerichtswesen üblichen Begriffen. Diese Bezeichnungen werden hier beibehalten, auch wenn die jeweiligen Beteiligten keine offizielle Rolle innehatten.
} 
Cherokee) ${ }^{308}$, die anderen drei aus Südkorea, Neuseeland und Jordanien. ${ }^{309}$ Ebenfalls aus den USA kamen die drei „Ankläger“. ${ }^{310}$ International besetzt war wiederum die Gruppe von 23 Beobachtern. Niemand aus diesen direkt beteiligten Gruppen war hawaiischer Herkunft - so sollte die Unabhängigkeit des „Tribunals“ gewährleistet und zugleich nach außen dokumentiert werden. Vertreter der „Beklagten“ blieben den Verhandlungen fern und wurden während der einzelnen Veranstaltungen durch einen freigehaltenen Stuhl repräsentiert. ${ }^{311}$

Das von den Vereinten Nationen ebenfalls für 1993 ausgerufene „International Year of the World's Indigenous People“ sollte verstärkte Aufmerksamkeit auf die Situation indigener Bevölkerungen in aller Welt lenken - die hawaiischen Veranstalter des Tribunals nutzten die zufällige Parallelität der Ereignisse und machten von den daraus sich ergebenden Möglichkeiten der Vernetzung mit anderen indigenen Bewegungen Gebrauch (Blaisdell u. Kelly 1993:9). Durch Einladungen an Vertreter indigener Gruppen aus den kontinentalen USA und dem Pazifik ${ }^{312}$ unterstrichen sie die internationale Ausrichtung ihrer Veranstaltung, deren Ziele sie wie folgt beschrieben:

„1. To elicit and assemble a comprehensive record of the crimes committed by the US government and its subsidiaries ... against the Kanaka Maoli people and nation.

2. To educate the people of Hawaii, the US and the world community of these crimes.

\footnotetext{
${ }^{308}$ Die amerikanischen „Richter“ waren Milner S. Ball (Prof. f. Verfassungsrecht, University of Georgia), Ward Churchill (Prof. f. American Indian Studies, University of Colorado), Richard Falk (Prof. f. Internationales Recht, Princeton University), Lennox Hinds (Prof. f. Rechtswissenschaft, Rutgers University), Oda Makoto (Autorin und Literaturkritikerin) und Sharon Venne (Rechtsanwältin).

${ }^{309}$ Hyung-Kyung Chung (Theologieprofessorin, Ewha Women’s University Seoul), Te Moana Nui a Kiwa Jackson (Vorsitzender des Maori Legal Service, Wellington) und Asma Khader (Anwalt, Amman).

${ }^{310}$ Maivân Clech Lâm (Prof. f. Rechtswissenschaft, City University N.Y.), José Morin (Anwalt und Direktor der North Star Foundation), Glenn Morris (Direktor, Fourth World Center for the Study of Indigenous Law and Politics, University of Colorado).

311 „Tribunal brings out litany of ills foisted on Hawaiians“, Christopher Neil (HA 14.8.93:A5); eine Einladung an Präsident Clinton, an dem Tribunal teilzunehmen bzw. einen Vertreter zu entsenden, hatte das Organisationskomitee am 31.7.1993 abgesandt, also nicht einmal zwei Wochen vor dem ersten Verhandlungstag (Abdruck des Schreibens in Da Skupes [1993,1<7>:89]). Es steht zu vermuten, dass die Veranstalter die Anwesenheit staatlicher Vertreter nicht wirklich erwartet hatten, auch wenn offen bleibt, ob der Präsident der USA bei zeitigerer Einladung vielleicht reagiert hätte.

${ }^{312}$ Diese von den Veranstaltern als „International Witnesses“ bezeichneten Teilnehmer kamen aus Guam, den Philippinen, Amerikanisch Samoa, Tonga, Puerto Rico sowie verschiedenen Indianernationen der USA (Blaisdell u. Kelly 1993:8; Blaisdell et al. 1994:9). Eingeladen, aber nicht erschienen, war ebenfalls ein Vertreter aus Kuba.
} 
3. To analyze the causes, consequences and implications of these crimes.

4. To establish an historical, moral and legal basis for remedies and to propose effective strategies for appropriate redress“ (Blaisdell u. Kelly 1993:10).

Bei den meisten offiziellen Veranstaltungen im Rahmen von 'Onipa'a 1993 standen der Blick in die Vergangenheit und das Gedenken an den Umsturz und den Verlust staatlicher Unabhängigkeit im Vordergrund. Im Kontext des Tribunals sollten Umsturz und Annexion hingegen Ausgangspunkt für eine in die Zukunft gerichtete Politik und die weitere Konsolidierung der Hawaiischen Bewegung sein. ${ }^{313}$ Durch seine am Völkerrecht orientierte Konstruktion sollten der hawaiischen Autonomiebewegung Strategien zur Erlangung der Unabhängigkeit aufgezeigt und eröffnet werden: ${ }^{314}$ zum einen die Einforderung und Anwendung des in einer „Draft Declaration on the Rights of Indigenous Peoples“ festgeschriebenen Rechts auf Selbstbestimmung indigener Völker, ${ }^{315}$ zum anderen die Wiederaufnahme Hawai' is in die UN-Liste jener Gebiete, die von den Vereinten Nationen zur Dekolonisierung vorgesehen werden können (Blaisdell u. Kelly 1993:10).

Im Einführungsheft zum Ho'okolokolonui Kānaka Maoli nahmen die Autoren dessen späteren Urteilsspruch auf gewisse Weise vorweg:

„The Tribunal Hawai' $i$ is unique. It is a first for Hawai' $i$, for the US and for the World Community. It will bring to light at one time, experiences and insights not previously set down in any book nor allowed to be openly expressed before the whole world without being first filtered by the perpetrator - the US colonial government.

Through the voices of the unheard victims in the past and present, covering over 200 years, the Tribunal will deliver not merely evidence that demands a verdict, but a verdict that compels redress.

\footnotetext{
${ }^{313}$ Als konkrete und zentrale Ziele wurden in diesem Zusammenhang genannt: die Verbesserung der Lebensumstände der Hawaiier, die Wiedergutmachung des durch die USA begangenen historischen, moralischen und juristischen Unrechts an den Hawaiiern sowie eine Umkehr der USPolitik der politischen und militärischen Dominanz, der ökonomischen Ausbeutung und kulturellen Homogenisierung (Blaisdell u. Kelly 1993:10).

${ }^{314} \mathrm{Zu}$ den juristischen Aspekten des Tribunals siehe Merry (1997).

${ }^{315}$ Die Draft Declaration, erarbeitet von der United Nations Working Group on Indigenous Populations in Genf, wurde im Jahr 2007 von der UN-Vollversammlung ratifiziert. Der vollständige Text der „Declaration on the Rights of Indigenous Peoples“ ist verfügbar unter United Nations (2007).
} 
The US, a modern Goliath, will be confronted by our Kānaka Maoli people, a modern David, before the court of world opinion for all to judge” (Blaisdell u. Kelly 1993:10).

Auch schien es hier nicht so sehr auf Fragen der Verhandlungsführung und juristischer Beurteilung relevanter Tatbestände anzukommen, sondern eher um eine effektive Kommunikation bzw. Präsentation von Informationen zur kolonialen Vergangenheit und Gegenwart Hawai'is zu gehen. ${ }^{316}$ Das Presseecho blieb - im Gegensatz zu der ausführlichen Berichterstattung über die Ereignisse im Januar 1993 - eher gering. ${ }^{317}$ Die lokale Presse glaubte hinsichtlich des „Urteilsspruchs“ nicht an Überraschungen - ein Kommentator des Honolulu Advertiser schrieb denn auch bereits am dritten Tag des Tribunals: „There’s not much suspense attending the verdict in the ,International Tribunal' review of the Hawaiian monarchy now under way.“ ${ }^{318}$

\section{Das Tribunal}

Zu keinem Zeitpunkt gab sich die Veranstaltung den Anschein, durch das Recht der Vereinigten Staaten oder der Vereinten Nationen legitimiert zu sein vielmehr sahen die Veranstalter wie auch die „Richter“ ihre juristischen Grundlagen in einem Mandat der Kānaka Maoli sowie der Überzeugungskraft der vorgetragenen Argumente:

„It [das Tribunal; U.M.] refuses ... to define law in a formalistic or colonialist manner. It is guided by five mutually reinforcing conceptions of law from which it draws freely in developing its findings on the charges and its conclusions and recommendations for redress" (Ka Ho‘okolokolonui 1993:2-3).

Die fünf aufgeführten Rechtsquellen sind: das überkommene Recht der Kānaka Maoli; Internationales Recht; Verfassung, Gesetze sowie Gerichtsentscheide der Vereinigten Staaten und des Staates Hawai‘i; ferner ein „Law of Peoples as

\footnotetext{
${ }^{316}$ Die kompletten sechsbändigen Protokolle des Tribunals wurden im Verlag Nā Maka o ka 'Āina, Honolulu, veröffentlicht; im ebenfalls vorhanden Video über das Tribunal stehen allerdings weniger die Aussagen unbekannter Betroffener als vielmehr die Stellungnahmen bekannter Vertreter der Bewegung im Vordergrund (Nā Maka o ka 'Āina 1994).

${ }^{317}$ Insgesamt druckten die beiden großen Tageszeitungen im Laufe des August elf Artikel und Kommentare mit Bezug auf das Tribunal, nur sechs Artikel berichteten direkt von den Verhandlungen (Index to the Honolulu Advertiser and Honolulu Star-Bulletin 1993-1994, Bd. 1-2, S. 562, 1197).

318 „The ,Tribunal’: an educational morality play“; (HA 14.8.93:A10).
} 
Nations“; ${ }^{319}$ und schließlich ein „Inherent Law of Humanity.““320 Das Tribunal bezog sich bei der rechtlichen Beurteilung der den Vereinigten Staaten zur Last gelegten Vergehen zwar auf den juristischen Rahmen westlich geprägter Rechtsprechung, legte dabei aber ein aus den oben genannten Rechtsquellen gespeistes pluralistisches Recht zugrunde. Es folgte damit einer in ethnopolitischen Bewegungen verbreiteten Tendenz, spezifische eigene Belange zwar unter Zuhilfenahme pluralistischer Rechtschöpfungen zu bewerten, die Ergebnisse aber in der verklausulierten Form institutionalisierten westlichen Rechtsverständnisses publik zu machen und den eigenen Bewertungen somit Nachdruck zu verleihen (Merry 1997:43).

Die Anklage beruhte auf sieben den USA zur Last gelegten Rechtsbrüchen, die in der Klageschrift („Statement of Charges“) niedergelegt waren:

„1. Na Kaomi Ku'oko‘a (Impermissible interference in the internal affairs of a sovereign people and nation) [...]

2. Ke Kokua ana i Na Kaomi Ku'oko'a (Aiding and abetting a foreign coup d'etat against the government of a sovereign people and nation) [...]

3. Ka Ho'ohui 'Aina 'ana i ka Lahui Kanaka Maoli (Annexation of a sovereign people, their nation and their territory without their free and informed consent) [...]

4. Ka Pu'e 'ana o ke Aupuni 'e ma luna o ka Lahui Kanaka Maoli (Imposition of statehood on a people, their nation and territory without their free and informed consent) [...]

5. Ka 'Aihue i ka 'Aina ma o ka Pae'aina Kanaka Maoli (Illegal appropriation of the lands, waters and natural resources of the Kanaka Maoli) [...]

6. Ka Hana 'Ino o ka Waiwai a ka Lahui Kanaka Maoli (Economic colonization and dispossession of the Kanaka Maoli) [...]

7. Ka Pepehi a Ke Ku'e i Na Mea Pono Kanaka Maoli (Acts of genocide and ethnocide against the Kanaka Maoli) [...]

8. Na Hana 'Ino i Ka 'Aina ma o ka Pae'aina Kanaka Maoli (Destruction of the environment of Ka Pae'aina) [...]

\footnotetext{
319 „By initiative of peoples and nations, the experience of international people's tribunals has itself became a source of law. The framework of these efforts is contained in the 1976 Algiers Declaration on the Rights and Duties of Peoples“ (Ka Ho'okolokolonui 1993: :3).

320 „In addition to other sources there exists a higher law based on the search for justice in the relations among persons and peoples and their nations; as well, there is a law establishing the conditions for harmony between human activity and nature, drawing on ideas of stewardship that exist in many of the world's great cultural traditions, and that are especially embodied in the cultures of indigenous peoples“ (Ka Ho‘okolokolonui 1993:3).
} 
9. Na Hana 'Ino i ke Aloha ma o Ka Pae‘aina Kanaka Maoli (Violation of international and domestic trust responsibility) [...]“ (Kānaka Maoli Tribunal Kōmike 2004:341-55).

Diese Anklagepunkte (die hawaiische Sprache bleibt auf die Überschriften der ausführlich begründeten Kapitel begrenzt) fügten den von der Autonomiebewegung erhobenen bekannten Vorwürfen gegen die USA keine wesentlich neuen Aspekte hinzu. Sie stellten den Rahmen für die Anhörung von Zeugen dar, zu denen bekannte Vertreter der Bewegung gehörten wie Mililani Trask und Kekuni Blaisdell, Wissenschaftler wie Lilikalā Kame'eleihiwa, Jon Osorio oder Marion Kelly sowie zahlreiche Hawaiier, die als direkt Betroffene ihre eigene Situation vor dem Tribunal schilderten. ${ }^{321}$

Nach insgesamt sieben Verhandlungstagen auf fünf Inseln, an denen die Mitwirkenden des Tribunals auch die Orte aktueller sowie historischer Landrechtsauseinandersetzungen besuchten, wurde am 20. August 1993 in Honolulu das Urteil verkündet. Dieses Urteil, veröffentlicht im „Interim Report“ des Tribunals (Ka Ho'okolokolonui 1993:8-10) ${ }^{322}$, bestätigt im ersten Teil („Recognitions and Findings“) sämtliche Anklagepunkte unter deutlicher Bezugnahme auf internationale Verträge sowie UN-Resolutionen und stellt die andauernden Rechtsverletzungen durch die Institutionen der Vereinigten Staaten und des Staates Hawai'i fest. Darüber hinaus benennen die Richter im zweiten Teil des Urteils („Recommendations“) Möglichkeiten der Wiederherstellung des Rechtsfriedens, von denen als wichtigste hier zu nennen sind: die Anerkennung der Souveränität der „Lahui Kānaka Maoli“, der hawaiischen Nation, durch die USA und die internationale Staatengemeinschaft, die Rückgabe der beanspruchten Landflächen an die hawaiische Nation sowie die vollkommene Aufhebung der staatlichen „blood quantum standards“ und Anerkennung des Rechts der hawaiischen Nation, ihre Bedingungen für die Erteilung der Bürgerrechte selbst zu bestimmen. Allerdings unternimmt das Tribunal keinen Versuch, Wesen, Form und Struktur der genannten „hawaiischen Nation“ genauer zu definieren: die „nationale Identität“ ergibt sich den Veröffentlichungen des Tribunals zufolge gewissermaßen zwangsläufig aus der Identität einer Gegnerschaft den mächtigen USA und ihren Vertretern gegenüber sowie einer „Kānaka Maoli Identität“, die in der hawaiischen Sprache und kulturellen Praktiken verankert ist. Die im Rahmen des Tribunals erfolgte symbolische Nutzung westlicher Rechtssprache und Rechtsformen bot darüber hinaus eine

\footnotetext{
${ }^{321}$ Eine ausführliche Auswahl von Zeugenaussagen findet sich bei Churchill u. Venne (2004) die im zweiten Teil ihres Buches zum Tribunal die Protokolle der Anhörungen auf verschiedenen Inseln wiedergeben; siehe aber z.B. auch Rampell (1993:27).

${ }^{322}$ Die „Findings and Recommendations“ des Tribunals finden sich vollständig abgedruckt bei Churchill u. Venne (2004:677-726).
} 
Folie, vor der die Idee einer hawaiischen Nation Gehalt und Gestalt gewinnen konnte (Merry 1997:44).

Die öffentliche Rezeption des Tribunals

Während sich die Tageszeitungen in ihren Nachrichtenteilen um eine informative Darstellung der Vorgänge bemühten, offenbarten die begleitenden Leitartikel und Kommentare eine Haltung, die jeder Veränderung der gesellschaftlichen und politischen Situation der Hawaiier, die sich nicht ausschließlich im Rahmen des bundesstaatlichen Status quo vollzieht, eine Absage erteilte. So veröffentlichte der Honolulu Star-Bulletin am ersten Tag des Tribunals eine Kolumne, deren Autor das Tribunal und die geringe Beteiligung hawaiischer Wähler am politischen Prozess in Hawai‘i miteinander in Beziehung setzt (und dabei indirekt einer verstärkten Ethnisierung der Politik das Wort redet, zugleich aber die Hawaiier auffordert, ihre Rechte als amerikanische Staatsbürger wahrzunehmen).

„After 18 months of planning, a broad group of native Hawaiian organizations in the coming week will prove what most of us who live in Hawaii already know. [...]

There will be workshops, speeches, demonstrations and lectures. The hope, according to yesterday's announcements, is for native Hawaiians to learn what has hobbled past progress and how to work together for a just future. [...]

The irony is that native Hawaiians' best hope for as radical and dramatic a new century of self-determination as can be imagined will come not from tribunals, nor from the United Nations, but from determined, organized native Hawaiians who sign up voters and put their own standard-bearers in the State Legislature.“ ${ }^{323}$

Nach der Beendigung des Tribunals kommentierte ein Leitartikel des Honolulu Advertiser die Ergebnisse und kam ebenfalls zu dem Schluss, dass die Zukunft der hawaiischen Selbstbestimmung im Rahmen der existierenden Rechtsordnung des Staates Hawai'i zu suchen sei:

„Strident language may prove helpful if Hawaiians follow the tribunal's recommendation to apply to the United Nations to be placed on a list of non-self-governing nations slated for decolonization.

But we guess that would be an exercise in futility. We're more inclined to think Hawaiians will achieve a reasonably satisfactory form of sov-

323 „Capitol view: voters to decide sovereignty“, Richard Borreca (H S-B 13.8.93:A3) 
ereignty by appealing to their Island neighbors, many of whom are already receptive to righting past wrongs, with quiet reason. “324

Hawaiier sollten also versuchen, eine „akzeptable Form der Souveränität“ zu erlangen, indem sie als Minderheit an eine wohlwollende Mehrheit appellieren, ihren berechtigten Forderungen entgegenzukommen. Allerdings vergaß der Autor zu erwähnen, dass viele Hawaiier genau diesen Weg seit Jahrzehnten verfolgten, ohne dass grundsätzliche Veränderungen stattgefunden hätten. Insgesamt lässt sich also auch hinsichtlich des Tribunals - wie bei 'Onipa'a - feststellen, dass einer grundsätzlich positiv eingestellten Berichterstattung Leitartikel und Kommentare - insbesondere des Honolulu Advertiser - gegenüberstanden, die letztlich den politischen Status quo auf den Inseln verteidigten. Die Autonomiebewegung hatte 1993 in beiden großen Tageszeitungen Honolulus zwar ein interessiertes Forum, aber keine Förderer der von ihr angestrebten Ziele.

Wie schon bei der staatlich geförderten Großveranstaltung 'Onipa'a 1993 hielt sich auch bei Ka Ho'okolokolonui Kānaka Maoli das Interesse der nationalen Medien in sehr engen Grenzen - wenn auch dieses Mal eine restriktive Öffentlichkeitsarbeit der Veranstalter nicht dafür verantwortlich gewesen sein dürfte. Wie Merry (1993:43) feststellte, trugen in diesem Fall wohl eher weltweit verbreitete Vorstellungen von den „glücklichen Inseln“ zur Ausblendung einer problematischen Gegenwart bei. Allerdings erschienen auch in Deutschland einzelne Beiträge über das Tribunal. So druckte die Berliner Tageszeitung einen Bericht, in dem das Tribunal und die gegenwärtige soziale Situation der Hawaiier vorgestellt wurden, auf den aber keine weiteren Nachrichten z.B. über den Urteilsspruch folgten. Und in der Frankfurter Allgemeinen Zeitung erschien nach dem Ende des Tribunals ein längerer Artikel, der über das Urteil, die hawaiische Geschichte und die Ziele der Bewegung informierte. ${ }^{325}$ Die Beispiele des Tribunals und der Gedenkveranstaltungen von 1993 zeigen, wie schwierig es selbst für regional bedeutende Aktionen der Autonomiebewegung ist, länger andauerndes nationales oder gar internationales Medieninteresse zu gewinnen.

Alles in allem wird es nach seinem offiziellen Abschluss schnell still um das Tribunal. Die achttägigen Verhandlungen und das gegen die USA gefällte Urteil hatten keine konkreten Auswirkungen auf die politischen Entwicklungen. Das gesammelte Material aus den Verhandlungen, Dokumente, Gutachten und Zeugenaussagen, wurde hinterlegt, um es für die Unterrichtung der

\footnotetext{
324 „Hawaii tribunal: ,truth is out’; what’s next?“, (HA 24.8.93).

325 „Tribunal gegen die USA auf Hawaii“, Stephan Dömpke (TAZ 17.8.93:9); „Die blaue UNFahne über Ruinen kündet vom Traum der Hawaiianer: Sehnsucht nach der polynesischen Kultur“, Horst Rademacher (FAZ 27.8.93:3).
} 
Öffentlichkeit nutzbar zu machen (Young 1995:149). ${ }^{326}$ Darüber hinaus darf aber der Konsolidierungseffekt, den die Veranstaltung für einen Teil der hawaiischen Bewegung sicherlich hatte, nicht unterschätzt werden - und ein Teil der Veranstalter sollte in den Folgejahren als „Tribunal Kōmike“ eine deutliche Rolle in den Auseinandersetzungen um das „Referendum“ oder die „Native Hawaiian Vote“ im Jahre 1996 spielen, deren gesetzliche Grundlagen ebenfalls im Jahre 1993 gelegt wurden.

\footnotetext{
${ }^{326}$ Wobei allerdings auch zu vermerken ist, dass eine weitergehende Bearbeitung des Materials über sehr lange Zeit nicht erfolgte - eine erste Zusammenstellung der Aussagen und Gutachten findet sich in dem 2004 erschienenen Band von Ward Churchill und Sharon H. Verne, die beide zum Richterkollegium des Tribunals gehörten.
} 


\subsection{Autonomiebewegung, Bundesstaat und USA im Ge- denkjahr 1993}

Es waren nicht nur die großen Veranstaltungen, die das zeitliche Umfeld des Jahres 1993 prägten. Vonseiten der organisierten Bewegung wurden verschiedene Protestveranstaltungen initiiert, die ebenfalls ein entsprechendes Echo in den Medien fanden. Auch Politiker und staatliche Institutionen griffen das herannahende Gedenkjahr auf, um mit Resolutionen und politischen Initiativen einen Beitrag zur Aufarbeitung des Umsturzes von 1893 zu leisten. Alle im Folgenden aufgezeigten Aktivitäten hatten einen direkten Bezug auf dieses übergreifende Thema oder aber gewannen ihre besondere Bedeutung durch den zeitlichen Zusammenhang mit dem Gedenkjahr.

\section{Die Autonomiebewegung}

Als am 20. Januar 1993 die reguläre Sitzungsperiode des Parlaments in Honolulu eröffnet wurde, fanden sich die Abgeordneten mit verschiedenen Gesetzentwürfen und Petitionen konfrontiert, die sich mit Fragen der hawaiischen Autonomie befassten. So zog eine Demonstration der Gruppierung „Kuleana Land Owners Group““327 schon am Eröffnungstag von Kāne'ohe (auf der Nordseite der Insel O`ahu, Ko`olau) nach Honolulu, um dem Parlament eine Petition zu übergeben. Hierin forderten sie ein Moratorium der Vergabe von Ländereien aus den Beständen der von verschiedenen Institutionen verwalteten Treuhandgebiete an nicht-hawaiische Nutzer; des weiteren verlangten die Demonstranten die Abhaltung eines Referendums unter der hawaiischen Bevölkerung, in dem diese über die weit divergierenden Vorstellungen innerhalb des Hawaiian Movement entscheiden sollte.

Am selben Tag übergab Mililani Trask im Namen Ka Lāhuis einen bereits ausformulierten Gesetzentwurf an die Legislatur, dessen Inhalt weit über die Forderungen der Hawaiier von der Ko‘olau Küste hinausging. Im Einklang mit dem Selbstverständnis Ka Lāhuis, die sich als bereits existierende hawaiische Nation versteht (Ka Lāhui 1993:2), verlangte dieser Gesetzentwurf nicht weniger als die Übertragung von ca. 650.000 ha aus dem Public Land Trust einschließlich der Hawaiian Home Lands an Ka Lāhui. ${ }^{328}$

Am 5. Juni 1993 veranstalteten Vertreter verschiedener SovereigntyGruppierungen ein „Hawaiian Sovereignty Economic Symposium“, bei dem

\footnotetext{
${ }^{327}$ Kuleana bezeichnet hier kleinbäuerlichen Landbesitz; siehe Lucas (1995:61).

${ }^{328}$ Der Demonstrationszug der Kuleana Land Owners Group und die Gesetzesinitiative Ka Lāhuis werden in der Presse zunächst gemeinsam erwähnt: „Legislature gets sovereignty request“, Kevin Dayton (HA 21.1.93:A12).
} 
die teilnehmenden Gruppen ${ }^{329}$ ihre jeweiligen Ziele präsentierten und die daraus resultierenden ökonomischen Konsequenzen diskutierten. Die Presse reagierte positiv auf dieses Symposium, wobei hier insbesondere die Berücksichtigung verbreiteter Befürchtungen innerhalb der Bevölkerung Hawai'is herausgestellt wurde. ${ }^{330}$ Auch hier zeigte sich der Honolulu Advertiser als Vertreter des ökonomischen (wie auch des politischen) Status quo und forderte in einem Leitartikel:

„What would our economic future be if Hawaiians achieved greater sovereignty or self-government? What this symposium should do is add to the thought that Hawaiian sovereignty represents a promise, not a threat, for Hawaii and its future [...] Sovereignty for Hawaiians does not automatically imply less for anyone else. The sovereignty movement will work best - will succeed in the good sense of the word - if everyone who lives here has a part and a stake in it.“331

Sicherlich subtiler als in vielen anderen Kommentaren dieser Zeitung wurde aber auch hier die hawaiische Bewegung als potenzielle Gefahr für das Gemeinwesen dargestellt und die Einordnung der Autonomiebewegung in den gesamtstaatlichen Politikzusammenhang gefordert.

Auch der seit 1985 jährlich von Ka Pākaukau veranstaltete „Sovereignty Sunday“ oder „Restoration Day“ machte 1993 lange vor der eigentlichen Feier Schlagzeilen. ${ }^{332} 150$ Jahre nachdem Kamehameha III. „Ka Lā Ho'iho'i Ea“, den Tag der wiedererrichteten Souveränität, zu einem Nationalfeiertag erklärte, ${ }^{333}$ verweigerte das städtische Grünflächenamt die Genehmigung zur Durchführung der Veranstaltung. Anstelle des von Ka Pākaukau vorgesehenen Einholens der Flagge der Vereinigten Staaten und des anschließenden zeremoniellen Hissens der Flagge des Königreichs Hawai‘i sollte in dem 1887 eröffneten ältesten Park der Stadt Honolulu ein Fest unter dem Motto „Parks add quality to life“ gefeiert werden.

\footnotetext{
${ }^{329}$ Ka Lāhui Hawai'i, Ka Pākaukau, I.A.H.A., 'Ohana Council sowie Ka Kane o ka Malo, eine eher periphere Gruppe um den Lehrer und Autor Michael Kioni Dudley.

330 „Sovereignty panel urges people to dream a bit“, Stu Glauberman (HA 4.6.93:A3); „Symposium airs proposals on Hawaiian Sovereignty“, Kris M. Tanahara (HA 6.6.93:A5); das Hawai'i Public Radio übertrug das Symposium.

331 „Sovereignty meet: a promise, not a threat“, Editorial (HA 5.6.93:A8).

332 „Fete pits Hawaiian groups against city: dueling observances may be headed for Thomas square showdown“, Christopher Neil (HA 28.6.93:A1, A2); „Double dibs on Thomas Square“, Devi Sen Lasker (H S-B 30.6.93:A7).

${ }^{333}$ Am 31.7.1843 ließ der britische Admiral Richard Thomas auf Anweisung Königin Viktorias im heutigen Thomas Square die Flagge des Königreichs Hawai'i aufziehen und machte so die von Lord George Paulet am 25.2.1843 erklärte Annexion des Archipels rückgängig.
} 
Als Sprecher der Veranstalter stellte Kekuni Blaisdell fest, dass es sich bei der Feier um eine Demonstration gegen die illegale Besetzung Hawai' is handele und fügte hinzu: „It appears that the City Parks Department is determined to perpetuate this dishonor by ignoring, indeed defying and distorting history by trivializing this major historic event with a birthday bash“. Die Interessen der Stadt formulierte ein Vertreter des Grünflächenamtes in schöner Offenheit: „What we don't want ... is a political forum. We were hoping they would like to present an educational thing“. ${ }^{334}$ Die Presse begleitete den Streit um Veranstaltungstermin und -ort mit einer für den Veranstalter des Restoration Day, Ka Pākaukau, sehr positiven Berichterstattung. Kekuni Blaisdell erhielt als Sprecher der Veranstalter die Gelegenheit, seine Ansichten ausführlich darzustellen und auch der historische Hintergrund der Feier wurde beleuchtet. Ein Kommentar des Honolulu Advertiser schloss sogar einen Monat vor der Veranstaltung mit den Worten:

„Seems to us if there is an irreconcilable conflict between the Restoration Day events and the parks festival on this particular date, the parks festival ought to move.“335

Mit mehr Teilnehmern als in den Jahren zuvor (ca. 200 Besucher fanden sich im Thomas Square ein) konnte die Feier schließlich am geplanten Ort stattfinden. Die Veranstaltung schloss neben der schon traditionellen Flaggenzeremonie auch Hula-Darbietungen, eine 'awa-Zeremonie und verschiedene Redebeiträge ein, deren Tenor vom Beitrag Blaisdells reflektiert wird: er rief USPräsident Clinton dazu auf „to follow the example of Admiral Thomas and restore our flag and our nation...“. 336

Nur kurz darauf bot sich Gelegenheit, den Präsidenten direkt anzusprechen. Clinton verbrachte einige Urlaubstage in Hawai'i und wandte sich in einer kurzen Rede am Strand von Waikīkī auch an die Inselbevölkerung. Er ging auf seine soeben beendete Asienreise und deren Bedeutung für die USA ein, sprach aber auch kurz den Umsturz von 1893 sowie die Bemühungen John Waihe'es, des damaligen Gouverneurs, zur Verbesserung der Lage der Hawaiier an und sicherte seine Unterstützung zu.

Der Auftritt Bill Clintons wurde von ca. 60 Demonstranten begleitet, die vor allem Ka Lāhui Hawai'i angehörten und auf mitgeführten Transparenten „Sovereignty“ sowie die Rückgabe von Land forderten. ${ }^{337}$ Als Sprechchöre, die „Justice for Hawaii“ forderten, den Präsidenten unterbrachen, wandte sich

\footnotetext{
${ }^{334}$ Für beide Zitate siehe: „Fete pits Hawaiian groups against city...“, Christopher Neil (HA 28.6.93:A1, A2).

335 „Thomas Square: restoration and celebration“, Editorial (HA 29.6.93)

336 „"Hawaiian flag-raising celebrates 1843 event", Christopher Neil (HA 1.8.93:A3).

337 „,Sovereignty group hopes voice was heard“, Staff (H S-B 12.7.93:A3, A6)
} 
dieser mit den Worten „I hope we can provide it“ direkt an die Demonstranten (US Government 1994:1066). Auch durch Äußerungen wie diese nährte der neu gewählte Präsident Hoffnungen auf eine veränderte Politik der Bundesregierung gegenüber den Forderungen der hawaiischen Minderheit.

Ein letztes denkwürdiges Datum des Jahres 1993 war schließlich der 18. Dezember, an dem 100 Jahre zuvor Präsident Grover Cleveland seine Mitteilung zur Wiedereinsetzung der hawaiischen Regierung an den US-Kongress verfasst hatte. Neben einer Gedenkveranstaltung vor dem 'Iolani Palast, an der Vertreter unterschiedlicher Gruppierungen teilnahmen, initiierte die Gruppe Na Kupa O Kuho‘one‘enu'u Pono einen „Unity March“, in dessen Verlauf ca. 250 hawaiische Demonstranten über eine der Hauptstraßen Waikīkīs zogen und in Sprechchören und auf Plakaten die hawaiische Eigenständigkeit forderten. Sie wollten hierdurch insbesondere auch Touristen mit den Ansprüchen der hawaiischen Minderheit konfrontieren. ${ }^{338}$ Das Beispiel dieser Demonstration zeigte einmal mehr, wie sehr die zeitweise Aneignung des Touristenzentrums Waikīkī durch hawaiische Demonstranten, die Störung des heilen Südseetraums, den Nerv der öffentlichen Meinung traf: Während die Nachricht über die Kundgebung im Zentrum der Hauptstadt auf den hinteren Seiten der Tageszeitungen blieb, erhielt die relativ kleine Demonstration einer mehr oder weniger unbekannten Gruppierung Raum auf der Titelseite des Honolulu Advertiser.

Neben den hier aufgeführten Ereignissen fanden weitere Informationsund Protestveranstaltungen sowohl auf O‘ahu als auch auf den anderen Inseln statt, deren Teilnehmerzahlen jedoch weit hinter jenen der hier genannten Veranstaltungen zurückblieben. Es wird deutlich, dass die von der Bewegung geplanten und durchgeführten Veranstaltungen im Wesentlichen das Aufmerksamkeitspotenzial der Hauptstadt und ihrer Umgebung suchten und wie verschiedene Beispiele zeigen, hier auch am ehesten von den Medien wahrgenommen wurden.

\section{Der Bundesstaat und die USA}

Das Gedenkjahr 1993 wurde von bundesstaatlichen und nationalen Institutionen nicht übergangen, jedoch hielt sich das staatliche Engagement in einem relativ begrenzten Rahmen. So wurden Gedenkveranstaltungen, die verschiedene Ausrichtungen der Bewegung und auch staatliche Stellen einbanden (wie z.B. Onipa'a), unterstützt, und Resolutionen, Gesetze oder gar Entschuldigungen vermittelten der Öffentlichkeit den Eindruck staatlichen Handelns.

Bereits zwei Jahre vor den Feierlichkeiten des Jahres 1993 verabschiedete die erste Kammer des Parlaments eine Resolution (H.R. 156), mit der dem

\footnotetext{
338 „Palace rally hears sympathetic words of 1893 president Cleveland“, (HA 19.12.93:A4); „Hawaiian marchers close off Kalakaua“, Kris M. Tanahara (HA 19.12.93:A1).
} 
herannahenden Gedenkjahr Rechnung getragen werden sollte. ${ }^{339}$ Die Resolution, die den Umsturz von 1893 als einen gegen die souveräne Nation Hawai`i gerichteten Akt militärischer Aggression charakterisierte, formulierte den Gedanken, dass die Entwicklung des politischen Status der hawaiischen Bevölkerung noch nicht abgeschlossen sei:

“... WHEREAS, the Legislature believes that the proper status of Hawaii's indigenous people within the political regime of the State of Hawaii and the United States of America has still not reached its final stage and is still in the process of evolution [...]

BE IT FURTHER RESOLVED that the Legislature encourages the promotion of debate revolving around the future of Hawaii as a Pacific Island society, within or without the United States of America...”

Wie Schweizer (2005:366) bemerkte, führte insbesondere der zuletzt zitierte Absatz zu lebhaften Debatten im Parlament, da verschiedene Abgeordnete ihn als eine Aufforderung zur Sezession Hawai' is von den USA lasen. Des Weiteren würdigte die Resolution das Gedenkjahr als eine Zeit der "special reflection to the rights and dignities of the native Hawaiians within the Hawaiian and the American societies...” und forderte dazu auf, die Rollen aller heute in Hawai‘i lebenden Bevölkerungsgruppen zu hinterfragen.

Im folgenden Jahr verabschiedeten Abgeordnetenhaus und Senat gemeinsam eine Resolution mit dem ausführlichen Titel: „Recognizing breaches of trust responsibility between the State of Hawai' $i$ and the Hawaiian people, extending a formal apology to the Hawaiian people, and recognizing and supporting the right of the Hawaiian people to sovereignty and self-determination“ (House Concurrent Resolution No. 300 H.D. 1 1992). ${ }^{340}$ Die Resolution erkannte die Unrechtmäßigkeit der politischen Entwicklungen seit 1893 an, beklagte die zunehmende Marginalisierung des hawaiischen Bevölkerungsteils und beschrieb die unzureichende Verwaltung hawaiischen Landes durch den Staat Hawai`i und die Regierung in Washington. Das Parlament erkannte darüber hinaus das Recht der Hawaiier auf „Souveränität“ an:

„,... the citizens of the State of Hawai'i renew their recognition of the inherent sovereignty of indigenous Hawaiians and commit themselves to assist the process of indigenous Hawaiians re-establishing their sovereign Hawaiian government as requested by the Hawaiian people,

\footnotetext{
${ }^{339}$ Die Resolution H.R. 156 liegt in der Bibliothek des Legal Reference Bureau in Honolulu vor. Der Text der Resolution ist ebenfalls vollständig abgedruckt bei Schweizer 2005:366-7 (FN 35).

${ }^{340}$ Die Resolution H.C.R. 300 H.D. 1 liegt einschließlich der zugehörigen Committee Reports in der Bibliothek des Legal Reference Bureau in Honolulu vor.
} 
with such powers, duties, land, ocean, and financial resources as decided by the Hawaiian people [...] This renewed recognition in no way prejudices the full expression of sovereignty and self-determination by the Hawaiian people as is recognized by international law, including the United Nation's policy on decolonization and the special rights of indigenous peoples ...“ (House Concurrent Resolution No. 300:14).

Neben der offiziellen Anerkennung einer Schuld des US-Staates Hawai'i gegenüber den Hawaiiern und einer formellen Entschuldigung beinhalten die Schlussformulierungen der Resolution eine weit reichende Aufforderung an den US-Kongress, den Hawaiiern das Recht auf Selbstbestimmung zuzugestehen:

„... the citizens of the State of Hawai'i call upon the President and the Congress of the United States of America to renew the recognition of and assist the re-establishment of a sovereign indigenous Hawaiian government, as requested by the Hawaiian people. As an initial step, redress requires recognition of indigenous Hawaiian rights of selfdetermination to a degree at least equal to those exercised by native American Indian and Alaskan tribes or nations ... Such renewed recognition, re-establishment, and trust obligation confirmation shall be without prejudice to the indigenous Hawaiian people's inherent right to the full exercise of sovereignty, which they have never surrendered ...“ (House Concurrent Resolution No. 300:14-5).

Die beiden Resolutionen, zwar ohne Gesetzeskraft, aber doch eine wirksame Willensbekundung der Legislative, trugen nicht nur der historischen Entwicklungen seit 1893, sondern ebenso dem bevorstehenden Gedenkjahr 1993 Rechnung. Es bleibt jedoch zu untersuchen, ob und inwieweit im Jahr 1993 und in der unmittelbaren Folgezeit seitens der staatlichen Institutionen und Verfassungsorgane konkrete politische Schritte erfolgten, die den obigen parlamentarischen Vorgaben und Ansprüchen genügten.

Bereits am 25. Januar 1993 wurde eine Resolution (H.R. No. 27) mit dem Titel "Supporting efforts to achieve sovereignty for native Hawaiians through federal initiatives" in das Abgeordnetenhaus eingebracht, deren Autoren die rechtliche Gleichstellung der Hawaiier mit den Indianern des USamerikanischen Festlands forderten. In diesem Zusammenhang stellten sie fest, dass alle Forderungen der Hawaiier nach Land oder Wiedergutmachung auf der 
Bundesebene zu regeln seien. ${ }^{341}$ Öffentliche Anhörungen, die der Aussprache im Ausschuss für hawaiische Angelegenheiten vorausgingen, machten die tiefe Spaltung zwischen Staat und Autonomiebewegung deutlich: Während Institutionen wie das Office of Hawaiian Affairs (OHA), das Department of Hawaiian Home Lands (DHHL) und das nicht-staatliche State Council of Hawaiian Homestead Associations (SCHHA) ${ }^{342}$ die Erklärung unterstützten, lehnten die Pro Hawaiian Sovereignty Working Group, Ka Pākaukau und Ka Lāhui Hawai'i die Resolution und die in ihr enthaltene starke Betonung der Verantwortung der Bundesregierung ab. Der zuständige Ausschuss, das Committee on Hawaiian Affairs, hob dann genau diese juristische Sicht besonders hervor: ,... [Hawaiian] sovereignty is a matter of federal obligation based on the trust relationship between the federal government and native Hawaiians“.${ }^{343}$ Diese Resolution war also durchaus dazu angetan, den Staat Hawai'i aus seiner Verantwortung gegenüber den Hawaiiern und ihren Autonomiebestrebungen gewissermaßen zu entlassen - und sie wurde schließlich am 16. April 1993 durch die erste Kammer des Parlaments angenommen.

Im Juli 1993 verabschiedete das Parlament in Honolulu zwei Gesetze, die direkte Auswirkungen auf die Autonomiebestrebungen des hawaiischen Bevölkerungsteils hatten. Zunächst bewilligte das Parlament US\$ 300.000 für die Organisation Hui Na‘auao. Bemerkenswert ist die Sprache des Gesetzestextes, der nach einer kurzen Auflistung wesentlicher Daten und Ereignisse der neueren hawaiischen Geschichte auf frühere Erklärungen des Parlaments verweist und feststellt:

„Because the actions taken by the United States were viewed as illegal and done without the consent of native Hawaiians, many native Hawaiians feel there is a valid legal claim for reparations. Many native Hawaiians believe that the land taken without their consent should be returned and if not, monetary reparations made, and that they should have the right to sovereignty, or the right to self-determination and selfgovernment as do other native American peoples.

\footnotetext{
${ }^{341}$ Eine Resolution fast gleichen Wortlauts wurde einen Monat später auch in den Senat eingebracht (S.R. No. 18) und am 19. April 1993 angenommen. Beide Resolutionen liegen in der Bibliothek des Legal Reference Bureau, Honolulu, vor.

${ }^{342}$ Das State Council of Hawaiian Homestead Associations (SCHHA) wurde 1987 als Organisation der hawaiischen Siedler auf Parzellen der Hawaiian Home Lands gegründet; im August 2008 hat die Organisation ihren Namen geändert und nennt sich nun „Sovereign Councils of the Hawaiian Homelands Assembly (SCHHA)“ - sie nimmt für sich in Anspruch mehr als 30.000 hawaiische Siedler zu repräsentieren.

${ }^{343}$ Standing Committee Report No. 1454 (16.4.1993), Bibliothek des Legal Reference Bureau, Honolulu.
} 
The legislature has also acknowledged that the actions by the United States were illegal and immoral, and pledges its continued support to the native Hawaiian community by taking steps to promote the restoration of the rights and dignity of native Hawaiians. The purpose of this Act is to provide funding for resources to educate the general public about Hawaiian sovereignty“ (Hawaii Session Laws 1993:1000).

Zwar erkannte das Parlament des Bundesstaates Hawai'i in diesem Text die Illegalität des Umsturzes 1893 an und bekräftigte vordergründig seine Absicht, für die Wiederherstellung der Rechte der Hawaiier einzutreten. Zugleich relativierte jedoch der erste Teil des Textes diese Aussagen, indem er die Formulierungen „Many native Hawaiians feel...“ bzw. „Many native Hawaiians believe..." den im Konjunktiv gehaltenen Forderungen vorausgehen lässt. - Die Gesetzesmacher benannten sonach Forderungen, die sie sich dennoch nicht eindeutig zu eigen machten.

Auch das zweite im Juli verabschiedete Gesetz (359, Senate Bill No. 1028) lässt sich als Beitrag des Parlaments zur Berichtigung der historischen Entwicklungen lesen, etablierte es doch die Hawaiian Sovereignty Advisory Commission (HSAC) ${ }^{344}$, die mit der Durchführung der Wahl einer hawaiischen Verfassungsversammlung betraut wurde. In den öffentlichen Anhörungen konkurrierte diese Gesetzesvorlage mit einem von Ka Lāhui formulierten Gesetzentwurf für die direkte und unmittelbare Übertragung ausgedehnter Landflächen an Ka Lāhui. Während der schließlich abgelehnte Gesetzentwurf Ka Lāhuis konkrete Forderungen erhob, die nur durch direkte Maßnahmen staatlicher Stellen zu erfüllen waren, setzte das schließlich verabschiedete Gesetz 359 (Senate Bill No. 1028) einen Prozess in Gang, der zwar einen sehr hohen Anspruch hatte, diesem letztlich aber nicht gerecht werden konnte oder gar sollte.

Hinsichtlich bundesrechtlicher Bestimmungen ergriff die Regierung in Washington keine konkreten Maßnahmen im unmittelbaren Umfeld des Jahres $1993 .^{345}$ Allerdings fiel die Abstimmung über den Etat des USVerteidigungsministeriums für das Haushaltsjahr 1994 zumindest zeitlich in den hier gesetzten Rahmen: In der Abteilung 10001 dieses umfangreichen Ge-

\footnotetext{
${ }^{344}$ Die Hawaiian Sovereignty Advisory Commission (HSAC) darf nicht verwechselt werden mit dem Sovereignty Advisory Council (SAC), das bereits am 26.6.1991 eingerichtet wurde und dessen Aufgabe lautete: „The council shall develop a plan to discuss and study the sovereignty issue“ (Hawaii Session Laws 1991:910). Hierfür stellte das Parlament US\$ 50.000 aus dem Budget des Office of Hawaiian Affairs (OHA) zur Verfügung. Im Januar 1992 lieferte das SAC dem Parlament einen vorläufigen Bericht (SAC 1992:10). Die Arbeit des SAC wurde über den vorläufigen Abschlussbericht hinaus nicht fortgesetzt (siehe hierzu auch Mardfin 1994:30-2).

${ }^{345}$ Denkbar wäre hier z.B. die rechtliche Gleichstellung der Hawaiier mit den indigenen Bevölkerungen des nordamerikanischen Kontinents oder auch die Einführung des Rechts der Hawaiier auf gemeinschaftliche Klageführung gegen bundesstaatliche Maßnahmen.
} 
setzes $^{346}$, die den Titel „Conveyance of Kaho'olawe Island, Hawai'i, to the State of Hawai'i“ trägt, beschloss das Ministerium die Beendigung der Bombenabwürfe der US-Navy sowie die Rückgabe Kaho'olawes an den Staat Hawai'i. Die Insel wurde am 7. Mai 1994 übergeben, um alsdann, wie in den Hawaii Revised Statutes bereits festgelegt, an eine noch zu schaffende souveräne hawaiische Verwaltung übertragen zu werden. ${ }^{347}$ Vertreter der hawaiischen Autonomiebewegung bewerteten das Ende der Bombardements und die Rückgabe der Insel sehr unterschiedlich. Für Davianna Pomaika‘i McGregor, langjährige Aktivistin der Protect Kaho'olawe 'Ohana, ${ }^{348}$ handelte es sich hierbei um einen der wichtigsten Vorgänge des Jahres:

„Perhaps the most significant action by the U.S. Congress and the Clinton administration in the centennial year of the overthrow was a measure in the military appropriations act to permanently stop the bombing of the sacred Hawaiian island of Kaho'olawe ... As a focal point of Hawaiian activism since 1976, stopping the bombing of Kaho'olawe is a major victory for the Hawaiian movement. Moreover, under state law, the island will be held in trust with other Hawai'i national lands and be turned over to a sovereign Hawaiian nation when it is reestablished and recognized by the U.S federal and Hawai' $i$ state governments. This is the first of the Hawaiian national lands which the State of Hawai'i has agreed to repatriate to the Hawaiian people's nation“ (McGregor 1994:8).

Völlig anders schätzte Mililani Trask (Ka Lāhui Hawai'i) die Bedeutung dieses Vorganges ein; in einem Interview mit der unabhängigen Wochenzeitung Honolulu Weekly stellte sie fest:

„What does the sovereignty movement get out of the Kaho'olawe deal? Nothing. At ,some point' in the next 10 years, they're supposed to turn over the bombed-out island, allegedly cleaned up, to the new nation.

\footnotetext{
${ }^{346}$ FY 1994 Department of Defense Appropriations Act Public Law 103-139, 107 Stat. 1418 hier zitiert nach der auf der Website der Kaho'olawe Island Reserve Commission (KIRC) abgelegten Fassung (KIRC 2009).

${ }^{347}$ „,[6K-9] Transfer. Upon its return to the State, the resources and waters of Kaho'olawe shall be held in trust as part of the public land trust; provided that the State shall transfer management and control of the island and its waters to the sovereign native Hawaiian entity upon its recognition by the United States and the State of Hawai 'i“ (Hawaii Revised Statutes o.J.:§6K-9 Internet: www.capitol.hawaii.gov/hrscurrent/Vol01_Ch0001-0042F/HRS0006K/HRS_0006K-0009.htm [21.1.2009]).

${ }^{348}$ Die Protect Kaho‘olawe 'Ohana ist eine im Jahre 1975 gegründete Organisation, die sich um die Rückgabe der Insel Kaho`olawe bemühte (Kaho`olawe, kleinste der Hauptinseln des hawaiischen Archipels, stand als militärisches Sperrgebiet unter der Jurisdiktion des US-Militärs).
} 
But whether it gets to the new nation or not, it will always be used as a state public park ... For the last 10 years, everybody keeps saying, ,The Hawaiian land base could be state and national parks, and you can be the curators.'

I agree with (Department of Hawaiian Homes Lands Chairman) Hoaliku Drake: Get the $\$ 400$ million (allocated by the feds to clean up Kaho'olawe), put it into the homesteads. People are homeless. If we ever needed federal bucks, it was there - for people, not for one more state park that you can’t even get transportation to“ (Ferrar 1994:5).

Am Beispiel der Insel Kaho'olawe zeigen sich einmal mehr große Unterschiede innerhalb der hawaiischen Bewegung: McGregors Feststellung ist vor dem Hintergrund der Protect Kaho'olawe 'Ohana zu sehen, die sich seit ihrer Gründung vornehmlich um die Beendigung der militärischen Nutzung der Insel bemühte und dabei eine besondere kulturelle Bedeutung Kaho'olawes für die Hawaiier und die Idee der „Aloha 'Āina“, der Liebe zum Land, in den Vordergrund rückte (cf. Protect Kaho'olawe 'Ohana 2009). Für Mililani Trask und Ka Lāhui Hawai' $i$ standen hingegen konkrete und grundsätzliche Veränderungen des politischen Status der hawaiischen Bevölkerung im Vordergrund der Bemühungen. - Was also die Bedeutung (revitalisierter) kultureller Vorstellungen für die Begründung der eigenen Vorgehensweisen betraf, so fanden sich die beiden Gruppierungen gewissermaßen an den entgegengesetzten Enden des Spektrums der Hawaiischen Bewegung.

Eine weit reichende Geste des Kongresses der Vereinigten Staaten stand am Ende des Gedenkjahres: Am 23. November 1993 setzte Präsident Clinton seine Unterschrift unter eine Resolution beider Häuser des USKongresses, die den Titel trug ,To acknowledge the $100^{\text {th }}$ anniversary of the January 17, 1893 overthrow of the Kingdom of Hawaii, and to offer an apology to Native Hawaiians on behalf of the United States for the overthrow of the Kingdom of Hawaii“(Senate Joint Resolution 1993). Diese Resolution erhielt durch die Unterschrift des Präsidenten als „Public Law 103-150“ Gesetzesrang. Verfasst von den damaligen US-Senatoren des Staates Hawai‘i, Daniel Akaka und Daniel Inouye, war sie bereits im Januar 1993 in den Senat eingebracht worden. Ihr Text setzt sich zusammen aus einer längeren Auflistung relevanter historischer Ereignisse, einem ausführlichen Zitat der Erklärung Königin Lili‘uokalanis vom 17. Januar 1893, mit der sie ihre Autorität gegenüber der USRegierung, aber nicht gegenüber der Provisorischen Regierung Hawai'is aufgab, sowie den Schlussbestimmungen, in denen sich die Anerkennung der historischen Rolle der USA sowie eine Entschuldigung des Kongresses finden:

„Section 1. Acknowledgement and Apology

The Congress - 
(1) on the occasion of the $100^{\text {th }}$ anniversary of the illegal overthrow of the Kingdom of Hawaii on January 17, 1893, acknowledges the historical significance of this event which resulted in the suppression of the inherent sovereignty of the Native Hawaiian people;

(2) recognizes and commends efforts of reconciliation initiated by the State of Hawaii and the United Church of Christ with Native Hawaiians;

(3) apologizes to Native Hawaiians on behalf of the people of the United States for the Overthrow of the Kingdom of Hawaii on January 17, 1893 with the participation of agents and citizens of the United States, and the deprivation of the rights of Native Hawaiians to selfdetermination;

(4) expresses its commitment to acknowledge the ramifications of the overthrow of the Kingdom of Hawaii, in order to provide a proper foundation for reconciliation between the United States and the Native Hawaiian people; and

(5) urges the President of the United States to also acknowledge the ramifications of the overthrow of the kingdom of Hawaii and to support reconciliation efforts between the United States and the Native Hawaiian people“ (Senate Joint Resolution 1993).

Mit diesem „Apology Law“, so eine häufige Bezeichnung, anerkannten die USA erstmals offiziell ihre Beteiligung am Sturz des hawaiischen Königreichs sowie ihre besondere Rolle bei späteren Ereignissen, die schließlich zur Inbesitznahme des Archipels durch die Vereinigten Staaten führten. Sie erfüllten damit auch eine Forderung der hawaiischen Autonomiebewegung, die schon 1988 in der Abschlusserklärung einer Native Hawaiian Rights Conference ${ }^{349}$ erhoben wurde und die fünf Voraussetzungen für eine hawaiische Autonomie nannte:

„(1) An apology by the United States government to Native Hawaiians and their government for the United States' role in the coup of 1893.

(2) A substantial land and natural resource base comprised of a reformed Hawaiian Homes program, a fair share of the ceded lands trust, the return of Kahoolawe and other appropriate lands.

(3) Recognition of a Native Hawaiian Government with sovereign authority over the territory within the land base.

\footnotetext{
${ }^{349}$ Teilnehmerorganisationen dieser Konferenz, die vom 7.-8.8.1988 stattfand, waren u.a. Ka Lāhui Hawai'i, das Institute for the Advancement of Hawaiian Affairs und die Protect Kaho`olawe ‘Ohana.
} 
(4) Recognition and protection of the subsistence and commercial hunting, fishing and gathering (including beach access), cultural and religious rights of Native Hawaiians, and the legitimate exercise of sovereign powers over such rights.

(5) An appropriate cash payment“ (zit. nach MacKenzie 1991b:91).

Konkrete Maßnahmen, wie in dieser frühen Erklärung der Bewegung gefordert, waren allerdings mit dem „Apology Law“ nicht verbunden. Insbesondere blieben weiterhin alle Entwicklungen, die auf die Eingliederung Hawai'is als Bundesstaat folgten, ausgeklammert. Das „Apology Law“ geht nicht auf kontroverse Interpretationen des Referendums von 1959 ein und beschränkt sich auf die Feststellung: ,... on August 21, 1959, Hawaii became the $50^{\text {th }}$ State of the United States“. Damit wurde zunächst allen Forderungen, die über eine Beilegung bestehender Konflikte im Rahmen des politischen Status quo (,reconciliation“) hinausgingen, eine Absage erteilt. ${ }^{350}$

${ }^{350}$ Zur geringen juristischen Bedeutung der „Apology Resolution“ siehe auch Nobles (2008:1223), Duus (2003). 


\section{Konflikte um Landrechte und Institutionen}

Das Land und seine Nutzung stehen in Hawai'i nicht erst seit der Herausbildung der Autonomiebewegung im Zentrum der Fragen um wirtschaftliche und politische Hegemonie. Der Erhalt einer hawaiischen Landbasis bzw. die Errichtung eines quasi-autonomen (oder, je nach politischer Ausrichtung der betreffenden Gruppierungen, auch völlig souveränen) hawaiischen Landgebietes hat seit den 1990er Jahren vorrangige Bedeutung unter den Zielen der hawaiischen Autonomiebewegung. ${ }^{351}$ In der politischen Sprache der hawaiischen Bewegung ist „Land“ zudem ein zentrales Zeichen, dessen vielschichtige Konnotationen auf inner-hawaiische Diskurse über die Vergangenheit und den Verlust des Landes - und damit schließlich der staatlichen Souveränität - verweisen. „Hawaiisches Land“ stand in den 1990er Jahren auf vielfältige Weise im Mittelpunkt hawaiischer Politik - Auseinandersetzungen über hawaiische Landrechte in Gegenwart und Vergangenheit, die gegenwärtige und zukünftige Nutzung staatlichen Landes oder auch die Finanzierung hawaiischer Institutionen aus den Pachteinnahmen des Staates bestimmten über weite Strecken die Konfliktthemen des Jahrzehnts. Die im Folgenden vorgestellten Fallbeispiele werden daher einen Überblick der hawaiischen Ansprüche sowie unterschiedlicher Formen der Konfliktführung geben.

Im ersten Abschnitt dieses Kapitels sollen nicht konkrete Landflächen und die um sie geführten Auseinandersetzungen im Vordergrund der Betrachtung stehen, sondern der Begriff „'Āina“ (,hawaiisches Land“) und seine Konnotationen bzw. Verwendung in politischen Zusammenhängen. Die Darstellung unterschiedlicher Konfliktfelder und damit auch verschiedener Strategien der

\footnotetext{
${ }^{351}$ Hier zeigt sich ein wichtiger Unterschied zwischen den Hawaiiern und den Native Americans des US-amerikanischen Kontinents: Während letztere vielfach auf staatsvertraglich abgesicherten, gegenüber den jeweiligen Bundesstaaten autonomen bzw. selbstverwalteten Territorien leben, besitzt der hawaiische Bevölkerungsteil im Bundesstaat Hawai`i aufgrund der spezifischen historischen Entwicklung keinen Anspruch auf ein autonomes Landgebiet.
} 
Autonomiebewegung gegenüber den staatlichen Stellen sind Gegenstand des zweiten Teils. Hierbei stehen die staatlichen Treuhandgebiete, deren komplexe Entstehungsgeschichte bis in das Jahr 1855, das Jahr der Māhele zurückreicht, nach wie vor im Zentrum der Auseinandersetzungen. Eine Darstellung diverser Konfliktstrategien der jeweiligen hawaiischen Gruppierungen verdeutlicht zugleich deren gegensätzliche Zielsetzungen. Schließlich stelle ich - drittens die private hawaiische Stiftung „Kamehameha Schools/Bishop Estate (KS/BE)“ vor, Ende des 19. Jahrhunderts errichtet und heute der mit Abstand größte Landeigner im Bundesstaat Hawai'i. Anhand der Kontroversen zwischen Kamehameha Schools/Bishop Estate (KS/BE) einerseits und hawaiischen Gruppierungen andrerseits lässt sich das Besondere inner-hawaiischen Mit- und Gegeneinanders verdeutlichen. Es handelt sich hierbei um Aspekte, die Konflikte zwischen hawaiischen Protagonisten begrenzen und abfedern können, aber auch um Beispiele problematischer politischer Auseinandersetzung zwischen Hawaiiern angesichts der Mehrheitsgesellschaft und ihren Exponenten.

\subsection{Hawaiisches Land damals, hawaiisches Land heute}

„I 'āina no ka 'āina i ke ali'i, a i waiwai no ka 'āina i ke kānaka. The land remains the land because of the chiefs, and prosperity comes to the land because of the common people" [Kursivschreibung im Original; U.M.] (Pukui 1997:125). Die im Sprichwort beschriebene Aufgabenteilung - der Adel trug für den politischen und spirituellen Erhalt des Landes Sorge, die gemeine Bevölkerung erwirtschaftete den Wohlstand des Landes - skizziert die Koordinaten, innerhalb derer sich die Einstellungen der hawaiischen Gesellschaft zum Land bis weit in das 19. Jahrhundert hinein bewegten. Heute evoziert die Verwendung des hawaiischen Wortes 'Äina anstelle der englischen Entsprechung im politisch-kulturellen Kontext Konnotationen, die uns besonders deutlich in der Fügung „Aloha 'Āina“ („Liebe zum Land“) begegnen. Sie wurde tonangebend im hawaiischen Diskurs um Land, als in den 1970er Jahren, nach fast 200 Jahren der Kolonialisierung, Investoren im Verbund mit der herrschenden politischen Klasse des Inselstaates immer größere Flächen für den Bau von Wohnund Tourismusprojekten an sich brachten (siehe hierzu Cooper u. Daws 1990). Es war vor allem die Protect Kaho'olawe 'Ohana, die den Ausdruck konzeptionell mit Inhalt füllte und einen wesentlichen Beitrag zu seiner Popularisierung leistete:

„Aloha 'Aina is a traditional concept that lays the foundations for Hawaiian religion, culture and lifestyle. Aloha means love and 'aina means land. The two words together express several levels of meaning. 
At the deepest level the presence of our ancestors and the gods of the land are acknowledged, respected and cherished through ceremonies both public and private. This intimacy with the 'aina is also expressed in the interdependent subsistence relationship between man and his island. Man is nurtured with taro from the land and fish from the sea, and in turn cultivates and nourishes the island. This relationship is finally symbolised by pride in our homeland - patriotism for this land Hawaii“ (Protect Kaho'olawe ‘Ohana in: McGregor 1989:88-9).

Im zuletzt genannten Sinn war der Begriff Aloha 'Āina, den Pukui und Elbert (1986:21) mit „love of the land or of one’s country, patriotism“ übersetzen und dem sie eine lange Geschichte bescheinigen, gegen Ende des 19. Jahrhunderts gebräuchlich und fand sich z.B. als Name einer hawaiischsprachigen Zeitung oder einer politischen Partei („Hui Aloha 'Āina - Hawai'i Patriotic League“).

Inwieweit es sich bei „Aloha 'Äina“ tatsächlich um das historische Bild ehemals realer Beziehungen zwischen Menschen und Land handelt, war neben der Kritik an anderen Traditionalismen Gegenstand einer Kontroverse (siehe Linnekin 1983; H.-K. Trask 1993). Wie dem im einzelnen auch sei, im Kontext der hier im Vordergrund stehenden kulturellen wie politischen Autonomiediskurse scheinen von hawaiischer Seite sowohl „Aloha 'Äina“ als auch das von Kame“eleihiwa (s.o.) angeführte „Mālama 'Āina“ („Sorge für das Land“; U.M.) bemüht zu werden, um die hawaiische Bevölkerung der Gegenwart über Generationen hinweg in einer einzigartigen und exklusiven Beziehung mit dem Land ideell zu verknüpfen. So schreibt z.B. Davianna Pōmaika'i McGregor, Professorin für Ethnic Studies an der University of Hawai'i, Mānoa, und Vertreterin einer eher integrativen, kulturell ausgerichteten Richtung der Autonomiebewegung, in einer Aufzählung von Kriterien zur zukünftigen Nutzung der Insel Kaho‘olawe: ${ }^{352}$

„A fifth principle recognizes that an inherent aspect of Hawaiian stewardship and use of cultural and natural resources is the practices of aloha 'āina and mālama 'àina, or respect and conservation of the land to ensure the sustainability of natural resources for present and future generations. These rules of behavior are tied to cultural beliefs and values regarding respect of the 'äina, the virtue of sharing and not taking too much, and a holistic perspective on organisms and ecosystems that emphasizes balance and coexistence. The Hawaiian outlook that shapes these customs and practices is lōkahi, or maintaining spiritual, cultural,

\footnotetext{
${ }^{352}$ Der Kriterienkatalog wurde ursprünglich von der Kaho‘olawe Island Reserve Commission, der Protect Kaho'olawe 'Ohana sowie der Edith Kalaniana'ole Foundation erstellt und stellt traditionelles hawaiisches Wissen und eine holistische Naturbetrachtung in den Vordergrund.
} 
and natural balance with the elemental life forces of nature“ (McGregor 2007:283).

Hier wird der Rückgriff auf das vorgeblich traditionelle hawaiische Verhältnis zum Land verknüpft mit gegenwärtigen Vorstellungen der Ökologiebewegung, eine Verbindung, die sich auch in einem der Studienschwerpunkte des Center for Hawaiian Studies an der University of Hawai' $i$, Mānoa, findet. Im Rahmen des MA Studienganges wird hier „Mālama 'Āina: living in harmony with the Land Resource Management" gelehrt. Des Weiteren stellt McGregor fest, dass die aufgeführten Kriterien eine ausgezeichnete Grundlage für die zukünftige Verwaltung aller hawaiischen Inseln darstellen könnten und impliziert damit einen zumindest ideellen hawaiischen Anspruch auf den gesamten Archipel. Auch Mililani Trask nutzt das Konzept der „Liebe zum Land“, um zwischen „Hawaiisch-Sein“ und Streben nach Autonomie eine ganz reale und notwendige Verbindungslinie zu ziehen:

„God put us on this earth and gave us a certain sacred-trust obligation to be guardians of this lands. Encoded in our cultural practices and value systems are the teachings necessary to maintain balance here in Hawai'i nei ... that only our people have been chosen to practice. This is the teaching of Hawaiian aloha 'äina, and lōkahi - living in balance with yourself, God, and the earth. ... It is the job of the Kanaka Maoli, the Hawaiian people, to maintain the balance of the earth and the heavens, to keep the connection with the land and with God. If we pass away, it's not just losing a traditional culture ... but it is also losing the 'aina, the pa' $i$ 'aina of Hawai' $i$ nei, this whole archipelago. More importantly, the world loses the keepers of the teachings of this knowledge. So this is really why we move for sovereignty and selfdetermination“ (Mililani Trask zitiert nach Mast und Mast 1996:394).

Indem M. Trask die Hawaiier hier gewissermaßen zu den „Hütern des Landes“ erklärt, hebt sie den hawaiischen Bevölkerungsteil aus der übrigen Inselbevölkerung heraus und schafft eine ideologische Basis für eine hawaiische Identität. Die besondere Bindung an das Land wird so zur Grundlage für den Kampf um politische Autonomie und kulturelle Selbstbestimmung.

Viele Äußerungen von Protagonisten unterschiedlicher Richtungen der Autonomiebewegung beziehen die Verwendung des hawaiischen Wortes 'Āina vornehmlich auf die Gesamtheit des "hawaiischen Landes“ als eines seiner realen Grundlage beraubten nurmehr abstrakten Konzeptes, während konkret zu benennende Landareale bzw. -einheiten in den politischen und juristischen Auseinandersetzungen und auch im Rahmen des Autonomiediskurses in aller Regel als (engl.) „land“/,lands“ benannt und umkämpft werden. So wird mit 
'Āina und den damit einhergehenden Konnotationen einer immerwährenden Einheit von hawaiischen Menschen, hawaiischer Kultur und hawaiischen Inseln ein ideelles Land geschaffen, das neben dem nur noch eingeschränkt verfügbaren realen Land Bestand hat. Dieses „,ideelle Land“ dient gleichsam einer Verortung der in den politischen und kulturellen Diskursen kreisenden „Hawaiianness“, in Verlautbarungen und Veröffentlichungen der Bewegung dokumentiert und perpetuiert es zugleich den hawaiischen Anspruch auf das „Land“.

Haunani-Kay Trask stellt die hawaiische Verbundenheit mit dem Land in den Zusammenhang der politischen Bestrebungen (1999:37): „,...practical enunciations of self-determination include the creation of different forms of self-government ...., the Native alternative practice of aloha 'aina (love of the land) in opposition to resort development, industrial parks, upper-income residential subdivisions, and military use.“353 Sie fährt fort: „Arguments for restitution and for international adjudication of the territorial conflict between Hawaiians and the state and federal governments are also examples of the sovereignty question in theoretical form“ (H.-K. Trask 1999:37). Der hier angesprochene Territorialkonflikt liegt an der Basis der hawaiischen Bemühungen um Autonomie. Er durchzieht in unterschiedlicher Gewichtung alle Autonomiediskurse und tritt auf verschiedensten Ebenen der konkreten Auseinandersetzungen um die Landnutzung, die Finanzierung hawaiischer Institutionen oder die Vergabe von Land an hawaiisch-stämmige Bürger im Rahmen des Hawaiian Homes Commission Act in Erscheinung. Und damit steht ebenso das Finanzgebaren der Treuhänder einer mächtigen, auf hawaiische Wurzeln zurückgehenden und in neuerer Zeit auch maßgeblich von Hawaiiern mitbestimmten privaten Institution wie des Kamehameha Schools/Bishop Estate in der Kritik.

Jenseits der politischen Rhetorik ist die Landfrage ein sehr konkreter Rechtsbereich, in dem Bundes-, Staats- und Territorialrecht mit den überkommenen Rechtsnormen des bis 1893 bestehenden Königreichs sich überschneiden. In Verbindung mit der Betrachtung der großen staatlichen Treuhandgebiete werde ich auf hawaiische Forderungen und Kritik aus den 1990er Jahren hinsichtlich der Nutzung dieser Landareale eingehen, um auch hier die Frage nach dem politisch-strategischen Einsatz von Vergangenheitsdiskursen sowie die Wende hin zu einer Kulturalisierung dieser zunächst so geschichtlich bzw. juristisch erscheinenden Fragestellungen zu verfolgen und nachzuzeichnen.

\footnotetext{
${ }^{353}$ In der ersten Ausgabe des Buches „From a native daughter“ (H.-K. Trask 1993:47-8) lautet dieser Satz: „,...theoretical enunciations of self-determination include arguments for the alternative of Aloha "Āina (love of the land) in opposition to resort development, upper-income residential subdivisions, and military use.”
} 


\subsection{Die staatlichen Treuhandgebiete im Bundesstaat Hawai'i}

Nach der Landteilung von 1855 verfügte Kamehameha III. über 1 Mio. ha Land, von dem er mehr als 600.000 ha zu so genannten „Government Lands“ machte, ,... lands of the Hawaiian government, subject always to the rights of tenants“" (zitiert nach MacKenzie 1991c:7). ${ }^{354}$ Diese Einschränkung besagte, dass auch das Regierungsland von der ansässigen Bevölkerung gemäß bestehender Gewohnheitsrechte weiter genutzt werden konnte. Es stand daher nur beschränkt für Verpachtung oder Verkauf zur Verfügung - ein Zustand, den die Verfassung der „Republik Hawaii“ änderte (Fuchs 1961:257). Dennoch betrug die Gesamtfläche des Staatslandes am Tag der Annexion (12. August 1898) immer noch fast 710.000 ha.

\section{Staatliches Land I: Der „Ceded Lands Trust“}

Der „Admission Act“ vom 18. März 1959, der die Aufnahme des Bundesstaats Hawai' $i$ in die Vereinigten Staaten regelt, sieht besondere Bestimmungen für den aus den Kron- und Regierungsländern des Königreichs hervorgegangenen - und nun von den USA an den neuen Bundesstaat rückübertragenen - öffentlichen Landbesitz vor. Von besonderer (und vielzitierter) Bedeutung ist dabei der Paragraph 5(f) dieses Dokuments, in dem zum einen die zulässigen Verwendungszwecke des Landes bzw. des aus ihm resultierenden Einkommens bestimmt werden, zum anderen der treuhänderische Charakter des staatlichen Besitztitels betont wird:

„The lands granted to the State of Hawaii [...] shall be held by said State as a public trust for the support of the public schools and other public educational institutions, for the betterment of the conditions of native Hawaiians, as defined in the Hawaiian Homes Commission Act, 1920, as amended, for the development of farm and home ownership on as widespread a basis as possible for the making of public improvements, and for the provision of lands for public use. Such lands, proceeds, and income shall be managed and disposed of for one or

\footnotetext{
${ }^{354}$ Spätere gerichtliche und gesetzliche Klärungen stellten fest, dass der jeweilige Monarch zwar uneingeschränkte Verfügungsgewalt über die „Crown Lands“ hätte, dieses Land aber zugleich an das Amt gebunden sei und damit nicht außerhalb der unmittelbaren Thronfolge weitergegeben werden konnte bzw. nach Abschaffung des Amtes durch den Umsturz von 1893 kein Anspruch der ehemaligen Monarchin Lili ‘uokalani auf Land oder Einkommen daraus bestand - die Zession des Landes an die USA im Jahre 1898 damit rechtmäßig sei: „Estate of Kamehameha IV“ 1864; Gesetzgebung vom 3.1.1865; „Territory of Hawaii v. Kapi`olani Estate“ 1908; zuletzt Lili‘uokalani v. United States, 1910 (Duus 2003:483; MacKenzie 1991c:16-17).
} 
more of the foregoing purposes in such manner as the constitution and laws of said State may provide, and their use for any other object shall constitute a breach of trust for which suit may be brought by the United States“ (U.S. Government 1959).

Es dauerte bis 1978, um die Treuhandschaft des Bundesstaates auch in der Staatsverfassung zu präzisieren - und seither heißt es in Artikel 12 der Hawaiischen Verfassung: „The lands granted to the State of Hawaii [...] shall be held by the State as a public trust for native Hawaiians and the general public".

Für die hawaiische Bevölkerung ist jedoch nicht nur die monetäre Frage von großer Bedeutung - auch eine Besitzübertragung und Übergabe der Verwaltung an hawaiische Institutionen oder Gruppierungen war seit dem Erstarken der hawaiischen Bewegung immer wieder Gegenstand von Auseinandersetzungen. In diesen Zusammenhang gehören die im Folgenden behandelten „Mosaiksteine“ des weitläufigen und vielschichtigen Gesamtbildes der „Public Lands“, deren Status quo von hawaiischer Seite durchgängig infragegestellt und angefochten wurde, wenn auch mit unterschiedlichen Strategien zu verschiedenen Zeiten. Die Beispiele sind neben ihrer Eigenbedeutung und dem damit einhergehenden Medieninteresse durchaus auch repräsentativ für ähnlich gelagerte Konfliktfälle mit Bezug auf den „Public Land Trust“.

Finanzen: das Office of Hawaiian Affairs und der „Ceded Lands Trust“

Durch die Gründung des Office of Hawaiian Affairs (OHA) und die Bestimmung, dass dessen Budget durch einen Anteil an den Einkünften aus dem „Public Lands Trust“ zu bestreiten sei, bekamen die Fragen um den Rechtsstatus der „Ceded Lands“ in Hinblick auf die hawaiische Bevölkerung eine ganz neue Dynamik. OHA stehen laut Gesetz 20\% der jährlichen Einnahmen des Staates aus dem „Ceded Lands Trust“ zu - eine Verpflichtung, der der Staat über viele Jahre hinweg nur unzureichend nachkam. Nachdem OHA zunächst 2 bis 3 Mio. US\$ jährlich erhalten hatte, führten Verhandlungen zu einer Nachzahlung von 131 Mio. US\$ im Jahre 1993 und zu einer Steigerung des Jahresetats auf 7 bis 8 Mio. US\$. ${ }^{355}$ Dass auch diese Zahlen weit hinter den berechtigten Ansprüchen der hawaiischen Institution zurückblieben, machte ein Gerichtsurteil von 1996 deutlich, das eine erneute Nachzahlung durch den Staat verlangte, deren Höhe nach unterschiedlichen Berechnungen zwischen 300 Mio. und 1,2 Mrd. US\$ lag. ${ }^{356}$ Die Verhandlungen über diese Ausgleichszahlung dauerten über die 1990er Jahre hinaus an, doch setzte das Parlament in der

\footnotetext{
${ }^{355}$ Eine Zusammenfassung der hier aufgeführten Kontroverse um Zahlungen des Staates an OHA findet sich bei Pratt u. Smith 2000:237-8.

${ }^{356}$ Siehe hierzu ausführlich: „The ceded lands ruling: will it break the bank?“, Alan Matsuoka, $\mathrm{H}$ S-B 13.1.1997 (http://starbulletin.com/97/01/13/news/story1.html [29.4.1998])
} 
Sitzungsperiode 1997 die jährlichen Zahlungen auf 15,1 Mio US\$ fest. Die parlamentarischen Beratungen wurden begleitet von hawaiischen Protestveranstaltungen, darunter eine vom Office of Hawaiian Affairs unter Beteiligung unterschiedlicher hawaiischer Organisationen ${ }^{357}$ durchgeführte 24-stündige Mahnwache vor dem Parlament, deren zentrale Aussage lautete: „The power of the Hawaiian people speaking with one voice saying keep the promise. No more will our destiny be denied.“ ${ }^{358}$ Rowena Akana, eine Treuhänderin des Office of Hawaiian Affairs, beschreibt den Protest als ,a demonstration of Hawaiians coming together as one people with one voice to tell state leaders that our rights as the host people of these islands are not expendable“ (ebd.). Der Protest, dessen Hauptschauplatz das Parlamentsgebäude war, verband die aktuellen Forderungen mit der hawaiischen Geschichte: er begann an der Kawaiaha'o Kirche, ehemals das Gotteshaus der Monarchen, setzte sich fort am Denkmal Kamehamehas I., dem Sinnbild für die Gründung des hawaiischen Königreiches, und endete am folgenden Tag am Standbild Lili‘uokalanis, einem Ort, der an den Verlust der hawaiischen Autonomie gemahnt.

Die Veranstaltung unter dem Motto „Aloha ke Akua: Ho'olōkahi me ke Aloha“ “359 hatte zum einen starke christlich-religiöse Bezüge, doch nahm ein während der Veranstaltung von Pastoren der Kawaiaha'o Kirche verlesenes Gebet an den christlichen Gott deutlich Bezug auf die politischen Ereignisse der vergangenen zwei Jahrhunderte:

„... Over the past two hundred years, native Hawaiians have welcomed with aloha our 'ohana [Familienverband; U.M.] from other lands. But now we are pushed to the pali [Abgrund; U.M.] of landlessness in the beautiful islands you gave to kupuna [Großeltern; U.M.] and hanauna hiwahiwa [verehrte Vorfahren; U.M.] in the beginning. We do not ask for return of all lands, only for protection of what rightfully remains ours. [...]

Your po'e Hawai‘i [hawaiische Menschen; U.M.] whom you have placed in these beautiful islands of Hawai'i from long ago are hurting and are in dire need of the resources of the 'àina you have entrusted to us for the improvement of our condition, and so that we can once again restore the 'iwi [Knochen, Leben; U.M.] and the fabric of aloha ke

\footnotetext{
${ }^{357}$ Unter den beteiligten Organisationen waren The Royal Order of Kamehameha, Independent Nation State of Hawai'i, Association of Hawaiian Civic Clubs, Council of Hawaiian Organizations, verschiedene lokale Siedlungsverbände (Hawaiian Home Lands) und zahlreiche kleinere Gruppierungen. - Jedoch war keine der bekannten Gruppierungen der Autonomiebewegung an der Demonstration beteiligt.

358 „Hawaiian community comes together for 24-hour unity vigil“, KWO Mai 1997:11.

359 „Die Liebe Gottes: Einheit und Frieden schaffen durch Zuneigung“ [U.M.]
} 
Akua [die Liebe Gottes; U.M.] back into our people and permeate our Hawai'i nei with your aloha...“360

Dieser Gebetstext deutet die Einrichtung der „Ceded Lands“, hier als „“āina you have entrusted to us for the improvement of our condition" beschrieben (ein Satz, der den Text des Paragraphen 5(f) des Admission Acts [s.o.] zitiert), gleichsam als eine göttliche Fügung - wenn nicht gar als ,verheißenes Land“. Er ist zugleich ein interessantes Beispiel für den Einsatz hawaiischer Ausdrücke in einem vornehmlich englischsprachigen Kontext, wie er ähnlich auch in Veröffentlichungen der hawaiischen Bewegung immer wieder zur Anwendung kommt. Ebenso emotional und ideell befrachtet wie z.B. das Wort 'áina, sorgen die o.a. hawaiischen Begriffe und Wendungen in ihrem jeweiligen Redezusammenhang für eine deutlich nachvollziehbare Einordnung sowohl der Sprecher als auch der Rezipienten als „po“e Hawai' $\mathrm{i}$ ““ und tragen so zur gegenseitigen Versicherung und Verstärkung einer fragilen kulturellen und ethnischen Zugehörigkeit bei.

Abgesehen von der Höhe der tatsächlich zugewiesenen Zahlungen aus dem Einkommen der in Rede stehenden Landflächen nach Paragraph 5(f) des Admission Acts bestehen erhebliche Einschränkungen bzw. Vorgaben hinsichtlich der Verwendung dieser Geldmittel durch das Office of Hawaiian Affairs. Denn das Gesetz zur Bildung des Office of Hawaiian Affairs schränkt den Kreis der Berechtigten analog zu den Regelungen des Hawaiian Homes Commission Act aus dem Jahr 1920 ein, so dass ausschließlich Native Hawaiians, d.h. Hawaiier mit einer zumindest 50 prozentigen hawaiischen Abstammung, zu den Nutznießern gehören. Der größte Teil der heute im Bundesstaat lebenden Hawaiier bleibt so vom Zugang zu den für OHA - und damit speziell für den hawaiischen Bevölkerungsteil - bestimmten Mitteln aus den Einnahmen der „Ceded Lands“ nach wie vor ausgeschlossen.

Ka Lāhui Hawai ' $i$, „Sovereignty“ " und die „Ceded Lands“

Für Ka Lāhui Hawai“i stellt der „Public Land Trust“ (einschließlich der Areale nach dem Hawaiian Homes Commission Act) die Grundlage eines autonomen hawaiischen Territoriums dar. Jonathan Kamakawiwo'ole Osorio, Professor am Center for Hawaiian Studies, UH Mānoa, und Mitglied von Ka Lāhui, fasst die Einstellungen der Gruppierung hierzu zusammen:

„In the minds of KLH [Ka Lāhui Hawai'i; U.M.] citizens and sympathisers, the return of these lands would be a significant first step in repairing the relationship between the United States and the Hawaiian people, would provide a suitable land base for the Native nation, and

360 „Unity Prayer“, Rev. Abraham Akaka u. William Kaina, KWO Mai 1997:12. 
would steer clear of any threat to private land holdings in Hawai 'i“ (Osorio 2003:218).

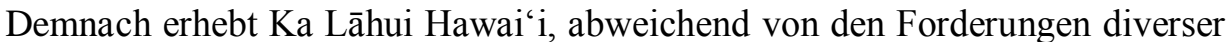
anderer Gruppierungen der Autonomiebewegung, weder einen Anspruch auf Land in Privatbesitz noch verlangt sie die völlige Souveränität des gesamten Archipels unter einer hawaiischen Regierung. In einem Beitrag zu den Anhörungen des Hawaii Advisory Committees anlässlich der Untersuchung der staatlichen Verwaltung der Hawaiian Home Lands im Jahre 1989 (siehe hierzu den folgenden Abschnitt) stellte Mililani Trask, damals Vorsitzende von Ka Lāhui, u.a. zu den „Ceded Lands“ fest:

„The central problem revolves around control and utilization of the valuable lands, natural resources and revenues of the Hawaiian homes and ceded lands which are the trust entitlements of the Hawaiian people. These trusts make the Native Hawaiians the wealthiest Native Americans in the United States. Despite this fact, the Native Hawaiians are the poorest, the sickest, and least educated of the State.

Neither the U.S. nor the State has ever been able to act as a responsible trustee in managing the Hawaiian homes or ceded lands ... Unless Native Hawaiians and Hawaiians are allowed to form self-governing entities, to be acknowledged and to control their lands and natural resources, they will continue to be denied equal protection of the laws and policies of the United States“ (zitiert in: Hawaii Advisory Committee 1991:41-2).

In ihrer Aussage verknüpft $\mathrm{M}$. Trask die Kontrolle über das hawaiische Land (hier sind ganz konkret die Areale des „Public Land Trust“ gemeint) mit dem materiellen Wohlergehen und der (völker-) rechtlichen Anerkennung der hawaiischen Bevölkerung durch die USA.

Um eine solche Forderung zu konkretisieren, reichte Ka Lāhui 1993 einen Gesetzentwurf ein, der die Übertragung des gesamten Public Land Trust an Ka Lāhui als die (noch ohne reale Landbasis) bereits bestehende souveräne hawaiische Nation vorsah. ${ }^{361}$

\footnotetext{
${ }^{361}$ Das Gesetzgebungsverfahren des Bundesstaates Hawai ‘i sieht die Einbringung ausformulierter Gesetzesentwürfe durch außerparlamentarische Interessensgruppen vor. Diese Entwürfe werden unter Einschluss der Öffentlichkeit in den jeweiligen Ausschüssen und von den beiden Kammern des Parlaments beraten. Zeitgleich stand ein von Gouverneur Waihe'e unterstützer Gesetzesvorschlag des Office of Hawaiian Affairs zur Diskussion („Senate Bill 1028“), der die Einrichtung einer „Hawaiian Constitutional Convention“ sowie deren Entscheidung über Art und Weise einer „Hawaiian Nation“ noch im Laufe des Jahres vorsah.
} 
Unmittelbar vor der Anhörung zu den Gesetzesinitiativen am 15. Februar 1993 fand eine Demonstration Ka Lāhuis statt, die vom 'Iolani Palast zum Ort des Hearings führte und an der sich nach unterschiedlichen Zählungen ca. 150-300 Menschen beteiligten. ${ }^{362}$ Die Demonstranten wandten sich vehement gegen das Office of Hawaiian Affairs und seinen Versuch, die Errichtung einer autonomen hawaiischen Verwaltungseinheit zu beschleunigen. So waren in dieser Frage der Landübertragung zwar alle zentralen Akteure (Ka Lāhui Hawai`i, das Office of Hawaiian Affairs sowie Gouverneur Waihe‘e) hawaiischer Abstammung, allerdings verorteten die Sympathisanten Ka Lāhuis sowohl das Office of Hawaiian Affairs als auch den Gouverneur eindeutig auf der „Haole-Seite“ der für außenstehende Betrachter oft so diffus und durchlässig erscheinenden Grenzziehung in den Konflikten um Land und Autonomie. Die Demonstration war dann auch weniger von der Unterstützung des eigenen Vorschlags geprägt, als vielmehr von der Gegnerschaft zum Office of Hawaiian Affairs und bun-

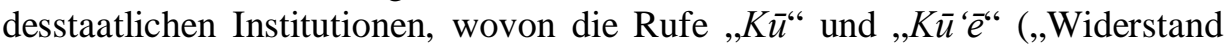
leisten“; U.M.) zeugten. Auch die Redebeiträge reflektierten diesen Gegensatz, wenn etwa der Großteil der ca. 50 Redner Stellung gegen einen Gesetzesvorschlag des Office of Hawaiian Affairs (Senate Bill 1028) bezog, der u.a. als ein Versuch gesehen wurde ,...to steal the sovereignty movement from Ka Lāhui Hawai' i“" (Kame'eleihiwa 1993:69). Die Proteste und Stellungnahmen verhinderten allerdings nicht, dass der Ausschuss lediglich S.B. 1028 befürwortete und den Text bereits am 18. Februar zur weiteren Beratung an das Parlament leitete. ${ }^{363}$

Der hier geschilderte kleine Ausschnitt aus dem „Auseinandersetzungsalltag“ Ka Lāhuis zeigt exemplarisch ein für diese Gruppierung typisches je nach Sachlage flexibles Vorgehen. Hinsichtlich des Diskurses um die Landrechtsfragen werde hierbei zwei „Argumentationsregister“ gezogen: Während sich die an die hawaiische Anhängerschaft gerichteten Äußerungen emotional aufgeladener kulturalistischer Symbole zur Begründung des eigenen Anspruchs bedienen (vgl. u.a. die Aussagen von Mililani Trask zu „Aloha 'Äina“), argumentiert Ka Lāhui gegenüber den relevanten nationalen und bundesstaatlichen Stellen in einer „kühlen“ juristischen Sprache und auf der Basis des bestehenden (Völker-) Rechts.

Die beiden oben aufgeführten Fallbeispiele zeigen das differenzierte und innerhalb der hawaiischen Bewegung durchaus auch kontroverse Bemü-

\footnotetext{
${ }^{362}$ Ich beziehe mich hier auf die Berichterstattung in den beiden großen Tageszeitungen Honolulus: „Hawaiians crowd sovereignty bills hearing“, Mary Adamski, H S-B 16.2.1993:A-1, A-6; „Sovereignty supporters demand land“, Esme M. Infante, HA 16.2.1993:A-2; „150 marchers vow to push for lands“, H S-B 16.2.1993:A-6.

363 „House panel oks Hawaiian ,con con'“, Kevin Dayton, HA 19.2.1993:A-1; die Beratungen dieses Gesetzentwurfs, der nie verabschiedet wurde, legten die Grundlage für die schließlich 1996 durchgeführte „Native Hawaiian Vote“.
} 
hen, den hawaiischen Anspruch auf das öffentliche Land zu untermauern und durchzusetzen. Dass der seit der Aufnahme des Bundesstaates in die USA andauernde rechtliche Status quo, und damit die Nutzung des „Public Land Trust“ durch den Staat Hawai'i, zumindest zweifelhaft ist, macht auch eine Passage des so genannten „Apology Law“ (Public Law 103-150 ${ }^{364}$ ) des US-Kongresses aus dem Jahr 1993 deutlich. Dort heißt es in erstaunlicher Offenheit:

„Whereas, although the Provisional Government was able to obscure the role of the United States in the illegal overthrow of the Hawaiian monarchy, it was unable to rally the support from two-thirds of the Senate to ratify a treaty of Annexation [...]

Whereas the Republic of Hawaii also ceded 1,800,000 acres of crown, government and public lands of the Kingdom of Hawaii, without the consent of or compensation to the Native Hawaiian people of Hawaii or their sovereign government;

Whereas the Congress, through the Newlands Resolution, ratified the cession, annexed Hawaii as part of the United States, and vested title to the lands in Hawaii in the United States [...]

Whereas the indigenous Hawaiian people never directly relinquished their claims to their inherent sovereignty as a people or over their national lands to the United States, either through their monarchy or through a plebiscite or referendum...“ (U.S. Congress 1993).

Diese Resolution hatte zwar keine unmittelbaren rechtlichen Auswirkungen und war auch nicht als rechtlich bindendes Dokument gedacht. Nach Auslegung verschiedener Rechtswissenschaftler kann der Text mit seinem Eingeständnis der Beteiligung am illegalen Umsturz und der Aneignung von hawaiischem Land ohne Zustimmung bzw. Entschädigung gleichwohl als Grundlage zur Durchsetzung hawaiischer Ansprüche auf eine Neuordnung des Landbesitzes und Übertragung von Landgebieten an eine zu schaffende (teil-) souveräne hawaiische Regierung dienen (Duus 2003:472f.).

Die mit der Māhele von 1855 geschaffenen öffentlichen Ländereien Hawai'is blieben also über alle Umwälzungen und Brüche der hawaiischen Geschichte hinweg erhalten, in Verträgen und Gesetzen immer wieder mit Auflagen versehen, die die Treuhandschaft und eben nicht die freie Verfügungsgewalt der jeweiligen Regierungen klarstellten und festschrieben. Nach wie vor ist die Problematik der hawaiischen Rechte an den „Ceded Lands“ aktuell und Fragen der Landübertragung sowie die damit unmittelbar verbundenen grundsätzlichen Probleme einer völkerrechtlichen Anerkennung der hawaiischen

${ }^{364}$ „Senate Joint Resolution 1993; Cal. No. 185. 103d Congress 1st Sess. (Rep. No. 103-126). 21. 1. 1993“" 
Bevölkerung durch die USA sind nach wie vor zentrale Themen einer seit den 1990er Jahren andauernden inner-hawaiischen Diskussion. Ein besonderes Kapitel stellen hierbei die Hawaiian Home Lands dar, die zwar eine wesentlich geringere Fläche als die „Ceded Lands“ umfassen und keine bedeutenden Einkünfte erzielen, als „hawaiisches“ Land aber im Bewusstsein der hawaiischen Bevölkerung einen wichtigen Platz einnehmen.

„Waiting List“, „Death List“: die Vergabe der Hawaiian Home Lands

Der Umgang mit den Hawaiian Home Lands war bis in die 1990er Jahre nicht frei von Beanstandungen. Ohne hier auf die zahlreichen spektakulären Verstöße gegen die Treuhandbestimmungen des Hawaiian Homes Commission Act im einzelnen einzugehen (siehe dazu Hawaii Advisory Committee 1991; U.S. Department of the Interior 2000; Murakami 1991:51-65), sei festgestellt, dass staatliche Kommissionen (ebd.), Betroffene und hawaiische Aktivisten (siehe u.a. Hasager und Prejean 1992) den Skandal der widerrechtlichen Nutzung des Landes im letzten Jahrzehnt des 20. Jahrhunderts immer wieder anprangerten. Es ging dabei um große Teile hawaiischen Siedlungslandes, das oft langfristig an nicht-hawaiische private Landeigner verpachtet wurde, und dies zu so günstigen Bedingungen, dass der Verdacht der Bevorteilung dieser Landbesitzer aufkommen musste. ${ }^{365}$

Vor dem Hintergrund dieser umfangreichen Landverpachtungen gewann die Vergabepraxis des Department of Hawaiian Home Lands (DHHL) besonderes Gewicht. Berechtigte Hawaiier mussten oft viele Jahre auf eine Landzuteilung warten, die viele von ihnen nicht mehr erlebten. Ebendies war der Grund für viel Unmut und Unzufriedenheit: Während dem Initiator des Hawaiian Homes Commission Act (HHCA) - Prinz Kūhiō Kalaniana'ole weithin Lob zuteil wurde, schlugen hawaiische Äußerungen einen ganz anderen Ton an, wenn es um die staatliche Verwaltung der Hawaiian Home Lands ging, sei es durch die Hawaiian Homes Commission (HHC) oder später durch das Department of Hawaiian Home Lands (DHHL).

Im Vordergrund des hawaiischen Unbehagens stand dabei in den 1990er Jahren immer wieder die lange Liste der auf Landzuteilung wartenden

\footnotetext{
365 „On the Big Island [Hawai'i], the Parker Ranch, said to be the nation’s largest, has for years leased large amounts of Hawaiian home lands for nominal rent; it pays DHHL $\$ 124,510$ annually for 32,845 acres, or $\$ 3.79$ per acre. Only 20,943 acres on the island are leased to homesteaders. On Maui, only 541 of 28,995 DHHL acres are homesteaded. But a company tied to Elmer Cravalho, a well-connected former speaker of the House and mayor of Maui, leases 15,620 acres for only $\$ 40,550$ (even less, until activists exposed the situation). [...] While only 831 acres of home lands on the island [Kaua'i; U.M.] are leased to homesteaders, a 14,558-acre chunk is leased by Kekaha Sugar, a subsidiary of Amfac, one of Hawaii’s original sugar kings. Kekaha also uses nearly all the area's available water“ (Novak 2004:195). Insgesamt waren 1991 mehr als 62\% der gesamten Fläche der Hawaiian Home Lands an Nicht-Hawaiier verpachtet (Tatibouet 1991).
} 
Berechtigten im Sinne des HHCA. So erhielten bis 1997 lediglich 6.428 Familien Siedlungsland bzw. Wohnraum auf Hawaiian Home Land, während fast 16.000 Berechtigte z.T. seit Jahrzehnten auf den Wartelisten standen (Pratt u. Smith 2000:84). Aufgrund des besonderen Rechtsverhältnisses, in dem Hawaiier gegenüber bestimmten Gesetzgebungen der Bundesregierung stehen, war es ihnen nicht möglich, den Rechtsweg gegen Beschlüsse (oder auch ausbleibende Entscheide) des DHHL zu beschreiten.

Es gab in den 1990er Jahren verschiedentlich Proteste gegen die als Unrecht empfundene Verfahrensweise des DHHL, wofür hier einige Einzelstimmen stehen mögen, die m.E. stellvertretend eine weit verbreitete Haltung gegenüber der „Waiting List“ zum Ausdruck bringen. So verfasste Charles Ka'ai'ai, ${ }^{366}$ im Jahr 1993 einen fiktiven Brief an den US-Senat, aus dem die Enttäuschung und auch der Ärger vieler Hawaiier gegenüber einer als rassistisch angesehenen Vergabepolitik spricht:

„My father was born in the year that the Hawaiian Home Lands Act was adopted. He is 70 years old and receiving Social Security. He lives in an old, run-down, rented home in a crowded, poor section of town. He is $100 \%$ blood Native Hawaiian and he is still on the damned waiting list at the Department of Hawaiian Home Lands. He is the direct beneficiary of a huge Land Trust and he has trouble making the rent. This nonsense has gone on long enough. I implore you to see that justice is served. Why should such suffering as has occured be tolerated in a democracy like the United States of America? This discrimination is racially motivated. Why else are the statistics so skewed?" (Ka'ai'ai 1993:71).

Im Entstehungsjahr des zitierten Textes fand das People's International Tribunal Hawai'i (Ka Ho'okolokolonui Kānaka Maoli) statt, dass sich auch ausführlich mit Fragen der Hawaiian Home Lands auseinandersetzte. Das Tribunal befragte hierzu Hawaiier auf allen Inseln des Archipels, um ein breites Meinungsbild zu gewinnen. Scharfe Worte fand Sondra Field-Grace, die zu den Besetzern von Hawaiian Home Lands bei Anahola, Kaua'i, gehörte, wenn sie den HHCA für die Missstände der „Waiting List“ verantwortlich macht:

„I would like to charge the United States and the State of Hawaii with murder of the 33,000 Native Hawaiians who died on the waiting list for Hawaiian Homelands. I believe it's murder. I believe they knew what they were doing. They knew when thy passed that act, that if a Hawai-

${ }^{366}$ Ka'ai'ai war zu dieser Zeit Vorsitzender der Pāhe'ehe'e Ridge Association, einer Siedlergemeinschaft auf Hawaiian Home Lands. 
ian intermarried two times they would have no rights to those lands. ... I've come to know in my heart that the Hawaiian Homes Commission Act is also genocide. To divide the race like that has caused so much hurt. ... It's murder, because they knew the Hawaiians weren't going to get on the land when they passed that act“ (Field-Grace zitiert nach Churchill u. Venne Hg. 2004:561-2).

Die Vorstellung, dass Berechtigte sterben können, während sie auf eine Entscheidung des DHHL warten, reflektiert auch ein Gedicht von Leilani Gamboa, in dem sie das vergebliche Warten der beiden Generationen vor ihr und das eigene Zurückschrecken vor der „Todesliste“ thematisiert. Die Verwendung von Vokabeln und Satzbau des „Hawaiian Pidgin“ (Hawai‘i Creole English) verleihen dem Gedicht zudem jene „hawaiische Authentizität“, die im internen hawaiischen Autonomiediskurs von erheblicher Bedeutung ist:

"Wait, / you'll get your turn. / They told tūtū ${ }^{367}$ to go - / she went home; / no humbug, her. // Wait, / you'll get your turn. /They told mama go - / she went home, too; / no humbug, her. // Wait, / you'll get your turn / they tell me. / No, I not going; / keep me off the / deathlist." (Gamboa 2003)

Eine Änderung des Status quo, d.h. der mangelhaften finanziellen Ausstattung des Department of Hawaiian Home Lands und der u.a. auch hierauf zurückzuführenden schleppenden Landvergabe zeichnete sich Mitte der 1990er Jahre ab, als die Verhandlungen zwischen Bundesstaat und DHHL über die Zahlung einer „Abfindung“ für die jahrzehntelange missbräuchliche Nutzung der Hawaiian Home Lands zu einem erfolgreichen Abschluss kamen. Unter dem Motto „Making it right: a fair and just settlement for all“ schreibt der Vorsitzende des DHHL, Kali Watson, in einem von der Institution herausgegebenen Faltblatt:

„Today we have the opportunity to finally make things right for native Hawaiians. After years of hard work, a settlement has been reached to correct past wrongs against the Hawaiian Home Lands (HHL) Trust ... This settlement, now in the form of legislative bills, would mark the end of our waiting period.

Senate Bill 1629 and House Bill 1828 settle all remaining HHL land claims, and are just and fair. More importantly, however, we can at last begin to heal the Trust and settle native Hawaiians on the land. These are exciting times for native Hawaiians and the people of Hawai'i...

\footnotetext{
${ }^{367}$ Großmutter; U.M.
} 
Let us enter the new millenium moving ahead - onipa'a“ (Watson in: Department of Hawaiian Home Lands 1995).

Die Legislatur von 1995 verabschiedete tatsächlich die entsprechenden Gesetze und machte damit den Weg frei für eine Vergleichszahlung in Höhe von US\$ 600 Mio., die in jährlichen Raten von US\$ 30 Mio. an das DHHL zu zahlen waren. ${ }^{368}$

Die Kontroversen um die Hawaiian Home Lands verweisen einmal mehr auf das komplexe Gemenge von Geschichte, Politik und ethnischer Zugehörigkeit - schließlich regelt ein Gesetz, dessen maßgeblicher Urheber ein bis in die Gegenwart gefeierter hawaiischer Adliger war, den Zugang zu ,hawaiischem“ Land über die Abstammung (50\% blood quantum-Klausel des Hawaiian Homes Commission Act) und legt somit die Grundlagen für Vorwürfe des Rassismus oder gar des Genozids. Aber auch Vertreter der Autonomiebewegung wie Ka Lāhui Hawai'i berufen sich in ihren Statuten auf diese im Hawaiian Homes Commission Act festgelegten Abstammungsregelungen, wenn es um die Vergabe zentraler Positionen in einer zukünftigen hawaiischen Nation geht. So sind die Konflikte um die Hawaiian Home Lands ein Beispiel für die oszillierenden Grenzziehungen der Autonomiebewegung zwischen „Hawaiiern“ und ihren Gegnern, zwischen einer rhetorisch immer wieder beschworenen Gemeinschaft aller Hawaiier und der realen politischen Situation, in der sich Hawaiier immer wieder als Gegner gegenüberstehen.

In den 1990er Jahren gaben sich nicht alle Hawaiier mit der vagen Aussicht auf die etwaige Zuteilung von Land zufrieden und nahmen das Recht in die eigene Hand. Es kam zu Landbesetzungen wie in Anahola, Kaua'i, mit denen die Besetzer zum einen ihre eigene Situation verbessern wollten, zum anderen aber auch auf die andauernde Problematik des Landvergabesystems aufmerksam machten. Eine besonders spektakuläre Landnahme erregte 1993 die Aufmerksamkeit der Medien und soll, nicht zuletzt wegen ihrer Implikationen für die Politikvorstellungen der Autonomiebewegung im folgenden Abschnitt kurz beleuchtet werden.

Die „Nation of Hawai 'i “ nimmt sich ihr Land: Makapu 'u Point, O‘ahu Im Mai 1993 besetzten Mitglieder des 'Ohana Council of the Hawaiian Kingdom ein Areal in der Nähe des Makapu'u Beach Parks an der Ostspitze der Insel O`ahu. Das besetzte Land stand unter der Verwaltung des Department of Hawaiian Home Lands (DHHL) und war an die Stadt Honolulu verpachtet, die es als öffentlichen Beach Park nutzte. In unmittelbarer Nachbarschaft befindet sich der Sea Life Park, mit 635.880 Besuchern im Jahre 1993 eine der

368 „Wrapping up the session: Hawaiian affairs; settlement holds promise of quick Home Land action“, Pat Omandan, H S-B 2.5.1995:A-10 
größten Touristenattraktionen auf der Insel O‘ahu (Hawaii State 1996:225). Da der Makapu‘u Beach Park darüber hinaus einer der beliebtesten Strände O`ahus ist, war der Aktion des 'Ohana Council große Beachtung gewiss. ${ }^{369}$

Die Landnehmer, deren Zahl im Laufe des Jahres auf 150 anwuchs, wollten das besetzte Land dauerhaft besiedeln und ein traditionelles hawaiisches Fischerdorf errichten. Ihr Anführer Dennis Kanahele ließ keinen Zweifel daran, dass ihnen das Recht zur Inbesitznahme des Landes zukam: „If we're indigenous people of Hawaii, we have every right to go back to our land, breathe our air and use our sea“. ${ }^{370}$

Eine Räumung des Geländes erfolgte aufgrund verschachtelter Eigentums- und Zuständigkeitsverhältnisse zunächst nicht und die Besetzung konnte bis weit in das folgende Jahr andauern. ${ }^{371}$ Eine Lösung des Konflikts zeichnete sich allerdings bereits zum Ende des Jahres 1993 ab, als das Department of Land and Natural Resources den Besetzern Ersatzflächen im benachbarten Waimānalo in Aussicht stellte. Zu diesem Zugeständnis von Seiten des Staates mag beigetragen haben, dass ein Großteil der Besetzer obdachlos war, was auch in den entsprechenden Vereinbarungen festgehalten wurde. Während es die staatlichen Stellen vermieden, auf die Argumentation Kanaheles und des 'Ohana Councils (Rechtmäßigkeit der Landnahme und Fortbestand der souveränen hawaiischen Nation) einzugehen, wandte sich Dennis „Bumpy“ Kanahele vehement gegen die Begründung staatlicherseits. Er verlangte stattdessen, sowohl die Landbesetzung von Makapu'u als auch die Inanspruchnahme des neu zugewiesenen Landes in den politischen Kontext der hawaiischen Autonomiebewegung zu stellen: „We came to you [gemeint ist der Bundesstaat Hawai' $i$; U.M.] not because of the homeless situation ... You should describe our rights and our situation“. ${ }^{372}$

Die Aktion des 'Ohana Council und der nachfolgende Umzug der Besetzer nach Waimānalo, einer überwiegend hawaiischen Siedlung, blieb innerhalb der hawaiischen Bewegung durchaus nicht unumstritten, wobei sich insbesondere Vertreter Ka Lāhui Hawai' is ablehnend äußerten. Mililani Trask (Ka Lāhui Hawai`i) äußerte sich einem Bericht des Honolulu Star-Bulletin zufolge zunächst zustimmend:

\footnotetext{
${ }^{369}$ Zunächst fand die kriminelle Vergangenheit des Anführers Dennis „Bumpy“ Kanahele stärkere Beachtung als die politischen Ziele der hinter Kanahele stehenden Gruppierung (siehe hierzu z.B. Young 1995:149; „Activist had a role in ruckus at Makapuu“, Devi Sen Laskar, H S-B 14.5.93:A-6).

${ }^{370}$ Zitat in: „Hawaiians settle in near Makapuu park“, Devi Sen Laskar, H S-B 14.5.93:A-6.

${ }^{371}$ Die Stadt Honolulu hatte das Land vom DHHL gepachtet, ließ den Nutzungsvertrag allerdings bereits im Juli 1993 auslaufen, so dass die Zuständigkeit wieder an den Staat zurückfiel. Das Department of Land and Natural Resources hatte bereits im Mai 1993 einen Antrag des 'Ohana Council erhalten, in dem dieses um die Genehmigung für die Anlage eines „Cultural Healing Village“ in Makapu'u ersuchte.

372 „Landboard oks relocation“, Stu Glauberman, HA 25.9.93:A-3.
} 
„We aggreed that in 1993, we couldn't just talk, testify and write letters, we had to do more ... We believe that peaceful occupation and civil disobedience are the only ways“. ${ }^{373}$

Doch Lilikalā Kame'eleihiwa erwähnte die Aktionen des 'Ohana Council in einem Rückblick auf die politischen Ereignisse des Jahres 1993 nur kurz und zögerte dabei auch nicht, unbestätigte Gerüchte zu nutzen, um die Gruppierung in ein schlechtes Licht zu rücken:

„In May, the 'Ohana Council moved on to Hawaiian Trust Lands located at Makapu'u Beach on O'ahu. Rumour has it that other homeless Hawaiians already living there were told that they must conform to 'Ohana Council rules or leave, but there has never been any official statement to that effect“" (Kame'eleihiwa 1993:70).

Um eine neutralere Sichtweise ist Young (1995:149-50) bemüht, doch zieht auch er die Legitimität des Landtausches in Zweifel, wenn er schreibt: „Some charge the group with getting preferential treatment in deference to Kanahele's reputation as someone who is not afraid to run afoul of the law."

Ein Grund für die hawaiische Ablehnung des „Erfolges“ von Kanahele und seinem 'Ohana Council mag in dem durchaus eigennützigen Vorgehen der Gruppe zu sehen sein, die das Erreichen eigener Ziele vor den politischen Erfolg der Bewegung stellte - die Errichtung eines Dorfes für die Mitglieder der „Nation of Hawai'i““ entsprach zwar den Zielsetzungen des 'Ohana Council, doch bleibt zu fragen, ob die Bewegung bzw. die Bemühungen um Autonomie davon in nennenswerter Weise profitieren konnten. Außerdem gelang den Mitgliedern des 'Ohana Council durch ihre außerhalb des Rechts stehende Aktion der Zugriff auf Land aus dem Bestand der Hawaiian Home Lands - Land, auf das viele Berechtigte über Jahre hinaus vergebens warten mussten und müssen.

373 „Hawaiians settle in near Makapuu park“, Devi Sen Laskar, H S-B 14.5.93:A-6. 


\section{3 „Kamehameha Schools/Bishop Estate“}

Anders als die im Vorhergehenden besprochenen Landgebiete steht das dritte hier behandelte Treuhandgebiet im Bundesstaat Hawai' $i$ nicht unter staatlicher Verwaltung. Es wird vielmehr als private Stiftung einer hawaiischen Adligen seit über 120 Jahren von einem in eigener Verantwortung stehenden Stiftungsrat verwaltet. ${ }^{374}$ Auch spielt der von der Stiftung verwaltete, bis auf Kamehameha I. zurückreichende Landbesitz für die Landforderungen der hawaiischen Bewegung so gut wie keine Rolle. Für die hawaiische Bevölkerung ist der gewaltige Besitzkomplex der Stiftung mit dem offiziellen Namen „Kamehameha Schools/Bishop Estate“ (KS/BE) dennoch in mehrerlei Hinsicht von großer Bedeutung: Zum einen finanziert der Bishop Estate, so der gebräuchlichere und im Folgenden neben der Abkürzung KS/BE verwendete Name, eine der herausragendsten hawaiischen Institutionen, die Kamehameha Schools. Diese Schule, deren weitläufiger Campus weithin sichtbar die Kamehameha Heights über der Stadt Honolulu dominiert, hat für viele Hawaiier sowohl in emotionaler wie auch bildungspolitischer Hinsicht einen hohen Stellenwert. Zum anderen aber ist der Bishop Estate als größter privater Landeigner in Hawai'i allzu oft auch Gegenspieler hawaiischer Landrechtsinteressen. So lässt sich hier am Beispiel des wirtschaftlichen und politischen Gebarens des Bishop Estate (dessen Stiftungsrat immerhin für einige Jahre nach 1993 ausschließlich mit Hawaiiern besetzt war) und der entsprechenden Gegenreaktionen von hawaiischer Seite das verschlungene Netz- und Linienwerk politischer Widersprüche, gesellschaftlicher Verwerfungen, aber auch tief- und weitgehender Übereinstimmungen innerhalb des hawaiischen Bevölkerungsteiles darstellen und einigermaßen entwirren. Doch zuvor seien der Bishop Estate selbst, seine Entstehung, seine Aufgabenfelder und heutige Bedeutung, näher vorgestellt.

\footnotetext{
${ }^{374}$ Neben „Kamehameha Schools/Bishop Estate“ existieren weitere Stiftungen hawaiischer Adliger, deren Finanzierung zu einem großen Teil auf dem in die Stiftung eingebrachten Landbesitz beruht. Keine dieser Stiftungen, obwohl ebenfalls mit bedeutenden Besitztümern ausgestattet, erreicht jedoch die politische und finanzielle Bedeutung von KS/BE. Zu nennen sind hier vor allem der „Lili‘uokalani Trust“, der von der letzten Königin Hawai‘is mit ihrem privaten Landbesitz ausgestattet wurde und der über das „Queen Lili“uokalani Children’s Center“ heute mehr als 2.000 Waisen und bedürftige Kinder hawaiischer Herkunft im Staat betreut. Der „Lunalilo Trust“", begründet von König William Lunalilo, betreibt heute in Honolulu ein Altenheim für Hawaiier und der „Queen Emma Trust“, bereits zu ihren Lebzeiten von Königin Emma, Gemahlin Kamehamehas IV., großzügig mit Finanzmitteln und in ihrem Testament auch mit Land ausgestattet, ist rechtlich in die „Queen’s Health Systems“ übergegangen, die das „Queen’s Medical Center“, das größte, für alle Bürger zugängliche Krankenhaus des Staates unterhalten (siehe ausführlicher hierzu: Kau 1991).
} 
Die Gründungsgeschichte des Bishop Estate reflektiert auf geradezu exemplarische Weise die Spannungen und gegenläufigen Tendenzen im Hawai ${ }^{\top} i$ der letzten Dekaden des 19. Jahrhunderts: Es war eine hochrangige hawaiische Adlige, die das ihr überkommene Land Kamehamehas I. einem Stiftungsrat aus Annexionisten übertrug, der mit dem Einkommen dieses Landes eine Schule einrichten sollte, in der hawaiische Kinder im Sinne der protestantischen Missionare auf ein Leben im westlich geprägten Königreich Hawai‘ $i$ vorbereitet werden sollten.

Bernice Pauahi Bishop (1831-1884), verheiratet mit dem amerikanischen Geschäftsmann Charles Reed Bishop unterzeichnete am 31. Oktober 1883 ihr Testament, die Grundlage des heutigen Bishop Estate. Selbst als Ali ‘ $i$ von höchstem Rang geboren, ${ }^{375}$ gehörte sie also nicht nur durch ihre Heirat der gesellschaftlichen Spitze des „,neuen“ Hawai'i der zweiten Hälfte des 19. Jahrhunderts an. Durchaus zu Hause in Vorstellungen westlicher Kultur und Lebensweise, nicht zuletzt durch ausgedehnte Reisen, war sie vor allem geprägt durch eine Bildung und religiöse Erziehung, die sie an der von Missionaren geleiteten Royal School genoss. In ihrem Testament bestimmte sie daher folgerichtig:

„I give, devise and bequeath all the rest, residue and remainder of my estate real and personal, wherever situated unto the trustees below named, their heirs and assigns forever, to hold upon the following trusts, namely: to erect and maintain in the Hawaiian Islands two schools, each for boarding and day scholars, one for boys and one for girls, to be known as, and called the Kamehameha schools. [...] I appoint my husband Charles R. Bishop, Samuel M. Damon, Charles M. Hyde, Charles M. Cooke, and William O. Smith, all of Honolulu, to be my trustees to carry into effect the trusts above specified“ (zitiert nach King u. Roth 2006:301). ${ }^{376}$

Der von Mrs. Bishop für die noch zu gründende Stiftung bestimmte Grundbesitz umfasste immense Gebiete auf allen Inseln des Archipels mit einer Ge-

\footnotetext{
${ }^{375}$ Pauahi war Urenkelin Kamehamehas I. und ihr Cousin König Lot, Kamehameha V., bestimmte sie auf seinem Sterbebett 1872 zu seiner bevorzugten Nachfolgerin. Bernice Pauahi Bishop und Lots Halbschwester Ruth Ke'elikōlani, die allerdings von Lot als Nachfolgerin abgelehnt wurde, waren als einzige Anwärter auf den Thron Nachfahren Kamehamehas I. in direkter Linie. Allerdings lehnte Mrs. Bishop ab und so wurde William Lunalilo, ebenfalls Nachfahre Kamehamehas I., durch Wahl der nächste Monarch des Königreichs.

${ }^{376}$ Es sei hier darauf hingewiesen, dass das ebenfalls nach Bernice Pauahi benannte Bishop Museum nicht zum Bishop Estate gehört. Das Museum wurde vielmehr im Jahre 1889 von Charles R. Bishop zu Ehren seiner verstorbenen Frau gegründet. Zum Museumskomplex in Kalihi, Honolulu, gehört heute auch das 1891 erbaute ursprüngliche Gebäude der „Kamehameha School for Boys“ („Bishop Hall“).
} 
samtfläche von 153.320 ha oder 1.533,2 km², die ihr ebenfalls durch Erbschaft zugefallen waren. ${ }^{377}$ Als Mitglieder des Stiftungsrates benannte Bernice Pauahi Bishop auschließlich Repräsentanten der neuen Zeit: Bis auf ihren Ehemann Charles Bishop waren alle genannten Treuhänder Nachkommen von Missionaren bzw. im Fall von Charles Hyde selbst missionarisch tätig und als erfolgreiche Geschäftsleute in Hawai'i arbeiteten sie später auf die Annexion der Inseln durch die USA hin. Einer der Treuhänder, William Smith, sollte darüber hinaus Mitglied des Committee of Safety werden, der treibenden Kraft des Umsturzes von 1893

Als Mrs. Bishop das Testament unterzeichnete, war noch nicht absehbar, wie reich der Bishop Estate einmal werden sollte: Im Jahr seiner Gründung hatte das Land einen Wert von US\$ 470.000 und brachte jährliche Einnahmen von US\$ 36.000, die kaum ausreichten, eine erste Gewerbeschule für Jungen zu unterhalten (King u. Roth 2006:32). Zu Beginn des 21. Jahrhunderts wurde der Marktwert des Landbesitzes, der 8\% des Staatsgebietes umfasst, auf ca. US\$ 10 Mrd. geschätzt ${ }^{378}$ und die hieraus erzielten Einnahmen betrugen im Jahre 1994 US\$ 224 Mio., wovon allerdings nurmehr US\$ 30 Mio. als Budget für die Kamehameha Schools zur Verfügung standen (Kau 1991:283; Rees 1995:4). Der Bishop Estate (KS/BE) ist damit die reichste wohltätige Stiftung in den USA; unter ihren Treuhändern, die der Oberste Gerichtshof des Staates Hawai' $i$ auf Lebenszeit benennt, fanden sich bis in die 1990er Jahre nur wenige Hawaiier, erstmals im Jahre 1993 setzte sich der gesamte Aufsichtsrat nur aus hawaiischen Treuhändern zusammen.

\section{Kamehameha Schools/Bishop Estate und hawaiischen Gruppen I: Kalama} Valley

Der Bishop Estate (KS/BE) war früh in Landrechtskämpfe mit der hawaiischen Autonomiebewegung verwickelt: In den Auseinandersetzungen um die Landrechte im Kalama Valley an der Südostküste O‘ahus standen sich in den ausgehenden 1960er Jahren der Bishop Estate als größter Landeigentümer auf der Insel und die dort ansässigen kleinen Farmer sowie hawaiische Unterstützergruppen wie das „Kōkua Kalama Committe““ gegenüber. Hier setzte KS/BE

\footnotetext{
${ }^{377}$ Allein 142.965 ha dieses Besitzes stammten aus der Hinterlassenschaft Ruth Ke'elikōlanis, (Hānai-Schwester Bernice Pauahis, Gouverneurin der Insel Hawai‘i, selbst Erbin eines großen Teils der ehemaligen Ländereien Kamehamehas I. und Zeit ihres Lebens westlicher Kultur und der englischen Sprache ablehnend gegenüberstehend); den größten Teil der übrigen 10.355 ha erbte sie von ihren Eltern (King u. Roth 2007:20-1, 26). Hānai bezeichnet ein Adoptionsverhältnis innerhalb des traditionellen hawaiischen Familienverbandes, der 'Ohana: hierbei wurden Kinder von den biologischen Eltern an andere, z.B. die Großeltern übergeben, die ihre Pflege und Erziehung übernahmen; das Verhältnis zu den biologischen Eltern blieb aber durchaus bestehen (siehe hierzu Pukui, Haertig u. Lee 1983:49-50).

${ }^{378}$ Der offizielle Wert auf der Basis der Landwerte von 1964 liegt bei US\$ 1,6 Mrd.
} 
rigoros seine Interessen durch und ließ das Land durch Polizeieinsätze räumen. In einem Artikel zur Entstehung der hawaiischen Autonomiebewegung beschreibt Haunani-Kay Trask die Lebensbedingungen der 55 Pächter und ihrer Familien, wobei sie ihre marginalen Lebensumstände als besonderen Wert herausstellt, und kontrastiert sie mit den Folgen der KS/BE-Entwicklungspläne:

„They lived in old wooden houses with their animals nearby ... Piggeries existed alongside food gardens and auto repair shops in a non-urban style variously described by residents as ,Hawaiian' and ,local'. Opposed to the austere subdivisions that were transforming $\mathrm{O}^{\text {‘ahu into a }}$ Southern California look-alike ..., Kalama Valley residents lived an open, relaxed life amongst relatives and friends in a community they felt to be a ,big family’“ (H.-K. Trask 1987:129).

Aber sie benennt auch die existierenden Gegensätze innerhalb der hawaiischen Bevölkerung, wenn sie z.B. auf die Rolle hawaiischer Institutionen wie des „Queen Lili‘uokalani Children’s Center“ und von Pastoren der „Wai‘alae-Koko Head Ecumenical Coalition“ bei der Hilfe für die ehemaligen Bewohner eingeht oder die Bewachung des geräumten Tales durch eine hawaiische Sicherheitsfirma aufzeigt: ,...the contradiction of a Trust specifically founded for the benefit of Hawaiian people proceeding to evict Hawaiians from Hawaiian land never bothered the ministers or the Center. Both groups perceived their duty to be social, not political“ (H.-K. Trask 1987:133). ${ }^{379}$

\section{Kamehameha Schools/Bishop Estate und hawaiische Gruppen II: Waiāhole Ditch}

Ein späteres Beispiel für die rigorose Ausrichtung der Politik des KS/BE an ökonomischen Zielen und die daraus resultierenden Konflikte zwischen hawaiischer Stiftung und hawaiischen Landnutzern nahm seinen Anfang in den 1973 beginnenden Auseinandersetzungen zwischen Landpächtern und dem Staat Hawai‘i über die Dauer von Pachtverträgen in den Gemarkungen Waiāhole und Waikāne auf der regenreichen Ostseite der Insel O'ahu. Zahlreiche Pächter waren hawaiischer Herkunft, doch ebenso viele hatten einen anderen ethnischen Hintergrund und die „Waiāhole-Waikāne Community Association“ vermied als Vertreterin der Betroffenen eine ethnisch ausgerichtete Festlegung. Dieser Konflikt, der schließlich die staatliche Anerkennung langfristiger Nutzungsberechtigungen bewirkte, wurde daher vor allem vor dem Hintergrund

\footnotetext{
${ }^{379}$ Bis zu welchem Grad der Bishop Estate (KS/BE) von Bernice Pauahi Bishop tatsächlich für die Ausbildung von Kindern ausschließlich hawaiischer Abstammung bestimmt wurde, ist aufgrund der Formulierung des Testaments strittig. Allerdings sind Nicht-Hawaiier in der auch gerichtlich bestätigten Praxis der Stiftung in aller Regel von Leistungen ausgeschlossen gewesen, auch wenn immer wieder Ausnahmen von dieser Regel erfolgten.
} 
existierender sozialer Gegensätze geführt (siehe hierzu Nakata 1999; Sanburn 1995).

Als die Oahu Sugar Company als eine der letzten großen Zuckerplantagen im Jahre 1994 ihren Betrieb einstellte, fand Waiāhole erneut den Weg in die Schlagzeilen und der nunmehr um Wasserrechte geführte Streit zwischen großen Landeigentümern auf der trockenen Leeseite der Ko'olau Berge und den Taro-Farmern in den Tälern von Waiāhole und Waikāne auf der Windseite hatte einen stärker ethnisch/hawaiisch geprägten Anstrich als die Kontroverse um Landnutzungsrechte einige Jahre zuvor. Gegenstand des Disputs waren die enormen Wassermengen (27 Mio. gallons bzw. $1.021 .950 \mathrm{~m}^{3} / \mathrm{Tag}$ ), die durch den Waiāhole Ditch, Teil eines für die großen Zuckerrohrplantagen angelegten Tunnelsystems, ${ }^{380}$ von der regenreichen Windseite O`ahus in die zentrale Ebene jenseits der Ko'olau Berge umgeleitet wurden und die nun zur Neuverteilung anstanden. Zu den großen Landeignern, die Ansprüche auf das Wasser des Waiāhole Tunnels anmeldeten, gehörte auch der Bishop Estate ${ }^{381}-$ er gab an, das Wasser zur Durchführung eines Wohnprojekts des gehobenen Bedarfs in Waiawa einschließlich der Einrichtung eines Golfplatzes zu benötigen (und damit für die Erwirtschaftung von Vermögen, das für die Erfüllung der wiederum den Hawaiiern zugutekommenden Bildungsaufgaben der Stiftung notwendig war). Dagegen standen als Interessenvertreter der Farmer und Naturschützer auf der Windseite O'ahus hawaiische Institutionen wie das Office of Hawaiian Affairs, das Native Hawaiian Advisory Council sowie die Native Hawaiian Legal Corporation. Und mit Ka Lāhui Hawai'i beteiligte sich neben der Waiāhole-Waikāne Community Association eine der bedeutendsten Gruppierungen der Autonomiebewegung an den Protesten gegen die Ansprüche des Bishop Estates. ${ }^{382}$

\footnotetext{
${ }^{380} \mathrm{Zu}$ diesem besonderen Aspekt der Wirtschaftsgeschichte Hawai'is - der Wasserverbrauch der großen Plantagen und die Umleitung von Wasser durch ausgedehnte Tunnelsysteme trug maßgeblich zu den wirtschaftlichen Schwierigkeiten hawaiischer Landeigner und zur mangelhaften Nutzbarkeit großer Landflächen außerhalb des Plantagenbesitzes bei - siehe ausführlich Wilcox 1996 (zu dem hier in Rede stehenden Tunnelsystem der Waiahole Water Company und der Oahu Sugar Company siehe pp. 98-108).

${ }^{381}$ Weitere Antragsteller von der Leeseite O`ahus waren zum einen der Campbell Estate, der Robinson Estate und das staatliche Department of Land and Natural Resources, die im Wesentlichen die Fortführung ihrer bisherigen Wasserrechte forderten; zum anderen waren es die Dole Food Company, das staatliche Department of Agriculture, das Honolulu Board of Water Supply und eben KS/BE, die Anträge auf neue Wassernutzung stellten. Durch die gemeinsame Antragstellung mit Vertretern privater Interessen sahen Kritiker die Unparteilichkeit des Staates in der Frage der Wasserrechte und als Treuhänder des Public Land Trust gefährdet (Tummons 1996:56).

${ }^{382}$ Bei den vorhergehenden Angaben und im Folgenden beziehe ich mich auf die nachstehenden Artikel: „The Bishop Estate question: profits or the people?“, Ian Y. Lind, H S-B 10.11.1997:A1, A-8; „OHA defends traditional Windward water rights“, Patrick Johnston, KWO, May 1995:1, 6; „Waiāhole Ditch water is troubled water“, Ke Kia“i, 1994, 5(10):1-3.
} 
Im Verlauf der Auseinandersetzungen verwiesen beide Seiten deutlich auf ihren hawaiischen Hintergrund - wenn auch in ganz unterschiedlicher Weise. Der Bishop Estate, der seine Ansprüche mit einem großen Aufgebot an finanziellen Mitteln durchzusetzen versuchte, überraschte seine Gegner mit dem Argument, dass er als Treuhänder der Ländereien Kamehamehas I. in der Tradition der Monarchen stehe und dass ihm aufgrund der Anerkennung traditioneller hawaiischer Landnutzungsrechte durch den Obersten Gerichtshof des Bundesstaates ähnlich absolute Rechte wie den ehemaligen Herrschern zukämen: „,... the trust of Bernice Pauahi, the legacy of the Kamehamehas, must be entitled to those traditional and customary prerogatives enjoyed by the Kamehameha alii...“. 383

Im Namen des Office of Hawaiian Affairs erklärte Vorstandsmitglied Kīna'u Boyd Kamali'i das Vorgehen OHAs gegen den Bishop Estate, indem sie zum einen auf die prinzipielle Gemeinsamkeit aller Hawaiier verwies, zum anderen aber die Verletzung essentieller hawaiischer Werte und Traditionen durch eine hawaiische Institution kritisierte. In einem Beitrag in der Zeitung des Office of Hawaiian Affairs Ka Wai Ola o OHA schrieb sie:

„It hurts when one Hawaiian agency must publicly challenge the actions of another Hawaiian institution; but there are clearly times when speaking up is in the best interest of all. [...]

In filing its objection, OHA is in reality making a plea to the Bishop Estate to reconsider its decision. Is developing Waiawa Ridge truly in the best interest of the beneficiaries under its trust? Granted, native Hawaiian students need classrooms, books, computers and teachers. They also need to be able to live on an island where the traditions and culture of their people are maintained and where verdant valleys and running streams can support taro growing, the restoration of fishponds, and the gathering of traditional foods from streams such as ' 0 'opu, hīhīwai and 'ōpae.

However, if the Hawaiian people are to survive and build a sovereign nation, they must maintain their ties to their history and their culture, their land and their water. The short-term payoffs generated by the development of golf courses and suburban residential areas will in the long term be destructive of values that are essential to the ultimate mission of the Bishop Estate.“ ${ }^{384}$

\footnotetext{
${ }^{383}$ Zitiert nach: „Estate claimed powers once held by Hawaii’s monarchs“, Ian Y. Lind, H S-B 10.11.1997:A-8

${ }^{384}$ „OHA objects to Bishop Estate water permit“, Kīna‘u Boyd Kamali ‘i, KWO April 1995:13.
} 
Zugleich entschärfte Kamali`i aber zumindest verbal den juristischen Angriff auf die Interessen des Bishop Estate, wenn sie zu Beginn des zweiten Absatzes die Klage des Office of Hawaiian Affairs zu einem Appell an den Bishop Estate umdeutete, das Richtige zu tun.

Dass der Bishop Estate sich in der Tradition der Ali ${ }^{\top} i$ und damit ebenfalls als Hüter hawaiischen Landes sah, macht auch eine Anzeige aus dem Jahr 1996 deutlich (der Streit um die Rechte am Wasserfluss des Waiāhole Ditch dauerte über das Jahr 1997 hinaus an). Neben einem Bild von „Ke Ali ‘i Bernice Pauahi Bishop“ und unter der halbseitigen Überschrift „The Mahele. The Overthrow. Land ,Reform'. And now Kuapā Isle.“" protestierte der Trust gegen den zwangsweisen Verkauf von Land an private Pächter, wie er von der Stadt Honolulu im Neubaugebiet von Kuapā Isle verlangt wurde. ${ }^{385}$ Dabei stellt der Bishop Estate sich und das von ihm verwaltete Land im Textteil der Anzeige in die lange Geschichte des hawaiischen Landverlustes:

„In the last two centuries, millions of acres of Hawaiian land have been taken from Hawaiians. The tragic history of abused Hawaiian ali‘ $\mathrm{i}$ property rights continues. [...]

Now, at Kuapā Isle, the City is acting to seize the ancestral lands of Ke Ali'i Pauahi, and ,redistribute' it to others. They call it ,mandatory conversion'. But it's all just a new chapter in an old history of stealing land from Hawaiians. [...] Isn't it time we stopped repeating our history, and started learning from it?، 386

Die hawaiischen Auseinandersetzungen mit dem Bishop Estate um die Rechte am Wasser des Waiāhole-Tunnels endeten erst 2002, als Kamehameha Schools/Bishop Estate nach jahrelangem Rechtsstreit und andauernden Protesten seinen Antrag auf Wasserzuweisungen aus dem Waiāhole-Tunnel zurückzog und damit den Forderungen hawaiischer Landbesitzer und hawaiischer Gruppen und ihrer Unterstützer von der Windseite O`ahus nachkam. Dieser Rückzug erfolgte nach der vollständigen Neubesetzung des Treuhändergremiums als Folge eines jahrelangen Rechtsstreits um die Verwaltung der mächti-

\footnotetext{
${ }^{385}$ Diese „mandatory conversion“, d.h. die Verpflichtung großer Landbesitzer nicht selbst genutztes und v.a. mit Eigenheimen bebautes Land an die Pächter zu verkaufen, ist Teil der bundesstaatlichen Strategie, die Zahl fester privater Besitztitel („fee simple“ im Gegensatz zum „leasehold title“) im Bundesstaat Hawai‘i zu erhöhen. Bereits in den 1970er Jahren trug die Umwandlung gepachteten Landes in feste Besitztitel durch Verkauf an die ehemaligen Pächter im Rahmen einer staatlichen „Landreform“ erheblich zum Anwachsen des Geldvermögens von KS/BE bei, so dass der Trust ab dieser Zeit auch als bedeutender Investor auf dem Kapitalmarkt auftrat.

${ }^{386}$ Ganzseitige Anzeige des Kamehameha Schools/Bernice Pauahi Bishop Estate in KWO, März 1996:5.
} 
gen Stiftung. Er kann daher durchaus im Kontext der Reorganisation sowohl der Verwaltung als auch des Verhältnisses des Bishop Estate zu seinen Nutznießern gesehen werden.

Die Krise des Bishop Estate und die Hawaiier

Abschließend folgt hier die Darstellung von Auseinandersetzungen im unmittelbaren Umfeld des Bishop Estate, die zwar nur einen mittelbaren Bezug zur Landfrage haben - kreisen sie doch vor allem um die interne Politik der Stiftung - gleichwohl aber einen besonderen Blick auf den größten hawaiischen Landeigner gewähren. Der politische und juristische Disput um den Bishop Estate ist im weiteren Rahmen dieser Arbeit auch deshalb von Interesse, da sich Hawaiier auf allen Seiten des komplexen Interessengemenges finden und so der Fokus auf die Dynamik eines zumindest in wesentlichen Zügen innerhawaiischen Konflikts gerichtet werden kann. Zudem wirft die besondere Problematik der öffentlichen Austragung eines solchen Konflikts um eine innerhalb der hawaiischen Gemeinschaft hochgeachtete Institution ein erhellendes Schlaglicht auf den nicht selten unübersichtlichen Frontverlauf innerhawaiischer Angelegenheiten.

Im Jahre 1995 prüfte die Steuerbehörde der Vereinigten Staaten, ob der Bishop Estate den Status einer wohltägigen und damit steuerbefreiten Stiftung aufrechterhalten könne. Das Medienecho reichte angesichts der Bedeutung des Bishop Estate über Hawai'i hinaus. So veröffentlichte das Wall Street Journal 1995 eine Artikelfolge, die im Honolulu Star-Bulletin nachgedruckt wurde und die mit dem Satz begann: „The giant Hawaiian trust that now owns 11 percent of Goldman, Sachs \& Co. bills itself as a charity. It's an increasingly tough sell“. ${ }^{387}$ Die Vorwürfe (darunter der Vorhalt der Finanzspekulation, die aber

387 „Bishop Estate invests unlike other charities“, Alix M. Freedman u. Laurie P. Cohen (The Wall Street Journal), H S-B 25.04.1995:A-1, A-7; Goldman, Sachs \& Co. war bis 2008 eine der weltweit führenden Investmentbanken. Ein frühes Beispiel der Kritik ist z.B. auch „A princess’ ransom: becoming a Bishop Estate trustee is the best Get-Rich-Quick scheme in the Pacific“, Desmond Byrne, Honolulu Weekly, 9.12.1992:4, 6. Einen gegenüber den Anzweiflungen kritischeren Standpunkt bezieht Rees (1995). Kritisiert wurden neben den Investitionspraktiken v.a. die Gehälter der Treuhänder: So steigerten sich die tatsächlichen Vergütungen der einzelnen Treuhänder von US\$ 13.000 im Jahre 1959 auf über US\$ 823.000 im Jahre 1995 und stellten damit die höchsten an Treuhänder wohltätiger Stiftungen gezahlten Vergütungen in den USA dar (Rees 1995: 4). Zugleich wurde u.a. bemängelt, dass nur eine relativ geringe Zahl hawaiischer Schüler wirklich in den Genuss einer Ausbildung in den Kamehameha Schools gelangte und eine Ausweitung der Schülerschaft auch nicht beabsichtigt war. Die massive und ausführlich begründete Kritik sollte in den folgenden Jahren zu einer ausgedehnten staatlichen Untersuchung der Geschäftspraxis der Stiftung und zu einem vollständigen Wechsel der in der Regel auf Lebenszeit bestellten Treuhänder führen. King u. Roth (2006) stellen die Untersuchung, deren Einzelheiten hier nicht weiter verfolgt werden, und ihre Ergebnisse aus Sicht der Kritiker ausführlich dar (beide Autoren gehörten zu den Verfassern einer Artikelserie im Honolulu Star-Bulletin, die die nachfolgenden Untersuchungen maßgeblich mit befördert hat). 
durchaus auch als die gelungene Vermehrung des Stiftungsvermögens gesehen wurde) waren z.B hinsichtlich der Treuhändervergütungen durchaus nicht frei von Anspielungen auf die ethnische Zugehörigkeit eben jener Treuhänder. So stellt z.B. Rees zum Zusammenhang der steigenden Einnahmen und Vergütungen sowie der wachsenden Zahl hawaiischer Trustees fest:

„As the revenues and commission fees increased, so too did the number of Hawaiian trustees. Today all five trustees have Hawaiian blood. The reaction to this turn of the screw in favor of Hawaiians living well has not been entirely favorable“ (Rees 1995:4).

Es ist nicht verwunderlich, dass sich bereits 1995 hawaiische Gruppen gegen die ihrer Meinung nach einseitige Berichterstattung über den Bishop Estate und seine hawaiischen Treuhänder wandten. H.K. Bruss Keppeler, Präsident der Association of Hawaiian Civic Clubs, wurde z.B. mit den Worten zitiert:

„Continuous slanted reports by the news media damage the good work and reputation of a charitable trust which has made significant and positive contributions of benefit not only to the Hawaiian people, but to the entire community“. 388

Und ein Statement der von Keppeler repräsentierten Gruppe der Hawaiian Civic Clubs, eher der politisch konservativen Richtung unter den hawaiischen Organisationen zuzuordnen, lautete dem Honolulu Star-Bulletin zufolge:

„The attacks, unrelenting series of innuendos, suppositions and denigrations, coupled with audacious suggestions on how the trustees of a private concern should operate, insult the dignity, intelligence and personal worth of every Hawaiian““. ${ }^{389}$

Sie wandten sich damit gegen die öffentliche Kritik am Träger einer Bildungsinstitution, zu deren Absolventen auch viele der politisch aktiven Hawaiier gehörten.

Das Jahr 1997 stellt einen Wendepunkt in der Geschichte des Bishop Estate dar: Im August dieses Jahres erschien der erste Teil der Serie „Broken Trust" in der Zeitung Honolulu Star-Bulletin, die umfangreiche, auch interne Informationen über die Geschäftspraxis der hawaiischen Stiftung veröffentlichte. Noch im gleichen Monat ordnete Gouverneur Cayetano die Einrichtung

\footnotetext{
${ }^{388}$ Keppeler zitiert in: „Hawaiian groups protest Bishop Estate coverage“, H A 11.05.1995:A-3

${ }^{389}$ Hawaiian Civic Club zitiert in: „Hawaiians plan protest of coverage“, Pat Omandam, H S-B 10.05.1995:A-7.
} 
einer staatlichen Untersuchungskommission zum Geschäftsgebaren der fünf Treuhänder an. Im Oktober des gleichen Jahres schließlich schlossen sich Lehrerschaft und ehemalige Schüler der Kamehameha-Schulen zu einem zuvor noch niemals da gewesenen Protest gegen die für das Bildungsprogramm zuständige Treuhänderin und die Bildungspolitik des KS/BE zusammen.

Vier der fünf Autoren von „Broken Trust“, deren erste Folge am 9. August 1997 erschien, ${ }^{390}$ waren hawaiischer Abstammung und sowohl angesehene Mitglieder der hawaiischen Gemeinschaft als auch des gesellschaftlichen Establishments. Diesen Umstand stellen Samuel King und Randall Roth, die beide zu den Autoren der Artikelserie zählten und die mit dem Buch „Broken Trust“ im Jahre 2006 eine zusammenfassende Chronik der Ereignisse veröffentlichten, besonders heraus:

„Five people had submitted „Broken Trust“ to the Star-Bulletin. Gladys Brandt, a former principal of the Kamehameha Schools and former chairwoman of the University of Hawai'i board of regents; Walter Heen, a retired judge of the State Intermediate Court of Appeals and a former state legislator and city councilman; Monsignor Charles Kekumano, a retired Catholic priest and chairman of the Queen Lili‘uokalani Trust; Samuel King, a senior Federal District Court judge; and Randall Roth, a professor at the University of Hawai'i Law School. [...]

Four of the five were Hawaiian: Brandt, Heen, Kekumano, and King. And these four were also küpuna, Hawaiian elders, with the wisdom of age: Heen was sixty-nine; Kekumano, seventy-eight; King, eighty-one; and Brandt, ninety. These did not seem like people who would try to garner publicity for themselves or hurt the sacred trust of a Hawaiian princess, an ali ‘i nui““ (King u. Roth 2006:153).

Diese Ausführungen tragen den im Raum stehenden Vorwürfen des Rassismus und der ungerechtfertigten Anwürfe von nicht-hawaiischer Seite gegen die „heilige Stiftung“ Rechnung. Und auch die Mitautorin Gladys Brandt ${ }^{391}$ erwähnt ihr Unbehagen in ihrem kurzen Vorwort zum Buch von King und Roth: „I did not enjoy my role as a critic, but I felt I had little choice. The resulting turmoil was painful but necessary“ (Brandt 2006:xi).

\footnotetext{
390 Die Artikelfolge ist im Internet verfügbar unter archives.starbulletin.com/specials/bishop1997.html (17.02.2009).

${ }^{391}$ Es soll hier auch auf die Umbenennung des Instituts für Hawai‘i Studien an der University of Hawaii at Mānoa zu Ehren von Gladys Brandt hingewiesen sein, dessen offizielle Bezeichnung (unter Verwendung ihres hawaiischen Namens) lautet: „Kamakakūokalani Center for Hawaiian Studies - Ka Hālau 'Ike o Hawai'i“'.
} 
Schon am 15. Mai 1997 machte ein Protestmarsch ehemaliger Schüler der Kamehameha-Schulen, die sich unter dem Namen „Na Pua a Ke Ali`i Pauahi“ - Kinder der Prinzessin Pauahi - zusammengefunden hatten, die Empörung über das Verhalten der mit dem Bildungsprogramm befassten Treuhänderin Lokelani Lindsey öffentlich. Der Demonstrationszug führte vom Königlichen Mausoleum in Mauna Ala, wo Pauahi Bishop bestattet ist, zum Verwaltungssitz des KS/BE in Honolulu und stellte den Zug damit auch räumlich in den Kontext des Willens Pauahis. Mit einem gewissen Sinn für Dramatik beschreiben King und Roth die Überlegungen im Vorfeld der Demonstration:

„What was being contemplated now would be a march by the Kamehameha 'ohana, the people for whom Bishop Estate was founded, against the trustees of Bishop Estate. The very idea was shocking, almost unthinkable. It was against tradition, against culture. [...] There were people in Hawai'i who were always ready to disparage Hawaiians. They would relish the spectacle of Hawaiians divided, openly in conflict with one another, about to turn the biggest, most visible, and most precious Hawaiian institution into a battlefield“ (King u. Roth 2006:134).

Doch die Demonstration gegen den Bishop Estate erfuhr bei Ankunft des Zuges eine Umdeutung, die dem Protestmarsch den Angriffscharakter nahm: Ein Redner des Absolventenjahrgangs 1941 formulierte ein Ziel der Demonstranten bei seiner Ansprache vor dem Verwaltungsgebäude des Bishop Estate wie folgt: „We are here to ho'oponopono. We are part of an ohana. [...] There are signs that they [die Treuhänder; U.M.] are listening and that they will do something for the school“ ${ }^{392}$ Durch die rhetorische und zugleich emotionale Einbindung der Trustees in den Verband einer hawaiischen 'ohana und die Darstellung der Demonstration als Angebot zum ho'oponopono, einem hawaiischen Modell der Konfliktbewältigung durch moderiertes und formalisiertes Gespräch der Kontrahenten, ${ }^{393}$ wurde der Konflikt im Vorhinein gleichsam begrenzt und durchbrach so nicht den Rahmen eines hawaiischen „Gemeinschaftsgefühls“. ${ }^{394}$

392 „Kamehameha marchers: ,We care’: about 300 gather to protest how Bishop trustees are running the school“, Gregg K. Kakesako, H S-B 15.05.1997 (Internet: archives.starbulletin.com/97/05/15/news/index.html [17.02.2009]).

${ }^{393}$ Siehe ausführlich zu dieser heute auch in der Therapie eingesetzten Konfliktlösungsstrategie Pukui, Haertig u. Lee (1983:60-70).

${ }^{394}$ Eine Demonstration von Lehrern der Kamehameha Schulen, die sich unter dem Namen Na Kumu o Kamehameha organisiert hatten, fand im Oktober 1997 statt. Sie wird hier nicht weiter berücksichtigt, da sich die organisierte Lehrerschaft aufgrund ihrer gemischen Zusammensetzung (das Testament Pauahis schrieb lediglich die protestantische Religionszugehörigkeit, nicht die 
Eineinhalb Jahre später prallten vor einer Gerichtsverhandlung am 2. Oktober 1998, die den Auftakt zur Entfernung der Treuhänder aus ihrem Amt bildete, die gegensätzlichen Meinungen zur Leitung des Bishop Estate aufeinander. Während auf der einen Seite „Na Pua a Ke Ali‘i Pauahi“ nun die endgültige Absetzung aller Treuhänder durch das Gericht forderte, traten die Gegendemonstranten für deren Verbleib und gegen jede Einmischung von außen ein. Die juristische Auseinandersetzung um Missmanagement und Fehlverhalten wurde in den Verlustdiskurs der hawaiischen Geschichte gestellt, wenn auf Seiten der Gegendemonstranten der Redner Palani Vaughan, ein bekannter Unterhaltungskünstler und ebenfalls Absolvent der Kamehameha Schulen, betonte:

„It's reminiscent of the overthrow when Hawaiians were split and polarized over the issue of whether our monarchy could fall ... As a result we lost our kingdom. If we're not careful, we could really lose an estate. ${ }^{395}$

Ihre Zuspitzung erfuhr diese Haltung in der Bezeichnung „Haole“, dem häufig abwertend benutzten Wort für die euro-amerikanischen Missionare sowie frühen Wirtschaftsoligarchen und ihre Nachkommen, das den Mitgliedern „Na Pua a Ke Ali‘i Pauahis“ zugerufen wurde und sie damit zu Verrätern an der hawaiischen Sache stempelte (King u. Roth 2006:239).

Der lang andauernde Rechtsstreit um die Verwaltung und die Treuhänder des KS/BE endete schließlich 1999 mit deren Rücktritt bzw. ihrer gerichtlich angeordneten Entfernung aus dem Amt, wobei die Treuhänder bis zuletzt den Standpunkt vertraten, dass es in diesem Prozess vor allem um einen Versuch von Nicht-Hawaiiern ging, die Macht über die Stiftung zu übernehmen bzw. zurückzugewinnen. Nicht unerwähnt bleiben soll hier, dass auch die neu ernannten Treuhänder schon bald wieder im Zentrum eines Sturms standen, als mit der Zulassung eines nicht-hawaiischen Schülers in einer Grundschule auf Maui 2002 die hawaiische Entrüstung im ganzen Staat Wellen schlug. Die aus diesen Auseinandersetzungen resultierende Rechtsprechung des Jahres 2005, die die gesamte Zulassungspolitik der Kamehameha Schulen aufgrund ihrer „preference for Hawaiians“ und damit der absoluten Ausgrenzung von NichtHawaiiern auf der Basis von „race“ für unzulässig erklärte, rief hawaiische Proteste auf allen Inseln hervor und wird von KS/BE unter Ausschöpfung der

ethnische Zugehörigkeit der Lehrer fest) in einem wesentlich neutraleren Rahmen bewegt als die (ehemalige) Schülerschaft der Schulen. Zu den Forderungen Na Kumu o Kamehamehas siehe ausführlich „Teachers speak up, defying trustees“, Greg Barrett, HA 19.10.1997:A1, A12; Statement der Organisation: „Faculty must participate in decisions“, HA 19.10.1997:B3.

${ }^{395}$ Vaughn zitiert nach: „Drive to oust trustees splits families, friends“, Craig Gima, H S-B 2.10.1998 (Internet: archives.starbulletin.com/98/10/02/news/index.html [17.02.2009]). 
Rechtsmittel bekämpft. Wie die „Rice v. Cayetano“-Entscheidung, mit der die Eingrenzung der Wählerschaft für das Office of Hawaiian Affairs auf hawaiischstämmige Wähler für nichtig erklärt wurde, ist auch dieser Gerichtsentscheid Ausdruck einer Tendenz in der Rechtsprechung, die Bürgerrechtsgesetzgebung der Vereinigten Staaten gegen die Hawaiier zu wenden. Diese juristische Gegenbewegung wird neben anderen Rechtsfragen Gegenstand des folgenden Kapitels zu den Rechtsdiskursen der 1990er Jahre sein. 



\section{Ethnie oder Nation: hawaiische Rechts- und Identitätsdiskurse}

Die im politischen Diskurs des Bundesstaates Hawai`i übliche Bezeichnung „Hawaiian Sovereignty Movement“ wie auch der in dieser Arbeit vornehmlich verwendete Begriff „Hawaiische Autonomiebewegung“ bilden beide die terminologische Klammer für eine Vielheit unterschiedlicher politischer Gruppierungen. Wie die vorangegangenen Ausführungen zeigten, unterscheiden sich diese Gruppierungen nicht nur hinsichtlich ihrer Ziele, auch ihre argumentativen und praktischen Strategien weichen nicht unerheblich voneinander ab. Doch bewirkten ihre jeweiligen Vorstellungen von politischer und kultureller Autonomie bzw. staatlicher Souveränität keine intensiven öffentlichen Grundsatzdiskussionen oder Auseinandersetzungen und schlossen wechselnde Koalitionen untereinander durchaus nicht aus. Gleichwohl trug ebendiese Verschiedenheit zu einem Eindruck der Uneinigkeit bei, der in der medialen Berichterstattung schon früh als eher diffuser Vorwurf anklang. So schrieb z.B. der Journalist John Heckathorn bereits 1988 zum Abschluss eines informativen und objektiv-ausgewogenen Artikels über die Protagonisten und die Grundlagen einer zukünftigen hawaiischen Nation in der Zeitschrift Honolulu:

„The establishment of a Hawaiian Nation might face its toughest tests not in Washington, but at home. Creating a Hawaiian nation in the midst of the Islands will be unsettling - to put it mildly - for the rest of the state's ethnic groups, even though, at this point, no one seems to be advocating taking private property or evicting non-Hawaiians. [...] Finally, the toughest test might not come from the outside; it might come from within the Hawaiian community itself, where internal squabbles are not uncommon. [...] Getting sovereignty is not going to be easy for Hawaiians; it is going to require persistent pressure, a putting aside of 
personal differences, and a willingness to compromise“ (Heckathorn 1988:91).

Die drei hier ausformulierten negativen (Vor-) Urteile sollten mehr oder weniger deutlich die Diskurse der folgenden Jahre in Medien und Politik durchziehen: die Uneinigkeit der Bewegung hinsichtlich ihrer Ziele, die angebliche Unfähigkeit hawaiischer Protagonisten, persönliche Differenzen hinter das gemeinsame Ziel zurückzustellen und schließlich die diffus-drohende Andeutung möglicher Enteignungen von Nicht-Hawaiiern - die tatsächlich von keiner der Autonomiegruppen gefordert wurden und für die es in der Rechtsgeschichte der USA auch keine Präzedenzfälle gibt.

Im Folgenden werde ich Beispiele aus Politik und Recht vorstellen, die an Grundthemen dieser Arbeit anknüpfen und sie zugleich umfassender darstellen. Das bisher gezeichnete Bild der hawaiischen Autonomiebewegung gewinnt damit mehr Perspektive, indes der gewissermaßen schon „historische“ Fokus auf die Bewegung der 1990er Jahre ausgeweitet wird.

Am Beginn der Darstellung steht das von zahlreichen hawaiischen Organisationen, politischen und kulturellen Gruppierungen sowie staatlichen Stellen unterstützte Informationsbündnis Hui $\mathrm{Na}$ 'auao und damit die grundsätzliche Herleitung des hawaiischen Anspruchs auf Autonomie. Im Anschluss daran konturiert die nähere Betrachtung eines Verfahrens vor dem Internationalen Schiedsgericht in Den Haag die erheblichen Unterschiede zwischen einem völkerrechtlich argumentierenden Votum für die staatliche Souveränität Hawai'is und der vorherrschenden, ethnisch und kulturell begründeten Forderung nach Autonomie und Besserstellung des hawaiischen Bevölkerungsteiles. Die Skizze eines Aufsehen erregenden Urteils des Obersten Gerichtshofes der Vereinigten Staaten im Jahre 2000 schließt sich an: Mit ihrer Entscheidung gegen das hawaiische Exklusivrecht, den Vorstand des Office of Hawaiian Affairs zu wählen, stellten die obersten Richter die historisch, kulturell und ethnisch begründeten Privilegien der Hawaiier im Bundesstaat Hawai‘i zur Disposition. Den Abschluss bildet ein Blick auf die so genannte „Akaka Bill“, ein Gesetzentwurf, der die Anerkennung des hawaiischen Sonderstatus durch die Bundesregierung forcieren soll. Die hierdurch ausgelösten Diskussionen werfen erneut ein Schlaglicht auf das Spannungsfeld zwischen historisch-politischen Entwicklungen, der argumentativen Bedeutung „hawaiischer Abstammung“ sowie den US-amerikanischen Vorstellungen von „Race“, das den Hintergrund für den Diskurs über hawaiische Identität und die hawaiische Autonomie bildet und dessen jeweilige thematische Akzente in den Einzelkapiteln dieser Arbeit beleuchtet wurden. 


\subsection{Autonomie oder Souveränität, Nationalität oder Ethni- zität?}

Die Forderungen der hawaiischen Autonomiebewegung hatten ziemlich bald ein umfangreiches Korpus der Rechtsliteratur zur Folge. Sie behandelte insbesondere Analogien zur staatsrechtlichen Situation der „Indian Nations“ in den kontinentalen USA (z.B. Benjamin 1996; Kahanu u. van Dyke 1995; Mardfin 1994), die mögliche Rolle des Office of Hawaiian Affairs als Keimzelle einer hawaiischen Nation (z.B. McKenzie 1991b) oder untersuchte die grundsätzlichen, nicht rein rechtlichen Voraussetzungen einer hawaiischen Autonomie (z.B. Duus 2003; Lâm 1996; Oshiro 1995; L. K. Friedman 1992). An diesen Fachdiskursen waren hawaiische Juristen, unter ihnen auch der Bewegung nahe stehende Rechtswissenschaftler, maßgeblich beteiligt, jedoch fanden die Subtilitäten ihrer juristischen Erörterungen in aller Regel nicht den Weg in die öffentlich geführten Diskussionen, wo weithin eher plakative Aussagen das Bild bestimmten.

Daneben hatten verschiedene Konferenzen und Tagungen, die sich vor allem an die hawaiische Bevölkerung wandten, die unterschiedlichen Positionen innerhalb der Autonomiebewegung zum Thema und präsentierten die diversen Pläne und Zielvorstellungen. Die zugehörigen Veröffentlichungen bieten durch ausgefüllte Fragebögen bzw. von den Organisatoren erstellte Tabellen die Möglichkeit, Zielvorstellungen zu vergleichen und den jeweiligen Gruppierungen zuzuordnen (siehe z.B. Kenya Information Services 1993; Pono Kaulike 1995). ${ }^{396}$ Dabei kristallisieren sich, sieht man von kleineren Unterschieden ab, zwei Entwicklungslinien heraus: die eine, als deren Vorbilder die indianischen Nationen in den kontinentalen USA gelten können und die im hawaiischen Diskurs als „nation within a nation“ bekannt ist, während die andere auf nichts weniger als die völlige Unabhängigkeit eines „hawaiischen

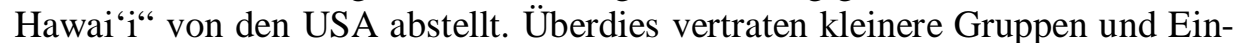
zelpersonen die Ansicht, das Gebiet des heutigen Bundesstaates solle geteilt oder unter hawaiischer Verwaltung in eine freie Assoziierung („,compact of free association“) mit den USA überführt werden, ähnlich dem völkerrechtlichen Status verschiedener Staaten in Mikronesien.

\footnotetext{
${ }^{396}$ Eine sehr ausführliche Darstellung der Autonomievorstellungen der größten Bewegungsgruppierungen (Nation of Hawai'i, Ka Pākaukau, Ka Lāhui Hawai'i, Institute for the Advancement of Hawaiian Affairs) sowie des Office of Hawaiian Affairs liefert der Politikwissenschaftler Anthony Castanha (1996). Kurze informative Interviews bekannter Protagonisten und eine Kurzfassung ihrer jeweiligen Standpunkte zur Autonomie finden sich auch in „Models for sovereignty“, Becky Ashizawa, H S-B 12.1.1993:A-6.
} 
Hui Na'auao: Koalition der Unterweisung

In der ersten Hälfte der 1990er Jahre wurde mit Hui Na'auao ein von zahlreichen Organisationen und Gruppen getragenes Informationsbündnis geschaffen, das eine breite hawaiische Öffentlichkeit über die diversen politischen Zielvorstellungen sowie die historischen und rechtlichen Grundlagen der seitens der Bewegung erhobenen Forderungen unterrichten sollte. Hui Na'auao (frei zu übersetzen als „Koalition der Unterweisung“) war ein Beispiel für die temporäre Kooperation sehr konträrer Gruppierungen und Institutionen. Gegründet 1991 und finanziell gefördert von der Administration for Native Americans (einer Behörde der US-Regierung), beschrieb sich das Bündnis selbst als: „An association engaged in promoting awareness of Hawaiian sovereignty and selfdetermination“ (Hui Na'auao 1992). Auf der Liste der 47 Mitgliedsorganisationen fanden sich staatliche, kirchliche und unabhängige politische Institutionen und Initiativen, das Office of Hawaiian Affairs, die Native Hawaiian Chamber of Commerce oder die Hawai'i Ecumenical Coalition ebenso wie Ka Pākaukau, die Protect Kaho'olawe 'Ohana oder der konservative Royal Order of Kamehameha. Zu den Unterstützern von Hui Na'auao zählte u.a. eine staatliche Behörde wie das Department of Hawaiian Home Lands, während die so unterschiedlich orientierten Kamehameha Schools/Bishop Estate, Ka Lāhui Hawai'i oder das 'Ohana Council unter der Rubrik „Interested Organizations“ geführt wurden (Hui Na'auao 1993). Bereits die wenigen hier genannten Institutionen und Organisationen veranschaulichen die sehr breite inhaltliche Fächerung der Mitglieder und Unterstützer von Hui Na'auao, die schließlich das gesamte Spektrum des hawaiischen Autonomiediskurses vertreten sollte.

Die Vermittlungsarbeit des Informationsbündnisses stützte sich vor allem auf Workshops, die in Zusammenarbeit mit lokalen Vereinigungen, Kirchengemeinden usw. auf allen Inseln durchgeführt wurden. Hierzu erklärte Hui $\mathrm{Na}$ ‘auao in seiner „Project Description“:

„Hui Na'auao conducts workshops and presentations which provide participants with an ancient to modern overview of Hawaiian history including evolutions in Native Hawaiian governance systems. ,Ho'ala', to awaken, is the first of Hui Na'auao's two-phased educational plan. Ho'ala examines pre and post contact Hawai'i as an inherently sovereign nation with its own culture, governance and land base... [...]

Forums currently conducted by Hui Na'auao feature a cross-section of individuals and organizations whose views represent some of the most advanced thinking on sovereignty and self-determination. Proponents of each model present their views in forums and panel discussions throughout the state. These forums provide the public with opportunities to interact with many organizations who seek, practice and/or are 
concerned about Hawaiian sovereignty and self-determination“ (Hui Na'auao o.J.a).

Einleitend wird in dieser Projektbeschreibung festgestellt, dass Kenntnisse und Meinungen aller Mitgliedsorganisationen in den gesamten Entwicklungsprozess des Curriculums eingeflossen sind - eine Umschreibung der grundsätzlichen Neutralität des Hui Na‘auao, die ja auch die Ausführungen im obigen Zitats deutlich machen. Hier wird auf allgemeine historische Grundlagen, die in der Bewegung entwickelten Autonomiemodelle und deren Vermittlung durch ihre jeweiligen Protagonisten abgehoben. In der Realität ließ sich dies allerdings nur begrenzt umsetzen: so fanden z.B. auf einem von mir besuchten Workshop am 29. April 1995 in der Kirche der United Church of Christ in Kahului, Maui, vor allem die Standpunkte der Rednerin vom Office of Hawaiian Affairs Gehör.

Zu den einzelnen Informationsblöcken stellte Hui Na ‘auao Arbeitshefte bereit, die in allgemein verständlicher Form das Informationsmaterial darbieten und mit konkreten Arbeitsaufgaben verknüpfen (Hui Na'auao o.J. b; 1994). Diese Hefte fanden in zahlreichen Veranstaltungen überall auf den Inseln Verwendung und die mit ihnen verbreitete Darstellung der hawaiischen Geschichte und möglicher zukünftiger Entwicklungen trug erheblich zu einer „hawaiischen Bewusstseinsbildung“ bei. Im Heft zum ersten Abschnitt Ho 'āla finden sich Materialien zum Verständnis der Autonomieforderungen, auf die ich noch näher eingehen werde. Neben historischen Karten aller hawaiischen Inseln mit ihren Grenzen und Namen der alten ahupua' $a$, der traditionellen Landnutzungsdistrikte, umfasst die Materialsammlung eine Liste der von 1846 bis 1863 zwischen dem Königreich Hawai‘i und europäischen Staaten geschlossenen Freundschafts- und Handelsverträge (ein Beleg für die ehemals international anerkannte Souveränität des Königreichs). Anschließend folgen der edierte Text des von Lili‘uokalani verfassten Schreibens zu ihrem Rücktritt 1893 und, auf 13 Seiten vollständig wiedergegeben, Präsident Grover Clevelands „Message to Congress relating to the Hawaiian Islands“ vom 18. Dezember 1893. Die Auswahl dieser Texte ist nicht ungewöhnlich, gehören sie doch zu einem „Kernbestand“ historischer Daten und Texte, die im Kontext der Autonomiebewegung als Belege für die internationale Anerkennung des souveränen Königreichs Hawai'i und die Unrechtmäßigkeit des Umsturzes sowie der Dokumentierung des hawaiischen Widerstandes dagegen dienen.

Insbesondere die absichtsvollen Hervorhebungen im letzten Text (Grover Clevelands „Message to Congress...") machen sehr deutlich, dass Hui Na'auao den Umsturz von 1893 und dessen völkerrechtliche Illegalität in den Vordergrund der Argumentation rückte. Durch Großdruck betont werden u.a. folgende Passagen des Dokuments: 
„...the ownership of Hawaii was tendered to us by a provisional government set up to succeed the constitutional ruler of the islands, who had been dethroned, and it did not appear that such provisional government had the sanction of either popular revolution or suffrage. [...]“ (Hui Na‘auao o.J.b:1-2).

„This military demonstration upon the soil of Honolulu was itself an act of war [Hervorhebung im Original; U.M.], unless made either with the consent of the Government of Hawaii or for the bona fide purpose of protecting the imperiled lives and property of citizens of the United States. But there is no pretense of any such consent on the part of the Government of the Queen, which at the time was undisputed and was both the de facto and the de jure government" (Hui Na'auao o.J.b:6).

Und schließlich auch die vielzitierten Textstellen:

„By an act of war committed with the participation of a diplomatic representative of Congress, the Government of a feeble but friendly and confiding people has been overthrown. A substantial wrong has thus been done which a due regard for our national character as well as the rights of the injured people requires we should endeavor to repair“ (Hui Na'auao o.J.b:11).

„She [Königin Lili‘uokalani; U.M.] surrendered not to the provisional government, but to the United States. She surendered not absolutely and permanently, but temporarily and conditionally until such time as the facts could be considered by the United States“ (Hui Na'auao o.J.b:12).

So werden in den Worten des damaligen höchsten Repräsentanten der Vereinigten Staaten die auch heute noch gültigen Argumente gegen die Rechtmäßigkeit des Umsturzes von 1893 zusammengefasst und dem Publikum ohne ausufernde Rechtsdiskussion leicht verständlich präsentiert. Zugleich erfährt der Umsturz eine Bewertung als das zentrale Ereignis der neueren hawaiischen Geschichte - ein Einschnitt, der (wie die Ankunft Cooks) die Geschichte in ein Davor und ein Danach teilt. Diese Bewertung wird vom allergrößten Teil der Autonomiebewegung geteilt, auch wenn es hinsichtlich der daraus abzuleitenden Konsequenzen durchaus differierende Auffassungen innerhalb der Bewegung gibt.

Darüber hinaus bietet der Leitfaden Anregungen zur Erarbeitung weiterer Themen, von denen ich hier zwei herausstellen möchte. Sie reichen über den unmittelbaren Kontext der rechtlichen und historischen Grundlagen hinaus 
und sind daher auch in Hinblick auf den hawaiischen Diskurs von Kultur, Abstammung, Identität und Autonomie von besonderem Interesse. Als „Activity \#3“ schlagen die Autoren unter anderem vor, nachzuvollziehen, wie sich hawaiische Religion sowie kulturelle Vorstellungen und Praktiken in der Auseinandersetzung mit den jeweils neuen Gegebenheiten änderten und warum sie über den Umsturz von 1893 hinaus weiter Bestand hatten (womit sie eine gewisse Kontinuität der genannten Aspekte als gegeben voraussetzen). Die Schlussfrage dieser „Activity \#3“ ist dann: „How will Sovereignty help perpetuate the Hawaiian culture?“ - Politische Autonomie wird hier nicht nur mit hawaiischer Kultur verknüpft, die Verfasser setzen auch voraus, dass die hawaiische Autonomie sich positiv auf den Fortbestand der hawaiischen Kultur auswirken wird bzw. legen im Umkehrschluss nahe, dass ihr Weiterbestand unter den Bedingungen des Status quo zumindest problematisch ist (auch über diese Auffassung besteht weitestgehend Einhelligkeit innerhalb der hawaiischen Bewegung). Indes bleibt hier festzuhalten, dass in diesen Textpassagen „Sovereignty“ ein Vehikel für die „hawaiische Kultur“ zu sein scheint und ihr nachgeordnet ist - anders als dies bei der Politisierung kultureller Phänomene und Praktiken durch die Bewegung zu beobachten war.

Neben dieser Verknüpfung der politischen Autonomie mit den erheblich weniger kontroversen Fragen hawaiischer Kulturpraktiken fällt unter den Unterrichtsmaterialien eine mehrseitige Anleitung zur Erstellung einer persönlichen Genealogie ins Auge: Unter dem Titel „He Mo'olelo 'Ohana Mo'okū'auhau...“ (Familienstammbaum; U.M.) findet der Leser einen Vordruck, in den er Namen und Daten seiner Vorfahren der vergangenen vier Generationen einsetzen kann. Der begleitende Text führt hierzu aus:

„The purpose ... is to find out how much you know about who you are and where you came from, as a Hawaiian. Questions you must ask yourself while you do these handouts are: how far back in time can you trace your 'ohana genealogy? What were they doing at the time of the 1893 overthrow of the Hawaiian Monarchy? The Mahele? The ending of the kapu system? The unification of the islands by Kamehameha? [...]

By answering these questions you are able to understand how much you really know or don't know about your link to a Hawaiian past. ... Those who do have their 'ohana genealogy are fortunate. A genealogy is a source of identity and place“ (Hui Na'auao o.J.b:o.S.).

Der Text verknüpft die Genealogie des Einzelnen mit den Wendepunkten der hawaiischen Geschichte, so dass über die persönliche Abstammungslinie historische Ereignisse Teil der individuellen und zugleich kollektiven Erfahrung und so wesentlicher Faktor der eigenen hawaiischen Identität werden. Eben diese 
Verknüpfung von Abstammung und Identität kommt im hawaiischen Autonomiediskurs zum Tragen; der weit überwiegende Teil der hawaiischen Bewegung hält die hawaiische Abstammung des Einzelnen denn auch für ein konstitutives Merkmal bzw. die Vorbedingung für eine reguläre Zugehörigkeit zu einem zukünftigen autonomen oder souveränen hawaiischen Gemeinwesen. Eine Minderheit unter den Exponenten der politischen Bewegung teilt diese Auffassung jedoch nicht: Aufgrund einer abweichenden Betrachtung der hawaiischen Historie und Bezugnahme auf andere Wendepunkte der Geschichte geben sie einem vom Territorialprinzip (ius soli) bestimmten Staatsbürgerschaftsrecht vor demjenigen des ius sanguinis den Vorzug. Diese gegensätzlichen Argumentationen und damit verbundenen Weiterungen von Abstammung, Ethnizität und politischer Geschichte in den Diskursen über Autonomie und „Hawaiianness“ stehen im Mittelpunkt des folgenden Abschnittes.

\section{Abstammung oder Nationalität: Wer kann ein Hawaiier sein im neuen Hawai' $i$ ?}

Hinsichtlich der Begründungen, die zur Legitimierung der Notwendigkeit eines autonomen/souveränen hawaiischen Gebildes vertreten werden, stehen sich im Wesentlichen zwei Lager mit konträren Auffassungen gegenüber. Für die einen beruht das hawaiische Recht auf „Sovereignty“ bzw. die Verpflichtung der USA zur Anerkennung der hawaiischen Autonomie auf der fortdauernden kulturellen und politischen Marginalisierung der Hawaiier nach dem Sturz der Monarchie, der zunehmenden Kolonialisierung und schließlich der Eingliederung des Archipels in die USA. Zwar unterscheiden sich die Protagonisten in den Schlussfolgerungen hinsichtlich der staatsrechtlichen Konsequenzen einig sind sie sich allerdings darin, dass eine allen gemeinsame hawaiische (biologische) Abstammung notwendige Voraussetzung für die Teilhabe am zukünftigen wie auch immer verfassten Gemeinwesen ist.

Ein anderer Standpunkt sieht die Legitimierung des Souveränitätsanspruches ebenfalls im Umsturz von 1893 als dem zentralen Wendepunkt der hawaiischen Geschichte. Im Vordergrund dieser Argumentation stehen jedoch weniger die negativen sozialen, politischen und kulturellen Folgen des Umsturzes für die Hawaiier, als vielmehr seine völkerrechtliche Illegitimität. Aus diesem primären Rechtsbruch resultiert dieser Argumentationslinie zufolge die Unrechtmäßigkeit aller weiteren Rechtsschritte, die von der Republik Hawaii, der Territorialregierung oder der Bundesregierung in Washington bis hin zum Referendum von 1959, das die Aufnahme in die USA besiegelte, eingeleitet wurden. Für die Vertreter dieser Richtung geht es in der Diskussion über die hawaiische Autonomie um nichts weniger als die Wiedererrichtung eines souveränen Nationalstaates Hawai‘i.

$\mathrm{Zu}$ den wichtigsten Exponenten der ersteren Richtung zählen, trotz gravierender Unvereinbarkeiten in vielen anderen Punkten, Ka Lāhui Hawai‘ $i$ 
und das Office of Hawaiian Affairs, die beide ein autonom verwaltetes hawaiisches Gebiet fordern und die Abstammungsgemeinschaft der Hawaiier als ein konstitutives Element eines solchen Gemeinwesens ansehen. Verfechter eines von den USA vollständig unabhängigen souveränen Hawai‘ $i$ sind u.a. Ka Pākaukau (Sprecher ist Kekuni Blaisdell), das Institute for the Advancement of Hawaiian Affairs (I.A.H.A.) mit seinem Leiter Pōkā Laenui und, seit seiner Gründung 1996, das Council of Regency of the Kingdom of Hawai'i, das von David Keanu Sai vertreten wird. Die Positionen der drei letztgenannten Gruppierungen werde ich im Folgenden näher betrachten, zeigen sich doch an diesen Beispielen, je nach ihrem Rechtsverständnis und ihrer jeweiligen Geschichtsdeutung, unterschiedliche Bewertungen der gemeinsamen Abstammung und ethnischen Zugehörigkeit.

Zunächst sollen kurz die Standpunkte von Ka Pākaukau und des Institute for the Advancement of Hawaiian Affairs (I.A.H.A.) rekapituliert bzw. im Falle des Council of Regency vorgestellt werden. Sowohl Ka Pākaukau als auch das Institute for the Advancement of Hawaiian Affairs (I.A.H.A.) beschreiben den Sturz der Monarchin Lili‘uokalani im Januar 1893 als den Beginn einer bis heute andauernden Kolonialisierung Hawai'is durch die USA. Beide sehen die erneute Aufnahme des US-Bundesstaates Hawai' ${ }^{\prime} \mathrm{i}$ in die UNListe abhängiger Territorien als einen ersten Schritt hin zu einem geordneten Prozess der Dekolonisation, an dessen Abschluss ein unabhängiges souveränes Hawai' $i$ stehen soll. Über die Regierungsform dieses Staatsgebildes werden dann dessen Bürger zur gegebenen Zeit bestimmen. Doch während Ka Pākaukau ethnische Hawaiier als Bürger dieses neuen Staatswesens klar bevorzugt, ${ }^{397}$ stellt das Institute for the Advancement of Hawaiian Affairs (I.A.H.A.) ein entkolonialisiertes Hawai‘i in die Nachfolge des Königreichs: Dies bedeutet, dass nicht die hawaiische Genealogie (zurückzuführen in die Zeit vor der Ankunft James Cooks), sondern die Abkunft von einem Bürger des Königreichs (vor 1893) entscheidendes Kriterium für die Zugehörigkeit sein würde womit eine ethnisch definierte Abstammungsgemeinschaft für die Staatsbürgerschaft an Bedeutung verlöre. ${ }^{398}$

\footnotetext{
${ }^{397}$ Nicht-Hawaiiern stünde die Mitgliedschaft unter bestimmten Bedingungen ebenfalls offen, sie wären aber nur eingeschränkt berechtigt, tragende Funktionen zu übernehmen.

${ }^{398}$ Zahlreiche Bürger des Königreichs waren naturalisierte Euro-Amerikaner, die ja zum Teil hohe Positionen in Verwaltung und Regierung innehatten. Ein weiterer Vertreter einer vollständigen Unabhängigkeit Hawai`is ist Michael Kioni Dudley. Als Nicht-Hawaiier war Dudley in der Autonomiebewegung von eher marginaler Bedeutung, hatte aber als Koautor des Buches „A call for Hawaiian sovereignty“ (Dudley u. Agard 1993) in der ersten Hälfte der 1990er Jahre eine starke Medienpräsenz. Auch Dudley überträgt das Staatsbürgerrecht des Königreichs in seine Projektion eines souveränen Hawai 'i, wobei er jedoch die repräsentative Rolle eines Staatsoberhauptes sowie dessen Wahl hawaiischstämmigen Bürgern vorbehalten sehen möchte (Castanha 2006:Kap. 7, S. 14).
} 
Anders als die beiden bereits genannten Gruppen geht das Council of Regency of the Hawaiian Kingdom davon aus, dass die erzwungene Abdankung der Königin Lili‘uokalani im Sinne des Völkerrechts unwirksam blieb und das Königreich Hawai'i somit unter der Fremdherrschaft der Vereinigten Staaten weiter bestand und besteht. Bei Zugrundelegung dieser Annahmen wären die aktuellen Diskussionen um die zukünftige Form einer hawaiischen Selbstbestimmung und Autonomie oder Souveränität hinfällig. ${ }^{399}$ Eine solche Argumentation geht zugleich von der Fortdauer des bis 1893 geltenden Staatsbürgerrechts aus und erteilt damit, da die Bürgerrechte des Königreichs ja nicht aufgrund der hawaiischen Abstammung vergeben wurden, einer ausschließlich ethnisch begründeten staatsbürgerlichen Teilhabe an einem souveränen Hawai‘i eine Absage. Eine große Öffentlichkeit war dem zuvor eher unbekannten Council of Regency (und seinem Repräsentanten David Keanu Sai) schließlich sicher, als es Partei eines Verfahrens vor dem Ständigen Schiedsgerichtshof in Den Haag wurde. ${ }^{400}$

Der Hintergrund dieser Auseinandersetzung vor einem internationalen Gericht mag zunächst nichtig anmuten: 1999 wurde der bereits mehrfach wegen ähnlicher Vergehen bestrafte Lance Larson, Anhänger des Council of Regency, wegen Fahrens eines nicht zugelassenen Autos verhaftet. Er klagte daraufhin zunächst vor einem US-Bundesgericht gegen die Inhaftierung und begründete seine Klage mit der Feststellung, dass die Gesetze der USA bzw. einer untergeordneten Instanz wie des Bundesstaates Hawai'i im souveränen Königreich Hawai'i keine Geltung hätten. Da er aufgrund ungültiger Gesetze eines fremden Staates verhaftet worden sei, hätte ihm das Königreich Hawai‘ $i$ den ihm als Bürger des Königreichs zustehenden Schutz vorenthalten. Der zuständige Richter in Hawai‘i wies die Klage ab, machte (interessanterweise) jedoch eine bindende Schlichtung vor dem Internationalen Schiedsgerichtshof zur Auflage, die in der Folge von beiden Beteiligten, Larson und dem Council of Regency, auch beantragt wurde.

Jonathan K. Osorio, Direktor des Center for Hawaiian Studies der University of Hawai'i Mānoa, nahm dieses Verfahren zum Anlass, grundsätzliche Unterschiede zwischen dem Council of Regency und Ka Lāhui Hawai'i herauszuarbeiten. Dabei stellte er die Frage nach der Bedeutung von Recht und Ethnizität für die Begründung des hawaiischen Rechts auf Autonomie bzw.

\footnotetext{
399 Siehe hierzu Council of Regency (2007) und Osorio (2003).

400 „Hague tribunal ponders the Hawaiian kingdom“, Pat Omandam, H S-B 2.1.2001 (Internet: starbulletin.com/2001/01/02/news/story1.html [4.4.2001]). Der Ständige Schiedshof (Permanent Court of Arbitration) mit Sitz in Den Haag ist eine von den Vereinten Nationen unabhängige zwischenstaatliche Organisation, die aufgrund von Abkommen aus den Jahren 1899 und 1907 operiert. Er erlässt Schiedssprüche, schlichtet und setzt Untersuchungskommissionen in Streitfällen zwischen Staaten, privaten und zwischenstaatlichen Organisationen auf der Grundlage internationaler Schiedsbestimmungen ein (siehe PCA 2007 und Europa 2006).
} 
Souveränität (Osorio 2003). Indem er die Auffassung des Council of Regency grundsätzlich ablehnte, stellte er fest:

„Americans look to law to define and protect their nation's fragile sense of racial diversity. Hawaiians have never needed laws to promote racial diversity and cannot now place their faith in law that discounts ancestry as either an irrelevant or unwarranted emblem of identity. To do so would entail relinquishing the last vestige of that which makes us Hawaiian in the first place. As to the necessity of law's existence, there is perhaps, less disagreement. Even as the Council of Regency proclaims the importance of recognizing Kingdom constitutions, few other sovereignty groups will contest the importance of having a constitution in the first place“ (Osorio 2003:234).

Wie für fast alle Vertreter der hawaiischen Autonomiebewegung bleibt auch für Osorio die hawaiische Abstammung das entscheidende Kriterium für die Zugehörigkeit zu einer hawaiischen Gemeinschaft. Anders als die Mutabilität juristischer Definitionen und Regeln - zum Zeitpunkt der Veröffentlichung seines Textes lag das für die rechtliche Situation der Hawaiier folgenreiche Urteil im Prozess Harold Rice gegen Gouverneur Ben Cayetano (siehe das nachstehende Kapitel) erst kurze Zeit zurück - stellt die biologische Abstammung in aller Regel ein stabiles, nicht verhandelbares Attribut dar. Auch wenn die von staatlicher Seite verfügte juristische Bewertung dieses Attributs Veränderungen unterliegt (hier wäre z.B. die „,50\% Abstammungsregel“ des Hawaiian Homes Commission Act zu nennen), stellt die Berufung auf die gemeinsame Genealogie in der politischen und juristischen Auseinandersetzung ein wirksames, in seiner Substanz kaum angreifbares Argument dar. In diesem Zusammenhang gewann ein mögliches Verfahren vor dem Haager Schiedsgericht besondere Bedeutung: Eine positive juristische Bewertung der Standpunkte des Klägers (und damit auch des Council of Regency) hätte die völkerrechtliche Anerkennung des Fortbestandes des Königreichs Hawai‘i durch ein internationales Gericht bedeutet - und so den Gegnern eines sich auf der Exklusivität hawaiischer Abstammung gründenden Autonomiemodells neue und starke Argumente geliefert.

Im Jahre 1999 nahm der Ständige Schiedshof in Den Haag den Fall „Lance Larson gegen Kingdom of Hawai'i“" tatsächlich an, womit das Gericht, dessen Auftrag sich ja auf die Schlichtung von Streitsachen mit staatlicher Beteiligung beschränkt, dem Antrag bereits eine gewisse juristische Substanz zugestand. Es hatte nun über eine Rechtsfrage zu verhandeln, die sich in den Worten der Klageschrift wie folgt liest: 
„The primary issue in contention between the parties is that of the liability of the Respondent Government of the Hawaiian Kingdom towards the Claimant [Lance Larson; U.M.] with respect to his injuries. As summarized in Claimant's Memorial, it is Claimant's position that the Respondent Government of the Hawaiian Kingdom has a duty to protect Claimant's rights as a Hawaiian subject, even in times of war and occupation. [...] Because the occupation of the Hawaiian Islands still continues, Claimant's rights continue to be violated. Until Claimant's rights are fully protected, his Government has not fulfilled its obligations towards him as a Hawaiian subject. Claimant now seeks redress against his Government because this obligation has not been fulfilled“ (PCA 2001:8-9).

Nach zweijähriger Verhandlungsdauer stellte der internationale Schiedshof das Verfahren am 5. Februar 2001 ein. In ihrer Begründung der Einstellung zweifelten die Richter allerdings nicht an der Legitimität des Council of Regency of the Hawaiian Kingdom und seines Anspruches, das existierende souveräne Königreich Hawai`i zu vertreten. Als Grund für die Einstellung des Verfahrens nannten sie vielmehr die bestehende Einigkeit zwischen Kläger und Beklagtem (Larson und dem Council of Regency) über die oben genannten Beschuldigungen und die daraus folgende fehlende Notwendigkeit einer Schlichtung. Hinsichtlich der Rolle der Vereinigten Staaten als Besatzungsmacht stellte der Beschluss lediglich fest, dass die Prozessordnung des Gerichts eine Bewertung dieser Frage nicht zuließe, da die USA weder Partei des Schlichtungsverfahrens waren, noch (auch dies eine Voraussetzung für das internationale Schlichtungsverfahren) der Verhandlung zugestimmt hatten (PCA 2001:44).

Die Vertreter des Council of Regency beharrten nach der Einstellung des Verfahrens weiterhin auf ihrer Sicht der völkerrechtlichen Situation Hawai'is. Schon bald reichten sie einen Untersuchungsantrag beim Sicherheitsrat der Vereinten Nationen in New York ein (Council of Regency 2007), der hierauf allerdings nicht reagiert hat.

Während das innerhalb der hawaiischen Bewegung eher randständige Council of Regency also seine Bemühungen um internationale Anerkennung eines Besatzungsstatus für das Königreich Hawai'i fortsetzte, standen für eine breite Mehrheit der Hawaiier andere juristische Auseinandersetzung mit ebenfalls weit reichenden staatsrechtlichen Implikationen im Vordergrund. In einem politischen Klima, das von immer wieder neuen juristischen Versuchen geprägt war, Sonder- und Gruppenrechte auszuhebeln, erhielten Bemühungen um die rechtliche Anerkennung der Hawaiier als „Native Americans“ im Sinne der US-amerikanischen Indianergesetzgebungen erneut starken Auftrieb. 


\subsection{Hawaiische Gruppenrechte auf dem Prüfstand}

Wurden im vorangegangenen Kapitel divergierende Interpretationen der Geschichte sowie die daraus resultierenden unterschiedlichen Bewertungen von „Ethnie“ bzw. „Nation“ behandelt, so geht es auch in diesem Kapitel um das schier unentwirrbar erscheinende Vorstellungsgemenge von „Genealogie“ und „Abstammung“ sowie die Einordnung der Hawaiier in die US-amerikanischen Konzeptionen von „Race“.

Fragen, die Eigen- und Fremddefinitionen der Hawaiier berührten, gewannen zur Jahrtausendwende stark an Bedeutung. Nicht nur die gerichtliche Anfechtung des in Hawai'i geltenden beschränkten Wahlrechts zum Office of Hawaiian Affairs (das aktive wie passive Wahlrecht stand nach den Statuten des Bundesstaates Hawai'i ausschließlich Hawaiiern zu) sorgte für große Aufmerksamkeit. Auch die verstärkten Bemühungen um die Anerkennung der hawaiischen Bevölkerung als „Native Americans“ durch den US-Kongress betrieben vor allem von den beiden US-Senatoren des Bundesstaates, Daniel Inouye und Daniel Akaka - bewirkten nicht nur eine Intensivierung juristisch bestimmter Autonomiediskurse. Ebenso berührten beide Vorgänge auch unmittelbar die Fragen nach dem hawaiischen Selbstverständnis im Spannungsfeld zwischen ethnisch und historisch begründeten Ansprüchen einerseits sowie den von der Gegenseite geäußerten Vorhaltungen des Rassismus. Es nimmt daher nicht wunder, dass gerade hawaiische Autoren diese „fremd-initiierten“ Verfahren aufgriffen und problematisierten (so Kauanui 2005; Osorio 2001; Wong-Wilson [M. Trask] 2005). ${ }^{401}$

\section{Hawaiische Gruppenrechte}

Der Begriff der „Gruppenrechte“ bzw. „group rights“ umfasst verschiedene Bereiche des materiellen Rechts und bezieht sich ganz allgemein auf den rechtlichen Sonderstatus von Individuen oder Personengruppen (so z.B. im Familienrecht oder Arbeitsrecht). Von immer größerer Bedeutung in der juristischen Diskussion - und nach wie vor umstritten - sind aber auch jene Rechte, die Individuen aufgrund ihrer Mitgliedschaft in kulturellen oder ethnischen Gemeinschaften zugestanden bzw. von diesen verlangt werden (siehe z.B. Katz 1998; Kymlicka u. Shapiro 1997; Kuppe 1990). Gruppenrechte aufgrund ethni-

\footnotetext{
401 Zudem hat Rohrer (2006) als nicht-hawaiische Autorin eingehend die Auswirkungen des Gerichtsentscheids „Rice v. Cayetano“ behandelt und sich dabei vor allem mit der historischen bzw. sozialen Konstruktion der Kategorie „Haole“ (die insbesondere die Nachfahren der frühen weißen Siedler umfasst, aber zunehmend auch auf spätere euro-amerikanische Einwanderer ausgedehnt wurde) sowie der Aneignung von „Indigeneität“ durch nicht-hawaiische Zuwanderer auseinandergesetzt. Im Folgenden beziehe ich mich in wesentlichen Punkten auf die vier hier genannten Autoren (M. Trask, Kauanui, Osorio und Rohrer).
} 
scher oder kultureller Zugehörigkeit haben bis in die jüngste Vergangenheit eine negative Bewertung erfahren, nicht zuletzt durch Systeme wie die bis 1994 geltende Rassentrennung in Südafrika oder auch die bis in die 1960er Jahre betriebene Politik der Segregation in den Südstaaten der USA, wo Gruppenrechte die weiße Bevölkerung privilegierten und Afrikaner bzw. Afroamerikaner von der gesellschaftlichen Teilhabe ausschlossen. Heute werden Gruppenrechte jedoch vor allem im Kontext ethnischer oder kultureller Minderheiten diskutiert, für die sie einen wesentlichen Beitrag zur Besserstellung (im Sinne einer Beseitigung von Schlechterstellung) und Gleichberechtigung oder auch zu ihrem Fortbestand leisten können. Ihren Niederschlag fand diese Vorstellung von Gruppenrechten z.B. in der 2007 von der Generalversammlung der Vereinten Nationen verabschiedeten „Declaration on the Rights of Indigenous Peoples“, in deren Artikel 21 es unter anderem heißt:

„1. Indigenous peoples have the right, without discrimination, to the improvement of their economic and social conditions [...]

2. States shall take effective measures and, where appropriate, special measures to ensure continuing improvement of their economic and social conditions. [...]“ (United Nations 2007:9).

Und weiter heißt es in Artikel 33 dieser Erklärung:

„1. Indigenous peoples have the right to determine their own identity or membership in accordance with their customs and traditions [...]

2. Indigenous peoples have the right to determine the structures and to select the membership of their institutions in accordance with their own procedures“ (United Nations 2007:12).

Insgesamt erkennen die Vereinten Nationen in dieser Erklärung die so genannten Gruppenrechte für indigene Bevölkerungen nicht nur an, sie fordern sie sogar ein. Für die Hawaiier bleibt die „Declaration on the Rights of Indigenous Peoples“ allerdings insofern fast bedeutungslos, als die Bundesregierung in Washington die „Native Hawaiians“ zwar als „racial category“ kennt, ${ }^{402}$ nicht aber als indigene Bevölkerung (analog zu den „Native Americans“) anerkennt. ${ }^{403}$ Die Erklärung der Vereinten Nationen wendet sich jedoch an die Nationalstaaten und hat explizit deren Verhältnis zu indigenen Gruppen zum Inhalt.

\footnotetext{
${ }^{402}$ So im Regelwerk des US-amerikanischen Zensus.

${ }^{403}$ Dass „Native Hawaiians“ trotz der fehlenden staatlichen Anerkennung in zahlreichen nationalen Gesetzen und Regelungen im Sinne einer indigenen Minderheit angesprochen werden, gehört zu den Inkonsequenzen des US-amerikanischen Rechtssystems.
} 
Anders als die Bundesregierung erkennt der Bundesstaat Hawai'i den besonderen Status der Hawaiier auf vielen Ebenen wiederum an. Nicht nur die (im weitesten Sinne) mit den Interessen der hawaiischen Bevölkerung befassten staatlichen Institutionen wie das Office of Hawaiian Affairs (OHA) oder das Department of Hawaiian Home Lands (DHHL) beruhen auf einer grundsätzlichen Unterscheidung der Hawaiier von der übrigen Bevölkerung des Inselstaates. Auch die verschiedenen hawaiischen Sonderrechte hinsichtlich der Nutzung von Land, Wasser und Ozean sowie staatliche Maßnahmen (sowohl vonseiten des Bundesstaates Hawai‘i als auch durch einzelne US-Regierungsstellen) zur Förderung und Unterstützung der hawaiischen Bevölkerung, beruhen letztlich auf der Voraussetzung eines besonderen Status der Hawaiier innerhalb der Gesamtbevölkerung. Erreicht und begründet wird dieser Sonderstatus in aller Regel durch Zugrundelegung unterschiedlicher Abstammungskonzepte, im Falle des Hawaiian Homes Commission Act von 1920, der die rechtliche Grundlage des Department of Hawaiian Home Lands (DHHL) darstellt, sogar unter Schaffung einer zusätzlichen „inner-hawaiischen“ Kategorie „Native Hawaiians“ mit einer zumindest fünfzigprozentigen hawaiischen Abstammung.

In einem politischen Klima, das in den USA seit den ausgehenden 1990er Jahren von einem stark rückwärtsgewandten Konservatismus geprägt war, gerieten diese hawaiischen Sonder- oder Gruppenrechte immer stärker unter Beschuss. Im weiteren US-amerikanischen Kontext war eine Ausprägung dieses Konservatismus die starke Opposition gegen die unter dem Schlagwort „Affirmative Action“ durchgeführte aktive Gleichberechtigungspolitik des Staates zugunsten verschiedener Minderheiten. Wie die „Affirmative Action“ im Allgemeinen, so galten auch die Sonderrechte der Hawaiier im besonderen ihren Gegnern als eine der amerikanischen Verfassung entgegenstehende Bevorzugung aufgrund von „Race“ bzw. der biologischen Abstammung. In ironischer Umkehrung der Begründung vieler dieser Programme wurden sie in diesen Deutungen als Ausprägung eines Rassismus dargestellt, der sich gegen die weiße Mehrheitsbevölkerung der USA richtete.

„Rice v. Cayetano“

„Rice-Decision“ oder einfach nur „Rice v. Cayetano“ waren die Kurzformeln für das Urteil des Obersten Gerichtshofs der USA in der Verfassungsklage des Farmers Harold Rice gegen den damaligen Gouverneur Hawai'is, Ben Cayetano - ein Urteil, das den Status einer der bedeutendsten hawaiischen Einrichtungen, des Office of Hawaiian Affairs, in grundlegenden Bereichen veränderte und ein Auslöser weiterer Klagen gegen andere hawaiische Institutionen war.

Als Harold Rice, ein Bürger des Bundesstaates Hawai'i und selbst nicht hawaiischer Abstammung, im Jahre 1996 seine Zulassung zur Wahl des OHA-Vorstandes beantragte, wurde er folgerichtig aufgrund der geltenden 
Gesetzeslage zurückgewiesen. ${ }^{404} \mathrm{Er}$ verklagte daraufhin den Bundesstaat Hawai‘ $i$, da er sich einer Diskriminierung aufgrund seiner „Rassenzugehörigkeit“ ausgesetzt sah und verlangte vor Gericht die Durchsetzung seines Wahlrechts als gleichberechtigter Bürger des Bundesstaates.

Harold Rice scheiterte mit seiner Klage, und auch das Berufungsverfahren wurde 1998 gegen ihn entschieden. Beide Gerichte des Bundesstaates Hawai'i griffen die weithin geltende Ansicht auf, bei den Hawaiiern handele es sich schon aufgrund ihrer spezifischen Geschichte und Kultur um eine besondere, den Indianern des Festlandes zwar nicht gleichgestellte, aber doch vergleichbare Bevölkerungsgruppe des Bundesstaates. Sie rechtfertigten die bestehende Praxis mit diesem besonderen Status und führten darüber hinaus an, die Hawaiier seien die alleinigen Nutznießer des Office of Hawaiian Affairs, Wählerschaft und Klientel stimmten somit überein. In der Revision wurde das Urteil der ersten Instanz insofern noch präzisiert, als es sich eben nicht um eine vornehmlich auf der „Rassenzugehörigkeit“ beruhende Einschränkung des Wahlrechts handele, sondern vielmehr um eine sowohl juristisch als auch politisch begründete Maßnahme vor dem Hintergrund der besonderen historischen Entwicklungen in Hawai‘ $i .{ }^{405}$

1999 nimmt der Supreme Court der Vereinigten Staaten eine Verfassungsklage von Harold Rice an und verhandelt sie noch im gleichen Jahr unter der Bezeichnung „Rice v. Cayetano, Governor of Hawaii“. Grundlage dieser Klage waren der 14. und 15. Zusatz zur Verfassung der Vereinigten Staaten, in denen allen US-Bürgern die Gleichheit vor dem Gesetz und das Wahlrecht ohne Ansehen der „Rassenzugehörigkeit“ zugesichert wird. Bei solchen Verfahren ist es für interessierte Gruppen möglich und üblich, so genannte „Amicus curiae“-Schriftsätze einzureichen, die der Unterstützung der jeweiligen Prozesspartei dienen, aber auch zur Erleichterung der Urteilsfindung durch das Gericht beitragen sollen. Solche Erklärungen wurden für den Beklagten, den Bundesstaat Hawai'i, von verschiedenen hawaiischen Institutionen und Gruppierungen, den Kongressabgeordneten Hawai‘ is, dem National Council of American Indians, dem Generalstaatsanwalt der Vereinigten Staaten sowie den Regierungen von sieben Bundesstaaten ${ }^{406}$, des abhängigen US-Territoriums Guam und des Commonwealth of the Northern Mariana Islands zu Protokoll gegeben. Während Kamehameha Schools/Bishop Estate einen eigenen Schriftsatz einreichte, hatten die übrigen hawaiischen Organisationen gemeinsame

404 An der Spitze des Office of Hawaiian Affairs (OHA) steht ein Vorstand, dessen hawaiischstämmige Mitglieder dem Gesetz entsprechend bis zum Urteil des Obersten Gerichtshofes in geheimer Wahl von der hawaiischen Bevölkerung des Archipels gewählt wurden.

${ }^{405}$ Siehe „Top court backs Rice in OHA vote challenge“, Helen Alton u. Christine Donnelly, H S-B 23.02.2000 (Internet: starbulletin.com/2000/02/23/news/story1.html [11.05.2004]).

${ }^{406}$ Bei diesen Bundesstaaten handelte es sich um Alabama, Kalifornien, Nevada, New Mexico, Oklahoma, Oregon und Washington (s. Kauanui 2005:22). 
Schriftsätze verfasst. Wie schon bei anderer Gelegenheit geschehen, arbeiteten auch in diesem Fall Institutionen und Gruppierungen zusammen, die durchaus sehr divergierende Meinungen vertraten. So kooperierte Ka Lāhui Hawai' i mit dem Office of Hawaiian Affairs (OHA) wie auch mit Hā Hawai'i, der Folgeorganisation des von Ka Lāhui Hawai'i erbittert bekämpften Hawaiian Sovereignty Elections Council (HSEC). Hier zeigte sich erneut, dass die vielfach berufene Uneinigkeit der Hawaiier hinsichtlich politischer Fragen gemeinsames Handeln in konkreten Auseinandersetzungen mit „externen“ Gegenspielern nur begrenzt beeinflusst. ${ }^{407}$

Auch eine durchaus ungewöhnliche Einlassung des Vertreters der USRegierung vor dem Obersten Gerichtshof, Seth P. Waxman, war für die Gegner der Klage von erheblicher Bedeutung. Waxman stellte in seinem Schriftsatz zugunsten der Regierung des Bundesstaates Hawai‘'i fest:

„The United States has concluded that it has a trust obligation to indigenous Hawaiians because it bears a responsibility for the destruction of their government and the uncompensated taking of their lands.“ “408

Damit folgte Waxman einer Argumentationslinie, die seit der „Apology Resolution“ des US-Kongresses von 1993 (US Public Law 103-150) der Begründung staatlicher Maßnahmen und Programme diente und die implizit von einer durchaus gegebenen Äquivalenz des hawaiischen Status mit der rechtlichen Stellung der Native Americans des amerikanischen Festlandes ausgeht - womit ein beschränktes Wahlrecht für die OHA-Wahlen also keine Verletzung der Gleichbehandlung im Sinne der US-Verfassung darstellte. Die USamerikanische Gesetzgebung billigt der hawaiischen Bevölkerung, trotz einer weiterhin fehlenden offiziellen Anerkennung als indigene und damit im Sinne der „Native Americans“ bevorrechtigte ethnische Gruppe, in zahlreichen Gesetzen de facto einen besonderen Status zu. So galten zur Zeit der Verhandlungen vor dem Obersten Gerichtshof mehr als 160 durch den US-Kongress verabschiedete Gesetze und Regelungen, durch die „Native Hawaiians“ als eigenständige Zielgruppe betroffen waren. Hawaiier wurden zum Beispiel aufgenommen in den „Native American Programs Act“, den „Native American

\footnotetext{
${ }^{407}$ Neben den oben bereits genannten waren an diesem „Amicus curiae“-Schriftsatz beteiligt: die Association of Hawaiian Civic Clubs, das Council of Hawaiian Organizations, die Native Hawaiian Convention, die Native Hawaiian Bar Association, die Native Hawaiian Legal Corporation, das Native Hawaiian Advisory Council, Hui Kālai‘āina, Alu Like Inc. und Papa Ola Lōkahi. Einen weiteren gemeinsamen Schriftsatz reichten ein das State Council of Hawaiian Homestead Associations, Hui Kāko'o 'Āina Ho'opulapula, Kalama'ula Homestead Association sowie die Hawaiian Homes Commission (Kauanui 2005:21-2).

408 „Feds: OHA vote perfectly legal“, Pat Omandam, H S-B 29.07.1999 (Internet: starbulletin.com/1999/07/29/news/story1.html [09.08.1999]).
} 
Languages Act“ und den „Native American Grave Protection and Repatriation Act“ (NAGPRA). Andere Bundesgesetze schufen Programme, die sich speziell an die hawaiische Bevölkerung wenden, wie der „Native Hawaiian Health Care Act“ oder der „Native Hawaiian Education Act“. All diese Gesetze und Regelungen legen eine sehr weit gefasste Definition der Betroffenen zugrunde: als „Native Hawaiians“ gelten jeweils Nachfahren aller Inselbewohner, die vor 1778 (dem Jahr der Ankunft James Cooks) auf den hawaiischen Inseln lebten. Im Vorfeld dieses bedeutenden Verfahrens vor dem US-Verfassungsgericht zeigte sich deutlich die inkonsequente und ambivalente Haltung der bisherigen US-Regierungen, die den Hawaiiern zwar einen besonderen Status als benachteiligte Minderheit im Rahmen sozialer und kultureller Gesetzgebungen (die ja vom US-Kongress ausgingen) zubilligten, ohne indes die Hawaiier als eine ethnisch und politisch definierte Bevölkerungsgruppe mit eigenen Rechtsansprüchen gegen den US-Kongress anzuerkennen, wie es bei „Native Americans“ und „Alaska Natives“ der Fall ist.

Diesem Exkurs zur rechtlichen Stellung der Hawaiier gegenüber den USA steht nun die Selbstdarstellung und Argumentation des Klägers entgegen. Im Umfeld des Prozesses war Harold Rice bemüht, seine Absichten in einem pro-hawaiischen Licht erscheinen zu lassen. So stellte er, unter dessen Vorfahren auch Bürger des Königreichs Hawai‘i waren, in einem Interview fest: ,,... if I had to make a choice, a citizen of U.S. or a citizen of Hawai'i, I’d never think twice, I'd be a citizen of Hawai'i“" (zit. nach Rohrer 2006:6). Zugleich berief er sich auf Keanu Sai, den Vertreter des Council of Regency of the Hawaiian Kingdom und Fürsprecher einer juristisch-politischen Definition des „Hawaiisch-Seins“. Er habe von Keanu Sai die Bestätigung erhalten, ein Bürger des unabhängigen Königreichs Hawai'i zu sein, da seine Vorfahren ihre hawaiische Staatsbürgerschaft nie aufgegeben hätten (ebd.). Auf diese Weise versuchte Rice in seiner Selbstdarstellung den Anschein zu erwecken, mit der von ihm angestrengten Verfassungsklage lediglich Rechte einzufordern, die ihm als Nachfahre von loyalen Bürgern des Königreichs zustünden - wobei dieser völkerrechtliche Aspekt des Wahlrechts zum Vorstand des Office of Hawaiian Affairs allerdings kein Bestandteil seiner Klage vor dem Obersten Gericht war. ${ }^{409}$

Seine hawaiischen Gegner wiesen denn auch auf andere Aspekte sowohl der Klage als auch der Person des Klägers selbst hin. Hierbei stand auch die von Rice wiederholt angeführte Ahnenreihe auf dem Prüfstand. So schreibt Mililani Trask, zum Zeitpunkt der Klage Mitglied des OHA-Vorstandes:

${ }^{409} \mathrm{Zu}$ den Verflechtungen kolonialistischer Attitüde und kultureller Hybridität in der Selbstdarstellung des Klägers siehe Rohrer (2006:6-8). 
„Freddy Rice is the petitioner in the Rice vs. Cayetano case. He is a third-generation haole with a long family history of anti-Hawaiian, proannexationist activities. When interviewed by the Star Bulletin, Rice said his ancestors supported Queen Lili‘uokalani before the 1893 illegal overthrow. This assertion was also made by Rice in the briefs he filed. However, research by the Native Hawaiian Legal Corporation reveals that Rice is a liar“ (M. Trask 1999:8).

Hierzu führte sie weiter aus, dass einer seiner Großväter jenem Komitee angehörte, das König Kalākaua 1887 zur Unterzeichnung der so genannten Bajonett-Verfassung zwang und der darüber hinaus auch an der Verfassung der Republik Hawai'i mitgewirkt habe. Harold Rices Vater, Senator des Territoriums Hawai‘i, soll schon 1893 einen Gesetzentwurf zur Sterilisation „sozial unangepasster Personen“ in das Parlament eingebracht haben, der überwiegend Hawaiier betroffen hätte. Neben dieser von Mililani Trask betriebenen „Dekonstruktion“ des Rice'schen Anspruches auf „Hawaiianness“ durch die Auflistung anti-hawaiischer Taten seiner Vorfahren, auf die er sich ja dezidiert berufen hatte, standen aber auch seine eigenen aktuellen Beweggründe für die Klage gegen das OHA-Wahlrecht in der Kritik. Rowena Akana, zum Zeitpunkt des Verfahrens ebenfalls Mitglied des OHA-Vorstandes, fragte sich in diesem Zusammenhang, unter Auflistung früherer Klagen des Harold Rice gegen hawaiische Sonderrechte und unter Wiederholung der bereits von M. Trask erhobenen Vorwürfe gegen dessen Vorfahren, ob hinter der Klage nicht andere Ziele als die Bestätigung einer eigenen „Hawaiianness“ stünden:

„Although Mr. Rice says he has nothing against OHA, he previously filed litigation against the Bishop Estate to challenge educational benefits for Hawaiians, and against the YWCA [Young Women’s Christian Association; U.M.] for holding Hawaiian language classes. Perhaps this third-generation missionary descendant, with ancestors in the provisional government that overthrew our queen, has a different agenda from the one he states“ (Akana 1999:6).

Mililani Trask meinte, dieses „andere Programm“ des Harold Rice auch in der finanziellen Unterstützung erkennen zu können, die der Rice’schen Klage durch die konservative Organisation „Campaign for a Colorblind America“, ansässig in Washington, D.C., zuteil wurde: ${ }^{410}$

${ }^{410}$ Die Organisation „Campaign for a Colorblind America“ wandte sich bereits vor der RiceKlage erfolgreich gegen die Existenz von Wahlbezirken mit hispanischen oder schwarzen Wählermehrheiten und kann als Teil einer Bewegung gesehen werden, die gegen die in der Folge der amerikanischen Bürgerrechtsbewegung initiierten Gesetze und Programme zur Gleichbehandlung und Förderung von Minderheiten vorging. Die Einschätzung des Vorsitzenden der 
„Rice is part of a conservative and reactionary haole movement sweeping across the continent. The goal of the Campaign for a Color-Blind America is to diminish political power and eradicate government entitlements currently provided to native peoples, Asians, black Americans and anyone else who is not white. What makes these efforts racist is that Rice and his coalition have the money and power to impose their beliefs and biases on others“ (M. Trask 1999:8). ${ }^{411}$

Die Gegenüberstellung des vom Kläger Harold Rice propagierten Selbstbildes und der Darstellung, wie sie in den angeführten Zitaten von Vertreterinnen des OHA deutlich wird, wirkt wie ein Echo des im vorangegangenen Kapitel beschriebenen Gegensatzes von „Nation“ und „Genealogie“ als Wurzel des „Hawaiisch-Seins“. So präsentierte sich Rice als in der Tradition des Königreichs stehend und zugleich als jemand, der den (ethnischen) Hawaiiern sehr wohl gesonnen gegenüberstand. Seine Gegner hingegen beschrieben ihn als Rassisten mit einer anti-hawaiischen Einstellung.

Diese grundsätzlich gegeneinander stehenden Einschätzungen reflektieren die zentrale Problematik der Begründung von „Hawaiianness““. Ohne hier eine letztgültige Entscheidung hinsichtlich der Richtigkeit der Einschätzungen zu treffen, kann fraglos festgestellt werden, dass beide Standpunkte auf einer jeweils unterschiedlichen Interpretation der politischen und sozialen Geschichte des hawaiischen Archipels gründen. Steht auf der einen Seite eine evolutionäre Sicht auf sich aneinanderreihende historische Ereignisse, wobei die Hawaiier eine der gleichberechtigten Bevölkerungsgruppen des Inselstaates waren und sind, so vertreten die Gegner dieses Geschichtsbildes eine Vorstellung der historischen Brüche, durch die eine hawaiische Bevölkerung, durch Abstammung und Kultur als einzige Bevölkerungsgruppe der Inseln auf besondere Weise an das Land gebunden, zunehmend an den Rand des politischen und

„Campaign for a Colorblind America“ wurde im Honolulu Star-Bulletin unkommentiert und zum Abschluss eines längeren Artikels über die Anhörung des Falles in Washington wiedergegeben (ein wiederholter Hinweis auf die ambivalente Haltung der Presse anlässlich dieses Auseinandersetzung): „Edward Blum, chairman of Campaign for a Colorblind America, said the group is appalled that African Americans and Hispanics in Hawaii are being turned away from the OHA voting polls because of their skin color. The goal of the nonprofit group is to challenge racebased public policies and educate the public about the injustices of racial preferences“ („Hawaiians say hearing went badly“, Pat Omandam, H S-B 06.10.1999, Internet: archives.starbulletin.com/1999/10/06/news/story1.html [3.02.2009]).

${ }^{411}$ Wie umfassend diese Angriffe auf „Affirmative Action“ und die als Unrecht angesehenen Sonderrechte für Mitglieder der so genannten Minderheiten waren (und sind), wird aus den Erfolgsmeldungen einer reaktionären Gruppierung wie „Adversity net - a civil rights organization for color blind justice“ deutlich (Adversity Net 2004: www.adversity.net/about_us [22.02.2009]). 
sozialen Spektrums der Inselgesellschaft gedrängt wurde. Und so konnte Miliani Trask zum Schluss ihres Artikels appellieren:

„One thing is sure: Regardless of the outcome of the suit, Hawaiians will never give up our struggle for justice, self-determination and the return of our traditional lands. Rice vs. Cayetano throws fuel on the fire, it extinguishes nothing!“ (M. Trask 1999:8).

Nicht einmal ein Jahr nach dem Erscheinen des Textes von Mililani Trask, am 23. Februar 2000, entschied der Oberste Gerichtshof der USA gegen den Bundesstaat Hawai'i und erklärte das bis dahin geltende Wahlrecht für den Vorstand des OHA auf der Grundlage des 15. Verfassungszusatzes für nicht verfassungsgemäß. Die Urteilsbegründung sah die Vorhaltung der Bevorzugung einer Gruppe aufgrund ihrer „rassischen“ Zugehörigkeit bestätigt und rechtfertigte so die Aufhebung der bestehenden Wahlrechtsbestimmungen. Die Urteilsbegründung beschränkte sich hierbei auf eine Definition der Hawaiier durch „Race“. Sie blendete konkurrierende konstitutive Merkmale der Kategorie „Hawaiian“ wie Geschichte, Kultur oder Genealogie konsequent aus - und verneinte damit die trotz fehlender Verträge zwischen dem hawaiischen Königreich und den USA womöglich gegebene Basis für eine politische Eigenständigkeit wie im Falle der „Indian Nations“. Diese Entscheidung des Verfassungsgerichtes und die in ihr zutage tretende Ignorierung eines besonderen Status der hawaiischen Bevölkerung verdient eine nähere Betrachtung. ${ }^{412}$

Schon bei der Darstellung der Vorgeschichte des verhandelten Falles traf das Gericht in der schriftlichen Begründung eine Feststellung, deren Diktion dem allgemeinen wie auch dem juristischen Sprachgebrauch in Hawai' $\mathrm{i}$ entgegensteht und die den Grundtenor des Urteils bereits vorwegnahm. Nach einer ausführlichen Rekapitulation der in den Gesetzen des Bundesstaates Hawai`i unterschiedenen juristischen Kategorien wie „Hawaiians“ und „Native Hawaiians“, heißt es dann in dem Text:

„Petitioner Rice, a Hawaiian citizen without the requisite ancestry to be a ,Hawaiian' under state law, applied to vote in OHA trustee elections“ [meine Hervorhebung; U.M.] (United States 2000:1).

Die in Hawai'i selbstverständliche und allgemein gebräuchliche Unterscheidung zwischen hawaiischen und nicht-hawaiischen Bürgern des Staates Hawai`i, die ja auch der Kläger seinen früheren Aussagen zufolge nicht in

\footnotetext{
${ }^{412}$ Ich beschränke mich hier auf die Zusammenfassung der Urteilsbegründung (United States 2000); für eine Betrachtung der vollständigen Begründungen sowohl der Mehrheit als auch der abweichenden Meinung eines Richters siehe Rohrer (2006).
} 
Zweifel zieht und die im offiziellen Kontext außerhalb des Inselstaates ebenfalls durchaus geläufig ist, wird durch die hier gebrauchte Wendung (,a Hawaiian citizen“ anstelle z.B. der Formulierung „,a citizen of Hawai'i“) verwischt und so mit einem gewissen rhetorischen Geschick unter der Hand in eine Gleichsetzung der Nachfahren der indigenen Bevölkerung mit jenen der späteren Zuwanderer verkehrt. Hinsichtlich der begründungsrelevanten Geltung des 15. Verfassungszusatzes, der in seiner Substanz aus der Zeit des Sezessionskrieges (1861-65) stammt und ursprünglich das Wahlrecht ehemaliger Sklaven sichern sollte, präzisierte das Gericht seine Ansicht dann wie folgt:

„The voting structure in this case is neither subtle nor indirect; it specifically grants the vote to persons of the defined ancestry and to no others. Ancestry can be a proxy for race. It is that proxy here. [...] The ancestral inquiry in this case implicates the same grave concerns as a classification specifying a particular race by name, for it demeans a person's dignity and worth to be judged by ancestry instead of by his or her own merit and essential qualities ... The State's electoral restriction enacts a race-based voting qualification“" (United States 2000:2-3).

Das Gericht stellte hiermit fest, dass Abstammung in diesem Fall mit „Race“ gleichzusetzen sei und die Restriktionen bei den Wahlen zum OHA somit einer rassistisch motivierten Ausgrenzung von Menschen ohne die entsprechende Abstammung gleichkämen oder wie es zum Abschluss des höchstrichterlichen Syllabus heißt:

„... it [eines der Gegenargumente des Bundesstaates Hawai' $i$ hinsichtlich der Übereinstimmung von Wählern und Nutznießern im Falle des Office of Hawaiian Affairs; U.M.] rests on the demeaning premise that citizens of a particular race are somehow more qualified than others to vote on certain matters. There is no room under the Amendment for the concept that the right to vote in a particular election can be allocated based on race“ (United States 2000:3).

Darüber hinaus erteilte das Oberste Gericht nicht nur der Ansicht eine Absage, den Hawaiiern stünden wie den Native Americans des Kontinents besondere Rechte zu (einer Ansicht, die ja, wie oben besprochen, auch vom Vertreter der US-Regierung geteilt wurde), auch die Auffassung, dass es sich beim Office of Hawaiian Affairs (OHA) um eine vom Bundesstaat quasi unabhängige Institution handele, wurde von den Richtern verworfen. ${ }^{413}$

${ }^{413}$ Dieses Urteil kann durchaus im Rahmen der Bemühungen konservativer Kräfte in den USA gesehen werden, die Besetzungspolitik und damit den Supreme Court selbst zur Durchsetzung 
So offenbarte dieses Urteil nicht nur die tief greifenden Probleme, die aufseiten der US-Instanzen hinsichtlich einer Akzeptanz der Hawaiier als ethnisch oder auch politisch definierte Gruppe bestehen (und die eine gut gemeinte Resolution im Jahre 1993 ganz offensichtlich nicht aus dem Weg geräumt hatte). Darüber hinaus machten sowohl das Verfahren als auch das Urteil selbst einmal mehr deutlich, wie wenig Bedeutung den unterschiedlichen Facetten der komplexen Aus- und Abgrenzungskriterien des „Hawaiisch-Seins“ in juristischen, aber auch politischen und gesellschaftlichen Kontexten außerhalb des Archipels beigemessen wird bzw. aufgrund der geltenden Rechtslage beigemessen werden kann. In Verfahren und Urteil kollidierten in selten offensichtlicher Weise eine auf rigorose Ausgrenzung bedachte US-amerikanische Spielart des Konzeptes „race“, eine im Bundesstaat Hawai'i historisch wie politisch begründete und gewollte Eingrenzung aufgrund von Abstammungskriterien und ein polynesisch-hawaiisches Konzept der Verbundenheit durch eine gemeinsame Genealogie. Letztere dient heute zwar als Abgrenzungskriterium, wirkte in der hawaiischen Vergangenheit aber vor allem als integrative Kraft.

Das Urteil hatte zunächst den Rücktritt des gesamten Vorstandes des Office of Hawaiian Affairs und alsdann eine Neuwahl zur Folge, an der erstmalig auch Nicht-Hawaiier teilnehmen konnten. Im übrigen erfolgten hierauf weitere juristische Versuche, Privilegien und Sonderrechte der hawaiischen Bevölkerung abzuschaffen oder zu beschneiden; diese Verfahren scheiterten auf dem Weg durch die Instanzen nur knapp vor dem Obersten Gericht bzw. warten noch auf eine Entscheidung. ${ }^{414}$

reaktionärer Ziele zu benutzen; siehe hierzu einen erhellenden Artikel des Juristen und Politikwissenschaftlers Bruce Ackerman, Universität Yale (2005). Im hier behandelten Zusammenhang ist auch ein aktuelles Urteil des Supreme Court vom 28. Juni 2007 zu sehen, das die gezielte Förderung schwarzer und hispanischer Schüler aufgrund der Verletzung des Gleichheitsgebots der Verfassung für verfassungswidrig erklärte („Empörung über Rassenurteil: US-Demokraten befürchten Rückschlag für Minderheiten“, Christian Wernicke, Süddeutsche Zeitung 30.06.2007:9).

${ }^{414}$ Nachdem untere Gerichte für die Kläger entschieden hatten, wies das Oberste Gericht am 14. Juni 2006 den Fall „Arakaki v. Lingle“ wegen des nicht ausreichenden Klagegrunds ab (Linda Lingle war die amtierende republikanische Gouverneurin des Inselstaates). Die insgesamt 16 Kläger hatten aufgrund ihres Status als Steuerzahler nichts weniger als die völlige Auflösung des Office of Hawaiian Affairs (OHA), der Hawaiian Homes Commission (HHCA) sowie weiterer, teilweise oder ganz aus Steuermitteln des Bundesstaates und der Bundesregierung finanzierter Hilfsprogramme für Hawaiier verlangt. Seit 2003 harrt darüber hinaus eine Klage gegen Kamehameha Schools/Bishop Estate der Entscheidung, mit der ein anonym bleibender nichthawaiischer Kläger gegen die Zulassungspolitik (und damit indirekt auch gegen die finanzielle Förderung des Universitätsstudiums hawaiischer Schüler durch Stipendien der Kamehameha Schools/Bishop Estate) der Stiftung vorgeht (Cummings Losch 2007:222-6). 


\section{3 „Federal Recognition“: Ende des politischen Wider- standes?}

Eine weitere weitreichende Konsequenz der Verhandlungen des Falls „Rice v. Cayetano" und der angesprochenen Folgeverhandlungen bestand in den nunmehr einsetzenden Bemühungen von politischer Seite zur Schaffung eines auch staatsrechtlich tragfähigen und belastbaren Rechtsstatus für die hawaiische Bevölkerung. Die Initiative zu diesem über die Jahre konsequent vorangebrachten Gesetzesvorhaben kam - und dies unterscheidet es von entsprechenden Bemühungen der 1990er Jahre - nicht aus der hawaiischen Autonomiebewegung, sondern geht auf den US-Senator des Bundesstaates Hawai‘i, Daniel Akaka, zurück. Akaka brachte seinen Vorschlag erstmals im Jahre 2000 in den US-Kongress ein, wobei das Ziel die Schaffung juristischer Tatsachen noch vor dem Urteil des Obersten Gerichthofes in Sachen „Rice v. Cayetano“ war. Das Gesetz passierte den Kongress damals nicht und Akaka hat den Vorschlag seither, in jeweils leicht veränderter Form, in jeder Sitzungsperiode des USKongresses erneut in die Beratungen eingebracht.

Die „Akaka Bill“, so die übliche Bezeichnung für das Gesetzesvorhaben, genoss seit ihrer ersten Veröffentlichung große Aufmerksamkeit in Hawai'i - wurde hier doch zum ersten Mal versucht, unter Umgehung des Bundesstaates und im Rahmen des regulären Gesetzgebungsverfahrens, auf nationaler Ebene Grundlagen für die hawaiische Autonomie zu schaffen. Die offizielle Bezeichnung der hier besprochenen Version der „Akaka Bill“ (S 310, H.R. 505) lautet „Native Hawaiian Government Reorganization Act of 2007“ (U.S. Congress 2007:2), ihr ausführlicher Titel gibt schon die Stoßrichtung des Vorhabens an:

„To express the policy of the United States regarding the United States relationship with Native Hawaiians and to provide a process for the recognition by the United States of the Native Hawaiian governing entity“ (U.S. Congress 2007:II).

Die Gesetzgebung sieht also Verwaltungsstrukturen auf nationaler Ebene vor, deren Aufgabe die Registrierung von „Native Hawaiians“ sein wird, die dann eine Übergangsregierung wählen sollen - ein Vorgang, der an das Hawaiian Sovereignty Elections Council und die misslungene Wahl zu einer hawaiischen Verfassungsversammlung im Jahre 1996 erinnert (cf. Cummings 2004:154). Nach der Errichtung einer dauerhaften Regierungs- bzw. Verwaltungsform („Government“) wären dann alle noch offenen Fragen (wie die Übertragung von Landtiteln oder der finanzielle Ausgleich für begangenes Unrecht) Gegen- 
stand von Verhandlungen zwischen der neuen hawaiischen Regierung und der US-Regierung oder dem Bundesstaat Hawai'i (U.S. Congress 2007:36-7).

In der „Akaka Bill“ findet eine Definition der Kategorie „Native Hawaiians“ Verwendung, die unterschiedliche Begriffsbestimmungen in sich vereint:

„,... the term ,Native Hawaiian' means-

(i) an individual who is 1 of the indigenous, native people of Hawaii and who is a direct lineal descendant of the aboriginal, indigenous, native people who-

(I) resided in the islands that now comprise the State of Hawaii on or before January 1, 1893; and

(II) occupied and exercised sovereignty in the Hawaiian archipelago, including the area that now constitutes the State of Hawaii; or

(ii) an individual who is 1 of the indigenous, native people of Hawaii and who was eligible in 1921 for the programs authorized by the Hawaiian Homes Commission Act (42 Stat. 108, chapter 42) or a direct lineal descendant of that individual“ (U.S. Congress 2007:14).

Diese relativ weit gefasste Definition geht von einer nachgewiesenen biologischen Abstammung von hawaiischen Vorfahren aus, wie sie auch der größte Teil der Autonomiebewegung als Voraussetzung für das „Hawaiisch-Sein“ reklamiert. Als „Stichtag“ nennt sie den 1. Januar 1893 (und nicht das in der Autonomiebewegung häufig bemühte Jahr 1778, als James Cook als erster Europäer die Inseln erreichte), und somit das Jahr des Umsturzes und das Ende eines souveränen hawaiischen Staates - sonst eher das relevante Datum für staatsrechtlich orientierte Autonomiegruppierungen wie das Institute for the Advancement of Hawaiian Affairs (I.A.H.A.) oder das Council of Regency.

Der Gesetzentwurf weist auf die Präzedenzfälle hin, in denen der Kongress die Hawaiier als eigenständige und besondere Gruppe faktisch bereits anerkannt hat und benennt zudem die auf hawaiischer Seite in Rede stehenden Rechte. Er macht somit auf das bestehende widersprüchliche Verhältnis zwischen den USA und den Hawaiiern als einer definierten Gemeinschaft aufmerksam, das durch die Errichtung einer „Nation in der Nation“ geklärt werden könnte und sollte. Diese in Abschnitt 4 des Gesetzentwurfs ausgeführte Argumentationslinie reflektiert die von verschiedenen Autonomiegruppierungen vorgebrachten Begründungen und soll hier vollständig zitiert werden:

„(a) Policy.- The United States reaffirms that-

(1) Native Hawaiians are a unique and distinct, indigenous, native people with whom the United States has a special political and legal relationship; 
(2) the United States has a special political and legal relationship with the Native Hawaiian people which includes promoting the welfare of Native Hawaiians;

(3) Congress possesses the authority under the Constitution ... to enact legislation to address the conditions of Native Hawaiians and has exercised this authority through the enactment of-

(A) the Hawaiian Homes Commission Act, 1920 ...

(B) the Act entitled „An Act to provide fort he admission of the State of Hawaii into the Union, approved March 18, 1959 ...

(C) more than 150 other Federal laws addressing the conditions of Native Hawaiians;

(4) Native Hawaiians have-

(A) an inherent right to autonomy in their internal affairs;

(B) an inherent right of self-determination and self-governance;

(C) the right to reorganize a Native Hawaiian governing entity; and

(D) the right to become economically self-sufficient; and

(5) the United States shall continue to engage in a process of reconciliation and political relations with the Native Hawaiian people.

(b) Purpose.-The purpose of this Act is to provide a process for the reorganization of the single Native Hawaiian governing entity and the reaffirmation of the special and legal relationship between the United States and that Native Hawaiian governing entity for purposes of continuing a government-to-government relationship“ (U.S. Congress 2007:15-6).

Neben der voraussehbaren Ablehnung durch jene konservativen Kräfte, die auch das „Rice v. Cayetano“-Urteil als Sieg ihrer Sache und damit des vermeintlichen Kampfes gegen Bevorzugung auf der Grundlage von „Race“ feierten, schlug dem Gesetzesvorhaben aber auch von hawaiischer Seite gleich zu Beginn Protest entgegen: ${ }^{415}$ Dies war vornehmlich dem Umstand geschuldet, dass der „Native Hawaiian Government Reorganization Act“ eine Weiterentwicklung der hawaiischen Situation ohne die direkte Beteiligung der maßgeblichen hawaiischen Initiativen vorsah. Des Weiteren wurde die mit der „AkakaBill“ verbundene Aufgabe völkerrechtlich begründeter Ansprüche auf die Sou-

${ }^{415}$ Als unerwarteter Gegner der „Akaka Bill“ zeigte sich auch die U.S. Commission on Civil Rights, die die Gefahr einer bevorzugten Behandlung durch das Recht („,preferential treatment“) gegeben sah (OHA 2007:1-2). Fragen hinsichtlich des Gesetzes hatte auch das USJustizministerium, das unter anderem Änderungen und Präzisierungen des Textes hinsichtlich Landfragen, Problemen des Strafrechts und schließlich - aufgrund der Erfahrungen in einigen Indian Nations auf dem Festland - ein Verbot von Kasinobetrieben und Glückspiel einforderte (Cummings Losch 2006:146-7). Der Gesetzentwurf von 2007 trägt all diesen Forderungen Rechnung (siehe U.S. Congress 2007:42 ff.). 
veränität Hawai'is angeprangert (siehe z.B. Cummings 2003:182; 2006:147). So heißt es auf einer Website mit dem Namen „Stop Akaka“.416

„Why is the Akaka Bill so dangerous?

Because it will legitimize the 1893 illegal overthrow of the Hawaiian Nation.

It will extinguish forever all Hawaiian claims for redress and bar all future claims.

It will complete the theft of Hawaiian lands from a legal standpoint. $[\ldots]$

Native Hawaiians are the only and last large group that has not signed away their autonomy and land in a claims extinguishment agreement with the US.

The Akaka Bill is an attempt not only to legitimize the theft of the Hawaiian Nation, but also to prevent the ever growing move for independence“ (Stop Akaka 2009).

Sieht man von der zugespitzten Sprache ab, so stimmen auch die meisten Argumente nicht mit dem Inhalt der „Akaka Bill“ überein: Zwar würde das neue Gesetz in der Tat die juristische Diskussion der völkerrechtlichen Stellung der hawaiischen Nation abschließen (und damit wären die Ziele von Autonomiegruppierungen wie Ka Pākaukau, I.A.H.A. oder dem Council of Regency de facto gescheitert), doch sieht der Gesetzentwurf Akakas keine Abtretung von hawaiischen Ansprüchen auf Land oder finanzielle Entschädigungen vor. Es bliebe vielmehr einer zukünftigen politischen Vertretung der Hawaiier überlassen, diese Fragen mit der US-Regierung auszuhandeln. Insgesamt blieben die Proteste von hawaiischer Seite relativ gedämpft und erreichten bei weitem nicht jene Intensität, wie sie früheren Vorhaben zur Schaffung hawaiischer Regierungsstrukturen entgegenschlugen - so z.B. dem Hawaiian Sovereignty Elections Council und der „Native Hawaiian Vote“ von 1996 oder einem bundesstaatlichen Gesetzesvorhaben zur Zusammenlegung von zwei Institutionen (dem Office of Hawaiian Affairs und dem Department of Hawaiian Home Lands) im Jahre 1998. ${ }^{417}$

\footnotetext{
${ }^{416}$ Auf der Website finden sich keine Hinweise auf die Autoren und Betreiber der Seite; allerdings wird auf das Blog „Free Hawai'i““ (http://freehawaii.info) verwiesen, das von einer gewissen „Koani Foundation“ betrieben wird, die allerdings ebenfalls keine näheren Angaben über sich veröffentlicht. Das Blog präsentiert neben politischen Informationen Fotos von Protesten gegen die „Akaka Bill“ und bietet T-Shirts und Aufkleber mit Stellungnahmen gegen den Gesetzesvorschlag an.

${ }^{417}$ Ein Exkurs ist hinsichtlich dieses letztlich gescheiterten Gesetzentwurfs angezeigt, der für kurze Zeit in der Geschichte der Bewegung völlig gegensätzliche Standpunkte im gemeinsamen Protest zusammenführte. Diese so genannte „Autonomy Bill“ erregte 1998 die hawaiischen
} 
Einer der wichtigsten Befürworter der „Akaka Bill“ ist das Office of Hawaiian Affairs, das sich auch in diesem Fall (wie schon bei der „Native Hawaiian Vote“ 1996) als Prototyp einer zukünftigen hawaiischen Regierung sieht. In einer fast 70-seitigen umfassenden Stellungnahme weist das Office of Hawaiian Affairs z.B. die Vorbehalte der U.S. Commission on Civil Rights zurück (OHA 2007; siehe oben) und erstellt mit der Aktion „Kau inoa“, in deren Rahmen Hawaiier aufgefordert sind, sich als Menschen mit hawaiischer Abstammung erfassen zu lassen, schon heute ein von der „Akaka Bill“ gefordertes Bevölkerungsregister (OHA 2008a). Alles in allem wird der seit nunmehr dreizehn Jahren diskutierte Gesetzentwurf als ein Schutz vor der weitergehenden Erosion hawaiischer Rechte gesehen. Und angesichts der Tatsache, dass mit Barack Obama nun erstmals ein US-Präsident regiert, der in Hawai' $\mathrm{i}$ aufgewachsen und mit der dortigen politischen und gesellschaftlichen Situation vertraut ist, haben sich die Chancen für das Vorhaben Daniel Akakas womöglich ein Stück weit verbessert. Obama selbst hatte bereits während des Wahlkampfes seine Unterstützung der „Akaka Bill“ öffentlich gemacht (OHA 2008b). ${ }^{418}$

Dass nun womöglich ein Bundesgesetz, verfasst durch Senator Daniel Akaka, einen hawaiisch-stämmigen Repräsentanten des Staates Hawai ${ }^{i} \mathrm{i},{ }^{419}$ und

Gemüter und sah die Schaffung einer neuen zentralen Verwaltungseinheit vor, die analog zum bestehenden Office of Hawaiian Affairs organisiert sein sollte. Dieser ,independent governance structure“ wären dann alle spezifisch hawaiischen Rechte und Pflichten im Staatsgefüge Hawai'is übertragen worden, einschließlich der Verfügung über die Hawaiian Home Lands sowie die Insel Kaho“olawe. Anders als die bis heute aktuelle „Akaka Bill“ hätte dieses Gesetz die Struktur einer „hawaiischen Regierung“ vorgegeben und darüber hinaus sämtliche hawaiischen Ansprüche gegen den Staat Hawai'i abgegolten. Eine gemeinsame Wurzel des Protests war allerdings auch die Tatsache, dass der Gesetzesvorschlag von einem Nicht-Hawaiier in das Parlament eingebracht wurde und die Diskussion über eine hawaiische Zukunft im Wesentlichen außerhalb der Autonomiebewegung stattgefunden hätte (siehe z.B. „What's good, bad in ,autonomy bill’, Ed Case, HA 11.1.1998:B3; „1000 protest House bill on Hawaiians“, William Kresnak u. Judy Ma, HA 31.1.1998:B1; „Hawaiians demand bill be thrown out“, Mike Gordon, HA 1.2.1998:A23, A26; „Furor painful to native son“, David Waite, HA 8.2.1998:A1-3). Zahlreiche prominente Vertreter der Bewegung und des Office of Hawaiian Affairs (die auch in diesem Fall an einem Strang zogen) äußerten sich zu der anstehenden Entscheidung in der lokalen Presse: So z.B. Mililani Trask, Ka Lāhui Hawai'i („Bill is no substitute for sovereignty“, HA 11.1.1998:B3); Frenchy Desoto, OHA („Case bill deserved to be criticized“, HA 9.2.1998:A9); Mahealani Kamau'u, Native Hawaiian Legal Corporation („Kill ,autonomy’ bill“, HA 23.1.1998:A14); Kina'u Boyd Kamali'i, ehemals OHA („Call it ,termination bill’“, HA 11.1.1998:B3).

${ }^{418}$ Siehe auch: „Obama would sign Akaka Bill as president“, B.J. Reyes, H S-B 22.01.2008 (Internet: archives.starbulletin.com/2008/01/22/news/story05.html [24.02.2009]).

${ }^{419}$ Senator Akaka steht selbst auch beispielhaft für die Multi-Ethnizität der heutigen hawaiischen Bevölkerung: In seiner Selbstdarstellung heißt es hierzu: „U.S. Senator Daniel Kahikina Akaka is America's first Senator of Native Hawaiian ancestry, and the only Chinese American member of the United States Senate“ (Akaka 2009). 
behandelt in einem US-Kongress ohne nennenswerte hawaiische Beteiligung, den Abschluss jahrzehntelanger politischer Arbeit und den Ausgangspunkt einer politischen Autonomie der Hawaiier darstellt, entbehrt nicht einer gewissen Ironie. Dieser Vorgang verweist geradezu exemplarisch auf die vielschichtige Vermischung von Politik, Genealogie und Ethnizität in Hawai‘i. 



\section{Widerstand und autonome Praxis: Zeitgenössi- sche hawaiische Kunst im Spannungsfeld von Poli- tik und „Contemporary Art“}

Die Auseinandersetzungen in den letzten Jahrzehnten des 20. Jahrhunderts haben keinen Wiedergänger des Königreichs Hawai‘ $i$ auferstehen und seine Stelle als Repräsentant der hawaiischen Bevölkerung antreten lassen. Aber sie haben politische, soziale und kulturelle Fragen, die aus der komplexen Geschichte und gegenwärtigen Situation der hawaiischen Bevölkerung resultieren, in das Bewusstsein einer breiten Öffentlichkeit gebracht und dafür gesorgt, dass die „Hawaiian Sovereignty“ und die vielfältigen mit ihr verknüpften Aspekte aus dem politischen Alltag Hawai'is nicht mehr wegzudenken sind. Mit dem abschließenden Blick auf die hawaiische Kunstszene der Gegenwart rundet sich gewissermaßen die Darstellung der hawaiischen Autonomiebewegung und damit der Blick auf einen zentralen Aspekt der hawaiischen Kultur am Übergang vom 20. zum 21. Jahrhundert. Stand die kulturelle Revitalisierungsbewegung der „Hawaiian Renaissance“ zunächst noch im Schatten der politischen Bewegung, die sich verschiedener Aspekte hawaiischer Kultur zur Untermauerung ihres Anspruches bediente, so hatten sich zum Ende des 20. Jahrhunderts die Gewichtungen in diesem „Geben und Nehmen“ verschoben: eine mehr und mehr autonom agierende Szene bildender Künstler hawaiischer Abstammung nimmt die von der Autonomiebewegung postulierten Fragestellungen und Ziele in ihre Produktion auf und propagiert so nicht nur hawaiische „Sovereignty“ oder Autonomie, sondern grenzt sich auch in einem zunehmend unübersichtlichen Kunstmarkt von nicht-hawaiischen Künstlern ab. ${ }^{420}$ Viele der

\footnotetext{
420 Ähnliche Tendenzen der Verselbständigung lassen sich auch für das hawaiische Literaturschaffen nachzeichnen. Trotz bereits früher erschienener Anthologien hawaiischer Literatur (z.B. Hall 1985; Balaz 1989) nimmt die Literatur der Hawaiier noch in Stephen Sumidas Untersuchung der „Literary Traditions of Hawai'i“ (1991) gegenüber den Werken von Autoren euro-
} 
zeitgenössischen hawaiischen Künstler verstehen sich als politische Künstler und reflektieren in ihren Werken die politischen und theoretischen Auseinandersetzungen der letzten Jahrzehnte. Die politische Bewegung der Hawaiier ist auf diese Weise eng verwoben mit einer öffentlichen hawaiischen Kultur der Gegenwart, die sich nach und nach von Rückgriffen auf Tradition und Vergangenheit löst und zugleich immer wieder neue und eigenständige Zeichen kultureller Autonomie setzt.

Die von den Mitgliedern einer Gesellschaft geschaffenen Kunstwerke können gedacht werden als Ausdruck von überkommenen wie neu hinzukommenden Werten einer Kultur. Kunst kann sich aber auch mit dem Verlust von Maßstäben und Idealen auseinandersetzen und gesteckte Grenzen bewusst durchbrechen. Die Betrachtung der Kunst und der Bedingungen, unter denen sie zustande kommt, eröffnet einen spezifischen Blick auf das Selbstverständnis der Kunstschaffenden und der sie tragenden Gesellschaft. Die hawaiische Künstlerin und Erziehungswissenschaftlerin Manulani Aluli Meyer formuliert diesen Anspruch an die bildende Kunst, nicht nur subjektives Zeugnis zu sein, sondern zugleich das allen Gemeinsame zum Ausdruck zu bringen, wie folgt:

„Art is not separate from life. It is not just something to be looked at but an experience we have with that which we express. When Hawaiians create art, what I believe you experience is our own genealogy. And what emerges is the depth of our truth about a flower, an event, or an idea ... We are re-writing, re-scripting, re-imagining history. It is simply our version of the truth and when we speak it we are changing our future because we are able to define our past and present“ (Aluli Meyer 2001:13).

Manulani Meyer beschreibt hier einen auch im westlichen Kunstverständnis durchaus gängigen theoretischen Ansatz, den sie allerdings in einen spezifisch hawaiischen Kontext kleidet. Ich werde in diesem Kapitel die zeitgenössische hawaiische Kunst, verschiedene ihrer Protagonisten und unterschiedliche Aspekte und Tendenzen des gegenwärtigen Kunstlebens vorstellen. Dabei wird

\footnotetext{
amerikanischer oder asiatischer Abstammung nur geringen Raum ein. Heute zeugt u.a. die seit 1998 erscheinende Literaturzeitschrift 'oiwi: a native hawaiian journal (cf. Manley 1999) und die Vielzahl der in ihr publizierenden Autoren vom Selbstbewusstsein und der gewachsenen Bedeutung der hawaiischen Literatur. Bevorzugtes Medium hawaiischer Autorinnen und Autoren ist die Lyrik, was u.a. auf die hawaiische Tradition der Mele (Lieder; U.M.) zurückgeführt wurde (Ho‘omanawui 2005). Wichtige Vertreter der hawaiischen Lyrik sind Haunani-Kay Trask (1994, 2002; cf. Sullivan 2004) und 'İmaikalani Kalāhele (2002; cf. Winduo 2003) - beide sind zugleich bekannte Vertreter der Autonomiebewegung (Trask) bzw. der hawaiischen Kunstszene (Kalāhele). Eine Zusammenstellung anerkannter hawaiischer Lyriker und Lyrikerinnen findet sich auch bei Wendt, Whaitiri u. Sullivan (Hg.) 2003.
} 
deutlich werden, in welchem Maße viele Künstler und ihre Kunstwerke mittlerweile zu einer tragenden Kraft der Autonomiebewegung geworden sind und wie ihr Streben nach künstlerischer Eigenständigkeit und Anerkennung ein Forum praktizierter kultureller Autonomie geschaffen hat.

Mit ihren spezifischen historischen, kulturellen und politischen Wurzeln hat sich die hawaiische Gegenwartskunst, ausgehend von den durch Rückgriffe auf die Traditionen der Vergangenheit gekennzeichneten Revitalisierungsbemühungen der 1970er und 1980er Jahre, zu einer aktuellen Stimme im Konzert der Kulturen Hawai' is entwickelt. Doch während z.B. die hawaiische Musik, der traditionelle Gesang (und damit mittelbar die zeitgenössische Lyrik der Hawaiier) sowie der traditionelle Tanz Hula auf Überlieferungsstränge zurückgreifen können, die sich in der Zeit der kolonialen und kulturellen Dominanz der euro-amerikanischen Siedler z.T. im Verborgenen erhalten haben, ${ }^{421}$ erlebten die bildenden Künste tief gehende Brüche in der Praxis wie auch der Überlieferung des Wissens um Materialien und Technologie, Inhalte und Bedeutungen. Für die hawaiischen Künstler der Gegenwart heißt dies, dass sie sich das Wissen und das Können ihrer Vorfahren durch eigene Forschungsarbeit wieder aneignen müssen, wenn wie im Falle der hawaiischen Tatauierkunst (eine der wichtigen polynesischen Kunstgattungen) die direkte Kenntnis der traditionellen Technik und der alten Muster weitestgehend verloren war. ${ }^{422}$ Oder es ist gar notwendig, wie es das Beispiel der Herstellung von Kapa (Tapa oder Rindenbaststoff) zeigt, nicht nur die Techniken und Formensprache neu zu beleben, sondern auch das benötigte Rohmaterial, die Rinde des Papiermaulbeerbaumes (Broussonetia papyrifera) oder Wauke, durch Anbau des entsprechenden Baumes überhaupt erst wieder auf den Inseln verfügbar zu machen. 423

Solche Zäsuren sind jedoch nicht nur hinsichtlich der alten Techniken und des zu rekonstruierenden Wissens auszumachen: Nur wenige der heutigen hawaiischen Künstlerinnen und Künstler haben ihr Wissen um die Kunst in einem ausschließlich traditionell-hawaiischen Kontext gewonnen. Ihr überwiegender Teil hat vielmehr eine akademische Ausbildung in den westlich orientierten Kunstakademien Hawai' is sowie der kontinentalen USA erfahren. Für viele von ihnen steht daher neben der künstlerischen Aufarbeitung aktueller

\footnotetext{
${ }^{421}$ Stillman (2006) hat dies z.B. für den Hula nachgewiesen.

${ }^{422}$ So hat der Tatauierkünstler Keone Nunes die heute von ihm praktizierte Technik, bei der er den alten hawaiischen Instrumenten nachempfundene Geräte nutzt, und die von ihm verwendeten Muster durch eigene Studien in Archiven und Museen sowie das Sammeln erhaltener Informationen unter den Küpuna, den alten Hawaiiern, entwickelt (Pers. Mitteilung, K. Nunes, 2006).

${ }^{423}$ Kawaikaula‘au Aona-Ueoka, heute eine bekannte hawaiische Künstlerin, hat schon früh mit dem Anbau von Wauke begonnen und war bemüht, die Kunst der Kapa-Herstellung wieder zu verbreiten (Ward 1995).
} 
gesellschaftlicher Themen auch die Auseinandersetzung mit den Maßstäben der westlichen Kunstwissenschaften und Ästhetik im Zentrum ihrer Bemühungen. Ihre Werke, die großenteils ,westlichen“ Gattungen wie Malerei, Fotografie oder Installation zuzuordnen sind, werden so nicht nur durch das konkrete Thema der jeweiligen Arbeit, sondern auch durch das „unterfütternde“ theoretisch-ideologische Programm in einen spezifisch hawaiischen Kontext gestellt.

Ausgehend von den polynesischen Wurzeln der hawaiischen Gegenwartskunst sowie einem kurzen Blick auf die Situation der hawaiischen Künstler in der Mitte der 1990er Jahre, werden alsdann charakteristische Tendenzen des zeitgenössischen hawaiischen Kunstschaffens konturiert, einschließlich ihrer Bezüge zur politischen Bewegung und der zunehmenden Bedeutung einer „hawaiischen Praxis“. Das hier im Vordergrund stehende Schaffen hawaiischer Künstler und Künstlerinnen der Gegenwart, das einerseits ganz selbstverständlich Gattungen wie Malerei und Fotografie mit umfasst, gründet andererseits in den polynesischen Kunsttraditionen des Archipels, die nicht nur von einem anderen Kunstbegriff als dem europäischen inspiriert waren, sondern auch über einen eigenen Gattungskanon verfügten, zu dem z.B. die Malerei nicht gehörte. ${ }^{424}$

Wichtige traditionelle Kunstwerke wurden für den hawaiischen Adel oder für die Verwendung in religiösen Kontexten hergestellt. Mit der Aufhebung des Kapu im Jahre 1819 und der zunehmenden Bedeutung westlicher Luxusgüter kamen weite Teile des hawaiischen Kunstschaffens zum Erliegen. Heute finden sich die großartigen Zeugnisse der alten hawaiischen Kunst verteilt in den Museen Hawai' is und der Welt, unter ihnen nurmehr an die 150 erhaltene Beispiele der alt-hawaiischen Schnitzkunst (siehe z.B. Kaeppler 1978; Cox u. Davenport 1974). ${ }^{425}$

Will man im heutigen Hawai'i Beispiele für die einzigartigen Tempelskulpturen sehen, so muss man das Bishop Museum in Honolulu besuchen, wo

\footnotetext{
${ }^{424}$ Ohne mich auf die weitläufige Diskussion des Begriffs „Kunst“ einzulassen, der wesentlich bestimmt ist von historischen und theoretischen Inhalten europäischer Provenienz und in seiner engeren Auslegung einem voreuropäischen Verständnis nicht notwendig gerecht wird, ist „Kunst“ hier im weitgefassten Sinne von „Oceanic Art“ verwendet, der indigene Vorstellungen von Ästhetik ebenso umfasst wie er versucht, die europäische Vorstellung von Originalität und Künstlerschaft zu integrieren (siehe z.B. Thomas 1995; D’Alleva 1998; Kaeppler, Kaufmann u. Newton 1994). Wesentliche Bereiche eines polynesischen Kunstlebens, die nicht-materialisierten Redekünste, der traditionelle Tanz und die Kunst des Tatauierens bleiben hierbei ausgeblendet. Sie werden in diesem Kapitel ebenfalls nicht weiter betrachtet.

${ }^{425}$ So u.a. in der Sammlung des Instituts für Ethnologie der Universität Göttingen, die neben singulären Kunstwerken wie einer Federskulptur des hawaiischen Kriegsgottes Kükāi 'limoku auch eine herausragende Zusammenstellung vor-europäischer Kapa (Tapa bzw. Rindenbaststoffe) besitzt (Hauser-Schäublin u. Krüger [Hg.]1998; National Museum of Australia 2006; Little u. Ruthenberg 2006).
} 
die einzigen auf den Inseln verbliebenen Originalstandbilder ausgestellt sind, ${ }^{426}$ oder man lasse Nachbildungen jener mächtigen Schnitzwerke im rekonstruierten Tempelbezirk von Pu'uhonua o Hōnaunau auf der Insel Hawai'i auf sich wirken. Weitere „Fundorte“, für viele Touristen vielleicht die Orte intensivster Begegnung mit den überkommenen Formen alt-hawaiischer Kunst, sind die Souvenirgeschäfte und die „Hawaiiana“-Abteilungen der großen Drugstores und Discounter in Waikīkī. Hier quellen die Regale über von billigen Nachbildungen aus Kunststoff (in aller Regel „Made in China“), versehen mit dem Aufdruck „Hawai'i“. Neben dem Kunstkitsch und den Billigrepliken der Touristenmärkte arbeiten aber auch Schnitzer traditionelle Arbeiten nach und finden ihre Abnehmer unter den Besuchern mit gut gefüllten Geldbeuteln. Diese z.T. sehr gut gemachten kleinformatigen Skulpturen und Gebrauchsgegenstände sind nichtsdestoweniger lediglich möglichst exakte Kopien der beeindruckenden Vorbilder und sollen hier nicht der hawaiischen Kunst der Gegenwart zugerechnet werden.

Bei meinem ersten Besuch auf den Inseln in der Mitte der 1990er Jahre waren hawaiische Künstler und ihre Werke in den kommerziellen Galerien und den Museen kaum auszumachen und die bundesstaatliche „State Foundation on Culture and the Arts“ wusste 1995 mit meiner Frage nach ihren Bewertungskriterien für die Förderung hawaiischer Kunst nur wenig anzufangen: schließlich sei alles, was sie in Hawai‘i förderten „Hawaiische Kunst“ ${ }^{427}$ So waren Künstler hawaiischer Abstammung zwar an der jährlichen Stiftungs-Ausstellung „Artists of Hawai'i““ beteiligt, sie bzw. ihre Werke bildeten jedoch keine erkennbare, gegen andere Arbeiten und ihre Urheber abgegrenzte oder sich abgrenzende Kategorie. ${ }^{428}$

Daneben aber arbeiteten zu Beginn der 1990er Jahre in Hawai'i auch Künstler hawaiischer Abstammung, die mit ihren Werken einen eigenen Bezug

\footnotetext{
${ }^{426}$ Andere Großskulpturen finden sich u.a. in der Eremitage, St. Petersburg, und im British Museum, London.

${ }^{427}$ Natürlich förderte die Stiftung auch Künstler hawaiischer Abstammung, sie fanden sich allerdings eingereiht in die allgemeinen Förderungskategorien wie „Ethnic Heritage“, „Visual Arts“ oder „Folk Arts“ (State Foundation on Culture and the Arts 1994).

${ }^{428}$ Es liegt hier nahe, einen Blick in den Südpazifik zu werfen, wo die Kunst der Māori in Aotearoa/Neuseeland zu diesem Zeitpunkt längst als eigenständige Kunst geachtet war. Dies mag auch an den ungebrochener überlieferten Kunsttraditionen der Māori liegen, die zudem mit ihrer bekannten Schnitzkunst und ihren Architekturtraditionen, in denen sich über die Schnitzereien hinaus Malerei und textile Techniken auf faszinierende Weise mischen, auf traditionelle Formen und Medien zurückgriffen, die einem euro-amerikanischen Kunstverständnis näher liegen als die weniger aufwendigen Traditionen Hawai'is, und die spätestens seit der großen im Metropolitan Museum of Art in New York gezeigten Wanderausstellung Te Maori: Maori art from New Zealand collections (1984) auch über den Kreis der ethnographisch Interessierten hinaus internationale Anerkennung gefunden hatte.
} 
zu den hawaiischen Traditionen herstellten und über die Grenzen des Archipels hinaus Anerkennung gefunden hatten. So ist der außerhalb Hawai' is aufgewachsene Maler Herb Kawainui Kāne, 1928 in Minnesota geboren und am Art Institute of Chicago ausgebildet, ${ }^{429}$ u.a. durch seine in zahlreichen Veröffentlichungen belegte Kunst auch einer breiten nicht-hawaiischen Öffentlichkeit bekannt. Er gehörte zu den Initiatoren und Erbauern des traditionellen Reisebootes Hōkūle'a, einem der Auslöser der „Hawaiian Renaissance“. Kānes z.T. großformatige Ölmalereien schildern in realistischer Darstellung Ausschnitte der hawaiischen Vergangenheit. Als bekannter Maler hawaiischer Abstammung, der erst spät in seinem Leben auf die Inseln übersiedelte, und als Mitbegründer der kulturellen Revitalisierung, der er zugleich als kritischer Beobachter gegenüberstand, vereint Kāne in seiner Person viele Widersprüche des aktuellen „Hawaiisch-Seins“.

Der wesentlich jüngere Bildhauer Sean Kekamakupa‘a Browne wurde 1953 in Hilo auf der Insel Hawai'i geboren. Auch er hat eine Ausbildung in den kontinentalen USA, Japan und Europa genossen, bevor er sie 1983 an der University of Hawai'i, Mānoa, abschloss. Anders als seine historischen Vorbilder arbeitet er überwiegend mit den Materialien Stein und Bronze und ist vor allem durch seine großen Skulpturen im öffentlichen Raum bekannt geworden. Seine Werke sind zwar in der Formensprache der alten hawaiischen Kunst und den überlieferten Traditionen verpflichtet, doch erklärte er im schriftlichen Teil seiner Abschlussarbeit für den Master of Fine Arts (M.F.A.):

„The contemporary artist sometimes uses objects from many cultures as sources for ideas in creating works of art. Although the artifacts of these cultures were designed for specific purposes, the contemporary interpretations, while still referring to their original meaning, become a part of the art context today.

The works produced for my thesis are sculptural interpretations of Polynesian ethnographic objects. It is my hope that the works in my thesis, as contemporary art objects, will act as informational references to past Polynesian cultures and yet maintain their presence as cultural symbols of our present society“ (Browne 1983:1).

Obwohl es sich bei den mit hawaiischen Namen versehenen Objekten seiner Abschlussarbeiten um Nachempfindungen alt-hawaiischer Objekte handelt,

\footnotetext{
${ }^{429}$ Mitglieder der „Hawaiischen Diaspora“ in den kontinentalen USA, Europa und darüber hinaus sind in Hawai' $\mathrm{i}$ als Hawaiier akzeptiert - allerdings ist die Aufrechterhaltung einer „hawaiischen“ Identität außerhalb des Archipels wiederum nicht ohne Probleme (siehe z.B. Halualani 2002:195 ff., die die Praxis der „Identitätsbehauptung“ in der hawaiischen Diaspora Kaliforniens und Nevadas untersucht und kritisch kommentiert). 1990 lebten dem US-Zensus zufolge 72.272 Bürger hawaiischer Abstammung in den kontinentalen USA (OHA 1996:14-5).
} 
kommt Browne ohne Verweise auf spezifisch hawaiische Bezüge aus; er stellt vielmehr die Relikte der polynesischen Kultur in eine Reihe mit Objekten aus anderen Kulturen. Die materialisierten Traditionen bleiben für ihn Inspiration einer Gegenwartskunst, die keine konkreten Aussagen zu politischen oder gesellschaftlichen Situationen machen muss.

Viele der seit Mitte der 1990er Jahre an der University of Hawai'i ausgebildeten Künstlerinnen und Künstler hawaiischer Abstammung sehen die Bezüge ihrer Werke zur hawaiischen Kultur bzw. zur Gegenwart der Hawaiier grundsätzlich anders. In einem gesellschaftlichen und vor allem auch akademischen Klima, das vom Wirken einer starken Autonomiebewegung geprägt war, spielten politische Bezüge in den Arbeiten der jeweiligen Künstler eine immer größere Rolle. So schreibt Charles H. Souza 1995 im schriftlichen Teil seiner M.F.A.-Abschlussarbeit, deren Hauptteil eine Ausstellung mit dem Titel „'O Ka Wa‘a [das Boot; U.M.]: remains and departure“ bildete:

„Much of the contemporary Hawaiian art uses images appropriated from the Hawaiian religious past to sign or announce its identity as Hawaiian. I have chosen, in my work, not to reproduce these images taken out of context and call them contemporary because I produce them now. I believe it is more harmful to pick and choose, as some have, parts that may be attractive from the tradition without taking the whole ... I have chosen, instead, not to address the past; but rather to deal with the present and the issues that shape our future as Hawaiians and parents of future generations of Hawaiians“ (Souza 1995:2-3).

Mit diesem Text bezieht Souza Stellung gegen eine „contemporary Hawaiian art“ (wie sie m.E. auch durch das Werk Sean K. Brownes verkörpert wird), die sich mehr mit den Formproblemen der Vergangenheit als den sozialen Problemen der Gegenwart auseinandersetzt. Seine Ausstellungsarbeit, deren Objekte vor allem aus den Materialien Keramik und Holz gestaltet sind, setzt sich in mehreren „Kapiteln“ mit der Ankunft der Hawaiier auf den Inseln und ihrer Marginalisierung durch die zugewanderten Amerikaner auseinander. So stellt eine der zentralen Arbeiten der Ausstellung (ein auf hölzernen Sockeln stehendes Doppelrumpfboot) auf seinen mit Immobilienanzeigen und Touristenangeboten beklebten Rümpfen in weißen Buchstaben und in englischer, japanischer und koreanischer Sprache die Fragen: „Where are the Hawaiian people? Who named these islands? Where are they?" Die Antwort hierzu steht, unverständlich für die meisten Besucher, auf den schlichten schwarzen Sockeln: „Eia no makou" (Wir sind hier; U.M.). In dieser Arbeit, wie auch in den anderen „Kapiteln“ der Ausstellung, zeigt sich ein expliziter politischer Anspruch, der das weit verbreitete und vom Markt beförderte westliche Verständnis einer nur 
sich selbst verpflichteten Kunst überlagert - ein Anspruch, der für viele der jüngeren hawaiischen Künstlergeneration zum wesentlichen Antrieb ihres Schaffens wurde.

Der 1960 geborene Herman Pi`ikea Clark weist der zeitgenössischen Kunst der Hawaiier konkrete gesellschaftspolitische Aufgaben zu. In seiner auch der Theorie einer hawaiischen Gegenwartskunst gewidmeten M.F.A.-Arbeit stellt er fest:

„Contemporary Hawaiian art, as was its traditional counterpart, hana no 'eau, should function beyond western aesthetics to respond to the needs of contemporary Hawaiian society. Because the condition for Hawaiian society at present is heavily political, much of contemporary Hawaiian art has a communicative function that advocates a particular political point of view. [...]

The potential of Hawaiian arts to provide a visual cultural vehicle for Hawaiian education becomes apparent. In this expanded role, Hawaiian art moves beyond the narrow halls of western aesthetics to fulfill again its function as a tool for the advancement of the Hawaiian people and their culture“ (Clark 1996:11-2).

Clark erhebt hier den Anspruch, dass eine zeitgenössische hawaiische Kunst die westlichen Kunstvorstellungen durch ihre politische Ausrichtung und auf dem Wege der konkreten Vermittlung kulturell-politischer Inhalte überwinden kann und soll. Als sichtbares Zeichen dieses Anspruches inszenierte er seine Abschlussausstellung, die er als ein „contemporary Hawaiian ritual“ beschreibt, nicht in den Räumen des Kunstinstituts der Universität sondern auf dessen Dach, um so eine sichtbare Abgrenzung gegen die Normen westlichen Kunstverständnisses zu setzen (die er innerhalb des Gebäudes ansiedelt) und diese Normen zugleich zu durchbrechen.

Zwei weitere Stellungnahmen bekannter hawaiischer Künstler sollen dieses Quartett von Aussagen zur zeitgenössischen hawaiischen Kunst abrunden: Der Fotograf Kimo Cashman und die bekannte Fotografin Anne Kapulani Landgraf äußerten sich im Rahmen einer Ausstellung zeitgenössischer Fotografie aus Hawai ${ }^{\circ} \mathrm{i}^{40}$ (d.h. Exponate auch von Künstlern und Künstlerinnen nichthawaiischer Abstammung) zu den von ihnen gezeigten Werken, wobei Cashman seine Arbeit mit deutlichen Worten in den direkten Bezug der politischen Autonomiebewegung stellt:

${ }^{430}$ ReViewing paradise: contemporary photography from Hawai $i$; The Honolulu Advertiser Gallery, Honolulu (27.04.-28.06. 1995). 
„I have been very involved with the Native Hawaiian movement for sovereignty ... My works speak with this movement. They are a response to the racism, oppression, and exploitation the Native Hawaiian people have been subject to since the coming of the white man to Hawaii“ (Cashman 1995:4).

Kapulani Landgraf, die durch ihre stillen Landschaftsaufnahmen bekannt geworden ist (z.B. Landgraf 1994), vermittelt in ihren Bildern einen weniger offensichtlichen politischen Bezug; mit ihnen und durch sie stellt sie die Frage nach dem Verbleib einer hawaiischen Identität dieser so viel fotografierten Orte:

„Landscapes, environmental portraits of an altered place. At another time in Hawai' $i$ these places had names and significance. [...]

I am interested in rediscovering the significance of a place ... My photographs, titled in Hawaiian, are an attempt to document and express elements of my culture's collective sense of identity ... We have a wealth of romanticized views of Hawai'i made by visitors and colonizers. Where are the images and photographs by the indigenous people?" (Landgraf 1995:9-10).

Bei allen Unterschieden in der Formulierung sowie der Akzentuierung der Aussagen, die von mehr oder weniger verhüllten politischen Forderungen bis hin zu klar formulierten Kampfansagen an die politische und kulturelle Hegemonie der US-amerikanischen Mehrheitsgesellschaft reichen, ist allen hier zitierten Künstlern, die in unterschiedlichen Medien arbeiten, verschiedenen Altersgruppen zugehören, doch eines gemeinsam: Sie alle verbindet die eigene, sich selbst immer wieder abverlangte Suche nach dem „Hawaiischen“ in der hawaiischen Kunst unserer Zeit sowie die Forderung nach politischer und kultureller Anerkennung. Diese auf ganz unterschiedliche Weise artikulierten Bemühungen flossen in einem Ausstellungsprojekt zusammen, an dem alle bisher in diesem Kapitel genannten Künstler außer Herb Kawainui Kāne beteiligt waren. Die 1997 im East-West Center in Honolulu eröffnete und im folgenden Jahr auch in Deutschland gezeigte Ausstellung Ho'okū 'ē verstand sich als eine explizit politische Kunstpräsentation und soll im Folgenden vorgestellt und unter den oben benannten Gesichtspunkten näher betrachtet werden.

„Ho 'okū ‘è (Resistance) “ 1997/98

Wie schon der Titel „Ho'okū' ‘“" [Widerstand leisten; U.M.] der Gemeinschaftsausstellung deutlich machte, verstanden die Ausstellungsmacher diese Kunstschau nicht nur als Querschnitt der aktuellen hawaiischen Kunst, sondern 
auch als ein politisches Forum. „Ho'okū'e““ war nicht die erste Gruppenausstellung politischer Künstler hawaiischer Abstammung: bereits 1993 präsentierte ein Zusammenschluss von Künstlern („Council of Contemporary Hawaiian Artists“) in Zusammenarbeit mit dem Bishop Museum die Ausstellung „Ho“o Pāna ' $i$ “" [Wiedergutmachung, Ausgleich suchen; U.M.], an der sieben Künstlerinnen und Künstler teilnahmen. Am Schluss des Begleitheftes zur Ausstellung findet sich ein Statement der beteiligten Kunstschaffenden, das nicht nur den politischen Bezug der Präsentation, sondern auch das Selbstverständnis der Ausstellenden umreißt:

„These contemporary works are about pain, hurt, mistrust, and the betrayal of a friendship between two peoples, Hawaiians and Americans. What we see here are expressions of a people rooted in 2.000 years of making gods to worship and using art to speak.

We gather here at this time to demonstrate our great pain, 'eha'eha, caused by the American invasion of the Hawaiian Nation, an act leading to the immoral overthrow of its sovereign, Her Majesty, Lili‘u Kamaka'eha.

We, the Hawaiian artists, are mākua, parents, who create foundations for those following us to build on. Our job is to defend our point of view and continually question the forces that shape our lives and our futures" (Council of Contemporary Hawaiian Artists 1993).

Waren an dieser Ausstellung vor allem Künstler der „,älteren“ Generation beteiligt, so hatte „Ho'okū' '`“" mit ihren 20 Teilnehmern nicht nur fast dreimal so viele Ausstellende, die meisten von ihnen gehörten auch bereits zu den „Nachfolgern“, denen die Künstler der vier Jahre zuvor gezeigten „Ho“o Pāna'i“"Schau Eltern, Mākua, und Mentoren gewesen waren. ${ }^{431}$ Die an „Ho'okū' ${ }^{\circ}$ “‘ beteiligten Künstler und ihre Arbeiten erhoben allesamt einen mehr oder weniger deutlichen politischen Anspruch, der allerdings nicht, wie bei den Gedenkfeiern 1993, in einen konkreten, aktuellen Zusammenhang gestellt wird. Ausformuliert findet er sich, gewissermaßen als ihr Motto, auf der Titelseite des Begleitprospekts zur Ausstellung:

\footnotetext{
${ }^{431}$ Am Projekt „Ho‘okū‘ ‘“‘ wirkten die folgenden Künstler mit: Hāli‘imaile Andrade, Kawai Kaulā'au Aona-Ueoka, Sean K.L. Browne, Kimo Cashman, Ka'ili Chun, Herman Pi‘ikea Clark, Bob Freitas, April A. Hōkūlani Drexel, 'Imaikalani Kalāhele, Malia Kāne Kuahiwinui, Anne Kapulani Landgraf, Kawika Mahelona, 'Alika McNicoll, Meleanna Aluli Meyer, Ipō Nahipali, Kauka de Silva, Ka'ohu Seto, Chuck Kawai'olu Souza, Mele Visser und Lani Brehme Yamasaki. Zur Ausstellung siehe auch die Artikel „Art by Hawaiians viewed from a Western perspective“, Joan Rose (HA 25.5.1997:E-6); „Contemporary Native Hawaiian artists address issues“, Joan Rose (HA 18.5.1997:E-6).
} 
„Inspired by the theme of Ho'okū' ${ }^{`} \mathrm{e} /$ resistance, twenty contemporary Native Hawaiian artists from the Island of $\mathrm{O}^{\prime}$ ahu address issues pertinent to Native Hawaiians. The artworks visualize the problems of colonialism, race, genocide, and ethnocide and speak to the inherent right of Native Hawaiians to re-claim control over their language, land, natural resources, history values and practices“ [Hervorhebung im Original; U.M.] (Ho`okū‘ē 1997).

Dieser kurze Text wirkt wie ein Echo der Kunst auf die von Seiten der Protagonisten der Autonomiebewegung erhobenen Forderungen. Von der Kunstkritik vor Ort wird dieser politische Anspruch aufgegriffen und zumindest im hier zitierten Beispiel durchaus positiv bewertet (wenn auch mit einem Seitenhieb im Sinne der vom westlichen Kunstbetrieb eher kritisch gesehenen Zweckgebundenheit künstlerischer Äußerungen): „What makes for strong politics does not neccessarily make for strong art, so it is particularly heartening to find signs of their convergence in this exhibition, in works that gather strength from the context, but also speak eloquently on their own“ (Morse 1997). Mit „Ho'okū' ${ }^{`}$ “" trat eine überwiegend junge hawaiische Kunst in ihrer ganzen Vielfalt und mit einer in ihrer Mehrstimmigkeit doch eindeutigen politischen Haltung an die Öffentlichkeit des Bundesstaates, wo sie inzwischen (trotz aller weiter bestehenden Schwierigkeiten der Anerkennung durch Öffentlichkeit und Kunstszene) einen festen Platz eingenommen hat.

1998 übernahm das Linden-Museum Stuttgart die Ausstellung „Ho'okū'e““ im Rahmen des Stuttgarter Kunstprojekts „Vielfaches Echo“, an dem neben den öffentlichen Kunstmuseen und Ausstellungsorten in der Stadt auch zahlreiche private Galerien beteiligt waren. Ziel des Projektes mit seinen insgesamt 18 Ausstellungen außereuropäischer, in aller Regel zeitgenössischer Kunst war es, Bezüge zwischen außereuropäischen und europäischen Kunstformen aufzuzeigen und die Möglichkeiten transkultureller Kommunikation auf der Kunstebene auszuloten (Arbeitsgemeinschaft vielfaches echo 1998 a:6).

Fünf Künstlerinnen und Künstler begleiteten die Ausstellung nach Stuttgart, um dort ihre Anliegen in Lesungen, Workshops und Diskussionsveranstaltungen vorzubringen, wobei sie durchaus auch auf Unverständnis und Ablehnung stießen. ${ }^{432}$ Zwar hatte auch das „Vielfache Echo“ in gewisser Weise ein Motto, wenn auf dem Informationsprospekt zu den Ausstellungen der Kunsthistoriker und Medientheoretiker Hans Belting zitiert wird:

${ }^{432}$ Die Ausstellung wurde begleitet von Hali‘imaile Andrade, Kawai Kaulā‘au Aona-Ueoka, Herman Pi'ikea Clark, 'Imaikalani Kalāhele und Chuck Kawai'olu Souza. 
„Eine Kunst ,außerhalb’ der gewohnten Kunstgeschichte, also auch ,außerhalb’ der westlichen Kunstszene, könnte einem ja schließlich auch in Formen begegnen, die man schon deswegen nicht mit Kunst identifiziert, weil sie unseren Kunstbegriff abweisen, oder die man allzu rasch mit einer westlichen Ästhetik verstehen will, die nur in ein Mißverständnis führen kann. Wir fragen uns nicht, ob sich Kunst noch auf einen einzigen Begriff bringen läßt, den wir, als Kinder der Aufklärung, hartnäckig für universal, und neuerdings für global, halten. Aber wir müßten uns fragen, ob nicht jeder Begriff von Kunst, über den wir streiten, schon an den Grenzpfählen unseres westlichen Denkens endet“ (Arbeitsgemeinschaft vielfaches echo 1998b).

Doch in der Auseinandersetzung mit den Werken der hawaiischen Künstler ging der hier artikulierte Anspruch einer aufgeschlossenen Konfrontation mit fremden Kunstwelten und die Infragestellung des eigenen westlichen Kunstverständnisses weit über die Reaktionen von Vertretern der Kunstszene hinaus, wie sie auf einer Diskussionsveranstaltung mit den Künstlern im Stuttgarter Linden-Museum geäußert wurden. Hier wurde deutlich, in welchem Dilemma sich die zeitgenössische hawaiische Kunst - außerhalb Hawai'is und durchaus auch in Hawai'i selbst - befindet: so kollidierte für manche Betrachter die selbstverständliche Verwendung traditioneller Materialien mit dem von ,ethnischen Traditionen“ losgelösten, modernen Anspruch der Kunstwerke; die ganz offensichtlich politische Dimension verschiedener Beiträge mit ihren Verweisen auf hawaiische Geschichte, hawaiisches Land und die Genealogie erschien vielen als einer Gegenwartskunst unangemessen; und schließlich, dies vielleicht ein eher deutsches Phänomen, erregten die starken Bezüge auf die Abstammung den Unmut der Teilnehmer. Insgesamt war die Kritik so deutlich, dass sich Ingrid Heermann, Organisatorin und Kuratorin der Stuttgarter Ausstellung, veranlasst sah, der nach den Veranstaltungen erschienenen Begleitpublikation einen kurzen Kommentar beizufügen:

„Diskussionen in Stuttgart haben gezeigt, dass es im europäischen Kontext nicht immer einfach ist, die Aussagen der modernen hawaiischen Künstler bezüglich der Ahnen und der Erde nachzuvollziehen. Unsere eigene Geschichte hat uns jeden Anlaß zu großer Vorsicht bei Konzepten gegeben, die in eine Nähe zu ,Blut und Boden' gerückt werden könnten, zum anderen erscheinen sie vielen zu romantisch und einer modernen Gesellschaft unangemessen. [...]

Die Arbeiten sind ... auch Reflektionen über das eigene kulturelle Erbe der Künstler. Und sie sind eine Aufforderung an den fremden Betrachter, dieses kulturelle Erbe zu respektieren, anstatt es durch oft unreflek- 
tierte Aneignung und Vermarktung weiter infrage $\mathrm{zu}$ stellen und $\mathrm{zu}$ zerstören“ (Heermann u.a. 1998).

Die anwesenden Künstler führten bei der Eröffnung der Ausstellung eine formelle Weihung der Ausstellung durch, die sich an das auf hawaiischen Veranstaltungen in Hawai' $i$ heute übliche und auf traditionelle Formen zurückgreifende „Hawaiian Protocol“ anlehnte. Mit Blüten- und Farnkränzen geschmückt, deren Rohmaterial anders als in Hawaii nicht gesammelt werden konnte, sondern auf Bestellung vom Blumenladen geliefert wurde, in neotraditionelle Überwürfe gekleidet und unter dem Klang von Muschelhörnern und hawaiischem Sprechgesang, führten sie die Besucher in die Ausstellungsräume. Obwohl das „Hawaiian Protocol“, eine der zentralen Manifestationen hawaiischer kultureller Eigenständigkeit, in Hawai`i in seiner Selbstverständlichkeit kaum Aufsehen erregt, lieferte gerade dieses Zeremoniell in Stuttgart der Kritikerin der Stuttgarter Zeitung das Material für die Einordnung der Ausstellung in das Feld des Exotisch-Fremdartigen. Unter dem Titel „Mögen die Kunstwerke sich wohl fühlen!“ führte sie unter anderem aus:

„Auf den ersten Blick mag man den Arbeiten ihre politische Dimension kaum ansehen, sie sind häufig schwer lesbar, haben ihre eigene kulturell geprägte Ikonographie. Fremdartig, wenn auch beeindruckend war denn auch die Zeremonie zur Vernissage. Mit einem rituellen Gebet, mit Tönen aus Muscheln und Gesang weihten die Künstlerinnen und Künstler die Ausstellung ein. Nicht damit die Stuttgarter etwas zu sehen haben, sondern damit sich ihre Arbeiten hier in der Fremde auch wohl fühlen. Denn, so hawaiischer Glaube, auch Gegenstände besitzen Leben“ ${ }^{433}$

In gewissem Sinne wiederholt sich hier das oben bereits angesprochene Dilemma der hawaiischen Gegenwartskunst: deutlicher als vielleicht in Hawai' $\mathrm{i}$ erscheint hier der für die Kunstszene existierende Konflikt zwischen dem Anspruch einer modernen Kunst und dem wahrgenommenen Bemühen um kulturelle Einbettung. Wie unkompliziert die Hawaiier selbst mit solch zentralen Elementen ihrer ja durchaus auch „neu gefundenen“ Kultur umgehen können, mag das Beispiel einer nicht-öffentlichen 'Awa-Zeremonie (Kawa-Zeremonie) zeigen, die die mitgereisten Künstler unter Leitung von 'İmaikalani Kalāhele vor der Einweihung der Ausstellung mit dem kleinen Kreis der an Ausstellung

433 „Mögen sich die Kunstwerke wohl fühlen!“, Adrienne Braun, Stuttgarter Zeitung, 06.05.1998:11. 
und Begleitprogramm beteiligten Museumsmitarbeiter durchführten. ${ }^{434}$ Nachdem die 'Awa in feierlicher Stimmung und unter Gesang in einer flachen Steinschale angerührt war, wurde sie allen Anwesenden in einer Kokosschale gereicht und mit großer Feierlichkeit getrunken. Gleich danach bekamen wir Nicht-Hawaiier Schokolade angeboten, um den, wie es hieß, unangenehmen Geschmack der 'Awa zu neutralisieren - die Ernsthaftigkeit der Zeremonie litt unter dieser „Innovation“ nicht.

Um schließlich an dieser Stelle das Zitat Manulani Meyers vom Anfang dieses Abschnitts wieder aufzugreifen, so war „Ho'okū'ē / Resistance Widerstand“, ob in Honolulu oder Stuttgart, in dem von ihr angesprochenen Sinn ein wichtiger Mosaikstein der hawaiischen „Version der Wahrheit“, der durch seine Wirkung nach innen (und mit allen Einschränkungen auch nach außen) die hawaiische Zukunft ein wenig verändern half. Diese Ausstellung, die in ihr gezeigten Werke und das Selbstverständnis der hawaiischen Gegenwartskünstler als Exponenten hawaiischen Kulturlebens sind wichtige Schritte auf dem Weg zu einer autonomen Praxis der hawaiischen bildenden Kunst.

Zeitgenössische hawaiische Kunst und das „Recht am Bild“

Im März 2006, fast ein Jahrzehnt nach der oben besprochenen Ausstellung „Ho'okū' $\overline{\mathrm{e}} /$ Resistance“ fand in Honolulu der erste von hawaiischen Künstlern organisierte „Maoli Arts Month (MAMo)“ statt. ${ }^{435}$ Während dieses hawaiischen Kunstmonats beteiligten sich mehr als 50 bildende Künstler, Sänger und Autoren hawaiischer Abstammung an zahlreichen Ausstellungen und Veranstaltungen. Politische Aussagen, auch wenn sie integraler Bestandteil vieler ausgestellter Kunstwerke waren, standen hier nicht im Vordergrund - im Programmheft des MAMo machte der „Buyer’s Guide“ mit seiner Auflistung der beteiligten Künstler deutlich, dass es den Veranstaltern ein wichtiges Anliegen war, die Aufmerksamkeit potentieller Käufer zu erregen. Dies war auch das Ziel des ersten „Native Hawaiian Arts Market“, der im Rahmen des MAMo auf dem Gelände des Bishop Museums in Honolulu stattfand und mit seinen verschiedenen Vorführungen (Tanz, Handwerk, traditionelles Essen) zahlreichen Künstlern und Kunsthandwerkern ein Verkaufsforum bot.

Mit der Ausstellung „Kū I Ka Ni`o“4336 bot das Bishop Museum etablierten Künstlern der hawaiischen Kunstszene die Möglichkeit einer Präsentati-

${ }^{434}$ Als Mitwirkender am Begleitprogramm zur Ausstellung konnte ich an dieser Zeremonie teilnehmen.

${ }^{435}$ Die Abkürzung „MAMo“ kann im Hawaiischen auch als mamo bzw. nā mamo gelesen werden und bedeutet dann „die Nachfahren“ oder auch „die Kinder“ (U.M.) - so stellte schon der Titel der Ausstellungsreihe die ausstellenden Künstler in die Reihe ihrer hawaiischen Vorgänger. Der „Maoli Arts Month (MAMo)“ fand 2009 zum vierten Mal in Folge statt; er hat sich als Forum der hawaiischen Kunst- und Kulturszene etabliert.

436 „Den Gipfel erreichen“ (U.M.). 
on ihrer Werke. Im Rahmen der Ausstellung fand auch die Verleihung eines Preises statt, mit dem die Veranstalter des MAMo sechs bekannte hawaiische Künstler für ihr Lebenswerk ehrten. ${ }^{437}$ Die von den Preisträgern vertretenen Kunstsparten reichten von Federarbeiten, der kunstvollen Anfertigung traditioneller Blütenkränze oder Lei, Flechtarbeiten und Malerei bis hin zur Schnitzkunst und deckten so ein breites Spektrum gegenwärtiger hawaiischer künstlerischer Produktion ab. In einem kurzen Text des Programmheftes begründeten die Veranstalter die Zuerkennung der Preise. Sie beschreiben die Geehrten als Lehrer und Erneuerer und betten sie zugleich, ungeachtet der jeweils gewählten künstlerischen Ausdrucksform, in die von den Ahnen überkommenen Traditionen ein:

„Each of these visionary artists have reached the pinnacle of achievement. They have led by example, tread upon paths long gone, now revealed. Each has transmitted the words and works of our ancestors, bringing them back into our consciousness. Collectively, they have educated and illuminated thousands“ (MAMo 2006:8).

Standen in der Ausstellung und auf der Preisverleihung im Bishop Museum also die bereits arrivierten hawaiischen Künstler im Vordergrund, so fand eine neue Generation junger Künstler ihren Platz in einem Kunstladen in Chinatown, wo die Schau „It's Na'au or Newa“ (ebenfalls organisiert vom Bishop Museum) ihre zum Teil deutlich politisch motivierten Kunstwerke präsentierte. Auch hier wurde eine alles verbindende gemeinsame hawaiische Vergangenheit im Einleitungstext herausgestellt:

„It's the now, the na'au, our guts, the seat of our emotions, the journey to our present, our reactions, our reflections. It's the newa, the foreva, the path to our past, the war club, our protection, our foundation“. ${ }^{438}$

Allerdings mussten die jungen Künstler die eigene Historie erst entdecken, ihr Weg führte in diesem Fall aus der Gegenwart in die Vergangenheit. Das Spiel mit den traditionellen hawaiischen Ausdrucksformen und Techniken blieb ihrer künstlerischen Aufarbeitung aktueller Fragestellungen nachgeordnet. Betrachtet man zeitgenössische hawaiische Kunst, so fällt auf, dass die Künstler das Zusammengehen politischer Inhalte mit künstlerischem Ausdruck nicht als problematisch empfinden, sondern im Gegenteil geradezu als selbstverständlich

\footnotetext{
${ }^{437}$ Die geehrten Künstler waren Rocky Jensen, 'Īmaikalani Kalāhele, Herb Kāne, Mary Lou Kekuewa, Mary McDonald und Ipō Nihipali.

${ }^{438}$ Informationsblatt zur Ausstellung, 2006.
} 
ansehen. Neben der Verwendung überlieferter Formen und tradierter Techniken ist ebenso die offensive und offensichtliche Thematisierung aktueller hawaiischer Themen ein Kennzeichen hawaiischer Gegenwartskunst. Doch während letzteres in aller Regel den Künstlern hawaiischer Abstammung vorbehalten bleibt, finden Formensprache und traditionelle Techniken auch über den Kreis hawaiischer Kunstschaffender hinaus Aufnahme und Verbreitung. Der nachhaltige Einspruch von hawaiischer Seite gegen diese „missbräuchliche“ Nutzung traditioneller Kunstformen und Designs hat sein genaues Gegenstück in dem Protest gegen die als widerrechtlich angesehenen Eingriffe in Landnutzungsrechte und politische Selbstbestimmung. Er zeigt aber auch, wie sehr (bei allem etwa vorhandenen materiell-geschäftlichen Interesse) die überkommenen Kulturgüter als Teil der kulturellen Identität und damit als schützenswertes politisches Kapital empfunden werden:

„All over the world, but especially in America, cheap products coming off assembly lines spew forth, Made in Hawai' $i$ ' products made to soothe tourists' senses once they arrive back home with all their ,authentic Hawaiian' gifts for their relatives, coworkers and neighbors.

They open their suitcases, and what do they see? Bolts of cloth with designs found only in Hawai'i. They put their cheap dancing hula doll on their dashboard and watch her bounce with every vibration. They put on their seat cover, which bears an ,authentic Hawaiian design,' so their 'okole ${ }^{439}$ feels more comfortable. They break out the cheap plastic hula skirt for their neighbor's little girl, so she can attempt our native dance while grandma oohs and aahs with the video camera. [...]

All the while they don't understand why these seemingly happy, barefoot, brown-skinned people would care that everything that tourists think is a product of Hawai' $i$ is actually a travesty of everything Native Hawaiians hold dear to their culture. ,But we are all Americans,' they say. ,Shouldn't these people be grateful that I spent my vacation money on their products?'“ (DeBolt 2006).

Anders als bei den Inuit in Kanada, den Indianern im Südwesten der USA oder den australischen Aborigines existiert in Hawai‘i kein „Gütesiegel“, das ein Produkt als Erzeugnis indigener, in diesem Falle also hawaiischer Künstler oder Kunsthandwerker ausweisen würde. Die als „hawaiisch“ angesehene Bildund Formensprache, die der oben zitierte Leserbriefschreiber ansprach, ist für jeden, der sie nutzen möchte, frei verfügbar und es wird reichlich Gebrauch davon gemacht - das kleine Segment der „Native Hawaiian Art“ droht in der Beliebigkeit des Kunst- und Souvenirmarktes unterzugehen. Die hawaiische

439 ‘'̄kole-Gesäß (U.M.) 
Künstlerin und Professorin am Center for Hawaiian Studies der University of Hawai' $i$, Mānoa, Maile Andrade fand hierzu deutliche Worte:

„For too long, Native Hawaiian artist Maile Andrade says, she has watched non-Hawaiian artists garner recognition and wealth for art they try to pass off as being culturally Hawaiian. ,It's a theft,' she says. ,They're taking from our culture and using it to make money and publicize their name. A lot of times the culture they depict isn't even accurate - some of it is inappropriate'“ (Wong 2006).

Doch welche Maßnahmen zur Absicherung des hier formulierten Alleinanspruchs auf hawaiische Handwerkstechniken sowie Gestaltungs- und Formensprache (und damit auch der Wahrung wirtschaftlicher Interessen) möglich und notwendig sind, blieb bisher innerhalb der Kunstszene umstritten. Eine vom Office of Hawaiian Affairs finanzierte Studiengruppe legte 2007 die Ergebnisse einer Untersuchung zu einer „Cultural Trademark“ für hawaiische Kunstprodukte vor (Hale Kū'ai Study Group 2007). Angestrebt wird eine „Marke“, die dem Käufer signalisiert, dass er es mit einen „authentischen“ hawaiischen Produkt zu tun hat. Hierbei soll es sich nicht um ein Copyright handeln, wie es z.B. von australischen Aborigines oder auch verschiedenen Indianergruppen in den USA ausgeübt wird, deren jeweilige Motive und Designs nicht ohne ihre Einwilligung benutzt werden dürfen. Den Hawaiiern bleibt diese sehr weit reichende juristische Möglichkeit verschlossen, da sie von den USA nicht als „Nation“ im Sinne der Indianer-Gesetzgebung anerkannt sind - die Durchsetzung der Autonomievorstellungen Ka Lāhui Hawai' is oder die Implementierung der „Akaka Bill“ des Senators Daniel Akaka würden diesen Zustand allerdings grundlegend verändern. ${ }^{440}$

Aber auch die Frage der Authentizität eines Objektes oder einer kulturellen Handlung ist angesichts des multi-kulturellen und multi-ethnischen Hintergrundes der heutigen hawaiischen Bevölkerung nicht unproblematisch - Fragen westlicher Wissenschaftler nach der „Authentizität“ revitalisierter hawaiischer Kulturpraktiken stießen in den 1990er Jahren immer wieder auf den vehementen Protest hawaiischer Aktivisten. ${ }^{441}$ Auch die Autoren der Hale Kū'ai Study Group tun sich angesichts der Vielfalt dessen, was hawaiische Kunst ist, und des Fehlens eines verbindlichen rechtlichen Rahmens, schwer:

\footnotetext{
${ }^{440}$ Zur kritischen Diskussion solcher Konzepte eines „Native Copyrights“ siehe Brown 1998; 2003.

${ }^{441}$ Vergleiche H.-K. Trasks vehementen und bekannten Angriff (zusammengefasst in H.-K. Trask 1999:126-35) gegen die Untersuchungen Linnekins (1983, 1988) oder Keesings (1989).
} 
„,Authenticity' of works made by native artists primarily refers to the lineage of cultural knowledge transmitted to the artist, and the artist's experience, capability, and faithfulness in representing such cultural knowledge in the artwork, which for the most part can be objectively assessed. However, as artists tend to be creative and independentminded, most were leery about having a cultural arts board judge whether their work is sufficiently, authentic' to receive certification. Judgment by recognized kumu, kupuna, and master artists might be accepted as valid, but would still be problematic given the many different styles, sources, and ranges of art forms and varieties of content“ [Hervorhebung im Original] (Hale Kū'ai Study Group 2007:10).

So steht vorläufig noch dahin, ob über die „Echtheit“ hawaiischer Kunst bzw. hawaiischen Kunsthandwerks entschieden werden darf und welches Gremium solche Bewertungen letztlich vornehmen soll. Festzuhalten bleibt, dass eine hawaiische Kunstszene, die noch vor nicht allzu langer Zeit eher Eingeweihten bekannt war, heute trotz allen internen Gegensätzen und Konflikten eine selbstverständliche Präsenz im kulturellen Leben Hawai`is hat.

\section{Rechte an der Vergangenheit und die „Heimkehr des Kükā 'ilimoku“}

In den aktuellen Diskussionen um eine „Native Hawaiian Trademark“, in denen es ja auch um wirtschaftliche Vorteile geht, stehen sich unterschiedliche Interessen potentiell betroffener Künstler und Kunsthandwerker gegenüber. Während die oftmals älteren Produzenten einer am Überkommenen ausgerichteten hawaiischen Kunst strenge Maßstäbe und eine möglichst ungebrochene Weitergabe der Traditionen einfordern, verlangen gerade jüngere Künstler auch die Anerkennung von kreativer Innovation und künstlerischer Freiheit in der Themenwahl (Hale Kū'ai Study Group 2007:9). Aber auch angesichts des Umgangs mit den Künsten und Kunsterzeugnissen der hawaiischen Vergangenheit stehen sich Hawaiier immer wieder in widerstreitenden Lagern gegenüber. Exemplarisch zeigte sich dies am Fall der hawaiischen Organisation Hui Mālama I Nā Kūpuna o Hawai'i Nei (im Folgenden Hui Mālama genannt), die seit dem 26. Februar 2000 für Schlagzeilen in Hawai‘i sorgte und auf unerwartete Weise auch Ausstellung und Begleitprogramm der Cook/ForsterSammlung des Instituts für Ethnologie der Universität Göttingen, die im Frühjahr 2006 in der Honolulu Academy of Arts präsentiert wurde, nicht unberührt ließ.

Hui Mālama sieht seine Aufgabe in der Suche nach so genannten „Human Remains“, menschlichen Überresten, die in den Museen auf der ganzen Welt zu vorgeblichen Forschungszwecken gesammelt wurden und dort bis heute aufbewahrt werden. Nach Aussagen der Organisation sind seit ihrer Gründung im Jahre 1989 mehr als 5.000 Knochen hawaiischer Vorfahren (Iwi 
Küpuna) aus Museen der USA, Europas, Australiens und Kanadas nach Hawai' ‘ zurückgeführt und dort bestattet worden. In diesem Kontext findet in den USA das Wort „Repatriation“ Verwendung, dessen deutsche Entsprechung „Repatriierung“ ich im Folgenden benutzen werde. Hui Mālama sieht die Rückführung von Gebeinen als eine zentrale Aufgabe an, da ihres Erachtens insbesondere der Umgang mit Verstorbenen und ihren Überresten alleinige Aufgabe von Familienmitgliedern sei. Kein Museum, in dessen Besitz sich die Knochen heute befinden, könne für sich in Anspruch nehmen, die Zustimmung einer Familie zur Aufbewahrung der Gebeine erhalten zu haben. Unrechtmäßig einbehaltene Gebeine müssten den Angehörigen, womit hier durchaus auch die Gemeinschaft aller Hawaiier gemeint ist, zurückgegeben werden. ${ }^{42}$

In den USA regelt seit 1990 der „Native American Graves Protection and Repatriation Act“ (NAGPRA) den Umgang mit „Human Remains“ von Native Americans und Native Hawaiians sowie Objekten, die etwa als Grabbeigaben in direktem Zusammenhang mit Bestattungen stehen. NAGPRA sieht im Wesentlichen eine Auflistung aller in Museen und staatlichen Institutionen vorhandenen „Human Remains“ vor und, falls dieses verlangt wird, darüber hinaus eine Rückgabe an die Herkunftsgruppen. Das Gesetz bezieht die Hawaiier ausdrücklich ein und nennt Hui Mālama neben dem Office of Hawaiian Affairs als eine der im Sinne des Gesetzes befugten hawaiischen Organisationen. ${ }^{443}$

Der hier zu besprechende Fall nahm seinen Anfang am 26. Februar 2000. An diesem Tag übergab das Bishop Museum 83 hawaiische Objekte, unter ihnen einzigartige Holzarbeiten von großem kulturellen, historischen und auch finanziellen Wert, als Leihgabe an Hui Mālama. ${ }^{444}$ Die Organisation klas-

\footnotetext{
${ }^{442}$ Diesen Anspruch formulierte Hui Mālama u.a. in einem Schreiben an die Botschaft der Bundesrepublik Deutschland aus dem Jahr 1993, das als Reaktion auf die Ablehnung der Rückforderung hawaiischer „Human Remains“, die in einem deutschen Museum gelagert sein sollen, geschrieben wurde und das mir in Kopie vorliegt.

443 „(9) ,Native American' means of, or relating to, a tribe, people, or culture that is indigenous to the United States. (10) ,Native Hawaiian' means any individual who is a descendant of the aboriginal people who, prior to 1778, occupied and exercized sovereignty in the area that now constitutes the state of Hawaii“ (zitiert nach Fine-Dare [2002:199], die den vollständigen Gesetzestext wiedergibt). NAGPRA ist eines der zahlreichen Beispiele in der USamerikanischen Gesetzgebung, in denen die hawaiische Bevölkerung trotz der nach wie vor fehlenden Anerkennung als „Tribe“ oder „Nation“ als Einheit behandelt wird. Zu den Auswirkungen NAGPRAs im Rahmen der Repatriierungsbewegung der nordamerikanischen Indianer siehe die Autoren des von D. Mihesuah herausgegebenen „Repatriation Reader“ (2000); zur Geschichte des Gesetzes siehe besonders Trope u. Echo-Hawk (2000).

${ }^{444}$ Bei den Objekten handelt es sich Gegenstände, die David Forbes 1904 zusammen mit skelettierten und mumifizierten Überresten Verstorbener aus einer Höhle in Kawaihae auf der Insel Hawai‘i „geborgen“ hatte und die das Bishop Museum 1907 erworben hatte; siehe Whitney (2000) zu den Hintergründen der Übergabe an Hui Mālama.
} 
sifizierte sämtliche Gegenstände als Grabbeigaben, was sie den NAGPRABestimmungen unterworfen hätte. Ohne andere hawaiische Organisationen (wie z.B. das Office of Hawaiian Affairs, das ebenfalls einen Anspruch auf die Objekte angemeldet hatte) zu konsultieren, entschied Hui Mālama die gesamte Sammlung an einem geheim gehaltenen Ort auf der Insel zu bestatten. Zwar erhoben sich schon bald hawaiische Stimmen, die gegen die geheime Bestattung der Objekte protestierten, doch blieben die Auseinandersetzungen zwischen den beteiligten Organisationen und dem Bishop Museum eine im wesentlichen inner-hawaiische Angelegenheit. ${ }^{445}$ Die Anzahl hawaiischer Gruppen, die Anspruch auf die Objekte erhoben, erhöhte sich in den folgenden zwei Jahren, doch hielt Hui Mālama den Ort der erfolgten Repatriierung weiterhin geheim und sah die Angelegenheit als abgeschlossen an.

2003 verhandelte schließlich ein offizielles NAGPRAPrüfungskomitee den Sachverhalt. Die Prüfung beantragt hatten die Royal Hawaiian Academy of Traditional Arts, die ebenfalls ein Interesse an den hawaiischen Objekten angemeldet hatte, sowie das Bishop Museum, das inzwischen unter einer neuen Leitung stand. Das Komitee entsprach den Wünschen der Antragsteller und empfahl die Rückgabe der Objekte an das Museum sowie eine Wiederaufnahme des Repatriierungsverfahrens (NAGPRA Review Committee 2003:8), was Hui Mālama und ihr Sprecher Edward Ayau allerdings weiterhin ignorierten.

Ab November 2004 beteiligte sich die neu gegründete Organisation $\mathrm{Na}$ Lei Ali'i Kawānanakoa - hinter der die Nachfahrin König Kalākauas, Abigail Kekaulike Kawānanakoa stand - an den Auseinandersetzungen um die Objekte aus dem Bishop Museum. ${ }^{446} 2005$ reichten Na Lei Ali‘i Kawānanakoa sowie die Royal Hawaiian Academy of Traditional Arts mit ihrem Sprecher La'akea Suganuma eine gemeinsame Klage gegen Hui Mālama bei einem Bundesgericht in Hawai'i ein. Mit dieser Klage wurden die Auseinandersetzungen um die Repatriierung gewissermaßen öffentlich - zugleich verengte sich mit dieser gerichtlichen Öffentlichkeit aber auch die Sicht auf die juristischen Aspekte des bisher vor allem inner-hawaiischen Disputs. Da Edward Ayau als Sprecher Hui Mālamas sich weiterhin beharrlich weigerte, den Ort der erfolgten Repatriierung bekanntzumachen, ordnete das Gericht im Dezember 2005 seine Verhaf-

\footnotetext{
445 „Hawaiian leaders urging artifacts’ return to museum“, Robbie Dingeman (HA 11.4.2000:A1). Für das Bishop Museum schien die Angelegenheit ebenfalls beendet zu sein, da es davon ausgehen konnte, seine aus NAGPRA resultierenden Verpflichtungen erfüllt zu haben. 446 „Campbell heir ups stakes for artifacts: her organization earns status as a ,native Hawaiian’ group“, Sally Apgar (H S-B 21.11.2004:A1, A8); „Kawananakoa a force in the dispute over Hawaiian artifacts“, Sally Apgar (H $\quad$ S-B 10.1 .2005 archives.starbulletin.com/2005/01/10/news/story7.html [31.5.2009]).
} 
tung an. ${ }^{447}$ Bereits im Februar erschien z.B. eine ganzseitige Anzeige in der monatlich erscheinenden Ka Wai Ola o OHA, wo es heißt:

„December 27, 2005, Judge David Ezra held Hui Mālama's executive director (Edward Halealoha Ayau), Kumu (Pua Kanahele) and board members (Charles Maxwell, William Aila, and Antoinette Freitas) in contempt of court for refusing to reveal the exact location of the moepu from Kawaihae, an act that would force Hui Mālama to violate its cultural and religious beliefs and go against the request of the majority of claimants in the Kawaihae case to leave the iwi kupuna and moepū undisturbed. Judge Ezra's action is a result of a larger effort by L. Suganuma (Royal Hawaiian Academy of Traditional Arts), A. Kawānanakoa (Na Lei Ali‘i Kawānanakoa), and W. Brown (BPBM [Bishop Museum; U.M.]) to re-enter the burial caves, retrieve the moep $\bar{u}$, and re-start the NAGPRA process that has already been declared complete. [...] The imprisonment of Edward Halealoha Ayau also represents a new threat to all Hawaiians who wish to uphold their religious and cultural beliefs without persecution““ ${ }^{448}$

Unterzeichnet war diese Erklärung von 32 hawaiischen Organisationen, 57 'Ohana oder Familiengruppen und 63 Einzelpersonen. M. E. bewirkte die Inhaftierung Ayaus gerade unter politisch interessierten Hawaiiern eine starke Solidarisierung mit dem Verhafteten und den Zielen Hui Mālamas, die bis weit in das Jahr 2006 vorhielt.

Einen Monat nach der Verhaftung Ayaus hielt sich La'akea Suganuma, einer der in der zitierten Erklärung genannten Kläger, in Deutschland auf: Im Rahmen der Vorbereitung eines Ausstellungsprojekts der Honolulu Academy of Arts und des Instituts für Ethnologie der Universität Göttingen, nämlich der Präsentation der gesamten einzigartigen Göttinger Cook/Forster-Sammlung in Hawai‘i, führte Suganuma als Leiter einer kleinen hawaiischen Delegation eine Segnungszeremonie für das Federbildnis des Kriegsgottes Kūkā'ilimoku durch - auch dies ein Beispiel für die Kraft erneuerter „Traditionen“ in einem religiös-zeremoniellen Kontext. ${ }^{449} \mathrm{Zu}$ diesem Zeitpunkt liefen die Vorbereitungen für die Ausstellung „Life in the Pacific of the 1700s“ bereits seit langem. Neben Restaurierung, Fotografie oder wissenschaftlicher Bearbeitung gingen dem

447 „Group defies artifacts order: Hui Mālama leader jailed“, Gordon Pang (HA 28.12.2005:A1, A2).

448 „Declaration calling for the protection of the iwi kupuna (ancestral remains) and moepū (funerary objects) of the Honokoa, Kawaihae burial caves“, Ka Wai Ola o OHA 2006, 23 (2):18.

449 „Gesegnete Reise: Tränen für den Kriegsgott Kuka‘ilimoku“, Britta Bielefeld (Göttinger Tageblatt 24.1.2006:11). 
Projekt auch langwierige Verhandlungen voraus - hegte man doch durchaus begründete Befürchtungen, dass von hawaiischer Seite Restitutionsforderungen erhoben werden könnten.

Doch kam es nicht zu solchen Schritten von hawaiischer Seite, zu klar belegt ist die Provenienz der Dinge, in vielen Fällen Geschenke an Cook bzw. Mitglieder seiner Mannschaft. Die Presse in Hawai'i stellte heraus, dass die Ausstellung Objekte zeigte, die nun erstmals seit über 200 Jahren wieder in ihre Herkunftsregion zurückkehrten: „Coming Home“ lautete z.B. der Schriftzug auf dem Titelblatt des Honolulu Weekly. ${ }^{450}$ Und sie feierte die Ausstellung als „must-see exhibit“, als eine einmalige Gelegenheit, Dinge aus der hawaiischen und polynesischen Vergangenheit in solch großartigem Zustand bewundern zu können:

„What's most striking about the Honolulu Academy of Arts' new exhibit ,Life in the Pacific of the 1700s' is that many of its 200-year-old Pacific Island artifacts don't actually look like artifacts. The 350 items are so exquisitely preserved that you could just imagine someone opening one of the exhibit's glass cases, taking out a wood fishhook and catching dinner with it““. ${ }^{451}$

„Repatriierung“ war im Zusammenhang der Ausstellung kein Thema - die Cook/Forster-Sammlung beinhaltet zwar sakrale Objekte aus dem voreuropäischen Hawai'i, darunter aber keine „Human Remains“ oder Grabbeigaben. Im Besucherbuch der Honolulu Academy of Arts beschäftigen sich allerdings einige Einträge, wohl nicht immer ganz ernsthaft, mit diesem Problem. So findet sich auch am 29. März, zwischen begeisterten Anmerkungen zahlreicher Besucher, die Frage: „So when do we rebury them?“ - ein Hinweis auf dieses große hawaiische Thema im Frühjahr 2006. Es war allerdings bemerkenswert, dass viele Hawaiier, zumal die Vertreter der Wissenschaft (z.B. des Center for Hawaiian Studies der University of Hawai'i) oder hawaiischer Kulturorganisationen, der Ausstellung und dem die Eröffnung begleitenden wissenschaftlichen Symposium in den ersten Wochen fernblieben. Diese nicht-öffentlich gemachte Reaktion bezog sich denn auch nicht auf Ausstellung und Tagung, vielmehr war ein Grund hierfür m. E. in der engen personellen Verknüpfung des Veranstalters, der Honolulu Academy of Arts, mit den Gegnern Hui Mālamas, La'akea Suganuma und Abigail Kekaulike Kawānanakoa, zu sehen. Die Kontroversen um die Deutung der hawaiischen Vergangenheit, die kulturelle Praxis der Gegenwart und letztlich die politische Autonomie der Hawaiier sprachen auch hier auf vielleicht unerwartete Weise ihr gewichtiges Wort mit.

450 „Coming home“, Marcia Morse, Honolulu Weekly 16 (8):[Titel] 7-8.

451 „Living History“, Sterling Kini Wong, Ka Wai Ola o Oha 23 (3):20. 
Das letztgenannte Beispiel der Auseinandersetzungen um den korrekten Umgang mit den Objekten aus dem Bishop Museum zeigte einmal mehr, wie weit Konflikte auch über den Umgang mit Zeugnissen der voreuropäischen Kultur Hawai'is heute noch bzw. wieder reichen. Die Diskussionen um Repatriierung und die Entwicklungen auf dem Gebiet der bildenden Künste machen aber auch deutlich, wie sehr die politischen Forderungen und Vorstellungen des „Hawaiian Sovereignty Movements“ inzwischen im Bewusstsein vieler angekommen sind und sich als Baustein einer hawaiischen Identität gefestigt haben. Die Idee der Autonomie, der „Sovereignty“, prägt heute nachhaltig - auch ohne die lautstarken Stimmen der Protagonisten der 1990er Jahre - auf jeweils eigene Weise fast alle Felder gesellschaftlicher Auseinandersetzung, sie hat (um am Ende dieses „Kunst-Kapitels“ in einem passenden Bilde zu bleiben) sozusagen ihren Weg vom Plakat zum Gemälde genommen, von der eingängigen Parole zum Gedicht.

Die Suche nach der Hawaiischen Nation geht auch in den ersten beiden Jahrzehnten des 21. Jahrhunderts weiter: in politischen Verhandlungen und juristischen Argumentationen, in den noch immer fast alltäglichen Auseinandersetzungen um Landrechtsfragen, in der wissenschaftlichen Beschäftigung mit Geschichte und Gesellschaft und nicht zuletzt auf dem weiten Feld künstlerischer Äußerungen sind die Ideen einer „hawaiischen Autonomie“ gegenwärtig und werden auf ganz unterschiedliche Weisen interpretiert, verhandelt und/oder realisiert.

Ich habe in dieser Arbeit versucht, die Entwicklung der hawaiischen Bewegung mit ihren kulturellen bzw. politischen Ausprägungen und in ihrer komplexen Beziehungen zum Leben der heutigen hawaiischen Bevölkerung nachzuzeichnen. Dabei standen Deutungen, Strategien und Praktiken der Bewegung angesichts der kulturellen und politischen Hegemonie der USamerikanischen Mehrheitsgesellschaft im Vordergrund. Es ging mir hierbei also nicht um eine Analyse der Authentizität dieser Vorstellungen bzw. die Untersuchung, inwieweit und auf welche Weise Imaginationen oder „Erfindungen" von Gemeinschaft, Tradition und Kultur die Bewegung, ihre Aktionen und Ziele bestimmen - eine solche Analyse schien mir angesichts der aktuellen politischen Situation Hawai'is noch immer wenig angemessen zu sein, wie auch Maria Lepowsky in ihrer Analyse von Widerstandsbewegungen in Kalifornien und Neuguinea (und im Rückblick auf frühere Auseinandersetzungen in Hawai`i) zu Recht anmerkte:

„When anthropologists or historians label elements of indigenous cultural practices as creations or inventions, no amounts of appeals to history or cultural relativism - ,All traditions/customs are created/invented' - will soften the reality that such labels attack the authen- 
ticity, and thus challenge the validity and political potency, of the movements of historically or contemporaneously oppressed minorities“ (Lepowsky 2004:60).

Wie sie weiter ausführte, laufen wir als Wissenschaftler dabei immer Gefahr, vom Gegenüber als Gegner - gewissermaßen als „Teil des Problems“ - wahrgenommen zu werden und dabei aus den Augen zu verlieren, welch grundlegende gesellschaftliche und politische Umwälzungen die jeweiligen Bewegungen anstreben.

In Hawai'i standen und stehen den Bemühungen um politische Autonomie und kulturelle Eigenständigkeit mächtige und einflussreiche Kontrahenten entgegen - dennoch ist Autonomie, „Sovereignty“, inzwischen ein integraler Aspekt aktueller gelebter hawaiischer Kultur geworden. Dieser allgegenwärtige Hintergrund eines „souveränen Hawaiisch-Seins“ eröffnet heute neue Perspektiven und Grundlagen für die Betrachtung und Untersuchung hawaiischer Kultur im 21. Jahrhundert. 


\section{Daten zur Geschichte Hawai' 'is (1778-2000)}

1778 Am 18. Januar erreichen James Cook und seine Mannschaft als erste Europäer den hawaiischen Archipel.

1779 Am 14. Februar wird Cook von Hawaiiern in der Kealakekua Bucht getötet, nachdem er Kalani`ōpu'u, einen der mächtigsten Ali ‘ $i$ Hawai'‘s wegen eines Diebstahls als Geisel genommen hatte.

1780 Kurz vor seinem Tod übergibt Kalani'ōpu'u die weltliche Macht an seinen Neffen Kiwala' $\bar{o}$, die zeremonielle Verantwortung für den Kriegsgott Kūkā'ilimoku erteilt er seinem Neffen Kamehameha.

1795 Kamehameha beginnt mit der Eroberung des hawaiischen Archipels (1810 ergibt sich auch Kaumuali'i, das Oberhaupt Kauais; Kamehameha ist nun Herrscher über alle Inseln des Archipels.

1802 Seit Ankunft der ersten Europäer und der darauf folgenden Verbreitung neuer Krankheiten hat sich die Bevölkerungszahl Hawai‘ is halbiert.

1810 Sandelholzhandel eröffnet den Ali ‘ i die Möglichkeit, Geld zum Erwerb westlicher Waffen und Luxusgüter zu erwirtschaften; traditionelle Wirtschaft wird vernachlässigt.

1819 Nach dem Tod Kamehamehas bricht seine Witwe Ka'ahumanu mit seinem Sohn und Nachfolger Liholiho (Kamehameha II.) das Kapu; die offizielle Religion wird aufgelöst, zahlreiche Tempel zerstört.

1820 Die ersten protestantischen Missionare aus Neuengland erreichen Hawai‘i. 
1820 Walfang im Pazifik; in den Jahrzehnten bis 1860 werden Honolulu und Lahaina (Maui) zu wichtigen Handelshäfen.

1823 Reise von Kamehameha II. und seiner Gemahlin Kamamalu nach London, wo beide an Masern erkranken und sterben.

Die Bevölkerung Hawai' is wird auf ca. 240.000 geschätzt.

1824 Kauikeaouli, der jüngere Bruder des verstorbenen Königs, besteigt als Kamehameha III. den hawaiischen Thron.

1840 Die erste Verfassung Hawai 'is wandelt die absolute Monarchie in eine konstitutionelle Monarchie um.

1845 Erste Proteste der Bevölkerung gegen den Verkauf von Land an Ausländer; Forderungen werden laut, alle Ausländer aus der Regierung des Königreichs zu entfernen.

1848 Die Māhele, die Aufteilung des Landes zwischen König, Ali ‘ $i$ und Maka 'āinana, beginnt.

1850 In Hawai`i ansässigen Ausländern wird Landbesitz gestattet.

1852 Privatbesitz von Land wird im Gesetz festgeschrieben; eine neue Verfassung trägt den Änderungen Rechnung.

1853 Die Bevölkerung Hawai ‘is beträgt noch ca. 72.000.

1854 Erste Verhandlungen über eine Annexion Hawai‘ is durch die USA. Nach dem Tod Kamehamehas III. folgt ihm sein Neffe Alexander Liholiho (Kamehameha IV.) auf den Thron; er bricht alle Annexionsgespräche mit den USA ab.

1863 Lot (Kamehameha V.) wird König von Hawai‘i. Kamehameha V. löst 1864 die bestehende Verfassung auf und setzt eine neue in Kraft, die die Position des Königs stärkt.

1873 Nach dem Tod Kamehamehas V. wird William Lunalilo der erste vom Parlament gewählte Monarch Hawai`is.

1874 David Kalākaua wird nach dem Tod Lunalilos zum Monarchen gewählt. 
1875 Abschluss von Gegenseitigkeitsverträgen mit den USA. Zucker aus Hawai`i kann zollfrei in die USA eingeführt werden. USA erhalten die Hoheit über Pearl Harbour.

1887 Die so genannte „Bajonett Verfassung“ wird unter dem Druck von bewaffneten Haole verabschiedet. Diese Verfassung schränkte die Befugnisse des Königs und die Rechte der hawaiischen Bevölkerung ein.

1889 Eine hawaiische Widerstandsgruppe um Robert Wilcox versucht die Rechte des Königs wiederherzustellen. Hui Kalai‘āina, eine hawaiische Partei, will die Verfassung von 1864 wieder einführen.

1891 Als Nachfolgerin Kalākauas, der am 20. Januar 1891 in San Francisco starb, bestieg seine Schwester Lili‘uokalani den hawaiischen Thron.

1893 Am 14. Januar 1893 wird Lili‘uokalani vor der Verkündung einer neuen Verfassung, die hawaiischen Interessen Rechnung tragen sollte, von einem aus Haole-Geschäftsleuten zusammengesetzten „Committee of Public Safety“ mit der Unterstützung von US Truppen gestürzt.

1894 Die Provisorische Regierung Hawai'is verkündet die Gründung der „Republik Hawai'i““.

1898 Am 7. Juli 1898 annektieren die USA den gesamten hawaiischen Archipel.

1917 Tod Lili‘uokalanis.

1920 Einrichtung der Hawaiian Homes Commission.

1941 Bombardierung Pearl Harbors durch japanische Flugzeuge; Hawai`i wird unter Kriegsrecht gestellt.

1943 Die US-Regierung stellt weite Bereiche in Hawai‘i unter Militärverwaltung.

1946 Die Vereinten Nationen setzten Hawai'i zusammen mit den USTerritorien Alaska, Amerikanisch-Sāmoa, Guam, der Panama-KanalZone, Puerto Rico und den Virgin Islands auf ihre Liste der „Non-SelfGoverning-Territories“.

1959 Hawai`i wird zum Bundesstaat der USA. 
1970 Gründung von „The Hawaiians“, einer Gruppe, die gegen die missbräuchliche Verwendung von Hawaiian Home Lands protestiert.

1972 Gründung von „A.L.O.H.A. (Aboriginal Lands of Hawaiian Ancestry)“; die Gruppe tritt für die Zahlung von Wiedergutmachung an die Hawaiier durch die US-Regierung ein.

1976 Am 3. Januar 1976 fordert die Protect Kaho'olawe 'Ohana mit einer ersten illegalen Landung auf der Insel Kaho‘olawe die Beendigung der militärischen Nutzung als Bombenziel ein.

Am 1. Mai 1976 bricht der Nachbau eines traditionellen hawaiischen Doppelrumpfbootes, die Hōkūle‘a, zu ihrer ersten Reise in den Südpazifik auf.

1978 Das Office of Hawaiian Affairs (OHA) wird als hawaiische Institution mit Verfassungsrang eingerichtet.

1987 Gründung von Ka Lāhui Hawai' $i$, einer der wichtigen Gruppierungen des „Hawaiian Sovereignty Movements“.

1993 Gedenkfeiern an den Sturz der hawaiischen Monarchie im Jahre 1893. Im November entschuldigt sich US-Präsident Clinton in einer „Apology to Native Hawaiians“ für die Beteiligung der USA am illegalen Umsturz von 1893.

2000 Der Oberste Gerichtshof der USA erklärt das bestehende Wahlrecht zum Office of Hawaiian Affairs für verfassungswidrig; auch NichtHawaiier müssen an der Wahl teilnehmen dürfen.

US-Senator Akaka bringt erstmals seinen Entwurf eines „Native Hawaiian Government Reorganization Act“ in den US-Kongress ein.

(nach: MacPherson Furrer 1993:157 ff.; Churchill u. Venne [Hg.] 2004:xxxv ff.; Horcajo 2009) 


\section{Literatur}

Ackerman, Bruce. 2005. The art of stealth. London Review of Books 27(4):3-9 Akaka, Daniel. 2009. Daniel Kahikina Akaka, U.S. Senator for Hawaii. (Internet: akaka.senate.gov/public/index.cfm?FuseAction=Akaka.Home [25.02.2009])

Akana, Rowena. 1999. What's your real agenda, Mister Rice? Ka Wai Ola o OHA Mei (May):6

Alaska Native Knowledge Network. 2006. Alaska Native Claims Settlement Act. (Internet: www.ankn.uaf.edu/NPE/ancsa.html [10.03.2008])

Alsheimer, Rainer, Alois Moosmüller u. Klaus Roth (Hg.). 2000. Lokale Kulturen in einer globalisierenden Welt. (Münchener Beiträge zur interkulturellen Kommunikation 9). Münster: Waxmann

Aluli Meyer, Manulani. 2001. „Hawaiian art: a doorway to knowing“. In: Cazimero, Momi, David J. de la Torre u. Manulani Aluli Meyer (Hg.). $N \bar{a}$ Maka Hou: contemporary native Hawaiian art. S. 13-4. Honolulu: Honolulu Academy of Arts

Andrews, Lorrin. 1865. A dictionary of the Hawaiian language to which is appended an English-Hawaiian vocabulary and a chronological table of remarkable events. Honolulu: Henry M. Whitney

Andrade, Ernest. 1996. Unconquerable rebel: Robert W. Wilcox and Hawaiian politics, 1880-1903. Niwot: University Press of Colorado

Aoudé, Ibrahim (Hg.). 1999. The Ethnic Studies story: politics and social movements in Hawaii. Social Process in Hawai 'i 39

Arbeitsgemeinschaft vielfaches echo. 1998a. Vielfaches Echo: zeitgenössische Kunst zwischen den Kulturen. Nürnberg: Verlag für moderne Kunst

Arbeitsgemeinschaft vielfaches echo. 1998b. vielfaches echo - ausstellungen stuttgart 24. april bis 14. juni 1998 (Faltblatt zur Ausstellung). Stuttgart: AG vielfaches echo 
Bailey, Beth and David Farber. 1992. The first strange place: race and sex in World War II Hawaii. Baltimore: Johns Hopkins University Press

Baker, David J. 1997. Ea and knowing in Hawai' i. Critical Inquiry 23:640-59

Balaz, Joseph P. (Hg.). 1989. Ho'omanoa: an anthology of contemporary

Hawaiian literature. Honolulu: University of Hawai'i Press

Barrère, Dorothy B. 1980. „The Hula in retrospect“. In: Dorothy B. Barrère, Mary Kawena Pukui u. Marion Kelly. Hula: historical perspectives . Pacific Anthropological Records No. 30. Honolulu: Bernice P. Bishop Museum

Beaglehole, J.C. (Hg.). 1967. The journals of Captain James Cook on his voyages of discovery: the voyage of the Resolution and Discovery 1776-1780, part one. Cambridge: Cambridge University Press

Beckwith, Martha. 1970. Hawaiian mythology. Honolulu: University of Hawai'i Press (1. Aufl. 1940)

Beechert, Edward D. 1985. Working in Hawaii: a labor history. Honolulu: University of Hawai'i Press

Bell, Roger J. 1984. Last among equals: Hawaiian statehood and American politics. Honolulu: University of Hawai'i Press

Bellwood, Peter. 1978. Man's conquest of the Pacific. Auckland: Collins

Benjamin, Stuart Minor. 1996. Equal protection and the special relationship: the case of native Hawaiians. The Yale Law Journal 106 (3):537-612

Bishop Museum. 1995a. Hawaiiloa and Hōkūlea prepare for 1995 voyage to South Pacific. Ka 'Elele 22 (1):2

Bishop Museum. 1995b. Members embark on voyaging adventure at launching of Hawai 'iloa exhibit. Ka 'Elele 22 (2):2

Blaisdell, Kekuni. 1989. „Hawaiian“ vs. „Kanaka Maoli“ as metaphors. Hawai 'i Review 13 (3):77-9

Blaisdell, Kekuni. 1997. Kanaka Maoli self-determination and reinscription of Ka Pae'Aina (Hawai 'i) on the U.N. list of non-self-governing territories. (Internet: www.inmotionmagazine.com/ngo2.html [17.9.1998])

Blaisdell, Kekuni. 1998. The indigenous rights movement in the Pacific. (Internet: www.inmotionmagazine.com/pacific.html [7.9.1998])

Blaisdell, Kekuni u. Marion Kelly. 1993. Ka Ho'okolokolonui Kanaka Maoli: program, schedule, and information. (Programmbroschüre). Honolulu: Ka Ho'okolokolonui Kānaka Maoli

Blaisdell, Kekuni et al. 1994. Ka Ho 'okolokolonui Kānaka Maoli 1993 Mana 'o. (Informationsbroschüre). Honolulu: Ka Ho'okolokolonui Kānaka Maoli

Blaisdell, Kekuni u. Noreen Mokuau 1994. „Kānaka Maoli, Indigenous Hawaiians“. In: Hasager u. Friedman (Hg.). 1994:49-67

Böhnke, Regina. 2002. Tenerife - zwischen Autonomie und Tourismus. (Interethnische Beziehungen und Kulturwandel 33). Münster: LIT 
Boyle, Francis Anthony. 1993. Addressing the Hawaiian Sovereignty Advisory Commission regarding U.S. Public Law 103-150 -- the Hawaii Apology Bill -- and its implications for the restoration of the Independent Nation State of Hawai' $i$ under international law. (Internet: hawaii-nation.org/boyleall.html [21.9.1998])

BPB. 2011. Stichwort Symbolische Politik (Kalus Schubert u. Martina Klein: Das Politiklexikon. Bonn: Dietz 2011) (Internet: www.bpb.de/nachschlagen/ lexika/ politiklexikon/18323/symbolische-politik [12.10.2012])

Brown, Michael F. 1998. Can culture be copyrighted? Current Anthropology 39 (2):193-206

Brown, Michael F. 2003. Who owns native culture? Cambridge: Harvard University Press

Browne, Sean K. L. 1983. Sculptured interpretations of Polynesian ethnographic objects (M.F.A. Thesis, University of Hawai'i, Mānoa)

Buck, Elizabeth Bentzel. 1993. Paradise remade: the politics of culture and history in Hawai ' $i$. Philadelphia: Temple University Press

Bushnell, O.A. 1993. The gifts of civilization: germs and genocide in Hawai ' $i$. Honolulu: University of Hawai'i Press

Cashman, Kimo. 1995. „Artist’s statement“. In: ReViewing paradise: contemporary photography from Hawai ‘ (27.04.-28.06. 1995; Fotokopien), S. 4. Honolulu: The Honolulu Advertiser Gallery

Castanha, Anthony. 1996. Roles of Non-Hawaiians in the Hawaiian sovereignty movement. M.A. Thesis, Political Science, University of Hawai'i at Mānoa (Internet: www.hookele.com/non-hawaiians/index.html [28.8.1998]) Cayetano, Ben. 1998. State of the State 1998. (Internet: gov.state.hi.us/ sos1998.html [14.10.1998])

Chapin, Helen Geracimos. 1984. Newspapers of Hawai'i 1834 to 1903: from „He Liona“ to the Pacific cable. The Hawaiian Journal of History 18:47-81 Chapin, Helen Geracimos. 1996. Shaping history: the role of newspapers in Hawai' $i$. Honolulu: University of Hawai'i Press

Chinen, Jon J. 1958. The great Mahele: Hawaii's land division of 1848. Honolulu: University of Hawai'i Press

Churchill, Ward u. Sharon H. Venne (Hg.). 2004. Islands in captivity: the record of the international tribunal on the rights of indigenous Hawaiians. Cambridge: South End Press

Cilano, Cara. 2005. Of blood and of the heart: an interview with Georgia Ka'apuni McMillen. The Contemporary Pacific 17 (2):387-402

Clark, Herman Pi'ikea. 1996. Ho'okumu hou: the reassertion of native Hawaiian culture in visual art (M.F.A. Thesis, University of Hawai'i, Mānoa) Clement, Russel. 1980. From Cook to the 1840 constitution: the name change from Sandwich to Hawaiian Islands. The Hawaiian Journal of History 14:50-7 
Coffman, Tom. 2003. The island edge of America: a political history of Hawai ‘ $i$. Honolulu: University of Hawai'i Press

Coffman, Tom. o.J. Nation within: the story of America's annexation of the nation of Hawai 'i. Kāne' ohe: EPICenter

Cooper, George u. Gavan Daws. 1990. Land and power in Hawaii: the democratic years. Honolulu: University of Hawai'i Press

Cornell, Stephen E. 1988. The return of the native: American Indian political resurgence. Oxford: Oxford University Press

Council of Contemporary Hawaiian Artists. 1993. Ho'o Pānai: an exhibition of contemporary Hawaiian art presented on the occasion of the centennial of the Overthrow of the Hawaiian nation (Begleitheft zur Ausstellung 08.01.28.02.1993). Honolulu: Bernice P. Bishop Museum

Council of Regency. 2007. Council of Regency of the Hawaiian Kingdom (Website). (Internet: www.hawaiiankingdom.org [28.5.2007])

Cox, Lindsay. 1993. Kotahitanga: the search for Māori political unity. Auckland: Oxford University Press

Cox, J. Halley u. William H. Davenport. 1974. Hawaiian sculpture. Honolulu: University of Hawai'i Press

Cudic, Sanda. 2001. Multikulturalität und Multikulturalismus in BosnienHerzegowina. (Europäische Hochschulschriften, XXXI 438). Frankfurt/Main: Peter Lang

Cummings, Tracie Ku'uipo. 2003. Political reviews. Polynesia: Hawaiian issues. The Contemporary Pacific 15 (1):179-83

Cummings, Tracie Ku'uipo. 2004. Political reviews. Polynesia: Hawaiian issues. The Contemporary Pacific 16 (1):153-8

Cummings Losch, Tracie Ku'uipo. 2006. Political reviews. Polynesia: Hawaiian issues. The Contemporary Pacific 18 (1):143-8

Cummings Losch, Tracie Ku'uipo. 2007. Political reviews. Polynesia: Hawaiian issues. The Contemporary Pacific 19 (1):222-33

D’Alleva, Anne. 1998. Arts of the Pacific Islands. New York: Harry N.

Abrams

Da Skupes. 1994. Focus: Ka Pakaukau and the Tribunal Komike. Da Skupes 2 (8):1

Davenport, William. 1969. The ,Hawaiian cultural revolution': some political and economical considerations. American Anthropologist 71:1-20

Daws, Gavan. 1968. Shoal of time: a history of the Hawaiian Islands. Honolulu: University of Hawai'i Press

Day, Richard R. 1985. „The ultimate inequality: linguistic genocide“. In: Nessa Wolfson u. Joan Manes (Hg.). Language of inequality (163-81). Contributions to the sociology of language 36. Berlin: Mouton

DeBolt, William Kalamakuaikalani. 2006. Made in Hawai' i. Ka Wai Ola o OHA 23 (3):3 
Department of Hawaiian Home Lands. 1994. 1994 Annual report. Honolulu: Department of Hawaiian Home Lands

Department of Hawaiian Home Lands. 1995. „Making it right: a fair and just settlement for all“ (Faltblatt des Department of Hawaiian Home Lands, Honolulu)

Dörner, Andreas. 1996. Politischer Mythos und symbolische Politik. Reinbek: Rowohlt Taschenbuch Verlag

Dracklé, Dorle u. Waltraud Kokot. 1996. „Neue Feldforschungen in Europa: Grenzen, Konflikte, Identitäten. In: Kokot u. Dracklé (Hg.). 1996:3-20

Dudley, Michael Kioni u. Keoni Kealoha Agard. 1993. A call for Hawaiian sovereignty. Honolulu: Nā Kāne O Ka Malo Press (1. Aufl. 1990)

Duncan, Janice K. 1973. Kanaka world travellers and fur company employees, 1785-1860. The Hawaiian Journal of History 7:93-111

Durie, M.H. 1998. Te Mana te Kāwanatanga: the politics of Mãori selfdetermination. Auckland: Oxford University Press

Duus, Brian. 2003. „Federal recognition of the Native Hawaiian government with a transfer to it of territory derived from the Ceded Lands“. Asian Pacific Law and Policy Journal 4 (2):467-515

Ellis, William. 1827. Narrative of a tour through Hawai 'i or Owhyhee. London: H. Fisher, Son, and P. Jackson (3. Aufl.)

Ethnic Studies 221. 1974. Hawaiians: organizing our people. A pamphlet produced by students of ES 221. Honolulu: Ethnic Studies Program, University of Hawai'i at Mānoa

Europa. 2006. EU-Gerichte und internationale Gerichte (Internet: europa.eu/scadplus/leg/de/lvb/l16007.htm [19.02.2009])

Esser, Hartmut. 1996. „Ethnische Konflikte als Auseinandersetzung um den Wert von kulturellem Kapital“. In: Heitmeyer u. Dollase (Hg.). 1996:64-99

Ferrar, Derek. 1994. 101 and counting. Honolulu Weekly 4(2):4-6

Ferrar, Derek u. Julia Steele. 1993. 100 years of subjugation. Honolulu Weekly 3(2):4-7

Fine-Dare, Kathleen S. 2002. Grave injustice: the American Indian repatriation movement and NAGPRA. Lincoln: University of Nebraska Press

Finney, Ben. 1979. Hokule' $a$ : the way to Tahiti. New York: Dodd, Mead Finney, Ben. 1991. Myth, experiment, and the reinvention of Polynesian voyaging. American Anthropologist 93 (2):383-404

Finney, Ben. 1994. Voyage of rediscovery: a cultural odyssey through Polynesia. Berkeley: University of California Press

Finney, Ben. 2006. „Renaissance“. In: K.R. Howe (Hg.), Vaka Moana - voyages of the ancestors: the discovery and settlement of the Pacific. S. 290-325. Honolulu: University of Hawai'i Press

Fleras, Augie u. Paul Spoonley. 1999. Recalling Aotearoa: indigenous politics and ethnic relations in New Zealand. Auckland: Oxford University Press 
Friedman, Jonathan. 1992. The past in the future: history and the politics of identity. American Anthropologist 94 (2):837-59

Friedman, Jonathan. 1993. Will the real Hawaiian please stand: anthropologists and natives in the global struggle for identity. Bijdragen tot de taal-, landen volkenkunde 149 (4):737-67

Friedman, Lesley Karen. 1992. Native Hawaiians, self-determination, and the inadequacy of the State land trusts. University of Hawai' $i$ Law Review 14 (2):519-80

Fuchs, Lawrence H. 1961. Hawaii pono: a social history. New York: Harcourt, Brace \& World

Fujikane, Candace. 1996. Archipelagos of resistance: narrating nation in Asian American, Native Hawaiian, and Hawaiis local literatures. (Ph.D. Thesis). Berkeley: University of California

Fujikane, Candace. 2000. Asian Settler Colonialism in Hawaii. Amerasia Journal 26 (2):xv-xxii

Fullard-Leo, Betty. 1993. The movement for Hawaiian independence. Aloha 16 (1):18-25

Gamboa, Leilani. 2003. Deathlist. 'Ōiwi: a native Hawaiian journal 3:151

Gill, Kawehi Kanui. 1992. Standing fast at 'Iolani Palace. Honolulu Weekly 2 (28):5

Goldman, Irving. 1970. Ancient Polynesian society. Chicago: University of Chicago Press

Groenemeyer, Axel. 2003. „Kulturelle Differenz, ethnische Identität und die Ethnisierung von Alltagskonflikten“. In: Groenemeyer u. Mansel (Hg.)

2003:11-46

Groenemeyer, Axel u. Jürgen Mansel (Hg.). 2003. Die Ethnisierung von Alltagskonflikten. Opladen: Leske + Budrich

Hā Hawai'i. 1998a. Hawaiian sovereignty: a process for the people. (Internet: http://ha-hawaii.com/ha.html [30.4.1998])

Hā Hawai' 'i. 1998b. Kahea I Na Hawai 'i: a call to all Hawaiians. (Internet: http://www.ha-hawaii.com/kahea.html [30.6.1998])

Hale Kū'ai Study Group. 2007. Cultural trademark study: findings and recommendations. Kailua: Hale Kū'ai (Internet: hawaiiantrademarkstudy.org/ Media/TrademarkStudyReport.pdf [25.5.2009])

Hale Kuamo‘o. 1996. Māmaka Kaiao: he puke hua 'ōlelo Hawai 'i hou. Hilo: Hale Kuamo'o / 'Aha Pūnana Leo

Hall, Dana Naone (Hg.). 1985. Mālama: Hawaiian land and water. Honolulu: Bamboo Ridge Press

Hall, Lisa Kahaleole. 2005. 'Hawaiian at heart' and other fictions. The Contemporary Pacific 17 (2):404-13

Halualani, Rona Tamiko. 2002. In the name of Hawaiians: native identities and cultural politics. University of Minnesota Press: Minneapolis 
Harkin, Michael J. (Hg.). 2004. Reassessing revitalization movements: perspectives from North America and the Pacific Islands. Lincoln: University of Nebraska Press

Hartwell, Jay. 1996. Nā Mamo: Hawaiian people today. Honolulu: ‘Ai Pōhaku Press

Hasager, Ulla u. Jonathan Friedman (Hg.). 1994. Hawai ' $i$ : return to nationhood. IWGIA Document 74. Kopenhagen: International Work Group for Indigenous Affairs

Hasager, Ulla u. Kawaipuna Prejean. 1992. „Administering Hawaiian Homelands: a trust broken - civil rights denied“. IWGIA-Newsletter 2:52-5 Hauser-Schäublin, Brigitta. 1995. Zur Verkörperung sozialer Ungleichheit als naturgegebene Verschiedenheit am Schnittpunkt zwischen Geschlecht, Klasse und Rasse. Zeitschrift für Ethnologie 120 (1):31-49

Hauser-Schäublin, Brigitta u. Gundolf Krüger (Hg.). 1998. James Cook. Gaben und Schätze aus der Südsee: die Göttinger Sammlung Cook/Forster (dt./engl.). München: Prestel

Hawaii Advisory Committee. 1991. A broken trust - the Hawaiian Homelands program: seventy years of failure of the federal and state governments to protect the civil rights of native Hawaiians. Washington: Hawaii Advisory Committee to the United States Commission on Civil Rights

Hawaii Constitution. 1997. Constitutions of the United States: national and state. Hawaii (revised and updated). Legislative Drafting Research Fund of Columbia University. Dobbs Ferry: Oceana Publications, Inc.

Hawaii Department of Health. 1997. Hawaii Health Surveillance Program 1989-1992:Survey report and data. (Internet:

www.hawaii.gov/health/hs_hsp.htm [3.5.1997])

Hawaii Revised Statutes. o.J. Hawaii Revised Statutes. Honolulu: Revisor of Statutes. (Internet: www.capitol.hawaii.gov/site1/docs/docs.asp [21.1.2009]) Hawaii Session Laws. 1979. Session laws of Hawaii: passed by the Tenth State Legislature regular session 1979. Honolulu: Revisor of Statutes Hawaii Session Laws. 1991. Session Laws of Hawaii. Sixteenth State Legislature. Regular and special sessions of 1991. Honolulu: Revisor of Statutes Hawaii Session Laws. 1992. Sixteenth State Legislature: regular session of 1992. Honolulu: Revisor of Statutes

Hawaii Session Laws. 1993. Session Laws of Hawaii. Seventeenth State Legislature, Regular Session of 1993. Honolulu: Revisor of Statutes

Hawaii Session Laws. 1994. Session Laws of Hawaii: Seventeenth State Legislature. Special session of 1993, regular session of 1994. Honolulu: Revisor of Statutes

Hawaii State Library. 1929-1994. Index to the Honolulu Advertiser and Honolulu Star-Bulletin. Honolulu: Department of Education, State of Hawaii 
Hawaii Visitors Bureau. 1992. Hawaii: the Aloha State. (Faltblatt). Honolulu: State of Hawaii/Hawaii Visitors Bureau

Hawaiian Affairs. 1978. Hawaiian Affairs Committee Report No. 59, 29.8.1978

Hazlehurst, Kayleen. 1993. Political expression and ethnicity: statecraft and mobilization in the Maori world. Westport: Praeger

Heckathorn, John. 1988. The Native Hawaiian nation: the hottest political issue of the 1990s. Honolulu 23 (6):56-9, 85-90

Heckmann, Friedrich. 1992. Ethnische Minderheiten, Volk und Nation. Stuttgart: Enke

Heermann, Ingrid u.a. 1998. Ho 'okū 'ē: Widerstand - moderne Kunst aus

Hawai' $i$. Stuttgart: Linden-Museum

Heitmeyer, Wilhelm u. Rainer Dollase (Hg.). 1996. Die bedrängte Toleranz: ethnisch-kulturelle Konflikte, religiöse Differenzen und die Gefahren politisierter Gewalt. Frankfurt/Main: Suhrkamp

Holt, John Dominis. 1995. On being Hawaiian. Honolulu: Ku Pa‘a Publishing Inc. (1. Aufl. 1964)

Honolulu. 1993. Sovereignty showdown: Ka Lāhui vs. the State. Honolulu 28 (5) :34, 65-70

Ho'okū'ē. 1997. Ho'okū'ē: resistance (Faltblatt zur Ausstellung 18.04.06.06.1997). Honolulu: East-West Center

Ho'omanawui, Ku'ualoha. 2005. He Lei Ho'oheno no nā Kau a Kau: language, performance, and form in Hawaiian poetry. The Contemporary Pacific 17 (1):29-81

Horcajo, Kainoa. 2009. Timeline (Internet:

www.oha.org/pdf/HwnHistoryTimeline.pdf [2.6.2009])

Hormel, Ulrike u. Albert Scherr. 2003. „Was heißt ,Ethnien’ und ,ethnische Konflikte’ in der modernen Gesellschaft?“. In: Groenemeyer u. Mansel (Hg.) 2003:47-66

House Concurrent Resolution. 1992. Recognizing breeches of trust responsibility between the state of Hawaii and the Hawaiian people and extending a formal apology. House Concurrent Resolution No. 300. Honolulu: House of Representatives, 16th Legislature 1992

House Majority Staff Office. 1993. Native Hawaiian Issues. Honolulu: Hawaii State Legislature

Howard, Alan. 1980. „Hawaiians“. In: Steven Thernstrom (Hg.). Harvard encyclopedia of American ethnic groups (449-52). Cambridge: Harvard University Press.

HSAC. 1994. Hawaiian Sovereignty Advisory Commission. Final Report, February 18, 1994. Honolulu: HSAC

HSEC. 1995. Hawaiian Sovereignty Electons Council: report to the legislature. Honolulu: HSEC 
HSEC. 1996. Hawaiian Sovereignty Elections Council: final report, December 1996. Honolulu: HSEC

Hui Na'auao. 1992. Hui Na'auao: sovereignty and self-determination - a community education project (Faltblatt). Honolulu: Hui Na'auao

Hui Na'auao. 1993. Hui Na'auao (Informationsmaterial). Honolulu: Hui Na'auao

Hui Na'auao. 1994. Ho'okahua I workshop: models and elements of sovereignty workbook. Honolulu: Hui $\mathrm{Na}$ 'auao

Hui Na‘auao. o.J. a. Project description. Honolulu: Hui Na'auao

Hui Na'auao. o.J. b. Ho ála workshop materials. Honolulu: Hui Na'auao I.A.H.A. 1987. The Hawaiian dialogue. Wai'anae: Institute for the Advancement of Hawaiian Affairs [eine gekürzte Version des Textes erschien auch in: MacPherson Furrer 1993:125-40]

I.A.H.A. 1992. Collection of papers on Hawaiian sovereignty and selfdetermination. Wai'anae: Institute for the Advancement of Hawaiian Affairs ' $\overline{\mathbf{I}} \cdot \overline{\mathbf{I}}$, John Papa. 1993. Fragments of Hawaiian History. Honolulu: Bishop Museum Press

'Īlio'ulaokalani. 2003. Palapala kūlike o ka 'aha pono Paoakalani Declaration 2003. (Internet: ilio.org/Ilioonline/ahapono04/Paokalani\%20Declaration.pdf [12.10.2004])

'Īlio'ulaokalani. 2004. About us. (Internet: ilio.org/Ilioonline/page_links/about_new.html [12.10.2004])

Jarves, James Jackson. 1843. History of the Hawaiian or Sandwich Islands. London: Edward Moxon

Ka Ho'okolokolonui Kānaka Maoli. 1993. Interim Report: Kanaka Maoli Nation, Plaintiff v. United States of America, Defendant. [Fotokopie] Ka Ho'okolokolonui Kānaka Maoli, 20.8.1993

Ka Lāhui. 1991. Ka Lāhui Hawai ‘ $i$ - The Sovereign Nation of Hawai ' $i$ : a compilation of legal materials for workshops on the Hawaiian nation. Honolulu: Ka Lāhui Hawai'i

Ka Lāhui. 1993. Ka Lāhui Hawai ' $i$ - The Sovereign Nation of Hawai 'i: a compilation of materials for educational workshops on Ka Lähui Hawai $i$. Hilo: Ka Lāhui Hawai‘i

Ka Lāhui. 1995. Ho 'okupu a Ka Lāhui Hawai ' $i$ : The Master Plan. Hilo: Ka Lāhui Hawai'i

Ka Pākaukau. 1993a. Ka Pākaukau's Position Statements. in: Hawaiian Sovereignty Economic Symposium: questionaire responses. Honolulu: Kenya Information Services

Ka Pākaukau. 1993b. E Ho 'olaule ‘a i Ka Lā Ho iho ‘i Ea o Ka Lāhui Kanaka Maoli (Flugblatt)

Ka‘ai‘ai, Charles. 1993. „Senator...“. In: MacPherson Furrer 1993:70-2 
Kaeppler, Adrienne. 1978. Artificial curiosities. Bernice P. Bishop Museum Special Publication 65. Honolulu: Bishop Museum Press Kaeppler, Adrienne, Christian Kaufmann u. Douglas Newton. 1994. Ozeanien: Kunst und Kultur. Freiburg: Herder

Kahanu, Noelle M. u. Jon M. van Dyke. 1995. „Native Hawaiian entitlement to sovereignty: an overview“. University of Hawai 'i Law Review 17:427-62

Kahananui, Dorothy M. (Hg.). 1984. Ka Mooolelo Hawaii. Honolulu: Committee for the Preservation and Study of Hawaiian Language, Art and Culture Kalāhele, 'İmaikalani. 2002. Kalahele: poetry and art. Honolulu: Kalamakū Press

Kamahele, Momiala. 2000. 'T̄lio'ulaokalani: defending native Hawaiian culture. Amerasia Journal 26 (2):38-65

Kamakau, Samuel Mānaiakalani. 1991a. Ka Po'e Kahiko: the people of old. Bernice Pauahi Bishop Museum Special Publication 51. Honolulu: Bishop Museum Press

Kamakau, Samuel Mānaiakalani. 1991b. Tales and Traditions of the People of Old: Nā Mo olelo a ka Po'e Kahiko. Honolulu: Bishop Museum Press Kamakau, Samuel Mānaiakalani. 1992a. The works of the people of old: na Hana a ka Po'e Kahiko. Bernice P. Bishop Museum Special Publication 61. Honolulu: Bishop Museum Press

Kamakau, Samuel Mānaiakalani. 1992b. Ruling chiefs of Hawaii. Honolulu: Kamehameha Schools

Kamau'u, Mahealani. 1993. „The historical precedence for sovereignty“. In: Macpherson Furrer (Hg). 1993:15-20

Kame' ${ }^{6}$ leihiwa, Lilikalā. 1992. Native Land and Foreign Desires: Pehea lā e Pono ai? Honolulu: Bishop Museum Press

Kame'eleihiwa, Lilikalā. 1993. The Hawaiian Sovereignty Movement: an update from Honolulu (January - August 1993). Journal of Pacific History 28 (3):63-72

Kanahele, George S. 1982. The new Hawaiians. Social Process in Hawaii 29:21-31

Kanahele, George S. 1986. Kü kanaka: stand tall. Honolulu: University of Hawai'i Press

Kānaka Maoli Tribunal Kōmike. 2004. „Ka Palapala O Na 'Olelo Ho‘ahewa (Statement of Charges). In: Churchill u. Venne (Hg.) 2004:341-55

Kane, Herb Kawainui. 1976. A canoe helps Hawaii recapture her past. $\mathrm{Na}$ tional Geographic 149 (4):468-89

Kaschuba, Wolfgang. 2001. „Geschichtspolitik und Identitätspolitik: nationale und ethnische Diskurse im Vergleich“. In: Beate Binder, Wolfgang Kaschuba u. Peter Niedermüller (Hg.). Inszenierung des Nationalen: Geschichte, Kultur und die Politik der Identitäten am Ende des 20. Jahrhunderts. (alltag \& kultur 7). Köln: Böhlau 
Katkin, Wendy F., Ned Landsman u. Andrea Tyree (Hg.). 1998. Beyond pluralism: the conception of groups and group identities in America. Urbana u. Chicago: University of Illinois Press

Katz, Stanley N. 1998. „The legal framework of American pluralism: liberal constitutionalism and the protection of groups“. In: Katkin, Landsman u. Tyree (Hg.) 1998:11-27

Kau, Catherine. 1991. „Native Hawaiian charitable trusts“. In: MacKenzie (Hg.) 1991:281-93

Kau, Catherine u. Melody K. MacKenzie. 1991. „Religious Freedom“. In: MacKenzie (Hg.) 1991:229-44

Kauanui, J. Kēhaulani. 2005. Precarious positions: Native Hawaiians and US federal recognition. The Contemporary Pacific 17(1):1-27

Kauanui, J. Kēhaulani. 2007. Diasporic deracination and „off-island“ Hawaiians. The Contemporary Pacific 19 (1):137-60

Keesing, Roger M. 1989. Creating the past: custom and identity in the contemporary Pacific. The Contemporary Pacific 1:19-42

Kent, Noel J. 1983. Hawaii: islands under the influence. New York: Monthly Review Press

Kenya Information Services. 1993. Hawaiian sovereignty economic symposium: questionnaire responses. Honolulu: Kenya Information Services

Keppeler, H. K. Bruss. 1992. „Native Hawaiian claims“. In: Roth (Hg.) 1992:195-203

Kernahan, Mel. 1993. A question of when. Pacific Islands Monthly March 1993:20

King, James. 1785. A voyage to the Pacific Ocean undertaken by the command of his Majesty for making discoveries in the northern hemisphere Vol. III (2nd ed.). London: H. Hughs

King, Samuel P. u. Randall W. Roth. 2006. Broken trust: greed, mismanagement \& political manipulation at America's largest charitable trust. Honolulu: University of Hawai'i Press

Kimura, Larry. 1976. „Wa'a Hōkūle'a“ (Ka Leo Hawaii: a fundraising project for Hui Aloha 'Āina Tuahine, University of Hawai 'i, Mānoa. LPCovertext).

KIRC. 2009. FY 1994 Department of Defense Appropriations Act Public Law 103-139, 107 Stat. 1418 (Internet: kahoolawe.hawaii.gov/rules/leg.htm\#Title [21.1.2009])

Kirch, Patrick V. 1985. Feathered gods and fishhooks. Honolulu: University of Hawai'i Press

Kokot, Waltraud u. Dorle Dracklé (Hg.). 1996. Ethnologie Europas: Grenzen, Konflikte, Identitäten. Berlin: Reimer

Kreisel, Werner 1984. Die ethnischen Gruppen der Hawaii-Inseln. (Erdkundliches Wissen 68). Wiesbaden: Franz Steiner Verlag 
Kuppe, René. 1990. Indigene Rechte und die Diskussion um ,Rechte für Gruppen'. Law \& Anthropology: Internationales Jahrbuch für

Rechtsanthropologie 5:1-23

Kuykendall, Ralph S. 1947. The Hawaiian kingdom: 1778-1854. Honolulu: University of Hawai'i Press

Kuykendall, Ralph S. 1953. The Hawaiian kingdom: 1854-1974. Honolulu: University of Hawai'i Press

Kuykendall, Ralph S. 1967. The Hawaiian kingdom: 1874-1893. Honolulu: University of Hawai'i Press

Kymlicka, Will u. Ian Shapiro (Hg.). 1997. Ethnicity and group rights. (Nomos 24. Yearbook of the American Society for Political and Legal Philosophy). New York: New York University Press

La Croix, Sumner J. u. Christopher Grandy. 1997. The political instability of reciprocal trade and the overthrow of the Hawaiian Kingdom. Journal of Economic History 57:161-189

Laenui, Pōkā. 1992. „The Hawaiian report““. In: I.A.H.A. 1992:1-22

Laenui, Pōkā. 1993. A primer on international activities as related to the quest for Hawaiian sovereignty. Wai'anae: Institute for the Advancement of Hawaiian Affairs (Internet: www.opihi.com/sovereignty/internat_law.txt [7.9.1998])

Laenui, Pōkā. 1994. Straight talk on Hawaiian sovereignty. Wai'anae: Institute for the Advancement of Hawaiian affaire

Laenui, Pōkā. 1996. Hawaiian sovereignty and the Native Hawaiian Vote.

(Internet: www.opihi.com/sovereignty/native.txt [28.8.1998])

Laenui, Pōkā. 1997a. Memorial. Adopted october 10, 1997 on the former grounds of Palace Square, Honolulu, Hawai 'i. (Internet: www.opihi.com/ sovereignty/memorial_1997.htm [28.8.1998])

Laenui, Pōkā. 1997b. Hawaiian loyalist rally committed to Hawaiian independence. (Internet: www.opihi.com/sovereignty/rally.htm [28.8.1998])

Lal, Brij V. u. Kate Fortune (Hg.). 2000. The Pacific islands: an encyclopedia. Honolulu: University of Hawai'i Press

Lâm, Maivân Clech. 1996. Indigenous Hawaiians’ options for selfdetermination under U.S. and international law. Law \& Anthropology: Internationales Jahrbuch für Rechtsanthropologie 8:200-11

Landgraf, Anne Kapulani. 1994. Nā Wahi Pana o Ko 'olau Poko. Honolulu: University of Hawai'i Press

Landgraf, Anne Kapulani. 1995. „Artist’s statement“. In: ReViewing paradise: contemporary photography from Hawai'i (27.04.-28.06. 1995;

Fotokopien), S. 9-10. Honolulu: The Honolulu Advertiser Gallery

Landye Bennett Blumstein. 2007. Alaska Native Claims Settlement Act Resource Center. (Internet: www.lbblawyers.com/ancsa.htm [10.03.2008]) 
Lee, Ann Feder. 1993. The Hawaii State Constitution: a reference guide.

(Reference guides to the state constitutions of the United States, 14). Westport: Greenwood Press

Lepowsky, Maria. 2004. „Indian revolts and cargo cults: ritual violence and revitalization in California and New Guinea. In: Harkin (Hg.) 2004:1-60

Lewis, George H. 1992. „Don’ go down Waikiki: social protest and popular music in Hawaii“. In: Reebee Garofalo (Hg.). Rockin’ the boat: mass music and mass movements (171-83). Boston: South End Press

Lewis, David. 1976. „Hokule“a“ follows the stars to Tahiti. National Geographic 150 (4):512-37

Lewis, George H. 1996. Beyond the reef: cultural constructions of Hawaii in mainland America, Australia and Japan. Journal of Popular Culture 30

(2):123-34

Lili‘uokalani. 1990. Hawaii’s story by Hawaii’s Queen. Honolulu: Mutual Publishing

Lind, Andrew W. 1967. Hawaii's people. Honolulu: University of Hawai‘ i Press

Lind, Andrew W. 1982a. Immigration to Hawai' i. Social Process in Hawaii 29:9-20

Linnekin, Jocelyn S. 1983. Defining tradition: variations on the Hawaiian identity. American Anthropologist 10 (2):241-52

Linnekin, Jocelyn S. 1988. Children of the land: exchange and status in a Hawaiian community. New Brunswick: Rutgers University Press

Linnekin, Jocelyn S. 1990a. Review: The Peopling of Hawai'i (Eleanor C. Nordyke). The Hawaiian Journal of History 24:206-7

Linnekin, Jocelyn S. 1990b. „The politics of culture in the Pacific“. In: Linnekin u. Poyer (Hg.) 1990:149-73

Linnekin, Jocelyn S. 1992. On the theory and politics of cultural construction in the Pacific. Oceania 62 (4):249-63

Linnekin, Jocelyn S. u. Lyn Poyer (Hg.). 1990. Cultural identity and ethnicity in the Pacific. Honolulu: University of Hawai'i Press

Little, Stephen u. Peter Ruthenberg (Hg.). 2006. Life in the Pacific of the 1700s: the Cook/Forster Collection of the Georg August University of Göttingen. Honolulu: Honolulu Academy of Arts

Lucas, Paul F. Nahoa. 1995. A dictionary of Hawaiian legal land-terms. Honolulu: Native Hawaiian Legal Corporation; University of Hawai'i Committee for the Preservation and Study of Hawaiian Language, Art and Culture MacPherson Furrer, Roger (Hg.). 1993. He Alo Ä He Alo: Hawaiian voices on sovereignty. Honolulu: American Friends Service Committee-Hawai‘i MacKenzie, Melody Kapilialoha. 1991a. „Introduction“. In: MacKenzie (Hg.) 1991d:ix-xi 
MacKenzie, Melody Kapilialoha. 1991b. „Self-Determination and SelfGovernance“. In: MacKenzie (Hg.) 1991 d:77-104

MacKenzie, Melody Kapilialoha. 1991c. „Historical background“. In: MacKenzie (Hg.) 1991:3-25

MacKenzie, Melody Kapilialoha (Hg.). 1991d. Native Hawaiian rights handbook. Honolulu: Native Hawaiian Legal Corporation

Malo, David. 1992. Hawaiian Antiquities (Moolelo Hawaii). Bernice P. Bishop Museum Special Publication 2. Honolulu: Bishop Museum Press

MAMo. 2006. Event \& buyers guide: first annual maoli arts month, march 3, april 2, 2006. (Broschüre)

Manley, Sharla. 1999. Indigenous writes: a new literary journal makes Hawaiian history. Honolulu Weekly (31.3.-6.4.1999):11

Mardfin, Jean Kadooka. 1994. Examining the idea of nationhood for the native Hawaiian people. (LRB Report No. 6). Honolulu: Legislative Reference Bureau

Mast, Robert H. u. Anne B. Mast. 1996. Autobiography of protest in Hawai ‘i. Honolulu: University of Hawai'i Press

Matsuda, Mari J. 1988. Native custom and official law in Hawaii. Law \& Anthropology: international yearbook for legal anthropology 3:135-46

Maunakea, Katherine. 1993. „An interview with Katherine Maunakea“. In: MacPherson Furrer 1993:25-32

McGregor-Alegado, Davianna. 1979. Hawaiian resistance, 1887-1889. (Thesis for the degree of Master of Arts, University of Hawai'i, Pacific Islands Studies)

McGregor-Alegado, Davianna. 1980. Hawaiians: organizing in the 1970s. Amerasia 7 (2):29-55

McGregor, Davianna Pōmaika‘i. 1989. „Ho‘omauke ea o ka lahui Hawai‘i: the perpetuation of the Hawaiian people“. In: Michael C. Howard (Hg.) Ethnicity and nation-building in the Pacific (74-97). Tokio: The United Nations University

McGregor, Davianna Pōmaika'i. 1990. „'Āina Ho'opulapula: Hawaiian homesteading“. The Hawaiian Journal of History 24:1-38

McGregor, Davianna Pōmaika'i. 1994. Sovereignty: Hawaiians and locals. Honolulu: D.P. McGregor (Kopie: UH Mānoa, Hamilton Library)

McGregor, Davianna Pōmaika'i. 2007. Nā Kua ăina: living Hawaiian culture. Honolulu: University of Hawai'i Press

Melbourne, Hineani. 1995. Maori sovereignty: the Maori perspective. Auckland: Hodder Moa Beckett

Meller, Norman. 1992. „Policy control: institutionalized centralization in the fiftieth state“. In: Smith u. Pratt (Hg.) 1992:13-28 
Merry, Sally Engle. 1997. „Legal pluralism and transnational culture: the Ka Ho‘okolokolonui Kanaka Maoli Tribunal, Hawai‘i, 1993“. in: Wilson (Hg.) 1997:28-48

Merry, Sally Engle. 2000. Colonizing Hawai ' $i$ : the cultural power of law. (Princeton Studies in Culture/Power/History) Princeton: Princeton University Press

Merry, Sally Engle u. Donald L. Brenneis (Hg.). 2003. Law \& empire in the Pacific: Fiji and Hawai' $i$. Santa Fe: School of American Research Press

Mihesuah, Devon A. (Hg.). 2000. Repatriation reader: who owns American Indian remains? Lincoln: University of Nebraska Press

Mills, Peter R. 2003. Neo in Oceania: foreign vessels owned by Hawaiian chiefs before 1830. The Journal of Pacific History 38 (1):53-67

Milner, Neal. 2006. Home, homelessness, and homeland in the Kalama Valley: re-imagining a Hawaiian nation through a property dispute. The Hawaiian Journal of History 40:149-76

Minerbi, Luciano. 1994. Native Hawaiian struggles and events. Social Process in Hawaii 35:1-14

Morse, Marcia. 1997. Ho'okū' ${ }^{`} \bar{e}$ : art, resistance and nature - all can return new life to seemingly barren land. Honolulu Weekly 7 (20):17

Murakami, Alan. 1991. „The Hawaiian Homes Commission Act“. In: MacKenzie (Hg.) 1991 d:43-76

Mykkänen, Juri. 2003. Inventing politics: a new political anthropology of the Hawaiian kingdom. Honolulu: University of Hawai'i Press

Nagel, Joane. 1993. Ethnic nationalism: politics, ideology, and the world order. International Journal of Comparative Sociology 34 (1-2):103-12

Nagel, Joane .1995. American Indian ethnic renewal: politics and the resurgence of identity. American Sociological Review 60(6):947-65

NAGPRA Review Committee. 2003. Minutes. Native American Graves Protection and Repatriation Review Committee twenty-fifth meeting: may 9 and 10, 2003, St. Paul, Minnesota. (Internet: www.nps.gov/history/Nagpra/REVIEW/meetings/RMS025.pdf [25.5.2009]) Nakata, Bob. 1999. „The struggles of the Waiāhole-Waikāne Community Association“. In: Aoudé (Hg.) 1999:60-73

Nation of Hawai'i. 1994. Proclamation of restoration of the independence of the sovereign Nation State of Hawai 'i, January 16, 1994. (Internet: hawaiination.org/proclamall.html [17.9.1998])

Nation of Hawai'i. 1995. Hawai 'i Constitution. 16 January 1995. (Internet: hawaii-nation.org/constitution.html [17.9.1998])

Nation of Hawai'i. 1998a. Present status of Hawaii. (Internet: hawaiination.org/overview.html [15.9.1998]) 
Nation of Hawaici. 1998b. Chronology. Critical events in the process of self.determination, restoration, and development. (Internet: hawaiination.org/chrono.html [17.9.1998])

Nation of Hawai‘i. 1998c. Pu'uhonua o Waimanalo. (Internet: www.hawaiination.org/puuhonua.html [30.4.1998])

National Museum of Australia. 2006. Cook's Pacific encounters: the CookForster Collection of the Georg-August University of Göttingen. Canberra: National Museum of Australia Press

Native Hawaiians Study Commission. 1983a. Report on the culture, needs and concerns of Native Hawaiians. Final report. Volume I. (Internet: www.eric.ed.gov/ERICDocs/data/ericdocs2sqV/content_storage_01/0000019b/8 0/30/2c/5e.pdf [12.03.2008])

Native Hawaiians Study Commission. 1983b. Report on the culture, needs and concerns of Native Hawaiians. Final report. Volume II. Claims of conscience: a dissenting study of the clture, needs and concerns of Native Hawaiians. (Internet: www.eric.ed.gov/ERICDocs/data/ericdocs2sql/ content_storage_01/0000019b/80/30/2c/61.pdf [12.03.2008])

Newlands, Francis. 1898. To Provide for Annexing the Hawaiian Islands to the United States (Internet: www.hawaii-nation.org/annexation.html [9.02.2009])

NHCAP. 1991. The Native Hawaiian Culture and Arts Program: annual reports 1990-1991. Honolulu: The Native Hawaiian Culture and Arts Program, Bishop Museum

Niethammer, Lutz. 2000. Kollektive Identität: heimliche Quellen einer unheimlichen Konjunktur. Reinbek: Rowohlt

Nobles, Melissa. 2008. The politics of official apologies. Cambridge: Cambridge University Press

Nordyke, Eleanor. 1989a. The peopling of Hawai ' $i$. Honolulu:University of Hawai'i Press

Nordyke, Eleanor C. 1989b. „Comment“. In: Stannard 1989:105-13

Nordyke, Eleanor C. u. Martha M. Noyes. 1993. 'Kaulana nā pua': a voice for sovereignty. The Hawaiian Journal of History 27:27-43

Novak, Viveca. 2004. „Hawai'i's dirty secret: the continuing denial of native Hawaiian land rights“. In: Churchill u. Venne (Hg.) 2004:191-208 [Erstveröffentlichung in: Common Cause Magazine Nov./Dec. 1989]

Obeyesekere, Ganath. 1993. The apotheosis of Captain Cook: European mythmaking in the Pacific. Princeton: Princeton University Press

OHA. 1989. Blueprint for native Hawaiian entitlements. Honolulu: Office of Hawaiian Affairs

OHA. 1993a. ,,...And the earth shall rise!": Hoolāhui - to rebuild a nation. A special supplement by the Office of Hawaiian Affairs, January 17, 1993. Honolulu: OHA 
OHA. 1993b. 'Onipa 'a Centennial Observance 1893-1993 (Programmheft). Honolulu: Office of Hawaiian Affairs

OHA. 1994a. Ho'olāhui hou: rebuilding a nation. Ka Wai Ola o OHA, Dec. 1994, 11(12):10-11

OHA. 1994b. 'Onipa 'a: five days in the history of the Hawaiian nation. Honolulu: Office of Hawaiian Affairs

OHA. 1996. Native Hawaiian Data Book 1996. Honolulu: Office of Hawaiian Affairs

OHA. 1997. Annual report for the Office of Hawaiian Affairs: fiscal year 1996 (July 1995-June 1996). Honolulu: Office of Hawaiian Affairs

OHA. 1998. OHA background. (Internet: oha.org/info/created.html [22.9.1998])

OHA. 2002. Native Hawaiian Data Book 2002: Statistical profile of the Hawaiian population in Hawai ' $i$. Honolulu: Office of Hawaiian Affairs (Internet: www.oha.org/pdf/databook_6_02.pdf [12.04.2008])

OHA. 2006. Native Hawaiian Databook: demographics. (Internet: www.oha.org/pdf/databook/2006/DataBook2006Demographics.pdf [12.03.2008])

OHA. 2007. Correcting the record: the U.S. Commission on Civil Rights and justice for Native Hawaiians. Honolulu: Office of Hawaiian Affairs (Internet: www.oha.org/images/stories/071113correcting.pdf [23.02.2009])

OHA. 2008a. Kau Inoa: to build a nation. (Internet: www.kauinoa.org/info.php [25.02.2009])

OHA. 2008b. Obama announces support for Akaka Bill (Internet:

$w w$ w.oha.org/index.php?option $=$ com_content\&task $=v i e w \& i d=540 \&$ Itemid $=2$ 24 [24.02.2009])

Okamura, Jonathan Y. 1980. Aloha Kanaka Me Ke Aloha 'Aina: local culture and society in Hawaii. Amerasia 7 (2):119-37

Oliver, Douglas L. 1989. Oceania: the native cultures of Australia and the Pacific Islands. Honolulu: University of Hawai'i Press

Olzak, Susan. 1983. Contemporary ethnic mobilization. Annual review of sociology 9:355-74

OMB. 1998. Revisions to the Standards for the Classification of Federal Data on Race and Ethicity. Executive Office of the President, Office of Management and Budget (OMB), Office of Information and Regulatory Affairs. (Internet: www.whitehouse.gov/WH/EOP/OMB/html/fedreg.html [17.9.1998])

Ortner, Sherry B. 1973. On key symbols. American Anthropologist 75 (5):1338-46

Orywal, Erwin u. Katharina Hackstein. 1993. „Ethnizität: die Konstruktion ethnischer Wirklichkeiten“. In: Thomas Schweitzer et al. (Hg.). Handbuch der Ethnologie. Berlin: Reimer; S. 593-609 
Oshiro, Lisa Cami. 1995. „Recognizing Nā Kānaka Maoli's right to selfdetermination“. New Mexico Law Review 25(1):65-96

Osorio, Jonathan Kamakawiwo ‘ole. 2001. ,What kine Hawaiian are you?’ A mo'olelo about nationhood, race, history, and the contemporary sovereignty movement in Hawai'i. The Contemporary Pacific 13(2):359-79

Osorio, Jonathan Kamakawiwo'ole. 2002. Dismembering lāhui: a history of the Hawaiian nation to 1887. Honolulu: University of Hawai'i Press

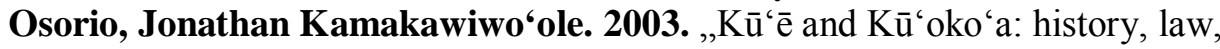
and other faiths“. In: Merry u. Brenneis (Hg.) 2003:213-37

Otaguro, Janice. 1992. Beat the drum slowly. Honolulu June 1992:31-3, 50-2

Paddock, Lisa. 1996. Facts about the Supreme Court of the United States.

New York: H.W. Wilson

Parker, Linda S. 1989. Native American estate: the struggle over Indian and Hawaiian lands. Honolulu: University of Hawai' i Press

PCA. 2001. Award: Larson v Hawaiian Kingdom. (Internet: www.pcacpa.org/upload/files/LHKAward.PDF [28.5.2007])

PCA. 2007. Permanent Court of Arbitration/Court permanente d'Arbitrage (Website). (Internet: www.pca-cpa.org [17.02.2009])

PIANGO. 1998. PIANGO Monthly v 1.1. (Internet: www.piango.org/docs/ Publications/monthly-1-1.html [30.05.2008])

Pono Kaulike. 1995. Self-determination: symposium for sovereignty. Honolulu: Pono Kaulike, Inc.

Pratt, Richard C. u. Zachary Smith. 2000. Hawai 'i politics and government: an american state in a Pacific world. Lincoln: University of Nebraska Press Prejean, Nakoa. 1994. „Kānaka Maoli and the United Nations“. In: Hasager u. Friedman (Hg.). 1994:276-85

Protect Kaho'olawe ‘Ohana. 1998. Kaho'olawe: Mo'olelo ‘Aina. (Internet: www.kahoolawe.org/moolelo.html [31.8.1998])

Protect Kaho'olawe 'Ohana. 2009. Vision of the Protect Kaho'olawe 'Ohana (Internet: $w w w . k a h o o l a w e . o r g / h o m e /$ ?page_id=2 \{21.01.2009])

Pukui, Mary Kawena u. Samuel H. Elbert. 1986. Hawaiian dictionary. Honolulu: University of Hawai'i Press

Pukui, Mary Kawena. 1997 [1983]. 'Ōlelo No 'eau: Hawaiian proverbs and sayings. Bernice P. Bishop Museum Special Publication No. 71. Honolulu:

Bishop Museum Press

Pukui, Mary Kawena, Samuel H. Elbert u. Esther T. Mookini. 1974. Place names of Hawaii. Honolulu: University of Hawai‘i Press

Pukui, Mary Kawena, E.W. Haertig u. Catherine A. Lee. 1983 (1972).

Nānā $i$ ke kumu: look to the source (vol. 1). Honolulu: Hui Hānai/Queen

Lili‘uokalani Children's Center

Pūnana Leo. 1998. History of the Pünana Leo. (Internet:

www.olelo.hawaii.edu/OP/orgs/apl/story.html [21.5.98]) 
Polynesian Voyaging Society. 1997. Newsletter - Ho'oilo 1997 (Internet: leahi.kcc.hawaii.edu/org/pvs/statewide97.html [30.4.1998])

Rampell, Ed. 1992a. Hawaiian nationalism - the wild card. Pacific Islands Monthly Nov. 1992:18-21

Rampell, Ed. 1992b. The coup against sovereignty: a historical perspective. Pacific Islands Monthly Nov. 1992:22-3

Rampell, Ed. 1993. America on trial. Pacific Islands Monthly Oct. 1993:26-7

Rampell, Ed. 1994a. Hawaiian lands, Hawaiian hands. Pacific Islands Monthly Feb. 1993:20-1

Rampell, Ed. 1994b. $101^{\text {st }}$ anniversary of overthrow observed. Pacific Islands Monthly March 1994:18

Rampell, Ed. 1995. The state of the Hawaiian Movement. Pacific Islands Monthly July 1995:14-5

Ralston, Caroline. 1984. Hawaii 1778-1854: some aspects of Maka'ainana response to rapid cultural change. Journal of Pacific History 19 (1):21-40

Rees, Robert. 1994. The politics of sovereignty. Honolulu weekly 10.8.1994, 4 (32):4-6

Rees, Robert. 1995. „Learning to love Bishop Estate“. Honolulu Weekly 1.2.1995:4-6

Rohrer, Judy. 2006. ,Got race?’ The production of Haole and the distortion of indigeneity in the Rice decision. The Contemporary Pacific 18(1):1-31

Root, Mary P.P. (Hg.). 1992. Racially mixed people in America. Newbury Park CA: Sage

Roth, Randall W. 1992. The price of paradise. Honolulu: Mutual Publishing

Russ, William Adam. 1992. The Hawaiian Republic (1894-98): and its struggle to win annexation. Selinsgrove: Susquehanna University Press (1961) SAC. 1992. Preliminary Report of the Sovereignty Advisory Council of the State of Hawaii to the Sixteenth Legislature, January 1992. Honolulu: Sovereignty Advisory Council

Sahlins, Marshall. 1958. Social Stratification in Polynesia. Seattle: University of Washington Press

Sahlins, Marshall. 1985. Islands of history. Chicago: University of Chicago Press

Sahlins, Marshall. 1990. „The political economy of grandeur in Hawaii from 1810 to 1830“. In: Emiko Ohnuki-Tierney (Hg.). Culture through time: anthropological approaches (26-56). Stanford: Stanford University Press

Sahlins, Marshall. 1992. Anahulu: the anthropology of history in the Kingdom of Hawaii. Vol. 1: Historical ethnography. Chicago: Chicago University Press Sahlins, Marshall. 1995. How natives think, about Captain Cook for example. Chicago: Chicago University Press

Salmond, Anne. 2004. The trial of the cannibal dog: Captain Cook in the South Seas. London: Penguin 
Sanburn, Curt. 1993. Keeping the lid on. Honolulu Weekly 3 (4):3 Sanburn, Curt. 1995. „Waiahole: the triumph of community“. Honolulu Weekly 25.1.1995:4-6

Schmidtke, Oliver u. Carlo E. Ruzza. 1993. Regionalistischer Protest als ,Life Politics': die Formierung einer sozialen Bewegung: die Lega Lombarda. Soziale Welt 44 (1):5-29

Schmitt, Robert C. 1971. New estimates of the pre-censal population of Hawaii. Journal of the Polynesian Society 80(2):237-43

Schmitt, Robert C. 1977. Historical statistics of Hawaii. Honolulu: University of Hawai'i Press

Schmitt, Robert C. 1989. „Comment“. In: Stannard 1989:114-21

Schütz, Albert J. 1994. The voices of Eden: a history of Hawaiian language studies. Honolulu: University of Hawai'i Press

Schweizer, Niklaus R. 2005. Turning tide: the ebb and flow of Hawaiian nationality. Bern: Peter Lang

Senate Joint Resolution. 1993. To acknowledge the 100th anniversary of the January 17, 1893 overthrow of the Kingdom of Hawaii, and to offer an apology to Native Hawaiians on behalf of the United States for the overthrow of the Kingdom of Hawaii. Senate Joint Resolution 19. Cal. No. 185; 103d Congress 1st Session

Sharp, Andrew. 1997. Justice and the Māori: the philosophy and practice of Mãori claims in New Zealand since the 1970s. Auckland: Oxford University Press

Silva, Noenoe K. 2004. Aloha betrayed: native Hawaiian resistance to American colonialism. Durham: Duke University Press

Simpson, MacKinnon. 1995. A voyaging heritage reborn. Spirit of Aloha 20 (4):6-9, 71

Sinnex, Cecil. 1993. Reflecting on the Overthrow of the Monarchy. MidWeek 9 (27):A8-A9

Smith, Anthony D. 1994. „The politics of culture“. In: Tim Ingold (Hg.). Companion Encyclopedia of Anthropology. London: Routledge, S. 706-33

Smith, Zachary A. u. Richard C. Pratt (Hg.). 1992. Politics and public policy in Hawai 'i. Albany: State University of New York Press

Stagner, Ishmael. 1985. Hula! (Pamphlets Polynesia). Lā'ie: Brigham Young University (Institute for Polynesian Studies)

Stannard, David E. 1989. Before the horror: the population of Hawai' $i$ on the eve of Western contact. Honolulu: Social Science Research Institute

Stannard, David E. 2005. Honor killing: how the infamous „Massie Affair“ transformed Hawai ' $i$. New York: Viking

State Foundation on Culture and the Arts. 1994. Annual report 1993-1994.

Honolulu: State Foundation on Culture and the Arts 
State of Hawaii. 1993a. Senate Resolution No. 18. The Senate, Seventeenth Legislature. State of Hawaii

State of Hawaii. 1993b. House Resolution No. 27 H.D.1. House of Representatives, Seventeenth Legislature. State of Hawaii

State of Hawaii. 1994a. State of Hawaii data book 1993-94: a statistical abstract. Honolulu: Dept. of Business, Economic Development and Tourism State of Hawaii 1994b. Senate Resolution No. 209 S.D.1. The Senate, Seventeenth Legislature. State of Hawaii

State of Hawaii. 1996. State of Hawaii data book 1996: a statistical abstract. Honolulu: Dept. of Business, Economic Development and Tourism

State of Hawaii. 1997. Hawaii Legislator's Handbook. Honolulu: Legislative Reference Bureau

State of Hawaii. 2006. State of Hawaii data book 2006. (Internet:

www.hawaii.gov/dbedt/info/economic/databook/db2006 [03.03.2008])

Stillman, Amy Ku'uleialoha. 1989. History reinterpreted in song: the case of the Hawaiian counterrevolution. The Hawaiian Journal of History 23:1-30 Stillman, Amy Ku'uleialoha. 2006. Modern Hula: a crucible of Hawaiian tradition. (Unveröffentlichter Vortrag, gehalten am 25.02.2006 auf dem Symposium „Changing contexts - shifting meanings“, Honolulu Academy of Arts, 23.-26.02.2006)

Stop Akaka. 2009. Why is the Akaka Bill so dangerous? (Internet: www.stopakakabill.com/Why_Is_It_So_Dangerous.html [23.02.2009])

Sturm, Circe. 2002. Blood politics: race, culture, and identity in the Cherokee Nation of Oklahoma. Berkeley: University of California Press

Sullivan, Robert. 2004. Review: Night is a sharkskin drum, by Haunani-Kay Trask. The Contemporary Pacific 16 (2):436-8

Sumida, Stephen Hiro. 1991. And the view from the shore: literary traditions of Hawai ' $i$. Seattle: University of Washington Press

Tachihata, Chieko. 1994. Resources: Hawaiian sovereignty. The Contemporary Pacific 5 (1):201-10

Takaki, Ronald. 1983. Pau Hana: plantation life and labor in Hawaii 18351920. Honolulu: University of Hawai'i Press

Tatar, Elizabeth. 1987. Strains of change: the impact of tourism on Hawaiian music. Bishop Museum Special Publication 78. Honolulu: Bishop Museum Tatibouet, Andre S. 1991. „Letter of transmittal“. In: Hawaii Advisory Committee 1991

Terrell, John. 1986. Prehistory in the Pacific Islands. Cambridge: Cambridge University Press

Theroux, Paul. 2002. Voices: Clay Bertelmann, Big Island. National Geographic 202 (6):12-5

Thomas, Nicholas. 1995. Oceanic Art. London: Thames and Hudson 
Thomas, Nicholas. 2003. Cook: the extraordinary voyages of Captain James Cook. New York: Walker \& Company

Tiffany, Martin. 1993. Hawaiian renaissance. Pacific Islands Monthly March 1993:21

Toivanen, Reetta. 2001. Minderheitenrechte als Identitätsressource: Die Sorben in Deutschland und die Saamen in Finnland. (Zeithorizonte 6). Münster: LIT

Trask, Haunani-Kay. 1987. The birth of the modern Hawaiian movement: Kalama Valley, O‘ahu. The Hawaiian Journal of History 21:126-53

Trask, Haunani-Kay. 1993. From a native daughter: colonialism and sovereignty in Hawai 'i. Monroe: Common Courage Press

Trask, Haunani-Kay. 1994a. „Kūpa'a 'Āina: Native Hawaiian nationalism in Hawai ‘i“. In: Hasager u. Friedman (Hg.). 1994:15-32

Trask, Haunani-Kay. 1994b. Light in the crevice never seen. Corvallis, Oregon: Calyx Books

Trask, Haunani-Kay. 1999. From a native daughter: colonialism and sovereignty in Hawai ' $i$, revised ed. Honolulu: University of Hawai'i Press

Trask, Haunani-Kay. 2000. Settlers of Color and ,Immigrant' Hegemony:

Locals in Hawai'i. Amerasia Journal 26 (2):1-24

Trask, Haunani-Kay. 2002. Night is a sharkskin drum. Honolulu: University of Hawai'i Press

Trask, Haunani-Kay. 2008. Evictions of beach villagers at Sand Island, Honolulu, 1980. (Internet: www.haunani-

kaytrask.com/gallery/eviction/index.htm [12.03.2008])

Trask, Haunani-Kay u. Mililani Trask. 1992. The Aloha Industry. Cultural Survival Quarterly 16 (4):50-1

Trask, Mililani. 1993. „Interview with Mililani Trask“. In: MacPherson Furrer (Hg.). 1993:113-24

Trask, Mililani. 1999. Rice, racism and reactionary trends. Ka Wai Ola o OHA Mei (May):8

Trope, Jack F. u. Walter R. Echo-Hawk. 2000. „The Native American Graves Protection and Repatriation Act: background and legislative history“. In: Mihesuah (Hg.) 2000:123-68

Tummons, Patricia. 1996. „Waiahole water wars“. Honolulu Weekly 25.9.1996:1, 4-6

Twain, Mark. 1990. Mark Twain in Hawaii: Roughing it in the Sandwich Islands; Hawaii in the 1860's (mit einem Vorwort von A. Grove Day). Honolulu: Mutual Publishing

United Nations. 1973. Charter of the United Nations (Internet: www.un.org/en/documents/charter/index.shtml [16.09.2012])

United Nations. 2002. Trust and non-self-governing territories, 1945-1999.

(Internet: www.un.org./Depts/dpi/decolonization/trust2.html [03.02.2008]) 
United Nations. 2007. Resolution adopted by the General Assembly: United Nations declaration on the rights of indigenous peoples. (Internet: www.un.org/esa/socdev/unpfil/documents/DRIPS_en.pdf [20.02.2009]) United States. 2000. Supreme Court of the United States. Syllabus. Rice v. Cayetano, governor of Hawaii: certiorari to the United States Court of Appeals for the Ninth Circuit. (Internet: www.supct.law.cornell.edu/supct/html/98818.zs.html [11.05.2004])

University of Alaska Anchorage Justice Center. 2001. ANCSA and Alaska Native Land. (Internet: justice.uaa.alaska.edu/rlinks/natives/ak_ancsa.html [10.03.2008])

U.S. Bureau of the Census. 1991. Statistical abstract of the United States: 1991 (111th ed.). Washington: U.S. Dept. of Commerce U.S. Bureau of the Census. 1995. Statistical abstract of the United States: 1995 (115th ed.). Washington: U.S. Dept. of Commerce U.S. Census Bureau. 2000. Census 2000. Tabe DP-1. Profile of general demographic characteristics. Geograhic area: Hawaii (Internet: censtats.census.gov/data/HI/04015.pdf [15.04.2008])

U.S. Congress. 1993. Senate Joint Resolution 19. To acknowledge the 100th anniversary of the January 17, 1893 overthrow of the Kingdom of Hawaii, and to offer an apology to Native Hawaiians on behalf of the United States for the overthrow... (Enrolled as agreed to or passed by both House and Senate) (Internet: thomas.loc.gov/cgi-bin/query/D?c103:2:./temp/ c103YYzB9H::

[17.02.09])

U.S. Congress. 2007. S. 310 (Report No. 110-260). 110th Congress, 2nd session. To express the policy of the United States regarding the United States relationship with Native Hawaiians... (Reported by Mr. Dogan, without amendment) (Internet: frwebgate.access.gpo.gov/cgi-

bin/getdoc.cgi?dbname=110_cong_bills\&docid=f:s320r.s.txt.pdf [23.02.2009]) U.S. Department of the Interior. 2000. From mauka to makai: the river of justice must flow freely. (Draft report on the reconciliation process between the Federal Government and Native Hawaiians). Washington: Department of the Interior, Department of Justice

U.S. Government. 1994. Public papers of the presidents of the United States: William J. Clinton 1993, book 1. Washington: US Govt. Printing Office U.S. Government. 1959. An Act to Provide for the Admission of the State of Hawaii into the Union (Internet:

www.capitol.hawaii.gov/hrscurrent/Vol01_Ch0001-0042F/04-Adm/ADM-.htm [9.02.2009])

Valeri, Valerio. 1972. Le fonctionnement du système des rangs à Hawaii. L'Homme 12 (1):29-66

Ward, Deborah L. 1995. Kapa re-emerges as art and potential industry. Ka Wai Ola o OHA Mei (May):11 
Washburn, Wilcomb E. 1996. „The Native American Renaissance, 19601995“. In: Bruce G. Trigger u. Wilcomb E. Washburn (Hg.). The Cambridge history of the native peoples of the Americas, vol.1. Cambridge: Cambridge University Press, S. 401-69

Webster, Yehudi. 1992. The racialization of America. New York: St. Martin's Press

Wendt, Albert, Reina Whaitiri u. Robert Sullivan (Hg.). 2003. Whetu

Moana: contemporary Polynesian poems in English. Honolulu: University of Hawai'i Press

West, Barbara A. Encyclopedia of the peoples of Asia and Oceania. New York: Infobase Publishing

Whitehead, John S. 1993. The anti-statehood movement and the legacy of Alice Kamokila Campbell. The Hawaiian Journal of History 27:43-63

Whitney, Scott. 2000. The museum and the cave. Honolulu May:29-32, 10611

Wilcox, Carol. 1996. Sugar water: Hawaii's plantation ditches. Honolulu: University of Hawai'i Press

Wilson, Richard A. (Hg.). 1997. Human rights, culture and context: anthropological perspectives. London: Pluto Press

Wilson, Robert. 2000. Reimagining the American Pacific. Durham: Duke University Press

Wilson, Terry P. 1992. „Blood quantum: Native American mixed bloods“. In: Root (Hg.) 1992:108-25

Winduo, Steven. 2003. Review: Kalahele, by Imaikalani Kalahele. The Contemporary Pacific 15 (2):507-10

Wong, Sterling Kini. 2006. Hawaiian made. Ka Wai Ola o OHA 23 (2):12

Wong-Wilson, Noe Noe. 2005. A conversation with Mililani Trask. The Contemporary Pacific 17(1):142-56

Wood, Houston. 1999. Displacing Natives: the rhetorical production of

Hawai 'i. Lanham: Rowman \& Littlefield

Young, Kanalu G.T. 1995. Political Reviews: Hawaiian issues. The Contemporary Pacific 7 (1):148-52

Zack, Naomi. 1993. Race and mixed race. Philadelphia: Temple University Press

Zack, Naomi. 1995a. „Introduction“. In: Zack (Hg.). 1995 b:Xv-XXv

Zack, Naomi (Hg.). 1995b. American mixed race: the culture of microdiversity. Lanham: Rowman \& Littlefield

Honolulu Star-Bulletin. www.starbulletin.com

Honolulu Advertiser. www.advertiser.com

Honolulu Weekly. www.honoluluweekly.com

Ka Wai Ola o OHA. www.oha.org 


\section{Versicherung}

Ich versichere, dass ich die eingereichte Dissertation „Auf der Suche nach der Hawaiischen Nation: Autonomiepolitik und kulturelle Revitalisierung im USBundesstaat Hawai'i““ selbständig und ohne unerlaubte Hilfsmittel verfasst habe. Anderer als der von mir angegebenen Hilfsmittel und Schriften habe ich mich nicht bedient. Alle wörtlich oder sinngemäß den Schriften anderer entnommenen Stellen habe ich kenntlich gemacht.

\section{Ulrich Menter}

Göttingen, 4. Juni 2009 